\title{
Characteristics of a Multi-functional Device and Capillary Flow of Refrigeration Systems
}

by

\author{
Ying Chen (陈 漟) \\ B. Eng., M. Eng.
}

Submitted in partial fulfillment of the requirements for the degree of

\section{Doctor of Philosophy}

in Mechanical Engineering

Ottawa-Carleton Institute for Mechanical and Aerospace Engineering Carleton University

Ottawa, Ontario, Canada

(C) Copyright by Ying Chen, 2007 


$\begin{array}{ll}\begin{array}{l}\text { Library and } \\ \text { Archives Canada }\end{array} & \begin{array}{l}\text { Bibliothèque et } \\ \text { Archives Canada }\end{array} \\ \begin{array}{l}\text { Published Heritage } \\ \text { Branch }\end{array} & \begin{array}{l}\text { Direction du } \\ \text { Patrimoine de l'édition }\end{array} \\ \begin{array}{l}\text { 395 Wellington Street } \\ \text { Ottawa ON K1A ON4 }\end{array} & \begin{array}{l}\text { 395, rue Wellington } \\ \text { Ottawa ON K1A ON4 } \\ \text { Canada }\end{array}\end{array}$

Your file Votre référence ISBN: 978-0-494-27091-2 Our file Notre référence ISBN: 978-0-494-27091-2

NOTICE:

The author has granted a nonexclusive license allowing Library and Archives Canada to reproduce, publish, archive, preserve, conserve, communicate to the public by telecommunication or on the Internet, loan, distribute and sell theses worldwide, for commercial or noncommercial purposes, in microform, paper, electronic and/or any other formats.

The author retains copyright ownership and moral rights in this thesis. Neither the thesis nor substantial extracts from it may be printed or otherwise reproduced without the author's permission.
AVIS:

L'auteur a accordé une licence non exclusive permettant à la Bibliothèque et Archives Canada de reproduire, publier, archiver, sauvegarder, conserver, transmettre au public par télécommunication ou par l'Internet, prêter, distribuer et vendre des thèses partout dans le monde, à des fins commerciales ou autres, sur support microforme, papier, électronique et/ou autres formats.

L'auteur conserve la propriété du droit d'auteur et des droits moraux qui protège cette thèse. $\mathrm{Ni}$ la thèse ni des extraits substantiels de celle-ci ne doivent être imprimés ou autrement reproduits sans son autorisation.
In compliance with the Canadian

Privacy Act some supporting forms may have been removed from this thesis.

While these forms may be included in the document page count, their removal does not represent any loss of content from the thesis.
Conformément à la loi canadienne sur la protection de la vie privée, quelques formulaires secondaires ont été enlevés de cette thèse.

Bien que ces formulaires aient inclus dans la pagination, il n'y aura aucun contenu manquant.

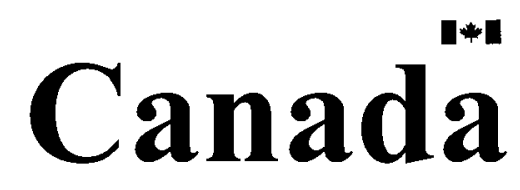




\section{Abstract}

A multifunctional accumulator-heat-exchanger-expander (AXE) device, which combines the functions of an accumulator, an internal heat exchanger, and an expander into one, is introduced into an automotive air-conditioning system to improve its performance while keeping the system compact. In such a device, non-adiabatic capillary flow plays an important role. The following work has been conducted to address the characteristics of flow as well as the device itself.

Two homogeneous models, ACAM and NACAM, have been successfully developed for adiabatic and non-adiabatic capillary flow, respectively. By developing a new correlation for the two-phase specific volume, they can handle a great range of mass flux for an arbitrary combination of parallel/counter flow and lateral/concentric heat exchangers. ACAM is a special case of NACAM where the thermal resistance across the tube wall is infinite. The prediction uncertainty is within $\pm 12.9 \%$ for adiabatic flow, $\pm 10.3 \%$ for lateral heat exchangers, and $\pm 20.0 \%$ for concentric heat exchangers.

To understand the influence of compressor speed on changes in pressures for a refrigeration system with and without an AXE, a series of experiments were conducted on an R134a-based test stand. The AXE was found to behave like a stabilizer, making the system less sensitive to the changes in compressor speed. Relative empirical correlations were developed with a deviation of less than $\pm 5.3 \%$. Oil effect is taken into account, and a "Quasi-Triangular Rule" is proposed to calculate the effectiveness of AXE without measured capillary outlet quality.

Further studies on carbon dioxide-based system are carried out. Heat transfer and fluid flow of a transcritical throttling process are investigated. A correlation that determines the optimum high pressure using the gas cooler outlet temperature, the effectiveness of internal heat exchanger, and 
the suction line inlet quality is developed. The prediction uncertainty is within $\pm 3.6 \%$.

The above work can be very helpful not only in understanding the capillary flow for various working conditions, but also in either designing or evaluating a currently used or next-generation refrigeration system. 


\section{Acknowledgements}

Years of endeavour that served to further the understanding of the characteristics of capillary flow formed this manuscript. This would not have been possible without the guidance and support of many people, to whom I would like to express my gratitude.

Thanks must first go to my thesis supervisor, Dr. Junjie Gu, for his enthusiastic participation and continuous advice. I consider myself very fortunate to have been able to work with him; he provided insight at key times while still allowing the project to develop in a directed-random-walk fashion.

The cooperation of Mr. Shujun Wang proved invaluable while in conducting the experiments on the R134a test stand over the course of two years. Also immeasurable was the help provided by Mr. Wei $\mathrm{Gu}$ during the development of the new $\mathrm{CO}_{2}$ experimental apparatus. The advice of Dr. Misheck Mwaba also helped me write better journal papers than I ever could have on my own.

I am also very grateful for Dr. Edgar A. Matida, and Dr. Mohammad Golriz for providing additional and valuable advice toward the clarification of theoretical models. Mr. Brian Crosland read the bulk of this manuscript, providing tremendous pertinent feedback. Lots of ideas also come from many other members in our research team through our regular group meetings.

Thanks also go to many members of the administrative and technical staff at the Department of Mechanical and Aerospace Engineering. Financial support from Materials and Manufacturing Ontario (MMO) is gratefully acknowledged.

Finally, I would especially like to thank my family, especially my wife, Yuan Liu. This 
achievement would not have been possible without their complete understanding and unconditional support.

Ottawa, Ontario

Ying Chen

March 30, 2007

Reproduced with permission of the copyright owner. Further reproduction prohibited without permission. 


\section{Contents}

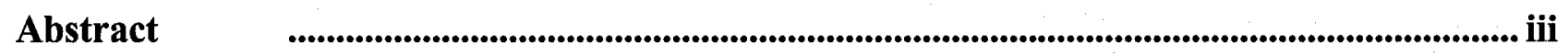

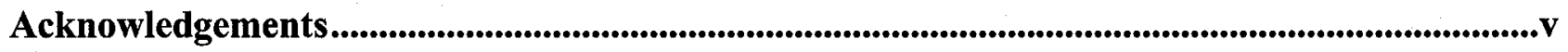

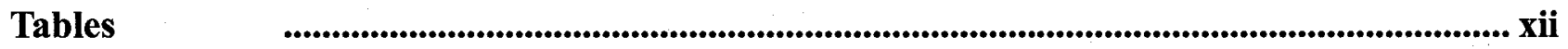

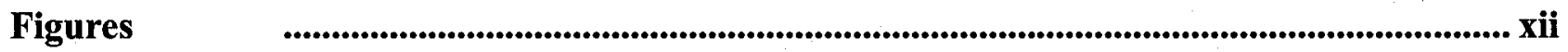

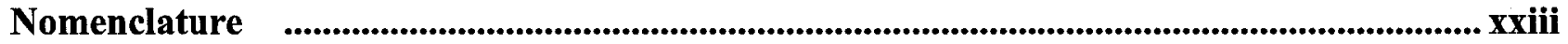

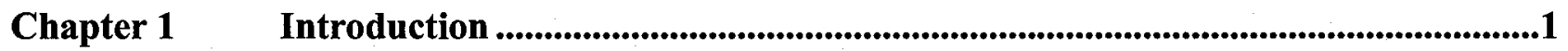

Classification of refrigeration systems................................................................... 4

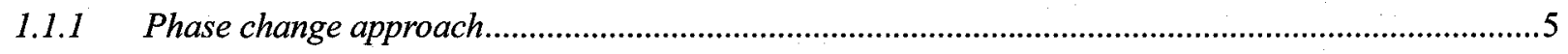

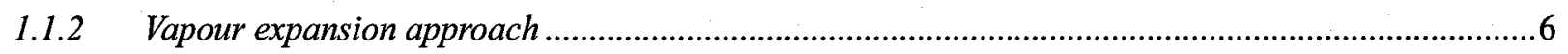

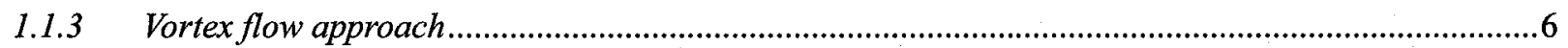

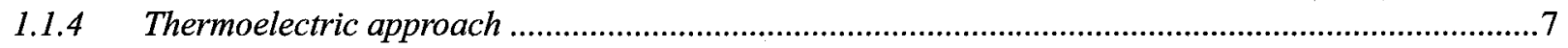

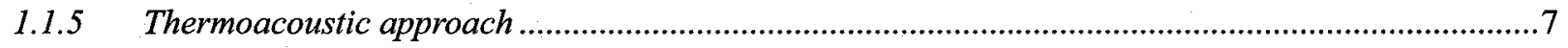

1.2 Two main driving forces — high efficiency and low environment impact ........................ 8

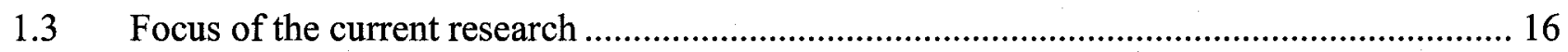

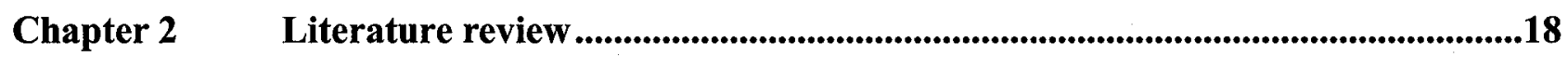

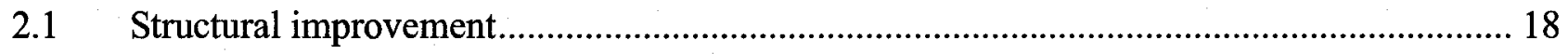

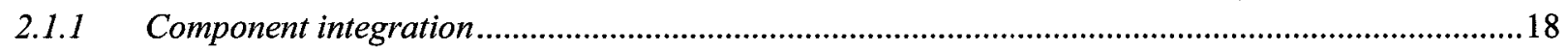

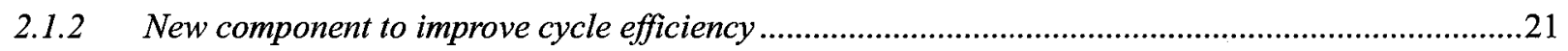

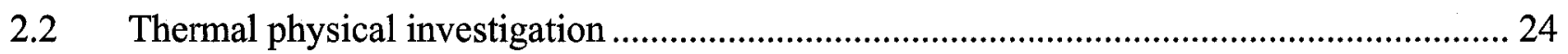




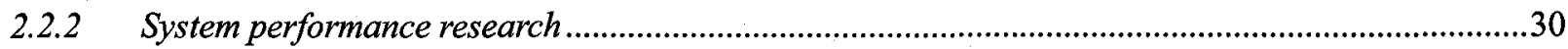

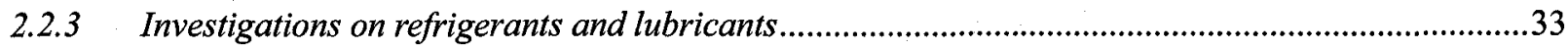

Chapter 3 Flow behaviour in adiabatic and non-adiabatic capillary tubes........................36

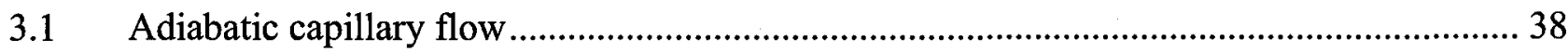

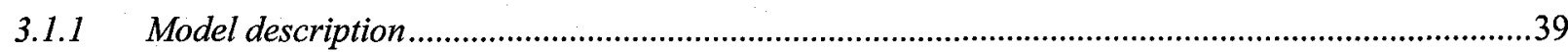

3.1.2 Parametric study and discussion ............................................................................................

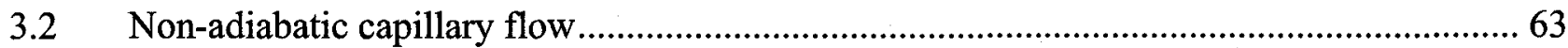

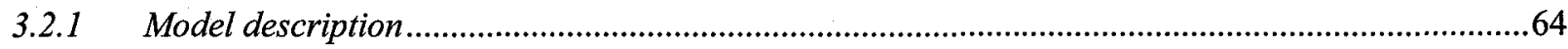

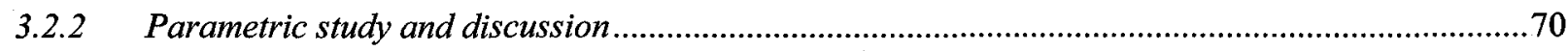

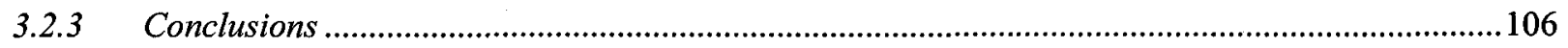

Chapter 4 Experimental study of the pressure behaviour of an AAC system without

AXE

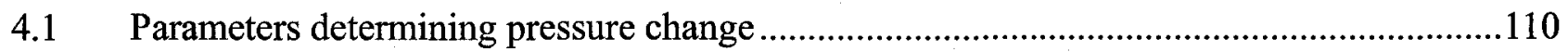

4.1.1 Pressure difference and mass flow rate …….............................................................................. 111

4.1.2 Evaporating temperature and compressor speed ....................................................................112

4.1.3 Determination of changes in mass flow rate ...............................................................................113

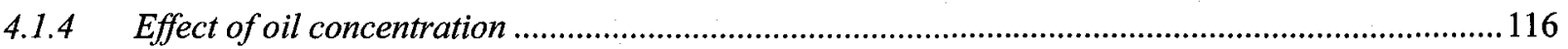

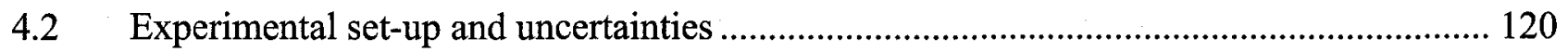

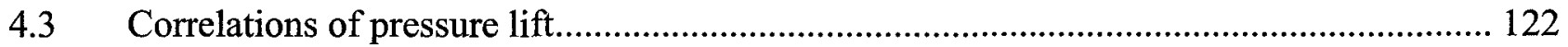

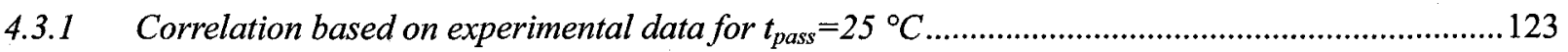


4.3.4 Verification of the model ACAM.

4.4 Conclusions

Chapter 5 Experimental study of the pressure behaviour of an AAC system with AXE 131

5.1 Determination of pressure drop across the AXE 134

5.1.1 Mass flow rate 138

5.1.2 Effect of oil concentration

5.1.3 Evaporating pressure

5.1.4 Overall pressure loss factor and pressure drop over AXE.

5.2 Influence of the compressor speed on system performance ....................................... 146

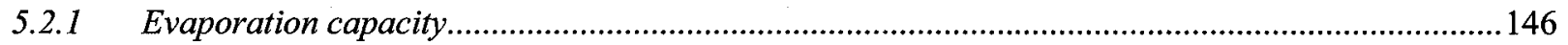

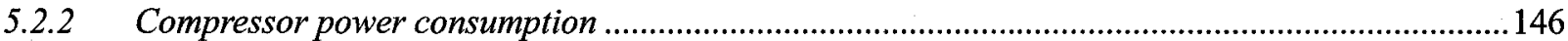

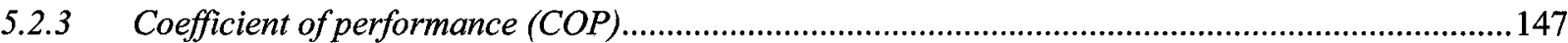

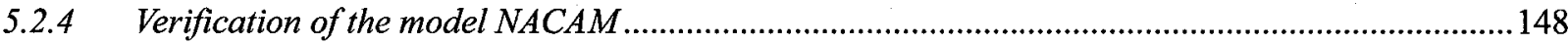

5.2.5 Feasibility of determining AXE effectiveness .............................................................................. 151

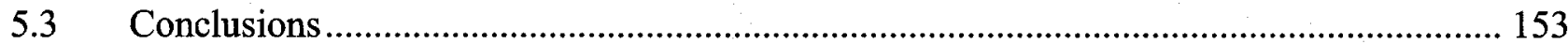

Chapter $6 \quad$ Non-adiabatic capillary flow in transcritical $\mathrm{CO}_{2}$ cycles..................................155

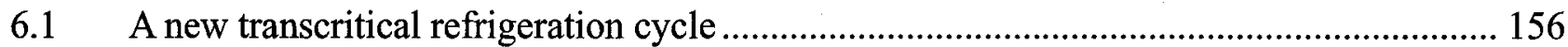

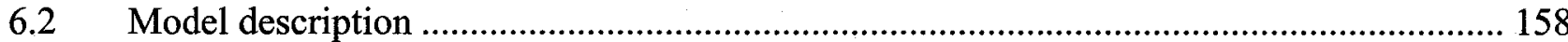


6.2.1 Assumptions

6.2.2 Governing equations.

6.2.3 Single-phase region 160

6.2.4 Two-phase region 161

6.3 Case studies 163

6.3.1 Case 1: Constant surrounding heat transfer coefficient. 163

6.3.2 Case 2: Varying surrounding heat transfer coefficient 173

6.4. Conclusions 178

Chapter 7 The optimum high pressure for transcritical refrigeration cycles 180

7.1 $\mathrm{CO}_{2}$-based transcritical refrigeration system 181

7.2 Determination of the optimum high pressure. 182

7.2.1 Theoretical deduction 183

7.2.2 Potential heat transfer capacity 183

7.2.3 Theoretical deduction of the optimum high pressure. 184

7.3 Simulation results and discussions 186

7.3.1 Influence of compressor efficiency 187

7.3.2 Potential heat transfer capacity 189

7.3.3 Influence of IHX effectiveness and the ambient temperature

7.3.4 Influence of the IHX inlet quality 194

7.3.5 Influence of evaporating temperature 195

7.3.6 Mathematical description of the optimum high pressure 196

Reproduced with permission of the copyright owner. Further reproduction prohibited without permission. 
7.4 Conclusions

Chapter 8 Conclusions and future work

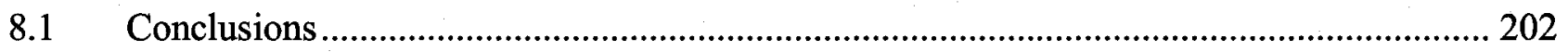

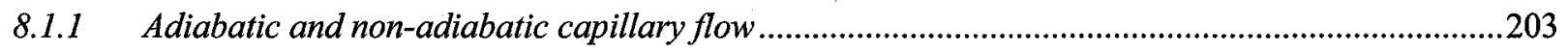

8.1.2 Changing trend of pressures for systems with and without $A X E$..............................................204

8.1.3 AXE behaviour and determination of the optimum high pressure of $\mathrm{CO}_{2}$-based systems ...............205

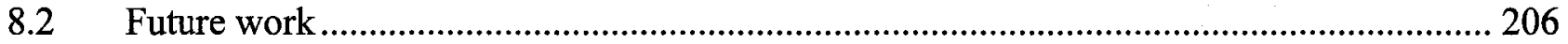

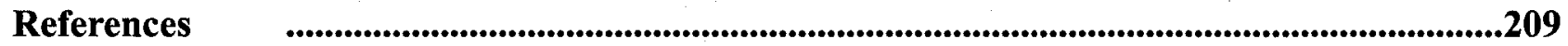

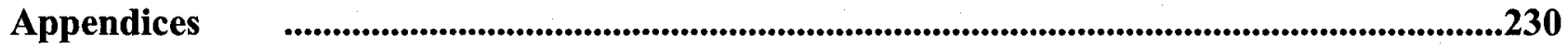

Appendix A Program to calculate AXE flow in R134a-based systems.................................. 230

Appendix B Program to correlate pressures and the compressor speed in an R134a AAC test stand 262

Appendix C Program to calculate AXE flow in $\mathrm{CO}_{2}$-based systems 272

Appendix D Program to calculate the optimum high pressure of a $\mathrm{CO}_{2}$-based systems 289 


\section{Tables}

Table 1-1 Vehicle fuel consumption of Canada and U.S.A. in 2004 ........................... 4

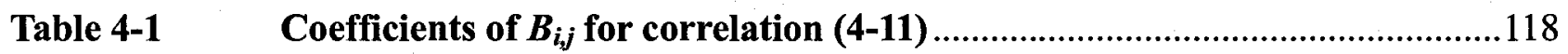

Table 5-1 Experimental results for R134a based AAC system with and without AXE 136

Table 5-2 Original and improved coefficients of Grebner's correlation ..................... 141

Table 7-1 $\quad P_{k, o p t}\left(\right.$ bar) vs. $x, \eta_{I H X}$, and $t_{a m b}=35{ }^{\circ} \mathrm{C}$ for $\eta_{i s}=0.9343-0.04478 r \ldots \ldots \ldots \ldots \ldots \ldots . . . \ldots 7$

\section{Figures}

Figure 1-1 Applications of refrigeration and air conditioning ..................................... 2

Figure 1-2 Schematic diagram of an AAC system .............................................. 11

Figure 1-3 Comparison between transcritical and subcritical cycles on a log $P$ - $h$ diagram

Figure 1-4 Comparison between transcritical and subcritical cycles on a $\boldsymbol{T}$-s diagram.. 13

Figure 3-1 Schematic and $\log P$ - $h$ diagram of an R134a-based AAC system with an IHX .

Figure 3-2 Illustration of a refrigeration system with an AXE ................................ 37

Figure 3-3 Adiabatic refrigerant flow in a capillary tube ........................................... 40

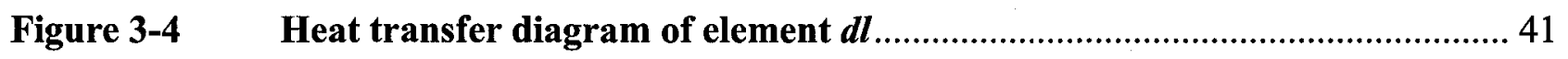


Figure 3-5 Comparison of different correlations in calculating the two-phase specific

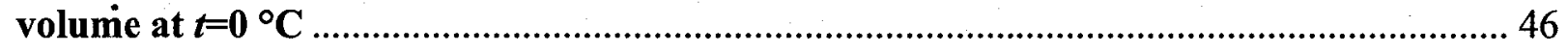

Figure 3-6 Flow diagram for adiabatic capillary model (ACAM) ................................. 49

Figure 3-7 A typical set of results of ACAM with $\dot{m}=9.52 \mathrm{~kg} / \mathrm{h}, L=1.526 \mathrm{~m}, t_{k}=37.65^{\circ} \mathrm{C}$,

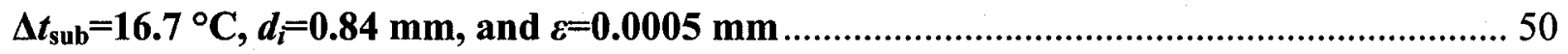

Figure 3-8 Comparison of the ACAM results with the experimental data of Melo et al and Wijaya for the mass flow rate 52

Figure 3-9 Comparison of prediction accuracy using Equation (3-19) with that using Equation (3-15) for various capillary lengths and condensation pressures 53

Figure 3-10 Mass flow rate vs. capillary inner diameter under different condensation pressures with $L=1.526 \mathrm{~m}, \Delta t_{\mathrm{sub}}=16.7^{\circ} \mathrm{C}, \varepsilon=0.0005 \mathrm{~mm}$, and $c=4.0 \%$ 54

Figure 3-11 Mass flow rate vs. capillary length under different condensation pressures with $d_{i}=0.84 \mathrm{~mm}, \Delta t_{\mathrm{sub}}=16.7^{\circ} \mathrm{C}, \varepsilon=0.0005 \mathrm{~mm}$, and $c=4.0 \%$ 55

Figure 3-12 Mass flow rate vs. condensation pressure for different capillary inner diameters with $L=1.526 \mathrm{~m}, \Delta t_{\mathrm{sub}}=16.7^{\circ} \mathrm{C}, \varepsilon=0.0005 \mathrm{~mm}$, and $c=4.0 \%$ 56

Figure 3-13 Mass flow rate vs. subcooling degrees for different condensation pressures with $L=1.526 \mathrm{~m}, d_{i}=0.84 \mathrm{~mm}, \varepsilon=0.0005 \mathrm{~mm}$, and $c=4.0 \%$ 57

Figure 3-14 Mass flow rate vs. surface roughness for different condensation pressures with $L=1.526 \mathrm{~m}, \Delta t_{\mathrm{sub}}=16.7^{\circ} \mathrm{C}, d_{i}=0.84 \mathrm{~mm}$, and $c=4.0 \%$ 59

Figure 3-15 Mass flow rate vs. subcooling degrees for different capillary inner diameters with $L=1.526 \mathrm{~m}, \Delta t_{\mathrm{sub}}=16.7^{\circ} \mathrm{C}, P_{k}=14 \mathrm{bar}$, and $c=4.0 \%$ 59 
Figure 3-16 Mass flow rate vs. oil concentration for different condensation pressures with $L=1.526 \mathrm{~m}, \Delta t_{\text {sub }}=16.7^{\circ} \mathrm{C}, d_{i}=0.84 \mathrm{~mm}$, and $\varepsilon=0.0005 \mathrm{~mm}$ 60

Figure 3-17 Heat transfer diagram of a finite element $d l$ of a concentric heat exchanger 63 Figure 3-18 Heat transfer diagram of a finite element $d l$ of a lateral heat exchanger....... 64

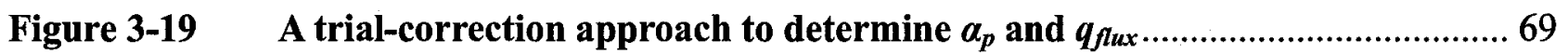

Figure 3-20 Flow diagram for non-adiabatic capillary model (NACAM) …………........... 71

Figure 3-21 Illustration of a lateral capillary tube-suction line heat exchanger ................. 72

Figure 3-22 Comparison of NACAM results with Peixoto for $L=2.057 \mathrm{~m}, t_{e}=-10.09{ }^{\circ} \mathrm{C}$, $d_{i}=0.787 \mathrm{~mm}, d_{i, \text { outer }}=6.314 \mathrm{~mm}$, and $\varepsilon=0.00045 \mathrm{~mm}$ 74

Figure 3-23 Comparison of NACAM results with Liu and Bullard for $L=1.880 \mathrm{~m}$, $t_{k}=54.44^{\circ} \mathrm{C}, t_{e}=15.56^{\circ} \mathrm{C}, \Delta t_{\text {sup }}=5.56^{\circ} \mathrm{C}, d_{i}=0.739 \mathrm{~mm}, d_{i, o u t e r}=9.525 \mathrm{~mm}$, and $\varepsilon=0.0004 \mathrm{~mm}$. 75

Figure 3-24 Comparison of NACAM results with Peixoto for $L=2.057 \mathrm{~m}, t_{e}=-10.09{ }^{\circ} \mathrm{C}$, $d_{i}=0.787 \mathrm{~mm}, d_{i, \text { outer }}=6.314 \mathrm{~mm}$, and $\varepsilon=0.00045 \mathrm{~mm}$ 76

Figure 3-25 Comparison of NACAM results with Melo et al. for $\dot{m}=7.42 \mathrm{~kg} / \mathrm{h}, L=3.000 \mathrm{~m}$, $t_{k}=52.43{ }^{\circ} \mathrm{C}, \Delta t_{\mathrm{sub}}=5.0^{\circ} \mathrm{C}, \Delta t_{\text {sup }}=1.81{ }^{\circ} \mathrm{C}, d_{i}=0.83 \mathrm{~mm}$, and $d_{i, o u t e r}=7.86 \mathrm{~mm}$ 77

Figure 3-26 Comparison of NACAM results with Melo et al. for $\dot{m}=5.49 \mathrm{~kg} / \mathrm{h}, L=3.000 \mathrm{~m}$, $t_{k}=35.53{ }^{\circ} \mathrm{C}, \Delta t_{\text {sub }}=5.2{ }^{\circ} \mathrm{C}, \Delta t_{\text {sup }}=1.79{ }^{\circ} \mathrm{C}, d_{i}=0.83 \mathrm{~mm}$, and $d_{i, o u t e r}=7.86 \mathrm{~mm}$. 78

Figure 3-27 A typical set of NACAM results with $\dot{m}=7.52 \mathrm{~kg} / \mathrm{h}, L=1.880 \mathrm{~m}, t_{k}=54.44{ }^{\circ} \mathrm{C}$, $\Delta t_{\mathrm{sub}}=8.0^{\circ} \mathrm{C}, t_{k}=15.56{ }^{\circ} \mathrm{C}, \Delta t_{\mathrm{sup}}=5.56^{\circ} \mathrm{C}, d_{i}=0.739 \mathrm{~mm}$, and $\varepsilon=0.0004 \mathrm{~mm}$ 80

Figure 3-28 Quality profile of results of NACAM under the same conditions as Figure 3-27 
Figure 3-29 A typical set of results of NACAM for a full-length heat exchange with $\dot{m}=9.94 \mathrm{~kg} / \mathrm{h}, L=1.526 \mathrm{~m}, t_{k}=37.65{ }^{\circ} \mathrm{C}, \Delta t_{\text {sub }}=16.7^{\circ} \mathrm{C}, t_{e}=0{ }^{\circ} \mathrm{C}, \Delta t_{\text {sup }}=0.38{ }^{\circ} \mathrm{C} d_{i}=0.84 \mathrm{~mm}$, $d_{i, \text { outer }}=9.00 \mathrm{~mm}$, and $\varepsilon=0.0005 \mathrm{~mm}$ 82

Figure 3-30 Quality and temperature profiles of results of NACAM under the same conditions as Figure 3-29 83

Figure 3-31 Influence of capillary inner diameter on the mass flow rate with $L=1.526 \mathrm{~m}$, $d_{i, \text { outer }}=8.00 \mathrm{~mm}, P_{k}=11.06 \mathrm{bar}, \Delta t_{\mathrm{sub}}=16.7^{\circ} \mathrm{C}, t_{e}=-10.09^{\circ} \mathrm{C}, \Delta t_{\mathrm{sup}}=4.79{ }^{\circ} \mathrm{C}, \varepsilon=0.0005 \mathrm{~mm}$, and $c=4.0 \%$ 84

Figure 3-32 Influence of capillary inner diameter on the temperature with $L=1.526 \mathrm{~m}$, $d_{i, \text { outer }}=8.00 \mathrm{~mm}, P_{k}=11.06 \mathrm{bar}, \Delta t_{\mathrm{sub}}=16.7^{\circ} \mathrm{C}, t_{e}=-10.09^{\circ} \mathrm{C}, \Delta t_{\text {sup }}=4.79{ }^{\circ} \mathrm{C}, \varepsilon=0.0005 \mathrm{~mm}$, and $c=4.0 \%$ 85

Figure 3-33 Influence of capillary length on the mass flow rate with $d_{i}=0.84 \mathrm{~mm}$, $d_{i, \text { outer }}=8.00 \mathrm{~mm}, P_{k}=11.06 \mathrm{bar}, \Delta t_{\mathrm{sub}}=16.7^{\circ} \mathrm{C}, t_{e}=-10.09^{\circ} \mathrm{C}, \Delta t_{\text {sup }}=4.79{ }^{\circ} \mathrm{C}, \varepsilon=0.0005 \mathrm{~mm}$, and $c=4.0 \%$ 88

Figure 3-34 Influence of capillary length on the temperature with $d_{i}=0.84 \mathrm{~mm}$, $d_{i, \text { outer }}=8.00 \mathrm{~mm}, P_{k}=11.06 \mathrm{bar}, \Delta t_{\mathrm{sub}}=16.7^{\circ} \mathrm{C}, t_{e}=-10.09^{\circ} \mathrm{C}, \Delta t_{\mathrm{sup}}=4.79{ }^{\circ} \mathrm{C}, \varepsilon=0.0005 \mathrm{~mm}$, and $c=4.0 \%$ . .88

Figure 3-35 Influence of condensation pressure on the mass flow rate with $L=1.526 \mathrm{~m}$, $d_{i}=0.70 \mathrm{~mm}, d_{i, \text { outer }}=8.00 \mathrm{~mm}, \Delta t_{\text {sub }}=16.7^{\circ} \mathrm{C}, t_{e}=-10.09^{\circ} \mathrm{C}, \Delta t_{\mathrm{sup}}=4.79^{\circ} \mathrm{C}, \varepsilon=0.0005 \mathrm{~mm}$, and $c=4.0 \%$ 89 
Figure 3-36 Influence of condensation pressure on the temperature with $L=1.526 \mathrm{~m}$, $d_{i}=0.70 \mathrm{~mm}, d_{i, \text { outer }}=8.00 \mathrm{~mm}, \Delta t_{\text {sub }}=16.7^{\circ} \mathrm{C}, t_{e}=-10.09^{\circ} \mathrm{C}, \Delta t_{\text {sup }}=4.79^{\circ} \mathrm{C}, \varepsilon=0.0005 \mathrm{~mm}$, and $c=4.0 \%$ 90

Figure 3-37 Influence of subcooling degrees on the mass flow rate with $L=1.526 \mathrm{~m}$, $d_{i}=0.84 \mathrm{~mm}, d_{i, \text { outer }}=8.00 \mathrm{~mm}, P_{k}=14.00 \mathrm{bar}, t_{e}=10.09{ }^{\circ} \mathrm{C}, \Delta t_{\mathrm{sup}}=4.79^{\circ} \mathrm{C}, \varepsilon=0.0005 \mathrm{~mm}$, and $c=4.0 \%$ .91

Figure 3-38 Influence of subcooling degrees on the temperature with $L=1.526 \mathrm{~m}$, $d_{i}=0.84 \mathrm{~mm}, d_{i, \text { outer }}=8.00 \mathrm{~mm}, P_{k}=14.00 \mathrm{bar}, t_{e}=10.09{ }^{\circ} \mathrm{C}, \Delta t_{\mathrm{sup}}=4.79^{\circ} \mathrm{C}, \varepsilon=0.0005 \mathrm{~mm}$, and $c=4.0 \%$ .92

Figure 3-39 Influence of surface roughness on the mass flow rate with $L=1.526 \mathrm{~m}$, $d_{i}=0.84 \mathrm{~mm}, d_{i, \text { outer }}=8.00 \mathrm{~mm}, P_{k}=14.00 \mathrm{bar}, \Delta t_{\mathrm{sub}}=16.7^{\circ} \mathrm{C}, t_{e}=-10.09{ }^{\circ} \mathrm{C}, \Delta t_{\mathrm{sup}}=4.79{ }^{\circ} \mathrm{C}$, and $c=\mathbf{4 . 0} \%$

Figure 3-40 Influence of surface roughness on the temperature with $L=1.526 \mathrm{~m}$, $d_{i}=0.84 \mathrm{~mm}, d_{i, \text { outer }}=8.00 \mathrm{~mm}, P_{k}=14.00 \mathrm{bar}, \Delta t_{\mathrm{sub}}=16.7^{\circ} \mathrm{C}, t_{e}=-10.09{ }^{\circ} \mathrm{C}, \Delta t_{\text {sup }}=4.79{ }^{\circ} \mathrm{C}$, and $c=4.0 \%$ .94

Figure 3-41 Influence of evaporating temperature on the mass flow rate with $L=1.526 \mathrm{~m}$, $d_{i, \text { outer }}=8.00 \mathrm{~mm}, P_{k}=14.00 \mathrm{bar}, \Delta t_{\mathrm{sub}}=16.7^{\circ} \mathrm{C}, \Delta t_{\mathrm{sup}}=4.79{ }^{\circ} \mathrm{C}, \varepsilon=0.0005 \mathrm{~mm}$, and $c=4.0 \% \ldots 95$ Figure 3-42 Influence of evaporating temperature on the in-tube temperatures with $L=1.526 \mathrm{~m}, d_{i}=0.84 \mathrm{~mm}, d_{i, \text { outer }}=8.00 \mathrm{~mm}, P_{k}=14.00$ bar, $\Delta t_{\text {sub }}=16.7{ }^{\circ} \mathrm{C}, \Delta t_{\text {sup }}=4.79{ }^{\circ} \mathrm{C}$,

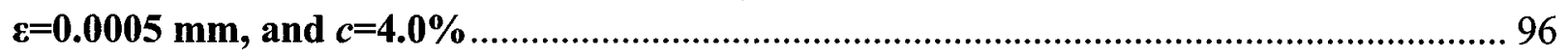

Figure 3-43 Influence of superheating degrees on the mass flow rate with $L=1.526 \mathrm{~m}$, 
$d_{i, \text { outer }}=8.00 \mathrm{~mm}, P_{k}=14.00 \mathrm{bar}, \Delta t_{\text {sub }}=10.0{ }^{\circ} \mathrm{C}, t_{e}=-10.09{ }^{\circ} \mathrm{C}, \varepsilon=0.0005 \mathrm{~mm}$, and $c=4.0 \% \ldots .97$

Figure 3-44 Influence of superheating degrees on in-tube temperatures with $L=1.526 \mathrm{~m}$, $d_{i}=0.84 \mathrm{~mm}, d_{i, \text { outer }}=8.00 \mathrm{~mm}, P_{k}=14.00 \mathrm{bar}, \Delta t_{\mathrm{sub}}=10.0^{\circ} \mathrm{C}, t_{e}=-10.09{ }^{\circ} \mathrm{C}, \varepsilon=0.0005 \mathrm{~mm}$, and $c=4.0 \%$ 98

Figure 3-45 Influence of suction line inlet quality on the mass flow rate with $L=1.526 \mathrm{~m}$, $d_{i, o u t e r}=12.70 \mathrm{~mm}, P_{k}=14.00 \mathrm{bar}, \Delta t_{\mathrm{sub}}=10.0{ }^{\circ} \mathrm{C}, t_{e}=-10.09{ }^{\circ} \mathrm{C}, \varepsilon=0.0005 \mathrm{~mm}$, and $c=4.0 \%$.. 99

Figure 3-46 Influence of suction line inlet quality on in-tube temperatures with $L=1.526 \mathrm{~m}$, $d_{i}=0.84 \mathrm{~mm}, d_{i, \text { outer }}=12.70 \mathrm{~mm}, P_{k}=14.00 \mathrm{bar}, \Delta t_{\mathrm{sub}}=10.0{ }^{\circ} \mathrm{C}, t_{e}=-10.09{ }^{\circ} \mathrm{C}, \varepsilon=0.0005 \mathrm{~mm}$, and $c=4.0 \%$ 100

Figure 3-47 Influence of suction line inner diameter on mass flow rate with $L=1.526 \mathrm{~m}, d_{i}$ $=0.84 \mathrm{~mm}, \Delta t_{\mathrm{sub}}=10.0^{\circ} \mathrm{C}, t_{e}=-10.09^{\circ} \mathrm{C}, \Delta t_{\mathrm{sup}}=4.79^{\circ} \mathrm{C}, \varepsilon=0.0005 \mathrm{~mm}$, and $c=4.0 \%$ 101

Figure 3-48 Influence of suction line inner diameter on temperatures with $L=1.526 \mathrm{~m}$, $d_{i, \text { outer }}=8.00 \mathrm{~mm}, P_{k}=11.06 \mathrm{bar}, \Delta t_{\mathrm{sub}}=16.7^{\circ} \mathrm{C}, t_{e}=-10.09^{\circ} \mathrm{C}, \Delta t_{\text {sup }}=4.79{ }^{\circ} \mathrm{C}, \varepsilon=0.0005 \mathrm{~mm}$, and $c=4.0 \%$ 102

Figure 3-49 Influence of flow arrangement on mass flow rate with $L=1.526 \mathrm{~m}$, $d_{i, \text { outer }}=8.00 \mathrm{~mm}, P_{k}=11.06 \mathrm{bar}, \Delta t_{\mathrm{sub}}=16.7^{\circ} \mathrm{C}, t_{e}=-10.09^{\circ} \mathrm{C}, \Delta t_{\mathrm{sup}}=4.79{ }^{\circ} \mathrm{C}, \varepsilon=0.0005 \mathrm{~mm}$, and $c=4.0 \%$ 103

Figure 3-50 Comparison of flow arrangement with $L=1.526 \mathrm{~m}, d_{i}=0.84 \mathrm{~mm}$, $d_{i, \text { outer }}=8.00 \mathrm{~mm}, P_{k}=11.06 \mathrm{bar}, \Delta t_{\text {sub }}=16.7^{\circ} \mathrm{C}, t_{e}=-10.09^{\circ} \mathrm{C}, \Delta t_{\text {sup }}=4.79{ }^{\circ} \mathrm{C}, \varepsilon=0.0005 \mathrm{~mm}$, and $c=4.0 \%$ .104

Figure 3-51 Influence of heat exchanger type on mass flow rate with $L=1.526 \mathrm{~m}$, 
$P_{k}=11.06 \mathrm{bar}, \Delta t_{\mathrm{sub}}=16.7^{\circ} \mathrm{C}, t_{e}=-10.09^{\circ} \mathrm{C}, \Delta t_{\mathrm{sup}}=4.79^{\circ} \mathrm{C}, \varepsilon=0.0005 \mathrm{~mm}$, and $c=4.0 \% \ldots \ldots .105$

Figure 3-52 Comparison of heat exchanger types with $L=1.526 \mathrm{~m}, d_{i}=0.84 \mathrm{~mm}$, $P_{k}=11.06 \mathrm{bar}, \Delta t_{\mathrm{sub}}=16.7^{\circ} \mathrm{C}, t_{e}=-10.09{ }^{\circ} \mathrm{C}, \Delta t_{\mathrm{sup}}=4.79{ }^{\circ} \mathrm{C}, \varepsilon=0.0005 \mathrm{~mm}$, and $c=4.0 \% \ldots \ldots .106$

Figure 4-1 Schematic of a heat exchanger model .....................................................113

Figure 4-2 Volumetric efficiency vs. compressor speed ...............................................114

Figure 4-3 Schematic diagram of two refrigeration cycles showing the effects of a change in compressor speed

Figure 4-4 Improvement to the Grebner's correlation

Figure 4-5 Prediction accuracy of the new correlation.

Figure 4-6 Compressor work consumptions before and after adjusting pressures ..... 120

Figure 4-7 Schematic of an R134a AAC experimental test stand with capillary tube... 121

Figure 4-8 Relationship between pressures and compressor speed

Figure 4-9 Predicted and measured temperature differences with compressor speed .. 124

Figure 4-10 Predicted and measured pressures with varying compressor speed............ 124

Figure 4-11 Comparison between predicted and measured pressures........................ 125

Figure 4-12 Improvement of correlation in predicting temperature differences............ 126

Figure 4-13 Influence of density at the compressor inlet............................................ 127

Figure 4-14 Comparison of data by experiments and ACAM ..................................... 128

Figure 5-1 Schematic of an R134a AAC experimental test stand with AXE ............... 135

Figure 5-2 Pressure drop of throttling process for systems with and without an AXE . 137

Figure 5-3 Mass flow rate for systems with and without an AXE .............................. 139 
Figure 5-4 Comparison between different correlations and experimental data 141

Figure 5-5 Evaporating pressure for systems with and without an AXE 142

Figure 5-6 Prediction of pressures for systems with and without an AXE for $t_{\text {pass }}=25^{\circ} \mathrm{C}$ using correlation (5-7) 143

Figure 5-7 Prediction of pressures for systems with and without an AXE using correlation (5-7) and the improved Grebner's correlation. 144

Figure 5-8 Comparison between predicted and measured pressures... 145

Figure 5-9 Cooling load for system with and without AXE ...................................... 146

Figure 5-10 Compressor power consumption for system with and without AXE. 147

Figure 5-11 COP behavior for system with and without AXE ................................... 148

Figure 5-12 Comparison of data by experiments and NACAM .................................. 149

Figure 5-13 Temperature profile of NACAM and ACAM flow with $l_{\text {capi }}=0.50 \mathrm{~m}$, $\varepsilon=0.00150 \mathrm{~mm}, d_{i}=1.65 \mathrm{~mm}, d_{i, o u t e r}=12.70 \mathrm{~mm}$, and $n=1526 \mathrm{rpm}$.

Figure 5-14 Illustration of throttling process with heat transfer in AXE ..................... 152

Figure 6-1 Schematic of a transcritical AAC system with AXE .............................. 156

Figure 6-2 Pressure-enthalpy diagram of a $\mathrm{CO}_{2}$ transcritical refrigeration cycle........ 157

Figure 6-3 Non-adiabatic capillary flow in a transcritical cycle................................ 159

Figure 6-4 Simulation results along capillary tube for $\mathrm{CO}_{2}$ with $Q_{e}=7 \mathrm{~kW}, P_{k}=120$ bar, $t_{\text {capi,in }}=45^{\circ} \mathrm{C}, t_{e}=0{ }^{\circ} \mathrm{C}, d_{i}=1.85 \mathrm{~mm}$, and $\varepsilon=0.0015 \mathrm{~mm}$

Figure 6-5 Capillary length vs. capillary inner diameter under different cooling pressures for $\mathrm{CO}_{2}$ with $Q_{e}=7 \mathrm{~kW}, t_{\text {capi,in }}=45^{\circ} \mathrm{C}, t_{e}=0{ }^{\circ} \mathrm{C}$, and $\varepsilon=0.0015 \mathrm{~mm}$ 165 
Figure 6-6 Cooling capacity vs. capillary length for $\mathrm{CO}_{2}$ with $d_{i}=1.85 \mathrm{~mm}, t_{\text {capi,in }}=45{ }^{\circ} \mathrm{C}$, $t_{e}=0^{\circ} \mathrm{C}$, and $\varepsilon=0.0015 \mathrm{~mm}$. 166

Figure 6-7 Cooling capacity vs. evaporating temperature for $\mathrm{CO}_{2}$ with $Q_{e}=7 \mathrm{~kW}$,

$$
P_{k}=120 \mathrm{bar}, d_{i}=1.85 \mathrm{~mm}, t_{c a p i, i n}=45^{\circ} \mathrm{C} \text {, and } \varepsilon=0.0015 \mathrm{~mm}
$$

Figure 6-8 Quality profile for different surface roughness for $\mathrm{CO}_{2}$ with $Q_{e}=7 \mathrm{~kW}$,

$$
P_{k}=120 \mathrm{bar}, d_{i}=1.85 \mathrm{~mm} \text {, and } t_{c a p i, i n}=45^{\circ} \mathrm{C} \text {. }
$$

Figure 6-9 Quality profile for different cooling loads for $\mathrm{CO}_{2}$ with $P_{k}=120$ bar,

$$
d_{i}=1.85 \mathrm{~mm}, t_{e}=0^{\circ} \mathrm{C}, t_{c a p i, i n}=45^{\circ} \mathrm{C}, \text { and } \varepsilon=0.0015 \mathrm{~mm}
$$

Figure 6-10 Heat transfer rate vs. different capillary inner diameter for $\mathrm{CO}_{2}$ with $Q_{e}=7 \mathrm{~kW}$,

$$
t_{\text {capi,in }}=45^{\circ} \mathrm{C}, t_{e}=0^{\circ} \mathrm{C} \text {, and } \varepsilon=0.0015 \mathrm{~mm}
$$

Figure 6-11 Behaviour of temperature and entropy of different capillary inner diameters for $\mathrm{CO}_{2}$ with $Q_{e}=7 \mathrm{~kW}, P_{k}=120 \mathrm{bar}, t_{c a p i, i n}=45^{\circ} \mathrm{C}, t_{e}=0^{\circ} \mathrm{C}$, and $\varepsilon=0.0015 \mathrm{~mm}$.

Figure 6-12 Three-dimensional presentation of the relationship between $d_{i}, Q_{e}$ and $L$ for $\mathrm{CO}_{2}$ with $P_{k}=120 \mathrm{bar}, t_{c a p i, i n}=45^{\circ} \mathrm{C}, t_{e}=0{ }^{\circ} \mathrm{C}$, and $\varepsilon=0.0015 \mathrm{~mm}$. 172

Figure 6-13 Simulation results along capillary tube for $\mathrm{CO}_{2}$ with $Q_{e}=7 \mathrm{~kW}, P_{k}=110$ bar,

$$
t_{c a p i, i n}=45^{\circ} \mathrm{C}, t_{c}=0^{\circ} \mathrm{C} \text {, and } \varepsilon=0.0015 \mathrm{~mm}
$$

Figure 6-14 Capillary length vs. capillary inner diameter under different cooling pressures for $\mathrm{CO}_{2}$ with $Q_{e}=7 \mathrm{~kW}, t_{c a p i, i n}=45^{\circ} \mathrm{C}, t_{e}=0{ }^{\circ} \mathrm{C}$, and $\varepsilon=0.0015 \mathrm{~mm}$

Figure 6-15 Cooling capacity vs. evaporation temperature for $\mathrm{CO}_{2}$ with $Q_{e}=7 \mathrm{~kW}$,

$$
P_{k}=120 \mathrm{bar}, t_{c a p i, i n}=45^{\circ} \mathrm{C} \text {, and } \varepsilon=0.0015 \mathrm{~mm}
$$

Figure 6-16 Profiles of friction factors for $\mathrm{CO}_{2}$ with $Q_{e}=7 \mathrm{~kW}, P_{k}=120$ bar, $t_{c a p i, i n}=45^{\circ} \mathrm{C}$, 
Figure 6-17 Heat transfer rate vs. capillary inner diameter for $\mathrm{CO}_{2}$ with $Q_{e}=7 \mathrm{~kW}$,

$$
t_{\text {capi,in }}=45^{\circ} \mathrm{C}, t_{e}=0^{\circ} \mathrm{C} \text {, and } \varepsilon=0.0015 \mathrm{~mm}
$$

Figure 7-1 COP behaviour for a $\mathrm{CO}_{2}$ transcritical system with an accumulator and an IHX..

Figure 7-2 Pressure-enthalpy diagram of transcritical $\mathrm{CO}_{2}$ refrigeration cycles 182

Figure 7-3 Compressor isentropic efficiency from different references. 188

Figure 7-4 Three-dimensional profile of COP curves under different cooling pressures and IHX efficiencies for $t_{I}=5.3^{\circ} \mathrm{C}\left(P_{e}=40 \mathrm{bar}\right), x=0.8$, and $t_{a m b}=35^{\circ} \mathrm{C}$.

Figure 7-5 The relationship between $q_{p o t n}, P_{k}$, and $x$ under different ambient temperatures for $P_{e}=40$ bar and $\eta_{I H X}=1$.

Figure 7-6 The high pressure turnings vs. $x$ and $t_{a m b}$ under different evaporating temperatures for $\eta_{1 H X}=1$

Figure 7-7 Two-dimensional illustration of the turning of the high pressure for $x=0.8 .191$

Figure 7-8 Three-dimensional profiles of COP under different high pressures and IHX efficiencies for $P_{e}=40$ bar, $x=0.8$, and $\eta_{i s}=0.9343-0.04478 r$.

Figure 7-9 Comparison of COP curves under different high pressures and IHX efficiencies for $P_{e}=40 \mathrm{bar}, x=0.8$, and $\eta_{i s}=0.9343-0.04478$.

Figure 7-10 Three-dimensional profiles of COP under different high pressures and IHX efficiencies for $P_{e}=40 \mathrm{bar}, t_{a m b}=35^{\circ} \mathrm{C}$, and $\eta_{i s}=0.9343-0.04478$. 194

Figure 7-11 Influence of high pressure on COP with varying evaporating temperature for 
$x=0.8, t_{a m b}=35{ }^{\circ} \mathrm{C}$, and $\eta_{i s}=0.9343-0.04478$.

Figure 7-12 Comparison between simulation results and experimental data 199 


\section{Nomenclature}

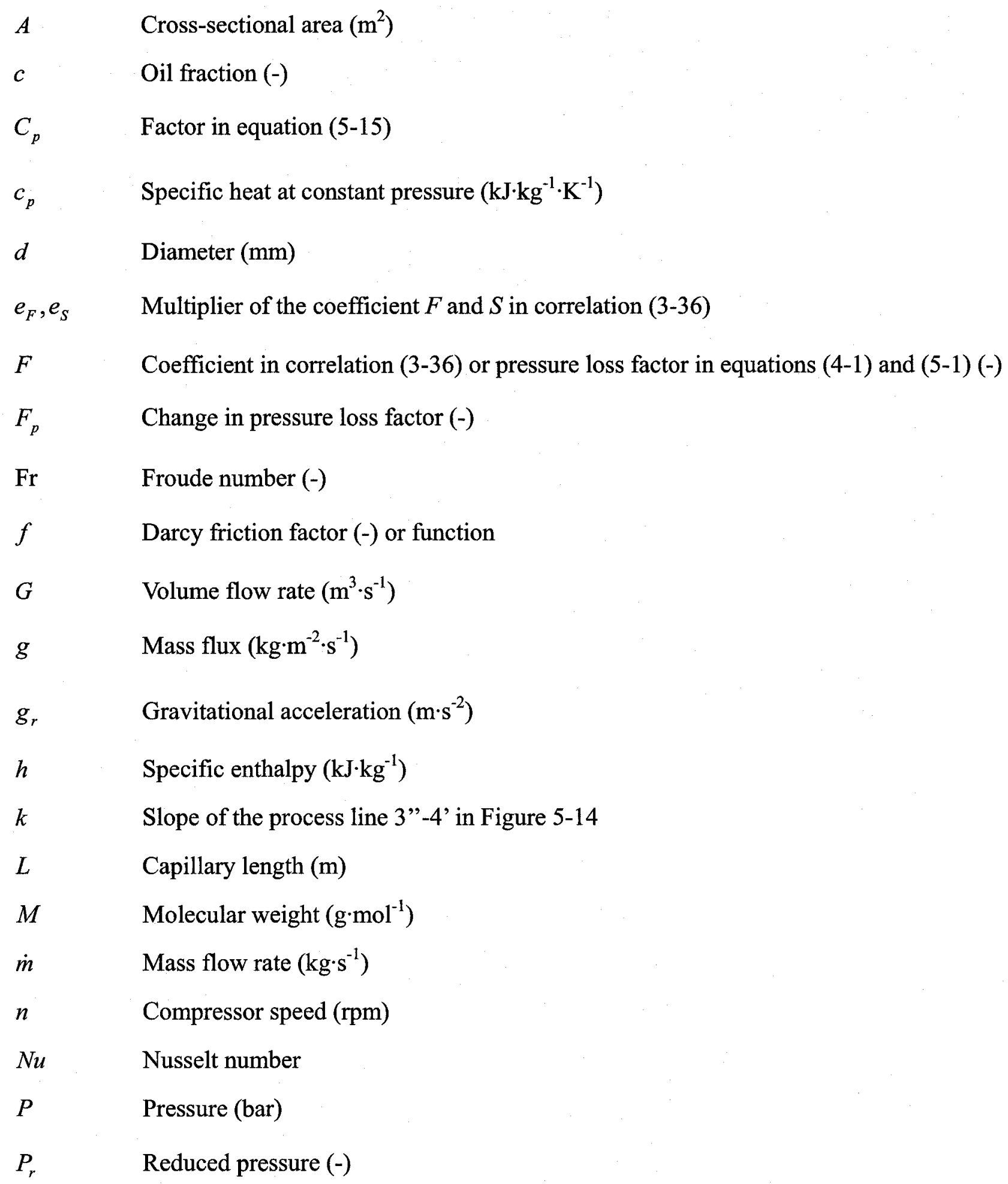




\begin{tabular}{ll} 
Pr & Prandtl number $(-)$ \\
$Q$ & Heat transfer rate $(\mathrm{W})$ \\
$Q_{e}$ & Cooling capacity $(\mathrm{kW})$ \\
$q_{0}$ & Specific cooling capacity $\left(\mathrm{kJ} \cdot \mathrm{kg}^{-1}\right)$ \\
$q_{f f u x}$ & Heat flux $\left(\mathrm{W} \cdot \mathrm{m}^{-2}\right)$ \\
$r$ & Pressure ratio $(-)$ \\
$\operatorname{Re}$ & Reynolds number $(-)$ \\
$S$ & Coefficient in correlation $(3-36)$ \\
$s$ & Specific entropy $\left(\mathrm{kJ} \cdot \mathrm{kg}^{-1} \cdot \mathrm{K}^{-1}\right)$ \\
$t$ & Temperature $\left({ }^{\circ} \mathrm{C}\right)$ \\
$T$ & Temperature $(\mathrm{K})$ \\
$U$ & Gross heat transfer coefficient $\left(\mathrm{W} \cdot \mathrm{m}^{-2} \cdot \mathrm{K}^{-1}\right)$ \\
$V$ & Velocity $\left(\mathrm{m} \cdot \mathrm{s}^{-1}\right)$ \\
$V_{d i s}$ & Displacement $\left(\mathrm{m}^{3}\right)$ \\
$v$ & Specific volume $\left(\mathrm{m}^{3} \cdot \mathrm{kg}^{-1}\right)$ \\
$x$ & Liquid refrigerant concentration $(-)$ \\
& Quality $(-)$ \\
\hline &
\end{tabular}

\section{Greek symbols}

$\begin{array}{ll}\alpha & \text { Heat transfer coefficient }\left(\mathrm{W} \cdot \mathrm{m}^{-2} \cdot \mathrm{K}^{-1}\right) \\ \delta & \text { Distance between the two tubes in a lateral heat exchanger }(\mathrm{m}) \\ \varepsilon & \text { Internal surface roughness }(\mathrm{mm}) \\ \varepsilon_{r} & \text { Relative surface roughness }(-) \\ \phi^{2} & \text { Friction factor multiplier }(-) \\ \varphi & \text { Heat flux }\left(\mathrm{W} \cdot \mathrm{m}^{-2}\right)\end{array}$




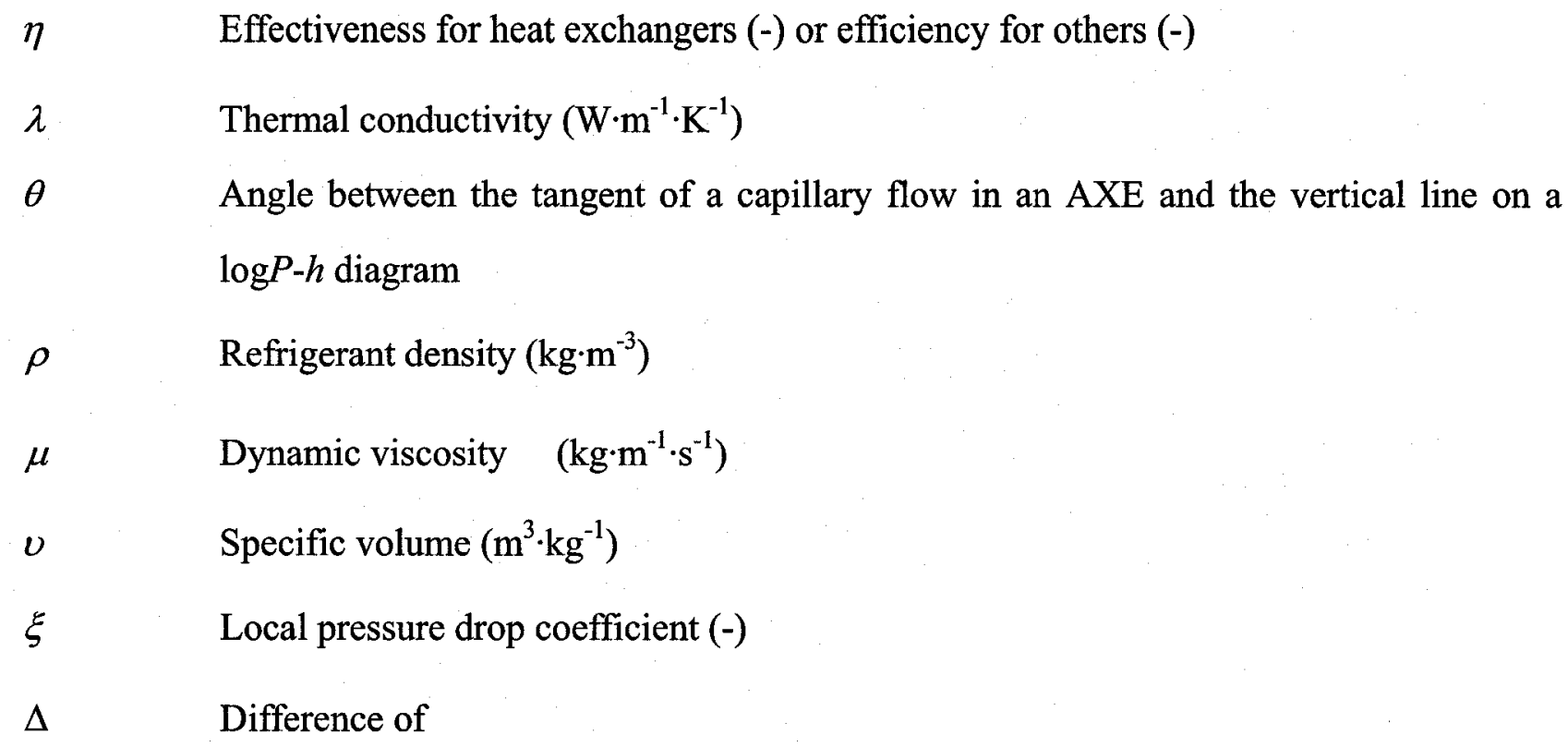

\section{Subscripts}

$\begin{array}{ll}\text { amb } & \text { Ambient } \\ \text { camp } & \text { Capillary } \\ c r & \text { Compressor } \\ e & \text { Critical } \\ f g & \text { Evaporator side } \\ g & \text { Liquid-gas } \\ h & \text { Gaseous } \\ i & \text { Hydraulic } \\ \text { in } & \text { Inner side of capillary tube or inertia } \\ i s & \text { Inlet } \\ k & \text { Isentropic } \\ l & \text { High pressure side } \\ & \text { Liquid }\end{array}$




\begin{tabular}{ll} 
liq & Transcritical liquid region \\
lo & Liquid only \\
max & Maximum \\
mech & Mechanical \\
min & Minimum \\
mix & Mixture \\
oil & Lubricant oil \\
opt & Optimum \\
out & Outlet \\
outer & Outer side of capillary tube \\
pass & Passenger compartment \\
pb & Pool boiling \\
potn & Potential \\
pure & Pure refrigerant \\
$r$ & Refrigerant \\
$s$ & Isentropic \\
$s a t$ & Saturated \\
$s l$ & Suction line \\
$s p$ & Single-phase \\
sub & Subcooling \\
sup & Superheating or Supercritical fluid region \\
$t p$ & Two-phase \\
tube & Capillary wall \\
$v$ & Vapour \\
\hline
\end{tabular}


weld Welding

\section{Acronyms}

$\begin{array}{ll}\text { AAC } & \text { Automotive air conditioning } \\ \text { ACAM } & \text { Adiabatic capillary model } \\ \text { AXE } & \text { Accumulator-heat-exchanger-expander } \\ \text { BTA } & \text { superheat related factor } \\ \text { CCOT } & \text { Clutch cycling orifice tube } \\ \text { CFC } & \text { Chlorofluorocarbon } \\ \text { COP } & \text { Coefficient of performance } \\ \text { CTA } & \text { moisture condensate factor } \\ \text { GWP } & \text { Global warming potential } \\ \text { HC } & \text { Halocarbon } \\ \text { HFC } & \text { Hydrofluorocarbon } \\ \text { IHX } & \text { Internal heat exchanger } \\ \text { MEMS } & \text { Micro electro-mechanical systems } \\ \text { NACAM } & \text { Non-adiabatic capillary model } \\ \text { ODP } & \text { Ozone depletion potential } \\ \text { RMS } & \text { Root-mean-square } \\ \text { TXV } & \text { Thermal expansion valve } \\ \end{array}$




\section{Chapter 1}

\section{Introduction}

With the continued advances in science and technology, humans are becoming more and more powerful in not only ensuring safe and healthy physical conditions but also making life easy and comfortable. During the course of this development, we have greatly changed the shape of our environment, the ecosystem of the whole earth, intentionally or unwillingly. From the nineteenth century to the present, this trend has been more and more obvious. Hundreds of huge cities have been erected, enormous highway systems spread to every corner of the earth, numerous dams bestride rivers, thousands of satellites hover in outer space, and large aircraft convey millions of people around the world; and so on and so forth. Meanwhile, deserts crawl over land that was once fertile soil, many species are facing extinction due to harmful industrial waste, air becomes thorny for breathing, global temperature continuously increases, disturbing the biological clocks of creatures, and people are more and more vulnerable to various diseases and cancers. We are at a critical point to protect our planet, to protect us from being devastated by ourselves. From the sustainable development point of view, we must find a way to save energy and protect the environment during the process when new technologies are being developed to better our living conditions.

As part of our everyday life, refrigeration and air-conditioning systems, including air-conditioners, refrigerators, freezers, chillers, food showcases, etc., are used extensively. Some of 
these applications are shown in Figure 1-1. Nowadays, the refrigeration industry has become a

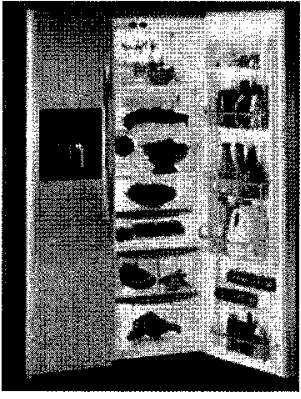

(a) Refrigerator ${ }^{1}$

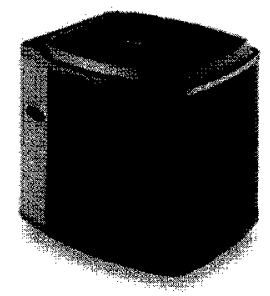

(d) Central air conditioning unit ${ }^{4}$

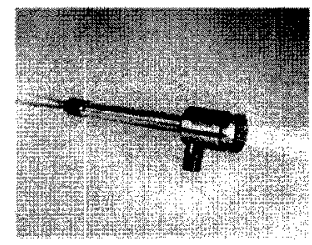

(g) Vortex tube cooler ${ }^{7}$

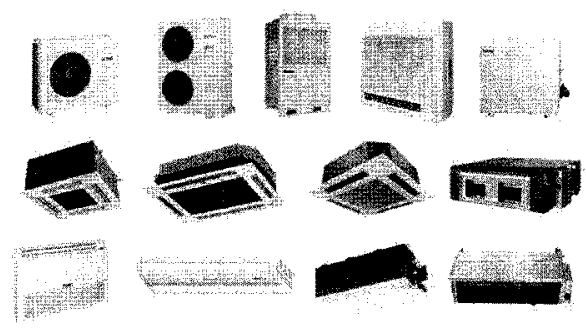

(b) Commercial air conditioning devices ${ }^{2}$

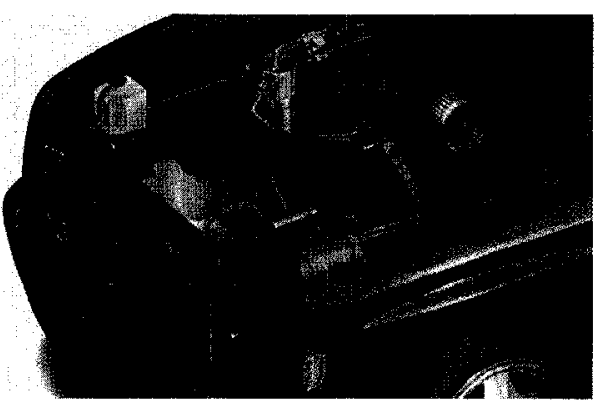

(e) Automotive air conditioner ${ }^{5}$

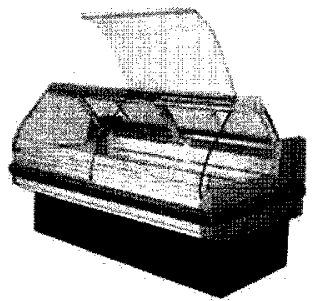

(h) Food showcase ${ }^{8}$

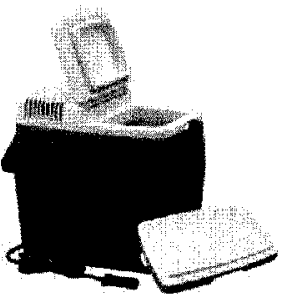

(c) Portable thermoelectric ice chest ${ }^{3}$

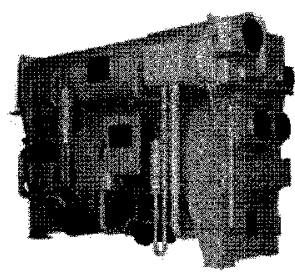

(f) $\mathrm{LiBr}-\mathrm{H}_{2} \mathrm{O}$ absorption chiller $^{6}$

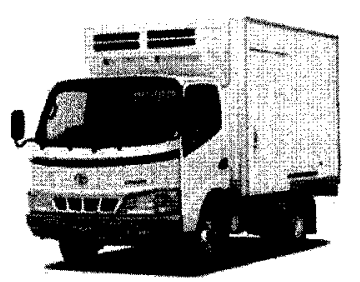

(i) Freezer vehicle ${ }^{9}$

Figure 1-1 Applications of refrigeration and air conditioning

${ }^{1}$ Source: http://www.frigidaire.com/products/refrigerators/side-by-side/Counter_Depth/prod_PHSC39EGS.asp

${ }^{2}$ Source: http://www.haier.com.au/products/commercialac.aspx

${ }^{3}$ Source: http://store.colemancampingstore.com/co16qtthcowi.html

${ }^{4}$ Source: http://www.ebuild.com/products/imageGallery.hwx/Q/productld.549720

${ }^{5}$ Source: http://www.climatictesting.com/Automotive_AC_System_Calorimeter.htm

${ }^{6}$ Source: http://saripuya.20un.com/absorption-chiller-manufacturers-pro.html

${ }^{7}$ Source: http://www.exair.com/vortextube/vt_frmain.htm

${ }^{8}$ Source: http://www.dragon-enterprise.com/products.asp?bigclassid= $16 \&$ smallclassid=66\#

${ }^{9}$ Source: http://www.env.go.jp/air/car/vehicles/die-018.htm 
symbol of modern society and these products consume a good portion of our energy supply.

The first artificial machine-powered refrigeration demonstration can be dated back to 1755 at the University of Glasgow in Scotland, where William Cullen used a pump to create a partial vacuum in a container full of ethyl ether, which then vaporized and absorbed heat from the surroundings (ASHRAE, 2006). The first practical refrigeration machine recognized by the industry was built by Jacob Perkins in 1834. Since then, refrigeration systems have kept changing people's lives greatly. Their domestic applications include refrigerators, air conditioners, heat pumps, heat pipes for consumer electronic systems, and thermoelectric coolers. Their commercial applications include chillers, central units for air conditioning, showcase refrigerators, mobile air conditioning systems, and refrigerator vehicle. They also have very important usage in medical and military fields. Refrigerators have become standard equipment for almost every family in advanced countries, and air conditioners are more and more popular in providing a steady comfortable living condition. In 2001, 80.8 out of 107.0 million households in the U.S.A. used electric air-conditioning systems, which consumed 183 billion $\mathrm{kWh}$ and cost $\$ 15.94$ billion (U. S. Department of Energy, 2004). In 2003, among the more than 11.5 million households in Canada, $43 \%$ were equipped with air-conditioning systems; almost every household used a refrigerator, and 30 percent used at least two; $61 \%$ of all households had freezers (National Resources Canada, 2005). If we suppose that Canadians use their air conditioners $1 / 4$ as often as their American counterparts and assuming similar electricity price, Canadians consume 2.8 billion $\mathrm{kWh}$ at a cost of $\$ 240$ million to air condition their houses. The averaged annual energy consumptions of refrigerators and freezers in 
2003 are $487.1 \mathrm{kWh}$ and $369.1 \mathrm{kWh}$, respectively, which correspond to 730 and 260 million $\mathrm{kWh}$ for each type and \$86 million in total. Based on the National Private Vehicle Use Survey in Canada (2000), there were 14.2 million private vehicles operated for personal use in 1996 . They traveled 247.6 billion kilometres and consumed 28 billion litres of fuel annually. Johnson (2002) showed the amount of fuel that was used for mobile air conditioning in the U.S.A.'s 213 million light duty vehicles was as much as 27 billion litres (they consumed 125.9 billion litres fuel in total), which is equivalent to $6 \%$ of domestic petroleum consumption. Table 1 shows the average fuel consumption of Canada and the U.S.A. in 2004 (Metschies, 2005). If taking a 1/5 factor as usage frequency difference between the two countries, the amount of money used to air condition Canadian vehicles is about $\$ 1.07$ billion.

Table 1-1 Vehicle fuel consumption of Canada and U.S.A. in 2004

\begin{tabular}{|c|c|c|c|c|c|c|}
\hline \multirow{4}{*}{ Country } & \multicolumn{2}{|c|}{ Fuel consumption } & \multicolumn{2}{c|}{ Fuel prices } & \multicolumn{2}{c|}{ Fuel Cost } \\
\cline { 2 - 7 } & $\begin{array}{c}\text { Commercial } \\
\text { vehicles } \\
\text { (Diesel) }\end{array}$ & $\begin{array}{c}\text { Passenger } \\
\text { cars } \\
\text { (Gasoline) }\end{array}$ & Diesel & Gasoline & Diesel & Gasoline \\
\cline { 2 - 7 } (Billion litres) & (Billion litres) & (USD) & (USD) & (Billion USD) & (Billion USD) \\
\hline Canada & 13.03 & 23.68 & 0.68 & 0.68 & 8.86 & 16.10 \\
\hline U.S.A. & 1670.31 & 185.81 & 0.57 & 0.54 & 952.08 & 100.34 \\
\hline
\end{tabular}

\subsection{Classification of refrigeration systems}

Fundamentally, a refrigeration system is equipment that conveys heat from a cold location (heat source) to a hot location (heat sink) at the cost of a certain amount of energy input. Such energy consumption could be from either thermal, mechanical, or electrical sources, or a 
combination of them. Based on different mechanisms, refrigeration systems can be divided into several categories.

\subsubsection{Phase change approach}

The vapour compression cycle is the most commonly used refrigeration method. The latent heat of the working fluids, in the forms of sublimation, melting, or vaporization, is used to cool down high temperature materials or regions. For most cases, continuous refrigeration is required; since sublimation and melting are difficult to use in a cycle, vaporization is the dominant method adopted.

Vapour compression systems are the most popular system. Absorption refrigerators are mostly used at locations where electricity is un-economic or industrial waste heat is available. Instead of using a compressor to pump the refrigerant from the low pressure side to the high pressure, the generator and absorber act as a thermal "compressor". Heat is supplied to the generator, evaporating the working fluid (of low boiling temperature) out of the weak solution and making the solution strong; then the solution is conveyed to the absorber to absorb the low pressure refrigerant vapour and become weak again. The refrigerant from the generator goes through a U-shape expansion tube and enters the evaporator to provide cooling capacity. Water is used as the working fluid in most vapour injection refrigerators, and thus the systems are adopted for air conditioning or cooling water for industrial processes. Since two flows at different pressures are required for an injector, it can be used in domestic refrigerators to improve system efficiency because there are two different 
temperature zones existing in the machine.

\subsubsection{Vapour expansion approach}

The vapour expansion cycle has four thermodynamic processes similar to the vapour compression cycle; the difference is that there is no phase change. The first and most important device is the air expansion refrigerator. The two main characteristics are that a gas turbine is adopted as an expansion device to recover work which is used for the compression process and the capability of providing temperatures lower than $-80^{\circ} \mathrm{C}$ with a one-stage structure. These make such an approach especially suitable for large-scale industrial applications.

\subsubsection{Vortex flow approach}

As a delicate, pure fluid mechanical device, a vortex tube takes advantages of the radial angular momentum difference between the swirl flow at the tube centre and that near the wall to generate two pressurized fluids with different temperatures and opposite flow directions. Figure 1-1 $(\mathrm{g})$ shows a commercial vortex tube. The basic physics inside is that the centered high-momentum flow dissipates part of its kinetic energy to the surrounding low momentum flow such that its temperature drops. Normally temperature drops of more than $50^{\circ} \mathrm{C}$ can be obtained between the inlet and the cold end with the inlet air at a pressure of 4 bar and a temperature of $20 \mathrm{C}$ (Stephan, 1983; Ahlborn, 2000). To generate flow separation, high speed inlet flow is required, and thus a compression device should be used as pre-processing equipment. Due to its quick start, easy 
of maintenance, and low temperature capabilities, vortex tubes are mostly used at small-scale applications where intermittent operation is a necessity. Combined with a heat regenerator and an injector, a vortex tube can provide even lower temperatures at better efficiencies.

\subsubsection{Thermoelectric approach}

Thermoelectric refrigeration is directly based on the Peltier-Seebeck effect that thermal differentials can be directly converted to electric voltage and vice versa for a closed loop composed of two different metals or semiconductors. The Peltier effect is the reverse of the Seebeck effect which creates a temperature difference from an electric voltage. Since semiconductors have more obvious Peltier effect than metals, they are widely used for refrigeration purposes. By placing thermal electric couplers in parallel and/or series, high cooling capacity and/or low temperature can be achieved. Thermoelectric refrigeration systems have many spectacular advantages: they are refrigerant-free, contain no moving parts, have no emission, are easy to maintain, the cold end temperature and its dropping speed can be adjusted by changing current, the cold end and hold end can be flipped by changing current direction, they are compact, etc. Currently the only drawback is their low efficiency at high cooling loads. Accordingly, they can be used in small capacity coolers such as shown in Figure 1-1(c), thermostats, and are especially convenient in cooling electric chips.

\subsubsection{Thermoacoustic approach}

In the last 30 years, thermoacoustic refrigeration was proposed to reduce our dependence on 
vapour compression systems. Sound waves are used to modulate gas parcels, making them oscillate within a short distance inside a tube equipped with a stack. Gas parcels contract and expand during the oscillations, allowing the transport of heat from a low temperature reservoir to a high temperature ambient environment.

Limited by the poor performance of heat exchangers, thermoacoustic refrigerators have a relatively low thermodynamic perfection at $0.1 \sim 0.2$ as opposed to $0.33 \sim 0.50$ for conventional cycles (Wetzel and Herman, 1997). However, by properly selecting the geometric parameters and working fluids, a competitive performance can be achieved (Herman and Travnicek, 2006). Besides, with its capability of reaching liquid nitrogen temperature with a one-stage structure (Dai $e t$ al., 2005), thermoacoustic refrigerator can be used for either refrigeration or cryogenics purposes where only small cooling loads need to be removed.

\subsection{Two main driving forces - high efficiency and low environment impact}

Every system is designed to fulfill a specific goal. Motors convert electrical or chemical energy into mechanical energy; bicycles convey people from one place to another; computers take commands as input, compile them into machine codes, perform high speed calculations, and output digital results. For a refrigeration system, the goal is to remove heat from a cold heat reservoir to a hot heat reservoir. Among the many kinds of refrigeration systems, the most important evaluation criterion is efficiency. To fulfill this purpose, new techniques focused on improving individual component efficiency as well as system performance have been proposed by researchers. For 
example, consider the most popular vapour compression systems: component improvements mainly include heat transfer enhancement, developing new compressors with high efficiency, and optimization of device size enhancing heat transfer; systematic research consists of introducing new components or new cycles such that the irreversibility of the cycle can be reduced (details will be covered in the next chapter). At the same time, some new refrigeration methods emerged, aimed at replacing currently used systems in some special applications, like vortex tube and thermoacoustic refrigeration. Although a new system that can provide both comparative efficiency and cooling capacity to its vapour compression rival has yet to arrive, they are leading the way for the development of refrigeration technology.

The main motive of replacing currently used vapour compression systems is because the refrigerants are not ecologically safe. As for this topic, it would be better to go over the refrigerant history, a history of safety to both human beings and nature. The first generation refrigerants were natural gases or materials used in chemistry labs such as carbon dioxide, ether, ammonia, sulfur dioxide, etc. Nearly all of these refrigerants were either flammable, toxic or both, and some were even highly reactive. Thus accidents were common in the early years of the refrigeration industry. As one exception, carbon dioxide has no chemical disadvantages. However, it operates at high pressures (over 100 bar) that made long-time stable operation a big challenge in the context of contemporary technologies. In 1928, halocarbons (HCs) or chlorofluorocarbons (CFCs) were synthesized by Thomas Midgley. The first commercialized CFC was R12 where "R" represents "refrigerant". Since then, CFC-based refrigeration systems have spread around the world quickly 
and dominated the industry for about 50 years. During this period, usage of other refrigeration systems declined dramatically except for some special locales.

In 1974 the era of CFCs faced a serious challenge. Molina and Rowland (1974) suggested that long-lived organic halogen compounds, such as CFCs, might behave in a fashion that catalyzes the destruction of ozone in the stratosphere. Besides, CFC refrigerants also contributed to global warming. An increase in global temperature can cause other changes, including a rising sea level and variance of precipitation. These changes are thought to be the cause of extreme weather events, such as floods, droughts, and hurricanes. To evaluate the impact of CFCs on the environment, two indices were proposed: Ozone Depletion Potential (ODP) and Global Warming Potential (GWP). On March 22, 1985, 21 countries passed the 1985 Vienna Convention for the Protection of the Ozone Layer to increase public awareness of the potentially harmful impact on human health and the environment through modification of the ozone layer, and take effective steps to protect them. About two years later (September 16, 1987), 46 countries signed the Montreal Protocol on Substances that Deplete the Ozone Layer. The Montreal Protocol is an international treaty designed to protect the ozone layer by phasing out a number of substances that are believed to destroy the ozone layer. A timetable was enacted on which those substances, most of them are CFCs and halons (substances used as blowing and/or extinguishing agents), had to be phased out and eventually eliminated. As a collective effort against global warming, the Kyoto Protocol to the United Nations Framework Convention on Climate Change was signed on December 11, 1997 and entered into force on February 16, 2005. According to the Climate Action Network (2006), more than 160 
countries and over $61 \%$ of global greenhouse gases emissions are covered by the Kyoto Protocol.

Inspired by the Montreal Protocol and the Kyoto Protocol, the refrigeration industry is endeavouring to reduce the refrigerant impact on the environment. Traditionally used refrigerants (such as R22 and R134a) are and will be phased out in favour of carbon hydrates (such as R290 and

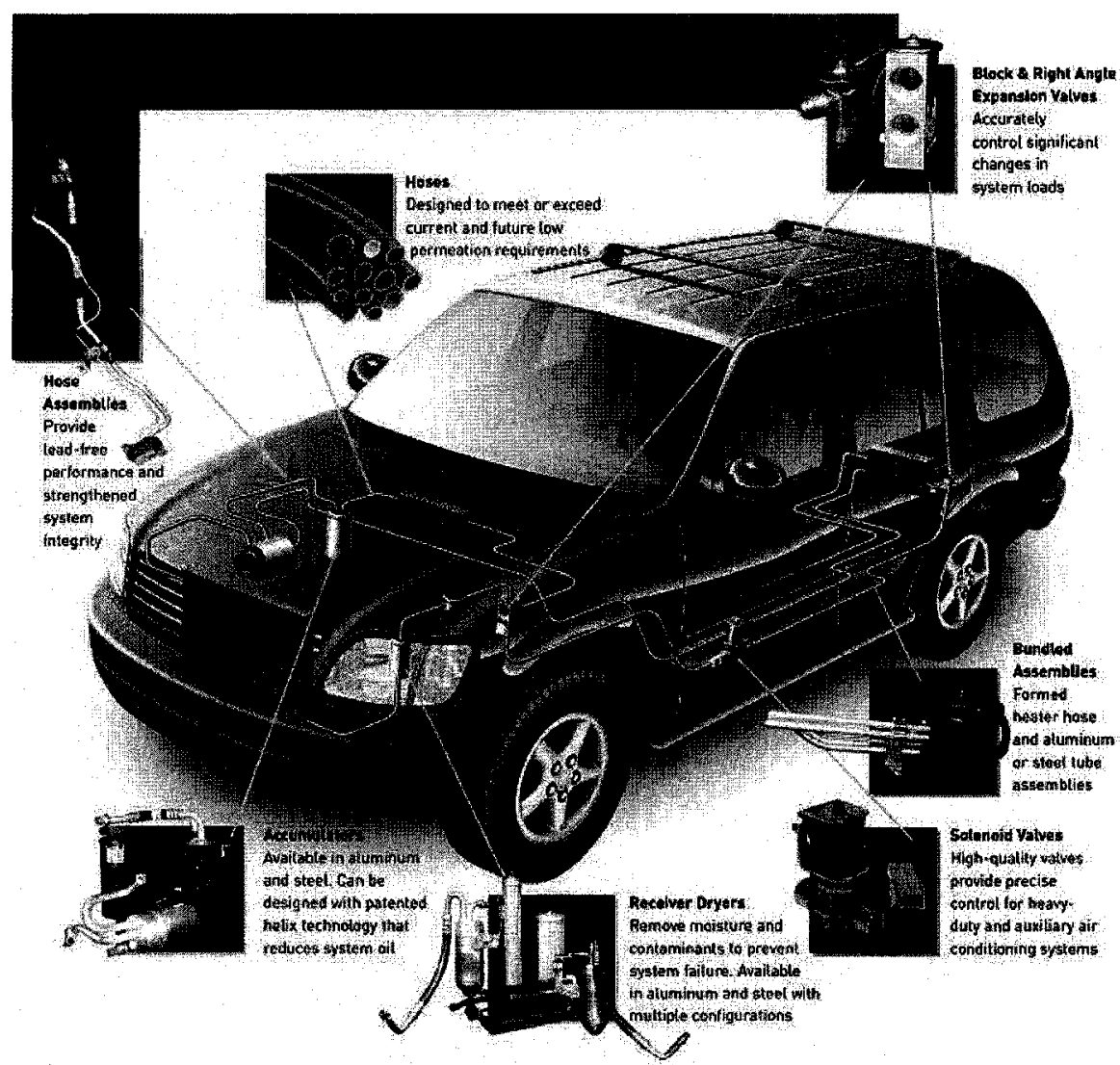

Figure 1-2 Schematic diagram of an AAC system ${ }^{10}$

R600a) and natural refrigerants (such as carbon dioxide). Among these alternatives, carbon dioxide distinguishes itself as a potential low-cost replacement with high specific heat, high thermal conductivity, low viscosity, and excellent availability. The transcritical $\mathrm{CO}_{2}$ system is of great

${ }^{10}$ Modified from http://www.parker.com/ead $/ \mathrm{cm} 2 . \operatorname{asp} ? \mathrm{cmid}=6366$ 
interest to the automotive industry, where it is known as an automotive air conditioning (AAC) system.

Figure 1-2 illustrates a typical AAC system, where two evaporators are adopted to provide a flexible conditioning method and better air distribution inside the passenger compartment. Unlike traditional refrigeration systems where the four components present are a compressor, a condenser, an expander (thermal expansion valve or capillary tube), and an evaporator, in AAC system an accumulator is used to accommodate greatly changing environmental conditions.

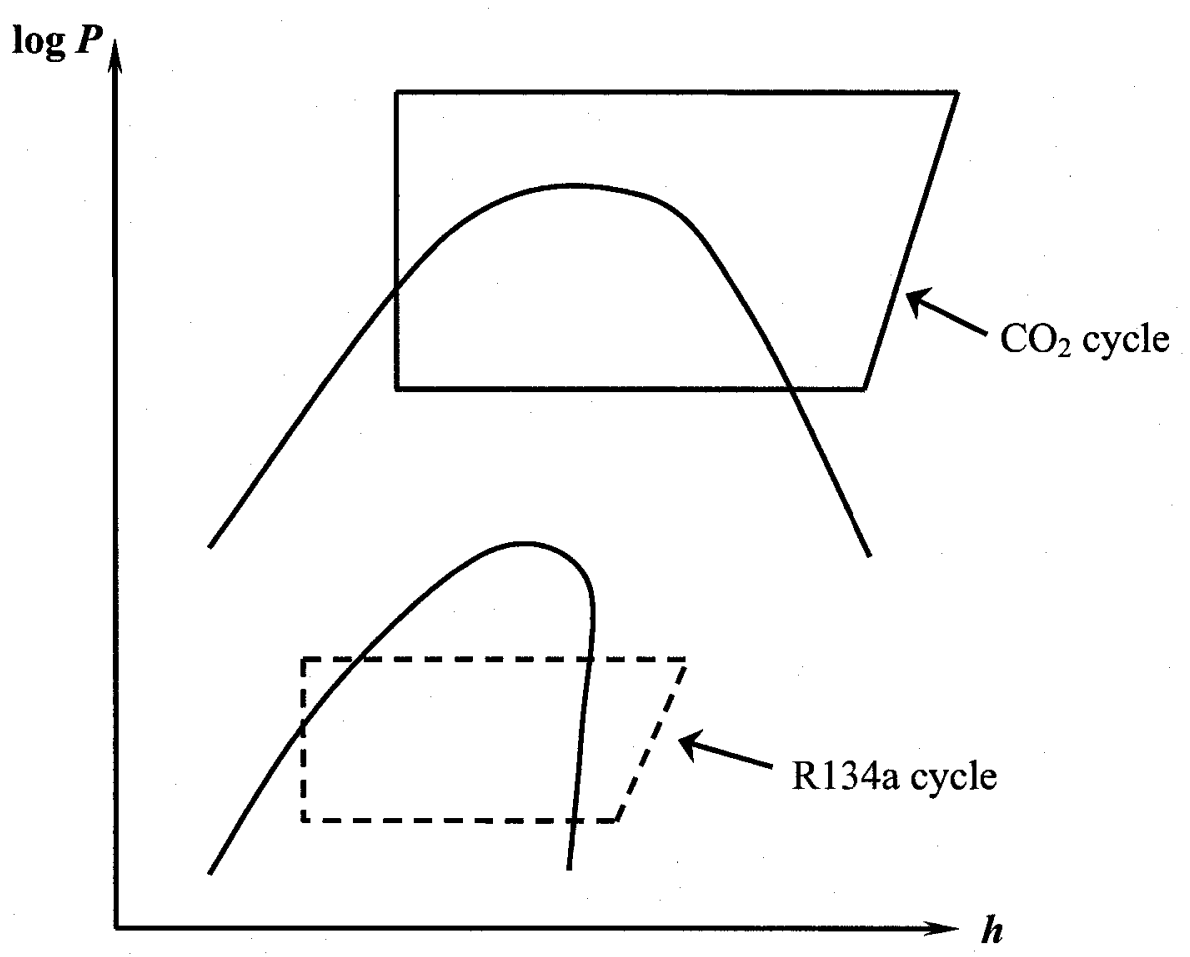

Figure 1-3 Comparison between transcritical and subcritical cycles on a $\log P-h$ diagram

Currently, the refrigerant used is R134a, a type of hydrofluorocarbon (HFC). It has no ODP and less GWP than CFCs. However, increasing concerns about global warming brought about the 
opinion that R134a should not be used long-term because of its GWP, which is 1300 times that of carbon dioxide. Now researchers in the AAC industry are actively working on alternative working fluids for clean refrigeration, and three main candidates, $\mathrm{R} 600 \mathrm{a}, \mathrm{R} 290$, and $\mathrm{CO}_{2}$, have been considered. Research interests turn to these fluids because of their low GWPs; particular attention is being given to $\mathrm{CO}_{2}$, which is also known as $\mathrm{R} 744$. $\mathrm{R} 600 \mathrm{a}$ and $\mathrm{R} 290$ have thermodynamic properties similar to R134a, but their volumetric cooling capacities are small; furthermore, they are flammable and thus only feasible for fixed location or large-scale applications.

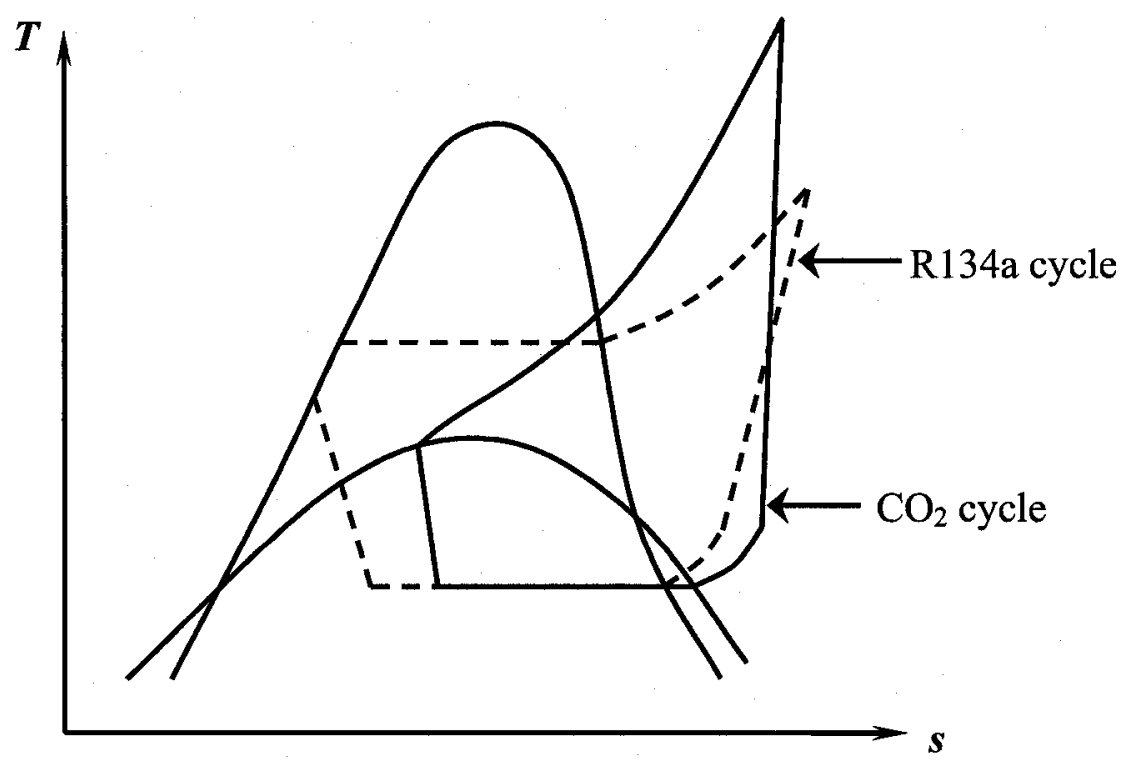

Figure 1-4 Comparison between transcritical and subcritical cycles on a $T$-s diagram

Compared with $\mathrm{R} 600 \mathrm{a}$ and $\mathrm{R} 290, \mathrm{CO}_{2}$ exists naturally and is a by-product of many processes such as the production of beer or $\mathrm{NH}_{3} . \mathrm{CO}_{2}$ is not produced specifically for use as a refrigerant; it can be captured from industrial processes, and then refined for use in refrigeration systems. If released, the overall volume of $\mathrm{CO}_{2}$ gas in the atmosphere does not change. Therefore, using $\mathrm{CO}_{2}$ as a refrigerant will not impact global warming. In addition to its environmental advantages, the $\mathrm{CO}_{2}$ 
has attractive thermal characteristics which make it a viable alternative refrigerant. From an engineering point of view, the main drawback of using $\mathrm{CO}_{2}$ as a refrigerant lies in its high operation pressures. For example, in a typical AAC system without an internal heat exchanger (IHX), the refrigerant evaporates at around $0{ }^{\circ} \mathrm{C}$, the corresponding low side pressures of R134a, R600a, and R290 are 2.93 bar, 4.70 bar, and 1.575 bar, respectively, while that of $\mathrm{CO}_{2}$ is 34.86 bar. When the ambient temperature rises to $35^{\circ} \mathrm{C}$, the high side pressures for the first three refrigerants are around 11.6 bar, 6.2 bar, and 15.3 bar accordingly. Since the critical temperature of $\mathrm{CO}_{2}$ is only around $31^{\circ} \mathrm{C}$, the cycle passes to the supercritical region, and pressure and temperature are no longer in one-to-one correspondence. However, the high side pressure is on the order of 100 bar. Pressure differences from the high-side to the low-side are as high as ten times. Figure 1-3 shows a schematic comparison between $\mathrm{CO}_{2}$ and $\mathrm{R} 134 \mathrm{a}$ systems on a $\log P$ - $h$ diagram, and Figure 1-4 is the corresponding comparison on a $T-s$ diagram. Even though safety issues are more closely considered in new systems, the explosion energy of a $\mathrm{CO}_{2}$ system is actually no greater than a traditional refrigeration system. $\mathrm{CO}_{2}$ works at much higher pressures than CFCs and HCFCs, which provides high volumetric cooling capacity (five times higher than R22 and R134a), and thus makes it possible to build a compact AAC system. The small viscosity provides more opportunity in introducing new small size heat exchangers into the system, which can further decrease the system dimensions. Though the wall of each component is thicker so as to ensure operational safety (partly compromising the deduction of system weight), the new system will be more compact and lighter to some extent. Besides, new material technology allows higher pressures in the thermodynamic cycle, which ensures the operational safety and system performance. 
Another obvious characteristic of a $\mathrm{CO}_{2} \mathrm{AAC}$ system is the essential existence of an IHX. Normally heat exchangers are applied in large-scale systems to increase the cycle efficiency, but rarely used in domestic and mid-scale refrigeration systems due to cost and weight considerations. The low critical temperature also means that the traditional condenser is actually a "gas cooler" because there is no liquid formed at the outlet of the component. If the refrigerant is directly throttled to the low side pressure, the quality at the evaporator inlet is around 0.5 , i.e. the specific cooling capacity $q_{0}$ is limited to a narrow range and only half of the potential heat might possibly be used to generate cooling capacity. In fact, for the sake of oil circulation, the quality at the evaporator outlet should be less than 1 so as to entrain some oil to the compressor, thus making $q_{0}$ even smaller. For a given cooling capacity $Q_{e}$, a smaller $q_{0}$ means more mass flow rate, which results in larger compressor and conveying tubes, and thus more weight. To solve this problem, an internal heat exchanger, exchanging heat between the gas cooler outlet to the expander inlet and the evaporator outlet to the compressor inlet is introduced. After proper selection, the system performance can be kept at least equal to traditional refrigeration systems, which will be discussed in the following section. Further, an internal heat exchanger could avoid sweating on the suction line and slugging the compressor.

For a typical refrigeration system, a compressor, a condenser, an expander, and an evaporator are four essential components; an accumulator is adopted in traditional AAC systems (e.g. using R134a as refrigerant) to adjust the system charge; an internal heat exchanger is introduced in $\mathrm{CO}_{2}$ AAC systems to better the system design and performance. The European AAC industry is expected 
to start switching to $\mathrm{CO}_{2}$ in 2009 and the North American industry is expected to follow three to five years later (Donkers and Vainio, 2004). Moreover, in commercial refrigeration applications, $\mathrm{CO}_{2}$ is also used as refrigerant or liquid/gas phase change secondary refrigerant at the low pressure stage in cascade systems (Billiard, 2002).

\subsection{Focus of the current research}

The current research will be focused on an AAC system equipped with a multi-functional device, AXE, which combines the functions of accumulator, internal heat exchanger, and expansion device into one simple component. Investigations on its behaviour and feasibility in both current and next generation refrigeration systems will be covered. Proper parameter ranges for operation will be discussed and relative suggestions will be provided. Detailed aspects of the research are as follows.

1. Develop a homogeneous numerical model for AXE, and investigate the relationships between thermodynamic parameters (such as high pressure, low pressure, viscosity, conductivity) and geometric parameters (such as inner diameter, outer diameter, length, roughness) to provide support in determining an optimal parameter range of operation.

2. Verify the simulation model by comparing with experimental data in open literature. At the same time, perform comparative experiments using traditional and newly-developed equipment and facilities that are set up in-house to simulate real driving conditions and 
further test the model.

3. Improve understanding for the mini-scale working mechanism of the novel multi-functional device.

4. Investigate influences when a refrigeration system shifts from subcritical to transcritical, and AXE behaviour in both regimes.

5. Investigate the influence of $\mathrm{AXE}$ on the system performance, especially the mass flow rate and operation pressures.

6. Expand the applicability of the AXE simulation model to make it tolerant to different: applications, either a low-capacity refrigerator or high-capacity air conditioner; working fluids, such as $\mathrm{R} 134 \mathrm{a}$ and $\mathrm{CO}_{2}$; flow arrangements, including parallel and counter flow; and heat exchanger types, which can be lateral or concentric. 


\section{Chapter 2}

\section{Literature review}

Often devices are added to an AAC system to make it run well, while increasing its complexity and weight at the same time. For the sake of compactness and high efficiency, many investigations have been undertaken. These works can be divided into two categories: improvement in system structure and investigation of componential mechanism.

\subsection{Structural improvement}

Since the traditional four parts have been well developed, there is little possibility to integrate some of them. Hence, the prior art focused on simplifying the structure of newly introduced devices, such as the IHX and the accumulator. Together with the expansion device, these three components are smaller than the other components. Accordingly, some integration research has been performed on how to reduce the number of components. On the other hand, new components are being proposed to improve the cycle efficiency.

\subsubsection{Component integration}

As for component integration of the three small devices, four possible integrating combinations are available, and they are a) accumulator-expander; b) accumulator-IHX; c) IHX-expander; and d) accumulator-IHX-expander. Several designs have been proposed by Mei and 
Chen (1993; 1997), Geiger et al. (2002), Dickson et al. (2002), Zhang et al. (2003), Makizono et al. (2003), and Yin et al. (2004). Except for Mei and Chen (1997), all the designs consider an integration of the IHX and the accumulator, while the expander is left as an independent device.

Mei and Chen (1993) put a segment of tube containing refrigerant from the condenser directly into the accumulator, and part of that was inside the liquid. By this means the hot liquid can be substantially cooled down which greatly reduces the quality at the evaporator inlet such that the tube surface area of the evaporator can be more effectively used. Four years later, Mei and Chen (1997) improved their system by integrating the expander with the accumulator-IHX, where a capillary functions as both an expander and part of a heat exchanger. The capillary is immersed in the accumulator liquid. Hot refrigerant enters it, being throttled and dissipating heat to the surrounding liquid.

According to Geiger et al. (2002), the main IHX has a multichannel tube wound into a radial spiral or a meander shape and is placed inside the accumulator. To get better performance, an additional multichannel IHX could be placed between the main IHX and the condenser/gas cooler.

An IHX was placed in the accumulator with different embodiments as illustrated by Zhang et al. (2003). The first type of heat exchanger to be used was concentric tubes, where the low temperature fluid goes through the internal tube. The heat exchanger is then placed underneath the deflector (a device preventing liquid from directly entering the J-tube). Another type is to wind a 
single radial spiral and place it on top of the reflector.

Makizono et al. (2003) divided the accumulator into two separate parts and designated the upper chamber for heat exchange. Most of volume of the IHX is used to contain the low temperature refrigerant, and the refrigerant from the condenser enters it and is cooled down. A plurality of parallel microchannels could be used to contain the high side refrigerant and shaped as a radial spiral. Detailed inlet and outlet structures were also provided.

Dickson et al. (2002) use a specific reservoir to store the liquid of the accumulator and wind the heat exchanging tubes around its outer wall. Vapor from the evaporator goes across the tubes and cools the high temperature fluid inside.

Yin et al. (2004) proposed another device to combine the IHX and the accumulator. A multichannel strip containing high temperature refrigerant was attached in parallel to the J-shape tube of the accumulator for most of the length. Fins were adopted to increase the heat transfer performance. Part of the IHX is immersed in the accumulator liquid.

Except for Mei and Chen (1997), all the other approaches were dealing with integration of the accumulator and the IHX. For those designs placing the IHX in the upper part of the accumulator, there is no theoretical difference with the discretized systems. For those designs immersing part of or the entire heat exchanger unit into the accumulator liquid, the temperature difference between the in-tube capillary flow and the outside flow is higher than the previous design. But the system 
performance change little, though the design can decrease the size of evaporator, which is meaningful for size-sensitive applications such as automotive refrigeration/air conditioning systems.

Mei and Chen (1997) proposed an illuminating complete integration of the three components into one simple device, but it still has its shortcomings. From a thermodynamic point of view, the specific cooling capacity is increased at the cost of vaporizing a certain amount of liquid, which generates extra gaseous flow to be compressed. The suction line refrigerant quality is mainly determined by the size of and the pressure drop over the accumulator. They do not change much in this case. Therefore, both the cooling capacity and the power consumption increase. Due to irreversibly losses, the system performance should be somewhat lower than a cycle without an IHX.

\subsubsection{New component to improve cycle efficiency}

As the key component of a refrigeration system, numerous related researches have been carried out on the compressor to improve its efficiency. One of the most important improvements is an intercooled two-stage compressor proposed by Yamasaki et al. (2001). Two-stage compressors are suitable for applications where large pressure lift is expected for two reasons: the poor compressor performance at a high pressure ratio, and the difficulty in compressing a "too-large" pressure difference (due to a lack of reliable sealing), which is especially important for $\mathrm{CO}_{2}$ cycles. In the thesis, because of its compact size, light weight, and high reliability, a hermetic $\mathrm{CO}_{2}$ rolling piston compressor was considered as the original model to be improved. A two-cylinder, two-stage 
compression design that ensures low vibration and low noise level was developed. The new compressor was shortly applied in domestic heat pump water heaters. In the patents that followed Matsumoto et al. (2004), and Sato el al. (2005), provided details about the design. According to Matsumoto et al. (2004) the refrigerant was compressed and discharged by the first rotary compression element, then drawn into the second element, being compressed and discharged thereby. The ratio of discharge port areas (the second element over the first element) was set to be smaller than the ratio of displacements. The ratio of area is between 0.55 and 0.85 of the ratio of displacement. Sato el al. (2005) further detailed the cooling device that cools down the refrigerant from the first compression element and thus decreases the compression work of the second element. $\mathrm{A} \mathrm{CO}_{2}$-driven cooling system that could provide higher efficiency than its $\mathrm{R} 134 \mathrm{a}$-based counterpart was proposed by Inoue et al. (2005), which consists of a newly designed two-stage compressor and internal heat exchanger. The system was expected to be applied in a wider range of applications including showcases and freezers.

Other efforts to improve compressor efficiency are specially focused on refrigerators. Different from other cycles where only one evaporating temperature is desired, two steady temperatures need to be kept for the freezer chest and the cooler chamber. Three approaches can be adopted to maintain two evaporating temperatures: there can be two refrigeration cycles, or non-azeotropic refrigerant mixtures can be used or else two capillary tubes with a proper mixing device can be placed at the compressor inlet. It is neither economical nor feasible to put two separate systems into one refrigerator shell which has space and weight limitations. So using two refrigeration cycles is 
not a good approach. Non-azeotropic refrigerants are a feasible solution. The component with the lower boiling point evaporates in the cooler chamber, and the other component with the higher boiling temperature evaporates in the freezer chest. There is little or no requirement of system modification, which makes it suitable for improving the performance of current refrigerators. But the biggest challenge is the long-term performance, which greatly relies on the composition of refrigerant mixtures. The composition will change with leakage, and the system performance deteriorates rapidly if the designed optimum composition ratio cannot be well maintained. Due to this, a double-circuit refrigeration cycle with a new compressor was proposed and analyzed by Chen et al. (2001). Gas Compensating Process (GCP) in a single-cylinder compressor was allowed such that returning refrigerant vapours at different pressures could be conveyed into the compressor without adding extra devices. The feasibility of using this GCP compressor in a domestic refrigerator for production of a larger cooling capacity was carried out. Based on a thermodynamic model, relative simulating programs were developed, in which refrigerant mass flow rates of the suction process and the compensating process were dependent parameters that varied with suction temperature, the position of the compensating chamber, piston shape and the physical characteristics of cylinder and suction valve. Accordingly, a curve of cooling capacity was drawn. Theoretical analysis showed that when the first intake temperature is constant, there exists an optimum size of the compensating chamber that maximizes the cooling capacity. The new compressor could greatly enhance cooling capacity.

It needs to be mentioned that the two-stage compressor could be used as an effective 
component for refrigerators. Because it intakes gas at two different pressures, the high pressure refrigerant from the condenser can be mixed with the discharged refrigerant from the first compression element and then sent to the second compression element. Further investigation is required to determine whether the two-stage compressor is better than the GCP or not.

\subsection{Thermal physical investigation}

Many researchers are endeavouring to improve the performance of heat exchangers and accumulators; the main input is from Lin et al., Bansal et al., Melo et al., García et al., Radarmacher et al., Hrnjak et al., Domanski et al., and Pettersen et al.. Their work can be categorized into three main areas: capillary flow study, system performance research, and investigation of refrigerants and lubricants.

\subsubsection{Capillary flow study}

Lin's work is closely related to experimental capillary flow behaviour, especially the pressure drop. The metastable flow phenomenon inside two capillary tubes using R12 as the working fluid was presented by Li et al. (1990). The tubes are $1.50 \mathrm{~m}$ long, and have inner diameters of $0.66 \mathrm{~mm}$ and $1.17 \mathrm{~mm}$, respectively. It was found that the underpressure of vaporization is low with large capillary inner diameter, greater subcooling degrees, and low mass flux. The metastable region is short with a large capillary inner diameter as well. The flash point, where the fluid begins to vaporize, does not change much with the back pressure, but the flow pressure in the two-phase region varies if the flow is choked. 
Based on their experimental results, the frictional pressure drop factor in these capillary tubes was further investigated (Lin et al., 1991). It was found that the single-phase frictional factor can be calculated by Churchill's equation (Churchill, 1977). For the two-phase region, a model was developed and can predict experimental data with uncertainties of about $15 \%$. They also proposed that the quality profile is non-linear and increases more rapidly near the capillary exit, which directly leads to a sharp pressure drop in that region.

When the refrigerant was changed from R12 to R134a and non-adiabatic capillary flow was taken into account, underpressure of vaporization was investigated experimentally by Chen and Lin (2001). A capillary-tube-suction-line heat exchanger was considered, where a capillary of $1.50 \mathrm{~m}$ length and $0.6 \mathrm{~mm}$ inner diameter was partially soldered to a suction line of $1.36 \mathrm{~m}$ length and 4 $\mathrm{mm}$ inner diameter. The first $0.14 \mathrm{~m}$ and the last $0.1 \mathrm{~m}$ of the suction line were unattached with the capillary. Experimental results showed the underpressure of vaporization decreases with an increase of heat transfer. A correlation based on experimental data and classic nucleation theory was then developed to predict the underpressure of vaporization of refrigerant flow for both adiabatic and non-adiabatic situations.

Bansal dabbled in many aspects of system analysis, but his most important contribution is on capillary flow study. An empirical approach was presented to develop simple correlations for sizing both adiabatic and non-adiabatic capillary tubes (Bansal and Rupasinghe, 1996). The maximum disagreement was found to be less than $\pm 8 \%$ for both cases. Later on they proposed a homogeneous 
model for expansion in a capillary (Bansal and Rupasinghe, 1998), which was used to investigate the relationships between thermodynamic and fluid flow parameters and could give valuable information in sizing capillary expansion devices and determining the critical choking condition.

Based on the adiabatic model, Xu and Bansal (2002) developed a non-adiabatic homogeneous model for capillary flow. R134a was used as the working fluid in this study. The model was developed based on assumptions that a lateral counter flow heat exchanger arrangement was considered, and no oil was entrained into the flow. The model accuracy was found to be within $\pm 20 \%$. By analyzing the flow behaviour using the model, the refrigerant tends to condense again within the heat exchanger region after it enters the two-phase region when the heat transfer effect is too strong, which can be treated as a fundamental description of "re-condensation". Such phenomenon was discussed in detail by them in a following study (Bansal and $\mathrm{Xu}, 2003$ ), where re-condensation was considered to be responsible for the discontinuities in some operation conditions. This is particularly obvious for high condensation temperatures. If re-condensation exists, a much longer capillary tube is required to throttle the refrigerant flow to a choking condition.

To obtain a parametric range of choking situations, Bansal and Wang (2004) presented a homogeneous adiabatic model. The model was validated by comparing with experimental data for $\mathrm{R} 22, \mathrm{R} 134 \mathrm{a}$, and R600a; the agreement is within $\pm 7 \%$. Through extensive parametric analysis, a new diagram called the "full range simulation diagram" was proposed as a visual approach to help 
understand the choking situation, which could be treated as a graphical explanation of fanno flow in capillaries.

Yang and Bansal (2005) investigated the influences of different parameters on a suction capillary-tube-suction-line heat exchanger using $\mathrm{R} 134 \mathrm{a}$ and $\mathrm{R} 600 \mathrm{a}$ as refrigerant. They found R134a outperforms R600a in both heat transfer rate and cooling capacity. If the inlet capillary tube is not well insulated before it attaches to the suction line, an $8 \%$ to $10 \%$ drop of heat transfer rate was observed.

As a further improvement in study on re-condensation phenomena, Bansal and Yang (2005) carried out a simulation work for non-adiabatic situations using R134a and R600a, respectively. A simple linear correlation was developed to express the relationship between the changes in pressure drop and the temperature along the capillary tube. Convergence problems were met when re-condensation occurs and the evaporating temperature is relatively low.

There are two aspects of Bansal's team's work that need further investigation. One is the heat transfer condition in concentric tube heat exchangers. Even in his latest non-adiabatic analysis on capillary flow, they only simulated the lateral tube heat exchanger of domestic refrigeration systems. Owing to a high two-phase heat transfer coefficient surrounding the inner tube, it could be reasonably expected that the concentric situation will give more heat transfer. The other is that most refrigerants being used in the discussion will be phased out in the near future, while the new 
features using $\mathrm{CO}_{2}$ is not available in his work yet. Because the $\mathrm{CO}_{2}$ cycle is transcritical, some particular characteristics should be considered. For example, Fang et al. (2001) pointed out that the inertial drag force plays an important role in the pressure drop inside a gas cooler, so it ought to be included in the momentum balance equation. Moreover, sharp changes of thermophysical parameters near the critical point will cause convergence problems, which is a big challenge for simulation. The first problem was taken into account by Melo et al. and García et al., as will be shown below. The second question is still an open area and not many achievements can be found in open literature.

Experiments on adiabatic capillary flow using different refrigerants including R12, R134a, and R600a were conducted and over 1000 sets of data were summarized by Melo et al. (1999). Five geometric and operational parameters, including capillary length, capillary inner diameter, subcooling, condensation pressure, and refrigerant type, were taken into account for eight capillaries. Capillary inner diameter was found to affect the mass flow rate greatly. Under the same condensation pressure, the mass flow rate of R134a is slightly lower than R12 and about 33\% higher than R600a. The Buckingham Pi theorem (1914) was adopted to derive a non-dimensional correlation to predict the mass flow rate and it is in good agreement with the measured data and other results from the open literature. The correlation is useful in the direct calculation of the mass flow rate of the system if the capillary size and a working condition are given.

For non-adiabatic flow, R600a was experimentally evaluated on concentric capillary-tube- 
suction-line heat exchangers in household refrigerators. Two correlations of the mass flow rate and the suction line outlet temperature were experimental determined, separately. The effects of each parameter were shown in column charts, which provide a direct comparison of their relative importance.

García et al. (2002a) developed a one-dimensional numeric method to analyze capillary flow for pure and mixed refrigerants. Metastable regions in liquid and two-phase region were considered, and the model is capable of calculating adiabatic and non-adiabatic flow for critical or non-critical conditions for steady as well as transient situations. Comparisons with experimental results showed that considering the metastable region will improve prediction accuracy of the mass flow rate (García et al. 2002b). Good agreements were obtained between simulated and measured data for both adiabatic flow and non-adiabatic concentric type flow.

García et al. (2006a) further improved upon their earlier mathematic model; different correlations for concentric and lateral heat exchangers were presented so as to obtain better prediction results. The same Newton-Raphson algorithm as used in García et al. (2002a) was adopted in iterations of mass calculation. Of all 196 measured mass flow rates, $96.4 \%$ of them are predicted within an error of $\pm 15 \%$ and averaged at $\pm 6.3 \%$. Of the 143 data points for suction line outlet temperature, $89.5 \%$ of them are predicted by variations within $\pm 2{ }^{\circ} \mathrm{C}$ and the mean value is $\pm 0.98{ }^{\circ} \mathrm{C}$. Divergences were also reported at those conditions at which re-condensation occurred. 


\subsubsection{System performance research}

Brown et al. (2002) compared the performance of two AAC systems using $\mathrm{R} 134 \mathrm{a}$ and $\mathrm{CO}_{2}$ as refrigerant. As suggested by Boewe et al. (1999) and Preissner et al. (2000), an additional IHX was adopted in the $\mathrm{CO}_{2}$ cycle. Calculation results showed that under various conditions the $\mathrm{CO}_{2}$ cycle performs $21 \%$ less efficiently when the ambient temperature is $32.2^{\circ} \mathrm{C}$ and $34 \%$ less effectively at $48.9^{\circ} \mathrm{C}$. The disparity was mainly attributed to the large entropy generation in the gas cooler for the $\mathrm{CO}_{2}$ cycle. Two points can be addressed. One is that the COP performance of the transcritical cycle can be further improved. In the comparison, the optimum discharge pressure might not have been achieved. The effectiveness of the IHX can be optimized to improve the cooling capacity while still maintaining a proper discharge temperature at the same time. The isentropic efficiencies for both cycles were the same in the comparison, which is conservative for real systems. In fact, a $\mathrm{CO}_{2}$ compressor will benefit from a lower compression ratio, making the isentropic efficiency at not worse than that of an R134a compressor. Moreover, as previously mentioned, a specially designed $\mathrm{CO}_{2}$ compressor can greatly improve the system performance (Inoue et al., 2005). The second point is that water heating might be another promising application field for $\mathrm{CO}_{2}$ systems because of the thermal driven force and excellent supercritical thermal physical properties of the fluid. Hwang and Radermacher (1998) compared the theoretical performance of R22-based and $\mathrm{CO}_{2}$-based systems for water heating and water chilling applications. They concluded that the $\mathrm{CO}_{2}$ cycle provides better performance. 
As for Radermacher, his team is concentrated on simulation and optimization and gave detailed models for accumulators, micro channel heat exchangers and space heat pumps. Zhao et al. (2001) built a $\mathrm{CO}_{2}$ test rig, and developed a semi-empirical model for predicting capacities of heat exchangers by introducing two new parameters, CTA (moisture condensate factor) and BTA (superheat related factor). The model results agree with experimental results of the evaporator within $13 \%$, while predicting the experimental data of the gas cooler within $20 \%$. The greater difference for the gas cooler might be caused by the significant property changes occurring at the supercritical area, especially near the critical point. His team also developed a numerical model for an accumulator using R134a as refrigerant (Schwarz et al., 2002). The model quantifies the pressure drop and discusses the influences of oil; it could not provide satisfactory coordination with the real device in monitoring liquid height.

Pettersen's team originated from Lorentzen who foresaw the renewed importance of the $\mathrm{CO}_{2}$ refrigeration system (Lorentzen and Pettersen, 1992; Lorentzen and Pettersen, 1993). They are focused on investigating micro scale heat transfer characteristics, especially related to $\mathrm{CO}_{2}$ refrigeration systems. His group developed a microchannel gas cooler (Pettersen et al., 1998) that showed competitive performance with traditional round tube gas coolers but were smaller and lighter. Compared with channels of normal size, microchannels have many heat transfer advantages. They provide an increased heat transfer surface area and a large surface-to-volume ratio, which results in much better heat transfer. Pettersen et al. (2002) further discussed the feasibility of manufacturing a domestic micro propane heat pump using MEMS (Micro Electro-Mechanical 
Systems) technology. Under certain conditions and with relatively low pressure drop, the heat transfer coefficient in a micro condenser is between $7,000 \mathrm{~W} /\left(\mathrm{m}^{2} \cdot \mathrm{K}\right)$ and $8,000 \mathrm{~W} /\left(\mathrm{m}^{2} \cdot \mathrm{K}\right)$, and in micro evaporator the number could even reach as high as $20,000 \mathrm{~W} /\left(\mathrm{m}^{2} \cdot \mathrm{K}\right)$. Although these values would be different when using $\mathrm{CO}_{2}$ as the working fluid, similar results can be expected because $\mathrm{CO}_{2}$ has low viscosity. This speculation was validated by Munkejord et al. (2002) and the two-phase heat transfer coefficient was found to be more than $10,000 \mathrm{~W} /\left(\mathrm{m}^{2} \cdot \mathrm{K}\right)$. One important result of their paper is the selection of proper refrigerant flux. Low mass flux is recommended for prevention of both a large pressure drop (that would cause smaller temperature difference) and dry out (that would greatly affect the heat transfer coefficient). The relationship between the two-phase flow distribution in a microchannel heat exchanger and its geometric parameters, including inlet pipe length and inlet pipe inner diameter, was discussed in detail by Vist and Pettersen (2004). As a staged product of their work, a comprehensive fundamental process and system design for a $\mathrm{CO}_{2}$ vapour compression system was provided by Kim et al. (2004). Microchannels support high mass flux with a relatively low temperature gradient; however, they also have possible weaknesses such as high pressure drop, flow mal-distribution, and high cost of manufacturing, depending on the refrigerant choice and the design being adopted. Compared with other gases, low viscosity $\mathrm{CO}_{2}$ fits microchannels better; the flow could be well distributed under proper design; and of course, the manufacturing cost could be lowered by mass production.

Hrnjak's group is more focused on understanding flow behaviour and improving heat exchanger effectiveness through experimental means. ElSherbini et al. (2003) made much 
instructive progress in the heat contact resistance of heat exchangers that could be included in the numerical model. The experimental results show as much as a $50 \%$ difference in contact heat resistance between brazed fin-tube joints and unbrazed joints. Hrnjak et al. (2001) also investigated the running performance of a transcritical heat pump and discussed the design optimization of gas coolers.

Liao et al. (2000) analyzed the optimum heat rejection pressure to maximize COP. Their work is based on a steady fluid flow model: the change of kinetic energy is not considered. This simplification is feasible for typical heat exchangers, while not exactly applicable to supercritical situations. Because of the special properties of $\mathrm{CO}_{2}$ (the specific volume changes greatly at high-side pressure and low-side pressure), kinetic energy should be incorporated. Zhao and Liao (2002) also performed another investigation on film condensation heat transfer inside mini triangular channels. The three-zone method is well developed and discussed in the paper, which provides an efficient way to unveil the physics of fluid flow in small geometries.

\subsubsection{Investigations on refrigerants and lubricants}

Grebner and Crawford (1993) measured the pressure-temperature-concentration relations and developed a model that covers R12/naphthenic mineral oil, R12/paraphinic mineral oil, R134a/PAG oil, and $\mathrm{R} 134 \mathrm{a} /$ Ester oil. Thermodynamic properties at three lubricant concentrations $(1 \%, 5 \%$, and $10 \%)$ were calculated using the model. 
Wang et al. (2006) investigated the effects of vapour quality and oil concentration on the performance of a swash plate compressor in detail. Vapour quality does not affect volumetric efficiency but does affect isentropic efficiency. When the quality increases from 0.8 to 0.9 , the heat exchanger's performance goes down. Only a portion of the liquid refrigerant evaporates. An evaporation ratio was proposed to embody this phenomenon; it decreases with compressor speed and quality.

Joudi et al. (2003) put five refrigerants, R12, R134a, R290, R600a and a mixture of R290/R600a (62/38 molar fraction), into a model separately to determine the most suitable alternative for R12. Results show the mixture is the best choice. After that, an experimental comparison was carried out between R12-based and R290/R600a-based systems. The R12 and R290/R600a systems behaved similarly: R12 was superior in pressures, $W_{\text {comp }}$, and COP, whereas the mixture outperformed pure R12 in subcooling, superheating, evaporator exit air temperature, and cooldown time. The system is a receiver/dryer type, and no IHX is taken into account.

Itard (1995) found that wet compression for heat pumps using R12, $\mathrm{R} 123, \mathrm{NH}_{3}$, and $\mathrm{NH}_{3} / \mathrm{H}_{2} \mathrm{O}$ (80/20) was not found to be beneficial to the system performance. Thermodynamic analysis shows that for non-azeotropic mixtures, solution recirculation offers better system performance. The reason might be that the excessive liquid refrigerant increases the heat transfer coefficient, and thus improving heat transfer. 
Voster and Meyer (2000) studied wet compression versus dry compression in heat pumps. In total 34 pure refrigerants and 31 non-azeotropic binary mixtures are considered. All re-entrant shape refrigerants and mixtures were found to yield better COPs at dry compression. Only R22, R32, and NH3 with bell-shaped $T-s$ curves reached higher COPs at wet compression. An R134a-based system cannot benefit from wet compression. For R22, R32, and NH3, though COP increases with wet compression, heat capacity decreases. 


\section{Chapter 3}

\section{Flow behaviour in adiabatic and non-adiabatic capillary tubes}

A capillary tube is a long-drawn tube with small inner diameter ranging from 0.5 to $3 \mathrm{~mm}$ and used as expansion device in refrigeration systems, especially in domestic refrigerators. In an AAC clutch cycling orifice tube (CCOT) system, an orifice tube converts hot, high pressure liquid from the condenser into cold, low pressure, two-phase flow before entering the evaporator. When in conjunction with an orifice tube, an accumulator located between the evaporator and the compressor stores excess fluid to accommodate various mass flow rates due to various climates, trapping the contaminates and absorbing moisture in the flow. The main characteristic of a CCOT system is that there are no moving parts in the expansion device, lowering the manufacturing cost and simplifying the maintenance. Since the tube diameter is fixed, the flow has to be metered by turning the compressor on and off, hence the name CCOT. Another approach to control the flow between the high side and the low side of a system is to use a thermal expansion valve (TXV). A thermocouple is attached at the evaporator outlet, and the temperature is used to meter the flow passing the TXV. The magnitude of the restriction is determined by the size of the opening of the valve. In the current research, the orifice tube is replaced by a capillary tube with larger inner diameter and greater length. There are two reasons for doing this. Firstly, it ensures the same pressure drop as was created using the orifice tube. Secondly, the longer tube provides more surface area, which allows it to function as a heat exchanger in the new device. For analysis, capillaries can 
be divided into two types: adiabatic and non-adiabatic. This chapter will discuss the capillary flow characteristics of both cases and examine the differences between them.
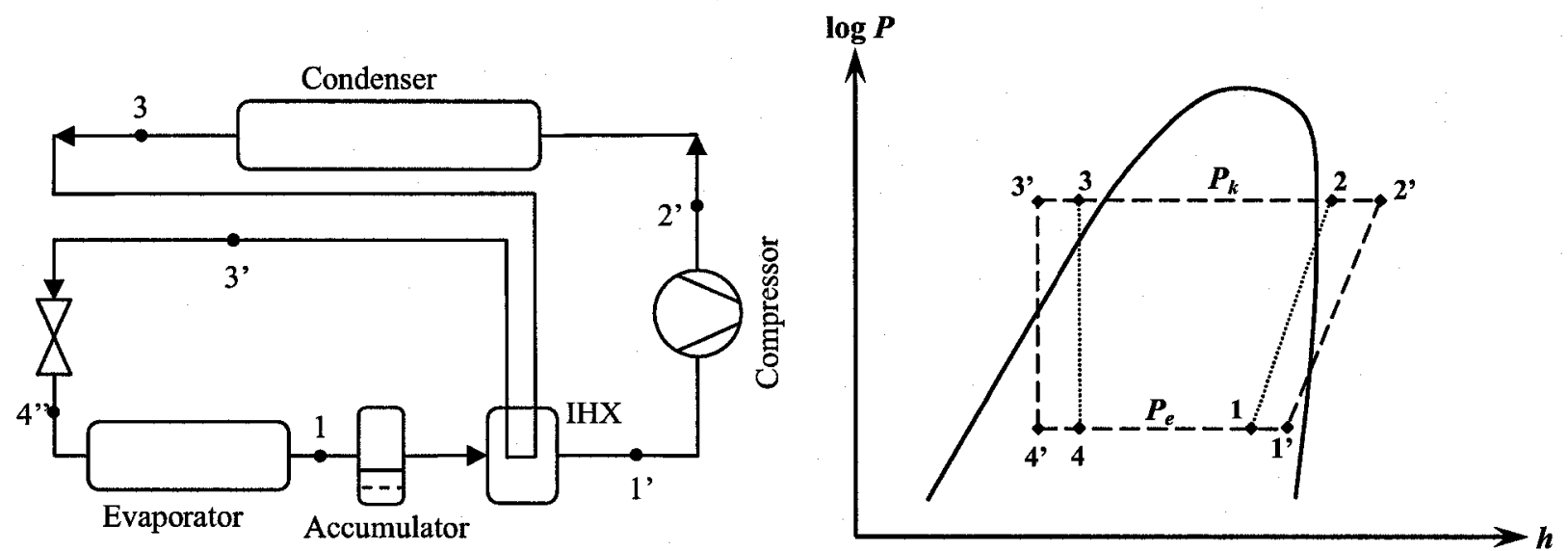

Figure 3-1 Schematic and $\log P$ - $h$ diagram of an R134a-based AAC system with an IHX

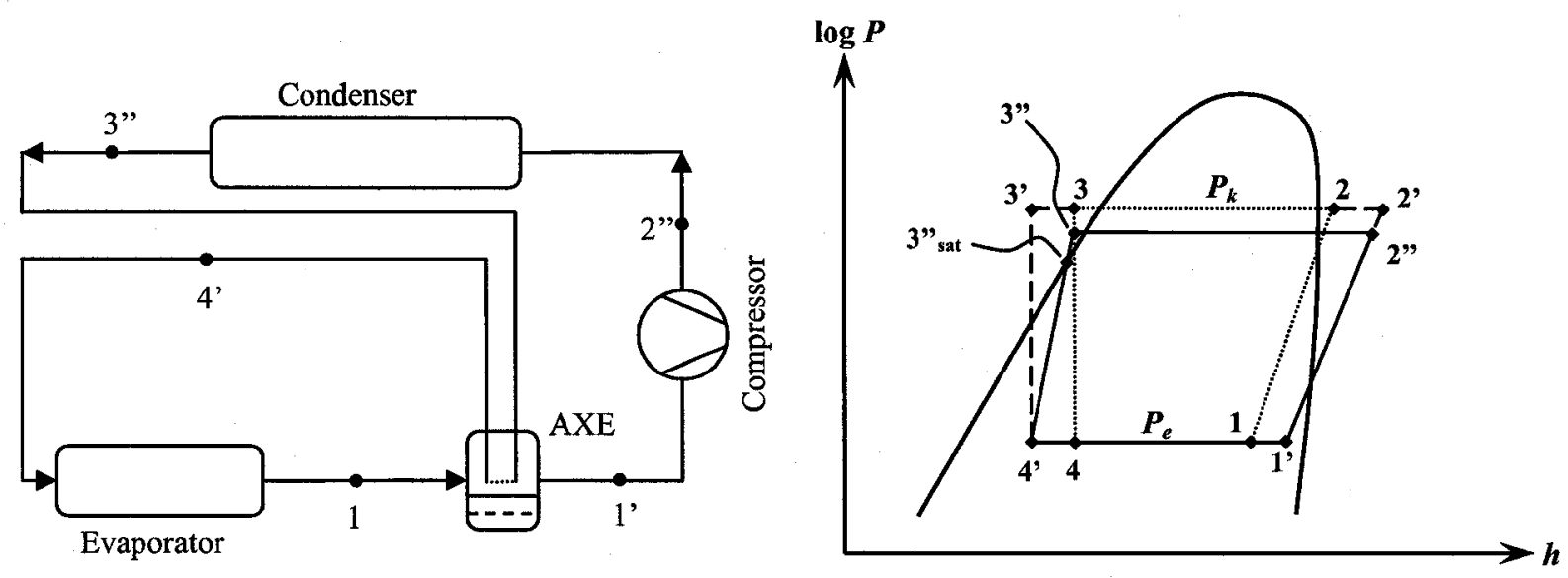

Figure 3-2 Illustration of a refrigeration system with an AXE

Figure 3-1and Figure 3-2 contain schematic diagrams as well as relative $\log P-h$ diagrams of two refrigeration systems. Figure 3-1 is an AAC system equipped with three separate devices, an accumulator, an IHX, and an expansion device. The cycle is represented by loop $1^{\prime}-2^{\prime}-3^{\prime}-4^{\prime}-1$ '. The hot refrigerant exiting the condenser (point 3) gives heat to the cold fluid from the evaporator (point 1), making the temperature of itself decrease to point 3 ' and that of the other flow increase to point 1'. If the IHX is removed, the rest of the system will be a traditional cycle that can be represented 
by the loop 1-2-3-4-1. While Figure 3-2shows a system in which the accumulator, the IHX, and the expander are integrated into a single new device: the AXE. The relative cycle is represented by loop 1'-2"-3"'-4'-1'. The AXE combines the function of heat transfer and expansion into one capillary tube, which can be assembled in the accumulator or the suction line, thus greatly simplifying the system structure and reducing its weight. Compared with the other two loops in the $\log P-h \operatorname{diagram}$ of Figure 3-1, this cycle has two new features: 1) a non-adiabatic expansion process corresponding to line 3"-4' emerges, and 2) the discharge pressure decreases. The high-pressure refrigerant in the capillary tube conveys heat to the suction line vapour while simultaneously being throttled to evaporation pressure, leading to the first feature. The second feature is a result of the heat transfer characteristics of the capillary tube, the condenser, and the evaporator; this will be discussed in detail in the following chapters. It is worth noting that the second feature is observed by following two steps that: 1) the orifice tube is replaced with a capillary such that its pressure drop is the same as that of the orifice tube, and there is no heat transfer occurring across the capillary wall; 2) the capillary tube is combined with the other two components and placed into the system. The pressure drop between points 3 " and 4' can be increased by increasing the capillary length in the second step.

\subsection{Adiabatic capillary flow}

Among the numerical studies of adiabatic capillary flow in open literature, three conservation laws were required: the conservation of mass, momentum, and energy. Most studies are based on homogeneous flow assumption. Though Wong and Ooi (1996) used a separate flow model to 
investigate the flow behaviour of R12, their technique was not widely applied to the field due to a lack of experimental support for determining the slip ratio of different refrigerants. Even if sufficient slip ratio data were available, the prediction improvement varies case by case and is not significant overall. For these reasons, a homogeneous model is adopted in the current research for the in-tube flow and the outside refrigerant flow. To consider the effects of non-equilibrium, especially during a phase changing process, a technique is used to calculate the two-phase specific volume.

\subsubsection{Model description}

The capillary tube under investigation is illustrated in Figure 3-3. The capillary tube can be divided into three distinct sections according to the flow status: the single-phase region, the metastable region and the two-phase region. Li et al. (1990) and Meyer and Dunn (1998) observed obvious non-equilibrium flow near the flash point, where the single-phase refrigerant starts vaporizing and enters the two-phase region. Note that for saturated two-phase flow, an extreme choking situation may exist at the outlet when the back pressure is low.

\subsubsection{Assumptions}

In order to simplify the true flow condition without losing the main physical characteristics, the following assumptions are made:

- One-dimensional flow.

- Horizontally placed capillary tube. 


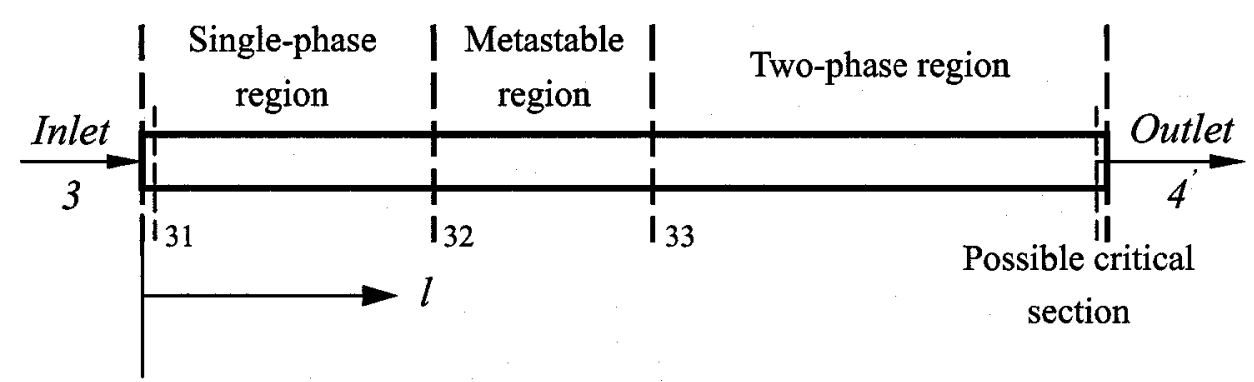

Figure 3-3 Adiabatic refrigerant flow in a capillary tube

- Steady state homogeneous flow.

- Incompressible flow in single-phase region.

- Constant inner diameter and uniform surface roughness throughout the tube.

\subsubsection{Governing equations}

It can be seen from Figure 3-3 that the total length of the capillary tube consists of three parts

$$
L=L_{s p}+L_{\text {meta }}+L_{t p}
$$

In the current research, the metastable region is considered to be part of two-phase flow. The reason for this decision is based on the new technique that adopted in determining the specific volume of the two-phase region embodies the late evaporation. Equation (3-1) can now be simplified as

$$
L=L_{s p}+L_{t p}
$$

For steady capillary flow, the mass flow rate does not change; therefore the mass balance equation is simply

$$
\dot{m}=\text { const }
$$




\subsection{Energy balance equation}

The energy conservation equation for an arbitrary section of length $d l$ (see Figure 3-4) can be written as

$$
h+\frac{1}{2} V^{2}=(h+d h)+\frac{1}{2}(V+d V)^{2}
$$

For a tube with constant inner diameter

$$
V=\dot{m} v=\frac{G v}{A_{i}}=g v
$$

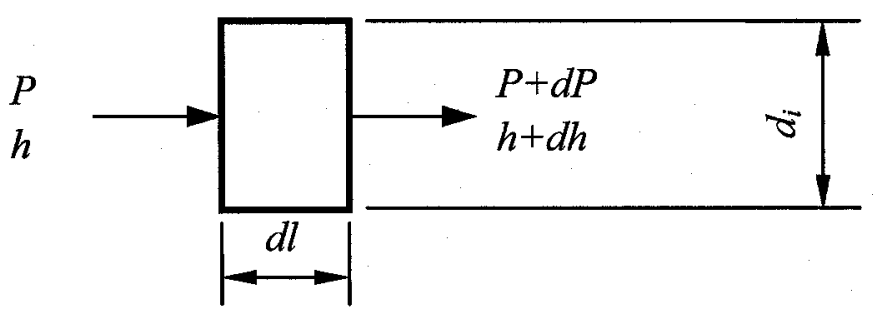

Figure 3-4 Heat transfer diagram of element $d l$

Substituting Equation (3-5) into (3-4) and simplifying produces

$$
d h+g^{2} v d v=0
$$

\subsection{Momentum balance equation}

The total pressure drop in a section $d l$ can be expressed as

$$
d P=-\frac{g^{2} v}{2}\left(f_{h} \frac{d l}{d_{i}}+\xi\right)-g^{2} d v
$$

Except for the inlet cross section, the local pressure drop coefficient $\xi$ is very small compared with the hydraulic drag term and will be considered negligible. Also, if the tube is straight, $\xi$ becomes zero by definition. 


\subsubsection{Single-phase region}

Many papers have been published on how to determine the Darcy-Weisbach friction factor $f$ in Equation (3-7) (Brittle and Pate, 1996; Churchill, 1977; Moody, 1944). Churchill's equation is adopted here for its good agreement with the Moody diagram.

$$
f=8\left[\left(\frac{8}{\operatorname{Re}}\right)^{12}+\left(A^{16}+B^{16}\right)^{\left(-\frac{3}{2}\right)}\right]^{\frac{1}{12}}
$$

where $A=2.457 \ln \frac{1}{(7 / \mathrm{Re})^{0.9}+0.27 \varepsilon / d_{i}}$

$$
B=\frac{37530}{\operatorname{Re}}
$$

The Reynolds number is given by

$$
\operatorname{Re}=\frac{g d_{i}}{\mu}
$$

\subsubsection{Two-phase region}

In this region, the hydraulic friction factor is expressed as (Lin et al, 1991)

$$
f=f_{t p}=\phi_{t p}^{2} f_{s p}\left(\frac{v_{s p}}{v_{t p}}\right)
$$

$f_{s p}$ is obtained using Equation (3-8), and the multiplier $\phi_{t p}^{2}$ is calculated by 


$$
\phi_{t p}^{2}=\left[\frac{\left(\frac{8}{\mathrm{Re}_{t p}}\right)^{12}+\left(A_{t p}^{16}+B_{t p}^{16}\right)^{\left(-\frac{3}{2}\right)}}{\left(\frac{8}{\operatorname{Re}_{s p}}\right)^{12}+\left(A_{s p}^{16}+B_{s p}^{16}\right)^{\left(-\frac{3}{2}\right)}}\right]^{\frac{1}{12}}\left[1+x\left(\frac{v_{g}}{v_{l}}-1\right)\right]
$$

For the two-phase flow region, different models were used to predict the average dynamic viscosity and then to calculate the relative Reynolds number in Equation (3-11), especially those from the research by McAdams et al (1942), Cicchitti et al (1960), Dukler et al (1964), and Lin et al (1991). Bittle and Pate (1996) recommended the McAdams model for capillary flow simulation; this model is also used by Xu and Bansal (2002). The Cicchitti model was adopted by Sinpiboon and Wongwises (2002). The Dukler model was used by Sinpiboon and Wongwises (2002) to predict the behavior of R12 in capillaries. To improve the accuracy of the McAdams and the Cicchitti models, which were found to generate an average deviation between the $\phi_{t p}^{2}$ and experimental results of about 24\%, Lin et al. (1991) proposed a simplified model. These four models are presented as follows.

McAdams et al.:

$$
\frac{1}{\mu_{t p}}=\frac{x}{\mu_{g}}+\frac{1-x}{\mu_{l}}
$$

Cicchitti et al.:

$$
\mu_{t p}=x \mu_{g}+(1-x) \mu_{l}
$$

Dukler et al.: 


$$
\begin{gathered}
\mu_{t p}=\mu_{g}+(1-\beta) \mu_{l} \\
\beta=\frac{x v_{g}}{x v_{g}+(1-x) v_{l}}
\end{gathered}
$$

Lin et al:

$$
\mu_{t p}=\frac{\mu_{g} \mu_{l}}{\mu_{g}+x^{n}\left(\mu_{l}-\mu_{g}\right)}
$$

In the Lin et al. model the exponent $n$ is determined using a curve fit of experimental data. For $0<x<0.25$ as investigated in the study, $n$ was taken as 1.4 .

Since the Lin et al. model is the latest improvement in a current study, and has been verified by both its authors and Wong and Ooi (1996), it is used in the present research.

Consider a finite element with length $d l$, the enthalpy and specific volume at its outlet are

$$
\begin{aligned}
& h_{o u t}=h_{l}+x \cdot h_{f g} \\
& v_{o u t}=v_{l}+x \cdot v_{f g}
\end{aligned}
$$

Substituting Equations (3-18) and (3-19) into the energy balance equation (3-6) and rearranging gives

$$
\frac{1}{2} g^{2} v_{f g}^{2} x^{2}+\left(h_{f g}+g^{2} v_{l} v_{f g}\right) x+h_{l}+\frac{1}{2} g^{2}\left(v_{l}^{2}-v_{i n}^{2}\right)-h_{i n}=0
$$

If the pressure at the element outlet is known, then the corresponding quality can be determined from the above equation to be 


$$
x=\frac{-\left(h_{f g}+g^{2} v_{l} v_{f g}\right)+\sqrt{\left(h_{f g}+g^{2} v_{l} v_{f g}\right)^{2}-2 g^{2} v_{f g}^{2}\left[h_{l}+\frac{1}{2} g^{2}\left(v_{l}^{2}-v_{i n}^{2}\right)-h_{i n}\right]}}{g^{2} v_{f g}^{2}}
$$

Then combining the equations (3-7) with (3-21) and using a trial-correct approach the outlet pressure and quality can be obtained. With these two values, all other thermodynamic properties (i. e. enthalpy, entropy, viscosity). can be determined.

Bansal and Rupasinghe (1998) used such techniques to investigate adiabatic capillary flow and compared the numerical data with the experimental results of Wijaya (1992), Melo et al. (1999), and Li et al. (1990), as well as the simulated results of Melo et al. (1992) and Wong et al. (1992). A fairly good agreement was observed with a deviation of less than $\pm 10 \%$. However, when the mass flux increases and becomes about ten times larger, the method generates a quality below zero; this could be attributed to the discontinuity at the interface between the single-phase and the two-phase region. At this point, a slight increase in the quality will result in an unrealistic increase of specific volume, which will then cause the kinetic energy increase greatly and thus the enthalpy drop drastically. In fact, due to the presence of the metastable region, the flash point will be located somewhere below the equilibrium saturated pressure where the fluid would normally vaporize. To enable the program to handle large mass flux situations, the specific volume as shown in Equation (3-19) was modified to take into account the metastable process. To achieve this, the following criteria must be satisfied in constructing a correlation of specific volume that is a function of quality

$$
\begin{aligned}
& f_{v}(x=0)=v_{l} \\
& f_{v}(x=1)=v_{g}
\end{aligned}
$$




$$
f_{v}^{\prime}(x=0)=0
$$

Forcing the first derivative of $f_{v}$ to zero ensures a smooth change in density when the fluid enters the two-phase region. For a super-heated gas, the density is dependant on two parameters (pressure and temperature) instead of one, so there is no need to assign a boundary condition at $x=1$. For the sake of backward compatibility, the function is supposed to have the form

$$
f_{v}(x)=x^{2-x^{\alpha}} v_{g}+\left(1-x^{2-x^{\alpha}}\right) v_{l}
$$

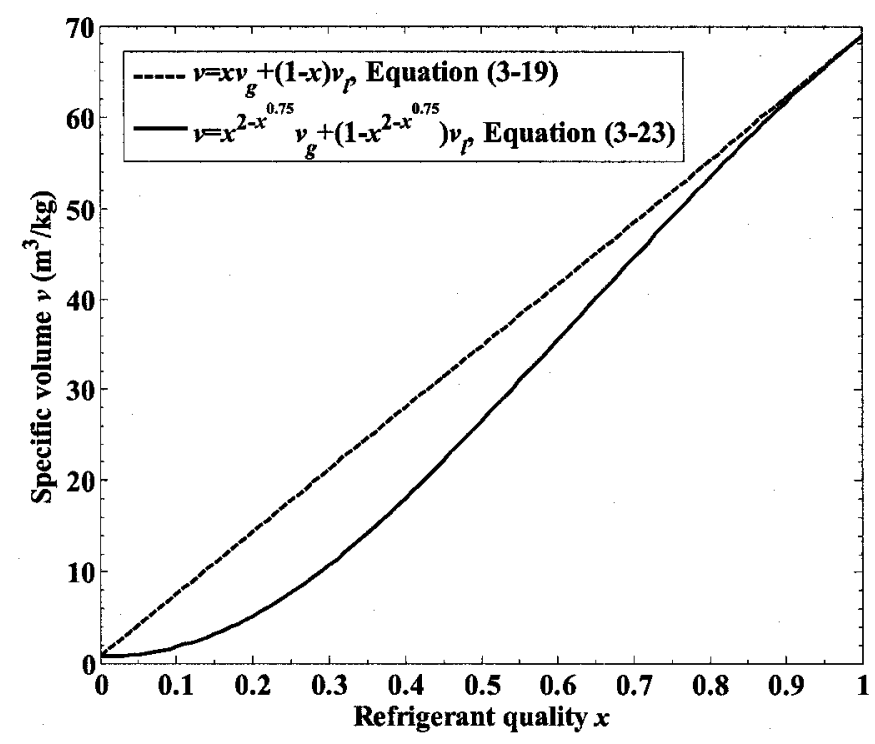

Figure 3-5 Comparison of different correlations in calculating the two-phase specific volume at $t=0{ }^{\circ} \mathrm{C}$

By fitting to a set of experimental data (Melo et al.,1999), $\alpha$ is found to be about 0.75 . Figure

3-5 illustrates the difference between Equation (3-23) and traditional used (3-19). The values calculated with (3-23) provide lower specific volumes over the entire range of quality, and the influence of $\alpha$ tapers off with the increasing refrigerant quality. Though the metastable flow region is not explicitly considered here, the low prediction can be treated as an indication of it. In fact, at a 
given point, the speed of the vapour is greater than that of the liquid, so the temperature of the vapour is higher than that of the liquid. Due to the one-to-one correspondence between pressure and temperature in the two-phase region, the vapour pressure is higher than the liquid. Accordingly, the mixture specific volume should be lower than the value at equilibrium.

\subsubsection{Judgment of extreme flow condition}

For in-tube two-phase flow, an extreme flow status may occur corresponding to a specific condition when the back pressure is lower than a critical value. In this case, the outlet velocity of refrigerant equals the local sonic velocity achieving the unit Mach number, and the mass flow rate through the capillary reaches maximum; the flow becomes choked, and the critical back pressure is called "choked pressure". For adiabatic analysis, entropy increases during the throttling process, making it a potential criterion to measure the occurrence of choking.

$$
s_{i, \text { out }}<s_{i, \text { in }}
$$

where $i$ stands for the $i$ th finite element of the capillary tube. When the outlet entropy is less than the inlet value, the extreme situation is achieved and relative critical parameters can be obtained via iterative calculations.

\subsubsection{Parametric study and discussion}

A program has been developed in MATLAB based on the governing equations described above. The required refrigerant properties, including thermophysical and transport properties, were generated by means of an in-house code R-Prop based on Peng-Robinson equation of state (PR 
EOS). Calculation results were compared to the software REFPROP 6.0 of the National Institute of Standards and Technology (NIST). R-Prop was found to be accurate with variations of less than $0.5 \%$. The flow diagram for the adiabatic capillary model (ACAM) is shown in Figure 3-6. The step size, i.e. the length of each finite capillary element, is not fixed. To save computing resources while also keeping sufficient accuracies, the step size is modulated such that the temperature difference remains between 0.2 and $2{ }^{\circ} \mathrm{C}$; it also decreases when the flow approaches the end of the single-phase region or the choking condition. A typical set of simulation results is shown in Figure 3-7. It can be seen that there are slight drops in enthalpy and entropy, which could be attributed to the discontinuity of thermodynamic properties between the single-phase and the two-phase regions. However, the resulting effect on the temperature and pressure are negligible. Although the model is applicable to any refrigerant, the present investigation is only limited to R134a. This is because experimental results for R134a are widely available in open literature, and because it is currently used in a number of applications which make the results more meaningful.

The mass flow rate that an adiabatic capillary tube can convey is influenced by many factors, including tube diameter, tube length, condensation pressure, subcooling degrees, surface roughness, and oil fraction. These parameters can be categorized as geometric, thermophysical, or operational factors. They are related to and influenced by each other. As mentioned by Bolstad and Jordan (1949) and Choi et al. (2004), when a choking situation is present (which generally occurs for typical steady-state applications), the effects of the evaporation pressure are negligible. This is also addressed by Bansal and Wang (2004). In the following analysis, one or two factors were varied at a 


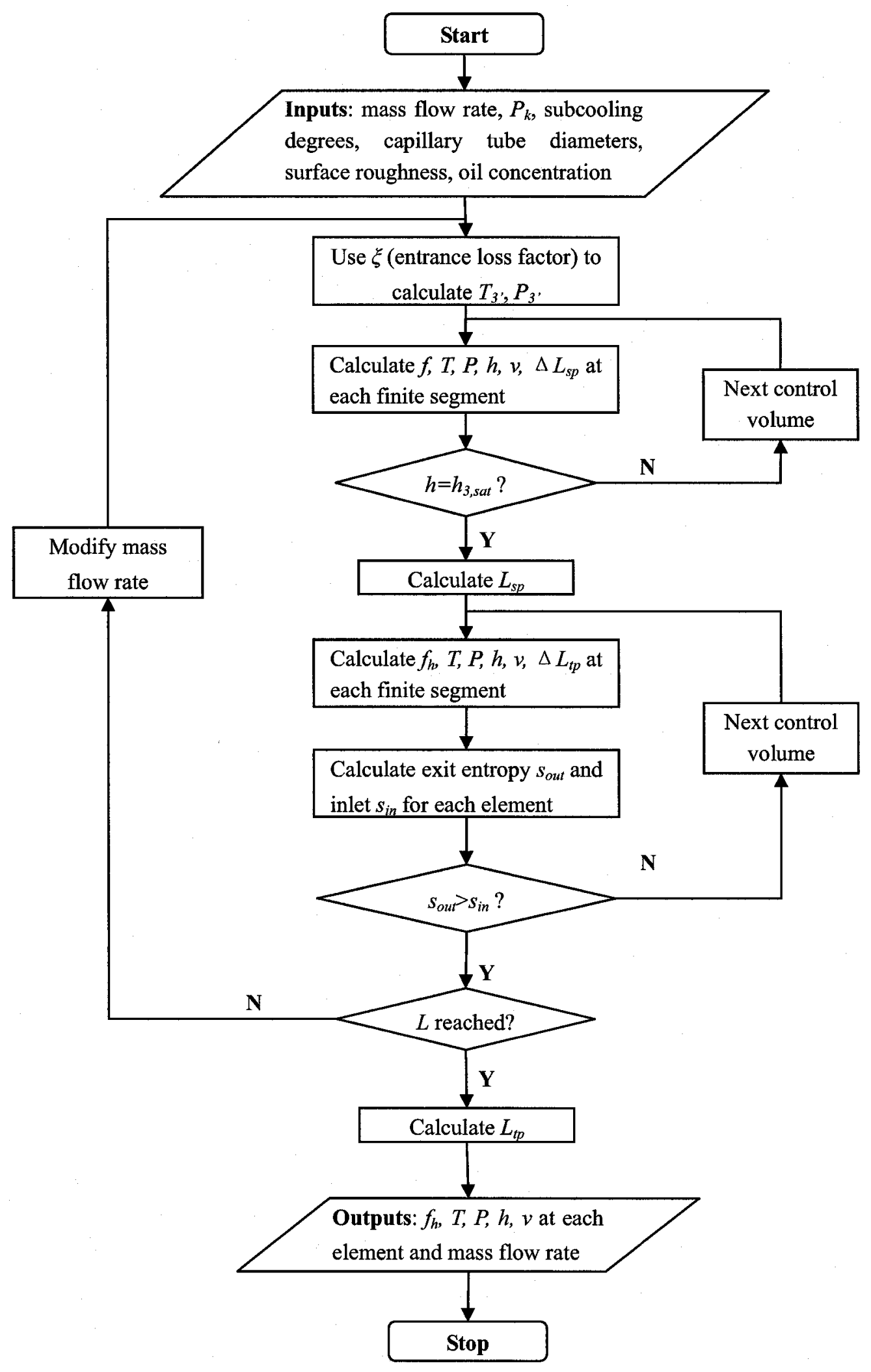

Figure 3-6 Flow diagram for adiabatic capillary model (ACAM) 


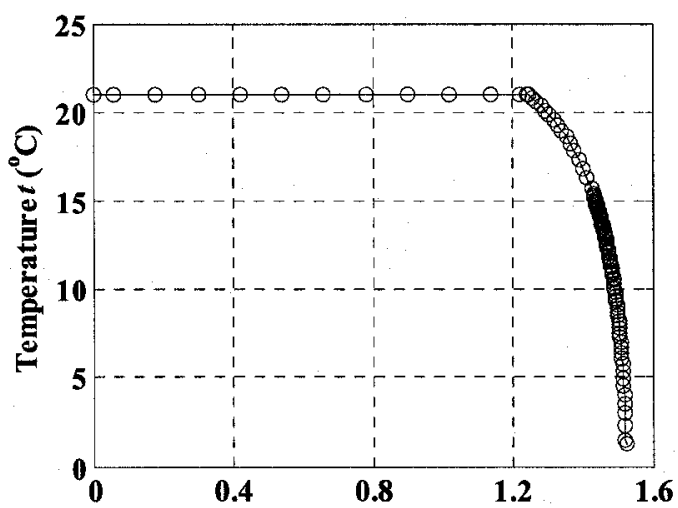

(a) Distance from capillary inlet (m)

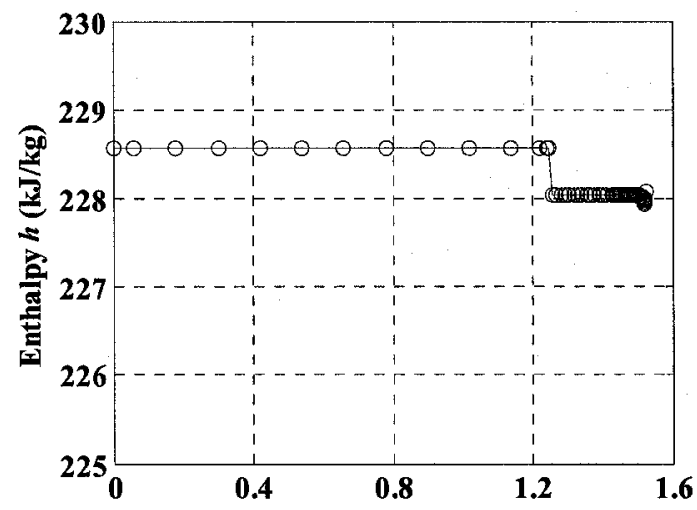

(c) Distance from capillary inlet (m)

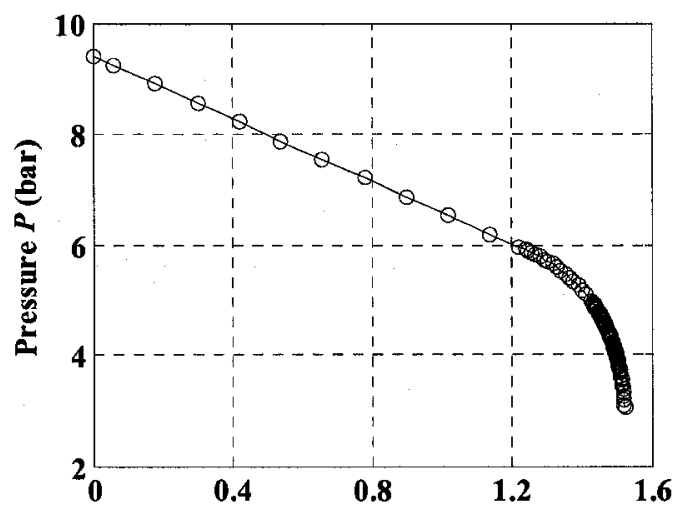

(b) Distance from capillary inlet (m)

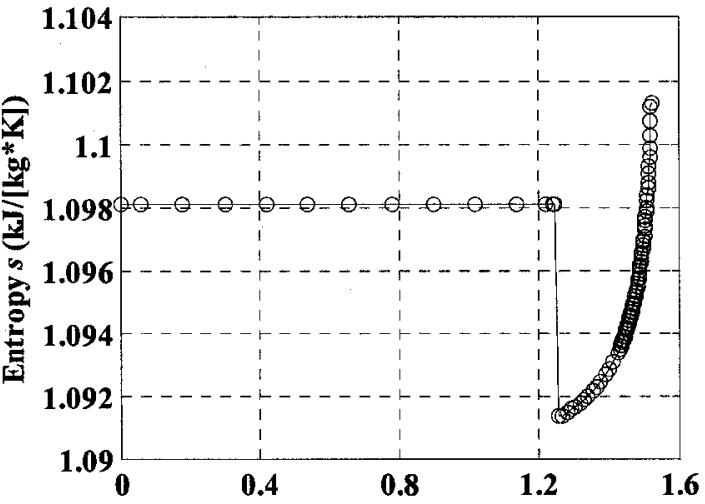

(d) Distance from capillary inlet (m)

Figure 3-7 A typical set of results of ACAM with $\dot{m}=9.52 \mathrm{~kg} / \mathrm{h}, L=1.526 \mathrm{~m}, t_{k}=37.65{ }^{\circ} \mathrm{C}$, $\Delta t_{\text {sub }}=16.7^{\circ} \mathrm{C}, d_{i}=0.84 \mathrm{~mm}$, and $\varepsilon=0.0005 \mathrm{~mm}$

time, and their effects on the capillary length were quantified. Since more than $50 \%$ of the total pressure drop occurs in the last $20 \%$ of the capillary tube length, which makes a slight change in length can result in a great variation in the pressure drop. For this reason the results comparison is oversensitive to capillary length. Bansal and Wang (2004) stated that a $0.001 \mathrm{~mm}$ increment in tube length can cause up to a $0.7 \mathrm{kPa}$ pressure drop near the choking point for a typical flow. In order to make more meaningful comparisons, the mass flow rate is thus used as a reference parameter instead of tube length. However, the changes along capillary length provide a straightforward and 
convenient way to show the behaviour of other parameters, such as pressure, temperature, etc., so it is also used for illustration under certain working conditions. For details about the influence of other parameters on tube length, some figures are provided in Chapter 6 for the investigation of $\mathrm{CO}_{2}$-based AAC systems.

\subsubsection{Validation of the ACAM}

In this section, the comparison between the ACAM results and some experimental results from open literature will be addressed, and then the accuracies of the ACAM using Equation (3-23) and that using (3-19) will be discussed.

Figure 3-8 is a comparison of the simulated results from ACAM with experimental data from open literature. Wijaya (1992) measured the mass flow rate inside capillary tubes with different lengths and working conditions. The condensation temperature varied from $37.8^{\circ} \mathrm{C}$ to $54.4^{\circ} \mathrm{C}$, and the subcooling degrees from $11.1^{\circ} \mathrm{C}$ to $16.7^{\circ} \mathrm{C}$. In this figure all data were generated for a tube inner diameter of $d_{i}=0.84 \mathrm{~mm}$ and $\Delta t_{\mathrm{sub}}=16.7^{\circ} \mathrm{C}$. The ACAM predicts results well, with deviations of less than $\pm 7 \%$, which provides the same order of accuracy as CAPIL model by Bansal and Rupasinghe (1998). The average prediction error is found to be $-0.77 \%$ while the root mean square (RMS) error is 3.58\%. Melo et al. (1999) tested different refrigerant flows (R12, R134a, and R600a) in eight capillary tubes with various geometric configurations (including internal diameters, lengths, and surface roughness combinations), under different working conditions to investigate their effects on the mass flow rate under choking conditions. For R134a experiments, the inner diameter ranges from $0.606 \mathrm{~mm}$ to $1.05 \mathrm{~mm}$, the length from $1.993 \mathrm{~m}$ to $3.027 \mathrm{~m}$, the surface roughness from 
$0.59 \mu \mathrm{m}$ to $1.08 \mu \mathrm{m}$, subcooling degrees from $3.4^{\circ} \mathrm{C}$ to $13.3^{\circ} \mathrm{C}$, and the condensation temperature from $36.65^{\circ} \mathrm{C}$ to $55.38^{\circ} \mathrm{C}$. The ACAM can predict most of their values with fairly good agreement (within $\pm 7.5 \%$ ), but for the first tube two out of three values are $10 \%$ overpredicted (one is $12.9 \%$ and the other is $12.4 \%$; the third is $0.9 \%$ ). The average prediction error for the whole range is $0.62 \%$ and the RMS error is $6.06 \%$.

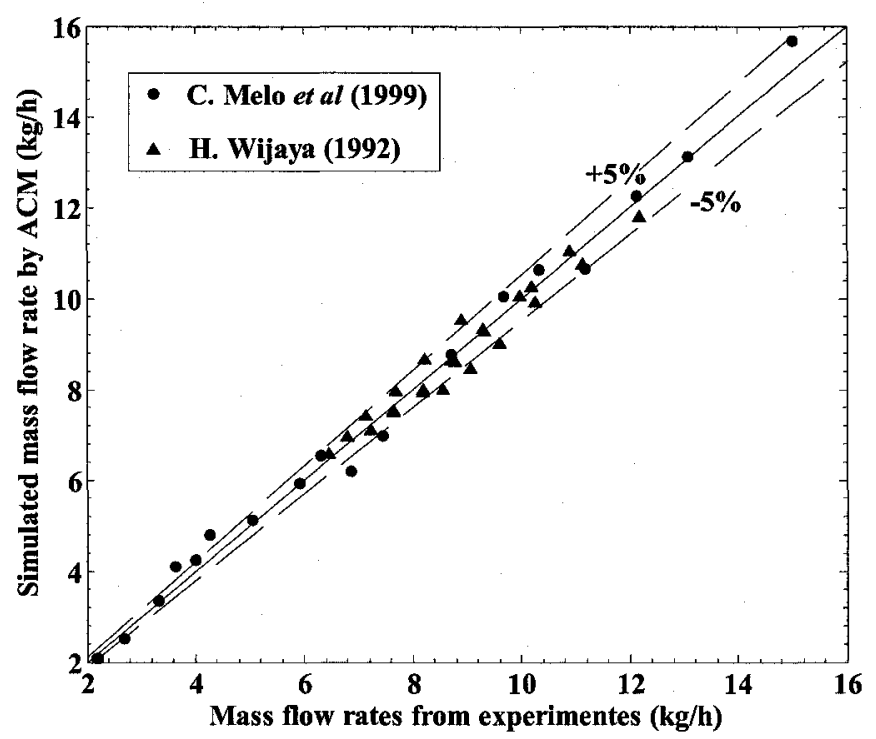

Figure 3-8 Comparison of the ACAM results with the experimental data of Melo et al and Wijaya for the mass flow rate

At this point it is interesting to not the difference in prediction error when ACAM is used with the old correlation (3-19) and the new one (3-20) for specific volume. Figure 3-9 shows the prediction errors of the two models in calculating mass flow rates using conditions from Wijaya's paper (1992). Those data in solid lines with circles are from Equation (3-19) and dash lines with squares are from Equation (3-15). They provide similar overall accuracies, with maximum deviations of $6.9 \%$ and $6.4 \%$, respectively. They also both overpredict when the condensation pressure is low and tend to underpredict when it is high. The average prediction error of the 
traditional correlation is $-3.04 \%$ and the corresponding RMS is $4.12 \%$.

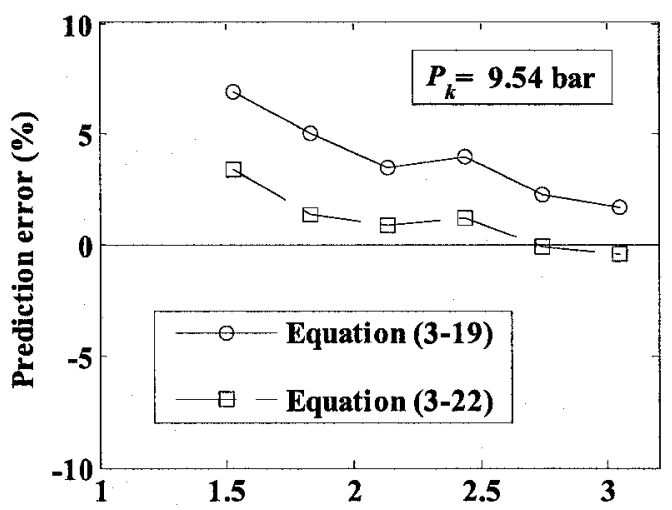

(a) Capillary length (m)

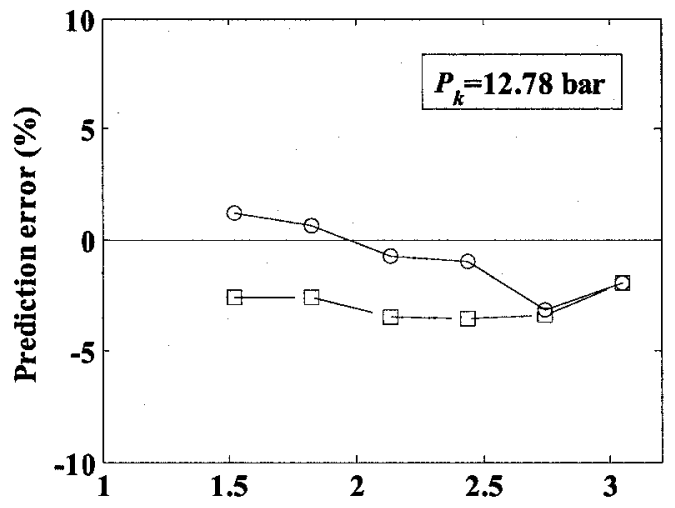

(c) Capillary length (m)

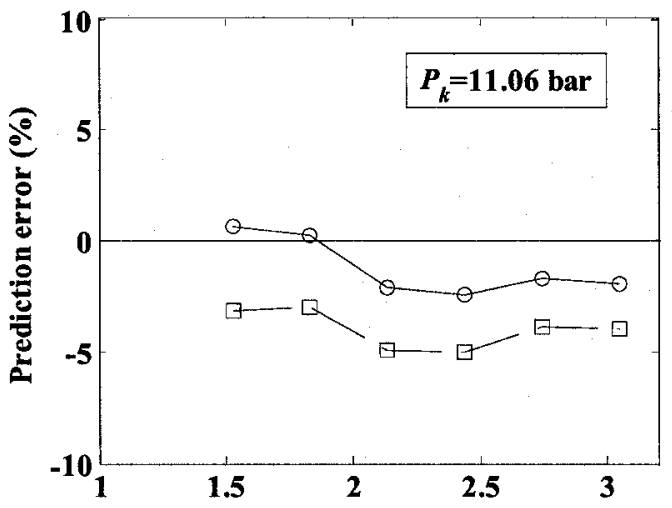

(b) Capillary length (m)

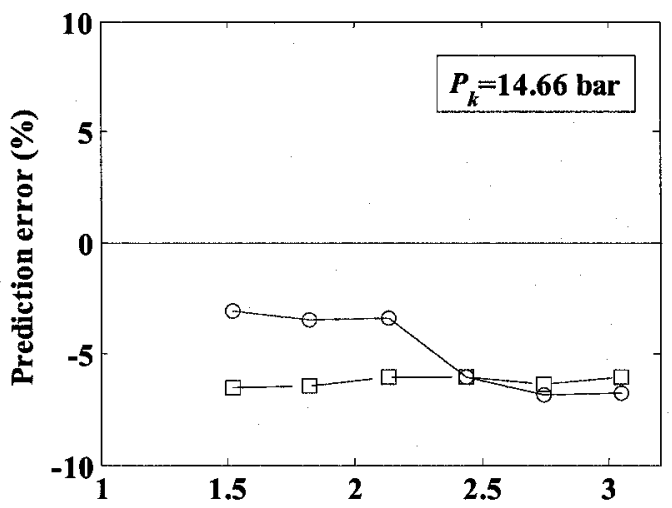

(d) Capillary length (m)

Figure 3-9 Comparison of prediction accuracy using Equation (3-19) with that using Equation (3-15) for various capillary lengths and condensation pressures

In the following chapters further verification will be provided by comparing the ACAM results with experimental data of the AAC system at Carleton University. It will prove that the ACAM can be used as a reliable tool to investigate the flow behaviour of capillary flow.

\subsubsection{Influence of tube inner diameter}

To analyze the relationship between mass flow rate and tube inner diameter, five different diameters were used at two different condensation pressures in the simulation; the results are shown 


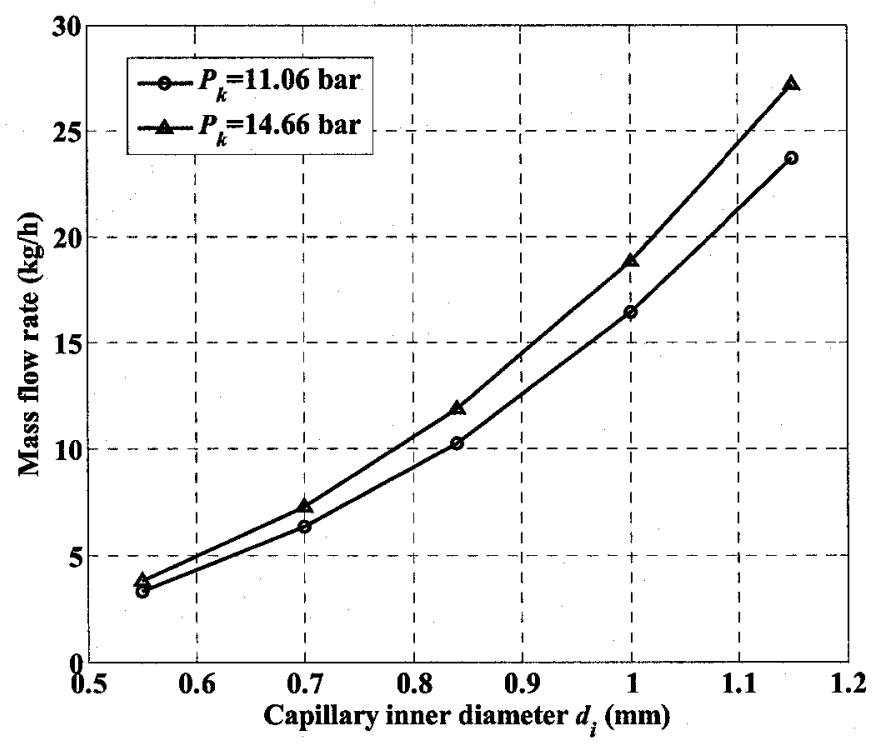

Figure 3-10 Mass flow rate vs. capillary inner diameter under different condensation pressures with $L=1.526 \mathrm{~m}, \Delta t_{\mathrm{sub}}=16.7^{\circ} \mathrm{C}, \varepsilon=0.0005 \mathrm{~mm}$, and $c=4.0 \%$

in Figure 3-10. As the diameter increases, the mass flow rate grows accordingly to maintain the pressure drop required by the cycle. This is reasonable because a larger diameter will create a slower fluid flow and thus a smaller pressure drop (if mass flow rate does not change). A longer distance will then be needed to achieve the same pressure drop. However, the length is fixed in this case, meaning the mass flow rate should be greater such that the pressure drop per unit length of capillary remains constant. This trend becomes more apparent as $d_{i}$ becomes larger, which means the throttling effect of the capillary decreases rapidly. As the pressure difference grows, a larger mass flow rate is achieved; this can also be seen by comparing two curves at fixed values of $d_{i}$. This means that by increasing the inlet pressure, more refrigerant can be pumped through the capillary tube.

\subsubsection{Influence of capillary length}

The opposite trend occurs when the capillary length is increased; an increase in capillary length 
has a negative effect on the mass flow rate as shown in Figure 3-11. For one curve with $d_{i}=0.84 \mathrm{~mm}$ and $P_{k}=11.06 \mathrm{bar}$, the mass flow rate drops from 10.25 to $7.09 \mathrm{~kg} / \mathrm{h}$. This can be explained as a friction effect. If the mass flow rate of $10.25 \mathrm{~kg} / \mathrm{h}$ did not change, the pressure drop at the location that is $1.526 \mathrm{~m}$ from the capillary inlet would be the same for any tube longer than that. However, since the choking situation only occurs at the outlet of a tube with constant inner diameter, the mass flow rate must be smaller than $10.25 \mathrm{~kg} / \mathrm{h}$ so that the choking section can move downward to the outlet of longer capillaries.

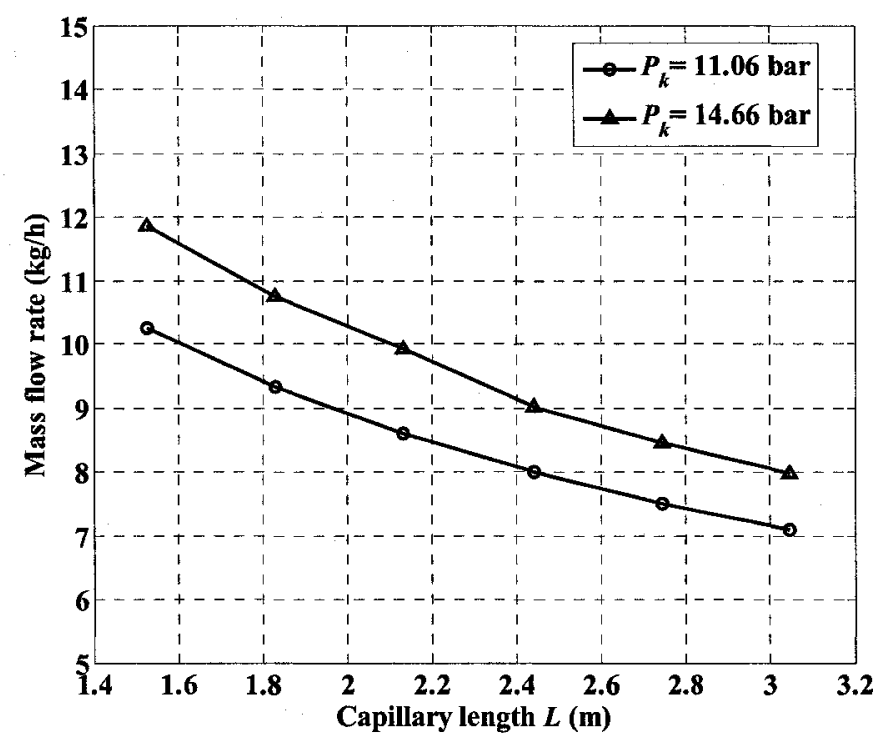

Figure 3-11 Mass flow rate vs. capillary length under different condensation pressures with $d_{i}=0.84 \mathrm{~mm}, \Delta t_{\mathrm{sub}}=16.7^{\circ} \mathrm{C}, \varepsilon=0.0005 \mathrm{~mm}$, and $c=4.0 \%$

Comparing the two curves it can be seen that mass flow rate decreases more quickly at higher condensation pressures. This is logical if we consider two extreme conditions of capillary length, i.e. short enough and long enough. If the length is short, (due to the reason addressed in the previous subsection) the mass flow rate is higher at higher $P_{k}$; if the length approaches infinity, then the flow chokes at the outlet, no matter what the inlet condition, and the mass flow rate will be extremely 
low. Therefore, the mass flow rate drops with capillary length from high to zero at higher and higher condensation pressures, and from low to zero at lower and lower condensation pressures.

\subsubsection{Influence of condensation pressure}

Condensation pressure is another important parameter for a refrigeration system. From a thermodynamic point of view, high pressures will create larger temperature differences between the inside refrigerant flow and the ambient temperature, and thus more irreversibility to the condenser.

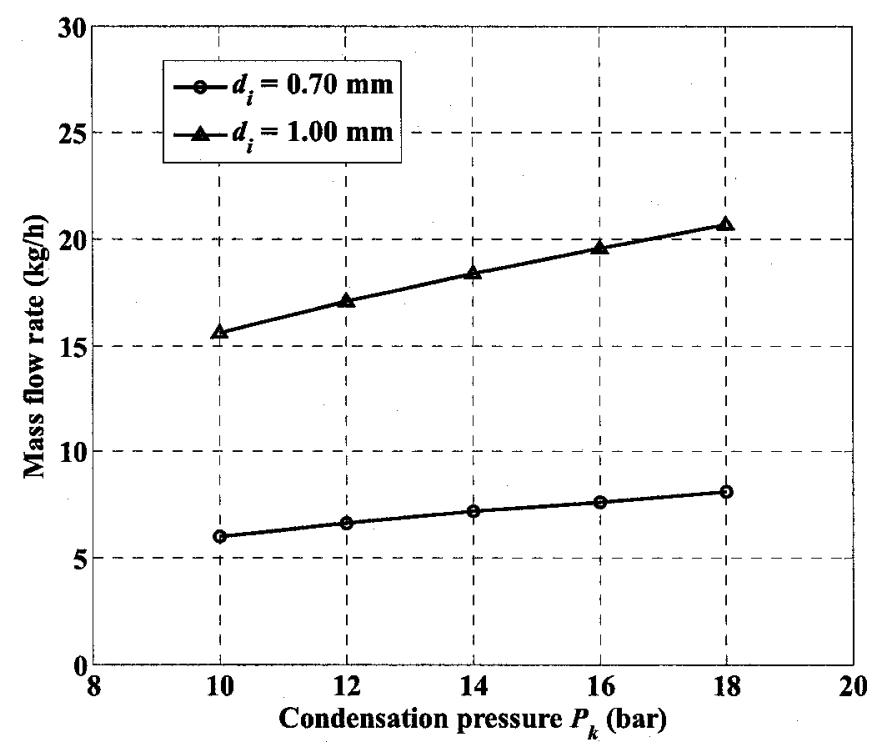

Figure 3-12 Mass flow rate vs. condensation pressure for different capillary inner diameters with $L=1.526 \mathrm{~m}, \Delta t_{\text {sub }}=16.7^{\circ} \mathrm{C}, \varepsilon=0.0005 \mathrm{~mm}$, and $c=4.0 \%$

On the other hand, if the discharge pressure is too low, it will weaken the compressor performance. Thus the cooling pressure should be within a proper range. Figure 3-12 shows the relationship between mass flow rate and condensation pressure with different capillary inner diameters. When $d_{i}$ is fixed, a higher $P_{k}$ will "pump" more refrigerant into the capillary. The relationship can be observed as linear. When $d_{i}$ increases from $0.7 \mathrm{~mm}$ to $1.00 \mathrm{~mm}$, the mass flow rate increases as well from 9.57 to $12.63 \mathrm{~kg} / \mathrm{h}$, which is in accordance with the statement in two 
subsections before (Section 3.1.2.2). As $P_{k}$ goes up, the increment of the mass flow rate gets greater, which means the mass flow rate is more sensitive to the diameter change at high condensation pressure.

\subsubsection{Influence of subcooling degrees}

Figure 3-13 shows the influence of subcooling degrees on the mass flow rate at different condensation pressures. Such influence can be determined by the ratio of the length of the single-phase flow to the total capillary length. Basically, the longer the single-phase region, the larger the mass flow rate is. For a given condensation pressure, more subcooling degrees means the refrigerant travels more distance before reaching the flash point, and there is less capillary length

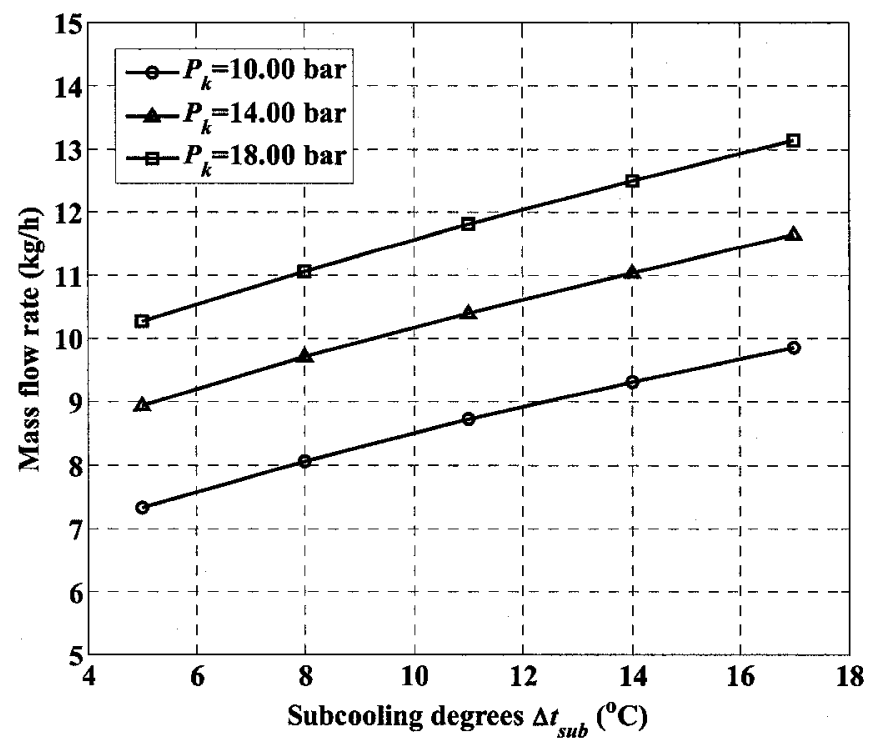

Figure 3-13 Mass flow rate vs. subcooling degrees for different condensation pressures with $L=1.526 \mathrm{~m}, d_{i}=0.84 \mathrm{~mm}, \varepsilon=0.0005 \mathrm{~mm}$, and $c=4.0 \%$

for it to vaporize. From Bansal and Wang (2004) it can be seen that the two-phase length is the more important value affecting the mass flow rate, so more refrigerant is required to generate the same pressure drop along less two-phase length. Meanwhile, if the subcooling degrees is fixed 
while the condensation pressure is increased, the single-phase length is increased, which also means a larger mass flow rate. Since the three curves are parallel to each other, it shows that the mass flow rate changes with subcooling degrees along similar trend lines for a given condensation pressure, which is a good feature for simulation. If the mass flow rate can be expressed as a function of subcooling degrees using regression based on a set of experimental data at a specific condensation pressure, then only one mass flow rate at any other pressure is sufficient to determine its behaviour with various subcooling conditions at that pressure.

\subsubsection{Influence of surface roughness}

The surface roughness $\varepsilon$ of the capillary has a smaller impact on the mass flow rate than the factors previously mentioned, as can be seen from Figure 3-14. Consider $P_{k}=14$ bar as an example: when $\varepsilon$ increases from $0.5 \mu \mathrm{m}$ to $1.5 \mu \mathrm{m}$, the mass flow rates drops $5.0 \%$, from $11.58 \mathrm{~kg} / \mathrm{h}$ to $11.00 \mathrm{~kg} / \mathrm{h}$. Just like Figure 3-13, the three curves in this figure are parallel, and thus a similar technique can be adopted in simulation to get a fast prediction of the mass flow rate at different surface roughnesses under different condensation pressures.

It should be noted that the relative roughness $\varepsilon_{r}$, which is defined as the roughness divided by the capillary inner diameter, in this figure varies from $5.95 \times 10^{-4}$ to $1.79 \times 10^{-3}$. It is interesting to investigate how the mass flow rate varies with different relative roughnesses while keeping other parameters the same. Figure 3-15 shows these results with $\varepsilon_{r}=4.55 \times 10^{-4} \sim 2.50 \times 10^{-3}$. Although the range is small, the mass flow rate does vary a lot, from around $4.60 \mathrm{~kg} / \mathrm{h}$ to around $17.88 \mathrm{~kg} / \mathrm{h}$. Compared with the maximum value of each line, the decrease in magnitude of the smallest value is 
uniformly $5.0 \%$. This implies that the changing trend of mass flow rate is independent of $d_{i}$.

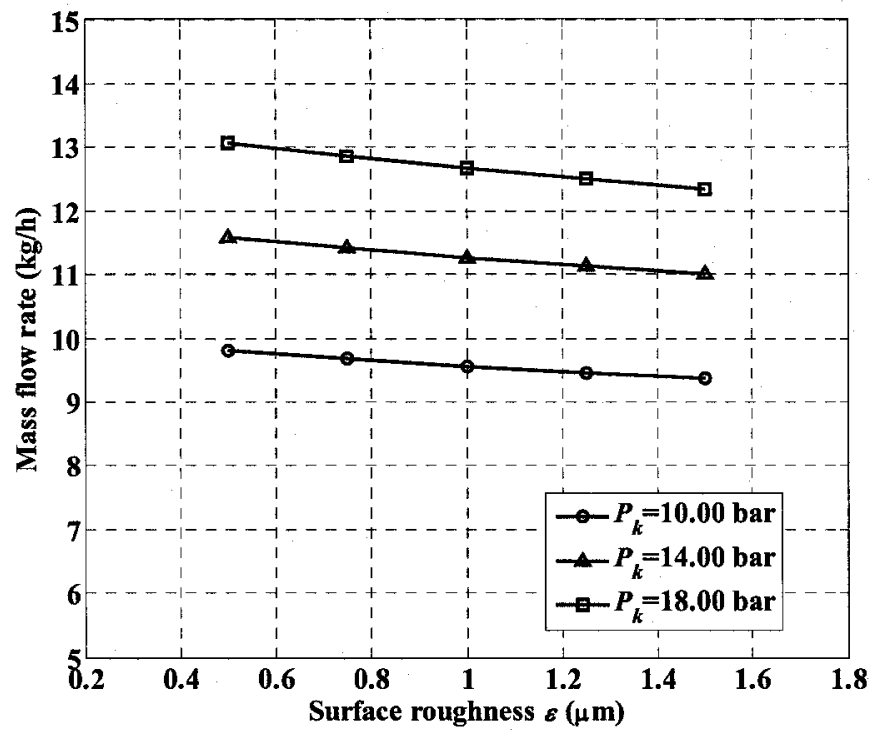

Figure 3-14 Mass flow rate vs. surface roughness for different condensation pressures with $L=1.526 \mathrm{~m}, \Delta t_{\mathrm{sub}}=16.7^{\circ} \mathrm{C}, d_{i}=0.84 \mathrm{~mm}$, and $c=4.0 \%$

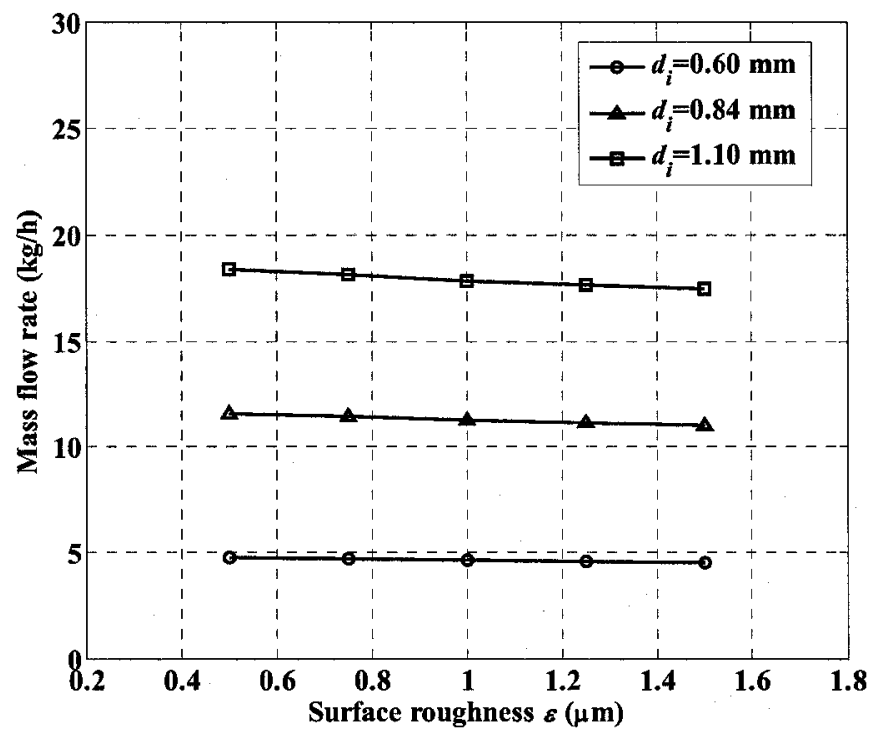

Figure 3-15 Mass flow rate vs. subcooling degrees for different capillary inner diameters with $L=1.526 \mathrm{~m}, \Delta t_{\mathrm{sub}}=16.7^{\circ} \mathrm{C}, P_{k}=14 \mathrm{bar}$, and $c=4.0 \%$

\subsubsection{Influence of oil effect}

In most refrigeration systems, a certain amount of oil is used to lubricate moving parts. As a 
side effect, part of it is carried by the refrigerant and circulates through the entire cycle. For heat exchangers, such as a condenser or an evaporator, oil adheres to the inner side of the heat transfer surfaces and becomes a film of thermal resistance. This effect will be detailed in the following section on non-adiabatic process. For adiabatic flows, the presence of oil makes effective mass flow rates of the refrigerant less than measured values. In fact, oil "smoothes" uneven tube surfaces and thus decreases roughness. As a result, the refrigerant experiences a smaller pressure drop, as if it had a smaller mass flow rate with unchanged surface roughness. Regarding this, the mass flow rate of refrigerant/oil mixture is determined by

$$
\dot{m}_{m i x}=\frac{\dot{m}_{\text {pure }}}{(1-c)}
$$

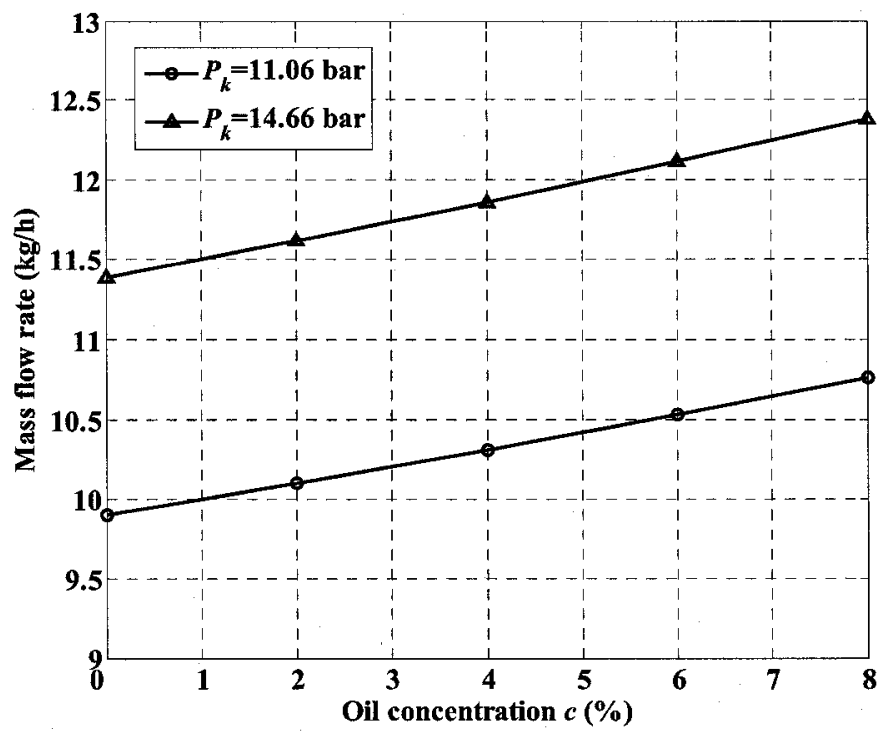

Figure 3-16 Mass flow rate vs. oil concentration for different condensation pressures with $L=1.526 \mathrm{~m}, \Delta t_{\text {sub }}=16.7^{\circ} \mathrm{C}, d_{i}=0.84 \mathrm{~mm}$, and $\varepsilon=0.0005 \mathrm{~mm}$

Another way to account for the oil effect is to consider the change in density of the fluid. The mixture density can be determined by 


$$
\frac{1}{\rho_{\text {mix }}}=\frac{c}{\rho_{\text {oil }}}+\frac{1-c}{\rho_{\text {pure }}}
$$

This provides a more accurate way to investigate the flow. However, due to the difficulty in determining the choking status of the mixture (i. e., to calculate either the critical entropy or the local sonic speed), Equation (3-25) was adopted in the ACAM. Figure 3-16 shows the change in the mass flow rate with different oil concentrations and condensation pressures. As oil concentration increases, the mass flow rate goes up accordingly. When the condensation pressure is increased, the single-phase region is elongated; this also results in an increase in the mass flow rate.

Up to this point we have discussed the relationships between the mass flow rate and geometric, thermodynamic, and operational parameters for adiabatic throttling processes. The single-phase length and the subcooling degrees a refrigerant flow experiences are two major criteria that underlie these parameters and affect the mass flow rate through the capillary. The modification of the specific volume of the refrigerant at the two-phase region is proposed as a convenient and reliable way to predict refrigerant behaviour without considering the complicated mechanism of the metastable flow. If choking conditions occur inside the metastable region, models in open literature are not sufficient to handle calculations of relative mass flow rate due to the lack of experimental support.

From parameter point of view, tube inner diameter and subcooling degrees are two key factors affecting the mass flow rate. Tube length and condensation pressure produce relatively small effects. Surface roughness and oil concentration have only a minor influence on the refrigerant flow. A 
larger tube inner diameter, more subcooling degrees, decreased tube length, higher condensation pressure, smaller roughness, and higher oil concentration together will increase the mass flow rate.

The following section will be focused on the refrigerant flow behaviour through a non-adiabatic capillary tube. 


\subsection{Non-adiabatic capillary flow}

Compared with adiabatic flow, the most important difference is that for non-adiabatic flow heat transfer occurs across the tube wall; the Fanno flow thus becomes a Fanno-Rayleigh flow because heat transfer and friction are both present. Figure 3-17 is an illustration showing flows inside a tube-in-tube heat exchanger, where a capillary with inner diameter $d_{i}$ is placed inside another tube with inner diameter $d_{i, o u t e r}$. High pressure, high temperature refrigerant from the condenser enters and is throttled to low pressure by friction and to low temperature by both friction and heat dissipation. At the same time heat is transferred to the low pressure, low temperature flow exiting
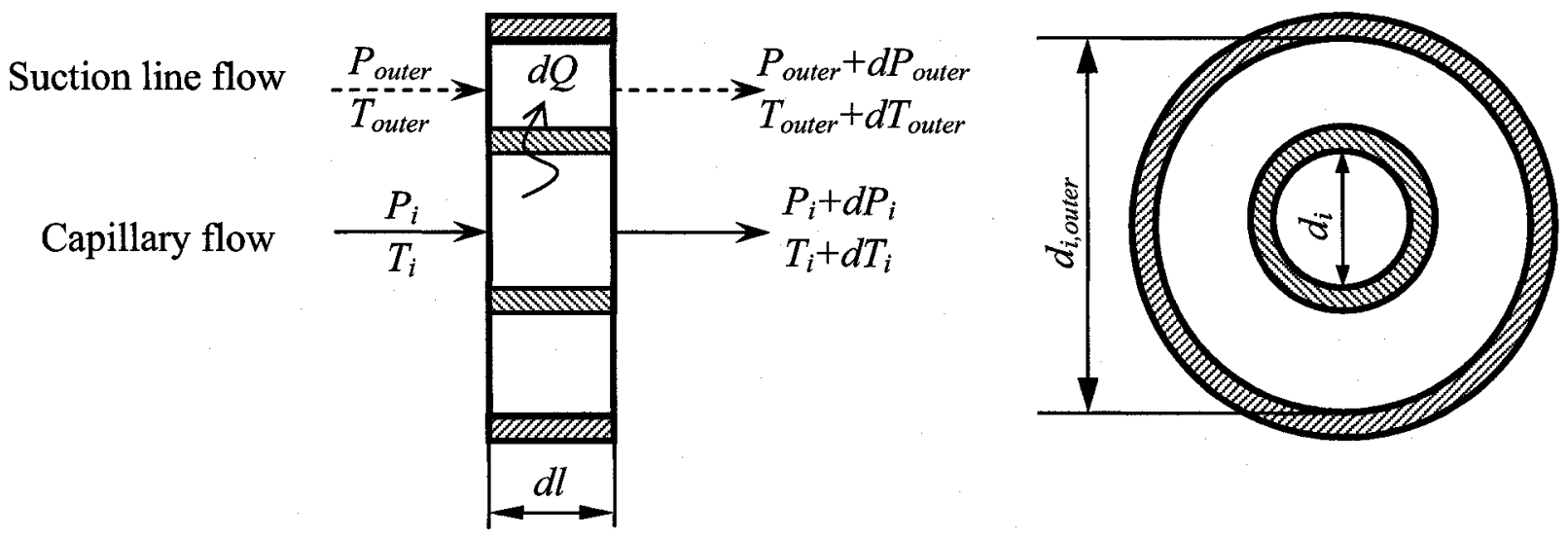

Figure 3-17 Heat transfer diagram of a finite element $d l$ of a concentric heat exchanger the evaporator. In Figure 3-2, the process undergone by the inside flow is represented by line 3"-4', and the outside flow is line $1-1^{\prime}$. In this figure the parallel configuration is shown; a counter flow pattern is adopted in some circumstances to improve the device efficiency. A simple way to construct an internal heat exchanger is to attach the capillary tube to the outside wall of the suction line, which is commonly seen in domestic refrigerators. Figure 3-18 is the flow diagram. In this case, the device has larger thermal resistance than that of Figure 3-17, but it is easier to 
manufacture and maintain. Both the heat exchanger type (concentric or lateral) and the flow arrangement (parallel or counter flow) will be covered in this section.
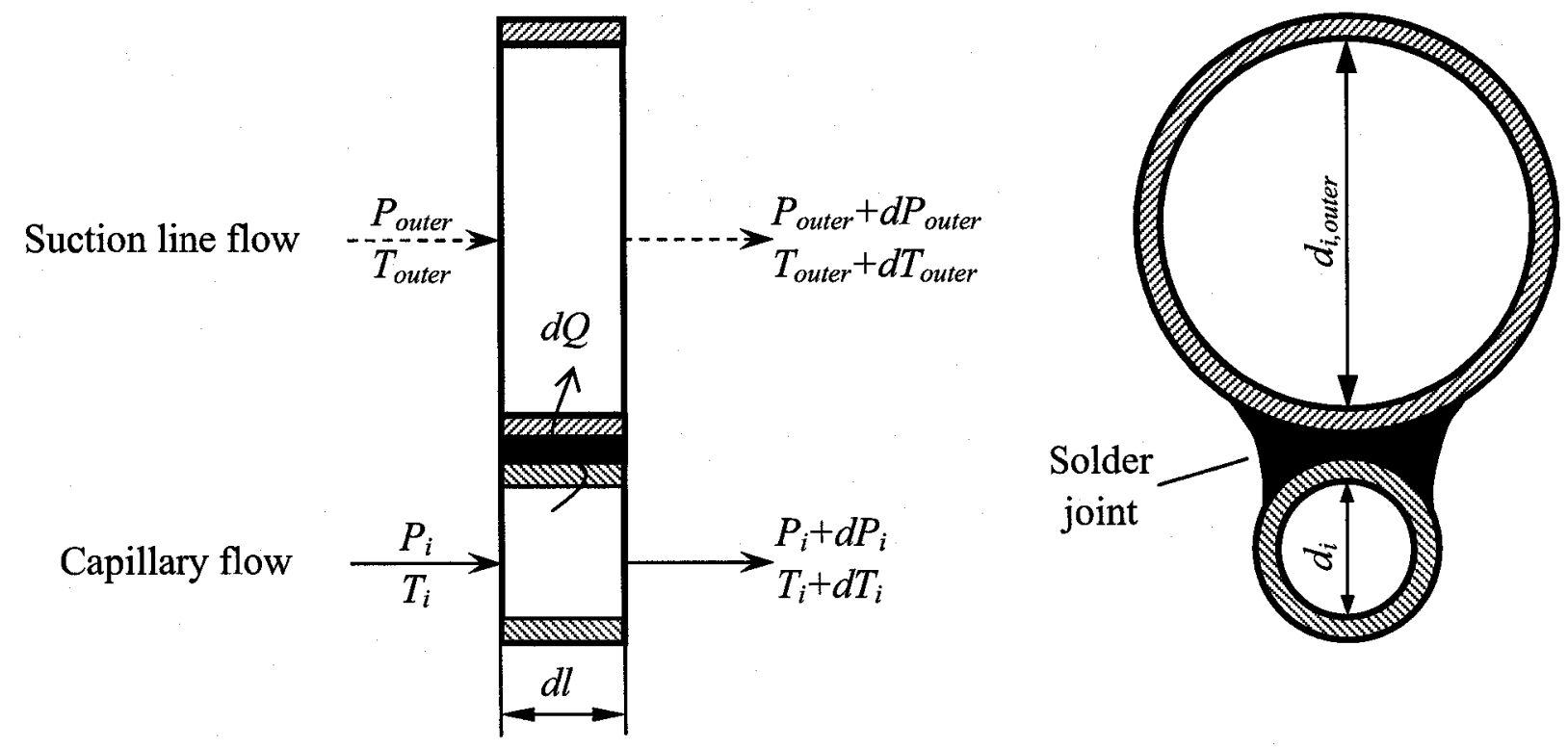

Figure 3-18 Heat transfer diagram of a finite element $d l$ of a lateral heat exchanger

\subsubsection{Model description}

\subsubsection{Governing equations}

Of the three conservation equations detailed in section 3.1.1.2, the mass and the momentum balances remain the same; only the energy balance needs to be modified to consider the effects of heat transfer. The three equations are

$$
\dot{m}=\text { const }
$$




$$
\begin{aligned}
& d P=-\frac{g^{2} v}{2}\left(f_{h} \frac{d l}{d_{i}}+\xi\right)-g^{2} d v \\
& h+\frac{1}{2} V^{2}=(h+d h)+\frac{1}{2}(V+d V)^{2}+d q
\end{aligned}
$$

where the specific heat transfer rate item $d q$ is positive when heat is transferred from the high temperature capillary flow to the low temperature suction line flow. This is defined by

$$
d q=\frac{U A_{i}\left(T_{i}-T_{\text {outer }}\right)}{\dot{m}}
$$

Substituting equations (3-5) and (3-28) into Equation (3-27) and rearranging gives

$$
d h+G^{2} v d v+\frac{4 U A_{i}\left(T_{i}-T_{\text {outer }}\right)}{G \pi d_{i}^{2}}=0
$$

For the tube-in-tube heat exchanger as shown in Figure 3-17, the gross thermal conductance is

$$
U A_{i}=\frac{d l}{\frac{1}{\alpha_{i} \pi d_{i}}+\frac{1}{2 \lambda_{\text {capi }} \pi} \ln \frac{d_{o}}{d_{i}}+\frac{1}{\alpha_{o} \pi d_{o}}}
$$

For the lateral arrangement heat exchanger in Figure 3-18, it is

$$
U A_{i}=\frac{d l}{\frac{1}{\alpha_{i} \pi d_{i}}+\frac{1}{2 \lambda_{\text {capi }} \pi} \ln \frac{d_{o}}{d_{i}}+\frac{\delta}{\lambda_{\text {weld }} w}+\frac{1}{2 \lambda_{\text {tube }} \pi} \ln \frac{d_{o, \text { outer }}}{d_{i, \text { outer }}}+\frac{1}{\alpha_{o} \pi d_{i, \text { outer }}}}
$$


where $\delta$ is the distance between the two tubes and $w$ is the thickness of soldering. Either the single-phase or the two-phase heat transfer coefficient of the capillary flow could be used for $\alpha_{i}$, while $\alpha_{i, \text { outer }}$ stands for the two-phase heat transfer coefficient of the suction line flow only.

\subsubsection{Single-phase region}

For the momentum balance, just as in section 3.1, the Churchill equation (3-8) is used to calculate the friction factors in the single-phase region.

Gnielinski's correlation (1976) was adopted to calculate the heat transfer coefficient of single-phase flow. It is given by

$$
N u=\frac{(f / 8)(\operatorname{Re}-1000) \operatorname{Pr}}{1+12.7(f / 8)^{1 / 2}\left(\operatorname{Pr}^{2 / 3}-1\right)}=\frac{\alpha_{i} d_{i}}{\lambda_{i}}
$$

\subsubsection{Two-phase region}

Taking the pressure drop into account, Lin et al's (1991) correlation (3-12) was used to calculate the two-phase friction factor.

For the two-phase flow, correlations to predict the heat transfer coefficient are different for condensation and evaporation. For condensation heat transfer, Jabardo et al (2002), Yang and Bansal (2004), Bansal and Yang (2005), and Tian and Li (2005) recommended Shah's correlation (1979) to predict the heat transfer coefficient, which is expressed as 


$$
\alpha_{i, t p}=\alpha_{l}\left[(1-x)^{0.8}+3.8 x^{0.76} \frac{(1-x)^{0.04}}{P_{r}^{0.38}}\right]
$$

where $\quad \alpha_{l}=0.023 \operatorname{Re}_{l}^{0.8} \operatorname{Pr}_{l}^{0.4} \frac{k_{l}}{d_{i}}$

$$
P_{r}=\frac{P}{P_{c r}}
$$

It needs to mention that Pr in Equation (3-32) is the Prandtl number, while $P_{r}$ in (3-33) is the reduced pressure.

For the evaporation condition, Liu and Winterton's model (1991) was adopted

$$
\alpha_{i, p p}=\left[\left(F \alpha_{l o}\right)^{2}+\left(S \alpha_{p b}\right)^{2}\right]^{0.5}
$$

where $\alpha_{l}$ is the heat transfer coefficient of the liquid phase (obtained by (3-34)), $\mathrm{F}$ and $\mathrm{S}$ are two factors, and $\alpha_{p}$ is the pool boiling heat transfer coefficient, expressed as

$$
\begin{aligned}
& F=\left[1+x \operatorname{Pr}_{l}\left(\frac{v_{g}}{v_{l}}-1\right)\right]^{0.35} \\
& S=\frac{1}{1+0.055 F^{0.1} \operatorname{Re}_{l}^{0.16}}
\end{aligned}
$$




$$
\alpha_{p}=55 P_{r}^{0.12}\left(-\log P_{r}\right)^{-0.55} M^{-0.5} q_{f l u x}^{0.667}
$$

If the tube is placed horizontally and the Froude number, (defined by $\left.F r=(g v)^{2}\left(g_{r} d_{i}\right)^{-1}\right)$, is less than 0.05 , then the coefficients $F$ and $S$ need to be multiplied by two factors, respectively.

$$
e_{F}=F r^{0.1-2 F r}
$$

and

$$
e_{S}=\sqrt{F r}
$$

To ensure the model is capable of dealing with a high mass flux situation, Equation (3-23) was used with an exponent $n=0.75$.

\subsubsection{Judgment of extreme flow condition}

As mentioned in section 3.1.1.5, an extreme flow status may occur under a specific condition when the back pressure is lower than a critical value. However, the entropy criterion that was used for adiabatic flow is not feasible for non-adiabatic flow. Due to the magnitude of heat dissipation, the entropy of the capillary flow may decrease. Thus the definition of sonic velocity is adopted to determine the critical situation, which gives

$$
V_{\text {sonic }}=\sqrt{\left(\frac{\partial P}{\partial \rho}\right)_{s}}=v_{\text {mix }} \sqrt{-\left(\frac{\partial P}{\partial v_{\text {mix }}}\right)_{s}}
$$

The refrigerant velocity can be calculated using Equation (3-5). As the pressure and temperature 
decrease, the fluid velocity and the local sonic velocity both increase. At the beginning of the two-phase region, $V_{\text {sonic }}$ is larger than $V$, but the fluid velocity increases faster. When $V=V_{\text {sonic }}$, the extreme situation occurs and relative critical parameters can be obtained via iterative calculations.

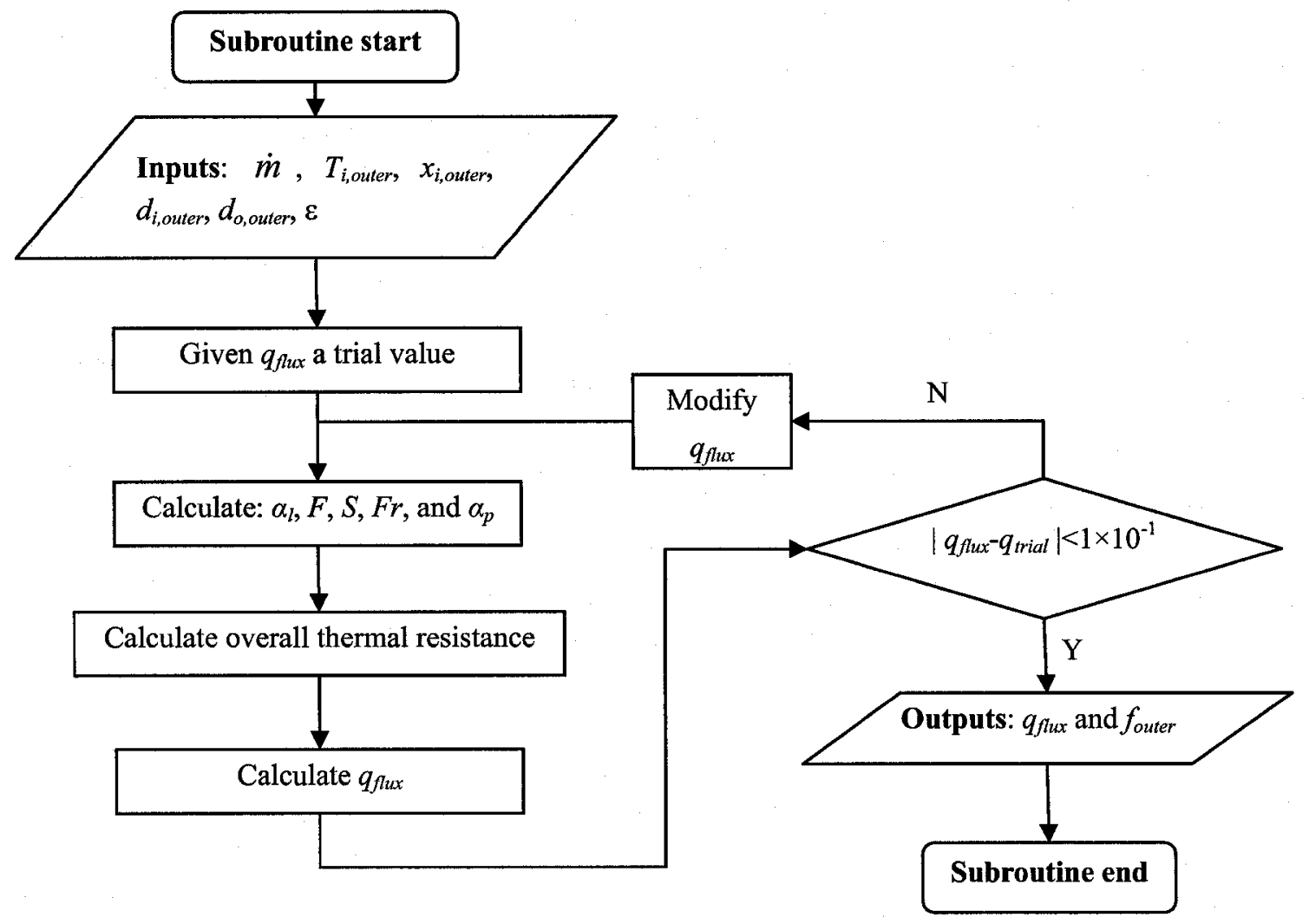

Figure 3-19 A trial-correction approach to determine $\alpha_{p}$ and $q_{f l u x}$

From Equation (3-39) it can be seen that the pool boiling heat transfer coefficient is dependent on the heat flux crossing the capillary tube wall. Since the heat flux can be calculated if these heat transfer coefficients are known, including $\alpha_{p}$, these two parameters are co-determined. To solve this problem, a trial-correction approach is applied, and Figure 3-19 is the flow diagram. 


\subsubsection{Parametric study and discussion}

The flow diagram for the non-adiabatic capillary model (NACAM) is shown in Figure 3-20. Since the suction line has a much larger inner diameter and thus relatively low flow velocity, normally no choking occurs at its outlet, therefore no critical judgment for the suction line flow was considered.

The capillary flow and the suction line flow are related through the heat transfer rate $q$, which is shown by dash lines. If a parallel flow arrangement is taken into account, the input values for the suction line flow are given. Otherwise, two trial values, i. e. outlet pressure (or temperature) and outlet quality, are assigned as inputs. When one simulation cycle of the capillary tube is finished, the output pressure (or temperature) and quality are compared with the inlet values: If they are equal (within a given accuracy), the program stops; if not, they are modified and returned as improved trial values to the next cycle until preassigned allowable accuracies are satisfied.

In the following discussion, the NACAM will be verified by comparing its results with several researchers. More validation will be provided in the following chapter, where data from our AAC test stand is discussed and compared. The model will then be used to investigate the relationships between various parameters. New characteristics related to heat transfer will be one focus of this section in addition to further comparison between NACAM and ACAM. 


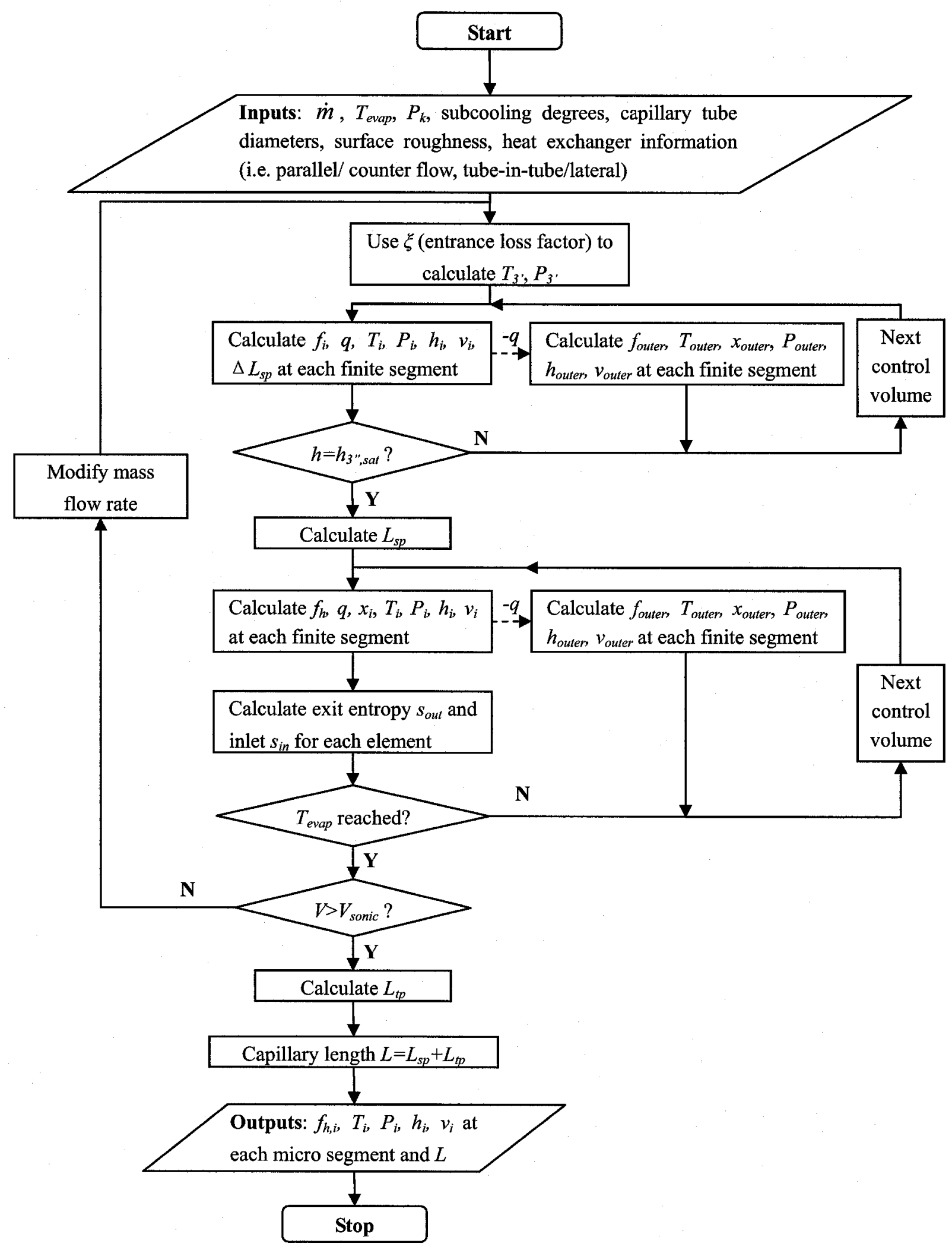

Figure 3-20 Flow diagram for non-adiabatic capillary model (NACAM) 


\subsubsection{Validation of the NACAM}

In order to validate the NACAM, its results are compared with some available experimental results from Peixoto (1995), Liu and Bullard (2000), and Melo et al (2000). Peixoto and Liu and Bullard performed experiments on lateral heat exchangers in a counter flow arrangement. For the capillary side, two adiabatic sections exist; first before and then after it contacts the suction line, which is illustrated in Figure 3-21. Refrigerant flow exiting the condenser goes into the capillary tube and experiences an adiabatic throttling process represented by process 3 "- 31 , where point 3 " is the condenser outlet as shown in Figure 3-2. The capillary tube is brazed to the suction line tube from point 31 to point 32 , where heat transfer occurs. After point 32 it undergoes another adiabatic process from 32 to 4' before entering the evaporator. Peixoto and Melo et al. conducted experiments
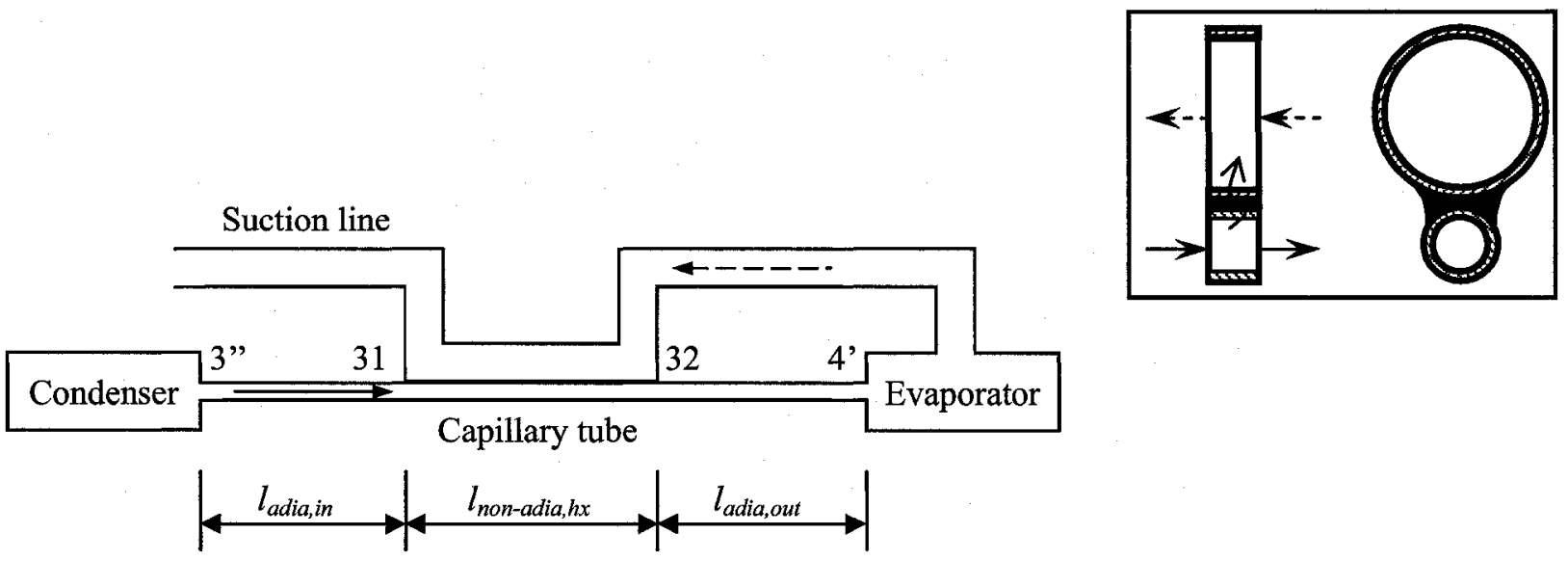

Figure 3-21 Illustration of a lateral capillary tube-suction line heat exchanger

on concentric heat exchangers with a counter flow arrangement, which has structure similar to a lateral heat exchanger. However, due to less thermal resistance between the two fluids, more heat flux can be achieved using tube-in-tube layout. Denoting "sp" as single-phase and "tp" as 
two-phase, for both types, determined by different working conditions, the flow might go through the heat transfer section in the form of sp-in-sp-out, sp-in-tp-out, or tp-in-tp-out.

\subsection{Lateral capillary-tube-suction-line heat exchangers}

Figure 3-22 compares the simulated results by NACAM with the experimental data of Peixoto (1995). The black round dots and the line with circles correspond to cycle with $\Delta t_{\text {sup }}=6.39^{\circ} \mathrm{C}$. The black squares and the line with rectangles are for $\Delta t_{\text {sup }}=9.49{ }^{\circ} \mathrm{C}$. It should be noted that the simulated results corresponding to the first two solid round points are approximations. NACAM couldn't generate matched results due to re-condensation (Bansal and Xu, 2003; Bansal and Yang, 2005). The flow mechanism assumed for NACAM cannot depict the true phenomenon occurring in the experiment. Similar results were obtained by Sinpiboon and Wongwises (2002). Under some operating conditions, the capillary refrigerant enters the two-phase region and dissipates heat to the suction line flow. If the specific heat transfer rate is large, the quality of capillary flow drops rapidly and thus becomes single-phase flow once again. This phenomenon is called "re-condensation". For the rest of the data, a slight over prediction can be observed with deviations from $1.41 \%$ to $7.60 \%$. The average value of the discrepancy is $5.54 \%$ and its RMS is $4.03 \%$. All results represent sp-in-tp-out flow. The larger the subcooling degrees, the smaller the prediction errors are.

In the research of Liu and Bullard (2000), the subcooling degrees are controlled using one of two apparatus: a separate glycol loop which was cooled by an R502 chiller subcools the refrigerant 


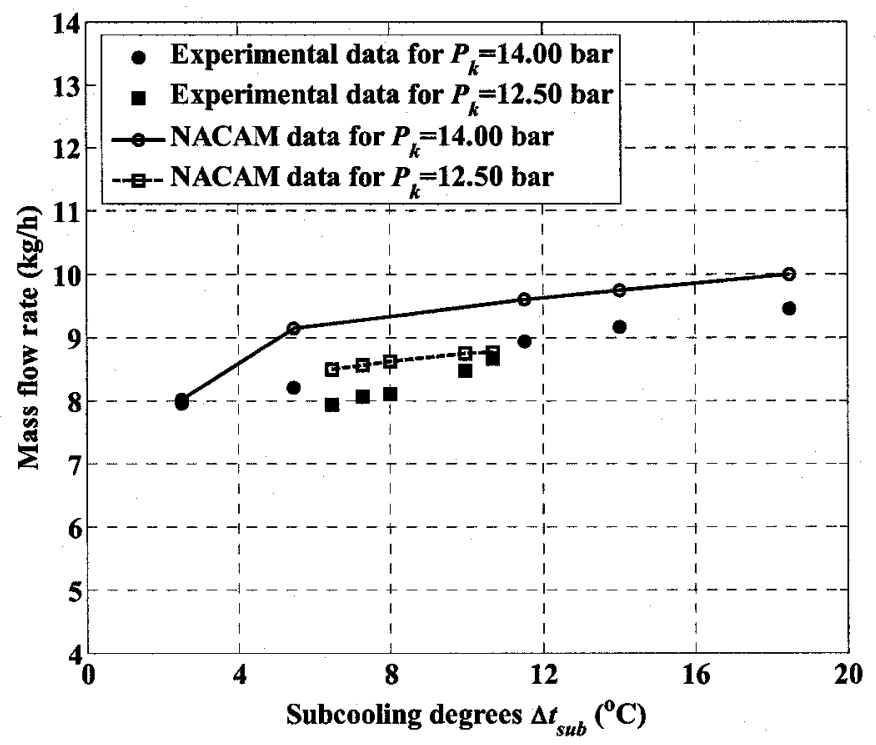

Figure 3-22 Comparison of NACAM results with Peixoto (1995) for $L=2.057 \mathrm{~m}, t_{e}=-10.09{ }^{\circ} \mathrm{C}$, $d_{i}=0.787 \mathrm{~mm}, d_{i, o u t e r}=6.314 \mathrm{~mm}$, and $\varepsilon=0.00045 \mathrm{~mm}$

in the condenser to as low as $27.78{ }^{\circ} \mathrm{C}$, or a glycol filled tank with a $2 \mathrm{~kW}$ heater is used to set a targeted amount of subcooling at the capillary inlet. Saturated flow at the capillary inlet was slowly cooled down to the maximum amount, then heated back to saturated liquid. The temperature change rate was controlled and kept very slow so as to achieve stable quasi-equilibrium conditions. Therefore, there are two sets of data for any one condition. Figure 3-23 shows the results of Liu and Bullard's (2000) experiments and the NACAM simulation. For the time sake, five cases with different subcooling degrees were considered, from 8 to $24^{\circ} \mathrm{C}$ with a step size of $4{ }^{\circ} \mathrm{C}$. Four out of five simulated results represent sp-in-sp-out flow, except for the $8{ }^{\circ} \mathrm{C}$ subcooling condition where the sp-in-tp-out mode was observed. It can be seen from the figure that NACAM under predicts the mass flow rate when $\Delta t_{\text {sub }}$ is less than $16.6^{\circ} \mathrm{C}$ and over predicts thereafter. The maximum prediction errors for increasing $\Delta t_{\text {sub }}$ and decreasing $\Delta t_{\text {sub }}$ are $10.3 \%$ and $4.8 \%$ respectively. For the increasing 
subcooling condition, all measured mass flow rates range from $8.17 \mathrm{~kg} / \mathrm{h}$ to $8.56 \mathrm{~kg} / \mathrm{h}$; for the decreasing subcooling condition, they are from $7.90 \mathrm{~kg} / \mathrm{h}$ to $8.22 \mathrm{~kg} / \mathrm{h}$. The simulated mass flow rate increases from $7.52 \mathrm{~kg} / \mathrm{h}$ at $8{ }^{\circ} \mathrm{C}$ subcooling to $8.56 \mathrm{~kg} / \mathrm{h}$ at $24^{\circ} \mathrm{C}$ subcooling. It is thus possible that there is some other mechanism dampening the influence of subcooling degrees on the mass flow rate.

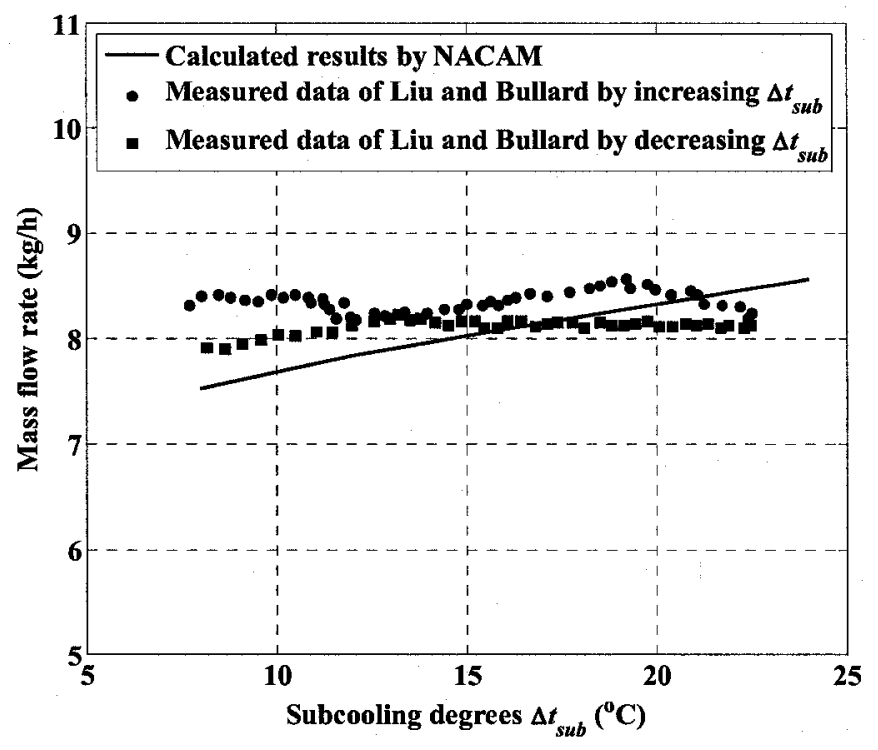

Figure 3-23 Comparison of NACAM results with Liu and Bullard (2000) for $L=1.880 \mathrm{~m}$, $t_{k}=54.44^{\circ} \mathrm{C}, t_{e}=15.56{ }^{\circ} \mathrm{C}, \Delta t_{\text {sup }}=5.56^{\circ} \mathrm{C}, d_{i}=0.739 \mathrm{~mm}, d_{i, o u t e r}=9.525 \mathrm{~mm}$, and $\varepsilon=0.0004 \mathrm{~mm}$

\subsection{Concentric capillary-tube-suction-line heat exchangers}

To further verify the NACAM, some experimental results reported by Peixoto (1995) and Melo et al. (2000) on concentric capillary-tube-suction-line heat exchangers are taken into account. As can be seen from Figure 3-24, two sets of data were compared. One was obtained for $P_{k}=11.50$ bar and $\Delta t_{\text {sup }}=5.39{ }^{\circ} \mathrm{C}$, and the other was for $P_{k}=10.60$ bar and $\Delta t_{\text {sup }}=7.09{ }^{\circ} \mathrm{C}$. For both conditions, re-condensation can be observed when $\Delta t_{\text {sub }}$ is between 4.0 and $6.0^{\circ} \mathrm{C}$, and sp-in-tp-out flow was 
observed for all subcooling degrees considered. For the higher condensation pressure, heat transfer occurs in the two-phase region inside the capillary tube when $\Delta t_{\text {sub }}<4.0^{\circ} \mathrm{C}$; NACAM under predicts the mass flow rate with errors ranging from $13.9 \%$ to $20.0 \%$. Heat transfer only occurs in the single-phase region when $\Delta t_{\text {sub }}>6.0^{\circ} \mathrm{C}$; in these cases the mass flow rates are $15.6 \%$ to $19.7 \%$ under predicted. For the lower condensation pressure, only single-phase heat transfer was observed for region where $\Delta t_{\mathrm{sub}}>6.0^{\circ} \mathrm{C}$. The relative errors fall into the range of $0.7 \%$ to $6.6 \%$.

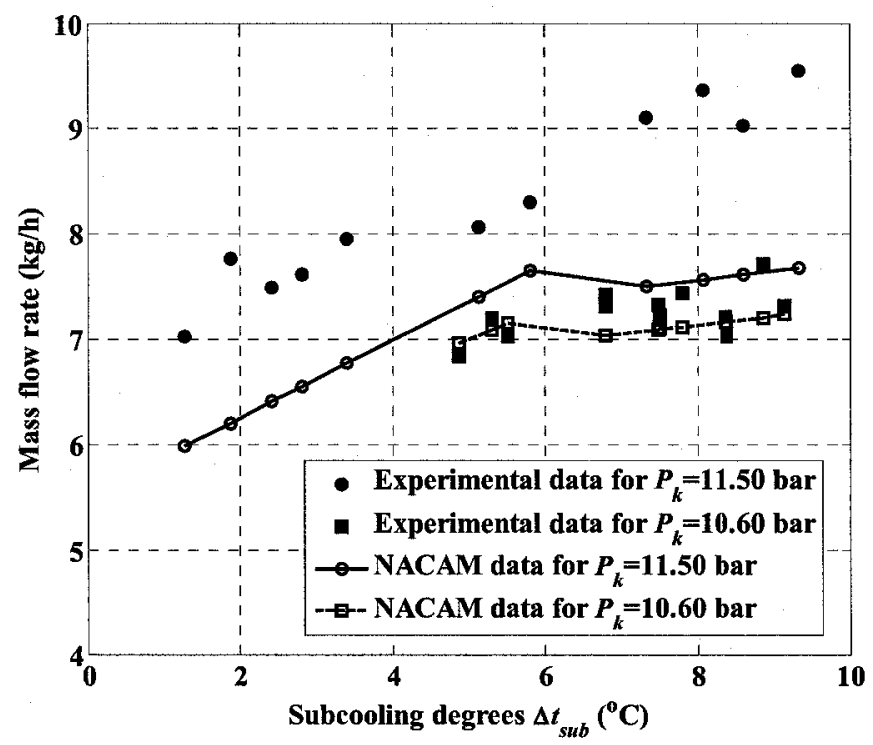

Figure 3-24 Comparison of NACAM results with experimental results for $L=2.057 \mathrm{~m}$, $t_{e}=-10.09^{\circ} \mathrm{C}, d_{i}=0.787 \mathrm{~mm}, d_{i, o u t e r}=6.314 \mathrm{~mm}$, and $\varepsilon=0.00045 \mathrm{~mm}$

Since there is no experimental data corresponding to the range $3.4{ }^{\circ} \mathrm{C}<\Delta t_{\text {sub }}<5.1{ }^{\circ} \mathrm{C}$ for $P_{k}=11.50 \mathrm{bar}$ and $\Delta t_{\mathrm{sub}}<4.0^{\circ} \mathrm{C}$ for $P_{k}=10.60$ bar were shown, so it is unclear whether there exists a situation where heat transfer occurs in both the single-phase and two-phase regions. To investigate this possibility, a subcooling value of $4.0^{\circ} \mathrm{C}$ was input into NACAM to simulate the mass flow rate for the high condensation pressure situation; two $\Delta t_{\text {sub }}$ values, $3.0^{\circ} \mathrm{C}$ and $4.0^{\circ} \mathrm{C}$, were chosen for 
the low pressure condition. The results show that re-condensation occurred for $\Delta t_{\mathrm{sub}}=4.0^{\circ} \mathrm{C}$ for both condensation pressures, and heat transfer occurred in the two-phase region for $\Delta t_{\mathrm{sub}}=3.0^{\circ} \mathrm{C}$. The corresponding mass flow rates are calculated as $6.24 \mathrm{~kg} / \mathrm{h}$ and $6.60 \mathrm{~kg} / \mathrm{h}$, respectively. Even when re-condensation occurs, the heat transfer starts in the two-phase region. Therefore, there might not exist a heat transfer mode that starts in the single-phase region and ends in the two-phase region for the capillary flow in this case.

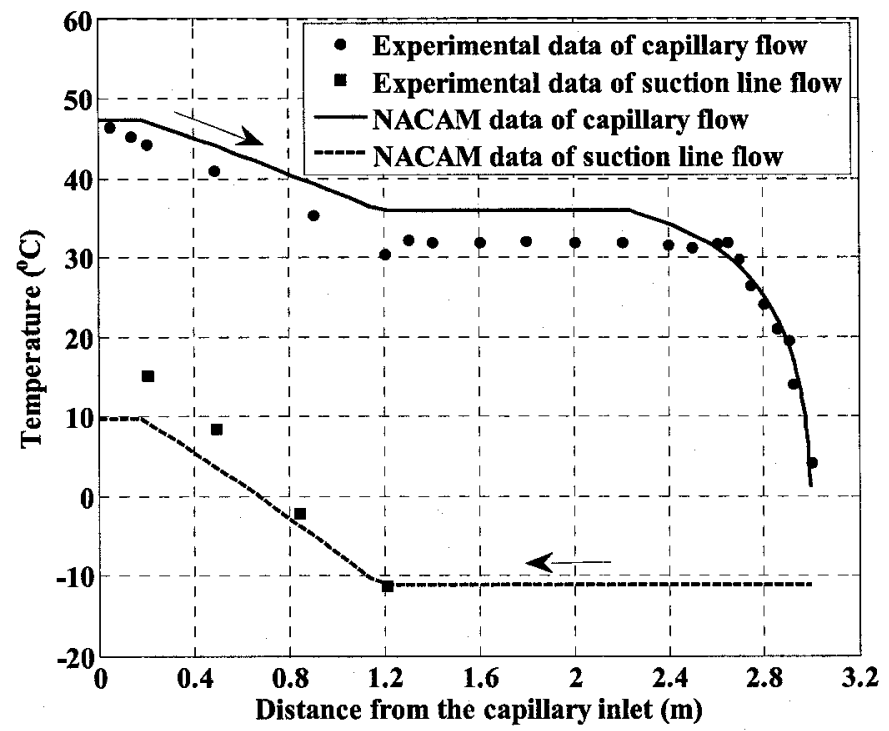

Figure 3-25 Comparison of NACAM results with Melo et al. for $\dot{m}=7.42 \mathrm{~kg} / \mathrm{h}, L=3.000 \mathrm{~m}$, $t_{k}=52.43{ }^{\circ} \mathrm{C}, \Delta t_{\mathrm{sub}}=5.0^{\circ} \mathrm{C}, \Delta t_{\mathrm{sup}}=1.81{ }^{\circ} \mathrm{C}, d_{i}=0.83 \mathrm{~mm}$, and $d_{i, \text { outer }}=7.86 \mathrm{~mm}$

Another feature of the Figure 3-24 is that the increase of the capillary mass flow rate with $\Delta t_{\text {sub }}$ slows down when the heat transfer process moves from the two-phase region to the single-phase region. The line slopes for $P_{k}=11.50 \mathrm{bar}$ and $P_{k}=10.60 \mathrm{bar}$ are $0.371 \mathrm{~kg} /\left(\mathrm{hr} \cdot{ }^{\circ} \mathrm{C}\right)$ and $0.295 \mathrm{~kg} /\left(\mathrm{hr}{ }^{\circ} \mathrm{C}\right)$ for heat transfer in the two-phase region, and only $0.0846 \mathrm{~kg} /\left(\mathrm{hr} \cdot{ }^{\circ} \mathrm{C}\right)$ and $0.0854 \mathrm{~kg} /\left(\mathrm{hr} \cdot{ }^{\circ} \mathrm{C}\right)$ in the single-phase region, respectively. This can be interpreted as the influence of different heat transfer 
coefficients for different flow phases. Due to the low heat transfer coefficient of single-phase flow, less heat is dissipated from the capillary refrigerant, which makes the process closer to adiabatic, allowing less flow to pass through the tube.

It might be of interest to note that those simulation data for re-condensation provide less deviation for $P_{k}=11.50$ bar than for $P_{k}=10.60$ bar. As discussed above, the prediction error is between $13.9 \%$ and $20.0 \%$ for $\Delta t_{\text {sub }}<4.0{ }^{\circ} \mathrm{C}$ and $\Delta t_{\text {sub }}>6.0^{\circ} \mathrm{C}$, but it is lowered to around $8.0 \%$ for subcooling in between these values. This shows that the current model can be used to predict refrigerant mass flow rate whether re-condensation occurs or not. It should be noted that in such a case the behaviours of other parameters, such as pressure, temperature, specific volume, etc. can not currently be described and thus require more investigation.

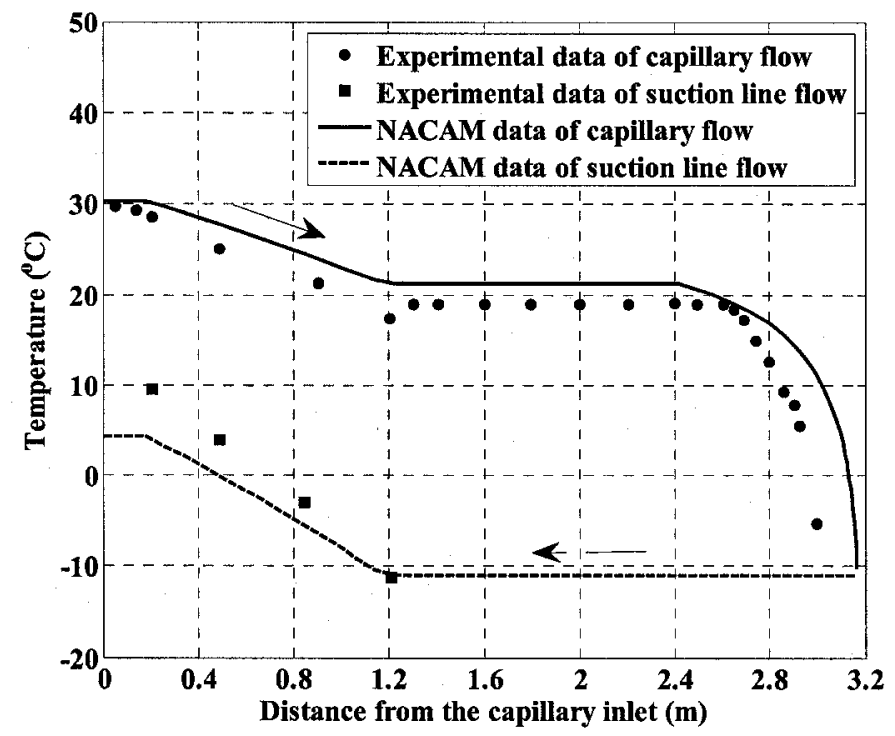

Figure 3-26 Comparison of NACAM results with Melo et al. for $\dot{m}=5.49 \mathrm{~kg} / \mathrm{h}, L=3.000 \mathrm{~m}$, $t_{k}=35.53{ }^{\circ} \mathrm{C}, \Delta t_{\mathrm{sub}}=5.2{ }^{\circ} \mathrm{C}, \Delta t_{\mathrm{sup}}=1.79{ }^{\circ} \mathrm{C}, d_{i}=0.83 \mathrm{~mm}$, and $d_{i, \text { outer }}=7.86 \mathrm{~mm}$ 
Figure 3-25 and Figure 3-26 are comparisons of temperature along the capillary tube between NACAM and Melo et al. (2000) for different mass flow rates. In both figures the simulated values are slightly higher than the measured data. In the first $20 \mathrm{~cm}$, there is no heat transfer, and the refrigerant should follow the isenthalpic line in the sub-cooled region. Accordingly, the temperature of the liquid refrigerant should not vary greatly. However, the experimental data show a steady decrease in this area. This might be attributed to the entrance effect, which was not included in the NACAM. In both figures, the simulated flash points occur earlier than in the experimental results, which could be explained as the presence of a metastable region. Compared with the model of Sinpiboon and Wongwises (2002), this is one obvious difference. Due to the improvement of NACAM, where specific volume is modified to smooth its change in the trans-phase area, the flow temperature drops faster than their model. This is especially evident in Figure 3-25, where the NACAM well follow the measured data. In Figure 3-26, the tube length is also slightly shorter than Sinpiboon and Wongwises's (2002) model.

For the flow in the suction line, similar to the model of Sinpiboon and Wongwises (2002), NACAM under predicts the temperature behaviour; this might be caused by cold leak to the environment. It is also possible that Liu and Winterton's (1991) correlation under predicts the heat transfer coefficient of the suction line flow. However, if the heat transfer is increased, the capillary length will greatly increase, which makes the model generate even more deviation.

Figure 3-27 shows the behaviour of a set of parameters for a capillary-tube-suction-line under 


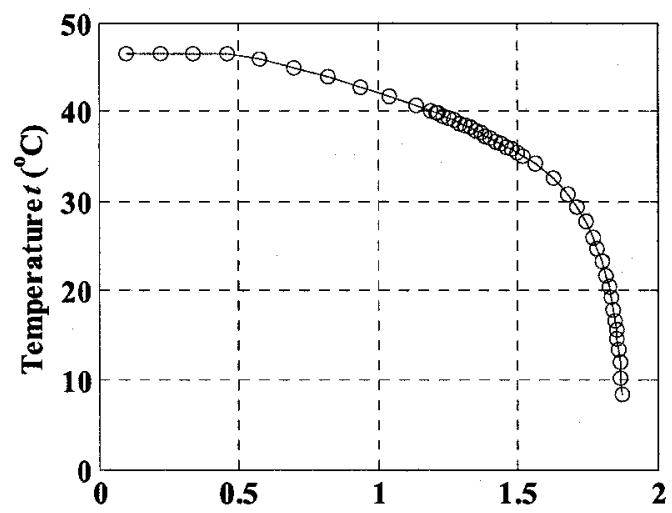

(a) Distance from capillary inlet (m)

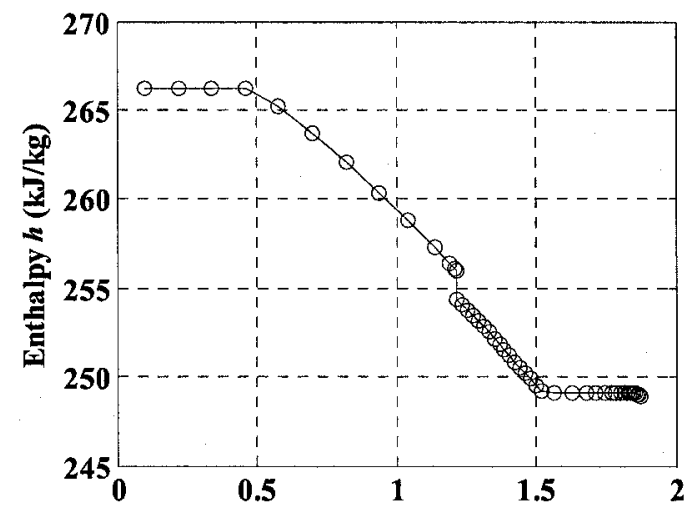

(c) Distance from capillary inlet (m)

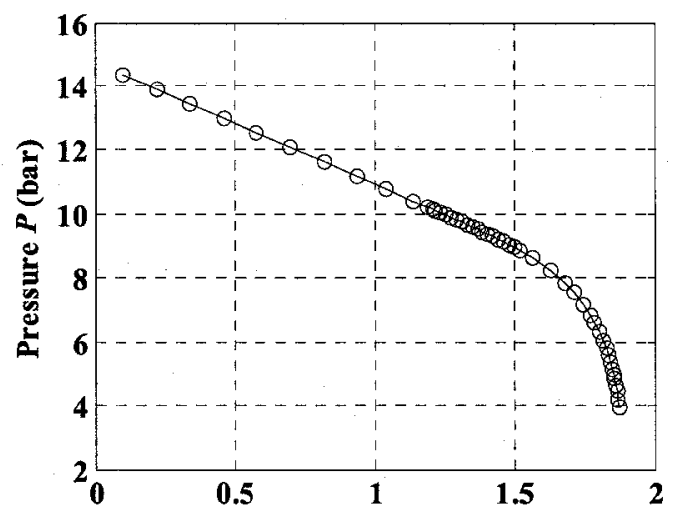

(b) Distance from capillary inlet (m)

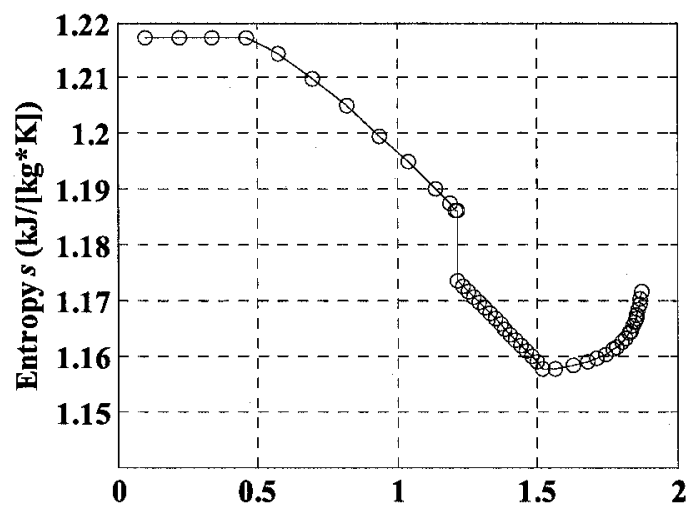

(d) Distance from capillary inlet (m)

Figure 3-27 A typical set of NACAM results with $\dot{m}=7.52 \mathrm{~kg} / \mathrm{h}, L=1.880 \mathrm{~m}, \boldsymbol{t}_{\boldsymbol{k}}=54.44^{\circ} \mathrm{C}$, $\Delta t_{\text {sub }}=8.0{ }^{\circ} \mathrm{C}, t_{k}=15.56^{\circ} \mathrm{C}, \Delta t_{\text {sup }}=5.56^{\circ} \mathrm{C}, d_{i}=0.739 \mathrm{~mm}$, and $\varepsilon=0.0004 \mathrm{~mm}$

a typical working condition. The heat exchange area starts at $0.533 \mathrm{~m}$ and ends at $1.536 \mathrm{~m}$. Outside of this area the refrigerant experiences an adiabatic process just like that shown in Figure 3-7. Larger entropy and enthalpy discontinuities can also be found around the flash point. It is interesting to note that the pressure decreases at nearly the same speed for both adiabatic and non-adiabatic processes in the single-phase region. Figure 3-28 details the corresponding quality profile. Two turning points can be clearly seen from the figure. The first point represents the start of 
flash while the second is the start of adiabatic two-phase expansion. Due to heat dissipation, the onset of flash is delayed, and the change rate of quality is relatively slow.

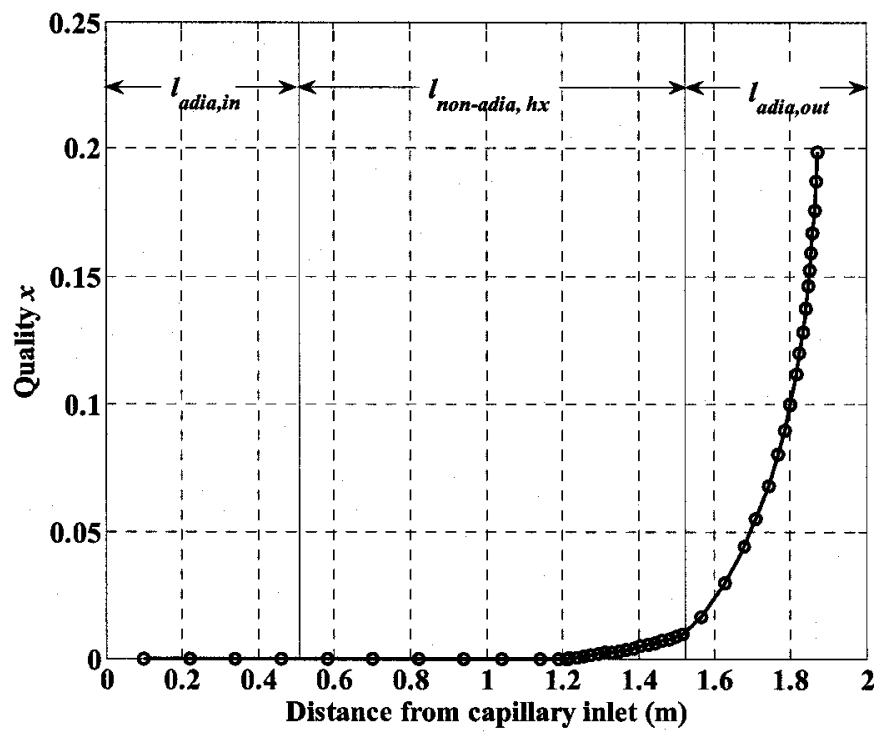

Figure 3-28 Quality profile of results of NACAM under the same conditions as Figure 3-27

To get a better idea of the differences between ACAM and NACAM, an investigation of a heat exchanger with full-length heat transfer between the capillary flow and the suction line flow was performed. The tube-in-tube counter flow model was adopted. For the sake of direct comparison, capillary tube length and diameters, the condensation pressure, subcooling degrees, and tube surface roughness were kept the same as in Figure 3-7. The evaporation temperature, superheated degrees, and suction line inner diameter were selected as $t_{e}=0{ }^{\circ} \mathrm{C}, \Delta t_{\text {sup }}=0.38{ }^{\circ} \mathrm{C}$, and $d_{i, \text { outer }}=9.00 \mathrm{~mm}$. Figure 3-29 shows the calculation results. Heat transfer makes all four parameters drop along the flow direction. The single-phase length is $10 \mathrm{~cm}$ longer than the adiabatic case. The mass flow rate is $9.94 \mathrm{~kg} / \mathrm{h}$, up from the adiabatic $9.52 \mathrm{~kg} / \mathrm{h}$. 


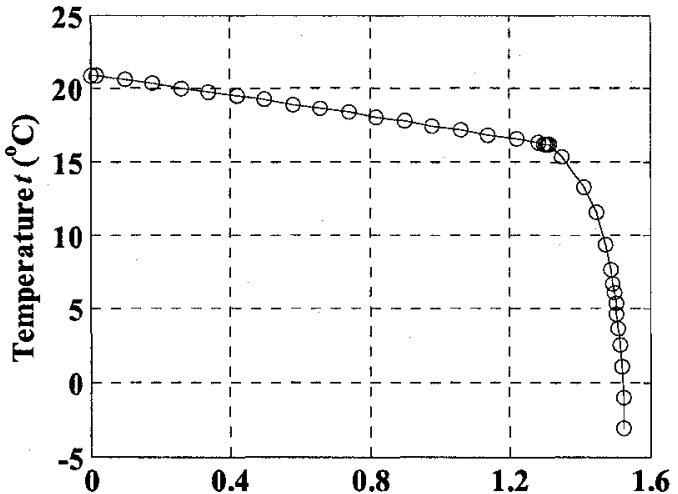

(a) Distance from capillary inlet $(\mathrm{m})$

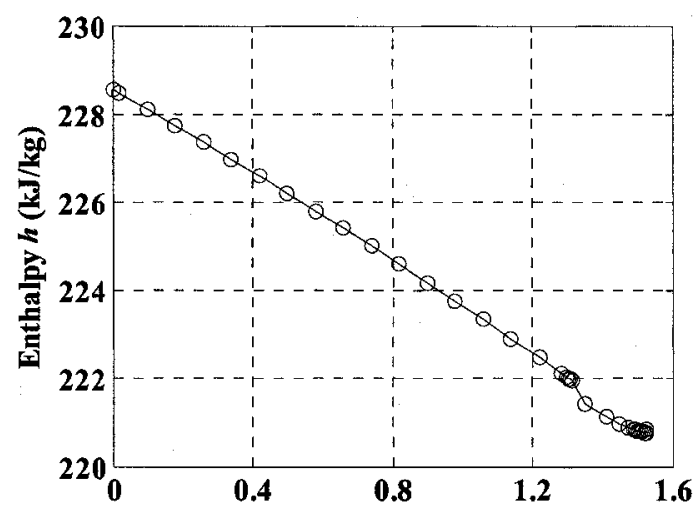

(c) Distance from capillary inlet (m)

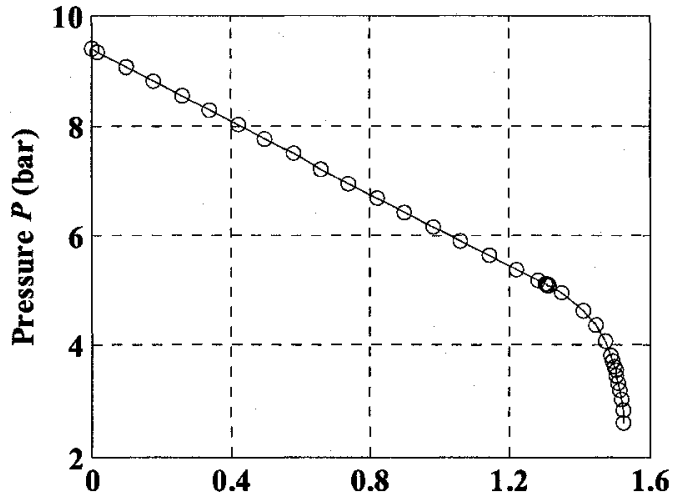

(b) Distance from capillary inlet $(\mathrm{m})$

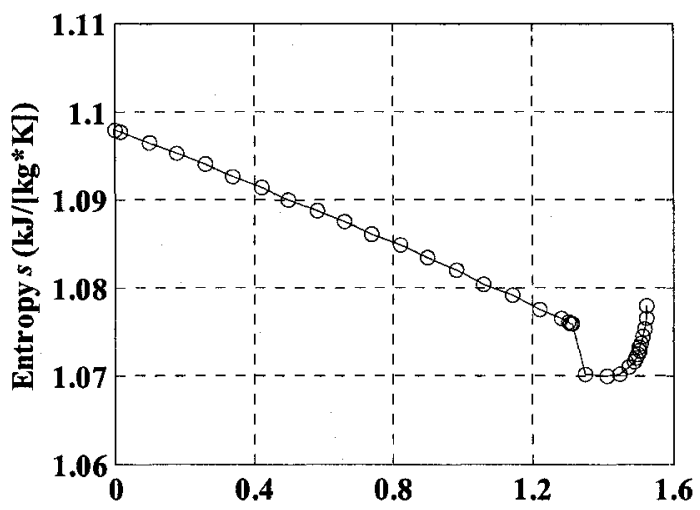

(d) Distance from capillary inlet (m)

Figure 3-29 A typical set of results of NACAM for a full-length heat exchange with $\dot{m}=9.94 \mathrm{~kg} / \mathrm{h}, L=1.526 \mathrm{~m}, t_{k}=37.65{ }^{\circ} \mathrm{C}, \Delta t_{\text {sub }}=16.7^{\circ} \mathrm{C}, t_{e}=0^{\circ} \mathrm{C}, \Delta t_{\text {sup }}=0.38{ }^{\circ} \mathrm{C} d_{i}=0.84 \mathrm{~mm}$, $d_{i, \text { outer }}=9.00 \mathrm{~mm}$, and $\varepsilon=0.0005 \mathrm{~mm}$

In Figure 3-30 (a), the exit quality of the capillary flow is slightly lower than the adiabatic case, dropping from 0.134 to 0.125 . In (b), the thermal driving force for the single-phase region increases from $11.9^{\circ} \mathrm{C}$ to $14.7^{\circ} \mathrm{C}$ along the flow direction. The higher slope of suction line flow corresponds to its relatively low specific heat capacity, which makes it easy to change under a certain amount of heat load. It is worth noting that reverse heat transfer from the suction line flow to the capillary flow might exist if the evaporation temperature or the superheated magnitude is relatively high. However, 
it won't affect the overall thermal performance of the device significantly because such heat transfer occurs at the capillary exit area where the temperature drops sharply within a very short length, and then the heat transfer is confined to the limited contact area.

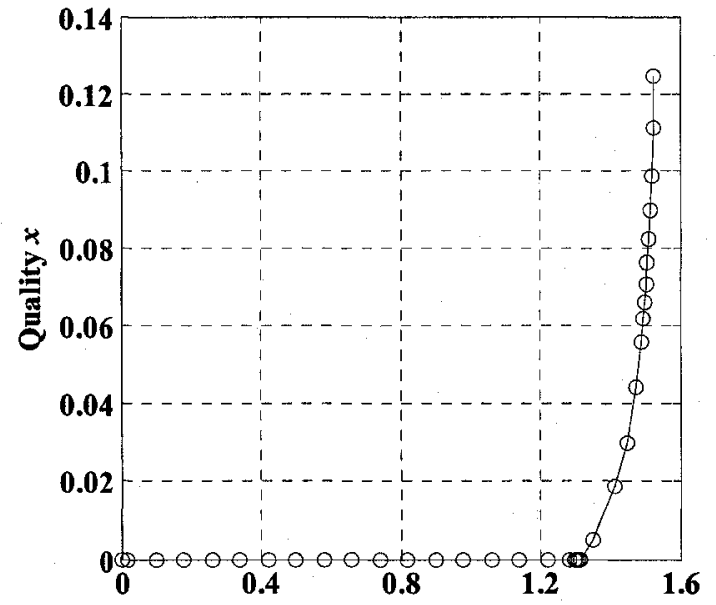

(a) Distance from capillary inlet (m)

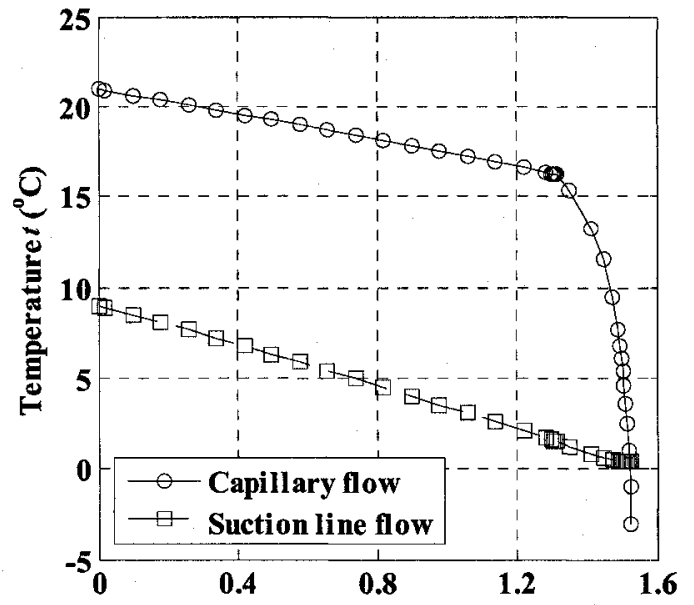

(b) Distance from capillary inlet (m)

Figure 3-30 Quality and temperature profiles of results of NACAM under the same conditions as Figure 3-29

\subsubsection{Parametric analysis}

In this section, comparisons between NACAM and ACAM will be conducted first, including changes in the influences of the inner diameter, the capillary length, the condensation pressure, the subcooling degrees, and the surface roughness. As new features of NACAM, further investigation on the influence of the evaporation pressure, the initial condition of the suction line flow (for two-phase flow it is the quality, and for superheated flow it is the inlet temperature), the inner diameter of the suction line, the flow arrangement (parallel flow or counter flow), and the heat exchanger type (tube-in-tube or lateral) will be performed. 


\subsection{Influence of capillary inner diameter}

When starting as a sub-cooled flow and subjected to heat dissipation, a capillary flow with the same mass flow rate as a similar adiabatic case is denser, making the friction resistance relatively smaller. More importantly, from the pressure-enthalpy diagram it can be seen that a capillary flow from ACAM model is nearly vertical; it declines to the bottom left, which means there is more room for the flow to go from the inlet to the saturated-liquid line. Determined by these two factors,

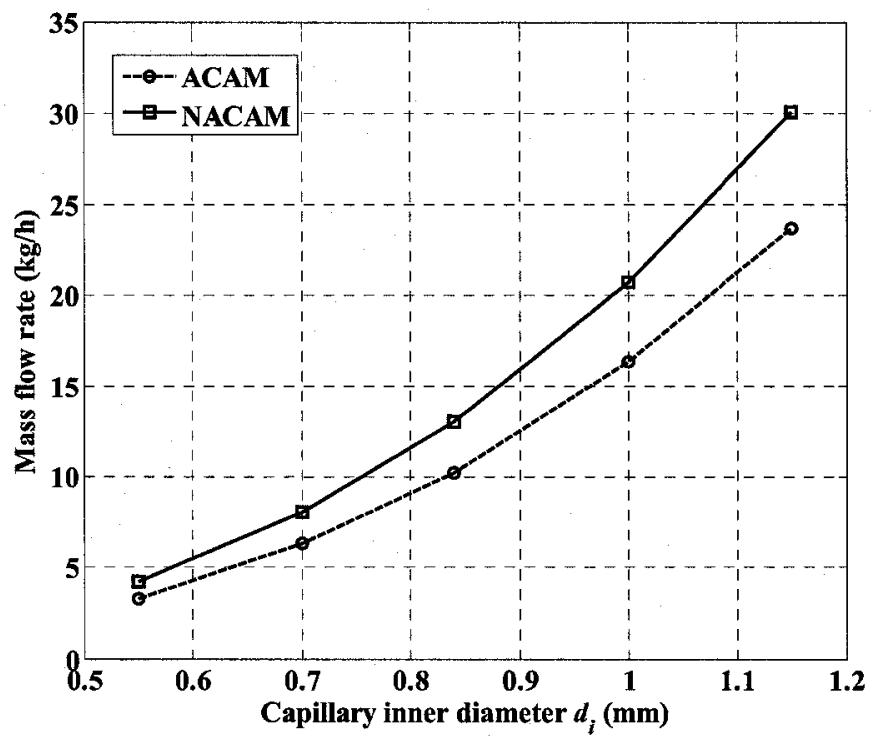

Figure 3-31 Influence of capillary inner diameter on the mass flow rate with $L=1.526 \mathrm{~m}$, $d_{i, \text { outer }}=8.00 \mathrm{~mm}, P_{k}=11.06 \mathrm{bar}, \Delta t_{\mathrm{sub}}=16.7^{\circ} \mathrm{C}, t_{e}=-10.09^{\circ} \mathrm{C}, \Delta t_{\mathrm{sup}}=4.79^{\circ} \mathrm{C}, \varepsilon=0.0005 \mathrm{~mm}$, and $c=4.0 \%$

the single-phase region will be longer than the corresponding adiabatic case. Therefore, with the same mass flow rate, a longer capillary is required to generate sufficient pressure drop. In other words, if the tube length is fixed, then more mass flow is expected. To verify this hypothesis, ACAM and NACAM were used to calculate the mass flow rate for the same capillary tube with a 
length of $1.526 \mathrm{~m}$, a surface roughness of $0.5 \mu \mathrm{m}$, a condensation pressure of $11.06 \mathrm{bar}$, a subcooling degrees of $16.7^{\circ} \mathrm{C}$, and an oil concentration of $4.0 \%$. For NACAM, the evaporation pressure and superheating degrees were also required, which were assumed to be 2.00 bar and $4.79^{\circ} \mathrm{C}$, respectively. The results are shown in Figure 3-31. For a given inner diameter, the NACAM results are higher than the ACAM results, proving the earlier assumption. Similar to Figure 3-10, the mass flow rate grows exponentially with the increase of capillary inner diameter.

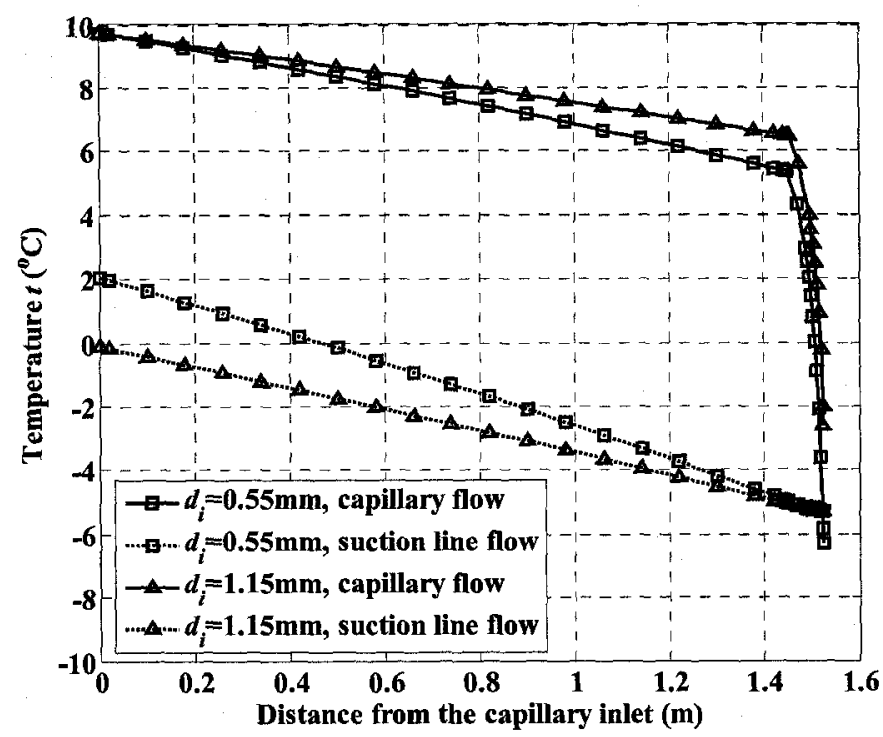

Figure 3-32 Influence of capillary inner diameter on the temperature with $L=1.526 \mathrm{~m}$, $d_{i, \text { outer }}=8.00 \mathrm{~mm}, P_{k}=11.06 \mathrm{bar}, \Delta t_{\mathrm{sub}}=16.7^{\circ} \mathrm{C}, t_{e}=-10.09^{\circ} \mathrm{C}, \Delta t_{\text {sup }}=4.79^{\circ} \mathrm{C}, \varepsilon=0.0005 \mathrm{~mm}$, and $c=4.0 \%$

Figure 3-32 is the temperature profile along the length of the capillary for different inner diameters using NACAM. When $d_{i}$ is enlarged from $0.55 \mathrm{~mm}$ to $1.15 \mathrm{~mm}$, the mass flow rate jumps from $4.20 \mathrm{~kg} / \mathrm{h}$ to $30.04 \mathrm{~kg} / \mathrm{h}$, and the capillary flow exit temperature increases from $-6.32{ }^{\circ} \mathrm{C}$ to $-2.63^{\circ} \mathrm{C}$, which corresponds to 0.05 bar increase of exit pressure. This shows that the pressure drop 
across the capillary tube is slightly influenced by $d_{i}$ and can not be kept constant. It is interesting that the single-phase length (about $1.45 \mathrm{~m}$ ) does not change for either case, even though the flash temperature varies.

Regarding temperature, another important feature is that with the same inlet temperatures for both the capillary and the suction line, the temperature difference between them increases with the capillary inner diameter. From a heat transfer point of view, this means that a smaller diameter tube will bring higher thermal effectiveness, though it also corresponds to a lower heat capacity $\left(Q=6.95 \mathrm{~W}\right.$ for $d_{i}=0.55 \mathrm{~mm}$ and $Q=36.96 \mathrm{~W}$ for $d_{i}=1.15 \mathrm{~mm}$ ). Such a phenomenon is logical and can be explained as follows: from Equation (3-28) it can be seen that the temperature difference is affected by the overall heat transfer coefficient and the mass flow rate; these two parameters lead the refrigerant in opposite directions. The overall heat transfer coefficient describes the capacity of the fluids to diminish a temperature difference between them. When $d q$ and $\dot{m}$ are fixed, a higher $U A$ will reduce the $\Delta T$. On the other hand, when $d q$ and $U A$ are fixed, $\Delta T$ is proportional to the mass flow rate. In this case, $\dot{m}$ also grows with $d_{i}$. If the rate of growth of $\dot{m}$ is greater than that of $U A$, a greater temperature difference is obtained. And if they grow with a similar speed, then $\Delta T$ will not change. Figure 3-32 belongs to the latter category and both $U A$ and $\dot{m}$ grow with the inner diameter. Because the specific heat transfer rate at $d_{i}=1.15 \mathrm{~mm}$ is slightly greater than that at $d_{i}=0.55 \mathrm{~mm}$, the temperature difference $(\Delta T)$ is smaller.

As for the temperature behaviour of one fluid, it can be determined by the following equation 


$$
d q=c_{p} \Delta T
$$

This equation is for fluids with constant specific heat capacity; $\Delta T$ is the temperature difference between one point of flow and another downstream point. In our case, the change of $\Delta T$ is much greater than that of the $c_{p}$, so it is qualitatively correct to correlate parameters $\Delta T$ and $d q$. As the $d q$ becomes small, the temperature difference reduces.

Due to the presence of heat transfer, the temperature drop of the capillary flow in the two-phase region is not as strongly affected by the mass flow rate as it was in the adiabatic case.

The total heat transfer capacity $Q$ is a product of the mass flow rate $\dot{m}$ and the specific heat transfer rate $q$. In this case, since the increase of $\dot{m}$ is greater than the decrease of $q, Q$ increases accordingly. This alone cannot guarantee an increase in the device effectiveness. Based on simulation results, increasing the suction line inlet temperature might be an effective way to improve the device performance in term of both the heat transfer rate and device efficiency.

\subsection{Influence of capillary length}

Figure 3-33 shows the influence of heat transfer on mass flow rate for capillaries with different lengths. As expected, more refrigerant can pass through the tube with the presence of heat dissipation. Compared with inner diameter, the influence is milder. But compared with Figure 3-11, where the increment of the mass flow rate drops from $15.7 \%$ to $12.6 \%$ with capillary length, the enhancement in this figure is relatively large at $27.4 \%$ to $28.5 \%$. 


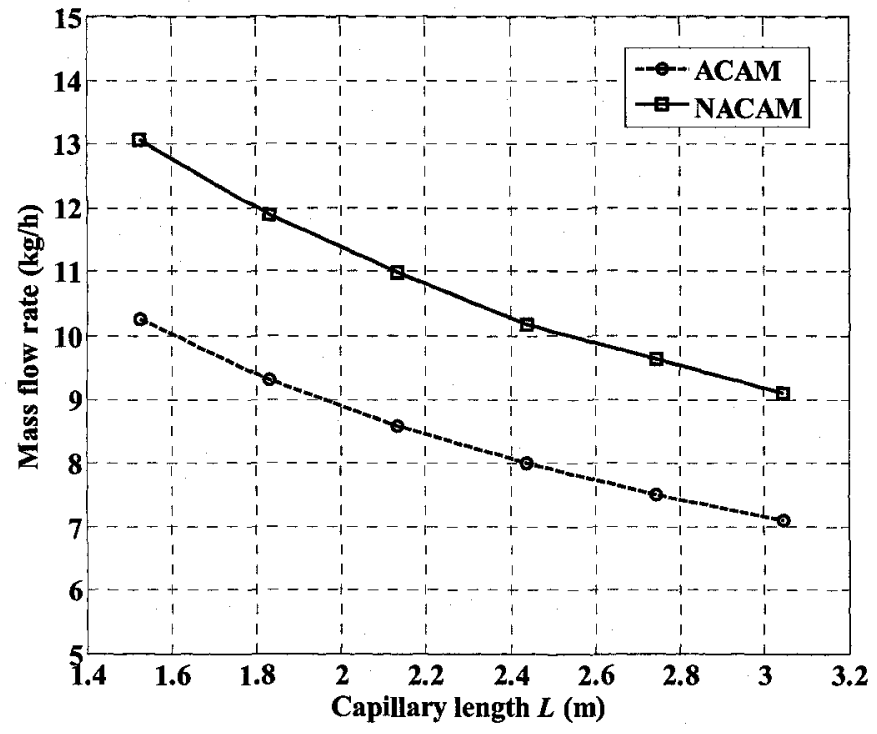

Figure 3-33 Influence of capillary length on the mass flow rate with $d_{i}=0.84 \mathbf{~ m m}$, $d_{i, \text { outer }}=8.00 \mathrm{~mm}, P_{k}=11.06 \mathrm{bar}, \Delta t_{\mathrm{sub}}=16.7^{\circ} \mathrm{C}, t_{e}=-10.09^{\circ} \mathrm{C}, \Delta t_{\text {sup }}=4.79^{\circ} \mathrm{C}, \varepsilon=0.0005 \mathrm{~mm}$, and $c=4.0 \%$

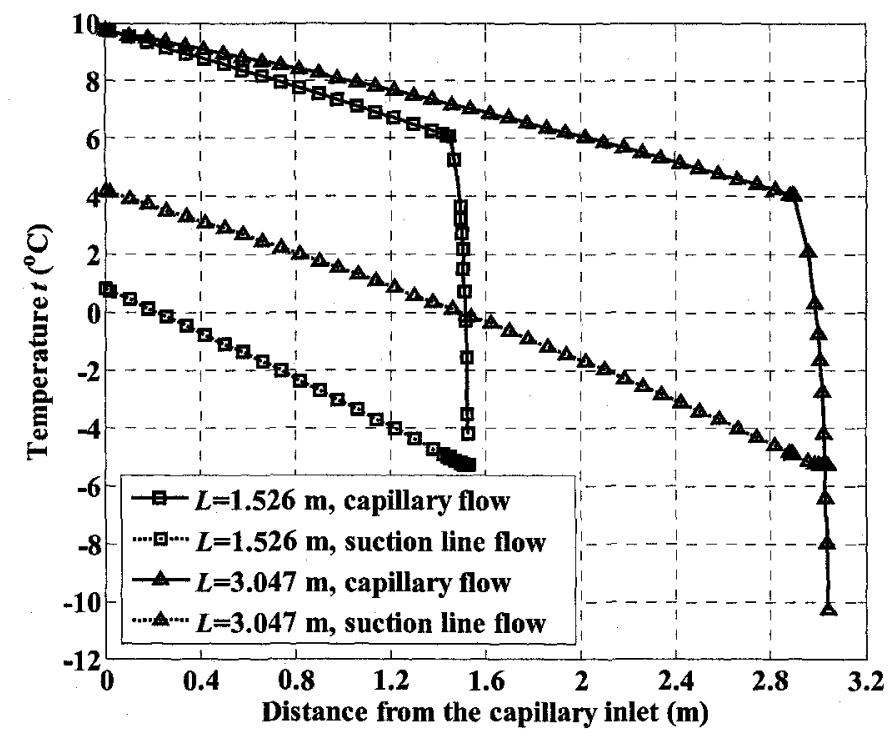

Figure 3-34 Influence of capillary length on the temperature with $d_{i}=0.84 \mathbf{m m}$, $d_{i, \text { outer }}=8.00 \mathrm{~mm}, P_{k}=11.06$ bar, $\Delta t_{\text {sub }}=16.7^{\circ} \mathrm{C}, t_{e}=-10.09{ }^{\circ} \mathrm{C}, \Delta t_{\text {sup }}=4.79^{\circ} \mathrm{C}, \varepsilon=0.0005 \mathrm{~mm}$, and $c=4.0 \%$ 
The temperature profile with different capillary lengths can be seen in Figure 3-34. The specific heat transfer rate decreases for the long tube length, thus the overall capillary flow temperature difference is smaller. At the same time, due to the influences of the three factors, the weaker heat transfer coefficient, the reduced temperature difference, and the increased thermal contact area, the heat transfer rate only increases slightly from $18.10 \mathrm{~W}$ to $19.57 \mathrm{~W}$. For $l_{\text {cap }}=3.047$ $\mathrm{m}$, the last $1.5 \mathrm{~cm}$ of the tube has the most rapid temperature drop from $-5.3{ }^{\circ} \mathrm{C}$ to $-10.3{ }^{\circ} \mathrm{C}$, and thus a slight reverse heat transfer occurs (about $0.02 \mathrm{~W}$ ).

\subsection{Influence of condensation pressure}

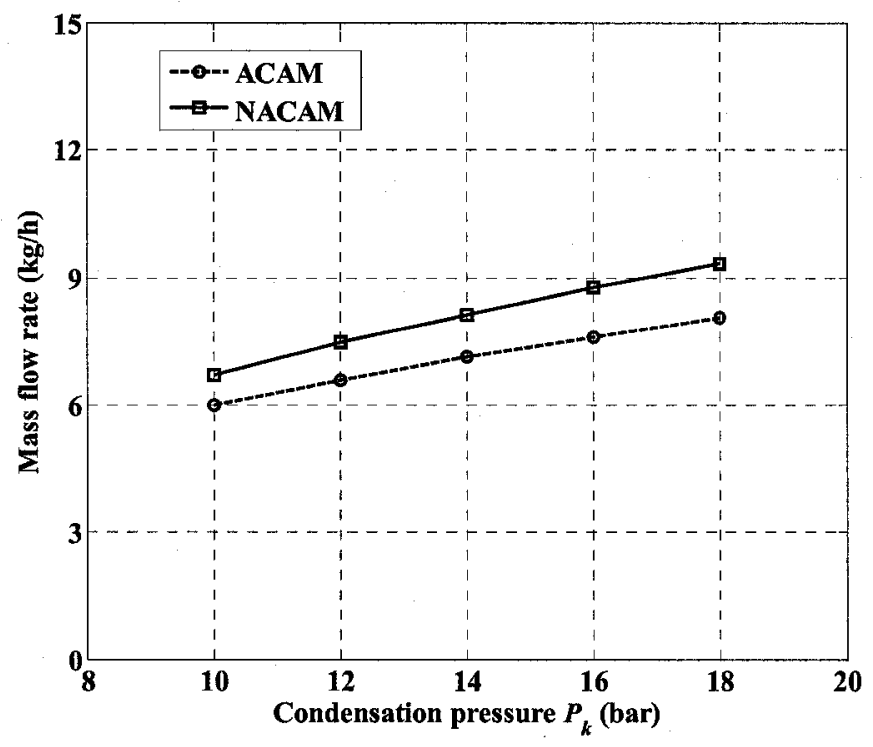

Figure 3-35 Influence of condensation pressure on the mass flow rate with $L=1.526 \mathrm{~m}$, $d_{i}=0.70 \mathrm{~mm}, d_{i, \text { outer }}=8.00 \mathrm{~mm}, \Delta t_{\text {sub }}=16.7^{\circ} \mathrm{C}, t_{e}=-10.09^{\circ} \mathrm{C}, \Delta t_{\text {sup }}=4.79^{\circ} \mathrm{C}, \varepsilon=0.0005 \mathrm{~mm}$, and $c=4.0 \%$

Keeping the working conditions of Figure 3-12 unchanged and letting the capillary flow exchange heat to the surrounding suction line flow with $d_{i, o u t e r}=8.00 \mathrm{~mm}$, the non-adiabatic flow 
will be more sensitive to a variation in condensation pressure. The calculation comparison is detailed in Figure 3-35. When $P_{k}$ increased from 10.00 bar to 18.00 bar, the ACAM mass flow rate increased from $6.01 \mathrm{~kg} / \mathrm{h}$ to $8.07 \mathrm{~kg} / \mathrm{h}$, providing an increase of $34.3 \%$; the NACAM mass flow rate increased from $6.70 \mathrm{~kg} / \mathrm{h}$ to $9.35 \mathrm{~kg} / \mathrm{h}$, providing a $39.6 \%$ increase.

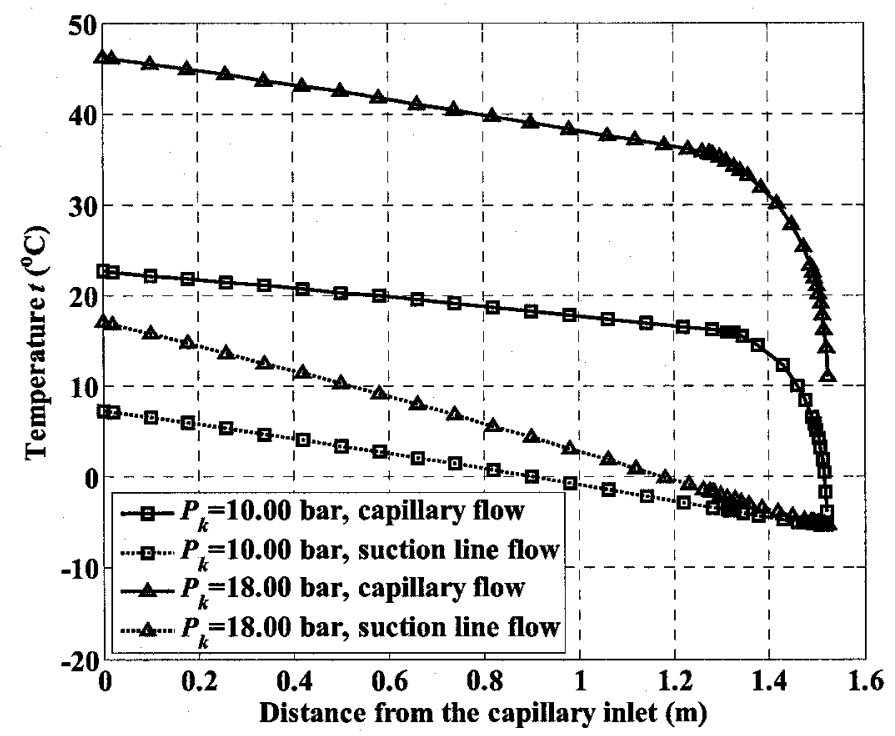

Figure 3-36 Influence of condensation pressure on the temperature with $L=1.526 \mathrm{~m}$, $d_{i}=0.70 \mathrm{~mm}, d_{i, \text { outer }}=8.00 \mathrm{~mm}, \Delta t_{\text {sub }}=16.7^{\circ} \mathrm{C}, t_{e}=-10.09^{\circ} \mathrm{C}, \Delta t_{\text {sup }}=4.79^{\circ} \mathrm{C}, \varepsilon=0.0005 \mathrm{~mm}$, and $c=4.0 \%$

Figure 3-36 shows the corresponding temperature profiles of the flows for $P_{k}$ equal to 10.00 bar and 18.00 bar. For the higher condensation pressure, the rate of temperature decrease is fast along the tube, which reveals large specific heat transfer rate. And for the same capillary length, this also means a greater temperature difference between the inlet and the outlet. For $P_{k}=10.00$ bar, the temperature difference between the capillary inlet and outlet $\Delta t=26.5^{\circ} \mathrm{C}$; for $P_{k}=18.00$ bar, $\Delta t=35.2^{\circ} \mathrm{C}$. As in Figure 3-32, the mass flow rate and the overall heat transfer coefficient increase 
at similar speeds, so the temperature difference between the capillary flow and the suction line flow is directly determined by $d q$ and thus is larger for 18.00 bar.

Therefore, though increasing the condensation pressure will increase the mass flow rate and the heat transfer rate, it will diminish the device effectiveness.

\subsection{Influence of subcooling degrees}

Compared with the influence of condensation pressure, subcooling degrees affect the flow strongly when the magnitude is small and weakly when it is large. As can be seen in Figure 3-37, the mass flow rate is increased by $26.1 \%$ when $\Delta t_{\text {sub }}$ is $5^{\circ} \mathrm{C}$ and $13.3 \%$ when $\Delta t_{\text {sub }}$ is $17^{\circ} \mathrm{C}$. This indicates that heat transfer makes the refrigerant flow less sensitive to the subcooling degrees.

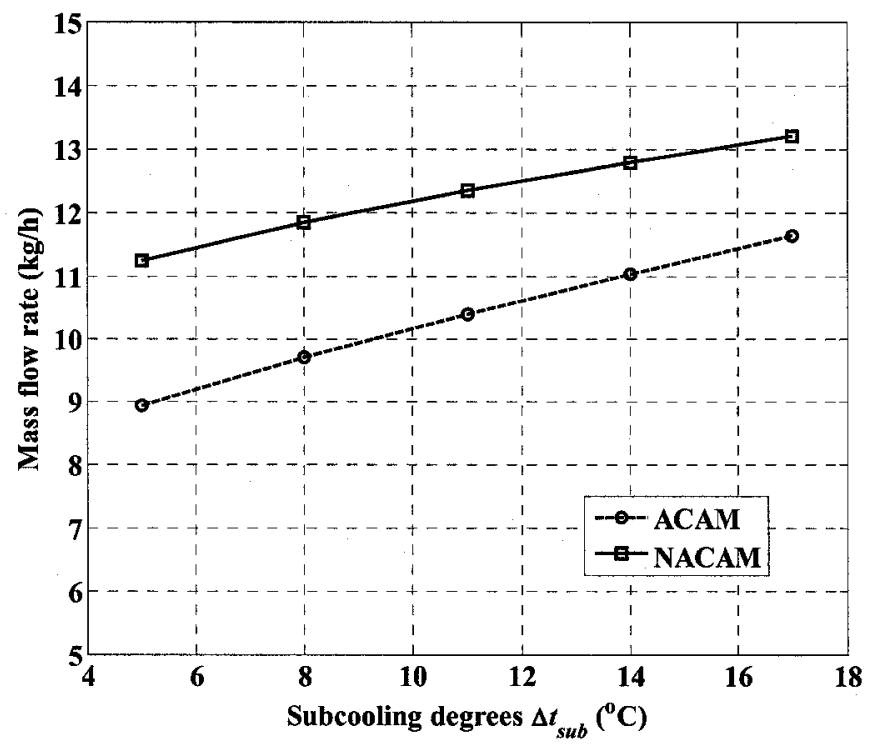

Figure 3-37 Influence of subcooling degrees on the mass flow rate with $L=1.526 \mathrm{~m}$, $d_{i}=0.84 \mathrm{~mm}, d_{i, \text { outer }}=8.00 \mathrm{~mm}, P_{k}=14.00 \mathrm{bar}, t_{e}=-10.09^{\circ} \mathrm{C}, \Delta t_{\text {sup }}=4.79{ }^{\circ} \mathrm{C}, \varepsilon=0.0005 \mathrm{~mm}$, and $c=4.0 \%$ 
In Figure 3-38, a greater decreasing rate of temperature corresponds to lower subcooling degrees, which means the specific heat transfer rate is high. Since the mass flow rate and the overall heat transfer coefficient vary at nearly the same rate, the temperature difference between fluids inside the two tubes is high at low subcooling degrees. Meanwhile, a higher capillary exiting temperature is obtained causing more energy loss due to the shockwaves. In this case, it seems that better device performance will be reached with more subcooling. However, this trend is valid only

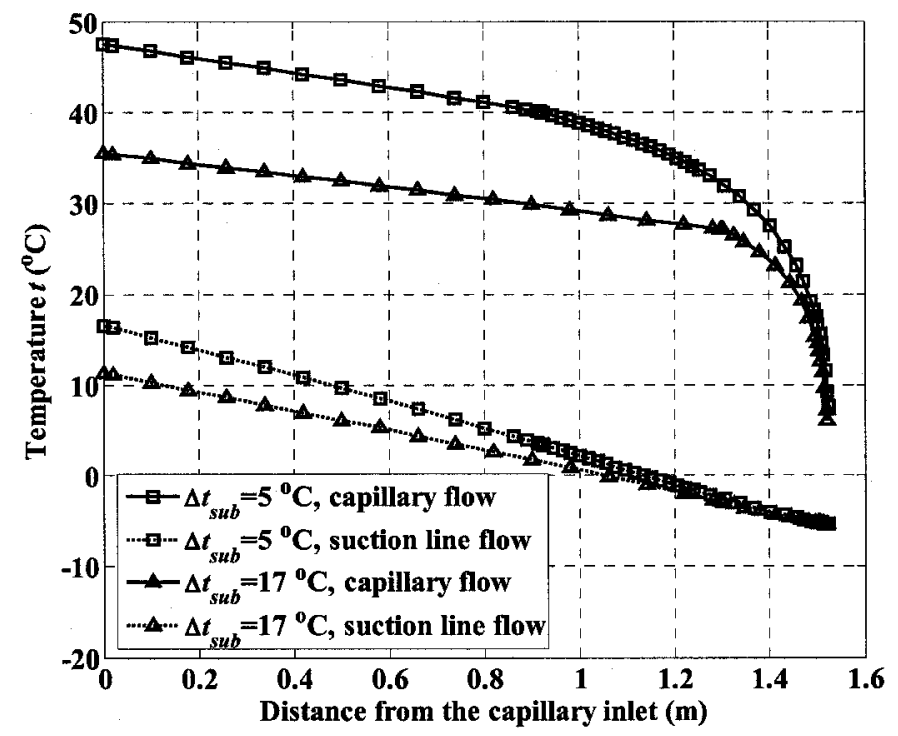

Figure 3-38 Influence of subcooling degrees on the temperature with $L=1.526 \mathrm{~m}, d_{i}=0.84 \mathrm{~mm}$, $d_{i, \text { outer }}=8.00 \mathrm{~mm}, P_{k}=14.00 \mathrm{bar}, t_{e}=-10.09{ }^{\circ} \mathrm{C}, \Delta t_{\text {sup }}=4.79^{\circ} \mathrm{C}, \varepsilon=0.0005 \mathrm{~mm}$, and $c=4.0 \%$

for a certain range. Suppose the subcooling degrees are high enough such that the capillary inlet temperature and the suction line inlet temperature are the same, reverse heat transfer then occurs over the whole capillary length, which is obviously harmful to the system performance. Moreover, a low temperature coolant and a huge contact area are required for the condenser; this requires more material, makes the component bulky, and might invalidate the feasibility of the system. Therefore, 
we may say that under the condition that no reverse heat transfer occurs in the heat exchanger, the greater the amount of subcooling degrees, the better the device will perform.

\subsection{Influence of surface roughness}

Figure 3-39 shows the relationship between surface roughness and mass flow rate for ACAM and NACAM. Since heat transfer occurs, the mass flow rate line of NACAM is higher than and parallel to the ACAM line. Two conclusions can be drawn from the figure: the mass flow rate is slightly affected by the surface roughness with only a $5.0 \%$ decrease when $\varepsilon$ increases from 0.50 $\mu \mathrm{m}$ to $1.25 \mu \mathrm{m}$; the changing trend of the mass flow rate is independent of whether or not heat transfer is occurring in the device. Compared with section 3.1.2.6, this trend is the same with different condensation pressures.

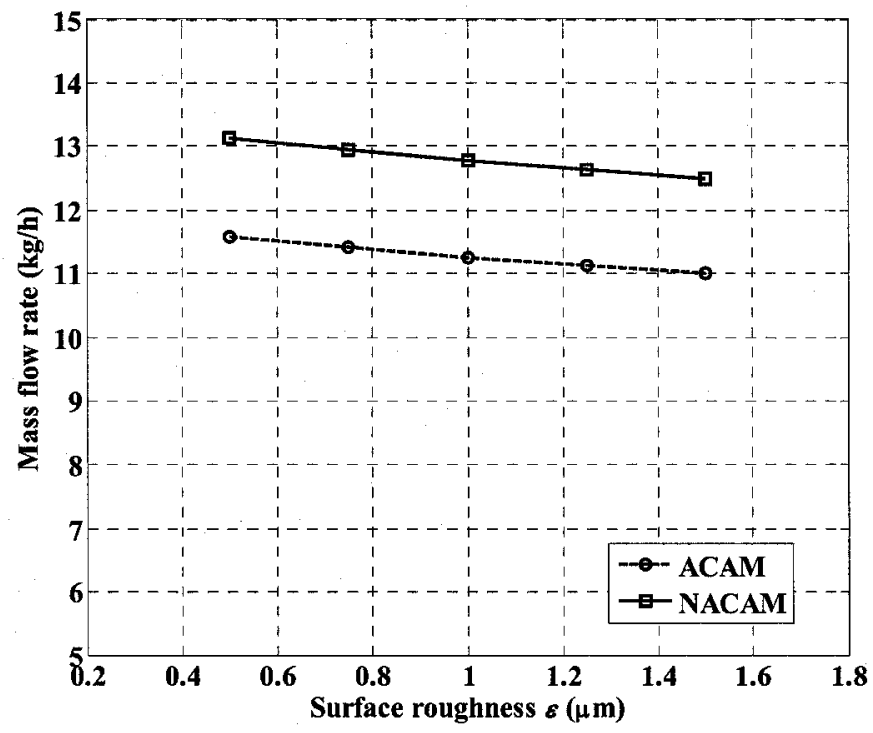

Figure 3-39 Influence of surface roughness on the mass flow rate with $L=1.526 \mathrm{~m}, d_{i}=0.84 \mathrm{~mm}$, $d_{i, \text { outer }}=8.00 \mathrm{~mm}, P_{k}=14.00 \mathrm{bar}, \Delta t_{\mathrm{sub}}=16.7^{\circ} \mathrm{C}, t_{e}=-10.09{ }^{\circ} \mathrm{C}, \Delta t_{\mathrm{sup}}=4.79{ }^{\circ} \mathrm{C}$, and $c=4.0 \%$ 
To investigate the temperature behaviour of the NACAM, two sets of data with $\varepsilon$ values of 0.50 $\mu \mathrm{m}$ and $1.25 \mu \mathrm{m}$ were considered. Figure 3-40 shows the results, where almost identical curves can be observed for both the capillary flow and the suction line flow. This means that although the surface roughness can affect the amount of refrigerant flowing through the tube, it will not change the temperature profile of the device.

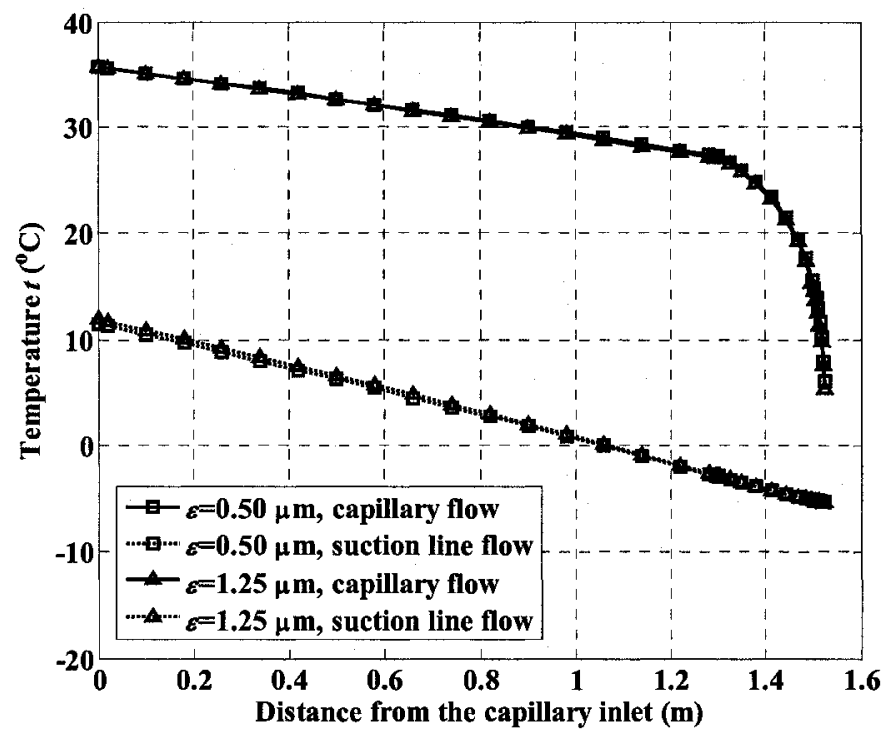

Figure 3-40 Influence of surface roughness on the temperature with $L=1.526 \mathrm{~m}, d_{i}=0.84 \mathrm{~mm}$, $d_{i, \text { outer }}=8.00 \mathrm{~mm}, P_{k}=14.00 \mathrm{bar}, \Delta t_{\text {sub }}=16.7^{\circ} \mathrm{C}, t_{e}=-10.09^{\circ} \mathrm{C}, \Delta t_{\text {sup }}=4.79{ }^{\circ} \mathrm{C}$, and $c=4.0 \%$

From sections 3.2.2.2.1 to 3.2.2.2.5 we have compared the differences between NACAM and ACAM. Since heat transfer exists in the NACAM, more thermal factors need to be taken into account, such as parameters of the second flow. From now on they will be addressed in detail.

\subsection{Influence of evaporation temperature}


To analyze the influence of evaporation temperature on the mass flow rate and the in-tube flow temperature distributions, five different evaporation temperatures are selected. They increase from $-15{ }^{\circ} \mathrm{C}$ to $5{ }^{\circ} \mathrm{C}$ with a step size of $5{ }^{\circ} \mathrm{C}$. To enrich the comparison, two different capillary inner diameters are considered. The results are shown in Figure 3-41 and Figure 3-42. In accordance with Figure 3-31, $d_{i}$ plays an important role in affecting the capillary capacity, while $t_{e}$ has a minor effect if the sizes of tubes are fixed. For $d_{i}=0.70 \mathrm{~mm}, \dot{m}$ only drops $5.0 \%$ over the range of evaporator temperatures; while for $d_{i}=0.84 \mathrm{~mm}$, the value is even lower at $4.7 \%$. This is because the

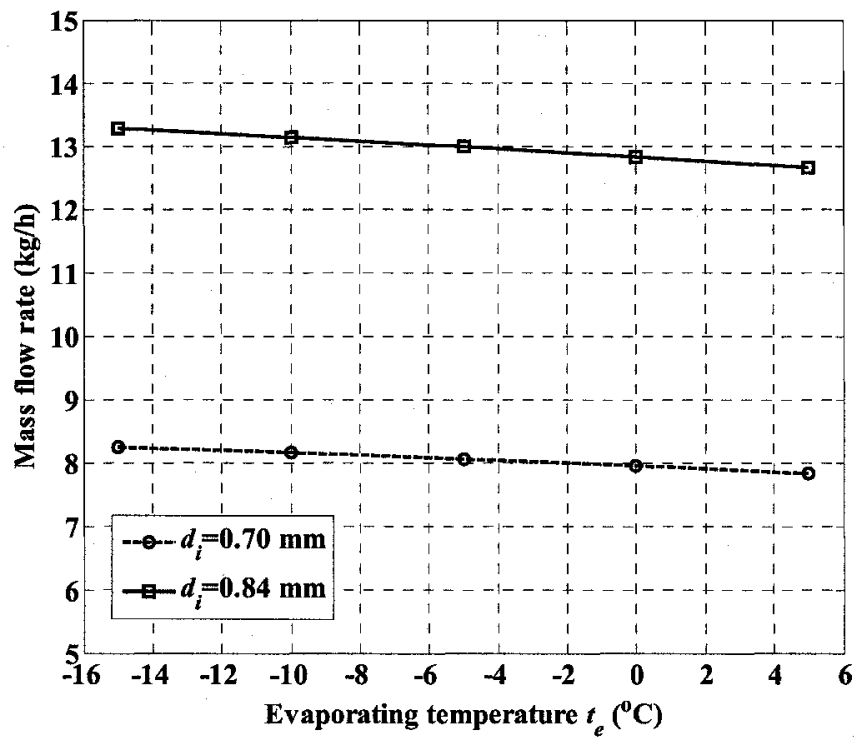

Figure 3-41 Influence of evaporating temperature on the mass flow rate with $L=1.526 \mathrm{~m}$, $d_{i, o u t e r}=8.00 \mathrm{~mm}, P_{k}=14.00 \mathrm{bar}, \Delta t_{\text {sub }}=16.7^{\circ} \mathrm{C}, \Delta t_{\text {sup }}=4.79{ }^{\circ} \mathrm{C}, \varepsilon=0.0005 \mathrm{~mm}$, and $c=4.0 \%$

heat transfer condition does not change significantly with the evaporation pressure if the suction line flow falls entirely into the super-heated region. In this case, the heat transfer coefficient in the suction line grows from $207 \mathrm{~W} /\left(\mathrm{m}^{2} \cdot \mathrm{K}\right)$ to $212 \mathrm{~W} /\left(\mathrm{m}^{2} \cdot \mathrm{K}\right)$ along the flow direction for $t_{e}=5{ }^{\circ} \mathrm{C}$, and from $192 \mathrm{~W} /\left(\mathrm{m}^{2} \cdot \mathrm{K}\right)$ to $204 \mathrm{~W} /\left(\mathrm{m}^{2} \cdot \mathrm{K}\right)$ for $t_{e}=-15^{\circ} \mathrm{C}$. An average of $5 \%$ reduction can be observed. 
Accordingly, the overall thermal conductance changes only slightly. The heat transfer coefficient increase along the suction line flow direction is caused by the increased flow velocity.

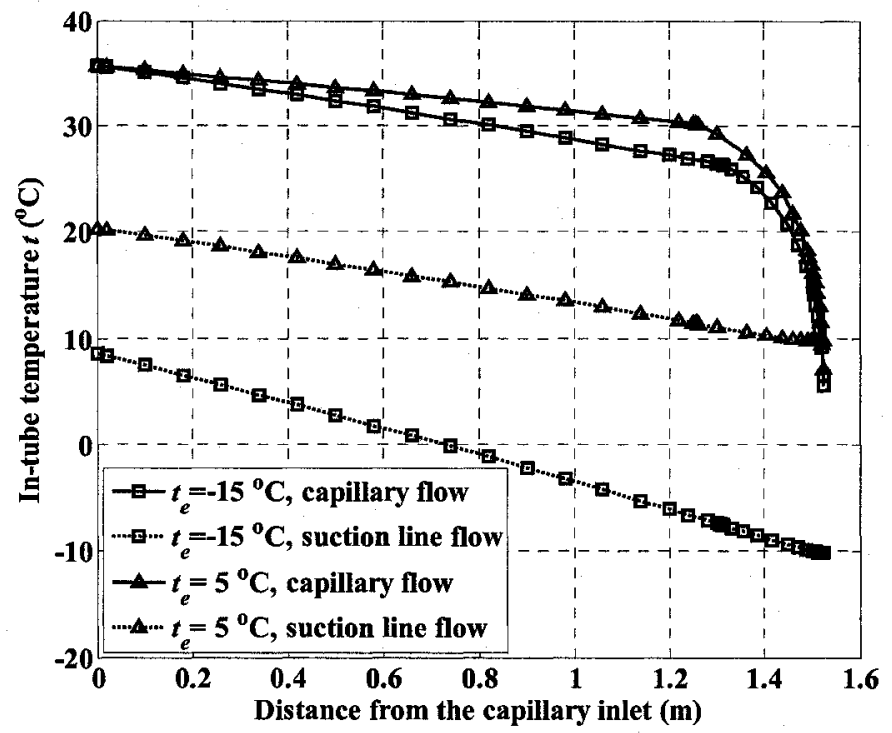

Figure 3-42 Influence of evaporating temperature on the in-tube temperatures with $L=1.526 \mathrm{~m}, d_{i}=0.84 \mathrm{~mm}, d_{i, \text { outer }}=8.00 \mathrm{~mm}, P_{k}=14.00 \mathrm{bar}, \Delta t_{\mathrm{sub}}=16.7^{\circ} \mathrm{C}, \Delta t_{\mathrm{sup}}=4.79^{\circ} \mathrm{C}$, $\varepsilon=0.0005 \mathrm{~mm}$, and $c=4.0 \%$

For the in-tube temperatures, a larger difference between the capillary flow and the suction line flow is obtained when $t_{e}$ is low. On the capillary flow side, the temperature profile is a little higher at a higher evaporation temperature; on the suction line flow side, it is much higher at higher $t_{e}$ due to their direct relationship. Therefore raising the evaporation temperature will benefit the device effectiveness; from the system point of view, this will better the overall performance.

\subsection{Influence of initial conditions of the suction line flow}

In section 3.2.2.2.1 the importance of the superheating degrees was mentioned; it will now be 
discussed in detail. For most domestic refrigerators, the flow at the suction line inlet is super-heated, which is the case we considered in the previous investigation. For CCOT-based AAC systems, the flow at the suction line inlet is in the two-phase region because a certain amount of liquid should be kept at all times so that lubricant oil can be carried to the compressor. Normally the quality at the suction line inlet is about $0.75 \sim 0.80$. Therefore, in this section the initial conditions are divided into two categories, the superheating case and the two-phase case.

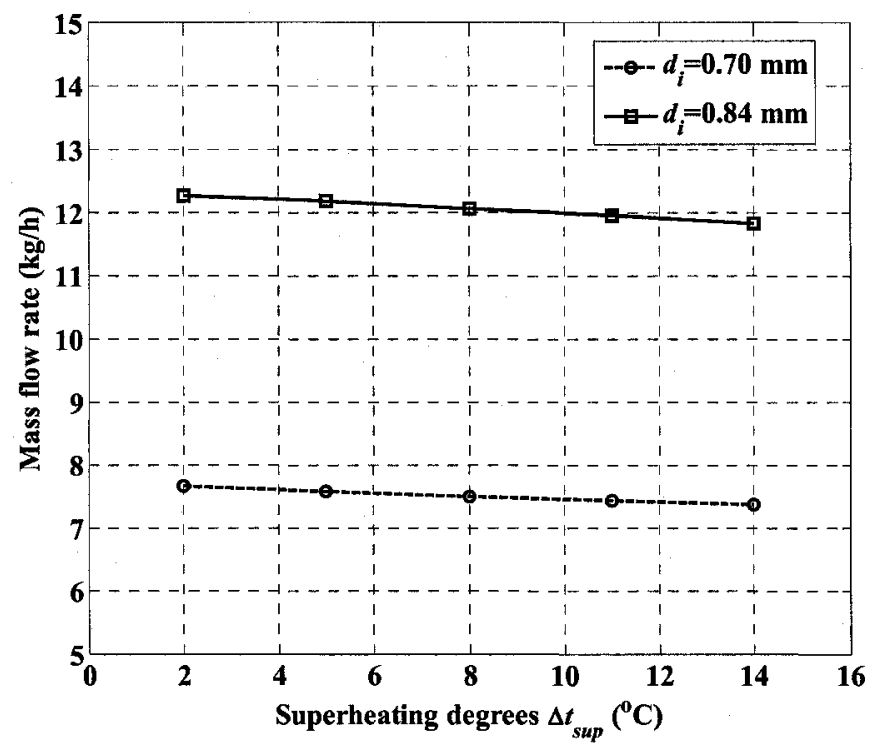

Figure 3-43 Influence of superheating degrees on the mass flow rate with $L=1.526 \mathrm{~m}$, $d_{i, \text { outer }}=8.00 \mathrm{~mm}, P_{k}=14.00 \mathrm{bar}, \Delta t_{\mathrm{sub}}=10.0^{\circ} \mathrm{C}, t_{e}=-10.09{ }^{\circ} \mathrm{C}, \varepsilon=0.0005 \mathrm{~mm}$, and $c=4.0 \%$

For the superheating case, Figure 3-44 shows the relationship between the mass flow rate and superheating degrees. Here, the evaporation temperature is fixed, so the heat transfer coefficient changes even less with a change in superheating degrees. As discussed in the previous section, this will help stabilize the mass flow rate. For different capillary inner diameters, $\dot{m}$ only decreases $3.6 \%$ to $3.8 \%$ for $\Delta t_{\text {sub }}$ values of 2 to $14{ }^{\circ} \mathrm{C}$. From Figure 3-44 it can be seen that a high $\Delta t_{\text {sub }}$ brings 
small rate of temperature change, but the fluid enters and exits the capillary tube at almost the same temperatures, respectively. Compared with the evaporation temperature, the influence of superheating is less important.

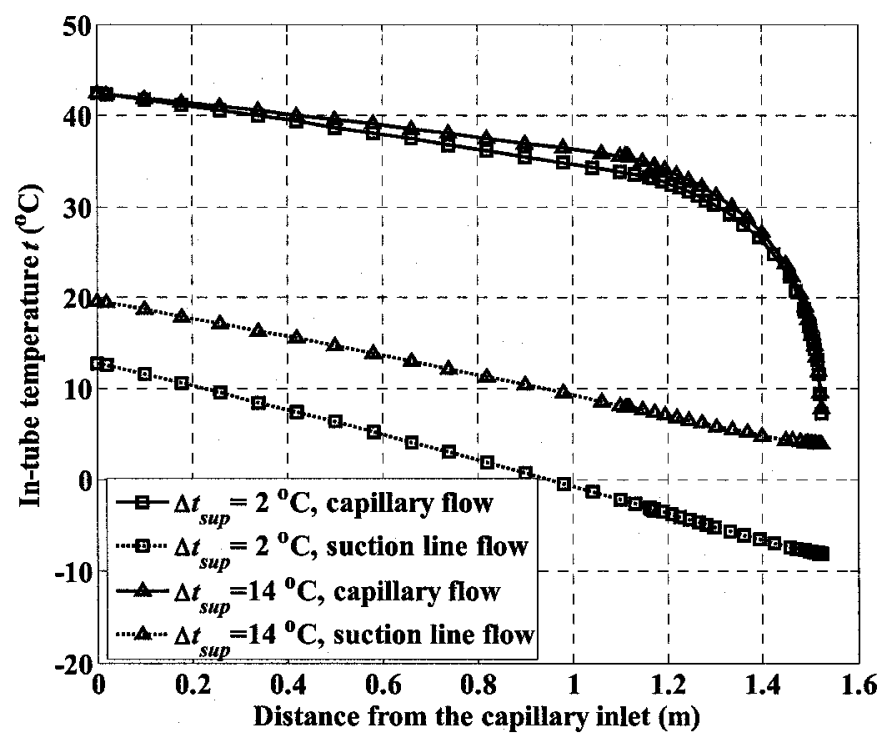

Figure 3-44 Influence of superheating degrees on in-tube temperatures with $L=1.526 \mathrm{~m}$, $d_{i}=0.84 \mathrm{~mm}, d_{i, \text { outer }}=8.00 \mathrm{~mm}, P_{k}=14.00 \mathrm{bar}, \Delta t_{\mathrm{sub}}=10.0{ }^{\circ} \mathrm{C}, t_{e}=-10.09{ }^{\circ} \mathrm{C}, \varepsilon=0.0005 \mathrm{~mm}$, and $c=4.0 \%$

In the two-phase region, due to the high value of the nucleate heat transfer coefficient, the flow condition differs significantly from a change in superheating degrees. To ensure the suction line outlet quality will be less than 1 (to achieve better heat transfer performance of the heat exchanger and lower compression work), the inner diameter of the suction line is intentionally enlarged to $12.70 \mathrm{~mm}$. The importance of the $d_{i, \text { outer }}$ will be discussed in detail in next section. Compared with $d_{i, \text { outer }}=8.00 \mathrm{~mm}$, the change will decrease the heat transfer rate of the device. Even so, Figure 3-45 shows a notable increase in the mass flow rate from around $7.5 \mathrm{~kg} / \mathrm{h}$ and $12.0 \mathrm{~kg} / \mathrm{h}$ in Figure 3-43 
to around $9.0 \mathrm{~kg} / \mathrm{h}$ and $14.9 \mathrm{~kg} / \mathrm{h}$. It increases slightly with the suction line inlet quality by $1.2 \%$ for $d_{i}=0.70 \mathrm{~mm}$ and $1.1 \%$ for $d_{i}=0.84 \mathrm{~mm}$. For the larger capillary inner diameter, the heat transfer coefficient increases from $484 \mathrm{~W} /\left(\mathrm{m}^{2} \cdot \mathrm{K}\right)$ to $562 \mathrm{~W} /\left(\mathrm{m}^{2} \cdot \mathrm{K}\right)$ along the flow direction for $x_{s l, i n}=0.600$, and from $536 \mathrm{~W} /\left(\mathrm{m}^{2} \cdot \mathrm{K}\right)$ to $612 \mathrm{~W} /\left(\mathrm{m}^{2} \cdot \mathrm{K}\right)$ for $x_{s l, i n}=0.782$. When the suction line inlet quality increases, the flow velocity increases due to the smaller density; thus the heat transfer coefficient increases.

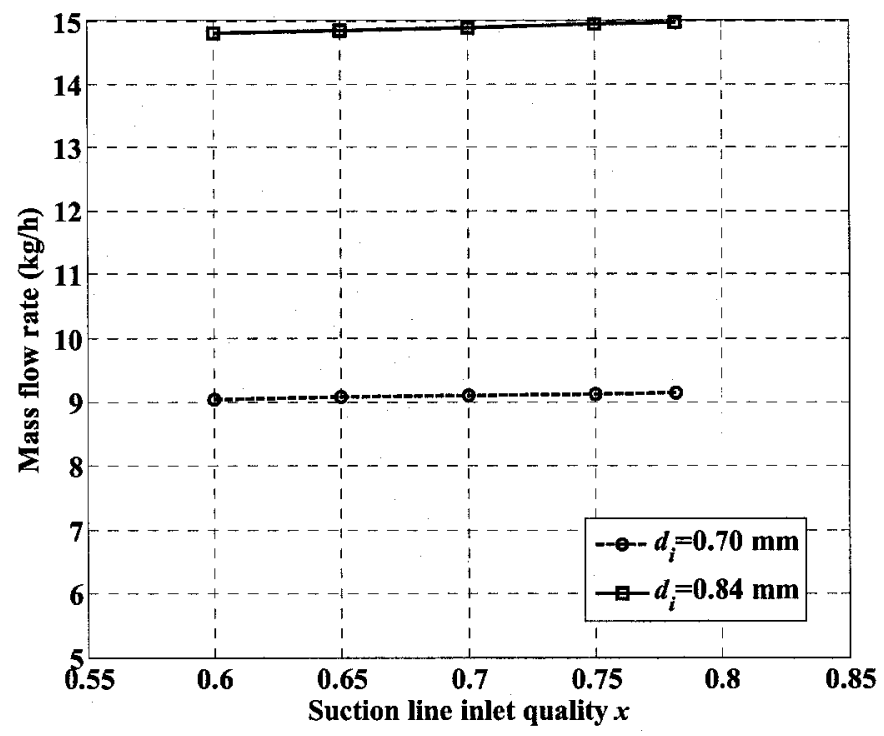

Figure 3-45 Influence of suction line inlet quality on the mass flow rate with $L=1.526 \mathrm{~m}$, $d_{i, \text { outer }}=12.70 \mathrm{~mm}, P_{k}=14.00 \mathrm{bar}, \Delta t_{\mathrm{sub}}=10.0^{\circ} \mathrm{C}, t_{e}=-10.09{ }^{\circ} \mathrm{C}, \varepsilon=0.0005 \mathrm{~mm}$, and $c=4.0 \%$

The temperature profile shown in Figure 3-46 differs slightly from previous figures in that it saddles in the single-phase region, showing the influence of the larger thermal driving force. At high suction line inlet quality $\left(x_{s, i, i}\right)$, a high specific heat transfer rate is obtained and a low temperature difference is achieved. Like Figure 3-44, the capillary flow temperature undergoes little change at the inlet and the outlet. Since the flow temperature remains the same throughout the 
suction line, a relatively high inlet quality will enhance the device performance. When the suction line flow enters the super-heated region, the heat transfer deteriorates sharply, so the inlet quality cannot be too high so as to avoid single-phase heat transfer. During a design process, the proper suction line inlet quality varies case by case and strongly depends on simulated outlet conditions.

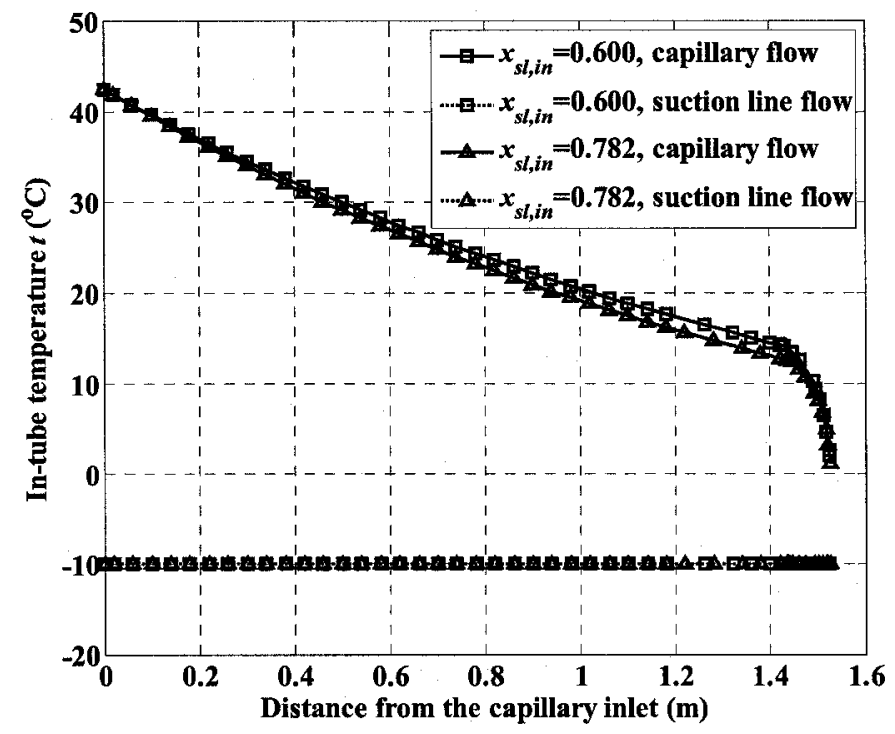

Figure 3-46 Influence of suction line inlet quality on in-tube temperatures with $L=1.526 \mathrm{~m}$, $d_{i}=0.84 \mathrm{~mm}, d_{i, \text { outer }}=12.70 \mathrm{~mm}, P_{k}=14.00 \mathrm{bar}, \Delta t_{\mathrm{sub}}=10.0{ }^{\circ} \mathrm{C}, t_{e}=-10.09{ }^{\circ} \mathrm{C}, \varepsilon=0.0005 \mathrm{~mm}$, and $c=4.0 \%$

From the above discussion it can be seen that the initial condition itself does not greatly affect the mass flow rate and the capillary temperature behaviour. The overall influence is on the order of $4 \%$ or less.

\subsection{Influence of inner diameter of the suction line}

Because the capillary and suction line flows are connected via heat transfer, a variation of inner 
diameter of the suction line causes a change in the specific heat transfer rate and thus affects the mass flow rate. Figure 3-47 details this relationship under two different condensation pressures. When $P_{k}$ is low, the variation will be small, and a large mass flow rate can be reached at low $d_{i, o u t e r}$. This is because the mass flux is inversely proportional to the square of the tube inner diameter. High mass flux corresponds to a small tube size, leading to a higher heat transfer coefficient. Meanwhile, the temperature profiles in Figure 3-48 show that the temperature drops faster with a smaller tube size, providing a larger specific heat transfer rate. Therefore, the total heat transfer rate is large for small tube size. When $P_{k}$ is $14.66 \mathrm{bar}$, the heat transfer rate $Q$ is $52.4 \mathrm{~W}$ for $d_{i, o u t e r}=6 \mathrm{~mm}$, and is only $15.8 \mathrm{~W}$ for $d_{i, o u t e r}=14 \mathrm{~mm}$.

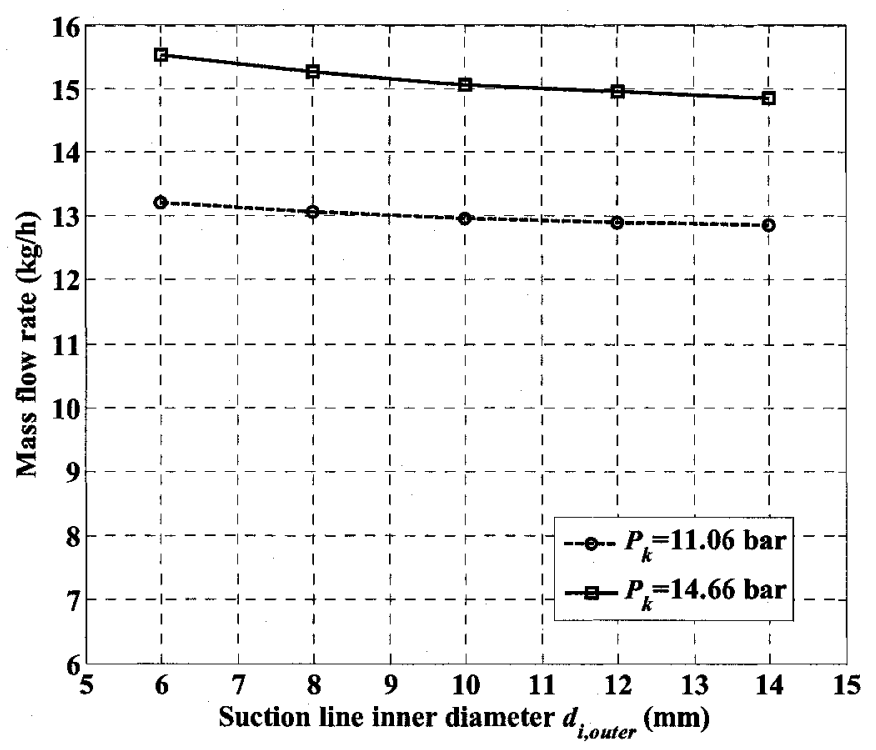

Figure 3-47 Influence of suction line inner diameter on mass flow rate with $L=1.526 \mathrm{~m}, d_{i}$ $=0.84 \mathrm{~mm}, \Delta t_{\mathrm{sub}}=10.0^{\circ} \mathrm{C}, t_{e}=-10.09^{\circ} \mathrm{C}, \Delta t_{\mathrm{sup}}=4.79^{\circ} \mathrm{C}, \varepsilon=0.0005 \mathrm{~mm}$, and $c=4.0 \%$

It can also be seen from Figure 3-48 that the majority of the temperature drop occurs in the last $10 \mathrm{~cm}$ to $15 \mathrm{~cm}$ of the capillary, where a lower exit temperature is achieved at a smaller suction line 
tube size. So decreasing $d_{i, \text { outer }}$ will benefit the heat transfer rate and the device effectiveness. If $d_{i, o u t e r}$ is large, the refrigerant flows so slowly that heat transfer barely occurs, and thus the capillary fluid temperature in the single-phase region becomes nearly flat. This is in accordance with the temperature profile at $d_{i, \text { outer }}=14 \mathrm{~mm}$ in the figure.

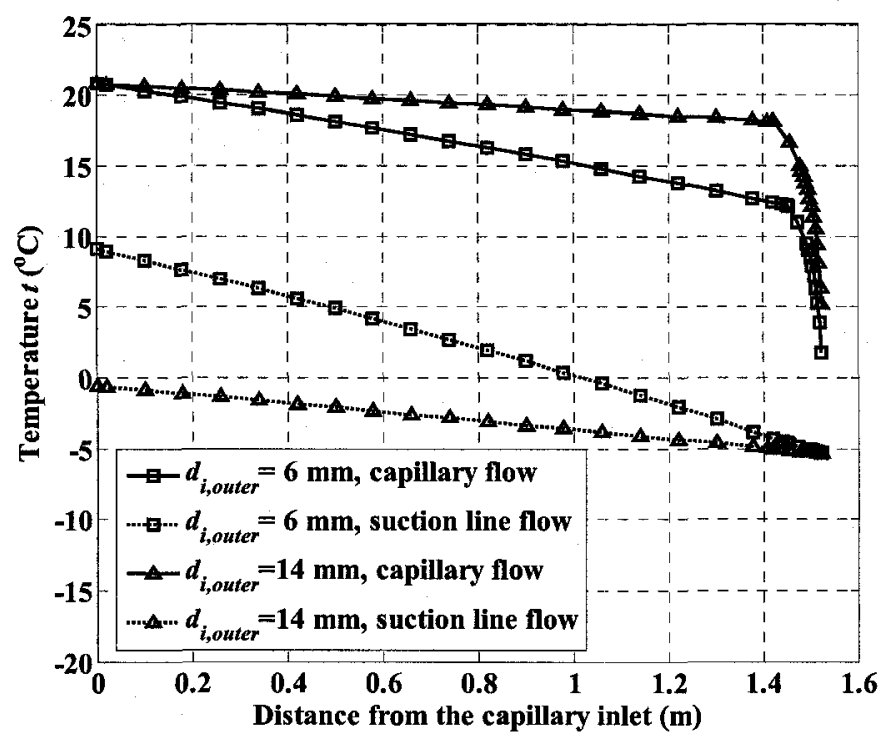

Figure 3-48 Influence of suction line inner diameter on temperatures with $L=1.526 \mathrm{~m}$, $d_{i, \text { outer }}=8.00 \mathrm{~mm}, P_{k}=11.06 \mathrm{bar}, \Delta t_{\text {sub }}=16.7^{\circ} \mathrm{C}, t_{e}=-10.09^{\circ} \mathrm{C}, \Delta t_{\text {sup }}=4.79^{\circ} \mathrm{C}, \varepsilon=0.0005 \mathrm{~mm}$, and $c=4.0 \%$

\subsection{Influence of flow arrangement}

If the fluid in the suction line is in the two-phase region, then its temperature remains almost the same throughout the heat transfer process, as can be seen in Figure 3-46. Therefore, whether the flow arrangement is counter flow or parallel flow does not affect the device performance. In this section, an investigation is conducted on a heat exchanger where the inlet flow of the suction line is $4.79^{\circ} \mathrm{C}$ super-heated. The behaviour of the mass flow rate and the temperature distribution along 
the flow direction are shown in Figure 3-49 and Figure 3-50, respectively. The mass flow rates for the two arrangements are nearly identical. Accordingly, the capillary fluids follow similar temperature tracks, and the two-phase expansion processes match especially well. For the parallel flow arrangement, a saddled curved is observed in the single-phase region. This is caused by the greater initial thermal driving forces. Combined with Figure 3-46, we can see that such phenomenon exists during heat transfer processes occurring in the two-phase region for both counter flow and parallel flow, and in the super-heated region for parallel flow only.

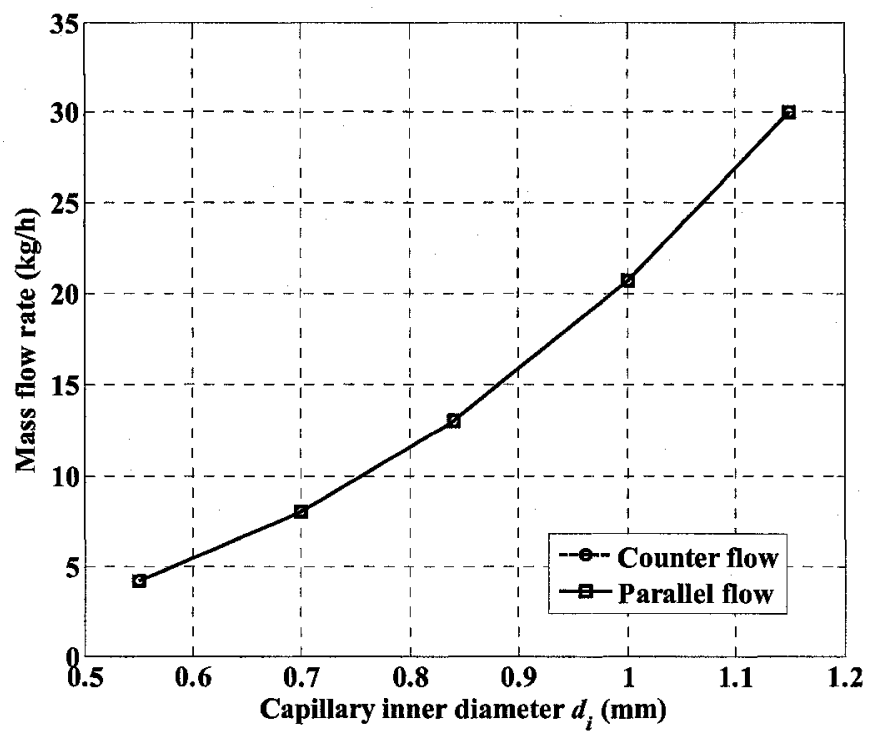

Figure 3-49 Influence of flow arrangement on mass flow rate with $L=1.526 \mathrm{~m}, d_{i, o u t e r}=8.00 \mathrm{~mm}$, $P_{k}=11.06$ bar, $\Delta t_{\text {sub }}=16.7^{\circ} \mathrm{C}, t_{e}=-10.09^{\circ} \mathrm{C}, \Delta t_{\text {sup }}=4.79{ }^{\circ} \mathrm{C}, \varepsilon=0.0005 \mathrm{~mm}$, and $c=4.0 \%$

For the suction line flow, the temperature increase along the flow direction is $5.8{ }^{\circ} \mathrm{C}$ for the parallel flow case and a slightly larger $6.1^{\circ} \mathrm{C}$ for the counter flow case; the heat transfer rates are $17.2 \mathrm{~W}$ and $18.1 \mathrm{~W}$, respectively. The small increase in heat load by $5.2 \%$ demonstrates the advantage of the counter flow arrangement. Besides, if a parallel flow arrangement is adopted, 
reverse heat transfer is more likely to occur at the capillary exit region.

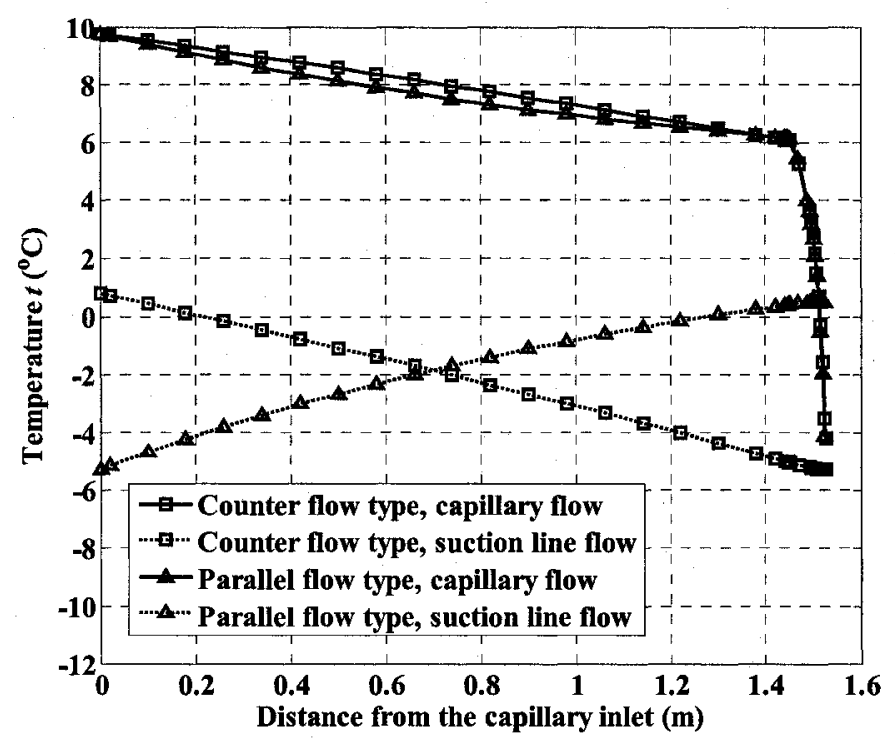

Figure 3-50 Comparison of flow arrangement with $L=1.526 \mathrm{~m}, d_{i}=0.84 \mathrm{~mm}, d_{i, \text { outer }}=8.00 \mathrm{~mm}$, $P_{k}=11.06 \mathrm{bar}, \Delta t_{\text {sub }}=16.7^{\circ} \mathrm{C}, t_{e}=10.09^{\circ} \mathrm{C}, \Delta t_{\text {sup }}=4.79^{\circ} \mathrm{C}, \varepsilon=0.0005 \mathrm{~mm}$, and $c=4.0 \%$

\subsection{Influence of heat exchanger type}

Different types of heat exchangers, such as the tube-in-tube or the lateral configurations, have different impacts on heat transfer rate, even though the mass flow rate may not change significantly. Thus these two types of heat exchangers were investigated. When the heat exchanger was reshaped from a concentric to lateral type, the capillary tube was pulled out and attached to the outside of the suction line by welding. It needs to be mentioned that the inner diameter of the lateral suction line is a little smaller than that in the concentric case to keep the cross sectional area of the suction line the same for both cases. For the solder thickness, $1 \mathrm{~mm}$ was adopted to mimic real situations. 


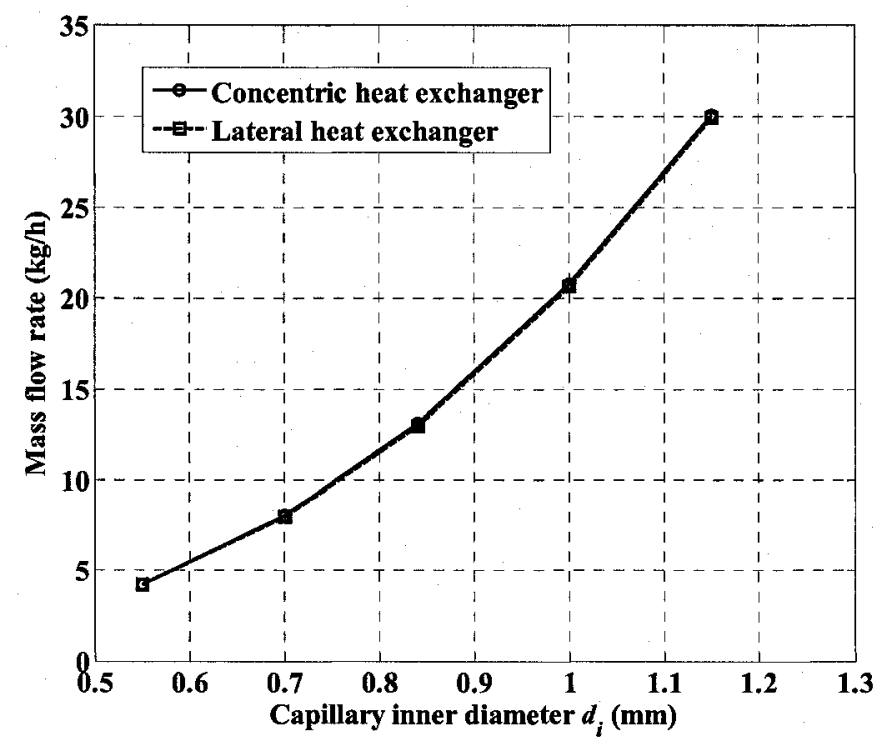

Figure 3-51 Influence of heat exchanger type on mass flow rate with $L=1.526 \mathrm{~m}, P_{k}=11.06 \mathrm{bar}$, $\Delta t_{\text {sub }}=16.7^{\circ} \mathrm{C}, t_{e}=-10.09{ }^{\circ} \mathrm{C}, \Delta t_{\text {sup }}=4.79^{\circ} \mathrm{C}, \varepsilon=0.0005 \mathrm{~mm}$, and $c=4.0 \%$

Figure 3-51 shows the behaviour of the mass flow rate for both types. It decreases slightly when a lateral configuration is adopted. This can be attributed to the relatively small change in the magnitude of the specific heat transfer rate when the suction line flow is super-heated. In other words, for both types the expansion processes are almost the same "far from adiabatic" process. At most a decrease of less than $1 \%$ is obtained. Meanwhile, due to the decrease of heat transfer area, the lateral heat exchanger has less capacity. For instance, with a $d_{i}$ of $0.84 \mathrm{~mm}, Q$ is $18.1 \mathrm{~W}$ for the concentric type and $13.9 \mathrm{~W}$ for the lateral case, a $23 \%$ decrease. In reality, if the heat exchanger is not well insulated and heat transfer occurs between the tube flows and the surroundings, and/or the tubes are not well contacted, then the lateral heat transfer type will perform even less efficiently. On the other hand, the lateral type is easier to make and maintain, so it is more suitable for applications 
where cost is of the most concern and "do-better" but not "do-the-best" is desirable.

The temperature of the capillary flow drops more slowly for the lateral case. This is because the contact area between the two tubes is limited and thus less heat can pass from one tube to the other.

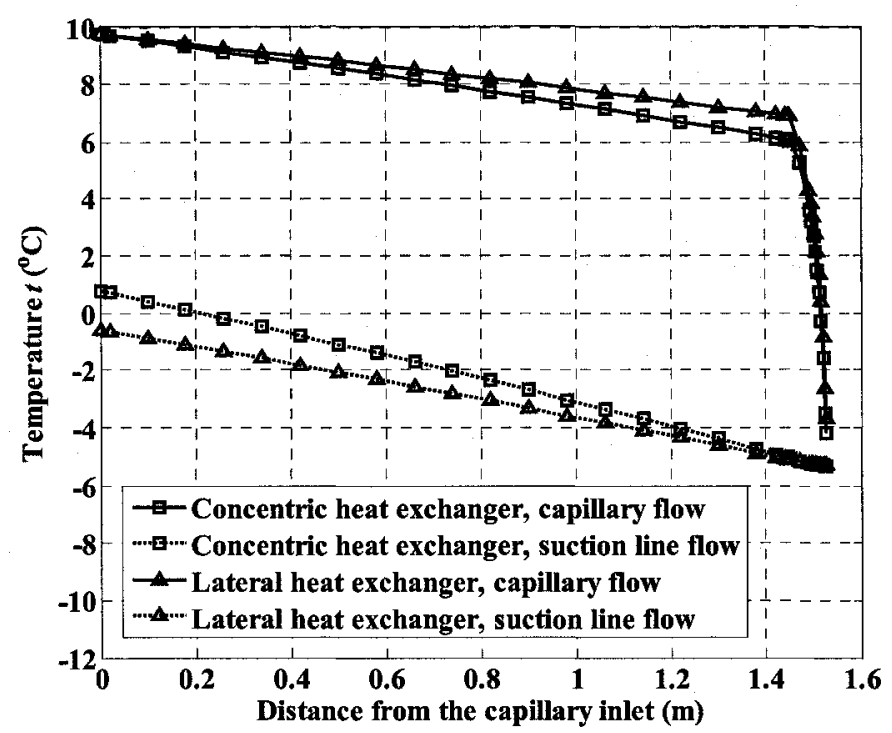

Figure 3-52 Comparison of heat exchanger types with $L=1.526 \mathrm{~m}, d_{i}=0.84 \mathrm{~mm}, P_{k}=11.06 \mathrm{bar}$, $\Delta t_{\text {sub }}=16.7^{\circ} \mathrm{C}, t_{e}=-10.09{ }^{\circ} \mathrm{C}, \Delta t_{\text {sup }}=4.79^{\circ} \mathrm{C}, \varepsilon=0.0005 \mathrm{~mm}$, and $c=4.0 \%$

\subsubsection{Conclusions}

The above discussion details the differences between the adiabatic model (ACAM) and the non-adiabatic model (NACAM). At the same time, relationships between the thermal factors and the mass flow rate have been further addressed. Relative temperatures of the tube flows provide more information about the heat transfer process. The specific heat transfer rate is the key parameter during analysis. It is determined through iterations using a trial-correction technique as shown in 
Figure 3-19. Since the mass flow rate and the overall thermal conductance change at similar rates, the change of the specific heat transfer rate reflects both the temperature difference between the two fluids and the temperature change of any one single fluid. The following conclusions can be drawn:

1. NACAM and ACAM follow similar trends with varying parameters, but due to the presence of heat transfer, NACAM allow greater mass flow to circulate in the system than ACAM.

2. The mass flow rate increases faster in NACAM than in ACAM as the capillary inner diameter is increased; slightly faster with increasing condensation pressure; at the same rate with increasing capillary length and surface roughness; and slower with increasing subcooling degrees. If the mass flow rate is large, the temperature difference increases as well, which leads to lower heat exchanger effectiveness. Though surface roughness affects the mass flow rate, it does not affect the temperature behaviour.

3. More superheating slightly decreases the mass flow rate, and a larger suction line inner diameter and higher evaporation temperature reduce it even more; higher suction line inlet quality slightly increases it. Regarding variations in the superheating, the evaporation temperature, and the suction line inlet quality, capillary outlet temperature does not change significantly. An increase in the suction line inner diameter however does increase the capillary outlet temperature.

4. For heat exchange between the capillary flow and the two-phase suction line flow, an 
"appropriate" inlet quality is required to achieve both better device performance and to ensure the proper delivery of lubricant.

5. Changing the flow directions does not change the mass flow rate, and only slightly affects the averaged temperature difference between the flows. But a counter flow arrangement is recommended because it reduces the chance of reverse heat transfer.

6. Under the condition that the heat exchanger is well insulated and tubes are well contacted, about $23 \%$ of heat transfer will be lost if a lateral type rather than a concentric type of heat exchanger is adopted. In this case, the mass flow rate will not be greatly affected; a smaller temperature difference between the tubes will be achieved when using the concentric type. 


\section{Chapter 4 Experimental study of the pressure behaviour of an AAC system without AXE}

In Chapter 3 the relationships between the mass flow passing through the capillary tube and various parameters for both adiabatic and non-adiabatic processes are addressed in detail. It can be seen from Figure 3-35 and Figure 3-41 that higher condensation pressure and lower evaporation pressure will increase the mass flow rate; $P_{k}$ is related with $\dot{m}$ strongly. Conversely, when the mass flow rate varies, the pressure drop across the capillary tube (which is the difference between the condensation pressure and the evaporating pressure) should change accordingly. But the important question is: will both of them change? If so, by how much?

Fundamentally, for a sub-critical refrigeration system wherein the entire cycle operates under the critical point of the working fluid, the high side and the low side pressures are mainly determined by the environmental temperature and the temperature of the conditioned zone, respectively. Depending on the manufacturing techniques used and the coolant(s) chosen, a temperature difference of $2{ }^{\circ} \mathrm{C}$ to $10^{\circ} \mathrm{C}$ is used for the condenser and evaporator during the design process. In reality, however, this value is changing, especially when the working condition is altered, or the mass flow rate is metered, or both. In such cases, it is difficult to predict the pressure behaviour with mass flow rate variations. Therefore, it is desirable to answer the above question using experimental techniques, which turn out to be the focus of this chapter. 
With the wide application of automotive air-conditioning (AAC) systems and variable displacement compressors (VDC), the influence of compressor speed on system performance becomes more and more important. As a common sense, it has a direct relationship to the mass flow rate. But in classical thermodynamics, it is often assumed that friction losses in the condenser and evaporator are negligible and that the specific cooling load and the specific work consumption are constant. The mass flow rate, then, will not affect the system's coefficient of performance. In fact, higher compressor speed corresponds to higher mass flow rate, which will cause a greater pressure drop for the same expansion device. Then the increase in pressure drop will be distributed to the low side and high side, causing a lower evaporating pressure and a higher condensation pressure. Therefore, compressor speed does affect the system performance. The pressure behaviour with compressor speed has been reported by many researchers including Tassou and Qureshi (1998), Shao et al. (2004), Boyde et al. (2000), Ratts and Brown (2000), Aprea et al. (2003), and Wang et al. (2006). However, not much attention was paid to the mechanism underlying the trend lines of discharge pressure and intake pressure with compressor speed. In this chapter, a further investigation is conducted on how the change in pressure drop is distributed to both the high pressure and low pressure sides.

\subsection{Parameters determining pressure change}

When a refrigeration system is not in operation, refrigerant temperature becomes the same as that of surroundings, and system pressure is the saturated value corresponding to the temperature if neglecting the oil effect. As the system starts running, a pressure difference is established gradually 
such that the low pressure (evaporator side) refrigerant absorbs heat from the conditioned zone and the high pressure (condenser side) refrigerant dissipates heat to the external environment. As the compressor speeds up, this pressure difference increases. Thus compressor speed directly influences the behaviour of pressure drop. But these two parameters are not related in a linearly proportional fashion. The growing magnitude of pressure lift decreases with the compressor speed. The mechanism underlying this phenomenon is discussed in this chapter.

\subsubsection{Pressure difference and mass flow rate}

For a typical sub-critical AAC system as depicted in a $\log P-h$ diagram (as in Figure 3-1), fluid dynamics says that the frictional pressure drop can be correlated to the mass flow rate by

$$
\Delta P=\frac{1}{2} f \rho V^{2}=\frac{f}{2 A^{2}} \frac{\dot{m}^{2}}{\rho}
$$

The pressure drop across an expansion device is proportional to the square of the mass flow rate and is inversely proportional to the inlet fluid density. It needs to be mentioned that such equation only considers the effects of friction, and thus might not hold for orifice tubes used in CCOT systems because the major part of the pressure drop is attributed to shock waves. Thus two points must be made before continuing: a. A relatively long capillary tube is investigated here, which has a greater inner diameter and length than an orifice tube. The size of the capillary tube is configured such that

friction induces the vast majority of the pressure drop. b. For situations where the flow is choked and shock wave is present, the dissipated energy is also proportional to the square of velocity. In such cases $f$ might need to be replaced by $F$ to represent the influences of not only friction but also 
shock waves. Given an AAC system with a typical cooling capacity of $4 \mathrm{~kW}$ : the mass flow rate is in the magnitude of $130 \mathrm{~kg} / \mathrm{h}$; a capillary tube with inner diameter of $1.8 \mathrm{~mm}$ is used; $P_{k}=18 \mathrm{bar}$; and $t_{\text {in,capt }}=30{ }^{\circ} \mathrm{C}$. The corresponding Reynolds number at the capillary inlet will be as high as $1.13 \times 10^{5}$. Therefore, it is acceptable to assume $f$ does not change significantly at such a scale. Details will be addressed in the following section discussing the experimental set-up.

For an R134a-based AAC system, the condenser and evaporator work partially or wholly in the two-phase region, which means condensation temperature directly corresponds to condensation pressure and has no relation to density. A similar relation applies to evaporating temperature and evaporating pressure. Thus a given pressure drop corresponds to a similar temperature difference. In this way, temperatures of the surroundings and the passenger compartment can be taken into analysis in determining states of the refrigerant flow. Based on this fact, the following correlation can be obtained.

$$
\Delta t=t_{k}-t_{e} \propto \Delta P
$$

and then

$$
t_{k}-t_{e}=f_{1}\left(\dot{m}^{2}, \rho\right)
$$

\subsubsection{Evaporating temperature and compressor speed}

For a heat exchanger with phase change as shown in Figure 4-1, the heat load could be expressed as

$$
Q=\dot{m}\left(h_{\text {out }}-h_{\text {in }}\right)=U A\left(t_{I}-t_{I I}\right)
$$

For condenser, the inlet is located in a super-heated region and thus the temperature is much higher 
than the saturated condensation temperature, which will make the equation either physically incorrect or in need of improvement. For an evaporator in an AAC system configured with a CCOT, the refrigerant exiting the evaporator goes into the accumulator where oil is entrained into the refrigerant flow and carried downstream to lubricate the compressor. Some liquid must be present at the accumulator inlet because it is needed to carry the oil. This fact holds for the entire evaporating process in the two-phase region; Equation (4-4) is thus accurate and can be rearranged as

$$
t_{e}=t_{\text {pass }}-\frac{h_{\text {out }}-h_{\text {in }}}{U A_{e}} \dot{m}
$$

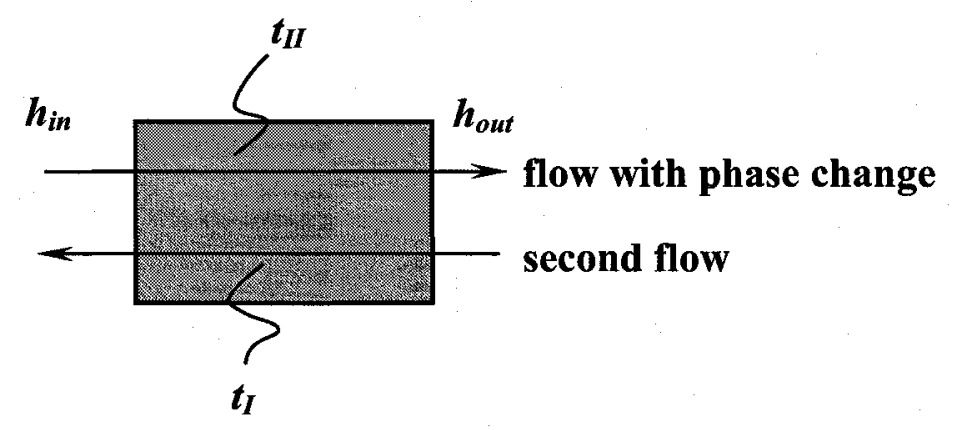

Figure 4-1 Schematic of a heat exchanger model

\subsubsection{Determination of changes in mass flow rate}

The mass flow rate is calculated by (Wang, 2000)

$$
\dot{m}=\eta_{v} \frac{n}{60} V_{d i s} \rho_{1}=f_{2}\left(\eta_{v}, n, \rho_{1}\right)
$$

Volumetric efficiency $\eta_{v}$ is normally considered to be a function of compression ratio $R$ and/or compressor speed $n$. However, based on experimental data, it is almost exclusively related to $n$ for a given compressor with $R=3.5 \sim 7, t_{\text {pass }}=25 \sim 35{ }^{\circ} \mathrm{C}$, and $t_{a m b}=40^{\circ} \mathrm{C}$. The same phenomenon can be observed from another correlation (SINDA/FLUINT, 2003). Figure 4-2 shows the behaviour of $\eta_{v}$ 
with changing compressor speeds. The density at the compressor inlet will not change significantly with $n$. For example, as $n$ increases, $P_{e}$ decreases; meanwhile, the quality at the evaporator outlet decreases, which makes point 1 follow almost exactly an isodense as shown in Figure 4-3. Here black solid lines represent a cycle running at compressor speed $n_{1}$, grey dashed lines are for $n_{2}$, and

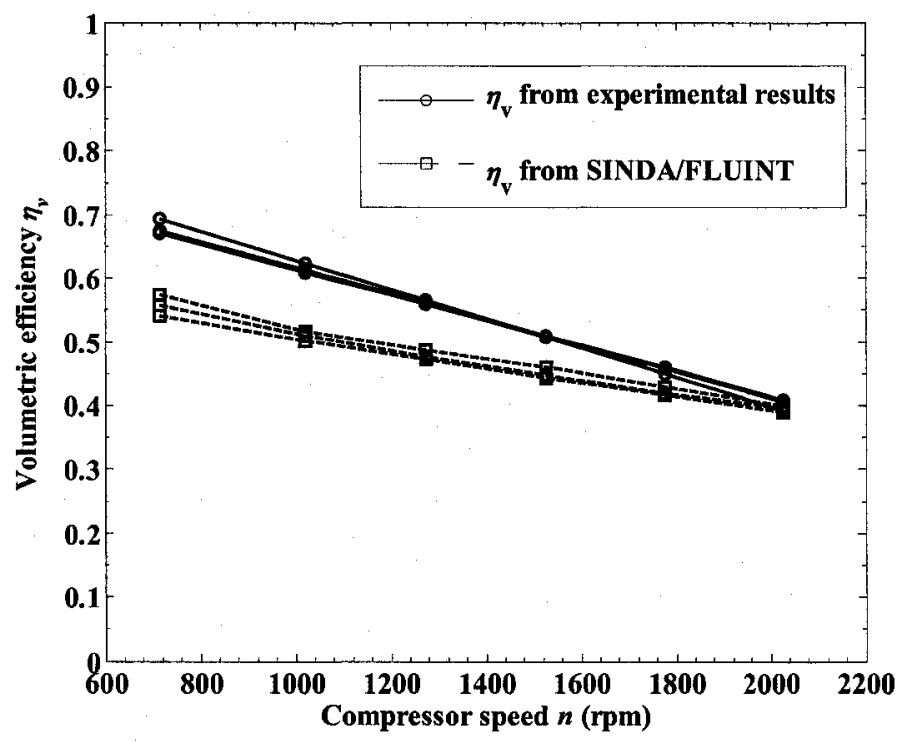

Figure 4-2 Volumetric efficiency vs. compressor speed

$n_{2}>n_{1}$. It can be seen that $\rho_{1}$ slightly influences the mass flow rate. The decrease of quality at the evaporator outlet can be regarded as the result of the decreased specific cooling capacity. The product of specific cooling capacity and mass flow rate is the cooling load, so these two factors are inversely proportional to each other for a given $Q_{e}$. In reality, $Q_{e}$ does increase with mass flow rate, but at a less rate; the specific cooling capacity then decreases, resulting in lower outlet quality. Some may argue that higher flow velocity should produce an increased heat transfer coefficient, and then higher outlet quality. However, for an R134a evaporator, the total heat transfer coefficient is dominated by that of the air side, which means a higher $n$ will not affect the heat transfer condition significantly compared to the contribution of the air flow rate from the passenger compartment. 
Therefore, the following expression can be obtained

$$
\Delta \dot{m}=\Delta\left(\eta_{v} \frac{n}{60} V_{d i s} \rho_{1}\right)=f_{3}(n)
$$

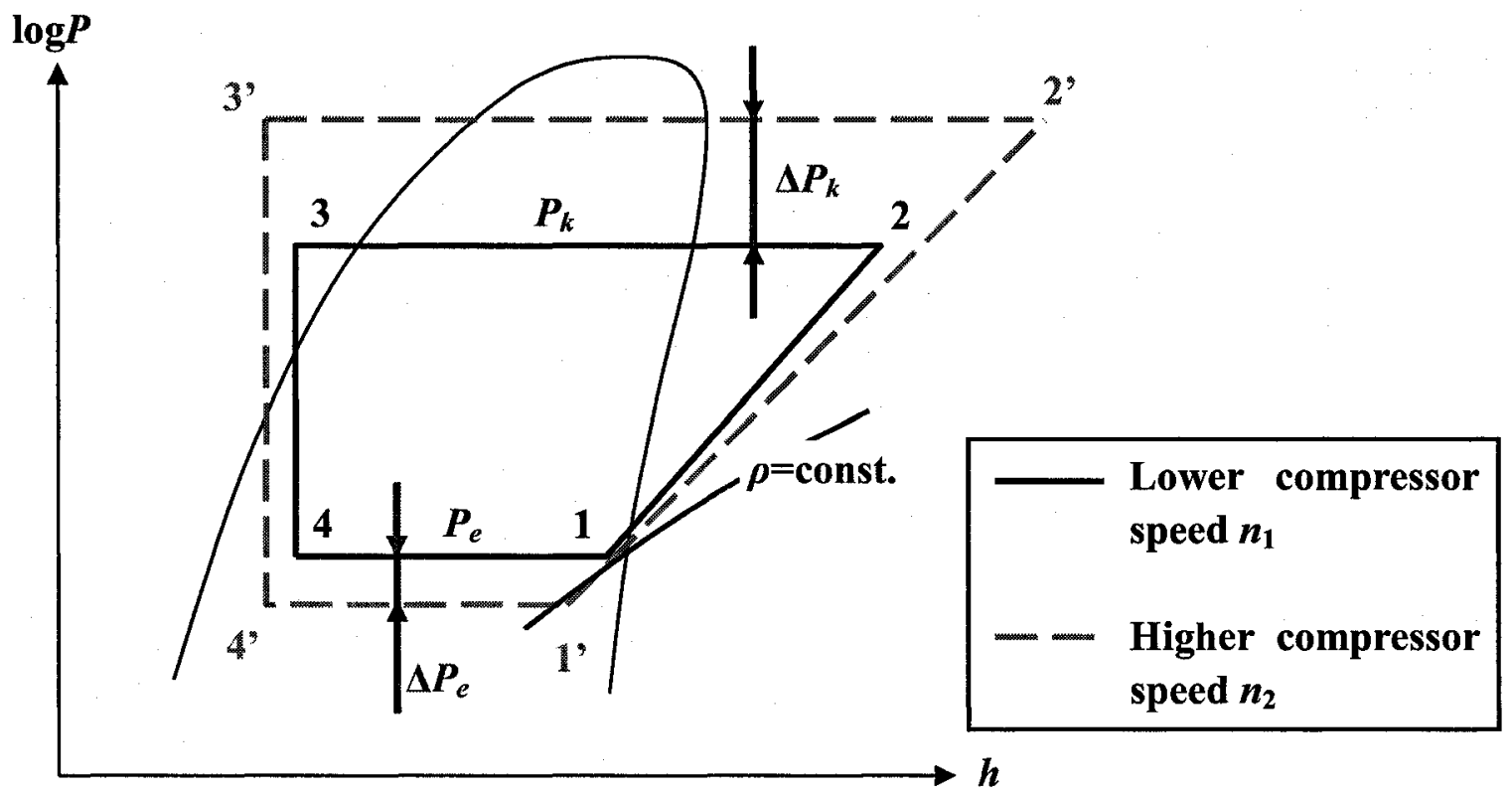

Figure 4-3 Schematic diagram of two refrigeration cycles showing the effects of a change in compressor speed

It is meaningful to further investigate how volumetric efficiency is affected by compressor speed because it is directly related to mass flow rate. Generally there are four factors that affect $\eta_{v}$ (Aprea et al., 2003): a. throttling losses across the suction and discharge valves; b. leakage past the rings of the piston and the leakage back through the suction and discharge valves; c. suction gas warming while mixing with remaining gas inside cylinder increases specific volume, and hence decrease the mass flow rate; and $\mathrm{d}$. motor electrical efficiency because some semi-hermetic compressor electric motors are cooled by means of the refrigerant fluid (Tassou and Qureshi, 1998). Two types of volumetric efficiency behaviour can be found, increasing with compressor speed by Tassou and Qureshi (1998), Shao et al. (2004), Boyde et al. (2000), and Ratts and Brown (2000); and decreasing with compressor speed by Aprea et al. (2003) and Wang et al. (2006). Tassou and 
Qureshi (1998) attribute the increase to optimized compressors that have maximum volumetric efficiencies at design speed. This is doubtful in that the first factor, throttling losses, must logically increase with compressor speed, thus decrease volumetric efficiency. Tassou and Qureshi (1998) did not address other factor(s) that could overweight this one to change this trend. Shao et al. (2004) attributed the increase to the better lubrication at higher compressor speed; they used an inverter compressor in their experiments. Boyde et al. (2000) took into account the factor that sealing works better at high compressor speed for polyester oil. Ratts and Brown (2000) stated that the temperature of the refrigerant mixture in the condenser drops with higher compressor speed. However, this temperature actually represents the value at the condenser exit and thus could be sub-cooled. On the other hand, Aprea, et al. (2003) observed similar trends to Wang et al. (2006) that volumetric efficiency goes down with compressor speed, which reflects the behaviour of the pressure drop across the suction and discharge valves.

Until now we have known the relationship between changes of the pressure drop, the mass flow rate, and the compressor speed from Equations (4-3), (4-5) and (4-7). If $f_{1}, f_{2}$, and $f_{3}$ are provided, then the pressures changes can be obtained.

\subsubsection{Effect of oil concentration}

To get accurate correlations, the thermophysical properties of the refrigerant must be accurate. Several researchers (Wang et al., 2006; Grebner and Crawford, 1993; and Pérez-Segarra et al., 2005) have reported that oil influences the system performance by increasing the refrigerant-oil mixture's pressure (i.e., in the two-phase region, given a temperature, the corresponding pressure of the 
mixture is higher than the saturated pressure of pure refrigerant). Grebner (1993) proposed a correlation to calculate mixture properties if any two of the three parameters (mixture temperature, mixture pressure, and refrigerant concentration) are given. The correlation has the form

$$
T=\left(1+T^{*}\right) T_{\text {sat }}
$$

where the nondimensional degree of apparent superheat

$$
\begin{gathered}
T^{*}=(1-w)(A+B P) \\
w=\frac{1-(1-c) x-c}{1-(1-c) x} \\
x=\frac{\dot{m}_{r, l}}{\dot{m}_{r, l}+\dot{m}_{r, g}} \\
c=\frac{\dot{m}_{o i l}}{\dot{m}_{r, l}+\dot{m}_{r, g}+\dot{m}_{o i l}}
\end{gathered}
$$

$T_{\text {sat }}$ is the saturated temperature of the pure refrigerant at pressure $P ; A$ and $B$ are two empirical coefficients. Grebner's results show that oil greatly affects the mixture properties if the liquid refrigerant concentration is less than $45 \%$. In an R134a system the compression process will be highly impacted because there is little liquid refrigerant in the flow. For example, if the refrigerant quality $x$ at the compressor inlet is 0.98 , and the oil concentration in the flow is $4 \%$, then $w$ is $32 \%$. Wang et al. (2006) investigated this effect in detail and proposed a parameter that they call evaporation ratio to evaluate how much liquid refrigerant evaporates during the compression process. Pérez-Segarra et al. (2005) split the volumetric efficiency into four partial components; the oil effect was considered along with the piston-cylinder leakage efficiency due to its direct relation to compressor lubrication. They investigated this effect both theoretically and experimentally.

After substituting experimental pressures and refrigerant concentrations into Grebner's 
correlation, fairly large discrepancies were found between predicted mixture temperatures and measured ones, especially in situations where $w$ is relatively low. This might be due to the fact that the correlation tries to cover four different sets of refrigerant-oil pairs and hence cannot generate satisfactory results for a single specific case. Knowing this, a new correlation between $P, T$ and $w$ was developed by the authors, which is expressed as

$$
T=A_{1} P^{-1.5}+A_{2} P^{-1}+A_{3} P^{-0.5}+A_{4}+A_{5} P^{0.5}+A_{6} P
$$

where

$$
A_{i}=B_{i, 1} w^{-1}+B_{i, 2}+B_{i, 3} w+B_{i, 4} w^{2}+B_{i, 5} w^{3}+B_{i, 6} w^{4}, \quad i=1,6
$$

Table 4-1 Coefficients of $B_{i, j}$ for correlation (4-11)

\begin{tabular}{|c|r|r|r|r|r|r|}
\hline $\boldsymbol{B}_{\boldsymbol{i}, \boldsymbol{j}}$ & \multicolumn{1}{c|}{$\boldsymbol{j}=\mathbf{1}$} & \multicolumn{1}{c|}{$\boldsymbol{j = 2}$} & \multicolumn{1}{c|}{$\boldsymbol{j}=\mathbf{3}$} & \multicolumn{1}{c|}{$\boldsymbol{j}=\mathbf{4}$} & \multicolumn{1}{c|}{$\boldsymbol{j}=\mathbf{5}$} & \multicolumn{1}{c|}{$\boldsymbol{j = 6}$} \\
\hline $\boldsymbol{i}=\mathbf{1}$ & -555.57 & 1529.83 & -1631.49 & 849.39 & -217.16 & 22.79 \\
\hline $\boldsymbol{i}=\mathbf{2}$ & 9941.60 & -27779.68 & 29642.06 & -15277.55 & 3861.57 & -383.49 \\
\hline $\boldsymbol{i}=\mathbf{3}$ & -57338.05 & 166134.44 & -180687.13 & 93312.84 & -23313.61 & 2281.07 \\
\hline $\boldsymbol{i}=\mathbf{4}$ & 135583.90 & -415835.15 & 466329.38 & -244495.89 & 61297.87 & -5973.48 \\
\hline $\boldsymbol{i}=\mathbf{5}$ & -135357.30 & 452121.67 & -529159.56 & 283692.82 & -71819.19 & 7021.51 \\
\hline $\boldsymbol{i}=\mathbf{6}$ & 46669.17 & -176319.04 & 217725.91 & -119919.13 & 30762.16 & -3027.66 \\
\hline
\end{tabular}

The coefficients of $B_{i, j}(i, j=1,6)$ are shown in Table 4-1. Though more coefficients are used in this correlation, it is not difficult to use them in a computer code. Moreover, two main advantages justify the inconvenience. First, it greatly improves the prediction accuracy. A comparison between Grebner's correlation and the new correlation is shown in Figure 4-4. Secondly, there is no need to calculate $T_{\text {sat }}$, which makes the correlation potentially capable to be expanded to refrigerant-oil mixture working at transcritical cycles.

To further validate the accuracy of the new correlation, measured pressures and refrigerant concentrations at the accumulator outlet are taken as inputs to calculate the mixture temperatures. 


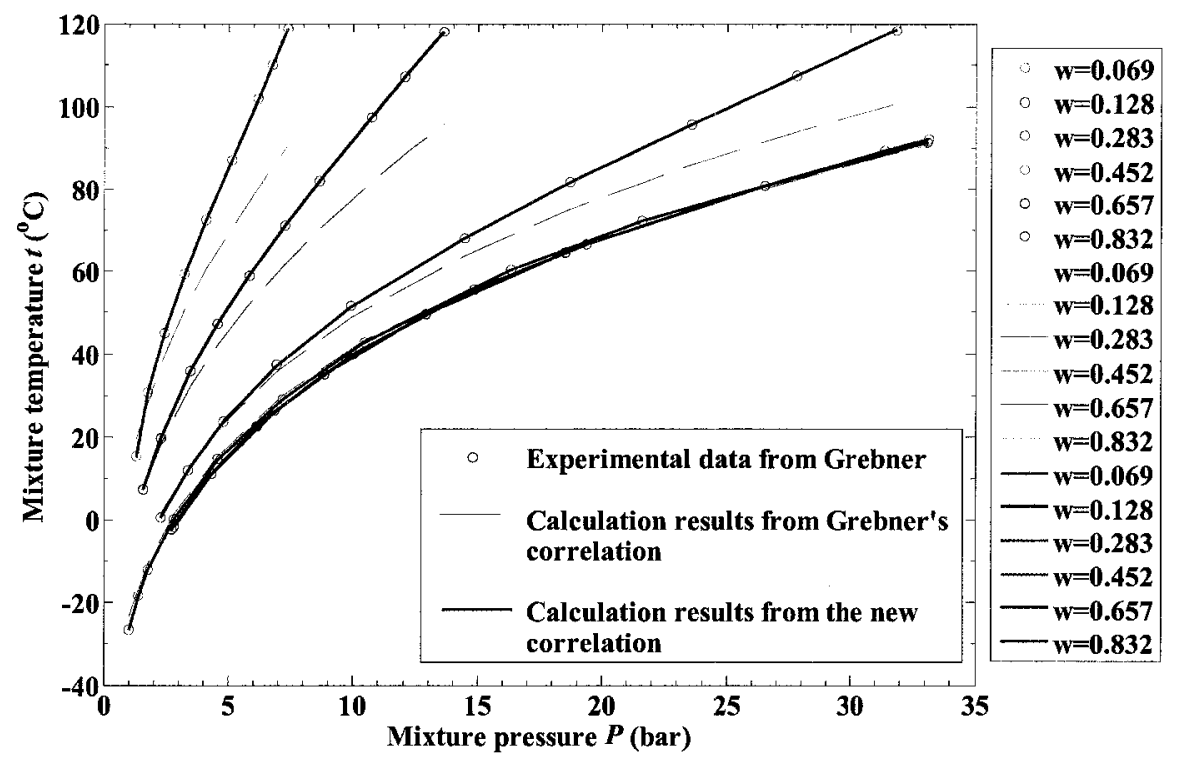

Figure 4-4 Improvement to the Grebner's correlation

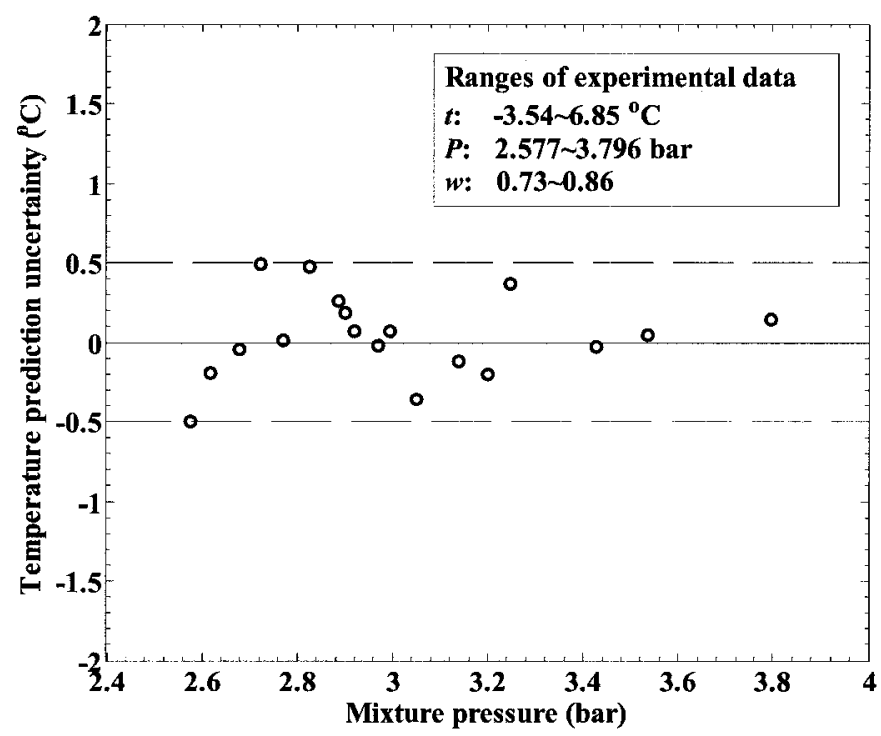

Figure 4-5 Prediction accuracy of the new correlation

Prediction uncertainties are shown in Figure 4-5. Keeping in mind the $\pm 0.2{ }^{\circ} \mathrm{C}$ accuracy of thermocouples, good agreement can be observed with all deviations being less than $\pm 0.5^{\circ} \mathrm{C}$.

As mentioned above, oil effects become obvious when the refrigerant concentration falls below $45 \%$, which corresponds to the compression process. Using adjusted pressures generated by the new correlation, reasonable estimates of the work consumption of the compression process can be 
obtained. Figure 4-6 shows the difference before and after the adjustment of measured pressures.

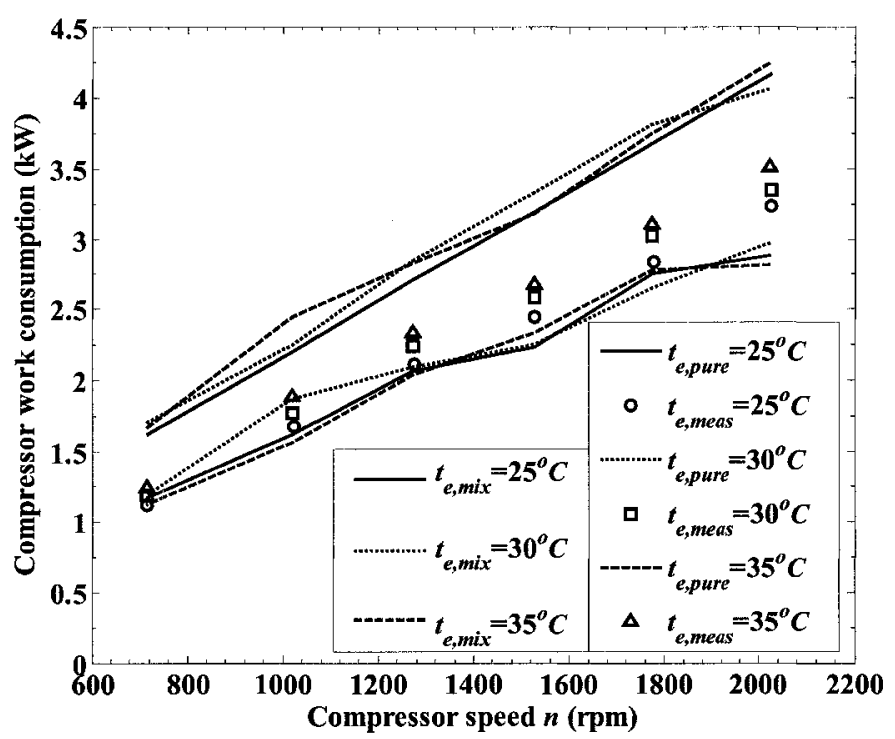

Figure 4-6 Compressor work consumptions before and after adjusting pressures

\subsection{Experimental set-up and uncertainties}

To investigate the changing trends of the evaporating pressure and the condensation pressure with compressor speed, experiments were conducted on an R134a-based AAC system adopting a CCOT mechanism. The test loop is shown in Figure 4-7. A compressor stand, a condenser stand with water-bath reservoir, an evaporator stand, and an accumulator stand are connected by tubing rigged with pressure and temperature sensors.

The compressor stand consists of an automotive swash plate compressor driven by a $10 \mathrm{hp}$ inverter-based variable-speed motor. A mass flow meter was mounted between the condenser and the expansion device to measure the total mass flow rate of both refrigerant and oil. A sight glass tube was placed right after the flow meter to ensure the refrigerant is in a sub-cooled state. Another flow meter was installed between the accumulator and the compressor to measure the vapour flow 
only.

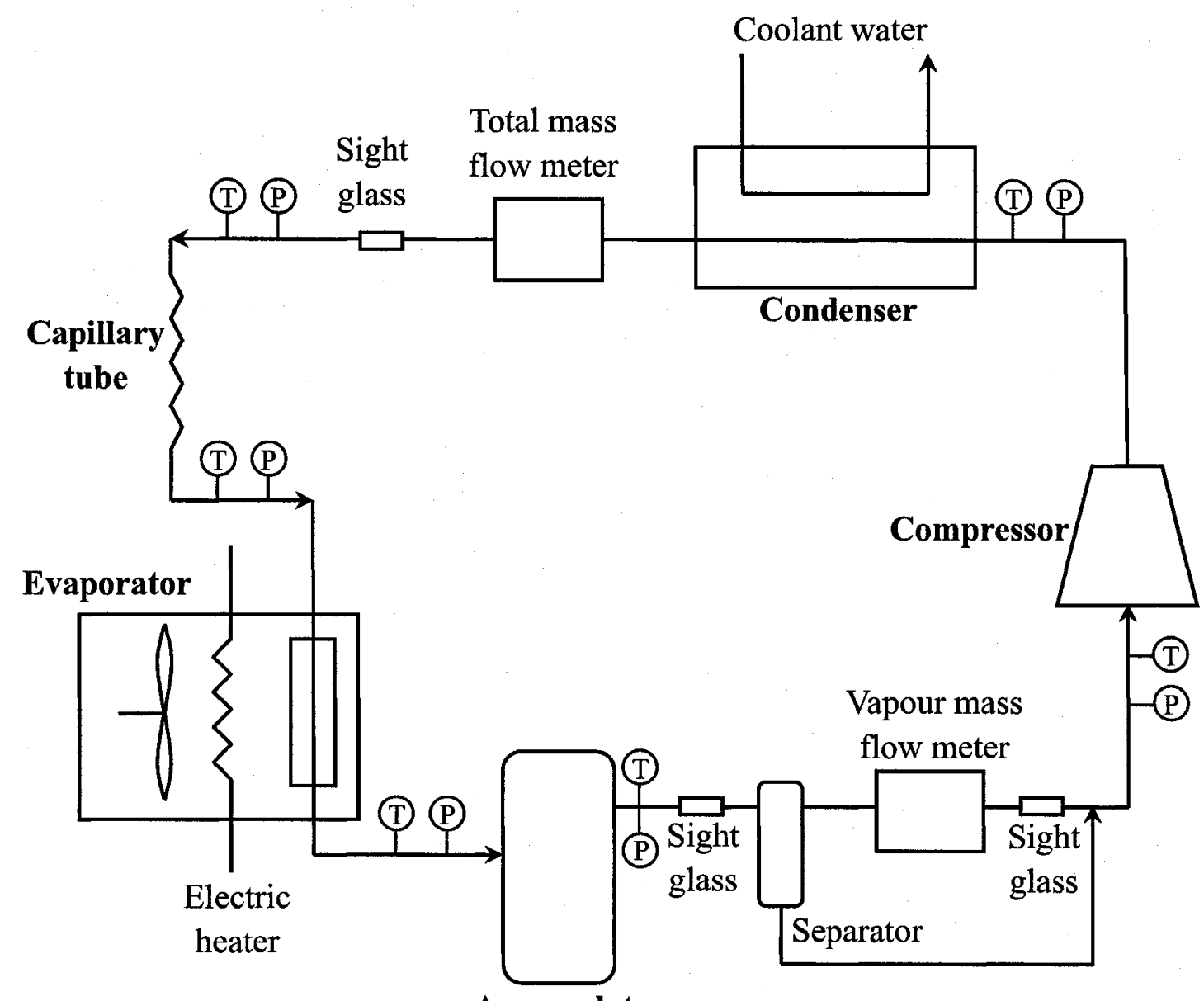

Accumulator

Figure 4-7 Schematic of an R134a AAC experimental test stand with capillary tube

Unlike a traditional AAC system where an orifice tube is adopted to create pressure balance between the high side and the low side, a capillary tube with a $1.65 \mathrm{~mm}$ inner diameter and $0.50 \mathrm{~m}$ length was used instead. It functions only as an expander in this chapter, but will be further used as a component of the heat exchanger in a successive investigation. $1.8 \mathrm{~kg}$ of refrigerant was charged into the system; the cooling water temperature was $40^{\circ} \mathrm{C}$; the passenger temperature was preset at 25,30 , and $35^{\circ} \mathrm{C}$, respectively; and the compressor speed was controlled at five speeds to mimic different driving conditions from idle to highway. Detailed information about the test rig can be 
found in the research by Wang et al. (2006).

Discharge and intake pressures were measured using $0-20 \mathrm{MPa}( \pm 10 \mathrm{kPa}$ full scale $)$ and 0 -15 $\mathrm{MPa}( \pm 10 \mathrm{kPa}$ full scale) pressure transducers. The thermocouples were found to be accurate to within $\pm 0.2{ }^{\circ} \mathrm{C}$. Mass flow rate fluctuations of two coriolis mass flowmeters were observed to be $\pm 0.1 \mathrm{~kg} / \mathrm{h}$. Refrigerant charge was controlled to be accurate within $\pm 0.001 \mathrm{~kg}$. The thermostats provided precise temperatures within $\pm 1^{\circ} \mathrm{C}$ variation.

\subsection{Correlations of pressure lift}

Under various working conditions, i.e. different $t_{p a s s}, t_{a m b}$, and $n$, the pressures $P_{e}$ and $P_{k}$ will change accordingly. Figure 4-8 is a typical set of pressure curves with varying compressor speed under different evaporation temperatures. Taking $P_{k}$ as an example, though different in magnitude, they follow similar trend, which means compressor speed influences $P_{k}$ in a similar manner no matter how $t_{\text {pass }}$ changes. This can be seen from Equation (4-5) as well. Taking the derivative of $t_{e}$ with respect to $n$ one gets

$$
\frac{d t_{e}}{d n}=-\dot{m} \frac{d\left(\frac{h_{\text {out }}-h_{\text {in }}}{U A_{e}}\right)}{d n}-\left(\frac{h_{\text {out }}-h_{\text {in }}}{U A_{e}}\right) \frac{d \dot{m}}{d n}
$$

For the same reason that leads to the decrease of quality at evaporator outlet (section 4.1.3), the enthalpy at the evaporator outlet $\left(h_{\text {out }}\right)$ is getting smaller with increasing compressor speed $(n)$. Since the condenser exit temperature is not sensitive to $n, h_{i n}$ does not change significantly. As a consequence, $\left(h_{\text {out }}-h_{\text {in }}\right)$ decreases with $n$ if other conditions are kept unchanged. At the same time, $U A_{e}$ decreases slightly and linearly with $n$, which might be caused by the decrease in the effective 
heat transfer area of the specific flow pattern, similar to the explanation of the behaviour of pressures and compressor speed by Tassou and Qureshi (1998). When the evaporator outlet quality decreases, more liquid film is formed along the inner side of the refrigerant tube, lowering the heat transfer effect slightly. Therefore, the first item on the right hand side of Equation (4-12) is almost linearly related to $n$, and $d t_{e}$ is shaped by $\frac{d \dot{m}}{d n}$. Equation (4-5) can thus be further expressed as $t_{e}=t_{\text {pass }}-f_{4}(n, \dot{m})$

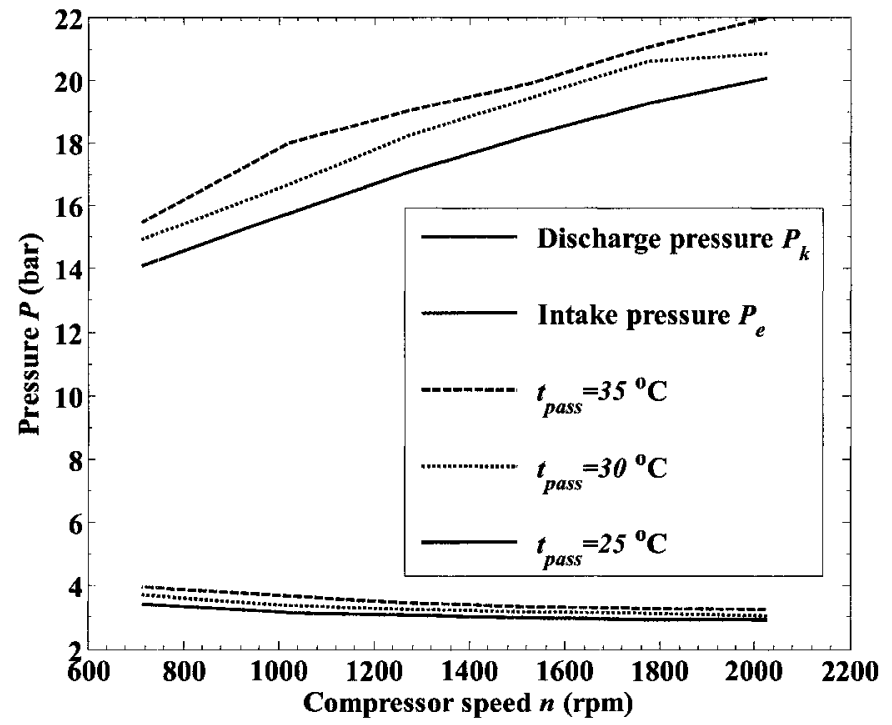

Figure 4-8 Relationship between pressures and compressor speed

\subsubsection{Correlation based on experimental data for $t_{\text {pass }}=25^{\circ} \mathrm{C}$}

Using experimental results obtained at $t_{\text {pass }}=25^{\circ} \mathrm{C}$ as sample data to determine the regression coefficients, the following correlation can be obtained

$$
\begin{aligned}
& t_{e}=t_{\text {pass }}-(790.32-0.077991 n) \dot{m} \\
& t_{k}=t_{e}+\left(2182.7 \dot{m}-9881.1 \dot{m}^{2}\right)
\end{aligned}
$$


where $\dot{m}=0.0071064-6.8413 \times 10^{-4} \sqrt{n}$

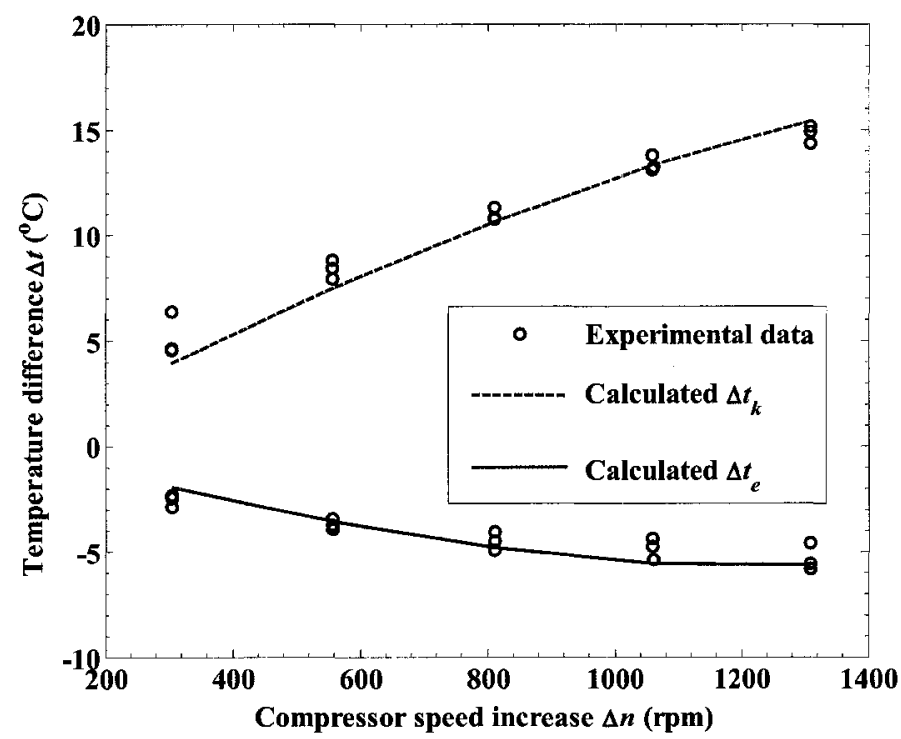

Figure 4-9 Predicted and measured temperature differences with compressor speed

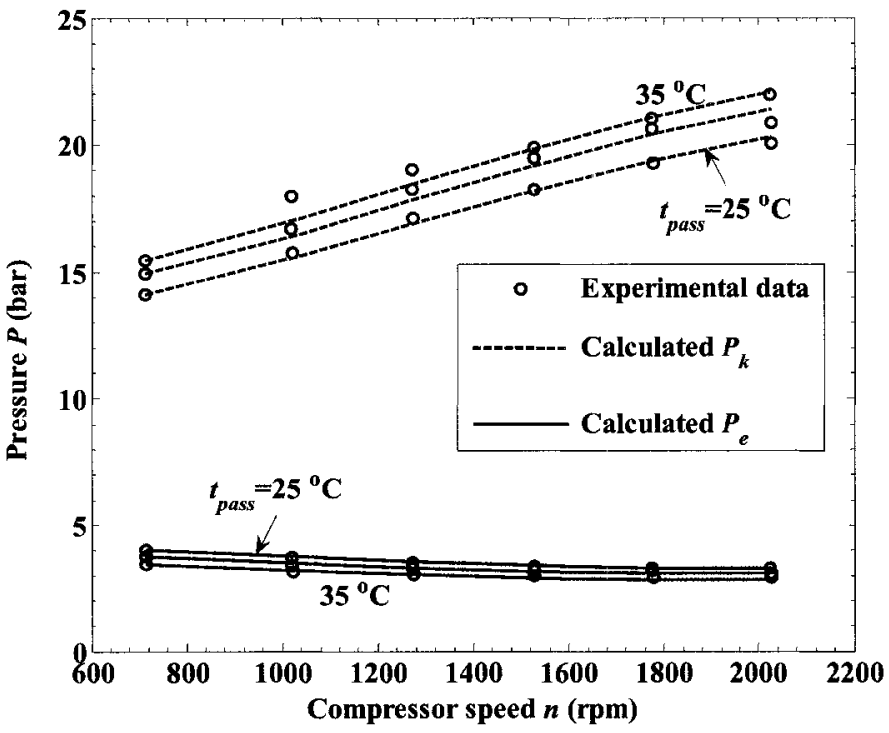

Figure 4-10 Predicted and measured pressures with varying compressor speed

Accordingly, the temperature differences are

$$
\begin{aligned}
& \Delta t_{e}=-(790.32-0.077991 n) \Delta \dot{m}+0.077991 \dot{m} \Delta n \\
& \Delta t_{k}=\Delta t_{e}+(2182.7-2 \times 9881.1 \dot{m}) \Delta \dot{m}
\end{aligned}
$$

It is worth mentioning that when $\Delta n$ is large, second order terms in the form of $(\Delta n)^{2}$, extracting 
from a Taylor Series, need to be included into the calculation to improve the accuracy.

Predicted temperature differences and measured data are compared in Figure 4-9. A slight over prediction of $\Delta t_{e}$ can be observed when $\Delta n$ is small, and a slight under prediction when $\Delta n$ is large, while predicted $\Delta t_{k}$ values are lower than measured at first and match well for the rest. These initial deviations can be attributed to the inaccuracies of the correlations, which do not represent starting points as well as those that follow. Using temperature and pressure differences, the corresponding pressures can be obtained. Results are shown in Figure 4-10, where the top dashed line and the bottom solid line correspond to $t_{e}=35^{\circ} \mathrm{C}$, the bottom dashed line and the top solid line are for $t_{e}=30^{\circ} \mathrm{C}$, and the two lines in the middle are for $t_{e}=25^{\circ} \mathrm{C}$. Prediction errors are well less than $\pm 5 \%$, as can been seen from Figure 4-11.

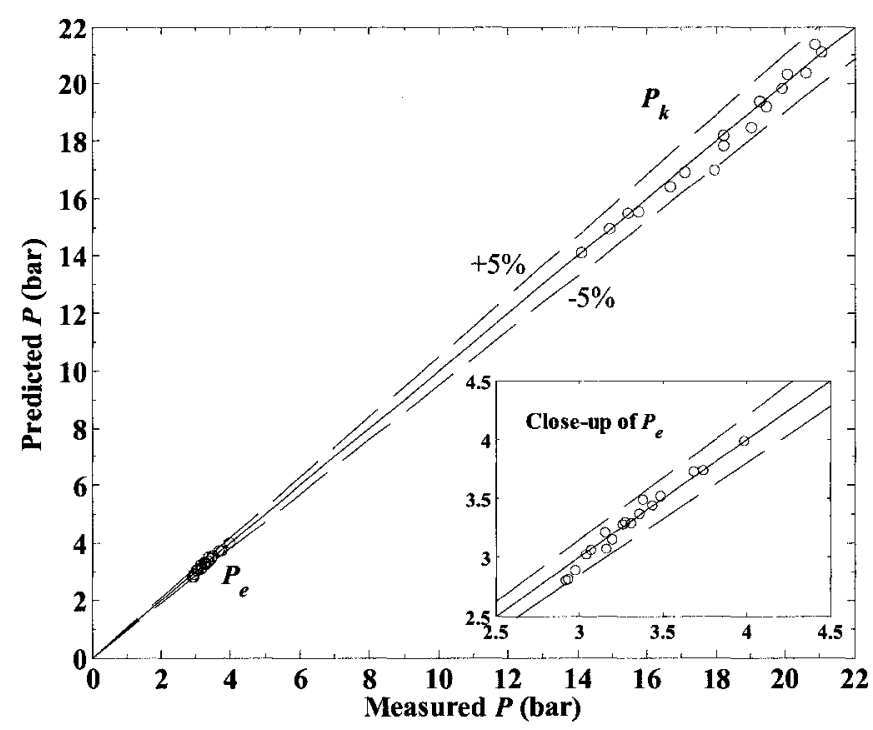

Figure 4-11 Comparison between predicted and measured pressures

\subsubsection{Improved correlation based on averaged experimental data}

To further investigate whether a new correlation will improve model accuracy or not, two 
averaged regression coefficients in terms containing $n$ were calculated and substituted into Equations (4-14) and (4-16), respectively. The improved correlations can be developed as follows.

$$
\begin{aligned}
& t_{e}=t_{\text {pass }}-(790.32-0.078408 n) \dot{m} \\
& \dot{m}=0.0071064-6.5971 \times 10^{-4} \sqrt{n}
\end{aligned}
$$

The coefficient in Equation (4-15) changes greatly, deteriorating the model, so it is not modified. The improvements of predicting temperature differences are shown in Figure 4-12. Though an improvement exists, it is not significant. This shows that correlation based on one set of specific experimental results can generate satisfactory results without more extensive experimental work.

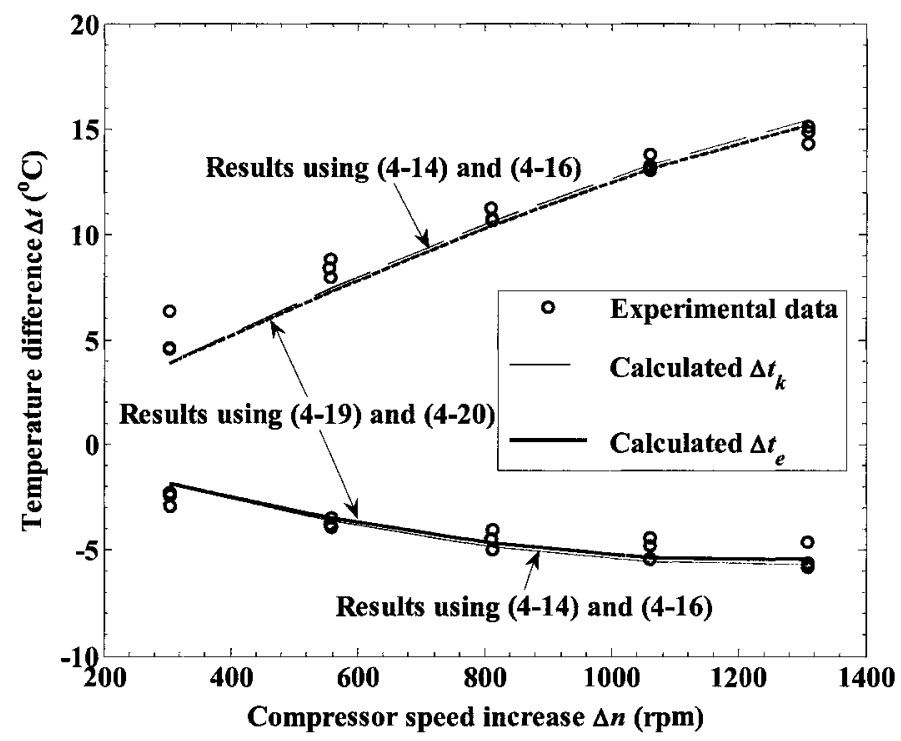

Figure 4-12 Improvement of correlation in predicting temperature differences

\subsubsection{Further discussion on the influence of suction density and ambient} temperature

As mentioned in subsection 4.1.3, the density at the compressor inlet weakly affects the mass flow rate. To verify the statement, it was not neglected in $f_{2}$, and correlation (4-16) can be 
re-expressed as

$$
\dot{m}=\rho_{1}\left(0.00024303-4.9523 \times 10^{-5} \sqrt{n}\right)
$$

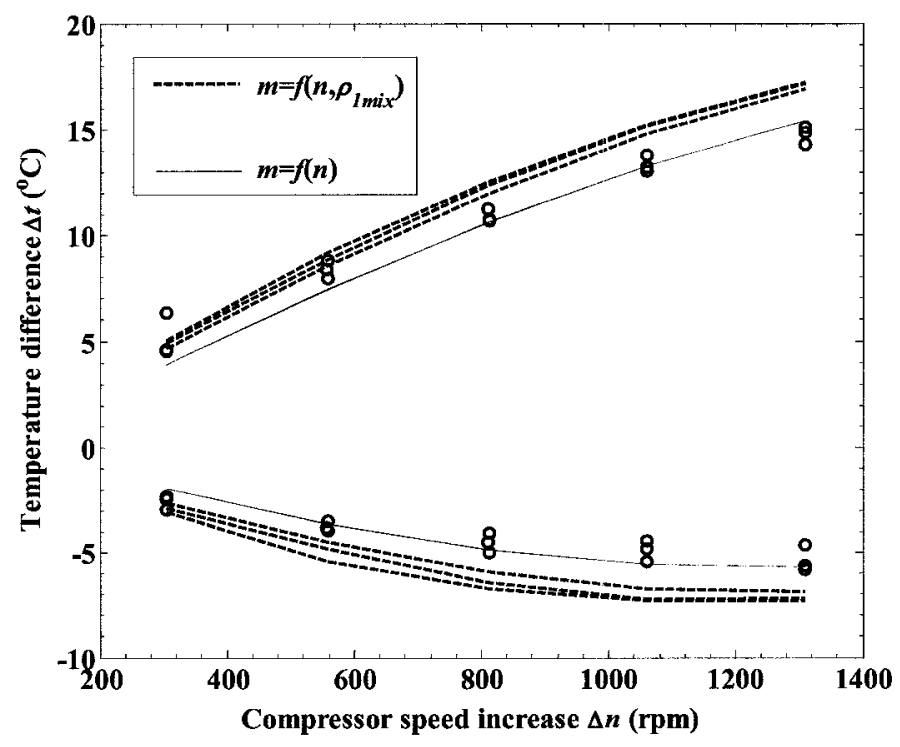

Figure 4-13 Influence of density at the compressor inlet

Figure 4-13 is the calculated temperature differences using (4-21). Though the first few points match more closely, more deviations are observed over the whole range. Two points can thus be made: the form $\dot{m}=\rho_{1}\left(c_{1}+c_{2} \sqrt{n}\right)$ does not work as well as $\dot{m}=c_{1}+c_{2} \sqrt{n}$ in Equation (4-16); correlating mass flow rate directly to compressor speed and including the influence of suction density into coefficients offers an easy way to depict flow behaviour without losing accuracy. From our experimental results it can be seen that the suction density is almost the same for various operation conditions because of the stabilizer AXE. So for other AAC systems with AXE, only Equation (4-16) needs to be rectified, and the other two equations (4-14) and (4-15) are generally applicable.

No influence of the ambient temperature can be seen from the equations derived in the above 
discussion. Actually $t_{a m b}$ was fixed at $40^{\circ} \mathrm{C}$, and was included in the coefficients in Equations (4-15) and (4-18).

\subsubsection{Verification of the model ACAM}

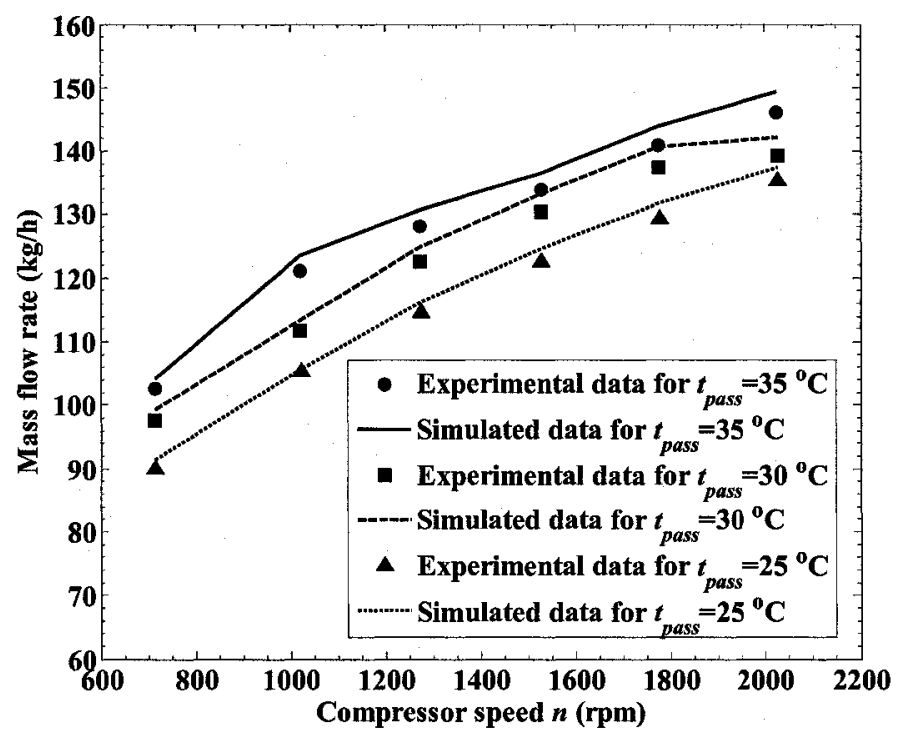

Figure 4-14 Comparison of data by experiments and ACAM

AAC systems have much higher mass flow rate than domestic refrigerators and air conditioners.

Available models in open literature are not capable of simulating flow behaviour beyond a capillary tube relatively small inner diameter. In our case, the capillary inner diameter $d_{i}=1.65 \mathrm{~mm}$, and the mass flow rate varies from 90 to $145 \mathrm{~kg} / \mathrm{h}$. ACAM was adopted to predict the mass flow rate; the results are shown in Figure 4-14, where a fairly good agreement can be observed. The experimental data were slightly over predicted by a maximum of $2.4 \%$ and an average deviation of $1.8 \%$. This validates ACAM to be an accurate model in adiabatic analysis; the deviations might be mainly caused by the inner diameter. We measured the inner diameter at the tube inlet, and assumed it to be the same everywhere throughout the capillary; it is possible a slight difference in diameter will 
result in a relatively large change in the mass flow rate. Based on observation, all predicted values are biased to one side, thus the true inner diameter might be slightly smaller than $1.65 \mathrm{~mm}$.

\subsection{Conclusions}

In this chapter a detailed investigation on the relationship between pressure drop and compressor speed was carried out. Based on the above discussion, the following conclusions can be drawn:

1. Oil effects must be taken into account so as to obtain reasonable parameter values, such as enthalpies and densities at the inlet or outlet of each component. This is especially important for the compression process.

2. Though a correlation based on one specific passenger temperature was developed, it can predict pressure changes well, with deviations being less than $\pm 5 \%$. More experimental data should slightly improve the correlation accuracy.

3. There are two main advantages to use a correlation between the behaviour of pressure drop $\left(P_{k}-P_{e}\right)$ and the compressor speed $n$. One is to provide a useful tool for simulation and optimization. Given $t_{\text {pass }}$ and an arbitrary $n$, the corresponding intake and discharge pressure can be determined, and vice versa. The other is to further understand how the system components affect these parameters. From Equations (4-19) and (4-20) it can be seen that the mass flow rate embodies the effect of the compressor; the coefficients represent the 
characteristics of other components (the coefficients in $f_{4}$ represent the evaporator, and $f_{1}$ the condenser). If a new compressor is incorporated into the system, only the correlation for $\dot{m}$ needs to change, while the others remain the same. This will greatly simplify the model in predicting system behaviour when only one component is replaced.

4. It needs to be addressed that $f_{1}$ also includes the contribution of the environment temperature, so further development is expected to clarify the contribution by the condenser.

5. ACAM proved to be a good model in predicting the mass flow rate for adiabatic flow. It slightly over predicts experimental data by less than $2.4 \%$. The true capillary inner diameter may be slightly smaller than $1.65 \mathrm{~mm}$. 


\section{Chapter 5}

\section{Experimental study of the pressure behaviour of an AAC system with}

AXE

Previously we have discussed the behaviour of pressures with a change in compressor speed in a CCOT AAC system where the orifice tube was replaced by a capillary with larger inner diameter and greater length than an orifice tube. In this chapter we will further investigate how such a system responds if a heat exchanger, normally called an internal heat exchanger (IHX), is incorporated into the system. Different from other researchers' work, a highly-integrated device AXE is adopted; AXE is an improved IHX especially suited to AAC systems.

Internal heat exchangers are widely used in various refrigeration systems, especially large commercial chillers, to recover part of the heat at the low pressure side between the evaporator and the compressor so that the heat load in the evaporator can be improved. Since it adds one more component to the system, IHXs are not used extensively in small-scale applications. However, in domestic refrigerators part of the capillary tube adheres to the suction line, forming a fundamental capillary tube-suction line heat exchanger; thus it is possible to increase the cooling capacity in these devices. There are several criteria to judge whether or not a heat regenerator is suitable for a system. The first is the COP. Normally, as the heat load is increased by the IHX, the compressor power consumption goes up as well because the intake temperature is higher and the isentropic efficiency is lower. Depending on various thermophysical properties of different refrigerants, the 
COP of IHX systems would increase if fluids that make the cooling load increase more significantly than the compressor work increase are utilized. According to Domanski et al. (1994), 24 out of 29 refrigerants under investigation allow the system performance to benefit from the introduction of an IHX. Aprae et al. (1999) presented a simple criterion to evaluate the influence of internal heat exchangers and graphically discussed two sets of working fluids: R502 with its substitutes and R22 with its substitutes. The assumptions the authors made when the simulation was carried out introduce uncertainties determined to be less than 5\%. Klein et al. (2000) extended the research to include more refrigerants, most of them mixtures. A non-dimensional relative capacity change index RCI was proposed to represent the effect of IHXs on system performance. Moreover, pressure drop across the IHX was taken into account and expressed in correlations as a reduced factor. Navarro-Esbrí et al. (2005) further investigated the influence of IHXs on refrigerant mass flow rate, and how pressure drop across the new device affects the system performance. Compared with the work of Aprae et al. (1999) and Klein et al. (2000), good agreement was observed except for slight over prediction for an R134a system using Klein's correlation. It needs to be noted that experimental data from Navarro-Esbrí et al. (2005) does not conflict with the simple criterion developed by Aprae et al. (1999). The second criterion is the working condition of the expansion device. Less vaporization occurs during the expansion process if heat regeneration is present, making the expansion device more stable (Domanski et al., 1994; Klein et al., 2000). The third factor is manufacturing and maintenance costs. More components lead to more weight, more complexity, and more initial costs, less system reliability and extra maintenance fees. 
All of the research mentioned above is based on systems having an IHX and an expansion device as two separate components. The schematic and related pressure-enthalpy diagram of these systems is shown in Figure 3-1. For some cycles, a receiver/dryer combination is adopted instead of accumulator, but the thermodynamic description does not change. This chapter is focused on the new device AXE that combines the functions of a traditional expansion device, accumulator, and suction line heat exchanger into one piece. A capillary tube with a $1.65 \mathrm{~mm}$ inner diameter and $0.5 \mathrm{~m}$ in length is inserted into the $\mathrm{J}$-shaped tube of the accumulator to throttle the refrigerant to the evaporation pressure, while simultaneously discharging heat to the surrounding suction vapour. The system composition and the corresponding pressure-enthalpy diagram of the new AXE cycle are shown in Figure 3-2. Contrary to previous systems where the suction line side pressure loss over the IHX becomes a concern, the J-tube side pressure loss over the AXE is negligible. This is because the inner diameter of the $\mathrm{J}$-shaped tube is $12.7 \mathrm{~mm}$, large enough that the inside vapour velocity is relatively low. Also, due to a delicate configuration of the capillary tube, the heat exchanger portion is as short as approximately $25 \mathrm{~cm}$. For both these reasons, the pressure loss of the low-side inside the AXE can be neglected during analysis. It needs to be addressed that Mei and Chen (1997) proposed an AXE with structure similar to our model, but these two designs differ in the placement of the capillary tube. Mei and Chen immerse the capillary in the over-feeding refrigerant liquid, which could ensure a relatively stable heat transfer rate. However, this will not affect the suction line inlet quality significantly because it is mainly determined by the pressure in the accumulator chamber and the size of the oil bleeding hole located at the bottom of the J-tube. From a thermodynamic point of view, this will increase the system flow rate while leaving the 
waste heat in the suction line unrecovered. In our research, the capillary tube is placed directly into the suction line portion. This will allow heat recovery to occur and effectively prevent the compressor from liquid slugging. Another obvious difference between the AXE system and non-AXE systems is the change in discharge pressure. The mechanism underlying this change will be discussed in this chapter.

To thoroughly investigate how the system pressure lift (i.e. pressure difference between the high-side pressure and the low-side pressure) behaves with changing compressor speeds for AAC systems with and without $\mathrm{AXE}$, two steps were taken. In the previous chapter a capillary tube was configured as a pure expansion device and no IHX was placed into the system. By this means traditional pressure behaviour was observed and the corresponding correlation was developed. This chapter will further the investigation on how AXE will affect the pressure drop and then the system performance.

\subsection{Determination of pressure drop across the AXE}

Beginning with the previous experimental test stand, the $0.5 \mathrm{~m}$ long capillary tube is folded and inserted into the J-tube. The new test loop is illustrated in Figure 5-1. All the measuring equipment has the same accuracies as stated in Chapter 4, section 4.2.

To make the results comparable, the environmental conditions were kept same as during the experiments on the non-AXE system: the refrigerant charge, the cooling water temperature, the three different passenger temperatures, and the five compressor speeds were controlled to be the same as in the previous chapter. 


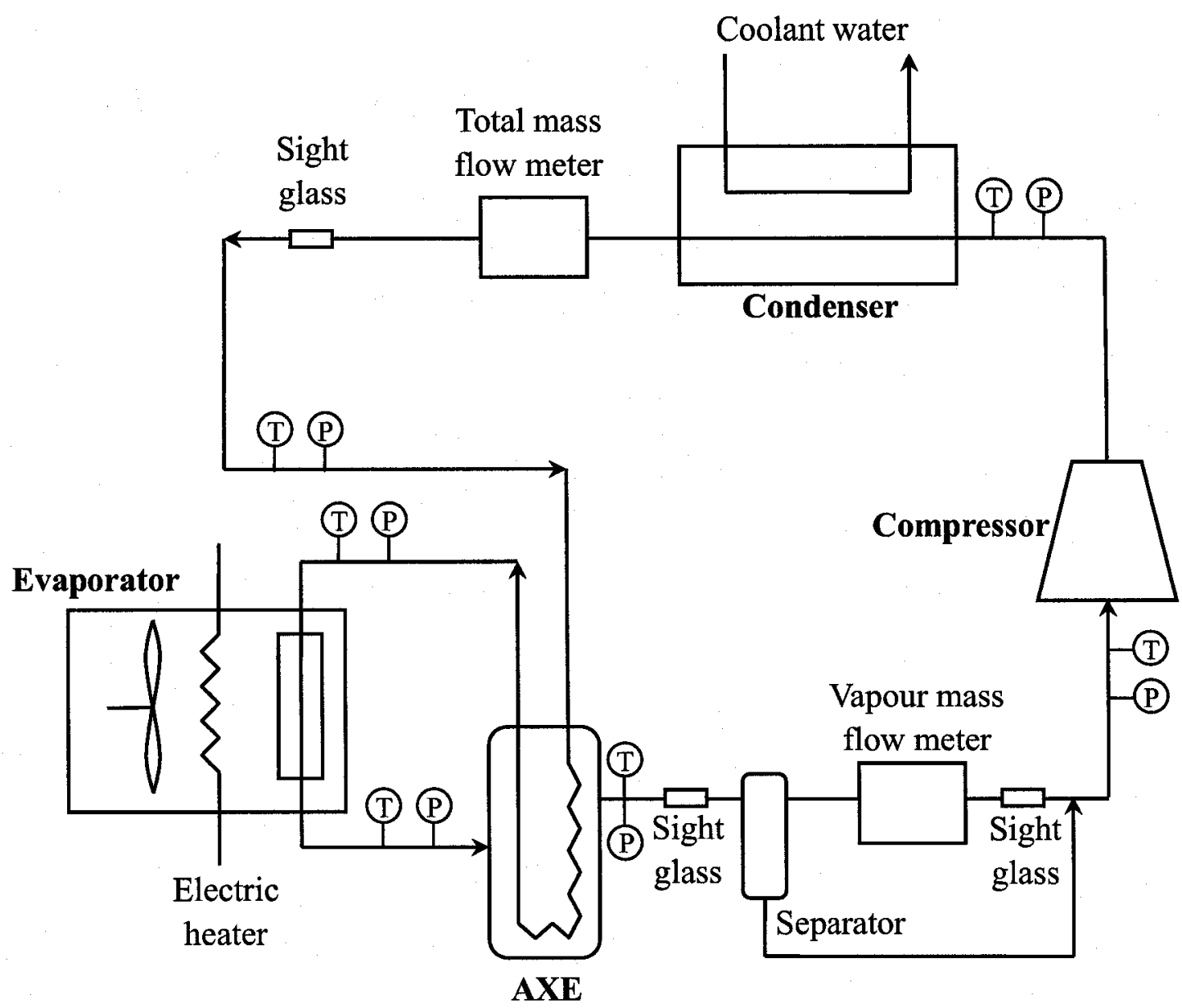

Figure 5-1 Schematic of an R134a AAC experimental test stand with AXE

The experimental data from the test stand with AXE have been obtained, and a set of results for $t_{a m b}$ being equal to $40^{\circ} \mathrm{C}$ are shown in Table 5-1 combined with results of Chapter 4 . Before performing any analysis, data processing techniques should be adopted. This is because the compressor speed is not fixed at an exact value. For example, at $t_{\text {pass }}=25^{\circ} \mathrm{C}$, the compressor speed $n$ is preset at $700 \mathrm{rpm}$. However, for the system without an IHX the measured value is $716.08 \mathrm{rpm}$; and for the system with an IHX the measured value is slightly different at $716.47 \mathrm{rpm}$. To investigate the influence of an IHX on pressures, we must compare pressures at exactly the same compressor speed, which means two measured compressor speeds should be the same. 
Table 5-1 Experimental results for R134a based AAC system with and without AXE

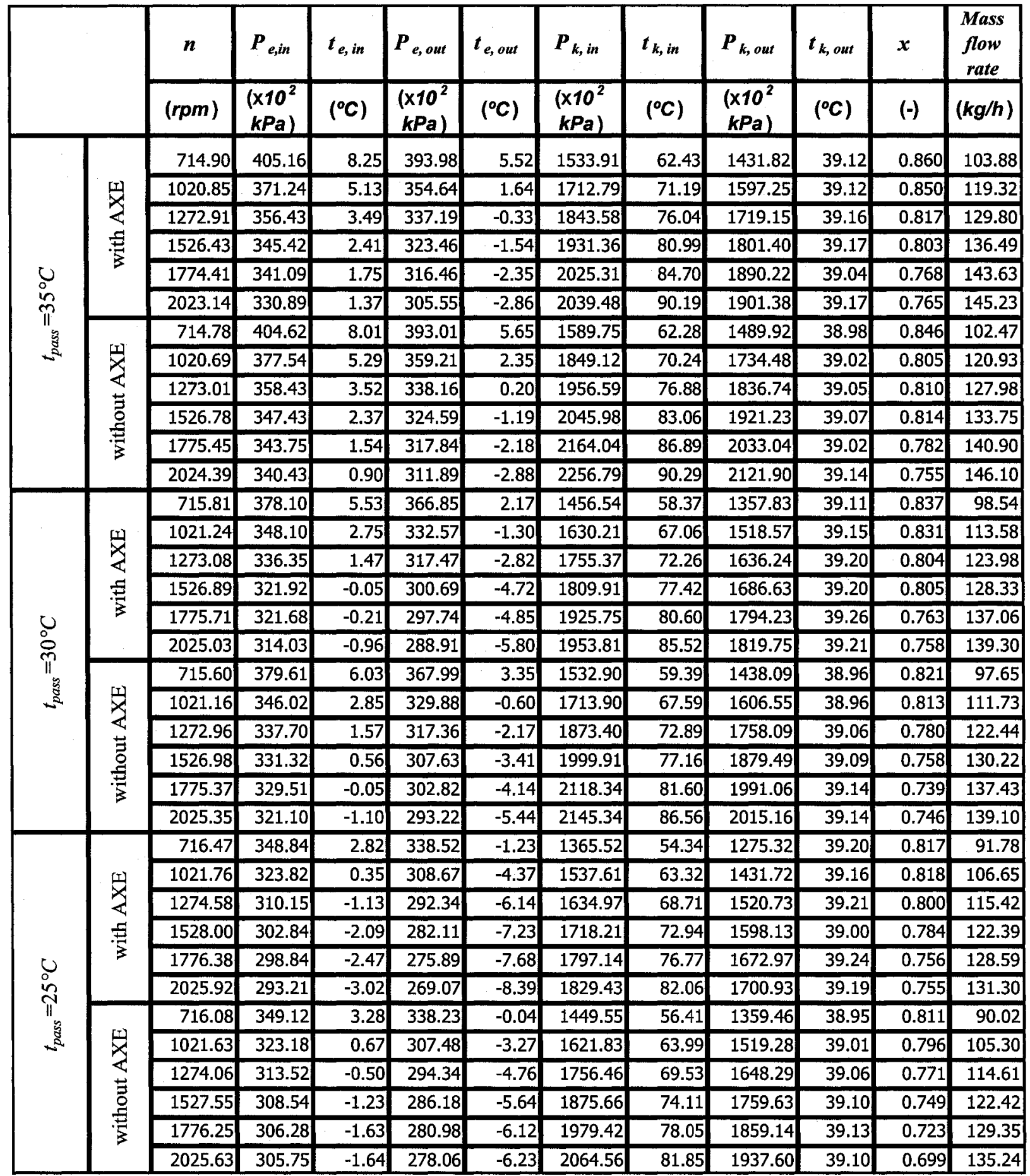

Though the measured compressor speeds differ slightly in this case, logically it needs to be

rectified to diminish uncertainties as much as possible. In this fashion, even though the compressor

speeds of the two systems differ greatly, the results can be adjusted such that they can be compared. 
To permit such a comparison, raw data were processed to elucidate the relation between pressures and the compressor speed.

The most obvious difference that can be observed is the behaviour of pressure drop over the expansion device as shown in Figure 5-2. Lower values are obtained for the AXE system over the compressor speed range from $700 \mathrm{rpm}$ to $2000 \mathrm{rpm}$. The pressure drop is directly related to the mass flow rate, AXE inlet density, and capillary geometry. How it is distributed between the high-side and the low-side is determined by the thermal characteristics of the condenser and the evaporator, respectively. Their relations can be described as follows (Chen et al., 2007):

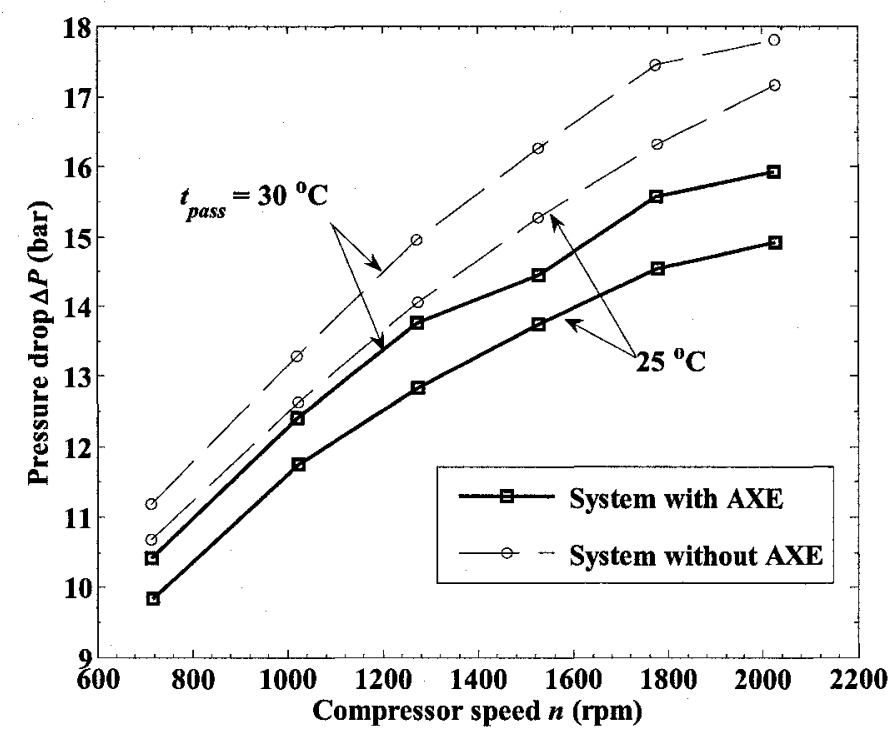

Figure 5-2 Pressure drop of throttling process for systems with and without an AXE

$$
\begin{aligned}
& \Delta P=\frac{1}{2} F \rho V^{2}=\frac{F}{2 A^{2}} \frac{\dot{m}^{2}}{\rho} \\
& \Delta t=t_{k}-t_{e} \Rightarrow \Delta P=P_{k}-P_{e} \\
& t_{e}=t_{\text {pass }}-\frac{h_{\text {out }}-h_{\text {in }}}{U A_{e}} \dot{m}
\end{aligned}
$$


The link between these expressions is the one-to-one correspondence between temperature and pressure in the two-phase region. The pressure drop study can thus be converted to a temperature related analysis, which links the pressure behaviour to the thermal resistances of the evaporator and the condenser and clarifies the underlying physical mechanism. To understand the meaning of changes in pressure drop, we need to investigate the relevant parameters individually.

As a geometric parameter, the cross-sectional area of the capillary tube was kept the same both in the basic and the AXE systems. Owing to sufficient heat transfer capacity of the condenser, the exit temperature stays almost constant at a sub-cooled state; accordingly, its density remains nearly unchanged. Therefore, only the mass flow rate $\dot{m}$, the temperatures $t_{e}$ and $t_{k}$, and the overall pressure loss factor $F$ are of interest in this chapter.

\subsubsection{Mass flow rate}

From the work of Navarro-Esbrí et al. (2005) mass flow rate was almost unchanged regardless of whether or not an IHX was present. According to the statement shown in section 4.1.3, the refrigerant specific volume at the compressor inlet does not change significantly with the compressor speed. This allows the new system to outperform its IHX counterparts by preventing the volumetric efficiency from decaying and the compressor from consuming more power. Figure 5-3 is a comparison of mass flow rates for the system with and without AXE. Therefore, the correlation developed in the previous chapter can be used in this analysis as well.

$$
\dot{m}=0.0071064-6.8413 \times 10^{-4} \sqrt{n}
$$




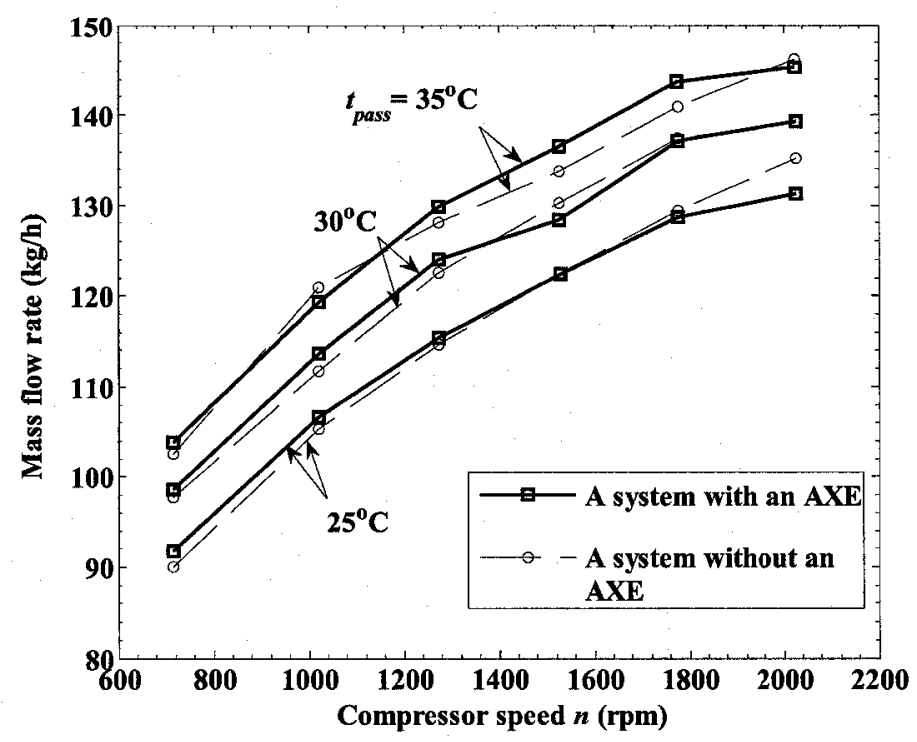

Figure 5-3 Mass flow rate for systems with and without an AXE

\subsubsection{Effect of oil concentration}

Many researchers have noticed the effects of oil in evaluating refrigerant themophysical properties (Grebner and Crawford, 1993; Pérez-Segarra et al., 2005; Wang et al., 2006; Chen and $\mathrm{Gu}, 2007)$. All of the work is based on Grebner's correlation which takes into account pressure, temperature, and liquid concentration of the refrigerant/oil mixture. It was found that the oil affects the system mostly in the compression process (Chen and Gu, 2007). Grebner's correlation (Grebner and Crawford, 1993) is expressed as

$$
T=\left(1+T^{*}\right) T_{\text {sat }}
$$

where the non-dimensional degree of apparent superheat is

$$
T^{*}=(1-w)(A+B P)
$$

where 


$$
\begin{aligned}
& w=\frac{1-(1-c) x-c}{1-(1-c) x} \\
& x=\frac{\dot{m}_{r, l}}{\dot{m}_{r, l}+\dot{m}_{r, g}} \\
& c=\frac{\dot{m}_{o i l}}{\dot{m}_{r, l}+\dot{m}_{r, g}+\dot{m}_{o i l}} \\
& A=C_{1}+\frac{C_{2}}{w^{0.5}}, B=C_{3}+\frac{C_{4}}{w^{0.5}}+\frac{C_{5}}{w}+\frac{C_{6}}{w^{1.5}}+\frac{C_{7}}{w^{2}}
\end{aligned}
$$

$T_{\text {sat }}$ is the saturated temperature of the pure refrigerant at pressure $P . A$ and $B$ are two empirical coefficients. Chen et al. (2007) found that the correlation under predicted the mixture temperature, especially when the liquid concentration is low. They developed a correlation that directly connects $P, T$, and $w$ without considering the saturated temperature $T_{\text {sat }}$, which is expressed as

$$
T=A_{1} P^{-1.5}+A_{2} P^{-1}+A_{3} P^{-0.5}+A_{4}+A_{5} P^{0.5}+A_{6} P
$$

where

$$
A_{i}=B_{i, 1} w^{-1}+B_{i, 2}+B_{i, 3} w+B_{i, 4} w^{2}+B_{i, 5} w^{3}+B_{i, 6} w^{4}, \quad i=1,6
$$

$B_{i, j}(i, j=1,6)$ is the coefficient derived by best-fitting the experimental data. The correlation provides high accuracy for the entire parameter range, and could be further expanded to transcritical refrigerants such as carbon dioxide because the saturated temperature does not exist in the supercritical region. However, its accuracy diminishes rapidly out of the range because the correlation is not based on physics but purely on mathematics. For this reason, it might be better to improve the coefficients of Grebner's correlation because it unveils a direct and linear relation between $T^{*}$ and $P$ at any given $w$. Coefficients are shown in Table 5-2, and a comparison between old and new coefficients is shown in Figure 5-4. Note that the units of $P$ and $T$ are psia and $\mathrm{R}$ respectively, while in the improved correlation they are bar and Celsius. 
Table 5-2 Original and improved coefficients of Grebner's correlation

\begin{tabular}{|c|c|c|c|c|c|c|c|}
\hline & $\boldsymbol{C}_{\boldsymbol{1}}$ & $\boldsymbol{C}_{\boldsymbol{2}}$ & $\boldsymbol{C}_{\boldsymbol{3}}$ & $\boldsymbol{C}_{4}$ & $\boldsymbol{C}_{5}$ & $\boldsymbol{C}_{6}$ & $\boldsymbol{C}_{7}$ \\
\hline Grebner's correlation & $-7.1525 \mathrm{E}-02$ & $5.9852 \mathrm{E}-02$ & $1.7133 \mathrm{E}-03$ & $-2.9749 \mathrm{E}-03$ & $1.7003 \mathrm{E}-03$ & $-3.7562 \mathrm{E}-04$ & $2.9984 \mathrm{E}-05$ \\
\hline Improved correlation & $-6.7001 \mathrm{E}-02$ & $5.2770 \mathrm{E}-02$ & $3.0335 \mathrm{E}-02$ & $-5.8017 \mathrm{E}-02$ & $3.7545 \mathrm{E}-02$ & $-9.5659 \mathrm{E}-03$ & $9.5208 \mathrm{E}-04$ \\
\hline
\end{tabular}

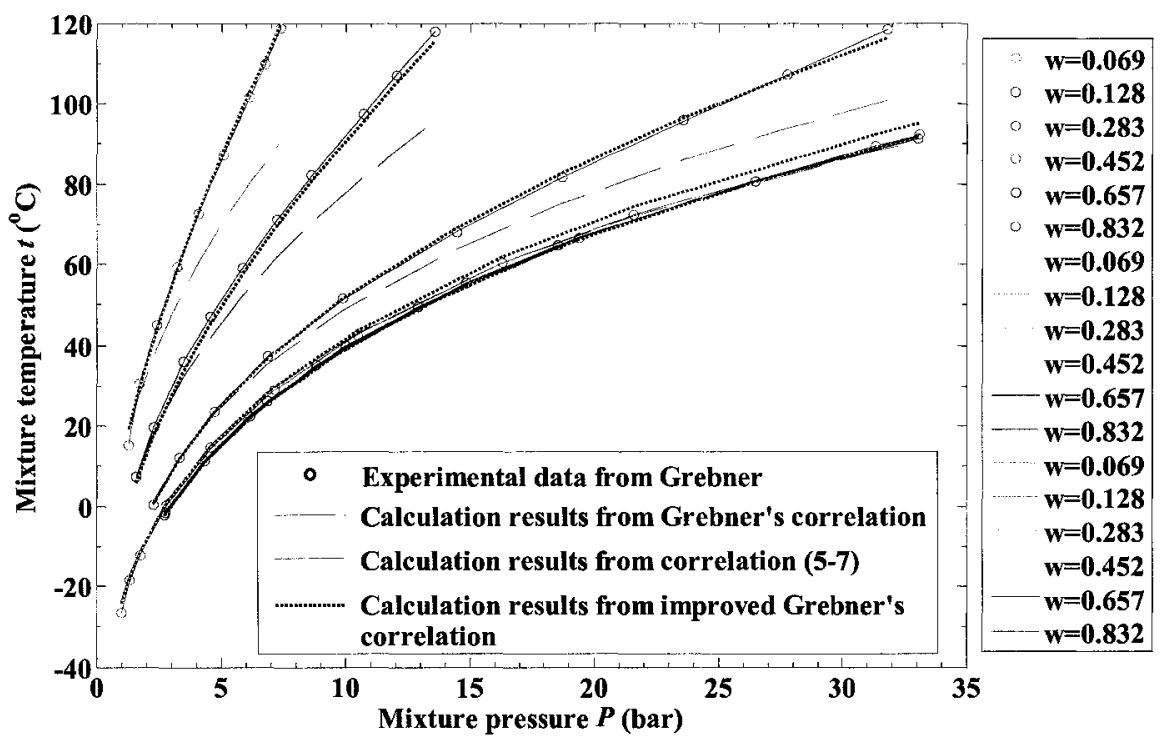

Figure 5-4 Comparison between different correlations and experimental data

Compared with correlation (5-7), the deviation of the improved Grebner's correlation is slightly larger but still satisfactory, and it keeps the right trends of curves well when $w<0.069, w>0.832$, and when the pressure is out of the parameter range. All the calculation results shown in this chapter were based on the improved Grebner's correlation. By directly substituting correlation (5-7) with the improved one, it was found that more computing time was consumed during the process of calculating the saturated temperature and overflow was more likely to occur. Accordingly, an acceleration algorithm was adopted to speed up the simulation process and a stronger damping factor was introduced between iterations to avoid overflow. 


\subsubsection{Evaporating pressure}

In Table 5-1 the evaporating pressure remains almost the same for basic and AXE systems at a specific passenger temperature. It decreases slightly at high compressor speed but the magnitude is within measurement uncertainty. The evaporating temperature and compressor speed for the basic system has been correlated as

$$
t_{e}=t_{\text {pass }}-(790.32-0.077991 n) \dot{m}
$$

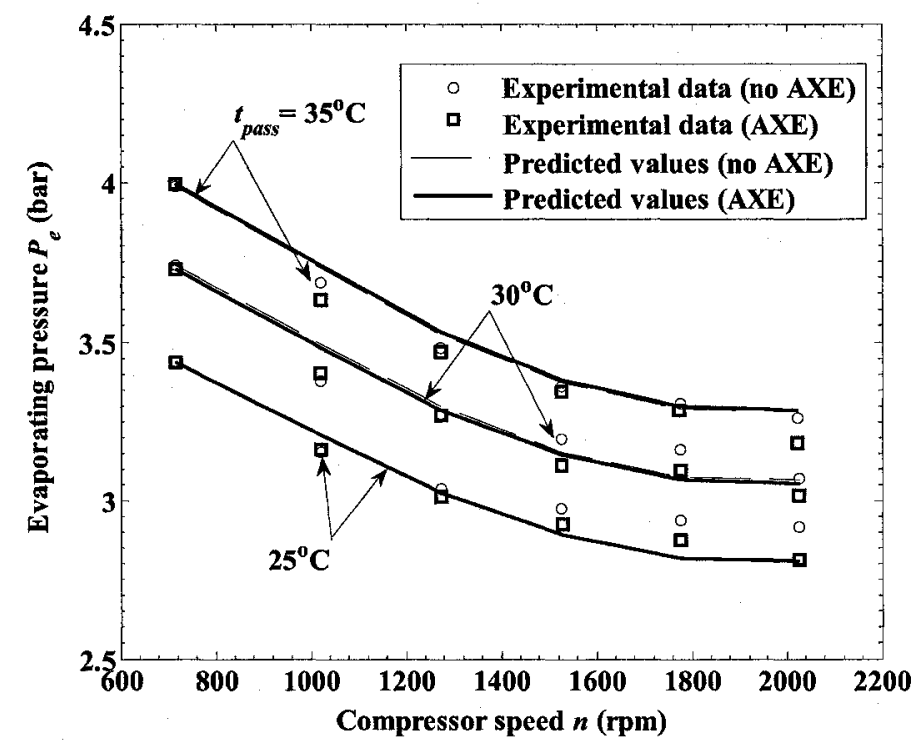

Figure 5-5 Evaporating pressure for systems with and without an AXE

Knowing $t_{e}$, the corresponding evaporating pressure can be easily determined. Figure 5-5 shows the prediction curves using correlation (5-9) for both systems. Fairly good agreement can be observed, and even better results are obtained for the AXE system than the basic system when $t_{\text {pass }}=25^{\circ} \mathrm{C}$. 


\subsubsection{Overall pressure loss factor and pressure drop over AXE}

The trend for evaporating pressure remains unchanged as shown in Figure 5-6, and hence the total change in pressure drop in the AXE system can be attributed to the change caused by heat transfer inside the enlarged capillary. The old correlation, as described below, is obviously not applicable here.

$$
t_{k}=t_{e}+\left(2182.7 \dot{m}-9881.1 \dot{m}^{2}\right)
$$

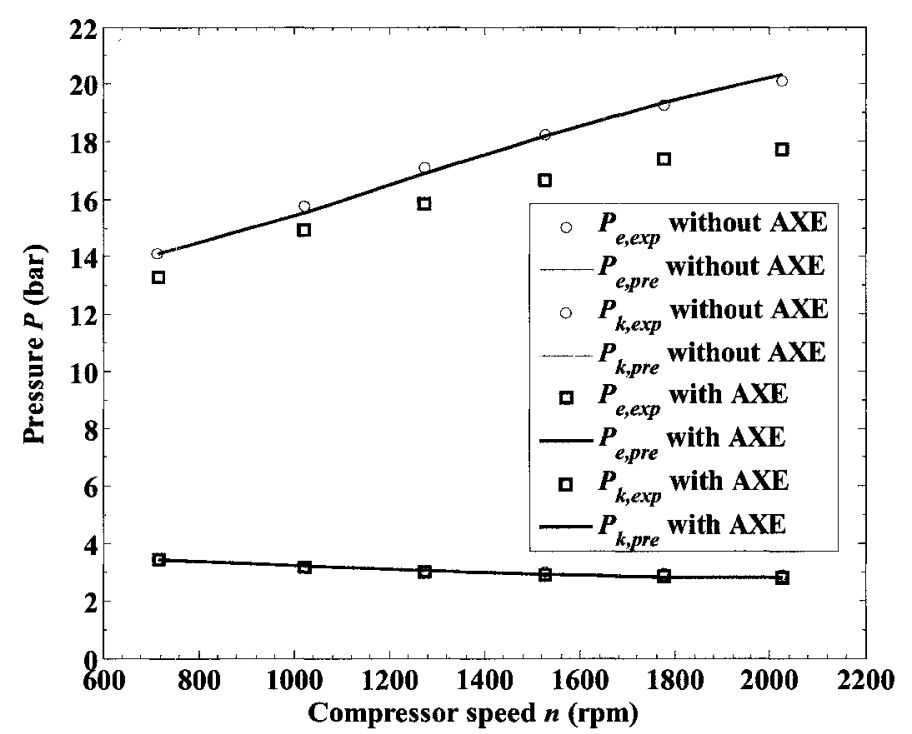

Figure 5-6 Prediction of pressures for systems with and without an AXE for $t_{p a s s}=25^{\circ} \mathrm{C}$ using correlation (5-7)

The prediction deviation can be seen in Figure 5-6. Because the same correlation is used for the systems with and without AXE, the prediction curves for the high-side pressure are identical. The same thing occurs for the low-side pressure. The maximum over prediction is found to be $2.60 \mathrm{bar}$ at $2000 \mathrm{rpm}$ which gives a $14.7 \%$ relative deviation.

To improve the prediction accuracy, the influence of heat transfer inside the capillary tube was taken into account because from a thermodynamic point of view it is the only factor that differs 
from the basic cycle. As heat is dissipated to the surrounding suction flow, the refrigerant becomes colder and denser, which slows down the flow velocity. The pressure drop reduces as well since it is proportional to the density and the velocity square. This can also be reasoned from Equation (5-1) as there is an inverse relation between pressure drop and density (with other parameters kept constant). The decreasing magnitude of the loss factor $F_{p}$ can thus be quantified as the decrease of pressure drop, which can be written as

$$
F_{p}=1-\frac{\Delta P_{A X E}}{\Delta P_{n o A X E}}
$$

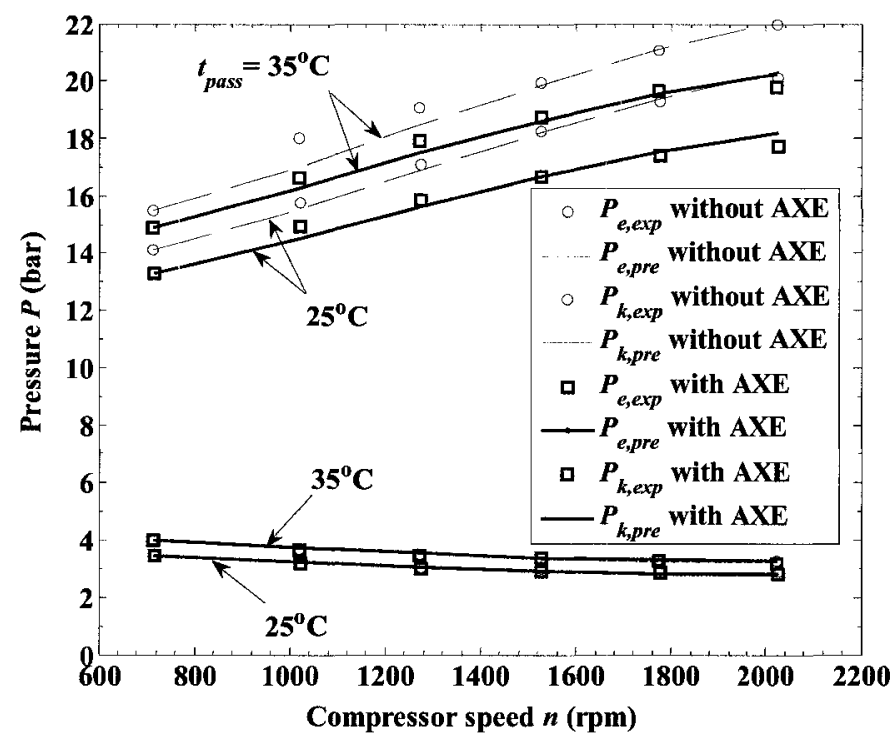

Figure 5-7 Prediction of pressures for systems with and without an AXE using correlation (5-7) and the improved Grebner's correlation

The new overall pressure loss factor becomes $F_{A X E}=F\left(1-F_{p}\right)$. Substituting this into Equation (5-1) one gets

$$
\Delta P_{A X E}=\frac{1}{2} F_{A X E} \rho V^{2}=\frac{F_{A X E}}{2 A^{2}} \frac{\dot{m}^{2}}{\rho}
$$

By performing a best-fit analysis on the experimental data, this friction change can be expressed as 


$$
F_{p}=0.0606718+1.3346683 \times 10^{-8} n^{2}
$$

Correspondingly, Eq. (9) will be modified to

$$
t_{k}=t_{e}+\left(0.9588878-4.1742367 \times 10^{-9} n^{2}\right)\left(2182.7 \dot{m}-9881.1 \dot{m}^{2}\right)
$$

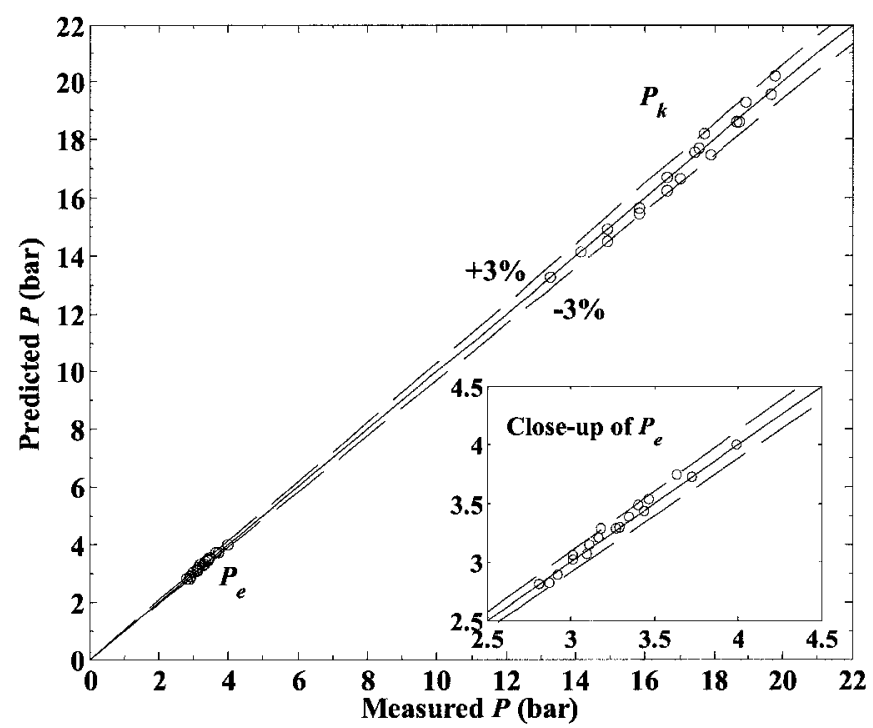

Figure 5-8 Comparison between predicted and measured pressures

Figure 5-7 is the pressure behaviour with changing compressor speeds for basic and AXE systems. Dashed lines were obtained using Equations (5-4), (5-9), and (5-10), while solid lines were obtained using (5-4), (5-9), and (5-14). A comparison between predicted and measured data is provided in Figure 5-8. Most of the simulated values are well kept within the boundary of $\pm 3 \%$ to the measured data except for one point which is offset by $3.1 \%$. 


\subsection{Influence of the compressor speed on system performance}

\subsubsection{Evaporation capacity}

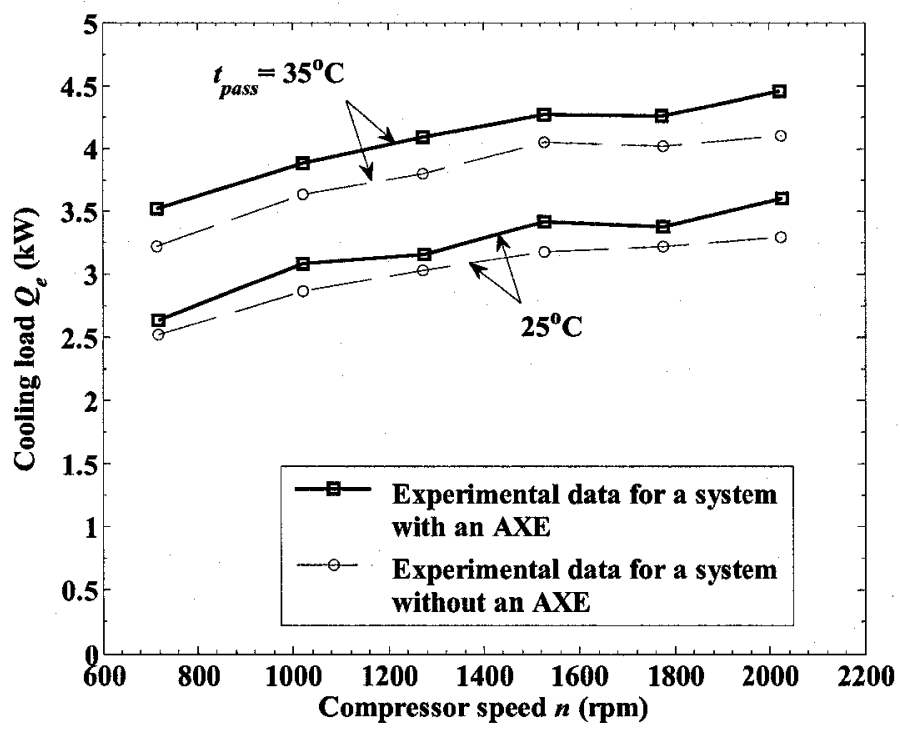

Figure 5-9 Cooling load for system with and without AXE

As a combined effect of unchanged mass flow rate and increased specific cooling capacity, the increase in cooling load is predictable. Figure 5-9 shows the increase for different passenger temperatures. The increase varies from $4.1 \%$ to $9.5 \%$ with an average of $6.8 \%$.

\subsubsection{Compressor power consumption}

The compressor requires less work input when an AXE is incorporated into the system. Figure 5-10 is a comparison of power consumption for systems with and without an AXE. There are two main reasons for the change. First, as mentioned above, the discharge pressure decreases (due to the reduction in friction), but the intake pressure varies little, leading to a smaller pressure ratio and thus better isentropic efficiency. Secondly, because of the heat transfer inside the AXE, the 
refrigerant quality is higher at the compressor inlet, meaning less liquid is involved in the compression process. According to Voster and Meyer (2000), an R134a-based system benefited from less liquid intake into the compressor, and the corresponding work consumption was cut down by $1.4 \%$ to $5.0 \%$ with an average of $2.9 \%$. At this point, the AXE outperforms traditionally used internal heat exchangers in its ability to lower power consumption. Though the magnitude is small, it demonstrates another merit of the novel device besides its advantages in keeping the system compact and simple.

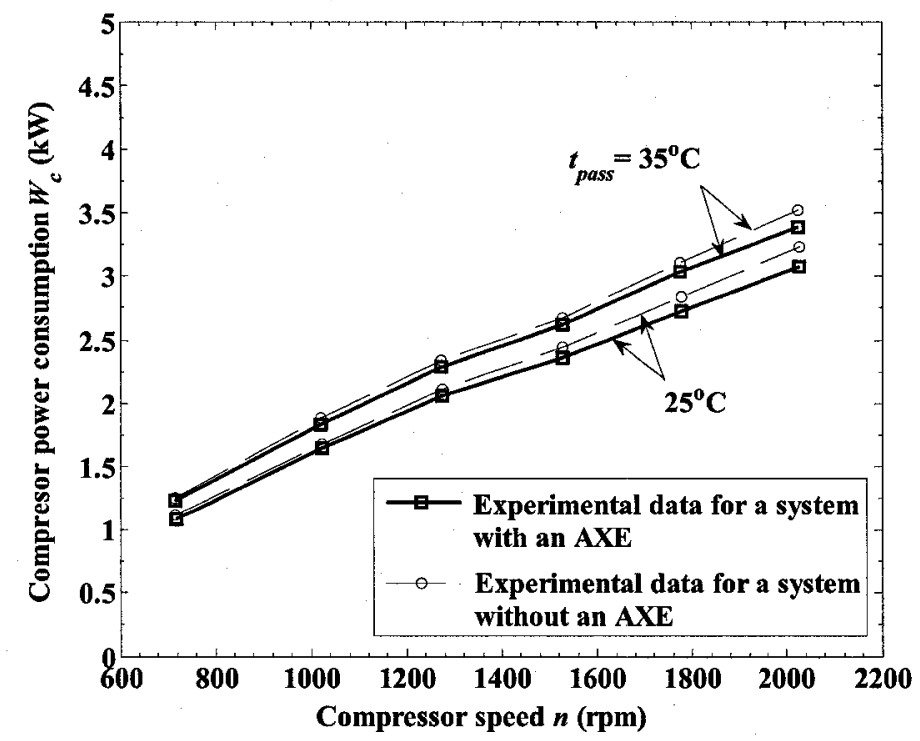

Figure 5-10 Compressor power consumption for system with and without AXE

\subsubsection{Coefficient of performance (COP)}

Better performance in both the evaporator and the compressor result in COP improvement.

Figure 5-11 details the increase, where the minimum is $4.5 \%$, the maximum $19.0 \%$, and the average 9.7\%. To get a better idea of the improvement, we can look at some statistical data for the U.S.A. In 
2000 , the amount of fuel that was used for mobile air conditioning in 213 million light duty vehicles was approximately 28 billion litres, which is equivalent to $6 \%$ of their domestic petroleum consumption (Johnson, 2002). Accordingly, 234.05 billion US dollars were spent for automotive air conditioning. Therefore, if AXE is adopted in vehicles, a huge amount of energy savings, equivalent to 22.70 billion dollars, will be achieved annually.

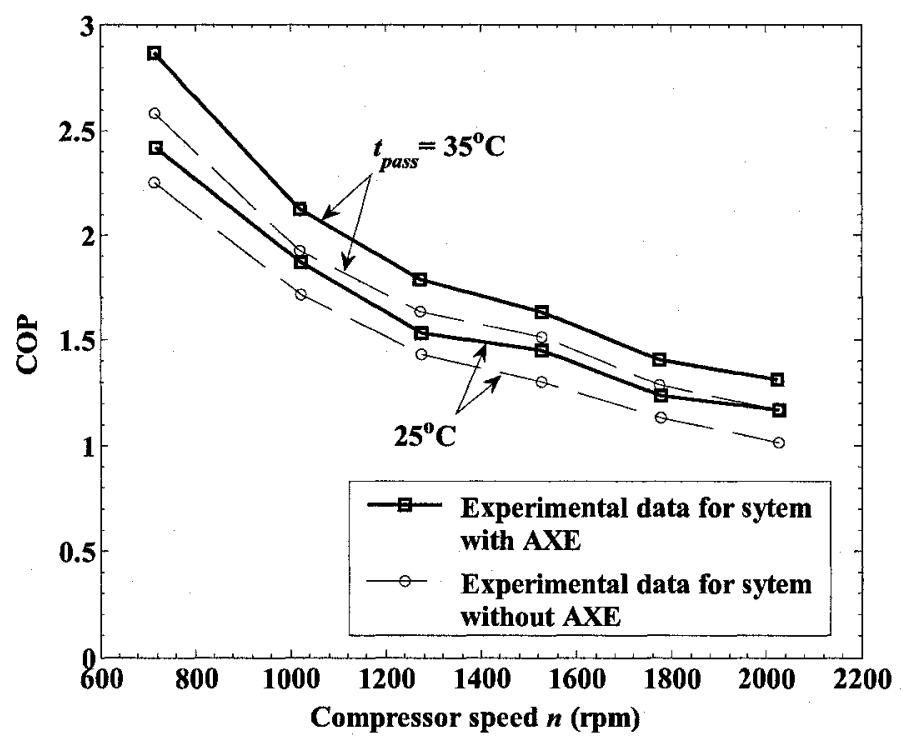

Figure 5-11 COP behavior for system with and without AXE

\subsubsection{Verification of the model NACAM}

Compared with the adiabatic expansion process as described in the previous chapter, the capillary fluid experiences a relatively low pressure drop along the same tube length due to increased heat transfer. In other words, the frictional resistance is less, allowing more refrigerant to pass through the capillary. To verify this, the NACAM was adopted to predict the mass flow rates under different conditions and compare them to measured data. The results are shown in Figure 
5-12, where a fairly good agreement can be observed for the compressor speed range, with an average over prediction of 3.5\%. The over prediction grows at low compressor speeds and/or low passenger temperatures. The maximum deviation is found to be $12.1 \%$ with $n=716 \mathrm{rpm}$ and $t_{\text {pass }}=25^{\circ} \mathrm{C}$.

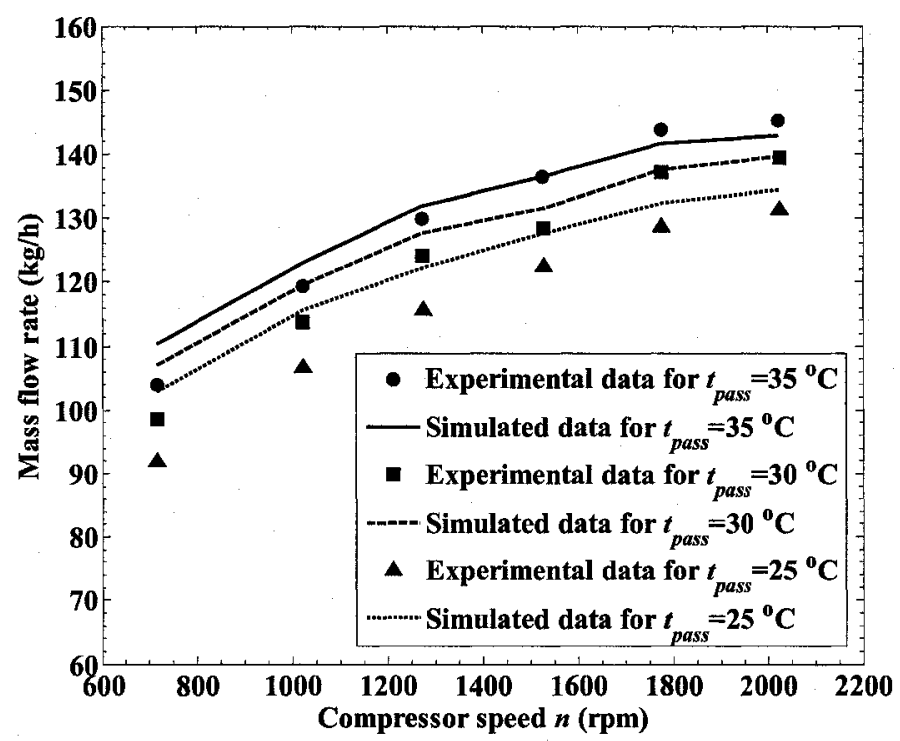

Figure 5-12 Comparison of data by experiments and NACAM

The global over prediction might be caused by the inner diameter, as discussed in section 4.3.4, and thus the true inner diameter should be a little smaller than the one substituted into the model. At low compressor speeds, less heat was transferred to the suction line flow than was predicted, causing the change of pressure drops not significant compared to the adiabatic case (as shown in Figure 5-7). Thus only a slight mass flow rate increase in the AXE system was measured. At low passenger temperatures, the difference between the capillary exit pressure and the evaporating pressure increases and more energy is dissipated through the shock wave, which might dampen the increase of the mass flow rate. Such an effect was not considered in NACAM, so over prediction 
occurred. Overall, NACAM is proven to be a useful model in predicting non-adiabatic flow rates.

Compared to Figure 4-14, the mass flow rate of NACAM is greater than that of ACAM when the compressor speed $n$ is low, and slightly smaller than the ACAM value when $n$ is high. It seems that the statement at the beginning of this section (more refrigerant passes through the capillary in NACAM than in ACAM) does not hold for the whole parameter range. But the phenomenon can be attributed to a change made to the capillary. For the adiabatic test, it was straight; for the non-adiabatic case, it was folded so as to fit the size of the AXE. This sacrifice was necessary for a compact device; from test results it was proven to be worthwhile. Accordingly, the equations (5-4), (5-9), and (5-14) will be generally applicable for the AXE with a similar folded configuration.

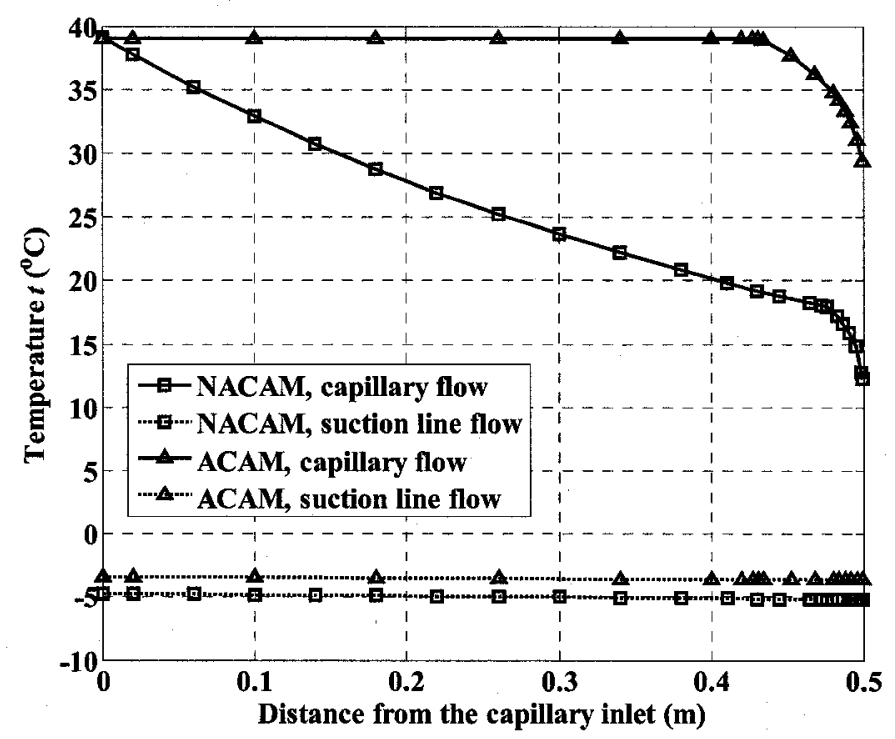

Figure 5-13 Temperature profile of NACAM and ACAM flow with $l_{\text {capi }}=0.50 \mathrm{~m}, \varepsilon=0.00150$ $\mathrm{mm}, d_{i}=1.65 \mathrm{~mm}, d_{i, \text { outer }}=12.70 \mathrm{~mm}$, and $n=1526 \mathrm{rpm}$.

NACAM: $\quad \dot{m}=131.4 \mathrm{~kg} / \mathrm{h}, P_{k}=16.32$ bar, $t_{\text {capi } i n}=39.20^{\circ} \mathrm{C}, t_{e}=-4.72{ }^{\circ} \mathrm{C}$, and $x_{s, j i n}=0.81$;

ACAM: $\quad \dot{m}=133.1 \mathrm{~kg} / \mathrm{h}, P_{k}=18.30 \mathrm{bar}, t_{\text {capj } i n}=39.09^{\circ} \mathrm{C}, t_{e}=-3.41^{\circ} \mathrm{C}$, and $x_{s, i n}=0.76$.

As discussed in section 3.2.2.1.1, if the specific heat transfer rate is too high, the refrigerant 
will go back and forth between the two-phase region and the single-phase region. Such so called re-condensation (Bansal and $\mathrm{Xu}, 2003$, Bansal and Yang, 2005) will cause instabilities. In our experiments, the refrigerant flow entered the capillary as a sub-cooled liquid and left as a two-phase mixture. This can be explained as the results of a proper combination of the capillary size and operation conditions. Figure 5-13 shows a typical temperature profile of the AXE flow and compares it with ACAM flow. As expected, the non-adiabatic flow enters the two-phase region later; the average temperature difference is smaller; and a greater pressure drop is achieved by friction.

\subsubsection{Feasibility of determining AXE effectiveness}

From the pressure decrease factor, $F_{p}$, the slope of process 3"-4' (Figure 3-2) can be obtained using a "Quasi-Triangular Rule". In Figure 3-2 line 3-4 represents a throttling process in a basic cycle and line 3" -4 ' is for the throttling/heat dissipating process in the AXE cycle. The length of 3-4 can be regarded as the length of the capillary because each point on the line has a unique corresponding distance from the capillary inlet. Similarly, the length of 3"-4' is also the length of the capillary tube of the AXE. In our experiments, the same capillary tube was adopted in both systems. Even though the relationship of the two tubes varies along the capillary flow direction, it can be expressed by a nonlinear factor $C_{p}$, where $0<C_{p}<\frac{1}{F_{p}}$. When $C_{p}=1$, two capillaries have a direct relationship. For a throttling process without choking, the temperature difference at both sides of capillary decreases along the inside flow direction, which leads to a decreased $C_{p}$. In the two-phase region, the change rate is greater. Near the capillary outlet, $C_{p}$ behaves asymptotic to $\frac{1}{F_{p}}$, 
which makes line 3"-4' vertical in Figure 3-2. From Equation (5-13) we know the proportion of $3-3$ " over 3-4; thus the slope of 3"-4' can be defined as

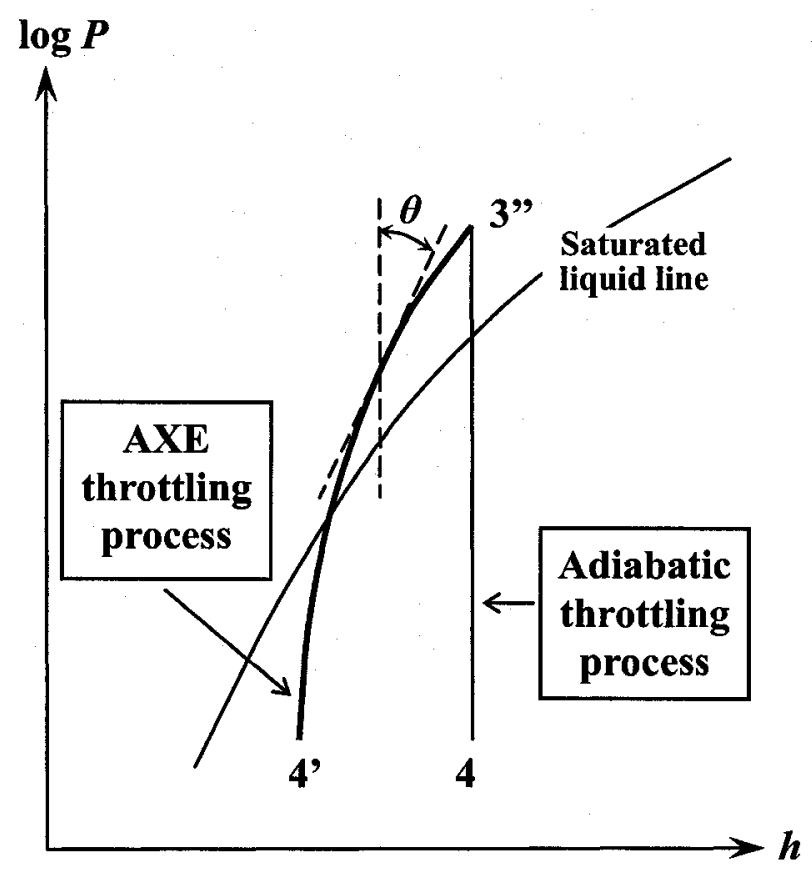

Figure 5-14 Illustration of throttling process with heat transfer in AXE

$k=\tan \left[\cos ^{-1}\left(1-C_{p} F_{p}\right)\right]$

Since $C_{p}$ varies along the capillary due to influence of local heat flux, it would be better if the analysis could be simplified. To do this, a finite element $d l$, located at a distance $l$ from the capillary inlet, is taken into account. Angle $\theta$, as shown in Figure 5-14, can be expressed as

$\cos \theta=\left(1-F_{p}\right)\left(1-\frac{l}{L}\right)$

where $L$ is capillary length. Then the averaged angle $\bar{\theta}$ can be derived as

$\cos \bar{\theta}=\frac{1}{L} \int_{0}^{L} \cos \theta d l=\frac{1-F_{p}}{L} \int_{b}^{L}\left(1-\frac{l}{L}\right) d l=\frac{1}{2}\left(1-F_{p}\right)$

Then the slope of process 3 "'-4' is approximated by an average 
$\bar{k}=\tan \left[\cos ^{-1}\left(\frac{1-F_{p}}{2}\right)\right]$

After knowing $k$, the enthalpy at point 4' can be determined. From heat balance, the power consumption of the heating fan in passenger chamber equals the cooling capacity. Now point 1 is computable. From the measured quality, pressure, and temperature at the compressor inlet, point 1' is known. Therefore, all the inlet and outlet information of AXE are available and the relative effectiveness can be calculated thereafter.

\subsection{Conclusions}

1. A correlation that describes the influence of compressor speed on system pressure behaviour for systems with AXEs has been developed with prediction deviation no more than $3.1 \%$.

2. Mass flow rate slightly increases with the presence of an IHX but with a lesser extent than simulation suggests.

3. New coefficients for Grebner's correlation were developed to improve the prediction accuracy for thermophysical properties of an R134a-PAG oil mixture.

4. The pressure drop across the AXE is smaller compared with that of an adiabatic throttling process. The decrease embodies the effect of lower friction due to heat exchange of the IHX. In other words, pressure drop per unit length of capillary tube goes down with increased heat transfer between the high-side in-tube flow and the low-side suction line flow. A factor $F_{p}$ is proposed to quantify the magnitude of the decrease.

5. High-side pressure decreases with the presence of AXE, and the trend can be described through $F_{p}$. Low-side pressure does not change much with the presence of AXE. Compared 
with the correlations in the previous chapter, where higher pressure drop corresponding to higher compressor speed will be distributed to the high-side and the low-side based on a correlation, the introduced AXE only affects the high-side pressure behaviour. In other words, mass flow rate affects both sides; heat transfer induced variation in pressure loss affects the high-side only.

6. By introducing the AXE into a refrigeration system, the cooling load slightly increases due to the increase of specific cooling capacity, while compressor power consumption is reduced due to the lower compression ratio and less liquid slugging. As a result, the COP is increased by $4.5 \%$ to $19.0 \%$; an average of $9.7 \%$.

7. A method has been proposed to use $F_{p}$ to determine the effectiveness of AXE by applying a "Quasi-Triangular Rule".

8. NACAM is proven to be a valid model in predicting refrigerant flow rate by an averaged $3.5 \%$ over prediction. The uncertainty increases when the passenger temperature and/or the compressor speed are low. 


\section{Chapter 6}

\section{Non-adiabatic capillary flow in transcritical $\mathrm{CO}_{2}$ cycles}

In recent years, the pursuit of environmentally-friendly refrigerants has caused CFC and HCFC refrigerants to gradually fade from use in the refrigeration industry. Many alternatives, such as R600a and R290, are being studied. Research interests in this field turn to fluids with a low GWP (Global Warming Potential) with particular attention given to carbon dioxide, which is also known as $\mathrm{R} 744$, as a candidate for refrigeration applications. As mentioned in the first chapter, $\mathrm{CO}_{2}$ exists naturally and is a by-product of many processes such as the production of beer or $\mathrm{NH}_{3} \cdot \mathrm{CO}_{2}$ is not produced for use as a refrigerant; it is captured from industrial processes, and then refined for use in refrigeration systems. Because of the lack of special production efforts, the overall volume of $\mathrm{CO}_{2}$ gas in the atmosphere does not change, provided that the by-product is also released or not sequestered in some way. Therefore, using $\mathrm{CO}_{2}$ as a refrigerant will not affect global warming. In addition to its environmental advantages, $\mathrm{CO}_{2}$ has attractive thermal characteristics that make it a viable alternative refrigerant.

Similar to what was discussed in previous chapters, an AXE can also be introduced into transcritical systems to improve the system performance, though the mechanism of the new system needs to be examined.

Many experimental and numerical studies on the fluid flow within capillary tubes have been performed. Some of them were dedicated to adiabatic processes (Bansal and Rupasinghe, 1998; 
Bansal and Wang, 2004; Zhang and Ding, 2004; Trisaksri and Wongwises, 2003), or refrigerants that will be replaced in the near future (Bansal and $\mathrm{Xu}, 2003$; Wongwises and Suchatawut, 2003). However, not much information on the basic physics governing the capillary tube flow with large inner diameter (between 1.5-2.5 mm) using $\mathrm{CO}_{2}$ as working fluid is available in the open literature. This chapter is intended to present a fundamental examination of the mechanism of capillary heat exchange and the flow behaviour in a transcritical cycle. A numerical model based on conservative laws of mass, momentum and energy is developed for this purpose. Detailed flow characteristics of $\mathrm{CO}_{2}$ in-tube flow are analyzed based on simulation results.

\subsection{A new transcritical refrigeration cycle}

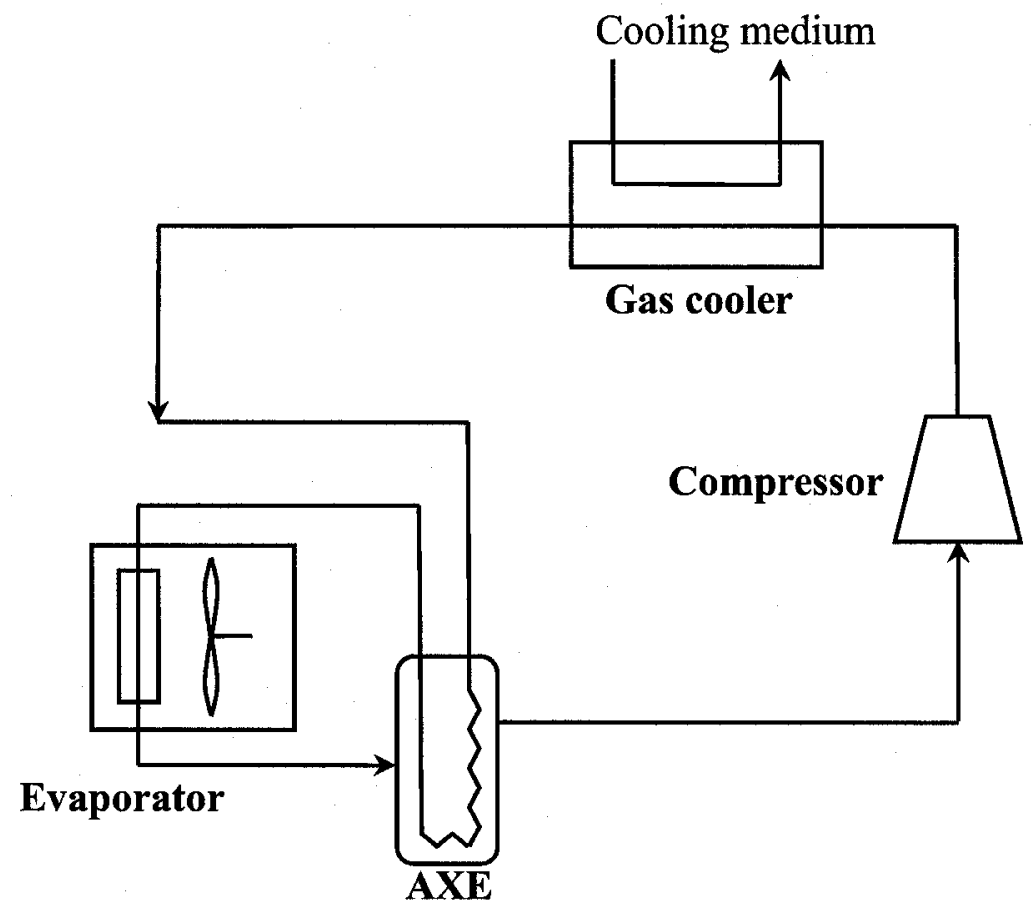

Figure 6-1 Schematic of a transcritical AAC system with AXE

Figure 6-1 is an illustration of a transcritical AAC system. The only difference between this and an R134a-based cycle is that a gas cooler other than a condenser is used. Because the discharge state of 
the refrigerant falls into the supercritical region, there is no condensation occurring during the cooling process, hence the title of "gas cooler". Because of this new feature, the high side pressure is not determined by the ambient temperature, and a question about the optimum discharge pressure (which will be detailed in the following chapter) thus arises.

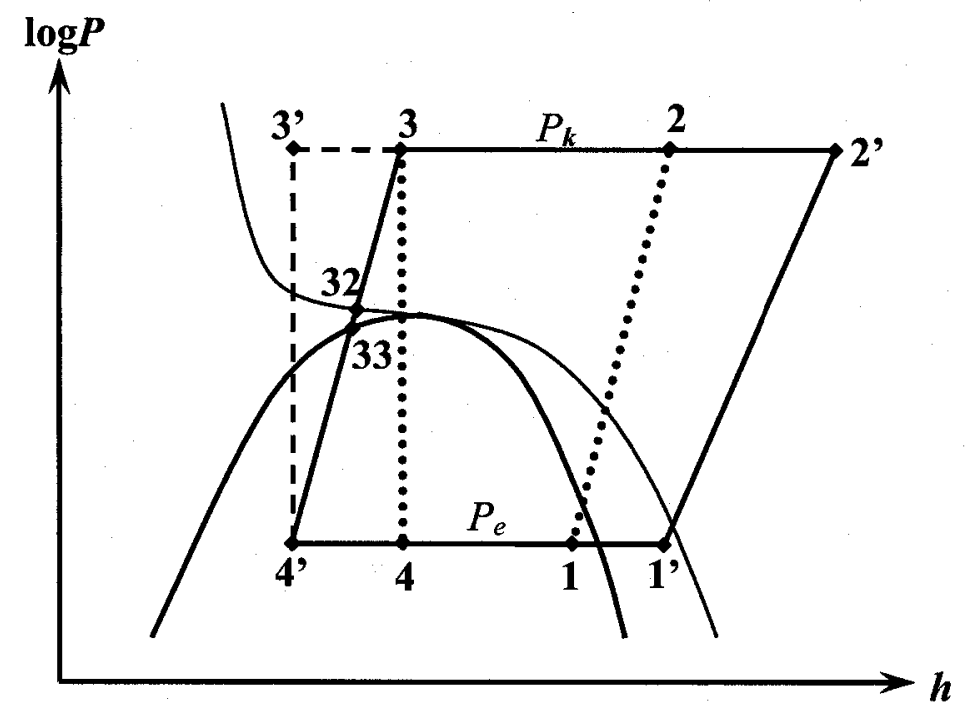

Figure 6-2 Pressure-enthalpy diagram of a $\mathrm{CO}_{2}$ transcritical refrigeration cycle

Figure 6-2 is the corresponding $\log P-h$ diagram of the transcritical cycle. 1-2-3-4-1 is a traditional loop without an internal heat exchanger. Because the critical temperature of $\mathrm{CO}_{2}$ is only around $31^{\circ} \mathrm{C}$, the specific cooling capacity $q_{0}$, which is represented by line $4-1$, is very small, yielding a relatively low COP. To improve the cycle efficiency, loop $1^{\prime}-2^{\prime}-3^{\prime}-4^{\prime}-1^{\prime}$ (with an internal heat exchanger) has been widely applied in automotive air conditioners recently (Pettersen et al., 1998). Although the compression work $w_{0}$ increases because of more superheating, the increase of $q_{0}$ is much greater. High pressure refrigerant from the gas cooler exchanges heat with low pressure refrigerant from the evaporator (processes 3-3' and 1-1' respectively) and then goes into the expander where it is throttled to a low pressure, low temperature and two-phase state. 
In this chapter, a theoretical model of the transcritical AXE flow will be established. A preliminary discussion will be carried out with a constant heat transfer coefficient in the suction line flow. Next, the inlet conditions of the suction line flow will be given and then the capillary flow characteristics will be investigated.

\subsection{Model description}

\subsubsection{Assumptions}

In order to simulate the real flow behaviour without losing the main physical characteristics, the following assumptions are made:

- One-dimensional flow.

- Horizontally placed capillary tube.

- Steady state homogeneous flow.

- Incompressible flow in single-phase region.

- Constant inner diameter and uniform surface roughness throughout the tube.

- Counter flow heat exchanger; the capillary is put in the vapour region of accumulator.

- Pure refrigerant (oil is not considered).

\subsubsection{Governing equations}

The non-adiabatic capillary tube discussed in this chapter is schematically shown in Figure 6-3. The capillary tube can be divided into three distinct sections according to the flow status: namely the supercritical flow region, the transcritical flow region and the subcritical two-phase flow 
region. For saturated two-phase flow, an extreme status may exist at the outlet when the back pressure is below a given threshold that is determined by the tube size and inlet conditions. This possible critical section is also shown in the figure. The total capillary length may be written as

$$
L=L_{\text {sup }}+L_{l i q}+L_{t p}
$$

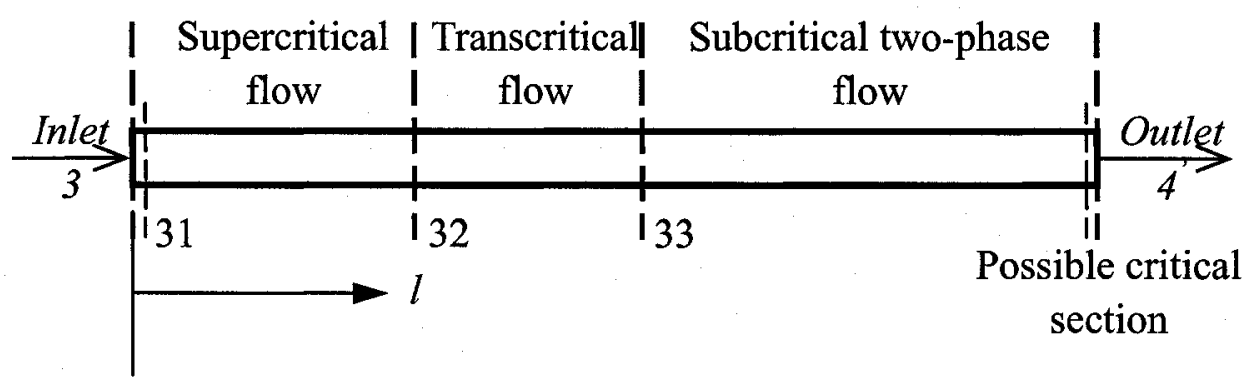

Figure 6-3 Non-adiabatic capillary flow in a transcritical cycle

Because the throttling process crosses the saturated curve (either saturated liquid or saturated vapour) near the critical point (as can be seen in Figure 6-2), the tube length corresponding to liquid flow is so small that it can be omitted. In fact, the status of the refrigerant in this region is very close to that of a high-density gas, so they can be treated the same without losing accuracy. Therefore Equation (6-1) can be written as

$$
L=L_{s p}+L_{t p}
$$

\subsubsection{Energy balance equation}

The energy conservation equation for a section of capillary tube of arbitrary length $d l$ is the same as Equation (3-27)

$$
h+\frac{1}{2} V^{2}=(h+d h)+\frac{1}{2}(V+d V)^{2}+d q
$$

where the specific heat transfer rate is 


$$
d q=\frac{U A_{i}\left(T_{i}-T_{\text {outer }}\right)}{\dot{m}}
$$

Substituting the above equation and $V=g v$ (Equation (3-5)) into Equation (6-3) gives

$$
d h+g^{2} v d v+\frac{4 U A_{i}\left(T_{i}-T_{\text {outer }}\right)}{g \pi d_{i}^{2}}=0
$$

The overall thermal conductance is thus

$$
U A_{i}=\frac{d l}{\frac{1}{\alpha_{i} \pi d_{i}}+\frac{1}{2 \lambda_{c a p i} \pi} \ln \frac{d_{o}}{d_{i}}+\frac{1}{\alpha_{o} \pi d_{o}}}
$$

\subsubsection{Momentum balance equation}

The momentum balance equation is the same as that obtained for a subcritical system, and Equation (3-7) is thus used in this study. For different flow regions, the friction factor is determined differently, which will be discussed separately in the following section.

\subsubsection{Single-phase region}

In momentum equation, the hydraulic drag factor $f_{h}$ is expressed as (Petrov and Popov, 1985)

$$
f_{h}=f_{s p}=f+f_{i}
$$

where the one-dimensional inertia factor can be expressed as

$$
f_{i}=\frac{8}{g c_{p}}\left[\frac{1}{v}\left(\frac{\partial v}{\partial T}\right)_{p}\right] d Q
$$

As discussed in section 3.1.1.3, Churchill's correlation (Churchill, 1977) was adopted to calculate the Darcy-Weisbach friction factor $f$. 
At supercritical status, thermophysical property variations greatly affect the pressure drop characteristics (Fang et al., 2001). During a cooling process, the absolute value of the inertia drag, which is negative, decreases the total hydraulic drag to a negative value so that the pressure may increase along the tube. Thus this inertia drag should be included in Equation (6-7).

Due to the drastic change of thermodynamic properties during the process $3-4$ ', the refrigerant flow velocity in the capillary varies greatly, so every term in Equation (6-5) should be kept.

Slightly different from section 3.2.1.2, the Petukhov-Kirillowv correlation was adopted to calculate the heat transfer coefficient $\alpha_{i}$ of the single-phase flow (Fang et al, 2001), which is given by

$$
\frac{\alpha_{i} d_{i}}{\lambda_{i}}=N u=\frac{(f / 8) \operatorname{Re} \operatorname{Pr}}{1.07+12.7(f / 8)^{1 / 2}\left(\operatorname{Pr}^{2 / 3}-1\right)}
$$

\subsubsection{Two-phase region}

In this region, the hydraulic friction factor is expressed as [18]

$$
f_{h}=f_{t p}=\phi_{t p}^{2} f\left(\frac{v_{s p}}{v_{t p}}\right)
$$

Here $f$ is obtained using Equation (3-8), and the multiplier $\phi_{t p}^{2}$ is calculated by

$$
\phi_{t p}^{2}=\left[\frac{\left(\frac{8}{\operatorname{Re}_{t p}}\right)^{12}+\left(A_{t p}^{16}+B_{t p}^{16}\right)^{-\frac{3}{2}}}{\left(\frac{8}{\operatorname{Re}_{s p}}\right)^{12}+\left(A_{s p}^{16}+B_{s p}^{16}\right)^{\frac{3}{2}}}\right]^{\frac{1}{12}}\left[1+x\left(\frac{v_{v}}{v_{l}}-1\right)\right]
$$


Different from the method used in 3.2., where $f_{i}$ and $f$ are calculated separately, $f_{h}$ is obtained by an empirical modification of $f$.

As for the heat transfer coefficient of the two-phase capillary flow, the main heat transfer resistance lies on the suction line side, so it could be regarded as infinite without losing accuracy (Xu and Bansal, 2002). For the suction line flow, it will be fixed at $400 \mathrm{~W} /\left(\mathrm{m}^{2} \cdot \mathrm{K}\right)$ in case 1 , and then the Steiner-Taborek's correlation (1992) will be used for case 2.

$$
\alpha_{t p}=\left[\left(F \alpha_{l}\right)^{3}+\left(S \alpha_{p}\right)^{3}\right]^{\frac{1}{3}}
$$

where $\alpha_{l}$ represents the local forced convective heat transfer coefficient (for liquid only) and can be calculated using Equation (6-9). $\alpha_{p}$ is the standard normalized nucleate pool boiling coefficient. The following equations are adopted to calculate the parameters in (6-12).

$$
\begin{aligned}
& \alpha_{p}=F_{p f}\left[\frac{\varphi}{\varphi_{0}}\right]^{n f}\left[\frac{d_{i}}{d_{0}}\right]^{-0.4}\left[\frac{\varepsilon}{\varepsilon_{0}}\right]^{0.133} F(M) \\
& F_{p f}=2.816 \operatorname{Pr}^{0.45}+\left[3.4+\frac{1.7}{1-\operatorname{Pr}^{7}}\right] \operatorname{Pr}^{3.7} \\
& n f=0.8-0.1 \exp (1.75 \operatorname{Pr}) \\
& F(M)=0.377+0.199 \ln (M)+0.000028427 M^{2} \\
& F=\left[(1-x)^{1.5}+1.9 x^{0.6}\left(\frac{v_{v}}{v_{l}}\right)^{0.35}\right]^{1.1}
\end{aligned}
$$

\subsubsection{Judgment of extreme flow status}

As discussed in section 3.2.1.4, for in-tube two-phase flow, an extreme flow status may occur corresponding to a specific condition when the back pressure is lower than a critical value. In this 
case, the local sonic velocity is adopted as a criterion for the critical situation, which gives

$$
V_{\text {sonic }}=\sqrt{\left(\frac{\partial P}{\partial \rho}\right)_{s}}=v_{m i x} \sqrt{-\left(\frac{\partial P}{\partial v_{\text {mix }}}\right)_{s}}
$$

Along the flow direction, the pressure and temperature of the capillary fluid decrease, while the quality increases. This causes the flow velocity $V$ to increase. When $V=V_{\text {sonic }}$, the extreme situation occurs and relative critical parameters can be obtained via iterative calculations.

\subsection{Case studies}

A program has been developed in MATLAB based on the governing equations outlined above. The required refrigerant properties, including thermophysical and transport properties, were taken from CoolPack version 1.46, a freeware developed by the Department of Mechanical Engineering, Technical University of Denmark.

\subsubsection{Case 1: Constant surrounding heat transfer coefficient}

A typical set of simulation results is shown in Figure 6-4 for $\alpha_{\text {outer }}=400 \mathrm{~W} /\left(\mathrm{m}^{2} \cdot \mathrm{K}\right)$. To further the investigation, the relationships between different parameters, such as capillary length, capillary diameter, cooling pressure, evaporating pressure, cooling capacity and vapour side heat transfer coefficient, are considered separately. These parameters could be categorized into geometric or thermophysical factors. One or two factors were varied at a time, and their effects were quantified. Unlike Chapter 3 where the mass flow rate is the major parameter under investigation, in this chapter other parameters, such as capillary length, cooling load and entropy will be addressed so as to diversify the discussion. 


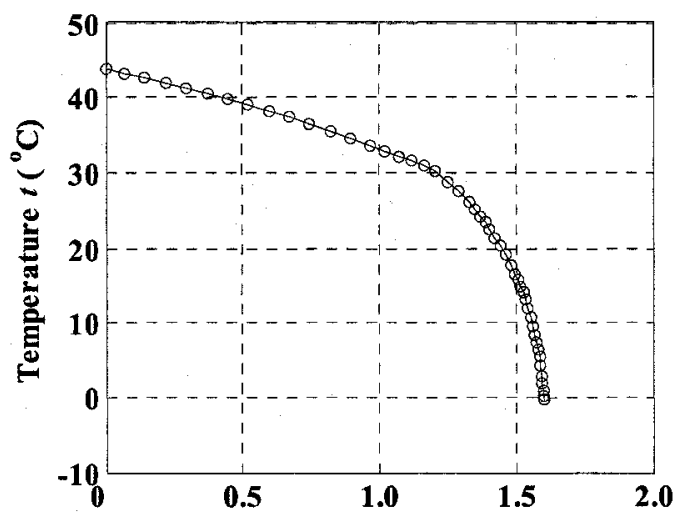

(a) Distance from capillary inlet (m)

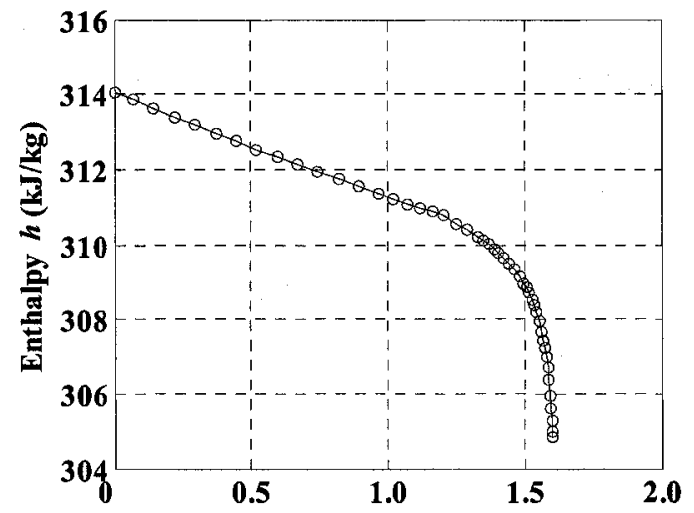

(c) Distance from capillary inlet (m)

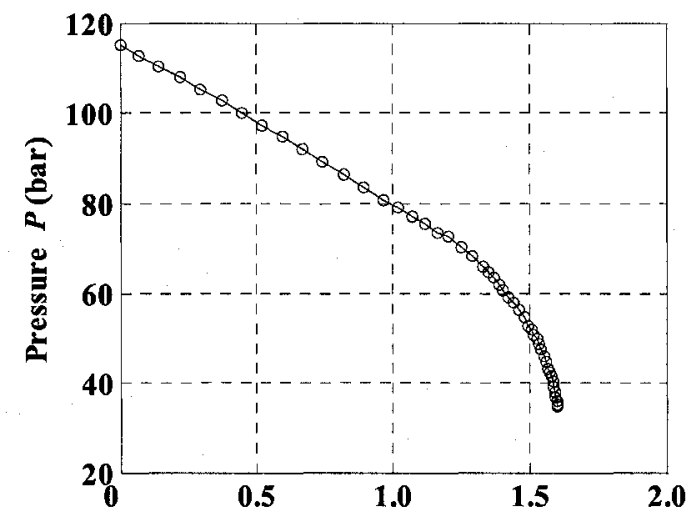

(b) Distance from capillary inlet (m)

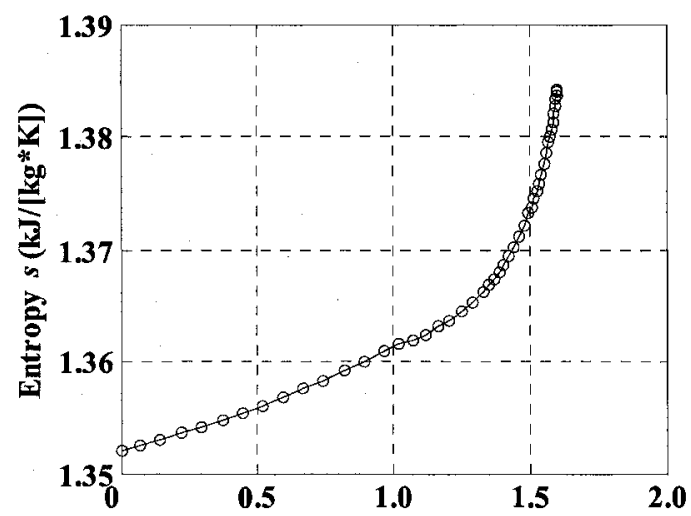

(d) Distance from capillary inlet (m)

Figure 6-4 Simulation results along capillary tube for $\mathrm{CO}_{2}$ with $Q_{e}=7 \mathrm{~kW}, P_{k}=120$ bar, $t_{\text {capi,in }}=45^{\circ} \mathrm{C}, t_{e}=0^{\circ} \mathrm{C}, d_{i}=1.85 \mathrm{~mm}$, and $\varepsilon=0.0015 \mathrm{~mm}$

\subsubsection{Inner diameter}

Like section 3.1.2.2, five different diameters were used at two different cooling pressures in the simulation; the results are shown in Figure 6-5. As the diameter increases, the capillary length also increases to maintain a certain pressure drop required by the cycle. This is because the larger diameter causes a slower fluid flow and a smaller pressure drop so that a longer distance is required to achieve the same pressure drop. This trend becomes more apparent as $d_{i}$ becomes larger, which means the throttling effect of the capillary decreases rapidly. As the pressure difference grows, 
longer capillary length is needed, which can also be seen by comparing two curves at fixed values of $d_{i}$.

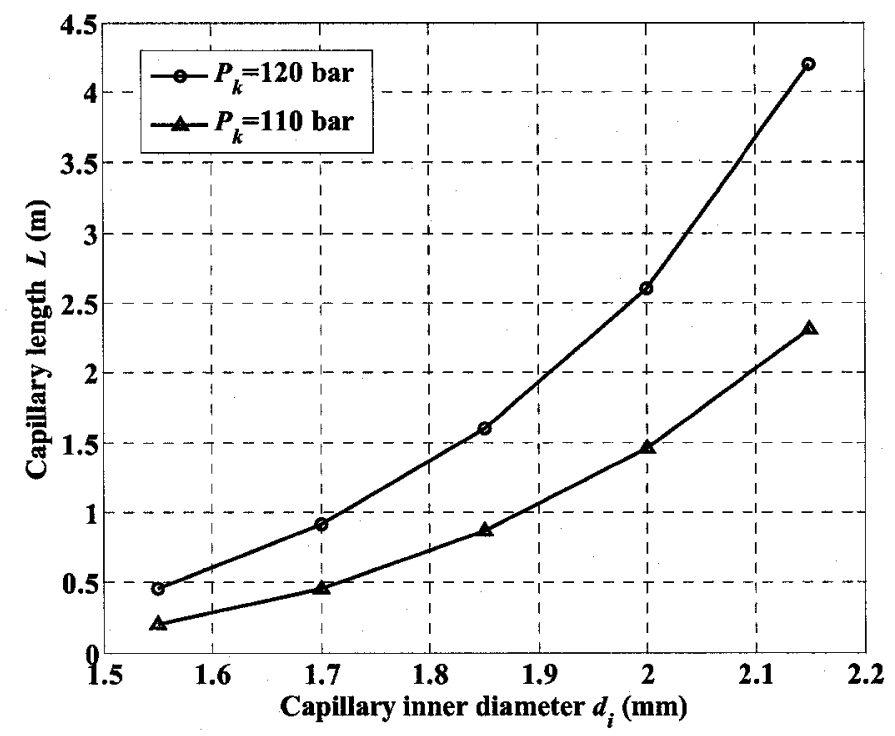

Figure 6-5 Capillary length vs. capillary inner diameter under different cooling pressures for $\mathrm{CO}_{2}$ with $Q_{e}=7 \mathrm{~kW}, t_{c a p i, i n}=45^{\circ} \mathrm{C}, t_{e}={ }^{\circ} \mathrm{C}$, and $\varepsilon=0.0015 \mathrm{~mm}$

\subsubsection{Cooling pressure}

For a transcritical $\mathrm{CO}_{2}$ cycle, due to manufacturing considerations, high pressures will create great challenges in material strength and operational safety. Moreover, it will reduce the system COP. On the other hand, if the cooling pressure is low, the pressure ratio will be low, which will weaken the compressor performance. Because of the restriction of the low critical temperature, the discharge pressure rarely goes under the critical value. Based on these considerations, the cooling pressure should also be kept within a specific range. Figure 6-6 shows the relationship between cooling capacity and capillary length at different cooling pressures.

Longer length $L$ is needed for higher cooling pressure while keeping $Q_{e}$ the same. This is reasonable because the pressure drop per unit length does not change in this case, so a larger 
pressure drop requires a longer capillary tube. On the other hand, if capillary length is fixed, cooling capacity increases as cooling pressure goes up. From Figure 6-2, it can be seen that increasing the distance between points 1 and 4 ' on the cooling curve, by means of increasing the high side pressure in the system, results in increases specific cooling capacity $q_{0}$. And from the momentum equation, larger pressure drop means higher mass flow rate. Therefore, $Q_{e}$ increases as a result of these two combined forces.

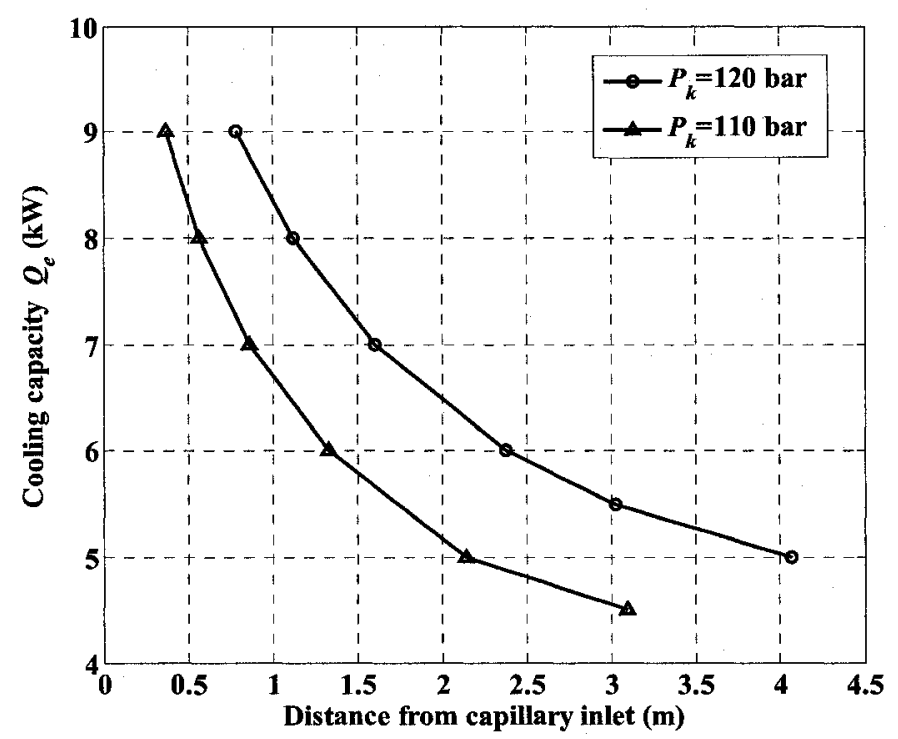

Figure 6-6 Cooling capacity vs. capillary length for $\mathrm{CO}_{2}$ with $d_{i}=1.85 \mathrm{~mm}, t_{\text {capi, in }}=45^{\circ} \mathrm{C}$, $t_{e}=0{ }^{\circ} \mathrm{C}$, and $\varepsilon=0.0015 \mathrm{~mm}$

\subsubsection{Evaporating pressure}

In the two-phase region, at a given pressure the corresponding refrigerant temperature can be found with its boiling-point relationship. Just as discussed in the previous subsection, the pressure difference directly affects the capillary length. With $Q_{e}$ unchanged, a lower evaporating temperature, corresponding to a larger pressure difference $\Delta P$, means longer length $L$. Different from traditional refrigerants, the specific cooling capacity of $\mathrm{CO}_{2}$ is larger at lower $t_{e}$ than a higher $t_{e}$, so $Q_{e}$ 
increases while the evaporating temperature decreases. The relationship between cooling capacity and capillary length under different $t_{e}$ is shown in Figure 6-7.

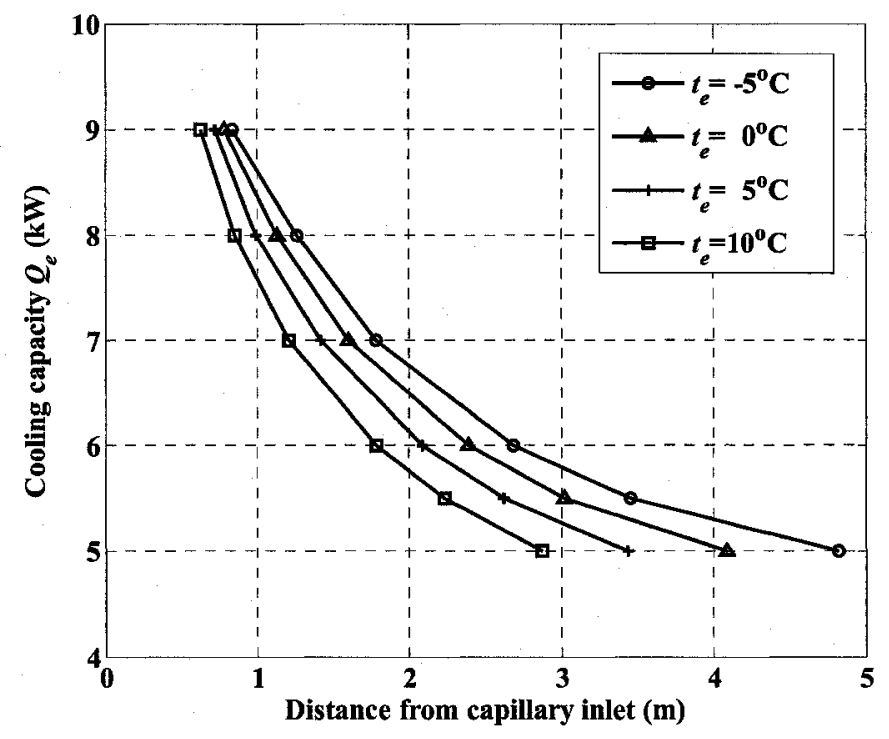

Figure 6-7 Cooling capacity vs. evaporating temperature for $\mathrm{CO}_{2}$ with $Q_{e}=7 \mathrm{~kW}, P_{k}=120 \mathrm{bar}$, $d_{i}=1.85 \mathrm{~mm}, t_{c a p i, i n}=45^{\circ} \mathrm{C}$, and $\varepsilon=0.0015 \mathrm{~mm}$

\subsubsection{Surface roughness of the capillary tube}

Roughness greatly affects the longitudinal friction factor; from the momentum equation it can be seen that $A$ decreases with coarser surfaces, making $f$ increase. A series of typical quality-capillary length curves are shown in Figure 6-8. As $\varepsilon$ is varied from $0.5 \mu \mathrm{m}$ to $2.0 \mu \mathrm{m}$, the resulting capillary length is shortened by $27 \%$, from $2.041 \mathrm{~m}$ to $1.492 \mathrm{~m}$. This means the capillary length is sensitive to the tube's internal surface conditions. A minor flaw in manufacturing or assembly could greatly influence the performance. Another notable phenomenon is that the quality is not greatly affected by the roughness of the capillary tube; thus the thermophysical properties at the outlet could be maintained within a certain range if the capillary length is properly selected. If other conditions are kept the same, this characteristic could be used as a judgment of whether the 
internal surface roughness is constant or not. If the quality is lower than normal values, there may be imperfections on the interior of the capillary tube.

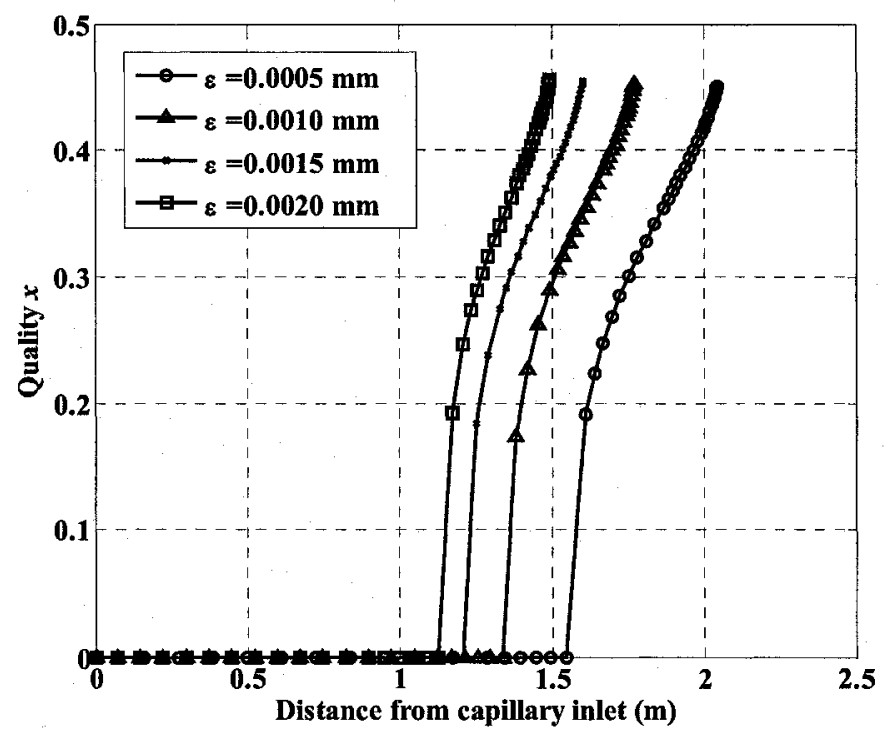

Figure 6-8 Quality profile for different surface roughness for $\mathrm{CO}_{2}$ with $Q_{e}=7 \mathrm{~kW}, P_{k}=120 \mathrm{bar}$, $d_{i}=1.85 \mathrm{~mm}$, and $t_{c a p i, i n}=45^{\circ} \mathrm{C}$

\subsubsection{Cooling capacity}

The relationship between quality and capillary length under different cooling capacities is shown in Figure 6-9. As expected, $L$ decreases gradually as $Q_{e}$ increases. In the two-phase region the quality curve becomes steeper as $Q_{e}$ goes up. This means a higher flow velocity will decrease the amount of heat transfer. The outlet quality increases to a maximum when $Q_{e}=7 \mathrm{~kW}$ before decreasing. From a system point of view, low quality means large specific cooling capacity, which means that less mass flow rate is needed to provide the same cooling capacity. However, when $Q_{e}$ is high, a choking situation occurs which will be discussed in the following section; when $Q_{e}$ is low, capillary length is too long to be practical for both economical and physical (size) reasons. So for a given $Q_{e}$, a proper $L$ determines the outlet quality if other factors remain unchanged. 


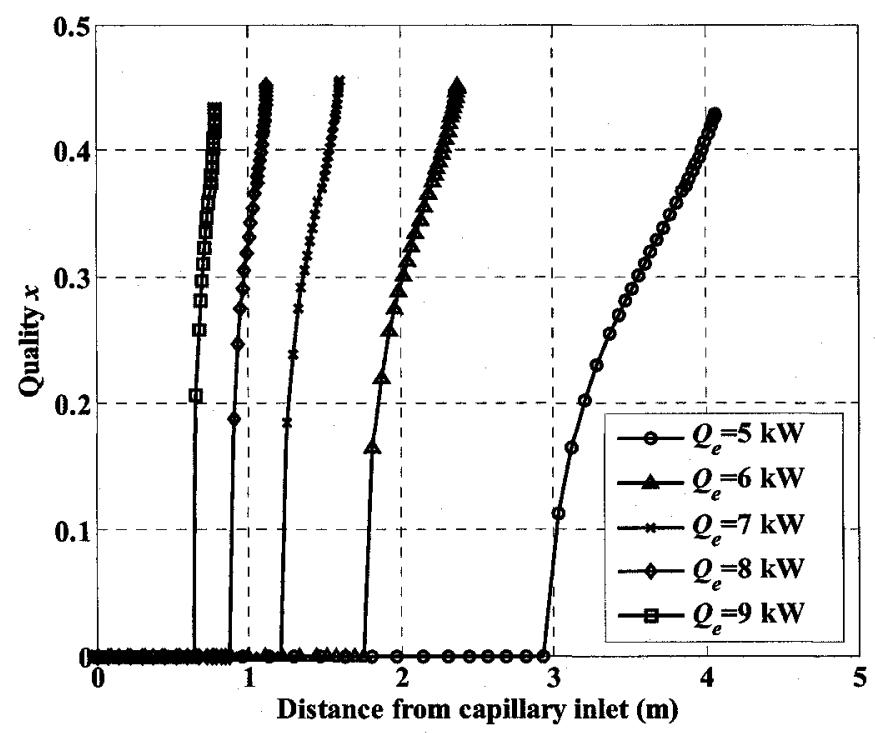

Figure 6-9 Quality profile for different cooling loads for $\mathrm{CO}_{2}$ with $P_{k}=120 \mathrm{bar}, d_{i}=1.85 \mathrm{~mm}$, $t_{e}=0^{\circ} \mathrm{C}, t_{\text {capi,in }}=45^{\circ} \mathrm{C}$, and $\varepsilon=0.0015 \mathrm{~mm}$

\subsubsection{Heat transfer rate}

The heat transfer profile of in-tube $\mathrm{CO}_{2}$ and the outside refrigerant flow is shown in Figure 6-10. When the inner diameter increases, the surface area of the capillary increases; from Figure 6-5 it is obvious that $L$ grows rapidly with increasing capillary inner diameter, so that the heat transfer is enhanced by larger $d_{i}$. Since a higher cooling pressure requires a longer capillary length, the heat transfer rate increases as expected with increasing $P_{k}$. On the other hand, when $d_{i}$ is fixed, the cooling pressure will also greatly affect the heat transfer. When a certain amount of heat transfer is available, $d_{i}$ grows while the cooling pressure decreases, which could be used to select appropriate capillary tubes with a standard inner diameter.

Compared with the cooling capacity ranging from $5 \mathrm{~kW}$ to $9 \mathrm{~kW}$, the heat transfer $Q$ is relatively small. This is mainly a result of the low outside heat transfer coefficient. For example, if $\alpha_{\text {outer }}$ changes from 400 to $1000 \mathrm{~W} /\left(\mathrm{m}^{2} \cdot \mathrm{K}\right)$ at $P_{k}=120 \mathrm{bar}$, and $d_{i}=1.85 \mathrm{~mm}$ while other parameters 
remain unchanged, $Q$ will increase from around $70 \mathrm{~W}$ to about $530 \mathrm{~W}$. Another inherent reason for a low $Q$ is that the temperature difference between in-tube refrigerant and suction line vapour decreases quickly as the refrigerant is cooled down in the capillary by the throttling process; this temperature difference is typically larger in a traditional internal heat exchanger. In either case, improving $\alpha_{\text {outer }}$ will effectively enhance the heat transfer of capillary.

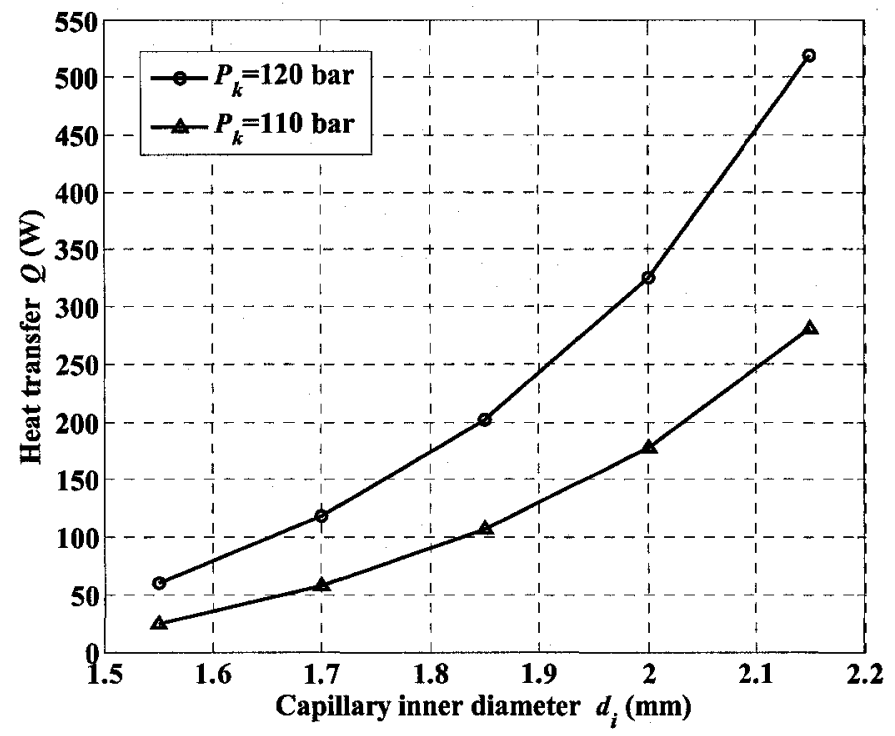

Figure 6-10 Heat transfer rate vs. different capillary inner diameter for $\mathrm{CO}_{2}$ with $Q_{e}=7 \mathrm{~kW}$, $t_{\text {capi }, i n}=45^{\circ} \mathrm{C}, t_{e}={ }^{\circ} \mathrm{C}$, and $\varepsilon=0.0015 \mathrm{~mm}$

\subsubsection{Choking analysis}

Figure 6-11(a) gives the temperature behaviour of carbon dioxide in the capillary. As $d_{i}$ becomes smaller, the required capillary length $L$ will decrease rapidly. Once $d_{i}<1.75 \mathrm{~mm}$, a choking situation occurs, which is exhibited as the outlet temperature being higher than the evaporating temperature $t_{e}$. This also means that the outlet pressure is higher than the back pressure in evaporator, the corresponding velocity reaches sonic velocity, and the mass flow rate reaches a maximum. Figure 6-11(b) shows the entropy variation characteristics under the same condition as Figure 6-11(a). 
Entropy $s$ grows quickly at small diameters as in adiabatic conditions. However, because the heat transfer rate between the high temperature internal refrigerant and the low temperature vapour increases as $d_{i}$ gets larger, the entropy $s$ finally decreases. As the temperature $t$ goes down, the driving force of heat transfer goes down too, and the process becomes more adiabatic. Another notable point is that entropy reaches a maximum at $d_{i}=1.85 \mathrm{~mm}$, which corresponds to the greatest entropy generation.
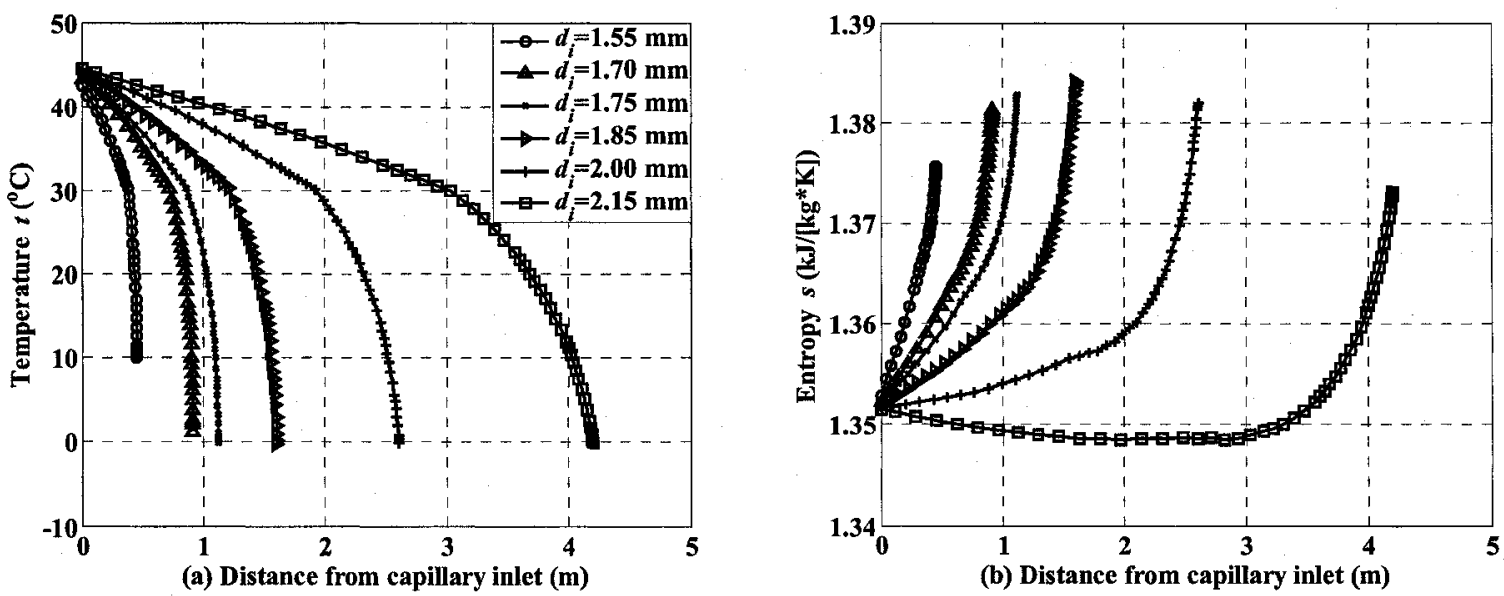

Figure 6-11 Behaviour of temperature and entropy of different capillary inner diameters for $\mathrm{CO}_{2}$ with $Q_{e}=7 \mathrm{~kW}, P_{k}=120$ bar, $t_{c a p i, i n}=45^{\circ} \mathrm{C}, t_{e}=0^{\circ} \mathrm{C}$, and $\varepsilon=0.0015 \mathrm{~mm}$

\subsubsection{Combined performance}

To obtain a better picture of the relationship between geometric parameters and cooling capacity, a three-dimensional chart is provided in Figure 6-12. Since it is impractical to have capillary length exceed $4.5 \mathrm{~m}$, and such high values could weaken the importance of the most relevant section where $L$ is in the $0.5-2 \mathrm{~m}$ range, lengths above $4.5 \mathrm{~m}$ are not shown in the chart. 


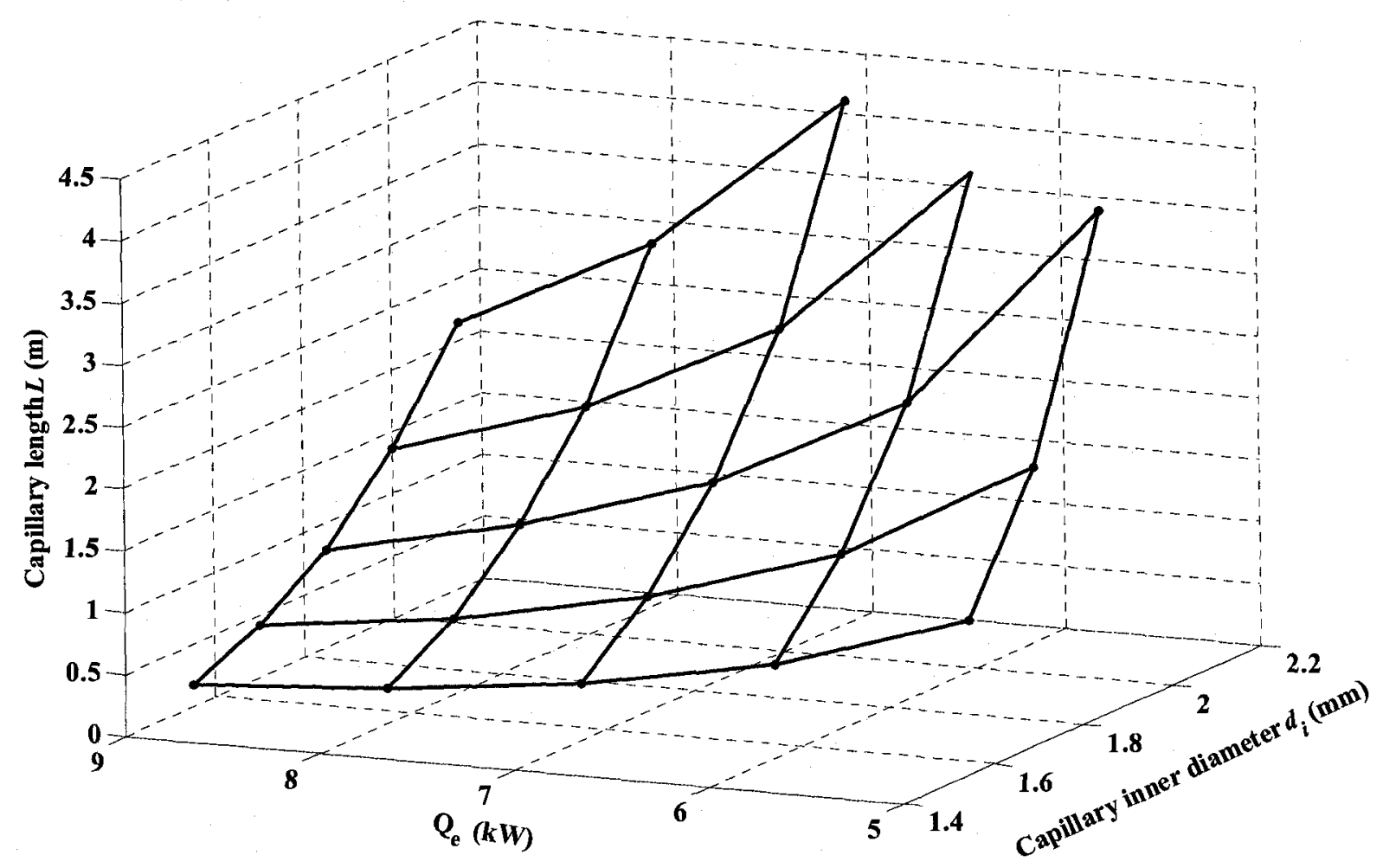

Figure 6-12 Three-dimensional presentation of the relationship between $d_{i}, Q_{e}$ and $L$ for $\mathrm{CO}_{2}$ with $P_{k}=120$ bar, $t_{c a p i, i n}=45^{\circ} \mathrm{C}, t_{e}=0{ }^{\circ} \mathrm{C}$, and $\varepsilon=0.0015 \mathrm{~mm}$

This figure is very useful for both system design and performance analysis. For instance, if cooling capacity is given by a client and the inner diameter is fixed by market, a corresponding capillary length could be directly obtained. On the other hand, when a machine is on site, the cooling capacity could be readily determined by the length of the capillary. In our case, at $Q_{e}=8 \mathrm{~kW}$, the proper range for the inner diameter is 1.4 to $1.9 \mathrm{~mm}$, corresponding to a capillary length between 0.5 and $1.5 \mathrm{~m}$; the optimum configuration relies on specific needs. It needs to be addressed here that the curved surface only shows the relationship when $t_{e}=0{ }^{\circ} \mathrm{C}$ and $P_{k}=120 \mathrm{bar}$; when these two parameters change, the surface changes accordingly. 


\subsubsection{Case 2: Varying surrounding heat transfer coefficient}

A typical set of simulation results are shown in Figure 6-13. The outside heat transfer coefficient is not fixed but variable. The initial states of outside refrigerant are at temperature $t_{s l, i n}=t_{e}$, and quality $x_{s l, i n}=0.8$ (these being the most common situations occurring in AAC systems). For the sake of convenience, the inner diameter of the suction line is fixed at $19.00 \mathrm{~mm}$.

\subsubsection{Inner diameter}

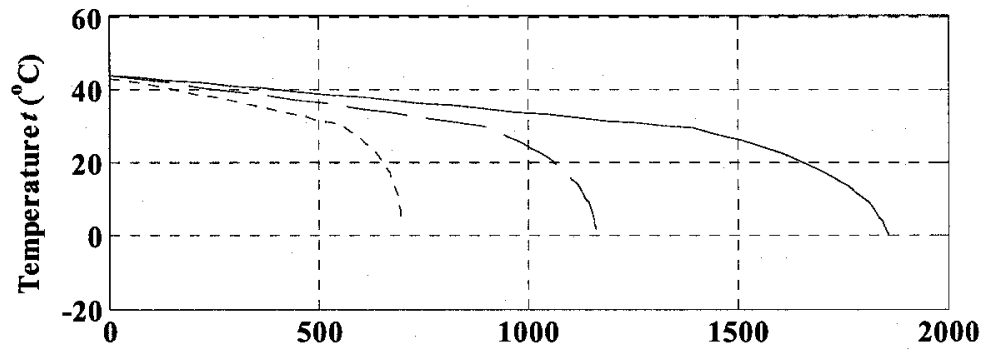

(a) Distance from capillary inlet $(\mathrm{mm})$

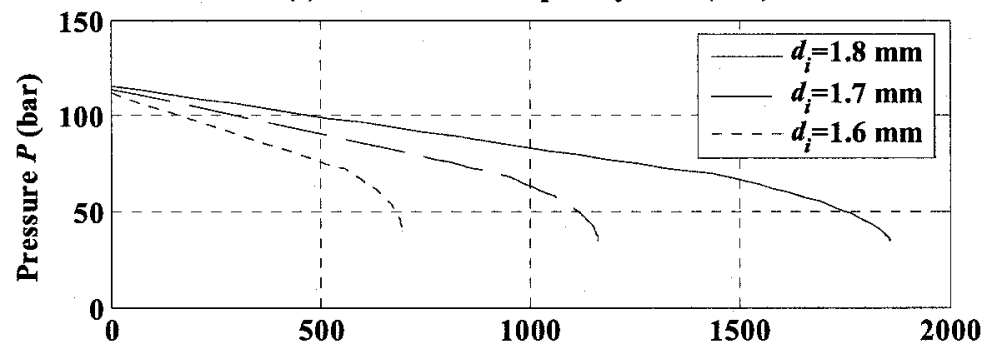

(b) Distance from capillary inlet (mm)

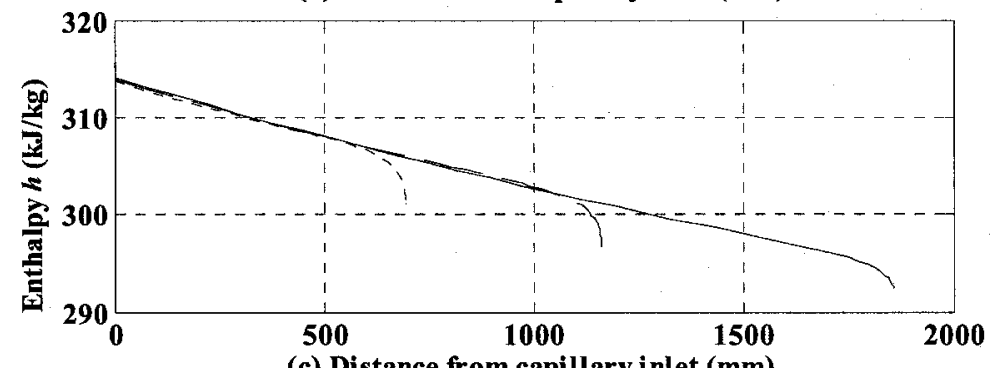

(c) Distance from capillary inlet $(\mathrm{mm})$

Figure 6-13 Simulation results along capillary tube for $\mathrm{CO}_{2}$ with $Q_{e}=7 \mathrm{~kW}, P_{k}=110 \mathrm{bar}$, $t_{\text {capi,in }}=45^{\circ} \mathrm{C}, t_{e}=0^{\circ} \mathrm{C}$, and $\varepsilon=0.0015 \mathrm{~mm}$ 
It can be seen from Figure 6-13 that a longer capillary tube is required as capillary inner diameter grows. To show this relationship clearly, five diameters and two cooling pressures were inputted into the simulation code and the results are shown in Figure 6-14. When $P_{k}=120$ bar the capillary length is longer than $4 \mathrm{~m}$ with some capillary inner diameters, and they are not shown in the Figure to ensure other data are clearly displayed. Compared to section 0 , a longer capillary length is obtained at a given $d_{i}$. For example, with $P_{k}=110$ bar and $d_{i}=2.0 \mathrm{~mm}, L$ is $1.45 \mathrm{~m}$ in Figure 6-5, while it is $2.25 \mathrm{~m}$ here. The increase can be attributed to the increase of the suction line heat transfer coefficient. Higher $\alpha_{\text {outer }}$ leads to the better heat transfer rate of the AXE. For a system with AXE, the specific cooling capacity is greater than that of a cycle without AXE. Also, if the cooling load is kept constant, then the mass flow rate is lower; from the momentum equation we thus know that more capillary length is required to fulfill the same pressure drop.

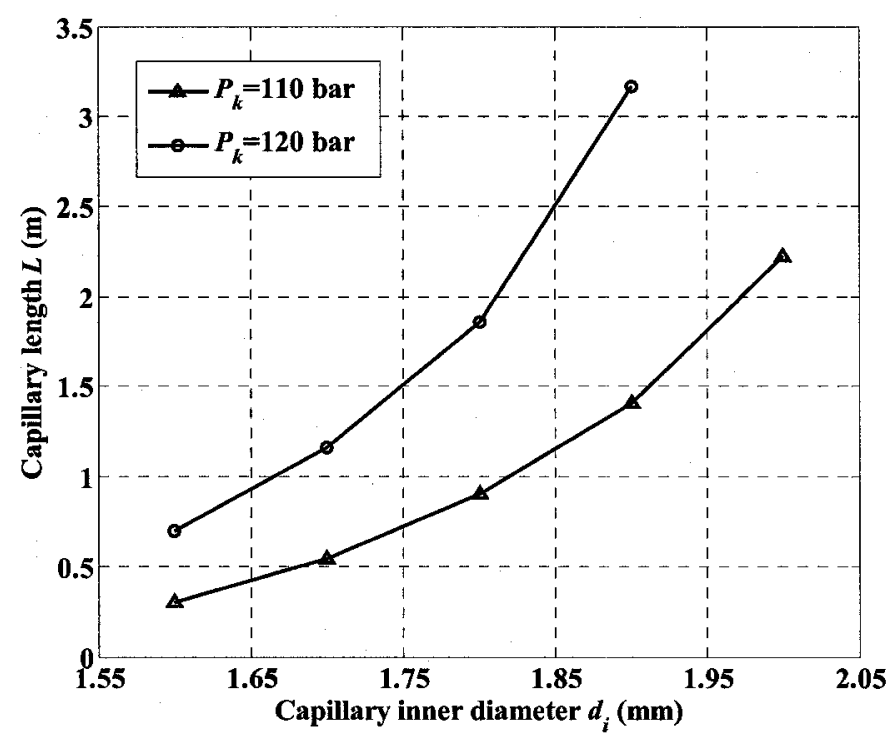

Figure 6-14 Capillary length vs. capillary inner diameter under different cooling pressures for $\mathrm{CO}_{2}$ with $Q_{e}=7 \mathrm{~kW}, t_{c a p i, i n}=45^{\circ} \mathrm{C}, t_{e}=0{ }^{\circ} \mathrm{C}$, and $\varepsilon=0.0015 \mathrm{~mm}$ 


\subsubsection{Cooling pressure}

From Figure 6-14 it can also be seen that a longer capillary is required to accommodate higher cooling pressure while keeping $Q_{e}$ and $d_{i}$ the same. The length is more sensitive to the high-side pressure with variable $\alpha_{\text {outer }}$. This again shows the double-damping effects of heat transfer on refrigerant flow for a system with a fixed cooling load. On one hand, more heat transfer "smoothes" the capillary tube, and thus a longer capillary is required. On the other hand, with fixed $Q_{e}$ the system flow rate is less, which also leads to longer capillary.

\subsubsection{Evaporating temperature}

Figure 6-15 shows the relationship between capillary length and evaporating temperature. With $Q_{e}$ unchanged, a lower evaporating temperature (corresponding to larger $\Delta P$ ) means longer $\mathrm{L}$.

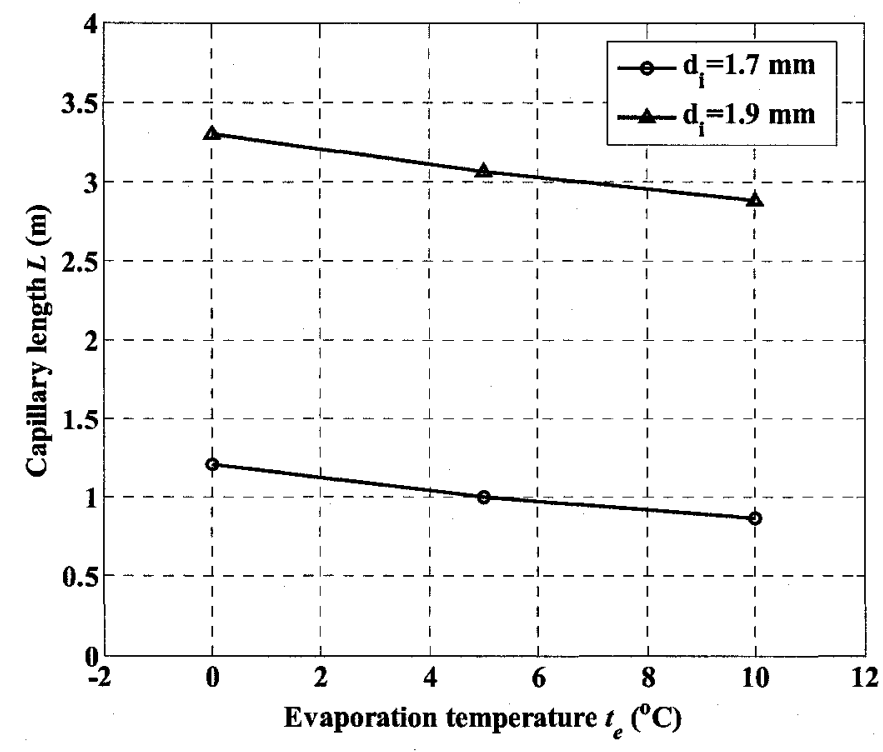

Figure 6-15 Cooling capacity vs. evaporation temperature for $\mathrm{CO}_{2}$ with $Q_{e}=7 \mathrm{~kW}, P_{k}=120 \mathrm{bar}$, $t_{\text {capi,in }}=45^{\circ} \mathrm{C}$, and $\varepsilon=0.0015 \mathrm{~mm}$ 


\subsubsection{Hydraulic drag factor}

As mentioned above, the inertia drag factor is introduced into the model to improve its accuracy. The profiles of $f_{h}, f_{i}$, and $f$ are illustrated in Figure 6-16. For the sake of easy comparison between these three factors, only single-phase data are used. Two conclusions can be drawn from the figure: a. the inertia factor is relatively small compared with friction term, about $1 / 15$ in this case; $b$. the drag factor varies near the saturated point. This could be attributed to the discontinuity of thermophysical properties such as viscosity.

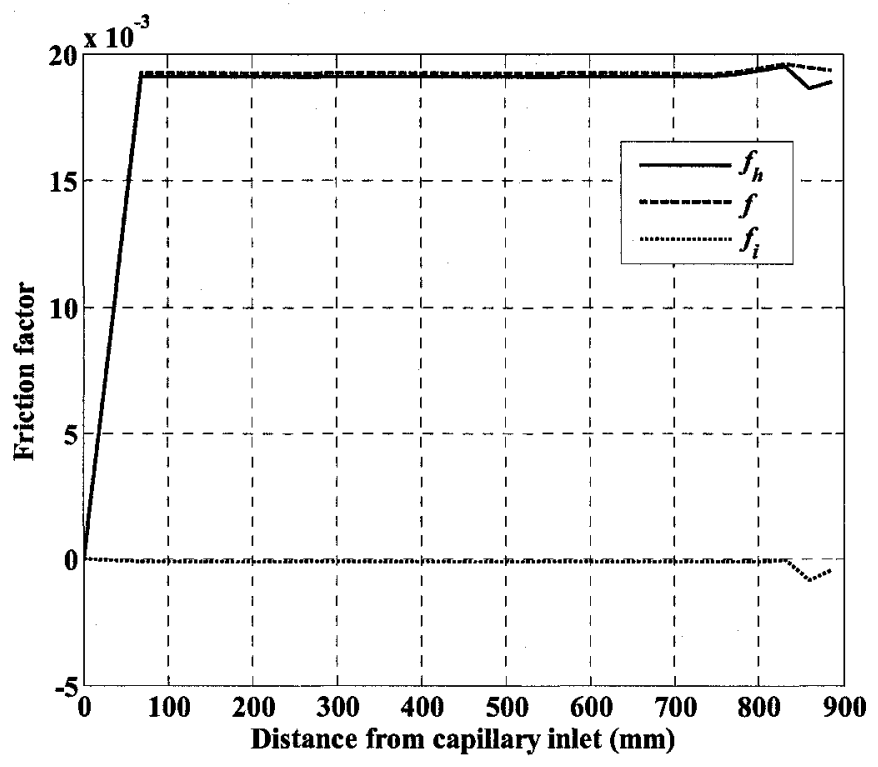

Figure 6-16 Profiles of friction factors for $\mathrm{CO}_{2}$ with $Q_{e}=7 \mathrm{~kW}, P_{k}=120 \mathrm{bar}, t_{c a p i, i n}=45{ }^{\circ} \mathrm{C}$, $t_{e}=0{ }^{\circ} \mathrm{C}$, and $\varepsilon=0.0015 \mathrm{~mm}$

\subsubsection{Heat transfer rate}

The heat transfer rate of the AXE is shown in Figure 6-17. Trends similar to those in Figure 6-10 can be observed, while the magnitude is greater in this case. With the cooling load unchanged, the mass flow rate is slightly smaller than that of Figure 6-10. However, larger contact area and overall heat transfer coefficient ensure a greater heat transfer rate. This also shows that the suction line heat transfer coefficient is higher than 400 
$\mathrm{W} /\left(\mathrm{m}^{2} \cdot \mathrm{K}\right)$, which is a merit of AXE.

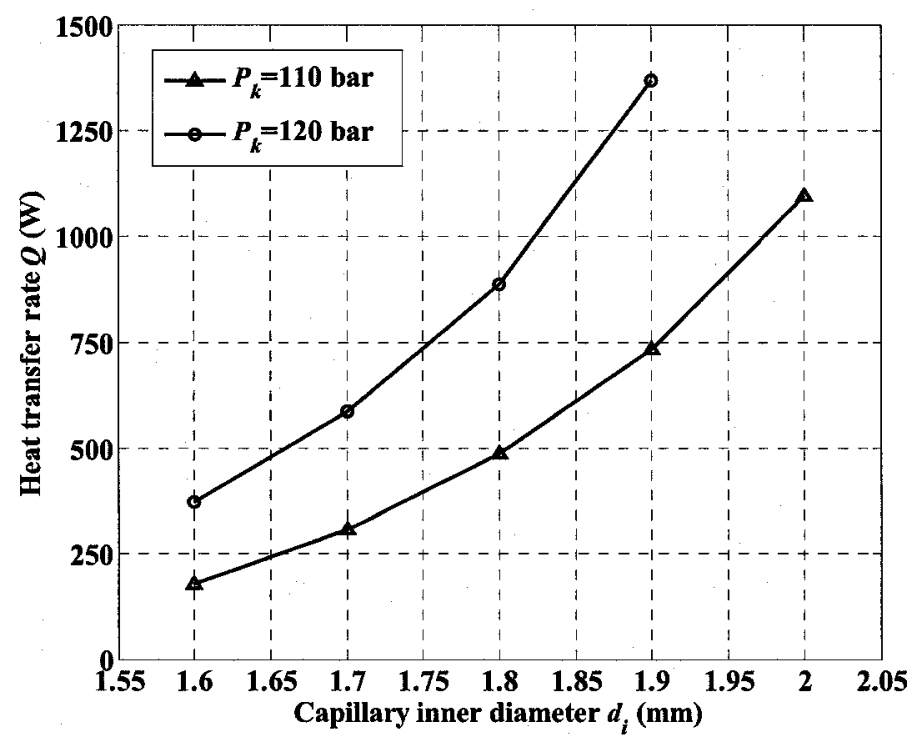

Figure 6-17 Heat transfer rate vs. capillary inner diameter for $\mathrm{CO}_{2}$ with $Q_{e}=7 \mathrm{~kW}$, $t_{\text {capi,in }}=45^{\circ} \mathrm{C}, t_{e}=0^{\circ} \mathrm{C}$, and $\varepsilon=0.0015 \mathrm{~mm}$

\subsubsection{Further comments}

In the above analysis, the quality of the outer refrigerant is initially fixed at 0.8 (not unity, as is commonly used in theoretical analysis). This is due to the specific characteristics of the accumulator: there is an oil-bleeding hole at the bottom of the J-shaped tube, and oil cannot be entrained into the J-tube if the refrigerant is dry. Because of the oil, there is actually no pure refrigerant flowing in the system, and the heat transfer coefficient is affected to some extent, which needs further investigation.

Another point relates to the improvement of the system performance if an AXE is present. In a $\mathrm{CO}_{2}$ cycle, replacing the IHX with an AXE allows the same amount of heat to be recovered but with a significant decrease in device weight. This is due to the high overall heat transfer coefficient of the AXE. Moreover, the AXE stabilizes the system pressures in an R134a-based system; a similar 
trend is expected for a $\mathrm{CO}_{2}$ cycle, which would require less compression work.

\subsection{Conclusions}

A non-adiabatic model for a transcritical refrigeration $\mathrm{CO}_{2}$ cycle was presented in this chapter.

The following conclusions can be drawn.

1. More heat transfer corresponds to lower quality at the capillary outlet.

2. Proper relation of tube diameter and length is a trade-off between system size and efficiency. The increase of inner diameter $d_{i}$, cooling pressure $P_{k}$, and outside heat transfer coefficient $\alpha$ will lead to longer capillary length $L$; while increasing evaporating temperature $t_{e}$, and cooling capacity $Q_{e}$ lead to a shorter $L$.

3. Due to the thermodynamic properties of $\mathrm{CO}_{2}$, a lower evaporating temperature $t_{e}$ brings about a larger $Q_{e}$, which is unlike traditional refrigeration cycles.

4. The inertia drag force was considered in the model and found to only slightly affect the pressure drop.

5. The heat transfer rates change with different kinds of capillary tubes under different conditions: for $\alpha_{\text {outer }}=400 \mathrm{~W} /\left(\mathrm{m}^{2} \cdot \mathrm{K}\right)$, it falls into the range of about 50 to $550 \mathrm{~W}$ with $Q_{e}=7 \mathrm{~kW}$, $t_{\text {capi, in }}=45^{\circ} \mathrm{C}, t_{e}=0{ }^{\circ} \mathrm{C}, d_{i}=1.85 \mathrm{~mm}$, and $\varepsilon=0.0015 \mathrm{~mm}$. For given suction line inlet conditions with $t_{s l, i n}=0^{\circ} \mathrm{C}, d_{i, \text { outer }}=19.00 \mathrm{~mm}$, the heat transfer rate varies from $200 \mathrm{~W}$ to $1400 \mathrm{~W}$ with the same conditions. The increase is due to a suction line heat transfer coefficient which is greater than $400 \mathrm{~W} /\left(\mathrm{m}^{2} \cdot \mathrm{K}\right)$.

6. An extreme condition can be determined by comparing the value of the local sonic velocity and 
the refrigerant flow velocity, which is based on the physical mechanism of the choking phenomenon.

The present model can be used for both system design and performance evaluation; it is also very helpful in understanding the supercritical flow behaviour inside the capillary. 


\section{Chapter 7}

\section{The optimum high pressure for transcritical refrigeration cycles}

Compared to current $\mathrm{AAC}$ systems, the introduction of $\mathrm{CO}_{2}$ will decrease the system performance if the structure remains unchanged. This is mainly because of the low critical temperature (about $31^{\circ} \mathrm{C}$ ) of $\mathrm{CO}_{2}$, which causes the quality at the inlet of the evaporator to be much higher than that of R134a. As a result, the specific cooling capacity is relatively low. Accordingly, an internal heat exchanger (IHX) is not optional but necessary for a $\mathrm{CO}_{2}$ system. A few research investigations focusing on how to achieve a maximum Coefficient of Performance (COP) under certain conditions have been reported. Some results show that the high pressure affects the COP significantly and an optimum high pressure exists (Pettersen and Skaugen, 1994; Kauf, 1999; Liao et al., 2000). Kauf (1999) proposed a graphic method to deduce a linear relationship between the optimum high pressure $P_{k}$ and the environment temperature $t_{a m b}$. Liao et al. (2000) investigated the relationship between $P_{k}, t_{a m b}$, evaporating temperature $t_{e}$, and the compressor efficiency $\eta_{c o m p}$. A correlation for $P_{k}$ in terms of appropriate parameters was then developed based on simulation results. However, though Liao et al. (2000) used a linear expression to describe compressor efficiency in terms of pressure ratio, there is another high-order expression shown in open literature (Sarkar, et al., 2004), which cannot be symbolized by the ratio of coefficients $K$ and $C$. In this chapter a thorough investigation between $P_{k}, t_{a m b}, t_{e}$, and IHX effectiveness $\eta_{I H X}$ was performed. The situation of two-phase refrigerant at the evaporator outlet was also considered. This was not 
addressed in the studies cited above. In this work theoretical analysis and simulation work were carried out; a correlation was developed based on simulation data; a comparison between simulation data and experimental results was also provided.

\section{$7.1 \mathrm{CO}_{2}$-based transcritical refrigeration system}

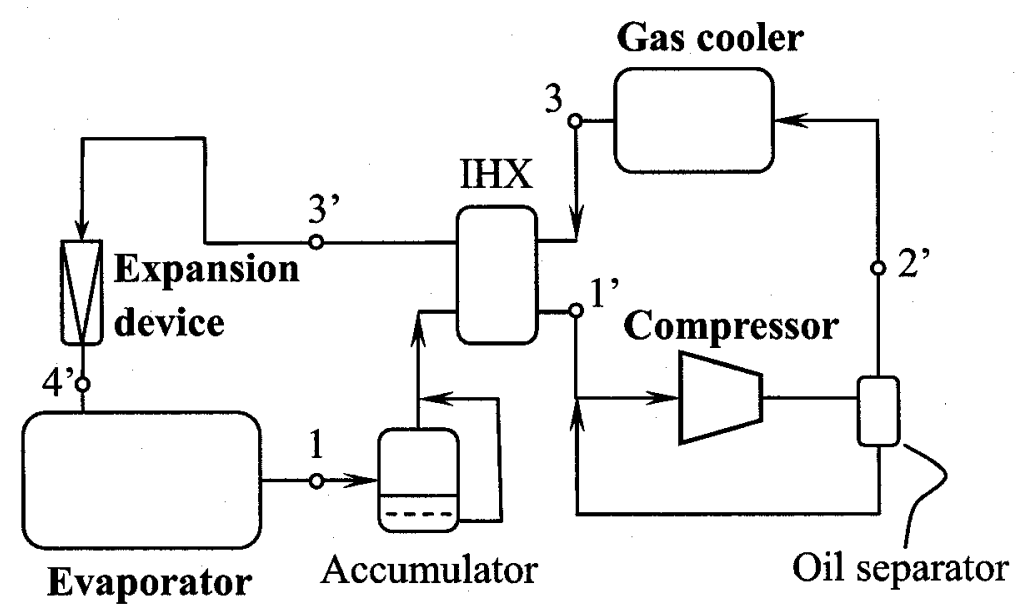

Figure 7-1 $\mathrm{COP}$ behaviour for a $\mathrm{CO}_{2}$ transcritical system with an accumulator and an IHX

For traditional refrigeration cycles, there are four main components: evaporator, compressor, condenser, and expansion device. In $\mathrm{CO}_{2}$ systems, a gas cooler replaces the condenser because the inside refrigerant runs at supercritical pressures and no liquid forms in the component. Figure 7-1 represents a typical diagram of a $\mathrm{CO}_{2}$ refrigeration cycle, and the system diagram is shown as loop $1-1^{\prime}-2^{\prime}-3-3^{\prime}-4^{\prime}-1$ in Figure 7-1. Superheated refrigerant (1') flows into the compressor where it is elevated to high pressure and high temperature (state $\left.2^{\prime}\right)$. Then the supercritical vapour flows through the gas cooler, rejecting heat to the environment while being cooled down to point 3 . Afterward it exchanges heat with the low-pressure refrigerant in the IHX and flows into the expansion device, where it is throttled to low pressure and low temperature (state 4'). Finally, the 
refrigerant in the evaporator absorbs heat and evaporates.

In the previous chapter an AXE was introduced into the system. This process is shown as loop 1-1'-2'-3-4'-1 in Figure 7-2. As a kernel feature of the AXE, the inside capillary tube makes the refrigerant move along a new line, 3-4', which is equivalent to the combination of a heat transfer process (3-3') and an adiabatic expansion process ( $\left.3^{\prime}-4^{\prime}\right)$. The following discussion is applicable to both loop 1-1'-2'-3-3'-4'-1 and loop 1-1'-2'-3-4'-1.

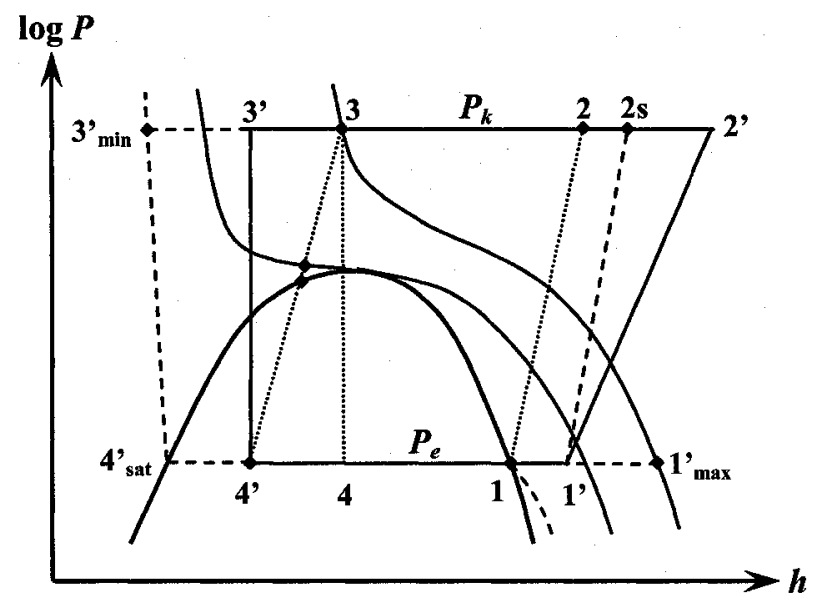

Figure 7-2 Pressure-enthalpy diagram of transcritical $\mathrm{CO}_{2}$ refrigeration cycles

\subsection{Determination of the optimum high pressure}

The analysis is based on the following assumptions:

- The $\mathrm{CO} 2$ is considered to be a pure fluid and the oil effect on altering the properties of $\mathrm{CO}_{2}$ is not taken into account.

- Pressure in the gas cooler and the evaporator is assumed to be constant. For the capillary tube used in the previous chapter, pressure drop and heat transfer are two goals of the device, thus the pressure is not constant. 


\subsubsection{Theoretical deduction}

The system performance COP is generally defined as

$$
\mathrm{COP}=\frac{Q_{e}}{W_{t}}=\frac{\dot{m} q_{0}}{\dot{m} w_{t}}=\frac{q_{0}}{w_{t}}
$$

From Figure 7-2 it can also be expressed as

$$
\mathrm{COP}=\frac{h_{1}-h_{4}}{h_{2^{\prime}}-h_{1}}
$$

A slight rise of the high pressure $P_{k}$ will lead to a big decrease of $h_{4}$, because the enthalpy of the refrigerant drastically changes in the region near the critical point. However, at higher pressure regions $P_{k}$ has a weak influence on $h_{4}$. On the other hand, a rise of $P_{k}$ has a nearly linear influence on $h_{2}$. As a result, for a system without an IHX, the COP will reach a maximum at a specific high pressure $P_{k}$. On the other hand, IHX effectiveness will affect $h_{4}$,and $h_{2}$, if it is included in the system, though its influence is relatively small compared with $P_{k}$. In order to find the maximum COP, the relationships between these parameters should be investigated.

\subsubsection{Potential heat transfer capacity}

$$
\eta_{I H X}=\frac{h_{3}-h_{3^{\prime}}}{q_{p o t n}}=\frac{h_{1^{\prime}}-h_{1}}{q_{p o t n}}
$$

where $q_{p o t n}$ represents the maximum specific heat transfer that can be used. Since $h_{3^{\prime}}=h_{4^{\prime}}$, after rearrangement it becomes

$$
\eta_{I H X}=\frac{h_{3}-h_{4^{\prime}}}{q_{p o t n}}
$$


From a heat transfer point of view, the hot refrigerant can be theoretically cooled down to $t_{3^{\prime}, \text { min }}$ which equals the evaporating temperature $t_{1}$. Its enthalpy also approximates the value of the saturated liquid with the same temperature, which is shown as point 4' ${ }_{\text {sat }}$ in Figure 7-2. This is valid even if a pressure loss exists in the component because the enthalpy of a liquid does not change much with pressure. The cold refrigerant can reach $t_{1, \max }$, which equals $t_{3}$. Because of the large property variance of a transcritical fluid, the corresponding enthalpy differences $\left(h_{3}-h_{3^{\prime}, \mathrm{min}}\right)$ and $\left(h_{1}, \max -h_{l}\right)$ are not equal. For energy balance, the maximum specific heat transfer would be the smaller of the two, that is

$$
q_{p o t n}=\min \left\{h_{3}-h_{3, \text { min }}, h_{1, \max }-h_{1}\right\}
$$

The discussion in this chapter takes into account the fact that the heat transfer condition deteriorates in the superheated area. Therefore, the extreme quality at the evaporator outlet was fixed as 1 for a $\mathrm{CO}_{2}$ refrigeration system with an IHX. This also accounts for the real situation of system oil cycling. Inside the accumulator the refrigerant in the tube should keep some liquid fraction so as to entrain oil and convey it to the compressor more smoothly. In a later section the $q_{p o t n}$ will be discussed in detail.

\subsubsection{Theoretical deduction of the optimum high pressure}

The enthalpy of 4' can be obtained from Equation (7-4)

$$
h_{4^{\prime}}=h_{3}-\eta_{I H X} q_{p o t n}
$$

Noting that from the energy balance $h_{1}{ }^{\prime}-h_{1}=h_{3}-h_{4^{\prime}}$, the enthalpy at the outlet of IHX can be expressed by 


$$
h_{1^{\prime}}=h_{1}+h_{3}-h_{4^{\prime}}=h_{1}+\eta_{I H X} q_{p o t n}
$$

For the compression process, the isentropic efficiency

$$
\eta_{c o m p}=\frac{h_{2 s}-h_{1^{\prime}}}{h_{2^{\prime}}-h_{1^{\prime}}}
$$

And the enthalpy at the outlet of compressor can be expressed as

$$
h_{2^{\prime}}=h_{1^{\prime}}+\frac{h_{2 s}-h_{1^{\prime}}}{\eta_{\text {comp }}}=\left(1-\frac{1}{\eta_{\text {comp }}}\right)\left(h_{1}+\eta_{I H X} \cdot q_{p o t n}\right)+\frac{h_{2 s}}{\eta_{c o m p}}
$$

Substituting Equations (7-6), (7-7), and (7-9) into (7-2) gives

$$
\mathrm{COP}=\frac{\eta_{\text {comp }}\left(h_{1}-h_{3}+\eta_{I H X} \cdot q_{\text {potr }}\right)}{h_{2 s}-h_{1}-\eta_{I H X} \cdot q_{p o t h}}
$$

It is easy to obtain $h_{1}=h_{1}\left(t_{1}, x\right)$ and $h_{3}=h_{3}\left(t_{3}, P_{k}\right) ; q_{p o t n}$ and $h_{2 s}$ are relatively complicated to obtain

$$
q_{p o t n}=\left\{\begin{array}{c}
h_{3}-h_{3, \text { min }}=q_{\text {potr }}\left(t_{1}, t_{3}, P_{k}\right) \\
h_{1, \text { max }}-h_{1}=q_{p o t n}\left(t_{1}, t_{3}, x\right)
\end{array}\right.
$$

So $q_{\text {potm }}=q_{\text {potn }}\left(t_{1}, t_{3}, P_{k} / x\right)$

and

$$
h_{2 s}=h_{2 s}\left(h_{1}, P_{k}\right)=h_{2 s}\left(t_{1}, x, \eta_{I H X}, q_{p o t n}, P_{k}\right)=h_{2 s}\left(t_{1}, t_{3}, x, P_{k}, \eta_{I H X}\right)
$$

Now Equation (7-10) can be expressed as a function of $t_{1}, x, t_{3}, P_{k}, \eta_{I H X}$, and $\eta_{\text {comp. }}$.

$$
\mathrm{COP}=\frac{\eta_{\text {comp }}\left[h_{1}\left(t_{1}, x\right)-h_{3}\left(t_{3}, P_{k}\right)+\eta_{I H X} \cdot q_{p o t n}\left(t_{1}, t_{3}, P_{k} / x\right)\right]}{h_{2 s}\left(t_{1}, t_{3}, x, P_{k}, \eta_{I H X}\right)-h_{1}\left(t_{1}, x\right)-\eta_{I H X} \cdot q_{p o t n}\left(t_{1}, t_{3}, P_{k} / x\right)}
$$

To find the optimum pressure, the derivative of COP with respect to $P_{k}$ is minimized, yielding: 


$$
\begin{aligned}
\frac{\partial \mathrm{COP}}{\partial \eta_{\text {comp }}} \frac{\partial \eta_{\text {comp }}}{\partial P_{k}} \frac{h_{1}-h_{3}+\eta_{I H X} q_{p o t n}}{h_{2 s}-h_{1}-\eta_{I H X} q_{p o t n}}+\frac{\eta_{\text {comp }}}{\left(h_{2 s}-h_{1}-\eta_{I H X} q_{p o t n}\right)^{2}} \times \\
\quad\left[\left(-\frac{\partial \mathrm{COP}}{\partial h_{3}} \frac{\partial h_{3}}{\partial P_{k}}+\eta_{I H X} \frac{\partial \mathrm{COP}}{\partial q_{p o t n}} \frac{\partial q_{p o t n}}{\partial P_{k}}+q_{p o t n} \frac{\partial \operatorname{COP}}{\partial \eta_{I H X}} \frac{\partial \eta_{I H X}}{\partial P_{k}}\right)\left(h_{2 s}-h_{1}-\eta_{I H X} q_{p o t n}\right)-\right. \\
\left.\left(\frac{\partial \operatorname{COP}}{\partial h_{2 s}} \frac{\partial h_{2 s}}{\partial P_{k}}-\eta_{I H X} \frac{\partial \mathrm{COP}}{\partial q_{p o t n}} \frac{\partial q_{p o t n}}{\partial P_{k}}-q_{p o t n} \frac{\partial \mathrm{COP}}{\partial \eta_{I H X}} \frac{\partial \eta_{I H X}}{\partial P_{k}}\right)\left(h_{1}-h_{3}+\eta_{I H X} \cdot q_{p o t n}\right)\right]=0
\end{aligned}
$$

When $q_{p o t n}=q\left(t_{1}, t_{3}, x\right)$, then $\frac{\partial q_{p o t n}}{\partial P_{k}}=0$, and the above expression can be simplified to

$$
\begin{aligned}
& \frac{\partial \operatorname{COP}}{\partial \eta_{\text {comp }}} \frac{\partial \eta_{\text {comp }}}{\partial P_{k}} \frac{h_{1}-h_{3}+\eta_{I H X} q_{p o t n}}{h_{2 s}-h_{1}-\eta_{I H X} q_{p o t n}}- \\
& \quad \frac{\eta_{c o m p}}{\left(h_{2 s}-h_{1}-\eta_{I H X} q_{p o t n}\right)^{2}}\left[\left(\frac{\partial \operatorname{COP}}{\partial h_{3}} \frac{\partial h_{3}}{\partial P_{k}}-q_{p o t n} \frac{\partial \operatorname{COP}}{\partial \eta_{I H X}} \frac{\partial \eta_{I H X}}{\partial P_{k}}\right)\left(h_{2 s}-h_{1}-\eta_{I H X} q_{p o t n}\right)-\right. \\
&\left.\left(\frac{\partial \operatorname{COP}}{\partial h_{2 s}} \frac{\partial h_{2 s}}{\partial P_{k}}-q_{p o t n} \frac{\partial \operatorname{COP}}{\partial \eta_{I H X}} \frac{\partial \eta_{I H X}}{\partial P_{k}}\right)\left(h_{1}-h_{3}+\eta_{I H X} \cdot q_{p o t n}\right)\right]=0
\end{aligned}
$$

Both (7-14) and (7-15) are quite complicated, and it is almost impossible to get the exact explicit derivative of enthalpy on pressure in the single-phase region because they are correlated with complicated semi-empirical equations such as Peng-Robinson (PR) and Benedict-Webb-Rubin (BWR) equations. It might be convenient for engineering purposes to obtain an explicit polynomial expression using numerical methods such as cubic-spline interpolation. However, direct simulation would be a simple choice in that both approaches using numerical methods will generate the same relationship. Therefore, it is meaningful to simulate the system behaviour under various working conditions.

\subsection{Simulation results and discussions}

A program has been developed in MATLAB based on the governing equations formulated in the previous section. The required thermophysical properties of the refrigerant were taken from 
CoolPack version 1.46. To learn how the five parameters $t_{l}, t_{3}, P_{k}, \eta_{I H X}$, and $x$ affect the system performance, and then find the optimum high pressure that generates the highest COP, one or two parameters are varied at one time while other conditions are kept the same. The gas cooler exit temperature is determined by

$$
t_{3}=-0.0015269 t_{a m b}^{2}-0.028866 t_{a m b}+7.7126
$$

This correlation was curve-fitted from Brown et al. (2002). Except where specified, the parameter ranges are: $t_{l}=-10^{\circ} \mathrm{C} \sim 10^{\circ} \mathrm{C}, t_{3}=35^{\circ} \mathrm{C} \sim 50{ }^{\circ} \mathrm{C}, P_{k}=80$ bar $\sim 135$ bar, $\eta_{I H X}=0 \sim 1$ and $x=0.7 \sim 1$.

\subsubsection{Influence of compressor efficiency}

In order to compare the current model with that of Kauf (1999), different expressions of the compressor efficiency from open literature (Liao et al., 2000, Brown et al., 2002; Robinson and Groll, 1998) were used. The detailed forms are as follows.

Robinson and Groll: $\quad \eta_{i s}=0.815+0.022 r-0.0041 r^{2}+0.0001 r^{3}$

Brown et al: $\quad \eta_{i s}=0.9343-0.04478 r$

Liao et al.: $\quad \eta_{i s}=1.003-0.121 r$

where the pressure ratio is defined by $r=\frac{P_{k}}{P_{0}}$.

Figure 7-3 shows the relationship between isentropic efficiency and pressure ratio. Liao et al.'s model was obtained by best-fitting the experimental data of a $\mathrm{CO}_{2}$ compressor from Danfoss A/S. Brown et al.'s model was obtained from a $\mathrm{CO}_{2}$ compressor by Rieberer and Halozan (1998) and is 
suitable for $r>2$. Robinson's was obtained from Jungnickel (1990). Starting from approximate values, Brown et al.'s model is similar to that of Robinson et al.'s, while Liao et al.'s model decreases much faster and goes below 0 at $r>8.29$, which is obviously impossible for commercial products. This is not a major concern since $r$ lies between 2 and 5 in most of the cases.

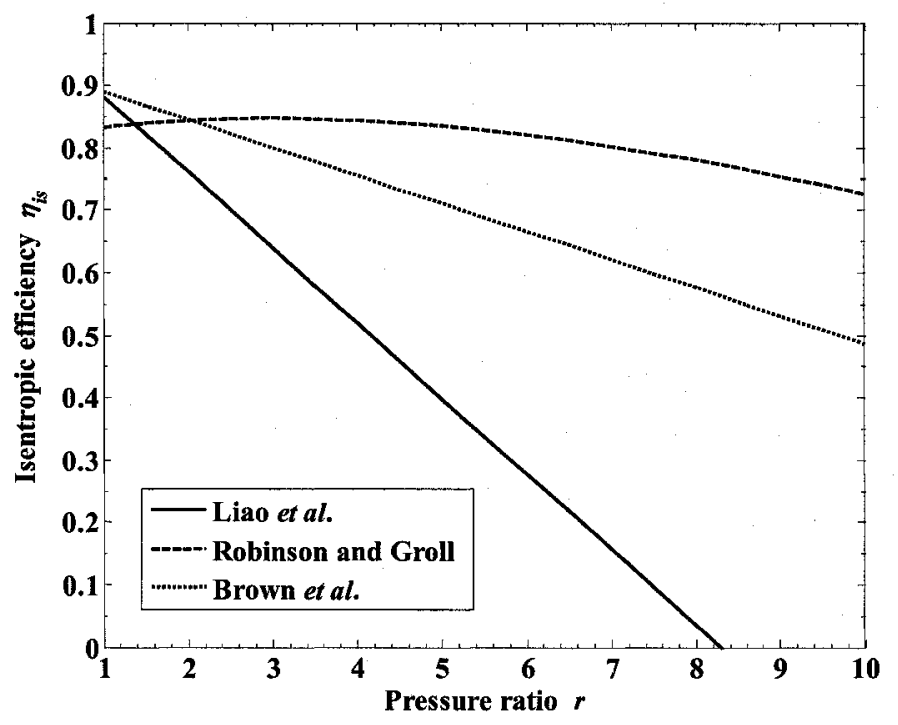

Figure 7-3 Compressor isentropic efficiency from different references

Substituting different $\eta_{c o m p}$ values into the model, a typical set of simulation data was generated and shown in Figure 7-4. Because (7-17) and (7-18) produce similar values when $r$ is less than 5, their associated COP curves have similar shapes and peak at similar $P_{k, o p t}$ values as well; correlation (7-19) has a lower COP and greater deviation in $P_{k, o p t}$. Since different types of compressors behave differently, it is hard to represent their characteristics by one or two parameters. Thus Brown et al.'s expression was adopted in the following simulations as a typical description for compressors. This simplification can reveal the main characteristics of $P_{k}$ values without losing generality.

It is noteworthy that the mechanical efficiency of the compressor $\eta_{\text {mech }}$ and the electric motor efficiency $\eta_{\text {motor }}$ were not included in analysis. For most of the cases they change little and are thus 
considered as constants. This will only affect the COP values proportionally and leave the $P_{k, o p t} \mathrm{~s}$ unchanged.

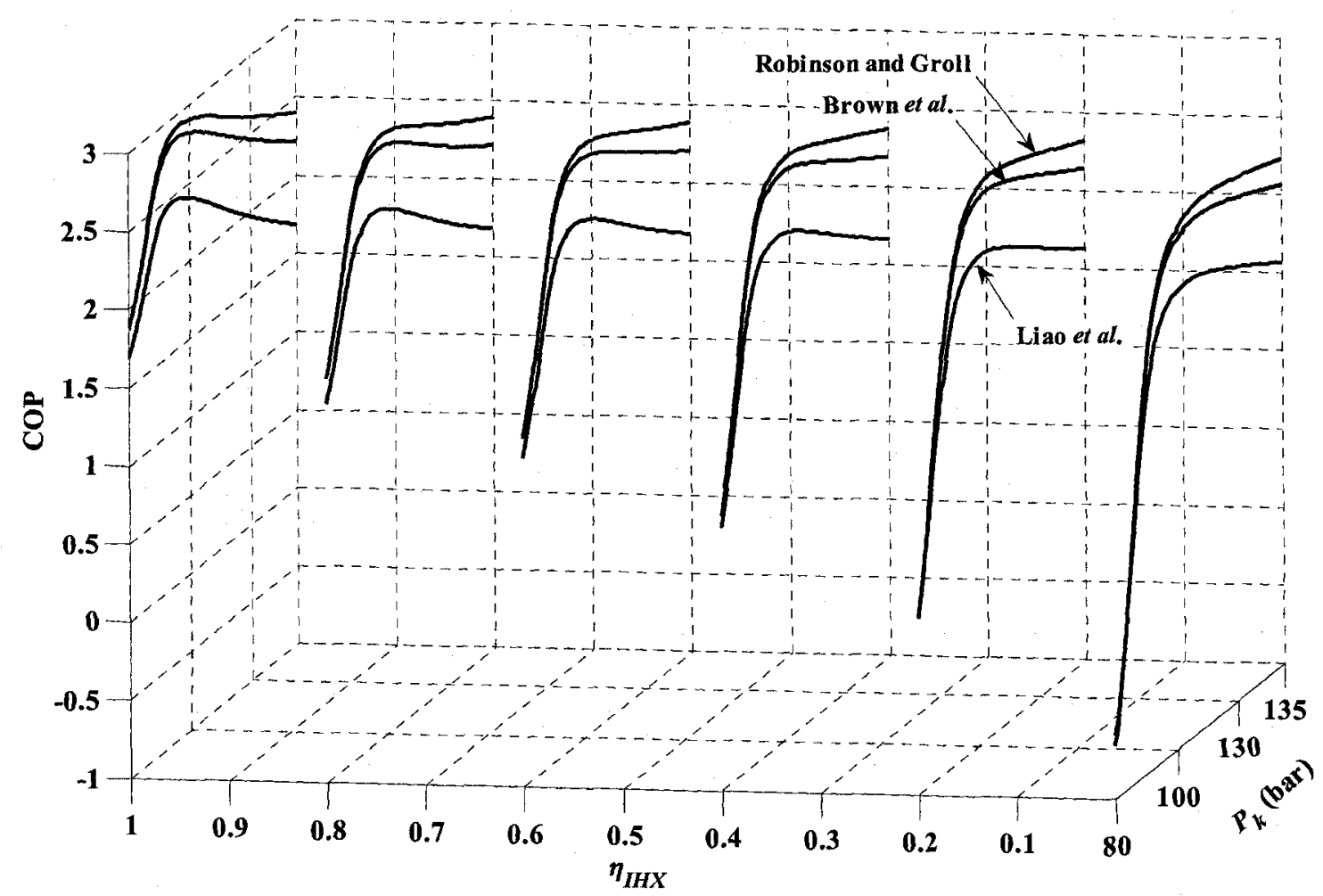

Figure 7-4 Three-dimensional profile of COP curves under different cooling pressures and IHX efficiencies for $t_{1}=5.3^{\circ} \mathrm{C}\left(P_{e}=40 \mathrm{bar}\right), x=0.8$, and $t_{a m b}=35^{\circ} \mathrm{C}$.

\subsubsection{Potential heat transfer capacity}

Because the IHX effectiveness changes the potential heat transfer capacity proportionally, it is fixed at 1 in this section. Figure 7-5 shows how the potential specific heat transfer changes with the high pressure, the inlet quality of IHX, and the ambient temperature. The straight lines that represent $q_{p o t n}$ are determined by $\left(h_{l^{\prime}, \max }-h_{1}\right)$, which is not related to $P_{k}$. The curves mean that $q_{p o t n}$ is determined by $\left(h_{3}-h_{3^{\prime}, \text { min }}\right)$, which describes how $h_{3}$ changes with $P_{k}$ along the isothermal line. In the $x$-direction, when a greater portion of the liquid leaves the evaporator, $\left(h_{1}, \max -h_{1}\right)$ increases, leading 
to an increase in $q_{p o t n}$ too. For $x \geq 0.95, q_{p o t n}$ is determined by $\left(h_{1}, \max -h_{1}\right)$. In the $P_{k}$ direction, some turning points exist, which show the change from $\left(h_{1, \text { max }}-h_{1}\right)$ to $\left(h_{3^{-}}-h_{3^{\prime}, \min }\right)$. In the $q_{\text {potn }}$ direction, a higher ambient temperature moves point 3 toward the right, increasing $q_{\text {potn }}$ accordingly.

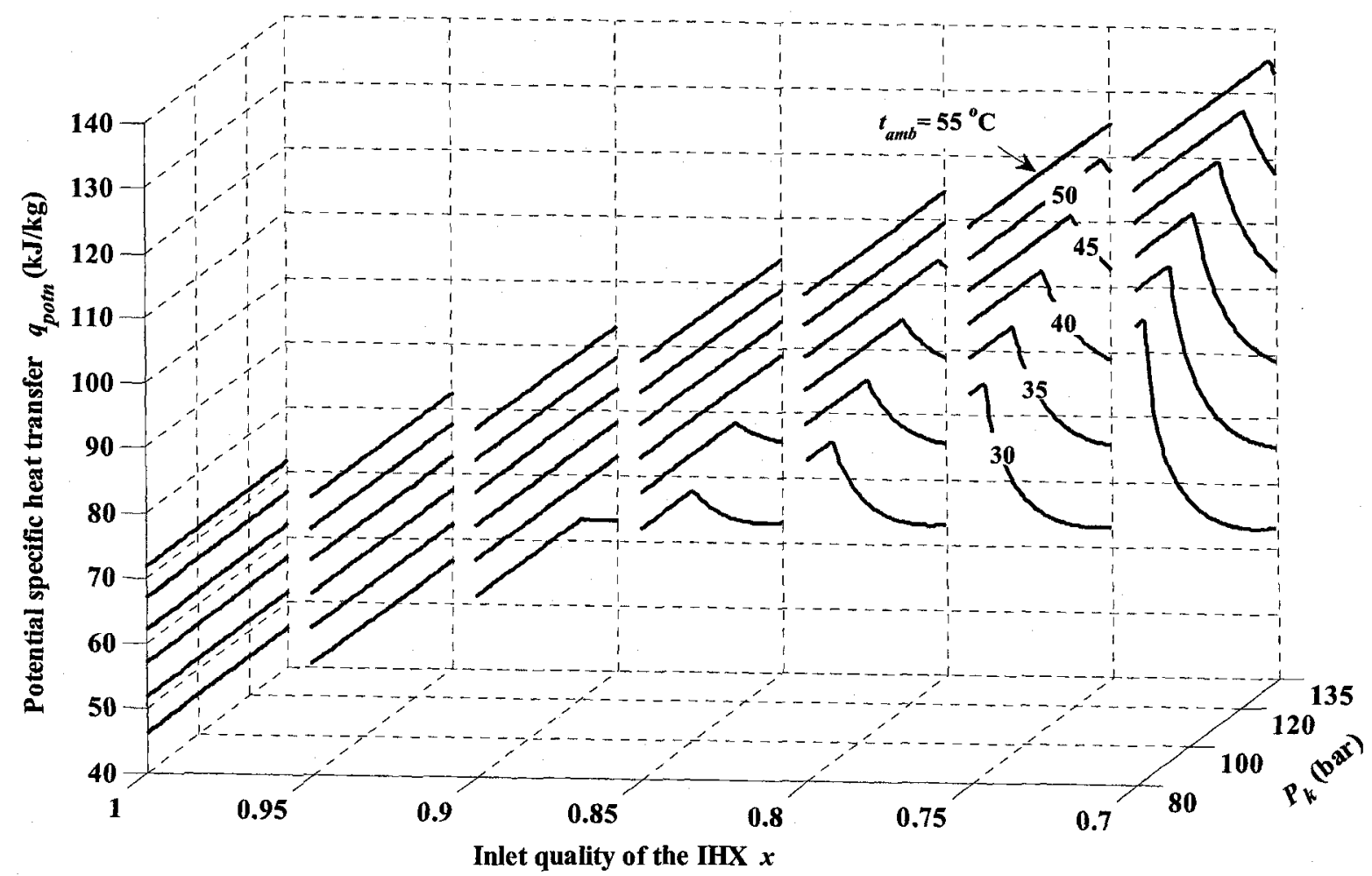

Figure 7-5 The relationship between $q_{p o t n}, P_{k}$, and $x$ under different ambient temperatures for $P_{e}=40$ bar and $\eta_{I H X}=1$.

In order to further investigate the characteristics of the turning points, the evaporation temperature was also changed to provide a wide parameter background. These simulation results are shown in Figure 7-6. To get a close-up of the figure, a projection of the data at $x=0.8$ has been put into Figure 7-7. For each line, the below region means $q_{p o t n}$ is determined by $\left(h_{1, \text { max }}-h_{1}\right)$, and for a given $t_{a m b}$ the $q_{p o t n}$ remains the same. For example, when $t_{l}=5{ }^{\circ} \mathrm{C}$ and $t_{a m b}=40{ }^{\circ} \mathrm{C}$, then $P_{k, \text { turn }}=118$ bar; wherever $P_{k}$ is when it is between 80 bar $\sim 118$ bar, $q_{\text {potn }}$ will remain constant at $100.21 \mathrm{~kJ} / \mathrm{kg}$. The region above each line means $q_{\text {potn }}$ is determined by $\left(h_{3}-h_{3}{ }^{\prime}, \min \right)$. Therefore, those 
lines can be regarded as

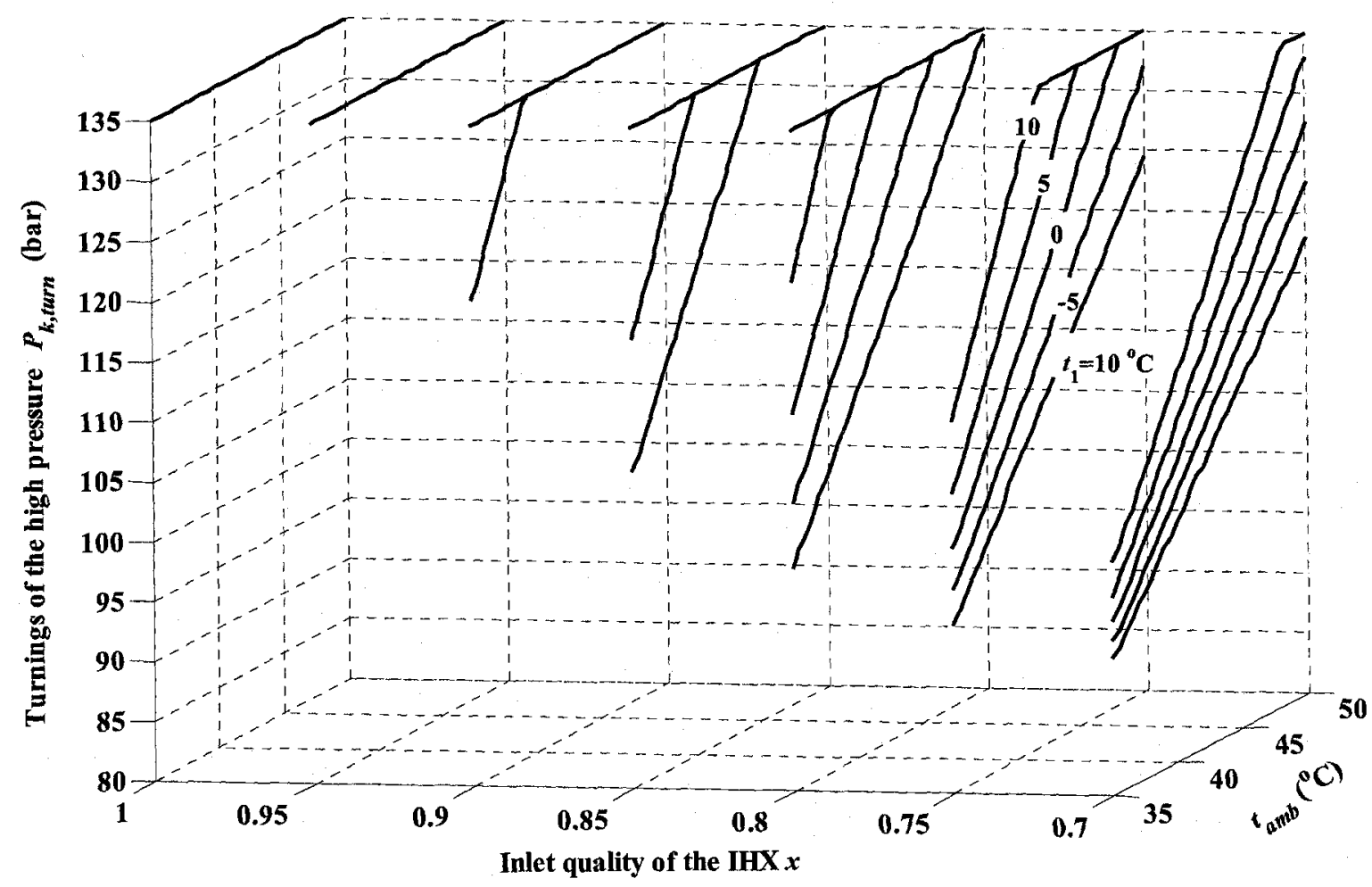

Figure 7-6 The high pressure turnings vs. $x$ and $t_{a m b}$ under different evaporating temperatures for $\eta_{I H X}=1$.

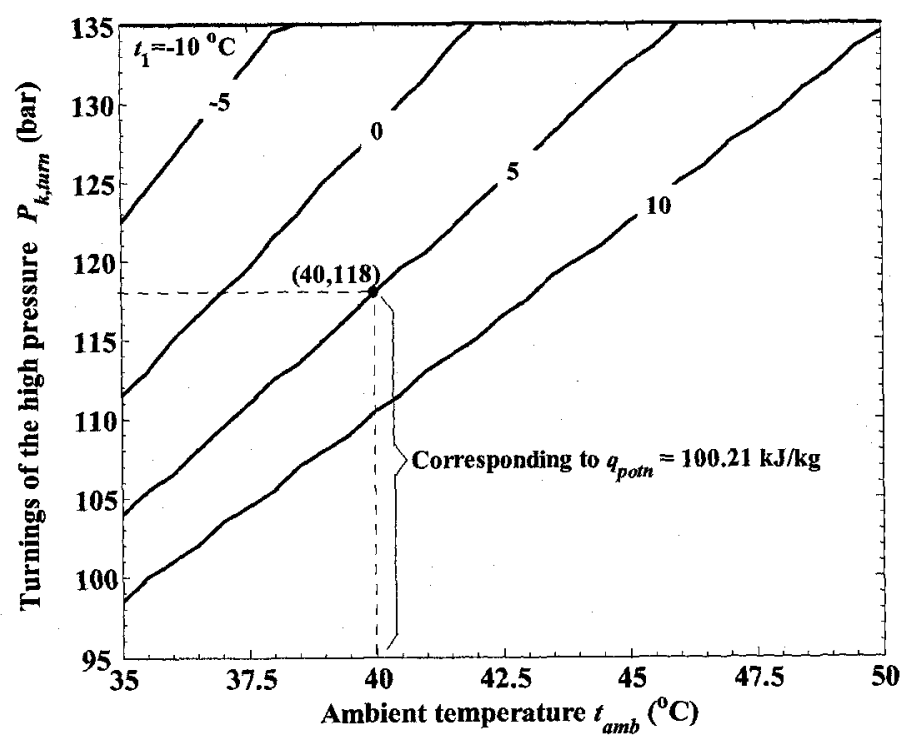

Figure 7-7 Two-dimensional illustration of the turning of the high pressure for $x=0.8$.

transit lines of $q_{p o t n}$ as well. From Figure 7-6 it can be seen that $P_{k, \text { tum }}$ increases as $x$ increases. For 
$x \geq 0.95$ and $t_{a m b}$ in the given range, $q_{p o t n}$ is determined by the enthalpy difference at the low pressure side. In this case, the specific heat does not change significantly, and the maximum temperature difference in the IHX is $\left(t_{1},-t_{1}\right)$. The heat transfer effectiveness $(7-3)$ can be thus deduced to the traditionally-used expression in terms of temperature differences.

\subsubsection{Influence of IHX effectiveness and the ambient temperature}

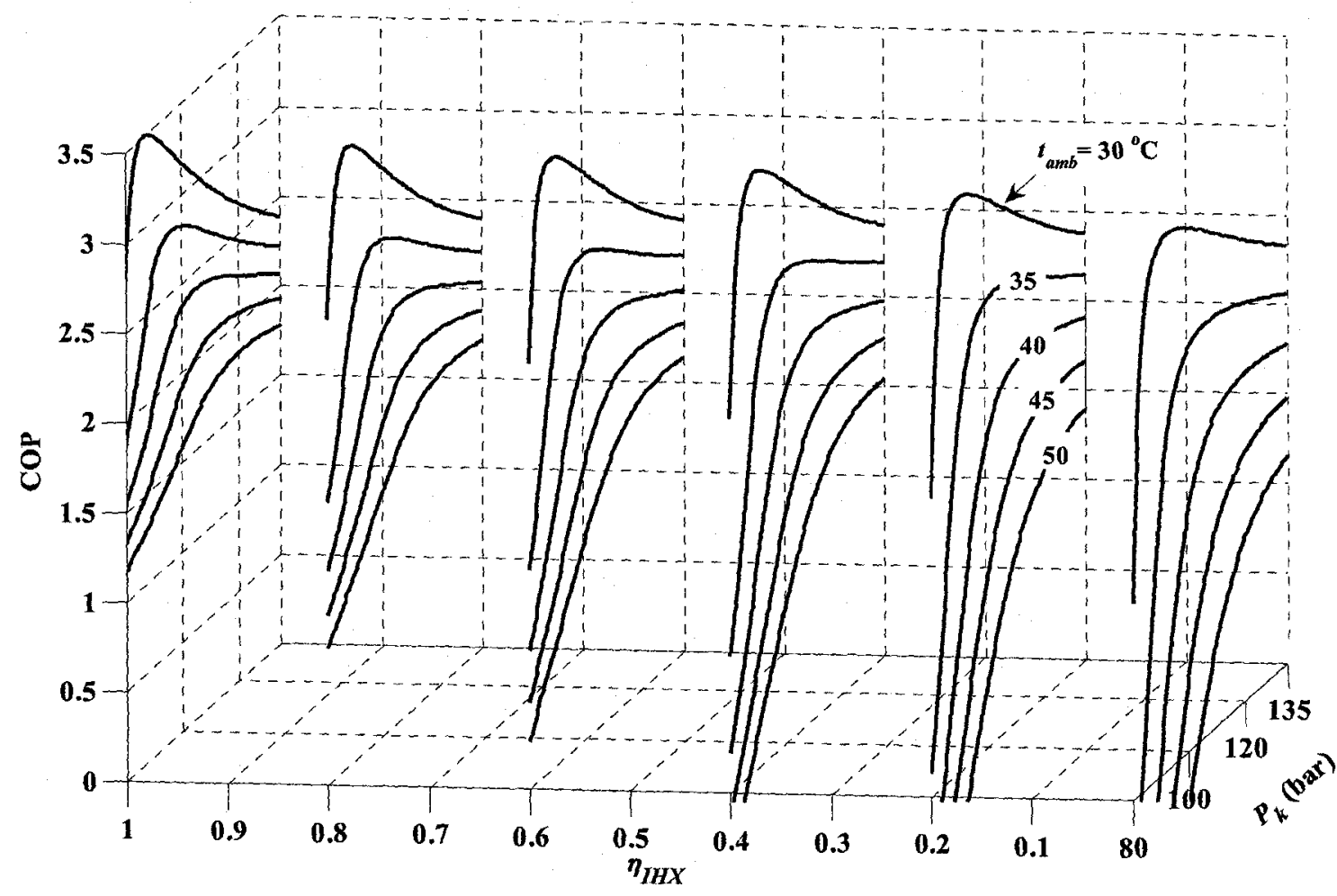

Figure 7-8 Three-dimensional profiles of COP under different high pressures and IHX efficiencies for $P_{e}=40$ bar, $x=0.8$, and $\eta_{i s}=0.9343-0.04478$.

Figure 7-8 shows the influence of $\eta_{I H X}$ and $t_{a m b}$ on COP. Along the $\eta_{I H X}$ direction, when $t_{a m b}$ and

$P_{k}$ are fixed, the system performs better with high $\eta_{I H X}$. This is the result of a large increase in specific cooling capacity and a small increase of specific work consumption. As $\eta_{I H X}$ approaches 0 (i e there is no IHX in system), the COP at $P_{k}=80$ bar is smaller than 0 , implying that there is no 
cooling capacity generated by the system. Therefore, an IHX is indispensable to ensure an acceptable COP.

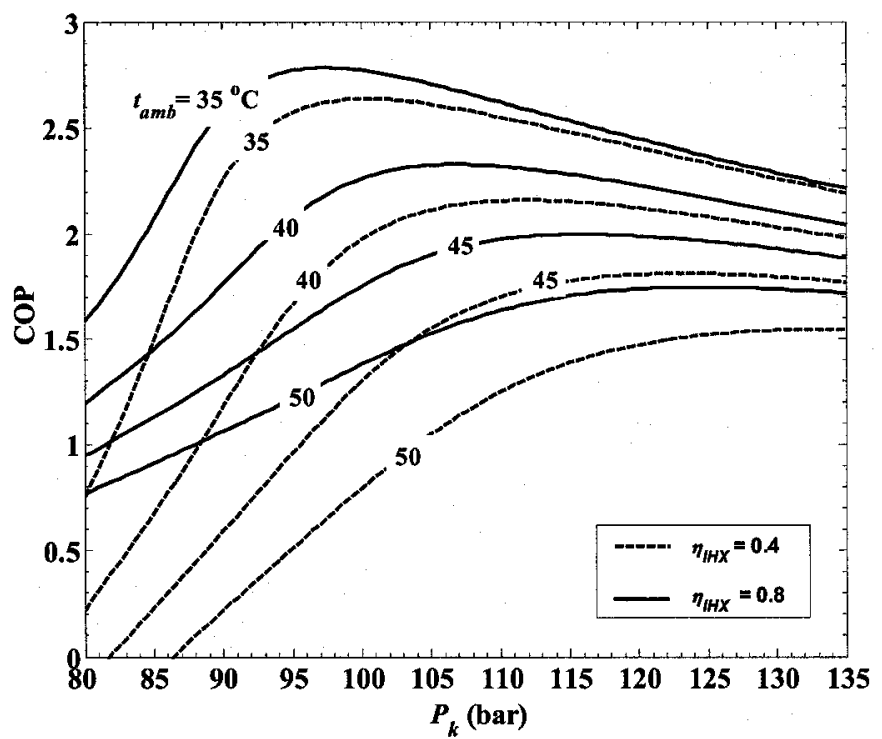

Figure 7-9 Comparison of COP curves under different high pressures and IHX efficiencies for $P_{e}=40$ bar, $x=0.8$, and $\eta_{i s}=0.9343-0.04478 r$.

To investigate the effect of $t_{a m b}$, two sets of data were extracted from Figure 7-8 and plotted in Figure 7-9. Three conclusions can be drawn from the figure: First, high ambient temperature leads to a low COP. In this case, an increase in $t_{a m b}$ represents a decrease in enthalpy $h_{3}$, and consequently a decrease in $q_{0}$. On the other hand, the work consumption of the compressor increases with higher $t_{a m b}$. As a result, the COP decreases. Second, $P_{k, \text { opt }}$ rapidly goes up with high $t_{a m b}$. Because the compressor work is approximately linear to the $t_{a m b}$, the COP change directly reflects the enthalpy change of $h_{3}$. Due to the sharp thermophysical property variations in the region just above the transcritical pressure, a small increase in ambient temperature will cause a large decrease in $h_{3}$. The high pressure that corresponds to the most intensive property change increases fast when $t_{a m b}$ increases. Further increases in $P_{k}$ lead to mild enthalpy changes with $t_{a m b}$, so the increase in COP is 
nearly proportional to the decrease in $t_{a m b}$. This is illustrated by the linear variance of COP at high $P_{k}$ near 135 bar. Finally, $P_{k, o p t}$ decreases with high $\eta_{I H X}$. To show how $\eta_{I H X}$ affects $P_{k, \text { opt }}$, examine $t_{a m b}=40{ }^{\circ} \mathrm{C} ; P_{k, o p t}$ drops from 121.25 bar to 105.00 bar while $\eta_{I H X}$ increases from 0 to 1 . Since low $P_{k}$, will decrease the pressure ratio, improve the compressor performance and safety of the system operation, a well-designed IHX is a very important factor in achieving high system performance.

\subsubsection{Influence of the IHX inlet quality}

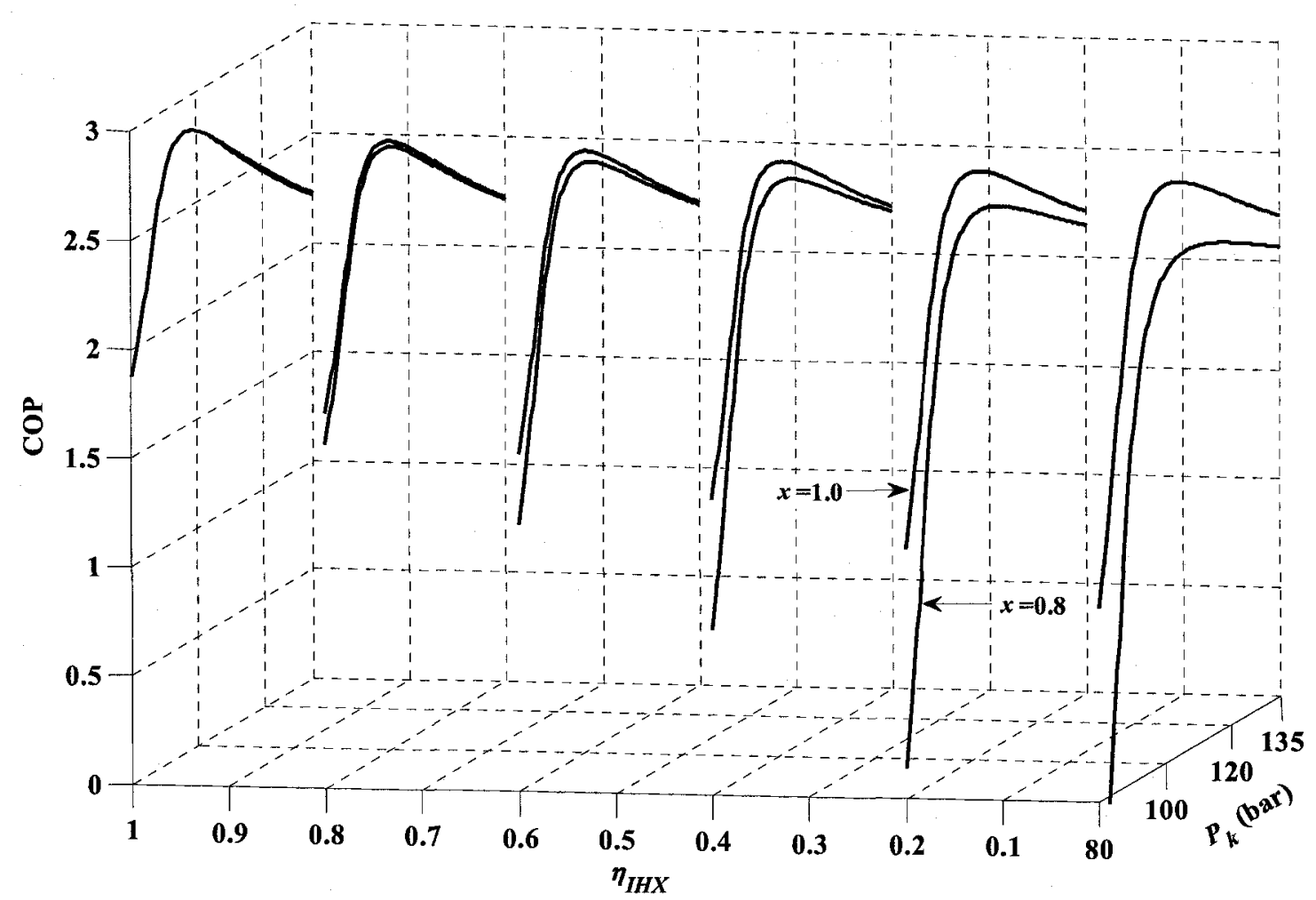

Figure 7-10 Three-dimensional profiles of COP under different high pressures and IHX efficiencies for $P_{e}=40 \mathrm{bar}, t_{a m b}=35^{\circ} \mathrm{C}$, and $\eta_{i s}=0.9343-0.04478 r$.

For an evaporator, the best design is to let the refrigerant exit at $x=1.0$ so as to fully utilize the latent heat of the refrigerant. However, variance of working conditions will affect the evaporator outlet quality; and for the consideration of size and weight in some applications, especially in the 
automotive industry, the evaporator is not big enough to evaporate all of the liquid. Thus the influence of IHX inlet quality should be taken into account. Figure 7-10 shows how COP varies with two variants, the IHX effectiveness and high-side pressure. If there is no IHX in the system, COP decreases largely, and the corresponding optimum high pressure changes greatly. Because of the decrease in specific cooling capacity, the system performance is lowered by smaller $x$, but this could be compensated by a high IHX effectiveness. For high $\eta_{I H X}$, the two COP curves are very similar, which means COP is not sensitive to $x$. This implies that the IHX acts as a performance stabilizer, and the $P_{k, \text { opt }}$ is easier to control as the variations in its magnitude are minimized.

\subsubsection{Influence of evaporating temperature}

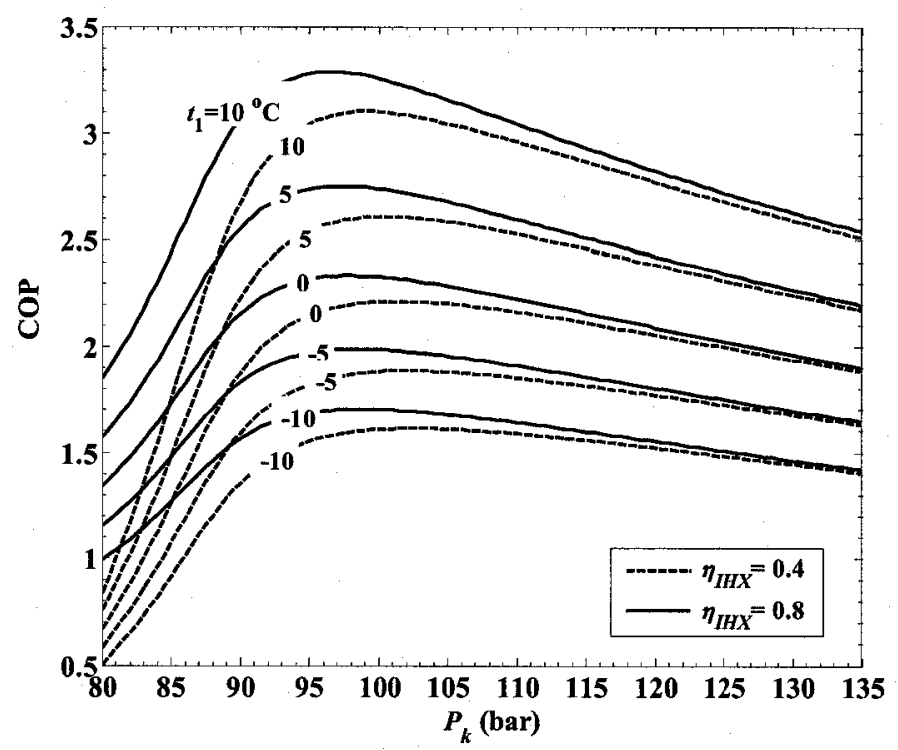

Figure 7-11 Influence of high pressure on COP with varying evaporating temperature for $x=0.8, t_{a m b}=35^{\circ} \mathrm{C}$, and $\eta_{i s}=0.9343-0.04478 r$.

In Kauf's research (1999) the evaporating temperature is shown to be less strongly related to $P_{k, o p t}$. To test whether this is true while considering an IHX and a non-saturated refrigerant at the 
evaporator outlet, the inlet quality is fixed at 0.8 , the ambient temperature at $35^{\circ} \mathrm{C}$, the $\mathrm{IHX}$ effectiveness at 0.4 and 0.8 separately, and the evaporation temperature varies from $-10{ }^{\circ} \mathrm{C} \sim 10^{\circ} \mathrm{C}$. The results are shown in Figure 7-11. For a given $\eta_{I H X}$, though COP differs over a wide range, they peak at almost the same high pressure. Another simulation was performed for $x=1$ while the other conditions remained unchanged. Results show that the optimum high pressure is also nearly independent of $t_{1}$; the biggest deviation of $P_{k, \text { opt }}$ is $0.20 \mathrm{MPa}$, and the relative error is $1.99 \%$.

\subsubsection{Mathematical description of the optimum high pressure}

Table 7-1 shows the optimum high pressures under different $x, t_{a m b}$, and $\eta_{I H X}$ for $t_{1}=5.3{ }^{\circ} \mathrm{C}$ and $\eta_{c o m p}=0.9343-0.04478 r$. To find the optimum high pressure at low $x$, high $t_{a m b}$, and low $\eta_{I H X}$, the range of $P_{k}$ has been expanded to include 80 bar $\sim 165$ bar. Using best-fitting techniques a correlation can be obtained

$$
\begin{aligned}
& P_{k, o p t}=A t_{a m b}+B \\
& \text { where } \quad A=A_{1} \eta_{I H X}^{3}+A_{2} \eta_{I H X}^{2}+A_{3} \eta_{I H X}+A_{4} \\
& B=B_{1} \eta_{I H X}^{3}+B_{2} \eta_{I H X}^{2}+B_{3} \eta_{I H X}+B_{4} \\
& A_{1}=-54.591 x^{3}+141.87 x^{2}-121.93 x+34.627 \\
& A_{2}=74.521 x^{3}-190.81 x^{2}+160.97 x-44.632 \\
& A_{3}=-23.574 x^{3}+577.57 x^{2}-45.691 x+11.42 \\
& A_{4}=3.0066 x^{3}-7.1147 x^{2}+5.1364 x-0.79788 \\
& B_{1}=2024.5 x^{3}-5278.4 x^{2}+4555.8 x-1301.2
\end{aligned}
$$




$$
\begin{aligned}
& B_{2}=-2831.8 x^{3}+7303.2 x^{2}-6220.7 x+1747.8 \\
& B_{3}=970.39 x^{3}-2437.1 x^{2}+2000.6 x-531.47 \\
& B_{4}=-119.51 x^{3}+294.89 x^{2}-230.94 x+57.489
\end{aligned}
$$

\begin{tabular}{|c|c|c|c|c|c|}
\hline$t_{a m b}\left({ }^{\circ} \mathrm{C}\right)$ & 30 & 35 & 40 & 45 & 50 \\
\hline \multicolumn{6}{|l|}{ (a) $x=0.7$} \\
\hline$\eta_{I H X}=0$ & 96.50 & 113.00 & 130.25 & 148.00 & 164.50 \\
\hline$\eta_{I H X}=0.2$ & 92.25 & 105.75 & 119.25 & 133.25 & 148.50 \\
\hline$\eta_{I H X}=0.4$ & 89.50 & 101.00 & 112.25 & 123.50 & 134.25 \\
\hline$\eta_{I H X}=0.6$ & 88.00 & 98.75 & 108.50 & 117.50 & 125.50 \\
\hline$\eta_{\text {IHX }}=0.8$ & 87.25 & 96.50 & 104.50 & 112.50 & 122.25 \\
\hline$\eta_{I H X}=1.0$ & 86.25 & 94.25 & 102.50 & 112.00 & 120.75 \\
\hline \multicolumn{6}{|l|}{ (b) $x=0.8$} \\
\hline$\eta_{\text {IHX }}=0$ & 92.50 & 106.75 & 121.25 & 136.25 & 152.00 \\
\hline$\eta_{I H X}=0.2$ & 90.25 & 103.00 & 115.50 & 128.50 & 141.75 \\
\hline$\eta_{I H X}=0.4$ & 89.00 & 100.50 & 112.00 & 123.00 & 134.25 \\
\hline$\eta_{I H X}=0.6$ & 88.00 & 98.50 & 109.00 & 119.25 & 128.75 \\
\hline$\eta_{I H X}=0.8$ & 87.00 & 97.25 & 106.75 & 116.00 & 124.50 \\
\hline$\eta_{\text {IHX }}=1.0$ & 86.50 & 96.25 & 105.00 & 113.00 & 120.75 \\
\hline \multicolumn{6}{|l|}{ (c) $x=0.9$} \\
\hline$\eta_{I H X}=0$ & 90.25 & 102.50 & 115.25 & 128.25 & 140.75 \\
\hline$\eta_{I H X}=0.2$ & 88.75 & 100.75 & 112.25 & 124.00 & 135.75 \\
\hline$\eta_{I H X}=0.4$ & 88.25 & 99.00 & 110.00 & 120.50 & 131.00 \\
\hline$\eta_{I H X}=0.6$ & 87.50 & 98.00 & 108.00 & 117.75 & 127.00 \\
\hline$\eta_{I H X}=0.8$ & 87.00 & 97.00 & 106.50 & 115.25 & 123.75 \\
\hline$\eta_{I H X}=1.0$ & 86.50 & 96.25 & 105.00 & 113.00 & 120.75 \\
\hline \multicolumn{6}{|l|}{ (d) $x=1.0$} \\
\hline$\eta_{I H X}=0$ & 88.50 & 100.00 & 111.50 & 123.25 & 134.50 \\
\hline$\eta_{I H X}=0.2$ & 88.00 & 99.00 & 109.75 & 120.25 & 131.00 \\
\hline$\eta_{\text {IHX }}=0.4$ & 87.50 & 98.00 & 108.25 & 118.25 & 128.00 \\
\hline$\eta_{\text {IHX }}=0.6$ & 87.25 & 97.25 & 107.00 & 116.25 & 125.50 \\
\hline$\eta_{\text {IHX }}=0.8$ & 86.75 & 96.75 & 106.00 & 114.75 & 123.00 \\
\hline$\eta_{\text {IHX }}=1.0$ & 86.50 & 96.25 & 105.00 & 113.00 & 120.75 \\
\hline
\end{tabular}

Table 7-1 $P_{k, o p t}$ (bar) vs. $x, \eta_{I H X}$, and $t_{a m b}=35^{\circ} \mathrm{C}$ for $\eta_{i s}=0.9343-0.04478 r$.

$P_{k}=80$ bar $\sim 165$ bar 
For $\eta_{I H X}=0 \sim 1.0, x=0.7 \sim 1.0, t_{1}=5.3^{\circ} \mathrm{C}, t_{a m b}=30^{\circ} \mathrm{C} \sim 50^{\circ} \mathrm{C}$, the model predicts the optimized high pressure with a satisfactory deviation of less than $0.94 \%$. If the range of $t_{1}$ is increased to $-10^{\circ} \mathrm{C} \sim 10^{\circ} \mathrm{C}$ and other conditions are kept the same (providing a wider application range for engineering practice), the maximum deviation goes up to $3.6 \%$.

Define $\eta_{I H X}=0$ and $x=1$, Equation (7-20) will be simplified to

$$
P_{k, o p t}=0.2304 t_{a m b}+1.929
$$

Compared with the slope 2.6 that was calculated by Kauf (1999), the value of 0.2304 in (7-21) is quite small. There are two main reasons. One is the different ways of defining the compressor efficiency. Expression (7-18) is used in this chapter, which might not be the one used by Kauf (1999) as different compressors behave in different ways. The other reason is the adoption of the gas cooler approach temperature. Kauf (1999) fixed $\left(t_{3}-t_{a m b}\right)$ to be $2.9{ }^{\circ} \mathrm{C}$; in practice its value varies with different working conditions. Due to this, expression (7-16) was adopted in this chapter. If $2.9^{\circ} \mathrm{C}$ is used as the approach temperature, the following expression can be obtained, which is quite close to the one by Kauf (1999).

$$
P_{k, o p t}=2.68 t_{a m b}+0.975=2.68 t_{3}-6.797
$$

To further validate the model, a comparison between simulation results and available data from Brown et al. (2002) and Pfafferott and Schmits (2004) was performed. The results are shown in Figure 7-12. The data set 1 are from Brown et al. (2002) and point A of experimental data 2 is from Pfafferott and Schmits (2004). Fair agreements can be observed from the figure. The differences between simulated curve 1 and data set 1 for $t_{a m b}=32.2{ }^{\circ} \mathrm{C}, 43.3{ }^{\circ} \mathrm{C}$ and $48.9{ }^{\circ} \mathrm{C}$ are $4.8 \%, 3.4 \%$ and $2.4 \%$, respectively. These deviations might be due to the constant $\eta_{I H X}, P_{k}$ and $P_{e}$ used in the 
calculation; in practice they change under different working conditions. From correlation (7-20) the $P_{k, o p t}$ is 111.64 bar for $t_{a m b}=43.3^{\circ} \mathrm{C}$, which means the system runs near its highest performance state. The most significant deviation in Figure 7-12 occurs at the experimental datum point A. The high COP value of 3.310 can be attributed to the abnormally small discharge enthalpy that corresponds

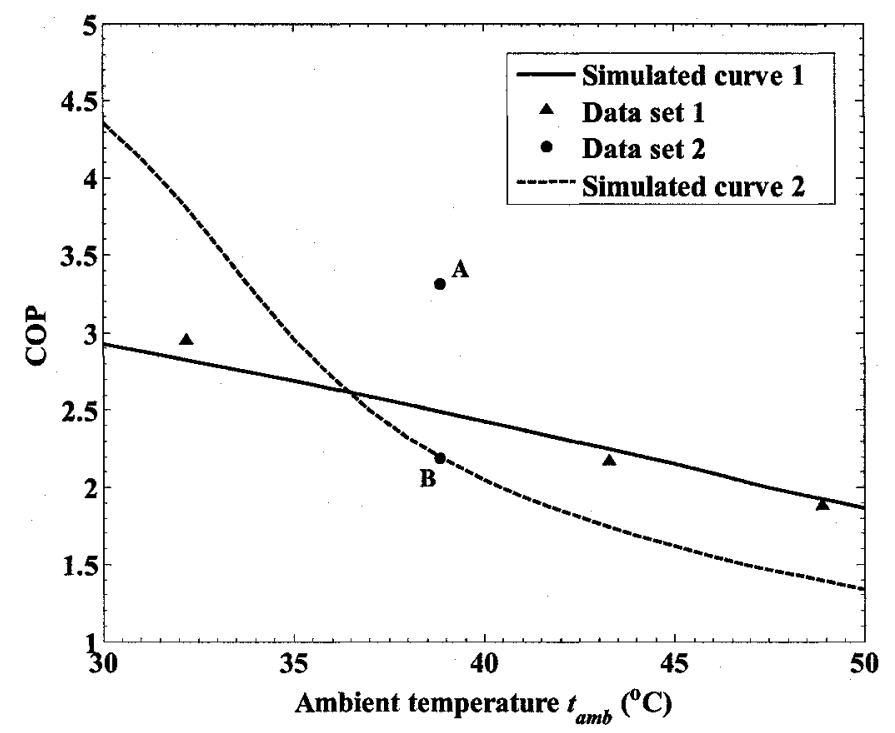

Figure 7-12 Comparison between simulation results and experimental data

(a) curve 1 and data 1 are generated for $P_{k}=117.0 \mathrm{bar}, P_{e}=42.21 \mathrm{bar}, x=0.93$, and $\eta_{I H X}=0.8$;

(b) curve 2 and data 2 are generated for $P_{k}=87.22$ bar, $P_{e}=46.04$ bar, $x=0.80$, and $\eta_{I H X}=0.9$

to small compression work consumption. The discharge entropy is $1.911 \mathrm{~kJ} /(\mathrm{kg} \cdot \mathrm{K})$, which is slightly smaller than the suction entropy of $1.921 \mathrm{~kJ} /(\mathrm{kg} \cdot \mathrm{K})$. This is normally impossible because the compression process is irreversible and thus will cause entropy generation. If Pfafferott and Schmits's (2004) isentropic efficiency is used to calculate the discharging entropy instead, the entropy increases to $1.948 \mathrm{~kJ} /(\mathrm{kg} \cdot \mathrm{K})$, and the corresponding COP is 2.191 , which is shown as point B in the figure. Through the comparison of experimental data and simulation results of Pfafferott and Schmits (2004), point B in the figure is more credible than point A. It can be seen that point B agrees well with the simulation result from this work. In this case, the optimum high pressure can 
be calculated by correlation (7-20); the value of 103.26 bar is much higher than the measured high pressure 87.22 bar. Thus, the system performance can be further improved by increasing the $P_{k}$.

\subsubsection{Relationship between IHX effectiveness and compressor discharge} temperature

From discussions in section 7.3.3, one can see the importance of a well-designed high-effectiveness IHX. However, system performance is also affected by other factors. High $\eta_{I H X}$ results in high suction temperature if other conditions are unchanged; the discharge temperature will increase quickly as well. This situation may be improved by lowering $t_{3}$, i.e. decreasing the temperature approach. The $\left(t_{3}-t_{a m b}\right)$ value is relatively high when $t_{a m b}$ is low (Brown $e t$ al., 2002), and thus if the value decreases, the suction temperature will decrease as well. Combined with what was stated in section 7.3.3, one can safely conclude that the best situation is to use a high-effectiveness IHX in a system that works at as a low $P_{k}$ as possible and a relatively low evaporating temperature. If $t_{e}$ is not favourable, a high discharge temperature might cause serious problem for compressor safety.

\subsection{Conclusions}

An IHX with high effectiveness is a very important factor for a transcritical $\mathrm{CO}_{2}$ system to achieve high COP. IHX can enhance the system performance and make it less sensitive to the quality at the evaporator outlet. A proper selection of IHX effectiveness is of great importance. When $t_{l}, t_{a m b}$ and $P_{k}$ are low, high $\eta_{I H X}$ is preferred; the compressor discharging temperature becomes an issue if $t_{1}$ is too high, hence $\eta_{I H X}$ needs to be chosen carefully. Because phase change 
might exist in an IHX, and the specific heat changes drastically in the region just above the critical pressure, traditional expressions used to describe the heat exchange effectiveness are no longer suitable. A practical effectiveness expression for IHX was used in this chapter based on enthalpy difference.

Detailed analysis on the relationship between the optimum high pressure $P_{k, o p t}$ and $t_{l}, t_{a m b}, \eta_{I H X}$, $\eta_{c o m p}$ was performed. Evaporating temperature has little influence on $P_{k, o p t}$. Based on simulation data, a correlation of $P_{k, o p t}$ was developed that can better predict the simulation values with a deviation of less than $3.6 \%$ in the whole range and $0.94 \%$ for $t_{l}=5.3^{\circ} \mathrm{C}$. It is worth mentioning that the correlation is applicable for a system with an oil separator whose compressor can be described by correlation (7-18). Different compressors will generate different performances, and the accuracy of the correlation will change accordingly. It is also important to note that the mechanical efficiency of the compressor $\eta_{\text {mech }}$ was not included in analysis. For most cases $\eta_{\text {mech }}$ changes little and is thus considered a constant. This will only modify the COP values proportionally but leave the $P_{k, o p t} \mathrm{~s}$ unchanged. 


\section{Chapter 8}

\section{Conclusions and future work}

This thesis has presented theoretical and experimental work on capillary flow behaviour in refrigeration systems with both low and high mass flux. AXE, as a new device harnessing the advantages of capillary flow, has been investigated particularly. While details have been shown in previous segregated chapters, it is important to look at the integrated findings and their impacts on our understanding of the flow mechanism. The main conclusions of the study are summarized as follows.

\subsection{Conclusions}

As an improvement on traditional internal heat exchangers, the newly-introduced multifunctional device, the accumulator-heat-exchanger-expander (AXE), could not only provide a COP improvement but also simplify system structure and maintenance. It can also stabilize the system pressures. As the first and most important step of investigating the AXE, its influence on an R134a-driven AAC cycle was investigated and compared to a cycle without AXE; a COP increase of $4.5 \sim 19.0 \%$ was found. The inside capillary flow model was fully established and verified for both refrigerators, where the mass flow rate is in the magnitude of $2 \sim 20 \mathrm{~kg} / \mathrm{h}$, and AAC systems, where the mass flow rate is $100 \sim 150 \mathrm{~kg} / \mathrm{h}$. The system shifts from subcritical to transcritical when the refrigerant is changed to the environmentally-friendly carbon dioxide, so some new features are 
introduced accordingly. The most important is that an optimum high pressure exists for a given working condition, which was modeled with three parameters. As a preliminary work, the behaviour of AXE in a $\mathrm{CO}_{2}$ cycle was studied. With a $0.5 \mathrm{~m}$ capillary tube, the recovered heat was simulated to be around $500 \mathrm{~W}$, which means a $7.1 \%$ improvement in cooling capacity. The following sections detailed the conclusions that can now be made.

\subsubsection{Adiabatic and non-adiabatic capillary flow}

In Chapter 3, an adiabatic simulation model (ACAM) and a non-adiabatic one (NACAM) were established and verified. ACAM can predict most experimental data with uncertainties of less than $\pm 7.5 \%$; it under-predicts by as much as $12.9 \%$ when the system flow rate is between $3.5 \mathrm{~kg} / \mathrm{h}$ and $4.5 \mathrm{~kg} / \mathrm{h}$. NACAM is able to simulate the mass flow rate of lateral heat exchangers with uncertainties of less than $\pm 10.3 \%$, and for a concentric configuration the deviation is less than $\pm 20.0 \%$. ACAM can be considered as a special case of NACAM where the overall thermal conductance is cut down to zero.

For adiabatic flow, single-phase length and the subcooling degrees are two main criteria in determining refrigerant flow rate. Changes to the inner diameter, condensation pressure, and surface roughness influence the length of the single-phase region and then the mass flow rate. In non-adiabatic conditions, mass flow rate increases due to the heat dissipation from the capillary flow to the suction line flow, while the temperature difference between them does not necessarily decrease. This can be attributed to the change in the specific heat transfer rate, which is 
co-determined by both the momentum and the energy balances.

The capillary inner diameter and the subcooling degrees are two main factors affecting the mass flow rate for both ACAM and NACAM. Thermal factors, flow arrangement, and heat exchanger type (NACAM only) affect the mass flow rate mildly; proper selections should be made so as to achieve better device performance.

\subsubsection{Changing trend of pressures for systems with and without AXE}

In Chapter 4 and Chapter 5, experimental work on an R134a-based AAC test stand was carried out. Relationships between the system pressure change and the compressor speed were correlated with and without presence of an AXE. To obtain the exact values of suction and discharge pressures, oil effect was taken into account, where a correlation without considering the critical point and another one improving the accuracy of Grebner's work were both obtained. The correlations can predict experimental results within $\pm 5.0 \%$. It was found they are not overly sensitive to the temperature of passenger compartment; they can be used for various $t_{\text {pass }}$ with good agreement within $\pm 5 \% \times(1+5 \%)= \pm 5.3 \%$ uncertainty.

The correlations are obtained based on a specific test stand; if a new compressor is installed, the mass flow rate will behave in a different way. However, the changing trend of pressures will remain the same. NACAM is capable of predicting both small and large mass flow rate situations after adopting the improved correlation for the two-phase specific volume. It slightly over predicts 
experimental mass flow rates by up to $3.5 \%$, which further validates its applicability as a useful tool in investigating capillary flow for various applications.

A "Quasi-Triangular Rule" was proposed to calculate the effectiveness of the AXE without knowing the measured quality at the capillary outlet.

\subsubsection{AXE behaviour and determination of the optimum high pressure of $\mathrm{CO}_{2}$-based} systems

In Chapter 6, $\mathrm{CO}_{2}$ was introduced as the working fluid in a refrigeration system and its behaviour in an AXE was discussed. Due to the thermodynamic properties of $\mathrm{CO}_{2}$, greater cooling capacity is expected at lower evaporating temperatures. The AXE is capable of recovering sufficient cooling leakage (occurring between the suction line and the environment) to increase the cooling capacity of the system. The inertia drag force was found to have only a minor influence on the flow. As an extreme situation, choking was also addressed. Relationship between the capillary length and multiple other parameters was considered, providing a visual approach in both system design and performance evaluation.

Inspired by its visual advantages and huge information capacity, three-dimensional charts were applied throughout the following Chapter 8 . In this chapter, we were focused on how to determine the optimum high pressure under various working conditions. As the three most influential factors, the capillary inlet temperature, the IHX effectiveness (which also works for AXE), and the suction 
line inlet quality was correlated to $P_{k, o p t}$. The corresponding prediction uncertainty is well controlled (within 3.6\%) for the whole parameter ranges. During the analysis, IHX effectiveness was taken into account for the first time. The results were verified by comparison to other researchers' work in open literature, which makes it a valuable tool in pinpointing the optimum discharge pressure under a specific working condition.

\subsection{Future work}

Though a huge amount of time has been spent on this research, more attractive aspects of the system are emerging accompanied by its many achievements. They are placed into future work as follows.

1. Though NACAM is strong enough in predicting flow behaviour in an AXE, a detailed description of the choking situation can be integrated into the model to make it applicable to all conditions. From the discussion in Chapter 7 it can be seen that when the capillary inner diameter is fairly large, the capillary length increases rapidly, which makes the AXE more like an IHX, and choking might not happen in such case. The improved NACAM will be able to handle this situation, which implies it could also be used as a model for condensers and evaporators.

2. At some specific conditions, re-condensation will occur, which should be avoided to keep the improvement to heat transfer performance. A detailed operating region describing how to 
prevent such phenomenon would be very helpful.

3. The influence of compressor speed on mass flow rate could be normalized using the Buckingham Pi theorem (1914). Substituting it into the pressure correlations will give a more general model.

4. The effect of the ambient temperature on the discharge pressures is implicit in Chapter 4 and Chapter 5 , and needs to be elucidated. The pressures are mainly determined by the temperatures of the ambient and the passenger compartment. An expansion device functions if sufficient pressure drop can be provided and fails if not. Therefore, the pressure loss factor could be modified to contain the influence of ambient temperature.

5. The flow arrangement and exchanger type could be introduced into the transcritical flow model to make it complete. More experimental data needs to be collected to verify and improve the model accuracy. The metastable region could be taken into account using techniques similar to those shown in Chapter 3. It needs to be mentioned that this time the technique will only improve the model accuracy but not enable it to handle a large mass flow rate situation. Because the supercritical $\mathrm{CO}_{2}$ enters the two-phase region from the dome of saturated lines (meaning the specific volume does not change significantly between the supercritical fluid and the two-phase flow), the convergence problems experienced in R134a cycles will not occur. 
6. The influence of compressor speed on the optimum high pressure can be added into the correlation to make it more versatile. This will be especially useful for either AAC systems or other systems equipped with variable displacement compressors.

7. The AXE model could be integrated into the system model so as to obtain an optimum parameter range from a systematic point of view. Due to the inherent difference between subcritical and transcritical cycles, there will be two separate system models.

The above work will not only help us to reach the underlying system physics but also presage a promising future of efficient and ecological life for the world. 


\section{References}

Agrawal, A., Eco-friendly refrigerants in the light of Montreal and Kyoto Protocols, 2002 Purdue Compressor Engineering \& Refrigeration and Air Conditioning Conference, R2-5, 2002.

Ahlborn, B. K., and J. M. Gordon, The vortex tube as a classical thermodynamic refrigeration cycle, Journal of Applied Physics, 88(6) 3645-3653, 2000.

Aprea, C., M. Ascani, F. de Rossi, A criterion for predicting the possible advantage of adopting a suction/liquid heat exchanger in refrigerating system, Applied Thermal Engineering, 19 329-336, 1999.

Aprea, C., F. de Rossi, A. Greco, and C. Renno, Refrigeration plant exergetic analysis varying the compressor capacity, International J. Energy Research, 27 653-669, 2003.

ASHRAE, Air conditioning and refrigeration chronology, ASHRAE archives, 2006.

Bansal, P. K., and R. Krüger, Test standards for household refrigerators and freezers I: preliminary comparisons, International J. of Refrigeration, 18(1) 4-20, 1995.

Bansal, P. K., and A. S. Rupasinghe, An empirical model for sizing capillary tubes, International J. of Refrigeration, 19 497-505, 1996. 


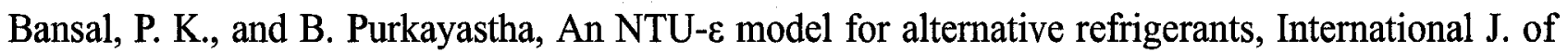
Refrigeration, 21(5) 381-397, 1998.

Bansal, P. K., and A. S. Rupasinghe, A homogeneous model for adiabatic capillary tubes, Applied Thermal Engineering, 18(3-4) 207-219, 1998.

Bansal, P. K., and B. Xu, A parametric study of refrigerant flow in non-adiabatic capillary tubes, Applied Thermal Engineering, 23 397-408, 2003.

Bansal, P. K., and G. Wang, Numerical analysis of choked refrigerant flow in adiabatic capillary tubes, Applied Thermal Engineering, 24 851-863, 2004.

Billiard, F., Use of carbon dioxide in refrigeration and air conditioning, International J. of Refrigeration, 25 1011-1013, 2002.

Bittle, R. R., and M. B. Pate, A theoretical model for predicting adiabatic capillary tube performance with alternative refrigerants, ASHRAE Transactions, 102(2) 52-64, 1996.

Boewe, D., J. Yin, Y. C. Park, C. W. Bullard, P. S. Hrnjak, The role of suction line heat exchanger in transcritical R744 mobile A/C systems, SAE International Congress and Exposition, Detroit, Michigan, Paper No. 1999-01-0583, 1999. 
Bolstad, N. M., and R. C. Jordan, Theory and use of the capillary tube expansion device: Part II, non-adiabatic flow, Refrigeration Engineering, 57(6) 572-583, 1949.

Boyde, S., S. Randles, P. Gibb, S. Corr, P. Dowdle, and A. McNicol. Effect of lubricant properties on efficiency of refrigeration compressors, Proceedings of the 2000 International Compressor Engineering Conference at Purdue, vol.1 311-317, 2000.

Brown, J. S., S. F. Yana-Motta, and P. A. Domanski, Comparative analysis of an automotive air conditioning systems operating with $\mathrm{CO}_{2}$ and R134a, International J. of Refrigeration, 25 19-32, 2002.

Brown, M. W., and P. K. Bansal, An overview of condensation heat transfer on horizontal tube bundles, Applied Thermal Engineering, 19 565-594, 1999.

Brown, M. W., and P. K. Bansal, An elemental NTU- $\varepsilon$ model for vapor-compression liquid chillers, International J. of Refrigeration, 24 612-627, 2001.

Buckingham, E., On physically similar systems: illustrations of the use of dimensional equations, Phys. Rev. 4(4) 345-376, 1914.

Chang, S. D., and S. T. Ro, Pressure drop of pure HFC refrigerants and their mixtures flowing in capillary tubes, International J. Multiphase Flow, 22(3) 551-561, 1996. 
Chen, D., and S. Lin, Underpressure of vaporization of refrigerant R-134a through a diabatic capillary tube, International J. of Refrigeration, 24 261-271, 2001.

Chen, Y., Y. Zhu, W. Geng, and J. Zhang, Simulation and Optimization of the Operation of Gas Compensating Compressor in Refrigerator Working on Double Circuit Cycle, International Conference on Energy Conversion and Application (ICECA 2001), 1108-1113, Wuhan, China, 2001.

Choi, J., Y. Kim, and H. Y. Kim, A generalized correlation for refrigerant mass flow rate through adiabatic capillary tubes, International J. of Refrigeration, 26 881-888, 2003.

Choi, J., Y. Kim, and J. T. Chung, An empirical correlation and rating charts for the performance of adiabatic capillary tubes with alternative refrigerants, Applied Thermal Engineering, 24 29-41, 2004.

Churchill, S. W., Friction-factor equation spans all fluid-flow regimes, Chemical Engineering, 84(7) 91-92, 1977.

Cicchitti, A., C. Lombardi, M. Silvestri, G. Soldaini, and R. Zavalluilli, Two-phase cooling experiments-pressure drop, heat transfer and burnout measurements, Energia Nucleare, 7 407-425, 1960. 
Climate Action Network, Kyoto protocol status of ratification, source link: http://www.climnet.org/EUenergy/ratification/calendar.htm, 2006.

Dickson, T. R., W. Whittle, and M. M. Stobbart, Internal heat exchanger accumulator, US patent No. 6,463, $757 \mathrm{~B} 1,2002$.

Domanski, P. A., D. A. Didion, and J. P. Doyle, Evaluation of suction-line/liquid-line heat exchange in the refrigeration cycle, International J. of Refrigeration, 17(7) 487-493, 1994.

Donkers, R. and M. Vainio, Phase-out of R134a from vehicle air conditioning systems in European Union: latest developments, SAE Automotive Alternative Refrigerant System Symposium, Scoltsdale, Arizona, June 29-July 1, 2004.

Dukler, A. E., M. Wicks, and R. G. Cleveland, Frictional pressure drop in two phase flow-Part A and B, AICHE Journal, 10 38-51, 1964.

ElSherbini, A. I., A. M. Jacobi, and P. S. Hrnjak, Experimental investigation of thermal contact resistance in plain-fin-and-tube evaporators with collarless fins, International J. of Refrigeration, 26 $527-536,2003$.

Fang, X., C. W. Bullard, and P. S. Hrnjak, Heat transfer and pressure drop of gas coolers, ASHRAE Trans, 107 (Part1) 255-266, 2001. 
Fiorelli, F. A. S., A. A. S. Huerta and O. M. Silvares, Experimental analysis of refrigerant mixtures flow through adiabatic capillary tubes, Experimental Thermal and Fluid Science, 26 499-512, 2002.

García, J. M., G. Hernández-Cruz, E. Ramos et al., Visualization of capillary boiling, Experiments in Fluids, 30 359-364, 2001.

García-Valladares, O., C. D. Pérez-Segarra, and A. Oliva, Numerical simulation of capillary-tube expansion devices behaviour with pure and mixed refrigerants considering metastable region. Part I: mathematical formulation and numerical model, Applied Thermal Engineering, 22 173-182, 2002a.

García-Valladares, O., C. D. Pérez-Segarra, and A. Oliva, Numerical simulation of capillary-tube expansion devices behaviour with pure and mixed refrigerants considering metastable region. Part II: experimental validation and parametric studies, Applied Thermal Engineering, 22 379-391, $2002 b$.

García-Valladares, O., Review of numerical simulation of capillary tube using refrigerant mixtures, Applied Thermal Engineering, 24 949-966, 2004.

García-Valladares, O., Numerical simulation of non-adiabatic capillary tubes considering metastable region. Part I: Mathematical formulation and numerical model, International J. of Refrigeration, in press, 2006a. 
García-Valladares, O., Numerical simulation of non-adiabatic capillary tubes considering metastable region. Part II: Experimental validation, International J. of Refrigeration, in press, $2006 b$.

Geiger, W., H. J. Krauss, M. Sickelmann, K. Staffa, C. Walter, H. Mittelstrass, Air conditioner with internal heat exchanger and method of making same, US patent No. 6,434,972 B1, 2002.

Gnielinski, V., New equations for heat and mass transfer in turbulent pipe and channel flow, International Chemical Engineering 16(2) 359-366, 1976.

Graham, D., J. C. Chato, and T. A. Newell, Heat transfer and pressure drop during condensation of refrigerant R134a in an axially grooved tube, International J. Heat and Mass Transfer, 42 1935-1944, 1999.

Grebner, J. J., and R. R. Crawford, The effects of lubricant on evaporator capacity for systems using mixtures of R12/mineral oil and R134a/synthetic oil, ASHRAE Transactions, 99(1) 380-386, 1993.

Gu, B., Y. Li, Z. Wang et al., Analysis on the adiabatic flow of R407C in capillary tube, Applied Thermal Engineering, 23 1871-1880, 2003.

Herman, C., and Z. Travnicek, Cool sound: the future of refrigeration? Thermodynamic and heat transfer issues in thermoacoustic refrigeration, Heat and Mass Transfer, 42 492-500, 2006. 
Hetsroni, G., M., Gurevich, A. Mosyak et al., Drag reduction and heat transfer of surfactants flowing in a capillary tube, International J. of Heat and Mass Transfer, 47 3797-3809, 2004.

Hrnjak, P. S., M. A. Shannon, T. M. Leicht et al., Detection of liquid mass fraction at the evaporator exit of refrigeration systems, International J. of Thermal Science, 40 773-786, 2001.

Hwang Y., and R. Radermacher, Theoretical evaluation of carbon dioxide refrigeration cycle, HVAC\&R Research, 4(3) 245-263, 1998.

Inoue, H., H. Yamasaki, M. Yamanaka, T. Yumoto, H. Nishikawa, Cooling system composed with rolling piston type 2-stage $\mathrm{CO}_{2}$ compressor, SANYO Technical Review, 37(1), 2005.

Itard, L. C. M., Wet compression versus dry compression in heat pumps working with pure refrigerants or non-azeotropic mixtures, International J. of Refrigeration, 18(7) 495-504, 1995.

Jabardo, J.M. Saiz, W. Gonzales Mamani, and M.R. Ianella, Modeling and experimental evaluation of an automotive air conditioning system with a variable capacity compressor, International J. of Refrigeration, 25 1157-1172, 2002.

Joudi, K. A., A. S. K. Mohammed, and M. K. Aljanabi, Experimental and computer performance study of an automotive air conditioning system with alternative refrigerants, Energy Conversion and Management, 44 2959-2976, 2003. 
Judge, J., and R. Radermacher, A heat exchanger model for mixtures and pure refrigerant cycle simulations, International J. of Refrigeration, 20(4) 244-255, 1997.

Kauf, F., Determination of the optimum high pressure for transcritical $\mathrm{CO}_{2}$-refrigeration cycles, International J. of Thermal Science, 38 325-330, 1999.

Kim, M. H., J. Pettersen, and C. W. Bullard, Fundamental process and system design issues in $\mathrm{CO}_{2}$ vapor compression systems, Progress in Energy and Combustion Science, 30 119-174, 2004.

Kim, S. G., M. S. Kim and S. T. Ro, Experimental investigation of the performance of R22, R407C and R410A in several capillary tubes for air-conditioners, International J. of Refrigeration, 25 $521-531,2002$.

Klein, S. A., D. T. Reindl, and K. Brownell, Refrigeration system performance using liquid-suction heat exchangers, International J. of Refrigeration, 23 588-596, 2000.

LaClair, T. J., and I. Mudawar, Thermal transients in a capillary evaporator prior to the initiation of boiling, International J. of Heat and Mass Transfer, 43 3937-3952, 2000.

Li, R. Y., S. Lin, Z. Y. Chen et al., Metastable flow of R12 through capillary tubes, International J. of Refrigeration, 13(3) 181-186, 1990. 
Liang, S. M., and T. N. Wong, Numerical modeling of two-phase refrigerant flow through adiabatic capillary tubes, Applied Thermal Engineering, 21 1035-1048, 2001.

Liao, S.M., T.S. Zhao, and A. Jakobsen, A correlation of optimal heat rejection pressures in transcritical carbon dioxide cycles, Applied Thermal Engineering, 20 831-841, 2000.

Lin, S., C. C. K. Kwok, R. Y. Li et al., Local frictional pressure drop during vaporization of R-12 through capillary tubes, International J. of Multiphase Flow, 17(1) 95-102, 1991.

Liu, Y., and C. W. Bullard, Diabatic flow instabilities in capillary tube-suction line heat exchangers, ASHRAE Transactions, 517-523, 2000.

Liu, Z., and R. H. S. Winterton, A general correlation for saturated and subcooled flow boiling in tubes and annuli, based on a nucleate pool boiling equation, International J. Heat and Mass Transfer 34 (11) 2759-2766, 1991.

Lorentzen, G.., and J. Pettersen, New possibilities for non-CFC refrigeration, in Proceedings of IIR International Symposium on Refrigeration, Energy and Environment, Trondheim, Norway, 147-163, 1992.

Lorentzen, G., and J. Pettersen, A new efficient and environmentally benign system for car air-conditioning, International J. of Refrigeration, 16(1) 4-12, 1993. 
Makizono, K., T. Hirata, Y. Kuroda, and Y. Yamauchi, Accumulator module, US patent No. 6, 530, 230 B2, 2003.

Matsumoto, K., N. Tsuda, H. Yamasaki, K. Sato, M. Tadano, Multistage rotary compressor and refrigeration circuit system, US patent No. 6,748,754 B2, 2004.

McAdams, W. H., W. K. Wood, and R. L. Bryan, Vaporization inside horizontal tubes. II. Benzene-oil mixture, Transaction ASME, 64 193, 1942.

Mei, V. C. and F. C. Chen, Liquid over-feeding air conditioning system and method, US patent No. $5,245,833,1993$

Mei, V. C., and F. C. Chen, Liquid over-feeding refrigeration system and method with integrated accumulator-expander-heat exchanger, US patent No. 5, 622, 055, 1997.

Melo, C., R. T. S. Ferreira, C. Boabaid Neto, J. M. Gonçalves, and M. M. Mezavila, An experimental analysis of adiabatic capillary tubes, Applied Thermal Engineering, 19 669-684, 1999.

Melo, C., J. M. Zangari, R. T. S. Ferreira, and R. H. Pereira, Experimental studies on non-adiabatic of HFC-134a through capillary tubes, in D. R. Tree (Ed.), 2000 International Refrigeration Conference at Purdue, 305-312, 2000. 
Metschies, G. P., International fuel prices $2005,4^{\text {th }}$ Edition, Deutsche Gesellschaft für Technische Zusammenarbeit (GTZ) GmbH, 2005.

Meyer, J. J., and W. E. Dunn, New insights into the behavior of the metastable region of an operating capillary tube, International J. of HVAC\&R Research, 4(1) 105-115, 1998.

Molina, M. J., and F. S. Rowland, Stratospheric sink for chlorofluoromethanes: chlorine atom catalysed destruction of ozone, Nature, 249 810-812, 1974.

Moody, L. F., Friction factors for pipe flow, Transaction of the ASME, 671-684, 1944.

Munkejord, S. T., H. S. Mæhlum, G. R. Zakeri, P. Nekså, J. Pettersen, Micro technology in heat pump systems, International J. of Refrigeration, 25 471-478, 2002.

National Resources Canada, National private vehicle use survey: October 1994-September 1996, summary report, Office of Energy Efficiency, 2000.

National Resources Canada, 2003 summary report: survey of household energy use, 2005.

Navarro-Esbrí, J., R. Cabello, and E. Torrela, Experimental evaluation of the internal heat exchanger influence on a vapour compression plant energy efficiency working with R22, R134a and R407C, Energy, 30 621-636, 2005. 
Ohadi, M. M., S. S. Lee, R. Radermacher et al., Critical review of available correlations for two-phase flow heat transfer of ammonia, International J. of Refrigeration, 19(4) 272-284, 1996.

Panzarella, C. H., S. H. Davis and S. G. Bankoff, Nonlinear dynamics in horizontal film boiling, J. of Fluid Mechanics, 402 163-194, 2000.

Peixoto, R. A., Experimental analysis and numerical simulation of capillary tube-suction line heat exchangers using refrigerant HFC-134a, in $19^{\text {th }}$ International Congress of Refrigeration, 437-444, 1995.

Pérez-Segarra, C. D., J. Rigola, M. Sòria, and A. Oliva, Detailed thermodynamic characterization of hermetic reciprocating compressors, International J. of Refrigeration, 28 579-593, 2005.

Petrov, N. E., and V. N. Popov, Heat transfer and resistance of carbon dioxide being cooled in the supercritical region. Thermal Engineering, 32(3) 131-134, 1985.

Pettersen, J., A. Hafner, and G. Skaugen, Development of compact heat exchangers for $\mathrm{CO}_{2}$ air-conditioning systems, International J. of Refrigeration, 21(3) 180-193, 1998.

Pettersen, J., Flow vaporization in microchannel Tubes, PhD thesis, Norwegian University of Science and Technology (NTNU), 2002. 
Pettersen, J., Flow evaporation of $\mathrm{CO}_{2}$ in microchannel tubes, Experimental Thermal and Fluid Science, 28 111-121, 2004.

Peixoto, R. A., Experimental analysis and numerical simulation of capillary tube-suction line heat exchangers using refrigerant HFC-134a, in: 19th International Congress of Refrigeration 1995, $437-444,1995$.

Preissner, M., B. Cutler, R. Radermacher, C. A. Zhang, Suction line heat exchanger for R134a automotive air-conditioning system, in Proceedings of 2000 International Refrigeration Conference at Purdue, West Lafayette, Indiana, 289 - 294, 2000.

Punčochář, M., and J. Drahoš, Limits of applicability of capillary model for pressure drop correlation, Chemical Engineering Science, 55 3951-3954, 2000.

Purkayastha, B., and P. K. Bansal, An experimental study on HC290 and a commercial liquefied petroleum gas (LPG) mix as suitable replacements for HCFC22, International J. of Refrigeration, 21(1) 3-17, 1998.

Qu, W., and T. Ma, Evaporation heat transfer of steady thin film in micro capillary tubes of micron scale, Heat Transfer-Asian Research, 31(7) 513-523, 2002.

Radermacher, R., and K. Kim, Domestic refrigerators: recent developments, International J. of 
Refrigeration, 19(1) 61-69, 1996.

Ratts, E. B., and J. S. Brown, An experimental analysis of cycling in an automotive air conditioning system, Applied Thermal Engineering, 20 1039-1058, 2000.

Richardson, D. H., H. Jiang, D. Lindsay et al., Optimization of vapor compression systems via simulation, Ninth International Refrigeration and Air Conditioning Conference at Purdue, R1-3, 2002.

Richter, M. R., S. M. Song, J. M. Yin et al., Experimental results of transcritical $\mathrm{CO}_{2}$ heat pump for residential application, Energy, 28 1005-1019, 2003.

Sami, S. M., and H. Maltais, Numerical modelling of alternative refrigerants to HCFC-22 through capillary tubes, International J. of Energy Research, 24 1359-1371, 2000.

Sami, S. M., D. E. Desjardins, and H. Maltais, Prediction of capillary tubes with alternative refrigerants to CFC-502, International J. of Energy Research, 25 1249-1261, 2001.

Sami, S. M., and H. Maltais, Experimental analysis of capillary tubes behaviour with some HCFC-22 alternative refrigerants, International J. of Energy Research, 25 1233-1247, 2001.

Sato, K., K. Matsumoto, H. Yamasaki, A. Tomiuka, K. Fujiwara, K. Yamaguchi, M. Yamanaka, 
Multistage compressor type rotary compressor and cooling device, US patent No. 6,907,746 B2, 2005.

Schwarz, T., M. Galluzzi, R. Radermacher et al., Model to investigate the performance of accumulators in vapor compression systems, Ninth International Refrigeration and Air Conditioning Conference at Purdue, R13-2, 2002.

Shah, M. M., A general correlation for heat transfer during film condensation inside pipes, International J. Heat and Mass Transfer, 22 547-556, 1979.

Shao, S., W. Shi, X. Li, and H. Chen, Performance representation of variable-speed compressor fro inverter air conditioners based on experimental data, International J. of Refrigeration, 27 805-815, 2004.

Shapiro, M., S. Ezekoye, and H. Baker, Fundamentals of Engineering Thermodynamics, Fourth Edition, Wiley Higher Education, 2002.

Shedd, T. A., and T. Y. Newell, Visualization of two-phase flow through microgrooved tubes for understanding enhanced heat transfer, International J. of Heat and Mass Transfer, 46 4169-4177, 2003.

Shedd, T. A., T. Y. Newell, and P. K. Lee, The effects of the number and angle of microgrooves on 
the liquid film in horizontal annular two-phase flow, International J. of Heat and Mass Transfer, 46 4179-4189, 2003.

SINDA/FLUINT user's manual, C\&R Technologies, 2003.

Sinpiboon, J. S. Wongwises, Numerical investigation of refrigerant flow through non-adiabatic capillary tubes, Applied Thermal Engineering, 22, 2015-2032, 2002.

Steiner, D. and J. Taborek, Flow boiling heat transfer in vertical tubes correlated by an asympototic model, Heat Transfer Engineering, 13(2), 43-69, 1992.

Stephan, K., S. Lin, M. Durst, F. Huang, and D. Seher, An investigation of energy separation in a vortex tube, International J. of Heat and Mass Transfer, 26(3) 341-348, 1983.

Tassou, S. A., and T. Q. Qureshi, Comparative performance evaluation of positive displacement compressors in variable-speed refrigeration applications, International J. of Refrigeration 21 (1) 29-41, 1998.

Thome, J. R., V. Dupont, and A. M. Jacobi, Heat transfer model for evaporation in microchannels Part I: presentation of the model, International J. of Heat and Mass Transfer, 47 3375-3385, 2004.

Tian, C., and X. Li, Transient behavior evaluation of an automotive air conditioning system with a 
variable displacement compressor, Applied Thermal Engineering, 25 1922-1948, 2005.

Tian, C., X. Li, and C. Dou, Experimental investigation and numerical simulation on the hysteresis zone of a variable displacement wobble plate compressor, International J. of Refrigeration, 28 988-996, 2005.

Trisaksri, V., and S. Wongwises, Correlations for sizing adiabatic capillary tubes, International J. of Energy Research, 27 1145-1164, 2003.

U. S. Department of Energy, 2001 Consumption and expenditures tables: Electric air-conditioning consumption tables, Energy information administration, 2004.

Vist, S., and J. Pettersen, Two-phase flow distribution in compact heat exchanger manifolds, Experimental Thermal and Fluid Science, 28 209-215, 2004.

Voster, P. P. J., and J. P. Meyer, Wet compression versus dry compression in heat pumps working with pure refrigerants or non-azeotropic binary mixtures for different heating applications, International J. of Refrigeration, 23 292-311, 2000.

Wang, J., and I. Catton, Evaporation heat transfer in biporous media, Heat and Mass Transfer, 37 275-281, 2001. 
Wang, S., J. Gu, and T. Dickson, Investigation of the effects of vapour quality and oil concentration on performance of a swash plate compressor, International J. Energy Research, 30 835-849, 2006.

Wang, S. K., Handbook of Air Conditioning and Refrigeration, Second Edition, McGraw-Hill, 2000.

Wetzel, M., and C. Herman, Design optimization of thermoacoustic refrigerators, International J. of Refrigeration, 20(1) 3-21, 1997.

Wijaya, H., Adiabatic capillary tube test data for HFC-134a, in: Proceedings of the IIR-Purdue Refrigeration Conference, West Lafayette, USA 63-71, 1992.

Wilson, M. J., T. A. Newell, J. C. Chato et al., Refrigeration charge, pressure drop, and condensation heat transfer in flattened tubes, International J. of Refrigeration, 26 442-451, 2003.

Wong, T. N., and K. T. Ooi, Refrigerant flow in capillary tube: An assessment of the two-phase viscosity correlations on model prediction, International Comm. of Heat and Mass Transfer, 22(4) 595-604, 1995.

Wong, T. N., and K. T. Ooi, Adiabatic capillary tube expansion devices: A comparison of the homogeneous flow and the separated flow models, Applied Thermal Engineering, 16(7) 625-634, 1996. 
Wongwises, S., and W. Pirompak, Flow characteristics of pure refrigerants and refrigerant mixtures in adiabatic capillary tubes, Applied Thermal Engineering, 21 845-861, 2001.

Wongwises, S., T. Songnetichaovalit, N. Lokathada et al., A comparison of the flow characteristics of refrigerants flowing through adiabatic capillary tubes, International Comm. of Heat and Mass Transfer, 27(5) 611-621, 2000.

Wongwises, S., and M. Suchatawut, A simulation for predicting the refrigerant flow characteristics including metastable region in adiabatic capillary tubes, International J. of Energy Research, 27 93-109, 2003.

Xu, B., and P. K. Bansal, Non-adiabatic capillary tube flow: a homogeneous model and process description, Applied Thermal Engineering, 22 1801-1819, 2002.

Yamasaki, H., M. Tadano, K. Sato, D. Matsuura, and T. Ebara, Hermetic $\mathrm{CO}_{2}$ Compressor, SANYO Technical Review, 33(2) 10-16, 2001.

Yana-Motta, S. F., J. A. R. Parise, and S. L. Braga, A visual study of R-404A/oil flow through adiabatic capillary tubes, International J. of Refrigeration, 25 586-596, 2002.

Yin, J., S. Memory, and R. M. DeKeuster, Integrated suction line heat exchanger and accumulator, US patent No. 6,681,597 B1, 2004. 
Yoo, S. H., J. H. Kim, Y. W. Hwang, M. S. Kim, K. Min, and Y. Kim, Heat transfer and pressure drop characteristics during the in-tube cooling process of carbon dioxide in the supercritical region, International J. of Refrigeration, 26 857-864, 2003.

Yoon, S. H., J. H. Kim, Y. W. Hwang et al., Heat transfer and pressure drop characteristics during the in-tube cooling process of carbon dioxide in the supercritical region, International J. of Refrigeration, 26 857-864, 2003.

Zhang, C., and G. Ding, Approximate analytic solutions of adiabatic capillary tube, International J. of Refrigeration 27 17-24, 2004.

Zhang, C. A., H. Mehraban, R. G. Gibbons Jr., T. Urdea, Accumulator with internal heat exchanger, US patent No. 6, 523, $365 \mathrm{~B} 2,2003$.

Zhao, T. S., and Q. Liao, Theoretical analysis of film condensation heat transfer inside vertical mini triangular channels, International J. of Heat and Mass Transfer, 45 2829-2842, 2002.

Zhao, Y., M. M. Ohadi, and R. Radermacher, Microchannel heat exchangers with carbon dioxide, Air-conditioning and Refrigeration Technology Institute Final Report, ARTI-21CR, 10020-01, 2001. 


\section{Appendices}

\section{Appendix A Program to calculate AXE flow in R134a-based systems}

\section{A-1 Main code}

This function calculates the outlet parameters of the capillary flow if the inlet condition is given. For ACAM flow, the inlet condition of the secondary flow can be ignored. Otherwise it needs to be considered.

function [mass,t6, P6, x6, h6, Q,t_outer,P_outer,x_outer,h_outer]

$=$ AXE(mass,P5,t5,x5,di,do,di0,epsilon,1_r,P_outer,t_outer,X_outer,di_outer,do_outer,U_sw_sp,U_sw_tp_b,U_sw_tp_c,Indicator,Mo del,Type,Oil)

$\%$ mass, $35, t 5, \times 5$ are tube inlet parameters, $t 6, P 6, x 6$, h6 are tube outlet parameters.

$\%$ mass outer, $u_{\text {_... outer, }} \mathrm{p}$... outer, $t$ outer, $\mathrm{x}$ _outer are for second flow, which could be air, water, or refrigerant from the evaporator.

$\%$..... if "outer" is water, mass, $u$, and take input, $\mathrm{p}=1 \mathrm{bar}, \mathrm{x}=1$.

$\%$-.m- if "outer" is air, mass, $u, t$, and $x$ take input, $p$ - Ibar. $x$ could be the relative humidity of the air.

$\%$..... if "outer" is refrigerant, mass, $u, t, p, x$ take inputs, and outputs are inlet parameters of the compressor.

$\%$...... dio is the tube inner diameter before entering the capillary

$\%[$ mass, $6, \mathrm{P} 6, \mathrm{x} 6, \mathrm{~h} 6, \mathrm{Q}, \mathrm{t}$ outer,p outer, $\mathrm{x}$ outer, h outer $] \mathrm{AXE}(160,18,40,-1,1.8,2.2,12.7,0.0015,0.6,1,0,0.8,12.5,18,3,5,2,0,1)$

$\%$ mass $=160 \mathrm{~kg} / \mathrm{h} ; \quad \mathrm{P5}=18 \mathrm{bar} ; \quad \mathrm{t} 5=40 \mathrm{C} ; \quad \times 5=-1$;

$\% \mathrm{di}=1.8 \mathrm{~mm} ; \quad$ do $=2.2 \mathrm{~mm} ; \quad$ di0 $=12.7 \mathrm{~mm} ; \quad$ epsilon $=0.0015 \mathrm{~mm}$;

$\% \mathrm{I}_{\mathrm{r}}=0.6 \mathrm{~m} ; \quad$ P_outer $=1 \mathrm{bar} ; \quad$ touter $=0 \mathrm{C} ; \quad$ _ outer $=0.8$;

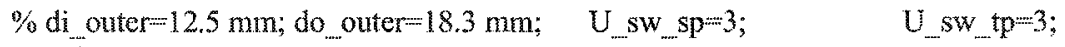

format short $\mathrm{g}$;

if $\left(\mathrm{x}_{\text {_outer }}>=0\right.$ \&\& $\mathrm{x} \_$outer $\left.<=1\right)$

$\left[\mathrm{hh}, \mathrm{ss}, \mathrm{vv}, \mathrm{P} \_\right.$outer $]=\mathrm{t}_{-} \mathrm{P} \_\mathrm{x}\left(\mathrm{t} \_\right.$outer, $0, \mathrm{x}_{-}$outer,0); \% Get correct pressure of outer pressure in bar.

end;

mass $=(1-0 i 1 / 100) *$ mass $/ 3600$;

$\%$ "Oil" represents the oil concentration which affects the effective mass flow rate and the effective CHTC

t_cond $\mathrm{l}=$ cputime;

if(Indicator $=0$ )

indicator ${ }^{\prime}$ Adiabatic';

elseif(Indicator $=1$ ) 
indicator $=$ 'Non-Adiabatic';

else

disp('The throttling process should be either adiabatic or non-adiabatic, please re-input...');

halt;

end;

if $($ Model $==0)$

model='AAC system';

elseif(Model $=1$ )

model='Refrigerator without weld';

elseif(Model==2)

model='Refrigerator with weld';

else

disp('The throttling process should be either for AAC or Refrigerator, please re-input...');

halt;

end;

if $($ Type $=0)$

type='Parellel';

elseif(Type $=1$ )

type='Counterflow';

else

disp('The HX should be either Parellel or Counter flow type');

halt;

end;

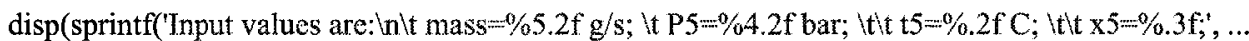

$1000^{*}$ mass,P5,t5,x5));

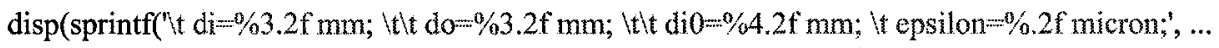
di,do,di0,1e3*epsilon));

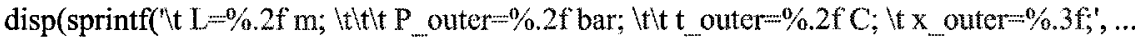

1_r,P_outer,t_outer,x_outer));

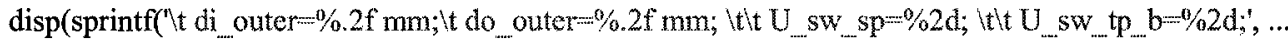

di_outer,do_outer,U_sw_sp,U_sw_tp_b));

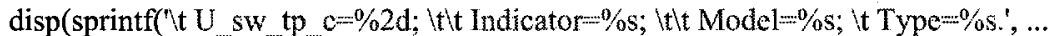

U_sw_tp_c,indicator,model,type));

$\% 1 \mathrm{r}=0.5 ; \mathrm{di}=1.8 ; \mathrm{do}=2.2 ;$

$\mathrm{di}=\mathrm{di} * 1 \mathrm{e}-3 ; \%(\mathrm{~m})$

$\mathrm{do}=\mathrm{do} * 1 \mathrm{e}-3 ; \%(\mathrm{~m})$

di0 $=$ di $0 * 1 \mathrm{e}-3 ; \%(\mathrm{~m})$

di_outer=di_outer* $1 \mathrm{e}-3 ; \%(\mathrm{~m})$

do_outer=do_outer*1e-3; \% (m) 


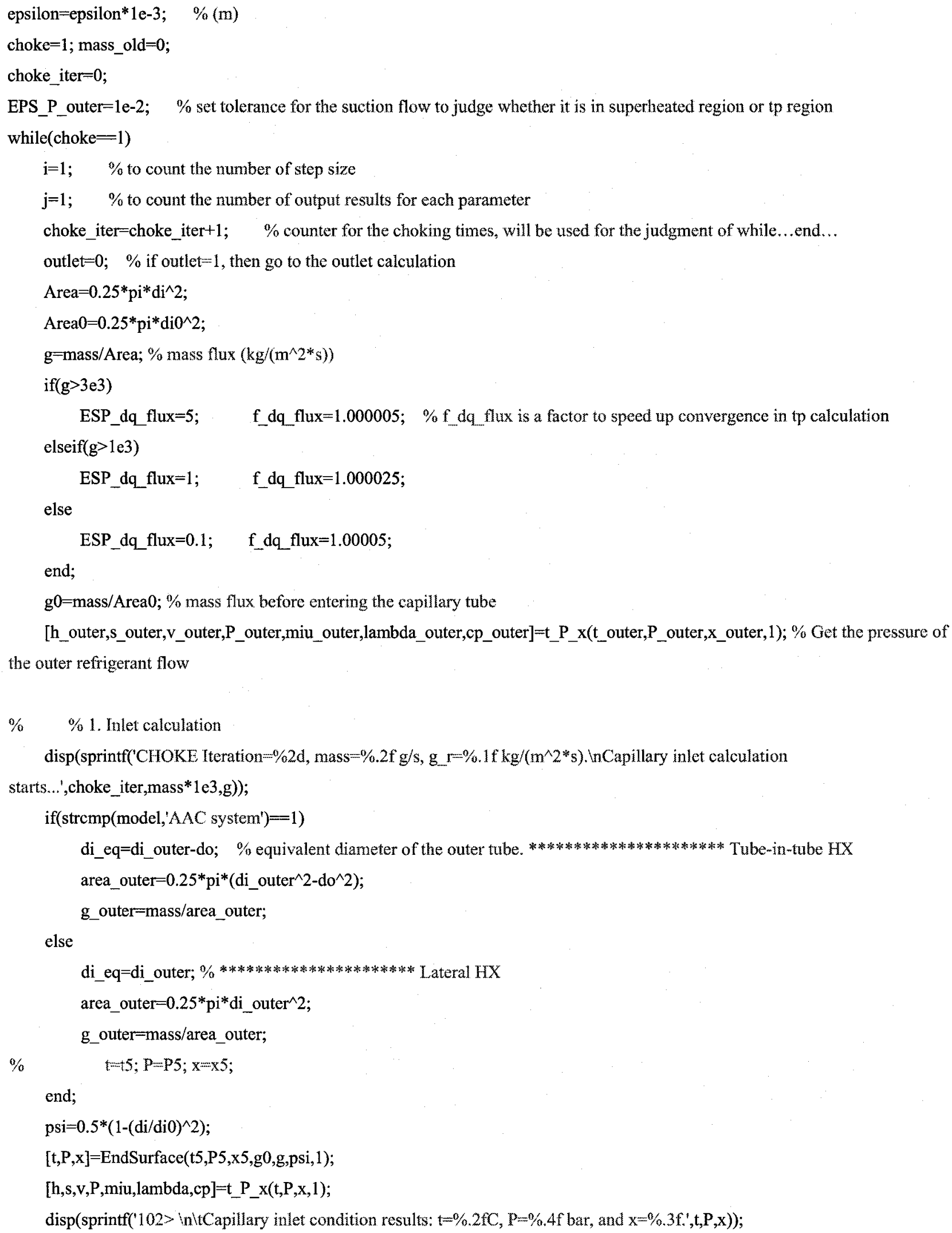




$$
\begin{aligned}
& \text { t_out }(\mathrm{j})=\mathrm{t} ; \quad \text { p_out }(\mathrm{j})=\mathrm{P} ; \quad \text { h_out }(\mathrm{j})=\mathrm{h} ; \quad \text { v_out }(\mathrm{j})=\mathrm{v} ; \quad \text { miu_out }(\mathrm{j})=\operatorname{miu} ; \quad \mathrm{x} \text { _out }(\mathrm{j})=\mathrm{x} \text {; } \\
& \mathrm{s} \_ \text {out }(\mathrm{j})=\mathrm{s} ; \quad \text { dQ_out }(\mathrm{j})=0 ; \quad \text { fh_out }(\mathrm{j})=0 ; \quad \text { f_out }(\mathrm{j})=0 ; \quad \text { fi_out }(\mathrm{j})=0 ; \quad \text { 1_out }(\mathrm{j})=0 \text {; } \\
& \text { t_outer_out }(j)=t \_o u t e r ; \quad \text { p_outer_out }(j)=P \text { _outer; } \quad \text { h_outer_out }(j)=h \_o u t e r ; \\
& \text { f_outer_out }(\mathfrak{j})=0 ; \quad \quad \text { v_outer_out }(\mathrm{j})=\mathrm{v} \text { _outer; } \quad \text { miu_outer_out }(\mathrm{j})=\text { miu_outer; } \\
& \text { s_outer_out }(\mathrm{j})=\text { s_outer; } \quad \text { x_outer_out }(\mathrm{j})=\mathrm{x} \text { _outer; } \\
& \text { if }(x<0) \\
& x_{-} \operatorname{out}(j)=0 \text {; } \\
& \text { end; } \\
& \mathrm{j}=\mathrm{j}+1 ; \quad \mathrm{i}=\mathrm{i}+1 \text {; }
\end{aligned}
$$

$\% 2$. Sub-cooled single-phase calculation

disp('Expansion process, single phase flow... );

1_sp $=0 ; \quad \mathrm{Q}=0 ; \quad \mathrm{dl}=1$ _r-1_sp;

$[\mathrm{h}, \mathrm{s}, \mathrm{v}]=\mathrm{t} \_\mathrm{P} \_\mathrm{x}(\mathrm{t}, \mathrm{P}, \mathrm{x}, 0)$;

t_cond2=cputime;

if $(x<0) \quad \% x$ much be less than 0 s.t. sp calculation is adopted

delta_P $=0.005$;

dl_sp $=0.02 ; \quad \%$ set the initial value of single-phase length

$\mathrm{dP}=1 ; \mathrm{dP} \_$old $=0$;

$\mathrm{t}$ old $=\mathrm{t}$; \% store old $\mathrm{t}$. If $(\mathrm{t}-\mathrm{t}$ old $)$ is too large, decrease the step size dl sp, or vice versa

while $($ abs $(\mathrm{dP})>2 \mathrm{e}-2$ \& outlet $=0$ )

$\%$ 1.1. In-tube HTC

$\left[\mathrm{U} \_r, f, f i 0\right]=U \_$refrig $\left(g, t, P, x, d i, e p s i l o n, 0, U \_s w \_s p\right) ; \% q$ flux is 0 for condensation.

1_sp=1_sp+dl_sp;

A_r $=\mathrm{pi}^{*} \mathrm{di}{ }^{*} \mathrm{dl} \_\mathrm{sp}$;

if(strcmp(model,'AAC system'));

R_r=1/(U_r*A_r); \% heat resistance of inside refrigerant

A_outer=pi*do*dl_sp;

R_t $=\left(1 /\left(2 * \mathrm{pi}^{*} 400 * \mathrm{dl} \_\mathrm{sp}\right)\right) * \log (\mathrm{do} / \mathrm{di}) ; \%$ heat resistance of copper capillary tube wall elseif(strcmp(model,'Refrigerator without weld'))

$\mathrm{R}_{-} \mathrm{r}=1 /\left(\mathrm{U}_{-} \mathrm{r}^{*} 0.5^{*} \mathrm{~A} \_\mathrm{r}\right) ; \%$ heat resistance of inside refrigerant

A_eff $=\operatorname{acos}\left(\left(0.5^{*}(\right.\right.$ do_outer-do $\left.)\right) /\left(0.5^{*}(\right.$ do_outer+do $\left.\left.)+1 \mathrm{e}-3\right)\right) *$ di_outer*dl_sp; \% $\%$ e-3 is the distance between

the two tubes

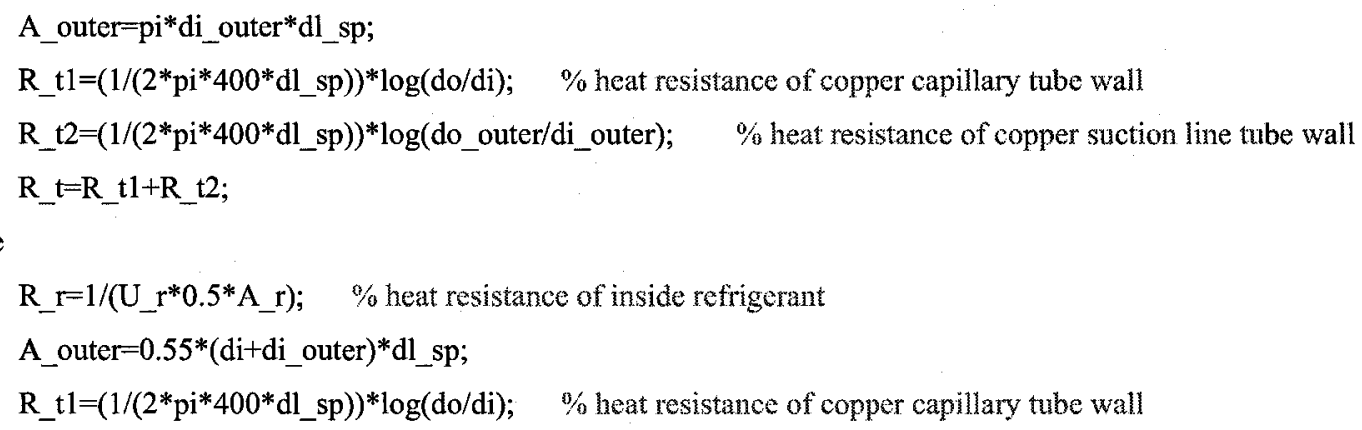


R_t2 $=\left(1 /\left(2 * \mathrm{pi}^{*} 400 * \mathrm{dl} \_\mathrm{sp}\right)\right)^{*} \log ($ do_outer/di_outer $) ; \quad \%$ heat resistance of copper suction line tube wall

$\mathrm{R} \_\mathrm{t} 3=1 \mathrm{e}-3 /(50 *$ do*dl_sp); $\% 50$ is the conductivity of solder (assume $60 \% \mathrm{Sn}$ and $40 \% \mathrm{~Pb}$ ) [6]

$\%$ "1e-3" in numerator is solder depth in $\mathrm{m}$, "do" in denominator is solder width in $\mathrm{m}$.

$R_{-} \mathrm{t}=R_{-} \mathrm{t} 1+R_{-} \mathrm{t} 2+R_{-} \mathrm{t} 3$;

end;

q_flux $=0 ;$ dq_flux $=0.1 * E S P \_d q \_$flux;

$\left[\mathrm{hh}, \mathrm{ss}, \mathrm{vv}, \mathrm{P} \_\right.$outer_r] $=\mathrm{t} \_\mathrm{P}$ _x $\left(\mathrm{t}\right.$ _outer, $0, \mathrm{x} \_$outer, 0$)$;

dP_outer=P_outer-P_outer_r;

if(strcmp(indicator,'Adiabatic') \&\&((1_sp< $<0.200) \|(1$ sp $>=1.200))) \% \%$ C. Melo non-adiabatic (2000)

if(strcmp(indicator,'Adiabatic') \&\&((1_sp<=0.533)||(1_sp>=1.536))) \%\% Pexito non-adiabatic (1995)

if(strcmp(indicator,'Adiabatic'))

if(strcmp(indicator, Adiabatic $) \& \&((1 \mathrm{sp} \times=0.508) \mid(1 \mathrm{sp}>=1.524))) \% \%$ Liu and Bullard lateral (2000)

if(abs(dP_outer) $>=$ EPS_P_outer) \% represents superheated inlet condition

[U_outer,f_outer]=U_refrig(g_outer,t_outer,P_outer,x_outer,di_eq,epsilon,q_flux,U_sw_sp); else

[U_outer,f_outer] $=U$ _refrig(g_outer,t_outer,P_outer,x_outer,di_eq,epsilon,q_flux,U_sw_tp_b);

end;

$\mathrm{dQ}=0 ; \quad \%$ for adiabatic process

else

while $\left(\operatorname{abs}\left(d q \_f l u x\right)>0.01 * E S P \_d q \_f l u x\right)$

q_flux_old=q_flux;

$\%$ 1.2. Out-tube HTC

$\left[\right.$ hh,ss,vv,P_outer_r] $=$ __P_x(t_outer, $0, x_{-} \_$outer, 0$)$;

dP_outer=P_outer-P_outer_r;

if(abs(dP_outer) $\left.>=E P S \_P \_o u t e r\right) \%$ outside flow is in sh region

[U_outer,f_outer]=U_refrig(g_outer,t_outer,P_outer,x_outer,di_eq,epsilon,q_flux,U_sw_sp);

else $\%$ outside flow is in tp region

$\left[\mathrm{U} \_\right.$outer,f_outer] $=U$ _refrig(g_outer,t_outer,P_outer,x_outer,di_eq,epsilon,q_flux,U_sw_tp_b); end;

$\%$ 1.3. Calculate the overall HTC and pressure drop of the single-phase region

R_outer=1/(U_outer*A_outer); \% outeside fluid

$\mathrm{UAo}=1 /\left(\mathrm{R} \_\mathrm{r}+\mathrm{R} \_\mathrm{t}+\mathrm{R} \_\right.$outer $)$;

$\mathrm{dQ}=\mathrm{UAo} *\left(\mathrm{t}-\mathrm{t} \_\right.$outer $) ; \quad \%$ for non-adiabatic process, $\mathrm{W}$

q_flux $=\mathrm{dQ} /$ A_outer;

$d q \_$flux $=q$ _flux $-q \_$flux_old;

end;

end;

$\mathrm{Q}=\mathrm{Q}+\mathrm{dQ} ; \quad \% \mathrm{~W}$

$\mathrm{dq}=-1 \mathrm{e}-3 * \mathrm{dQ} / \mathrm{mass} ; \quad \% / \mathrm{kJ} / \mathrm{kg}$

$\mathrm{dqw}=\mathrm{dq} *$ mass/A_r; $\quad \%$ heat flux through the wall 
$\mathrm{fi}=\mathrm{fi} 0 * \mathrm{dqw}$

$\mathrm{fh}=\mathrm{f}+\mathrm{fi}$;

$\left[t, P, h, v, m i u, s, d e l t a \_P\right]=s p \_i t e r\left(t, P, g, d l \_s p, d i, f h, f,-d q, d e l t a \_P, 2\right)$;

if(abs(dP_outer) $>=$ EPS_P_outer)

if(strcmp(type,'Parellel'))

[t_outer,P_outer,h_outer,v_outer,miu_outer,s_outer]=sp_iter(t_outer,P_outer,g_outer,dl_sp,di_eq,f_outer,0,dq,0,3);

else

[t_outer,P_outer,h_outer,v_outer,miu_outer,s_outer]=sp_iter(t_outer,P_outer,g_outer,dl_sp,di_eq,-f_outer,0,-dq,0,3);

end;

else

if(strcmp(type,'Parellel'))

[t_outer,P_outer,x_outer,h_outer,v_outer,miu_outer,s_outer] =tp_iter(t_outer,P_outer,x_outer,g_outer,dl_sp,di_eq,f_outer,f,dq,0,1);

else

[t_outer,P_outer,x_outer,h_outer,v_outer,miu_outer,s_outer] $=t p \_i t e r(t$ _outer,P_outer,x_outer,g_outer,dl_sp,di_eq,-f_outer,-f,-dq,0,1)

;

end;

end;

$\%$ use minus sign "-" before f outer, $\mathrm{f}$, and "dq" to convert the IXX from parellel to counterflow.

if $($ abs $(t-t$ old $)>2)$

dl_sp $=0.5 *$ dl_sp;

elseif(abs(t-t_old $)<0.3$ )

dl_sp=2*dl_sp;

end;

t_old $=$;

$\mathrm{dl}=1 \_\mathrm{r}-1 \_\mathrm{sp}$;

if $(\mathrm{dl}<1 \mathrm{e}-4)$

disp(sprintf( $>>95$ Tube length reached, $1=\% .3 \mathrm{fm}$, dl $=\% .4 \mathrm{fm} ., 1 \mathrm{r}, \mathrm{dl})$ );

outlet $=1$;

if(choke_iter $>1)$

choke $=0$;

end;

break;

end;

if $(t<100)$

[h_sat,s_sat,v_sat,P_sat] $=t \_P \_x(t, 0,1,0)$;

$\mathrm{dP}=\mathrm{P}-\mathrm{P}$ _sat;

ratio $=a b s\left(d P \_o l d / d P\right) ;$

if(ratio $=0$ )

if(ratio $>5$ )

dl_sp $=0.05 * \mathrm{dl} \_\mathrm{sp}$;

elseif(ratio $>3$ ) 


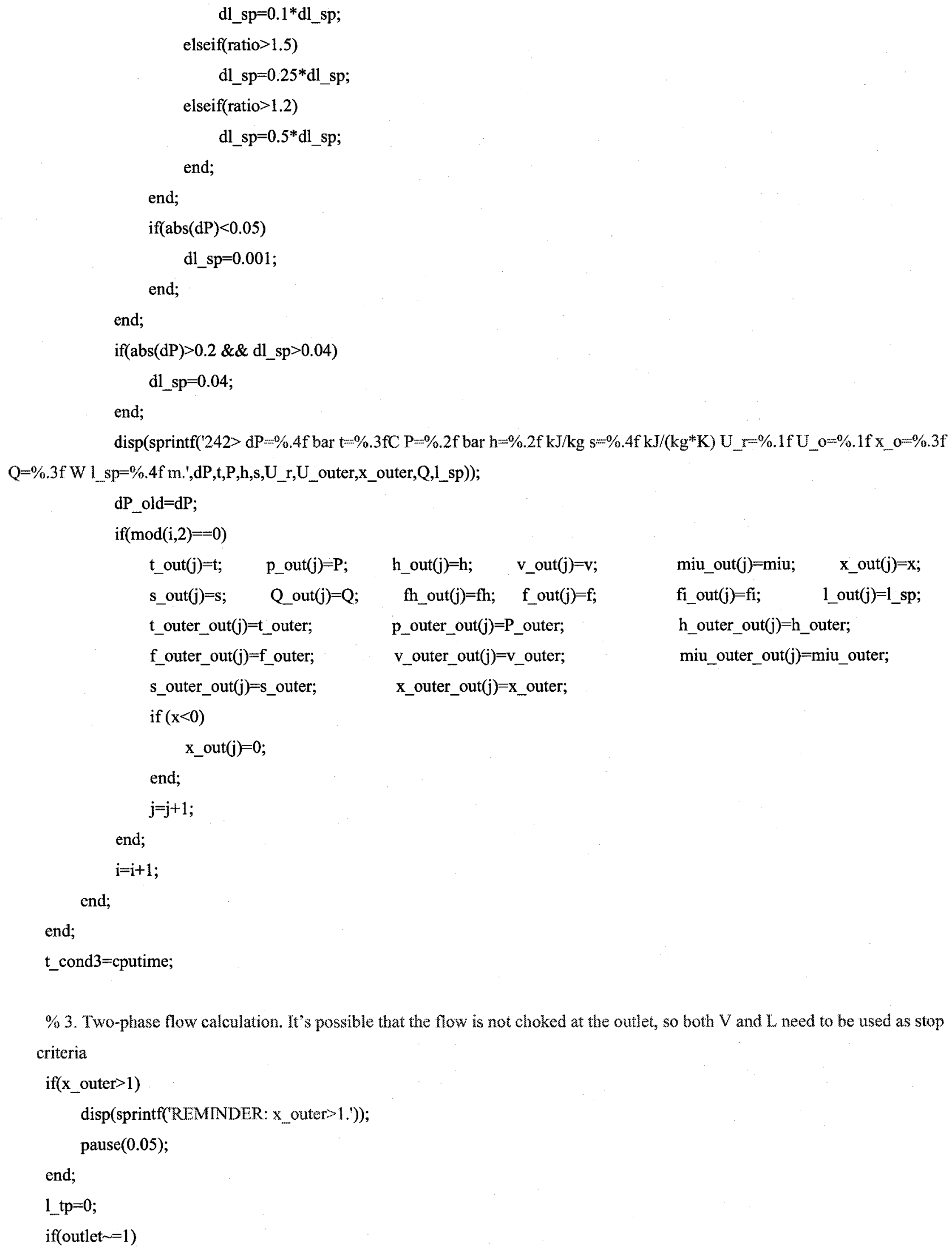




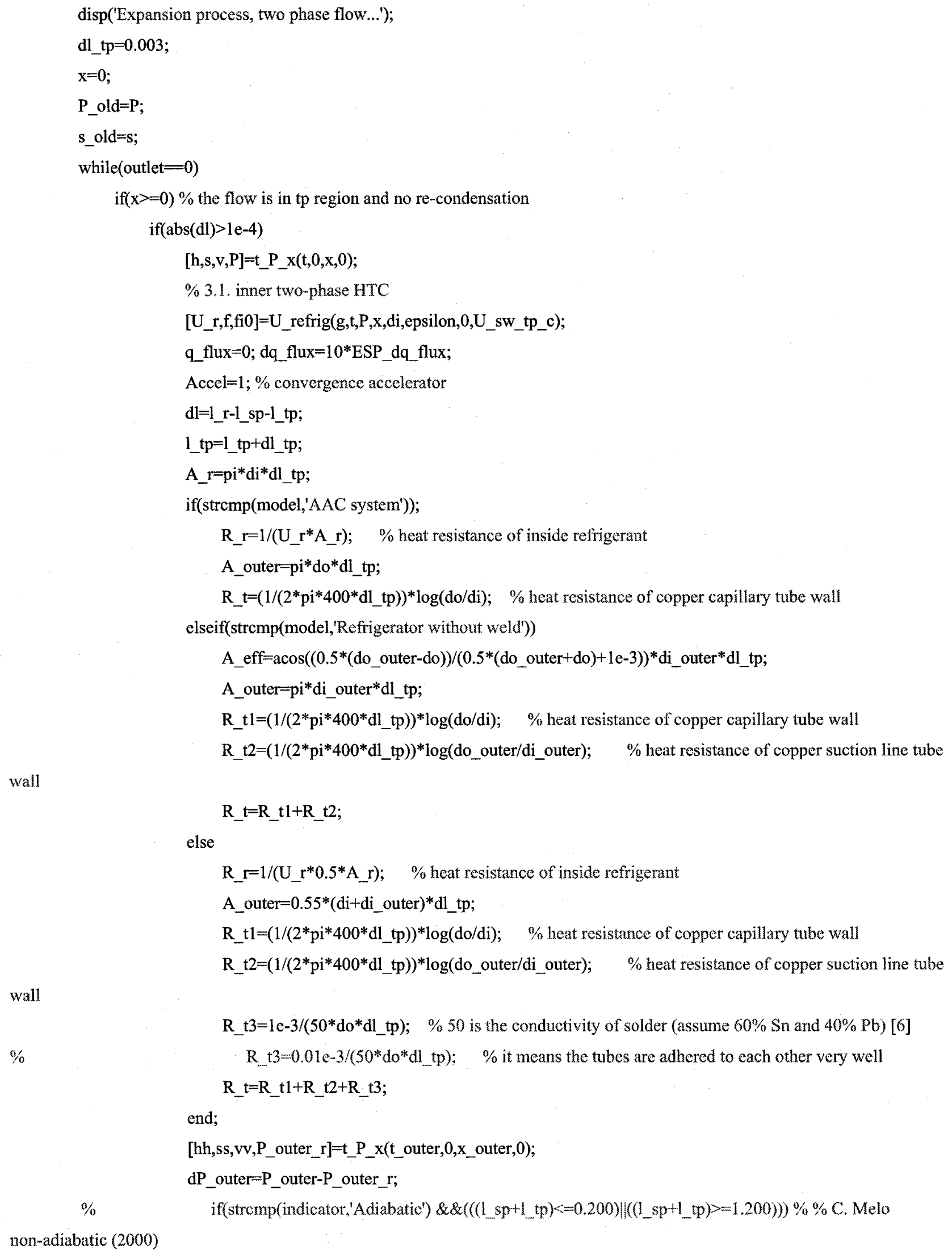


if(strcmp(indicator,'Adiabatic') $\left.\& \&\left(\left(\left(1 \_s p+1 \_t p\right)<=0.533\right) \mid\left(\left(1 \_s p+1 \_t p\right)>=1.536\right)\right)\right) \% \%$ Pexito non-adiabatic

(1995)

$\%$

$\%$

(2000)

[U_outer,f_outer]=U_refrig(g_outer,t_outer,P_outer,x_outer,di_eq,epsilon,q_flux,U_sw_tp_b);

end;

if(stremp(indicator,'Adiabatic'))

if(stremp(indicator, Adiabatic') \&\&(((1 sp+1 tp $)<=0.508) \mid((1 \mathrm{sp}+1$ tp $)>=1.524))) \% \%$ Liu and Bullard

if(abs(dP_outer) $>=E P S \_P \_$_outer)

$\left[\mathrm{U} \_\right.$outer,f_outer] $=U \_$refrig $\left(g \_o u t e r, t \_o u t e r, P \_o u t e r, x \_o u t e r, d i \_\right.$_eq,epsilon,q_flux,U_sw_sp); else

[U_outer,f_outer] $=U_{-}$refrig(g_outer,t_outer,P_outer,x_outer,di_eq,epsilon,q_flux,U_sw_tp_b); end;

$\mathrm{dQ}=0 ; \quad \%$ for adiabatic process

else

while(abs(dq_flux $)>$ ESP_dq_flux $)$

q_flux_old $=q \_$flux;

\% 3.2. outer two-phase HTC

if(abs(dP_outer) $>=$ EPS_P_outer)

[U_outer,f_outer] $=U$ _refrig(g_outer,t_outer,P_outer,X_outer,di_eq,epsilon,q_flux,U_sw_sp); U outer $=2.2 * U_{-}$outer;

else

$\%$ 3.3. total HTC and pressure drop for the twomphase flow

R_outer=1/(U_outer*A_outer); \% outeside fluid

$\mathrm{UAo}=1 /\left(\mathrm{R}_{-}+\mathrm{R}+\mathrm{R} \mathrm{t}+\mathrm{R} \_\right.$outer $)$;

$\mathrm{dQ}=\mathrm{UAo} *\left(\mathrm{t}-\mathrm{t} \_\right.$outer $) ; \quad \%$ for non-adiabatic process, $\mathrm{W}$

q_flux $=\mathrm{dQ} / \mathrm{A} \_$outer;

dq_flux $=q \_$flux-q_flux_old;

Converg $($ Accel $)=d q \_$flux;

Accel $=$ Accel +1 ;

q_flux=f_dq_flux*q_flux; $\% 1.0005$ is a small factor to speed up the convergence

$\%$ disp([dq flux dQ]);

if(Accel>50)

pause;

end;

end;

end;

$\mathrm{Q}=\mathrm{Q}+\mathrm{dQ} ; \quad \% \mathrm{~W}$

$\mathrm{dq}=-1 \mathrm{e}-3 * \mathrm{dQ} / \mathrm{mass} ; \quad \% \mathrm{~kJ} / \mathrm{kg}$

$\mathrm{dqw}=\mathrm{dq}^{*} \operatorname{mass} / \mathrm{A} \_\mathrm{r}$; 


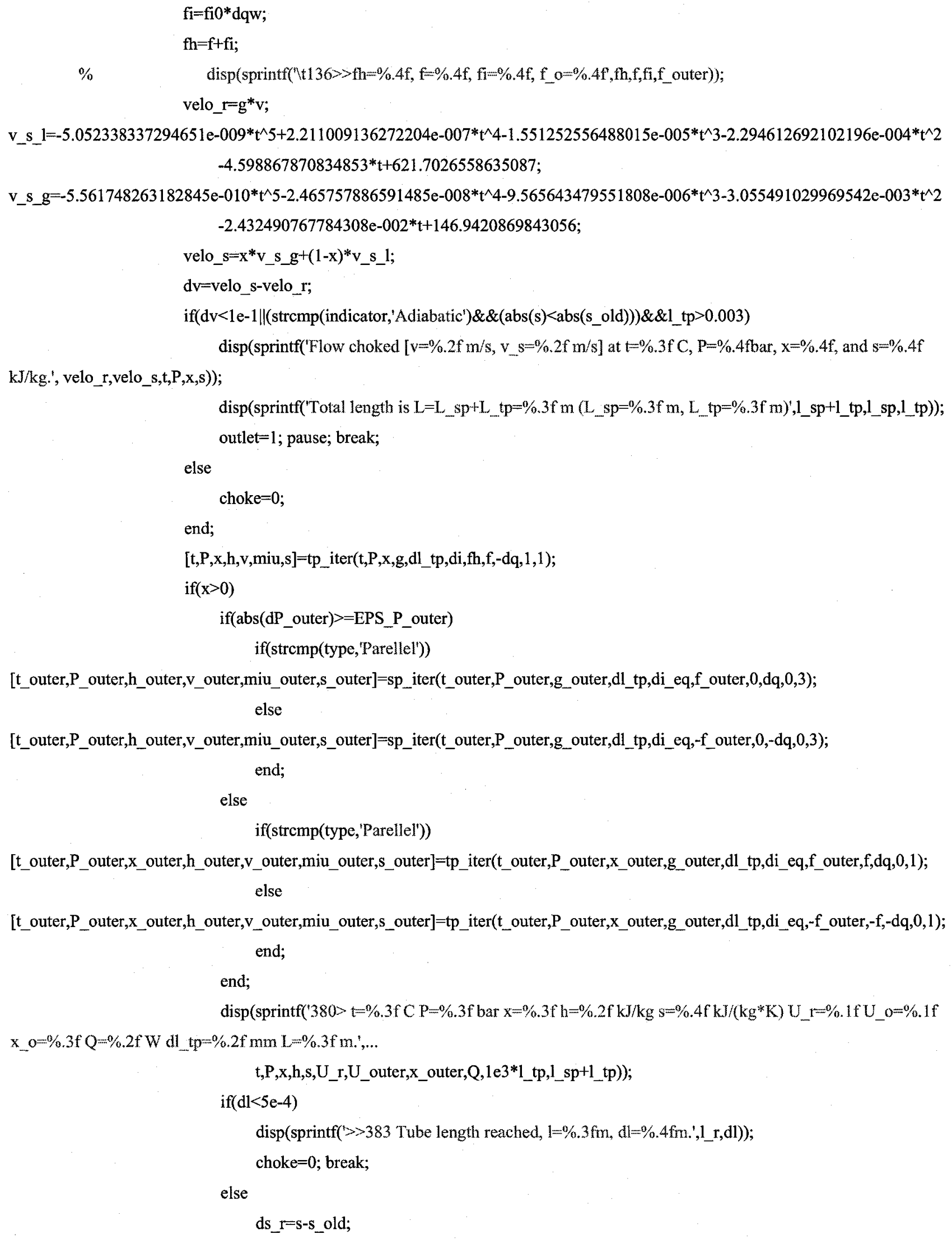




$$
\begin{aligned}
& \text { if(abs(ds_r) }>5 e-3) \\
& \quad \text { dl_tp }=2 e-3 ; \\
& \text { elseif(ds_r }>1.5 e-4)
\end{aligned}
$$

$\operatorname{disp}$ (sprintf('Flow choked $[\mathrm{v}=\% .2 \mathrm{f} \mathrm{m} / \mathrm{s}, \mathrm{v} s=\% .2 \mathrm{f} \mathrm{m} / \mathrm{s}]$ at $t=\% .3 \mathrm{f} \mathrm{C}, \mathrm{P}=\% .4 \mathrm{fbar}, \mathrm{x}=\% .4 \mathrm{f}$, and

$\mathrm{s}=\%$ \%.4f kJ/kg.', velo_r,velo_s,t,P,X,s));

disp(sprintf(Total length is $L=\% .3 \mathrm{fm}$ out of $\% .3 \mathrm{fm}(\mathrm{L} s p=\% .3 \mathrm{f} \mathrm{m}, \mathrm{L} t \mathrm{tp}=\% .3 \mathrm{f}$

m). $\left.\left.{ }^{\prime}, 1 \_s p+1 \_t p, 1 \_r, 1 \_s p, 1 \_t p\right)\right)$;

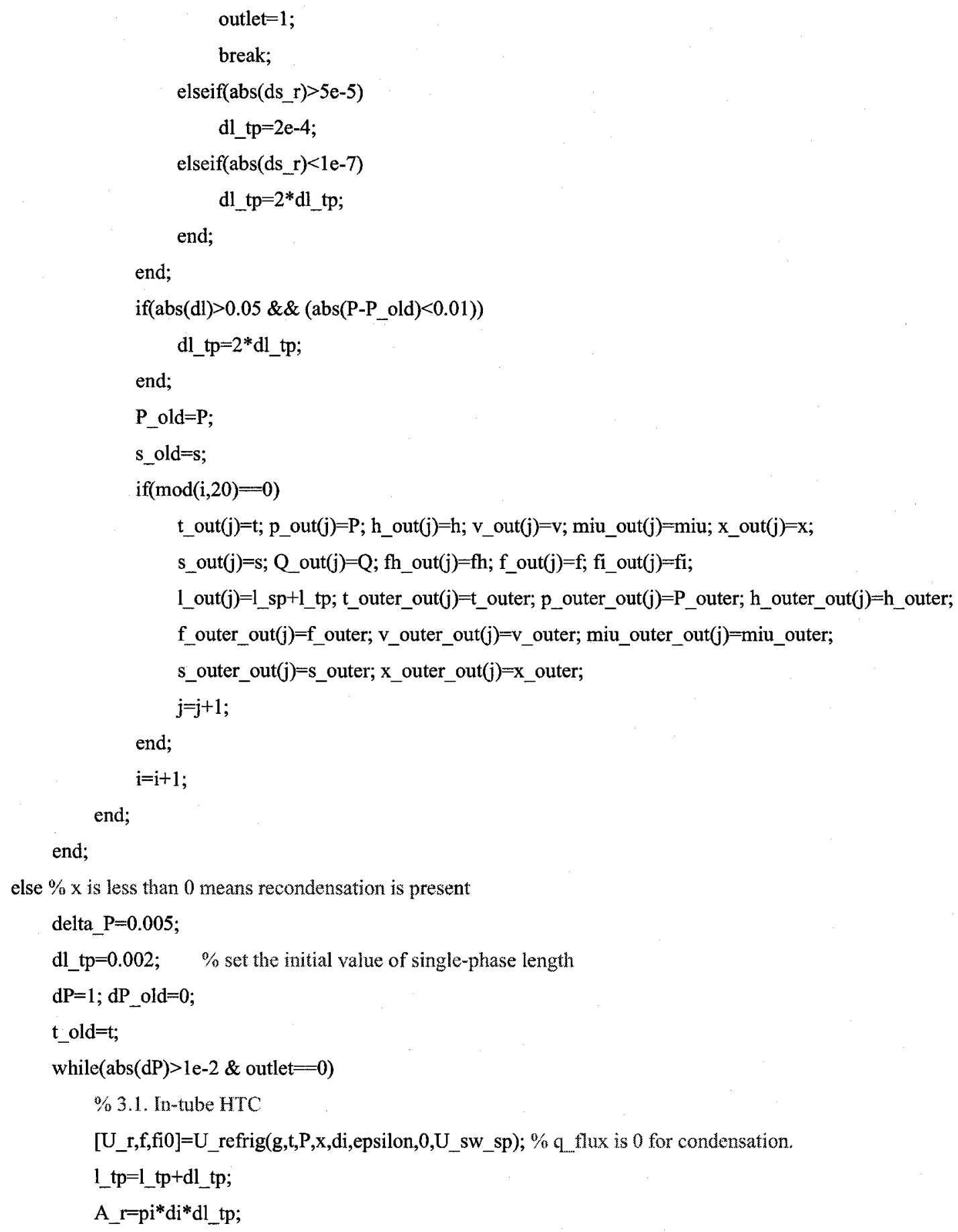


$\mathrm{R}_{-} \mathrm{r}=1 /\left(\mathrm{U}_{-} \mathrm{r}^{*} \mathrm{~A} \_\mathrm{r}\right) ; \quad \%$ heat resistance of inside refrigerant

if(strcmp(model,'AAC system'));

A_outer=pi*do*d1_tp;

$\mathrm{R}_{-} \mathrm{t}=\left(1 /\left(2 * \mathrm{pi}^{*} 400 * \mathrm{dl} \_\mathrm{tp}\right)\right) * \log (\mathrm{do} / \mathrm{di}) ; \quad \%$ heat resistance of copper capillary tube wall elseif(strcmp(model,'Refrigerator without weld'))

A_eff=acos $\left(\left(0.5^{*}(\right.\right.$ do_outer-do $\left.)\right) /\left(0.5^{*}(\right.$ do_outer+do $)+1$ e- 3$\left.)\right) *$ di_outer*dl_tp;

A_outer-pi*di_outer*dl_tp;

R_tl $=\left(1 /\left(2 * \mathrm{pi}^{*} 400 * \mathrm{dl} \_\mathrm{tp}\right)\right) * \log (\mathrm{do} / \mathrm{di}) ; \quad \%$ heat resistance of copper capillary tube wall

$\mathrm{R}_{-} \mathrm{t} 2=\left(1 /\left(2 * \mathrm{pi}^{*} 400 * \mathrm{dl} \_\mathrm{tp}\right)\right)^{*} \log ($ do_outer/di_outer $) ; \quad \%$ heat resistance of copper suction line tube

wall

wall

$R_{-} t=R_{-} t 1+R_{-} t 2 ;$

else

A_eff $=\operatorname{acos}\left(\left(0.5^{*}(\right.\right.$ do_outer-do $\left.)\right) /(0.5 *($ do_outer+do $)+1$ e-3 $\left.)\right) *$ di_outer*dl_tp;

A_outer=pi*di_outer*dl_tp;

$\mathrm{R}_{\mathrm{t}} \mathrm{t} 1=\left(1 /\left(2 * \mathrm{pi}^{*} 400 * \mathrm{dl} \_\mathrm{tp}\right)\right) * \log (\mathrm{do} / \mathrm{di}) ; \quad \%$ heat resistance of copper capillary tube wall

R_t $2=\left(1 /\left(2 * \mathrm{pi}^{*} 400 * \mathrm{~d} 1 \mathrm{tp}\right)\right) * \log ($ do_outer/di_outer $) ; \quad \%$ heat resistance of copper suction line tube

$\mathrm{R}_{\mathrm{t}} \mathrm{t}=0.01 \mathrm{e}-3 /\left(50 * \mathrm{do} * \mathrm{~d} 1_{-} \mathrm{tp}\right) ; \% 50$ is the conductivity of solder (assume $60 \% \mathrm{Sn}$ and $\left.40 \% \mathrm{~Pb}\right)[6]$

$R_{-} \mathrm{t}=\mathrm{R}_{-} \mathrm{t} 1+\mathrm{R} \_\mathrm{t} 2+\mathrm{R}_{-} \mathrm{t} 3$;

end;

q_flux $=0 ; d q \_$flux $=0.1 * E S P \_d q \_$flux;

[hh,ss,wv,P_outer_r] $=$ t_P_x(t_outer,0,x_outer,0);

dP_outer=P_outer-P_outer_r;

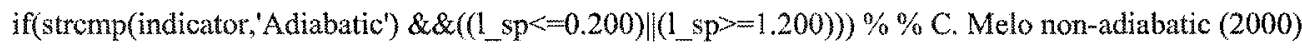

if(strcmp(indicator, Adiabatic) \&\&((1 sp<-0.533)\|(1 sp>- 1.536$))) \% \%$ Pexito nonmadiabatic (1995)

if(strcmp(indicator,'Adiabatic') \&\&(((1_sp+1_tp) $\left.\left.<=0.508) \mid\left(\left(1 \_s p+1 \_t p\right)>=1.524\right)\right)\right) \% \%$ Liu and Bullard

$(2000)$

$\%$

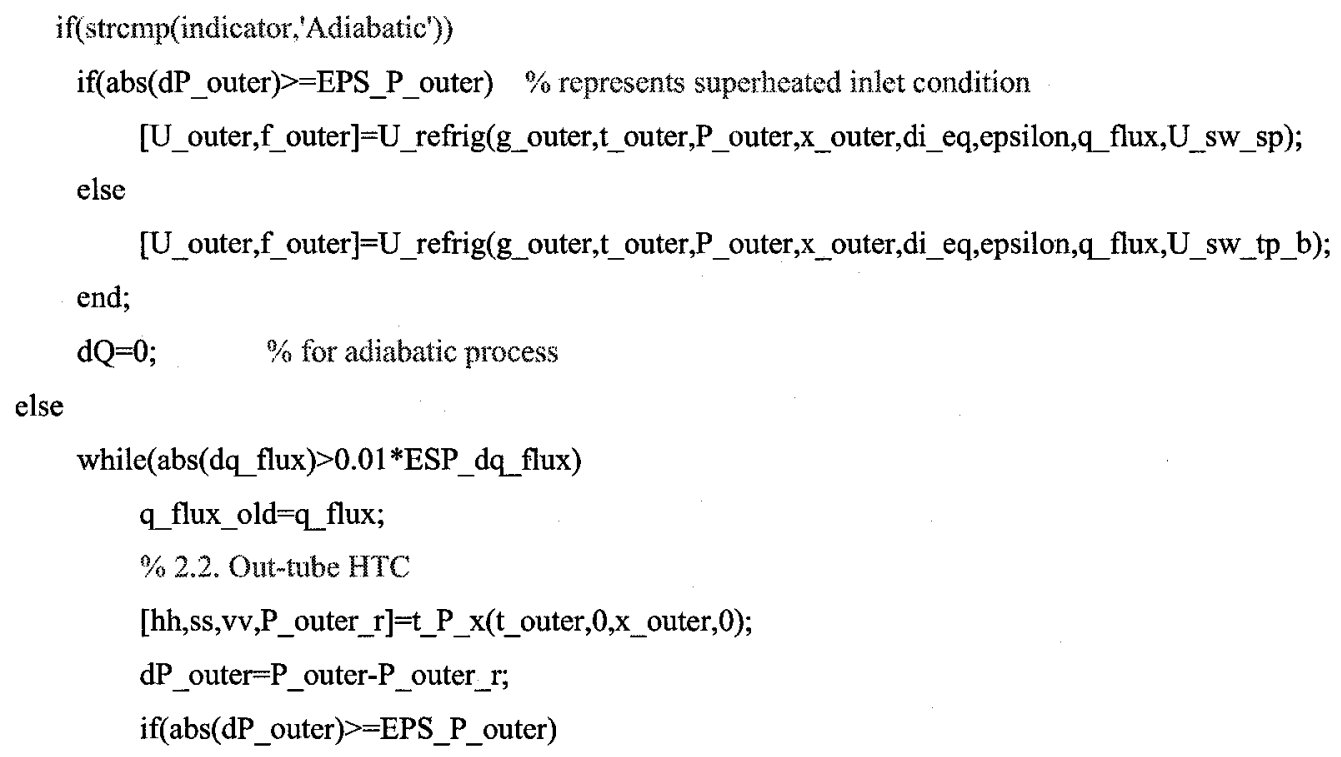


[U_outer,f_outer]=U_refrig(g_outer,t_outer,P_outer,x_outer,di_eq,epsilon,q_flux,U_sw_sp); else

[U_outer,f_outer] $=U$ _refrig(g_outer,t_outer,P_outer,x_outer,di_eq,epsilon,q_flux,U_sw_tp_b);

end;

$\% 2.3$. total HTC and pressure drop of the single-phase region

R_outer=1/(U_outer*A_outer); \% outeside fluid

$\mathrm{UAo}=1 /\left(\mathrm{R}_{-} \mathbf{r}+\mathrm{R} \_\mathrm{t}+\mathrm{R} \_\right.$outer $)$;

$\mathrm{dQ}=\mathrm{UAo} *\left(\mathrm{t}-\mathrm{t} \_\right.$outer $) ; \quad \%$ for non-adiabatic process, $\mathrm{W}$

q_flux $=d Q / A \_$_ff;

$\mathrm{dq}$ flux $=\mathrm{q}$ flux $-\mathrm{q}$ flux old;

end;

end;

$\mathrm{Q}=\mathrm{Q}+\mathrm{dQ} ; \quad \% \mathrm{~W}$

$\mathrm{dq}=-1 \mathrm{e}-3 * \mathrm{dQ} / \mathrm{mass} ; \quad \% \mathrm{~kJ} / \mathrm{kg}$

$\mathrm{dqw}=\mathrm{dq}{ }^{*}$ mass/A_r;

$\mathrm{fi}=\mathrm{fi} 0^{*} \mathrm{dqw}$;

$\mathrm{fh}=\mathrm{f}+\mathrm{fi}$;

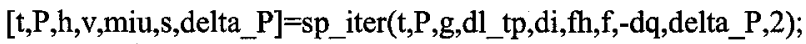

if(abs(dP_outer) $>=$ EPS_P_outer)

if(strcmp(type, Parellel'))

[t_outer,P_outer,h_outer,v_outer,miu_outer,s_outer]=sp_iter(t_outer,P_outer,g_outer,dl_tp,di_eq,f_outer,0,dq,0,3);

else

[t_outer,P_outer,h_outer,v_outer,miu_outer,s_outer]=sp_iter(t_outer,P_outer,g_outer,dl_tp,di_eq,-f_outer,0,-dq,0,3);

end;

else

if(strcmp(type,'Parellel'))

[t_outer,P_outer,x_outer,h_outer,v_outer,miu_outer,s_outer]=tp_iter(t_outer,P_outer,x_outer,g_outer,dl_tp,di_eq,f_outer,f,dq,0,1); else

[t_outer,P_outer,x_outer,h_outer,v_outer,miu_outer,s_outer]=tp_iter(t_outer,P_outer,x_outer,g_outer,dl_tp,di_eq,-f_outer,-f,-dq,0,1); end;

end;

if $($ abs $(t-t$ _old $)>2$ )

dl_tp $=0.5^{*} \mathrm{dl} \_\mathrm{tp}$;

elseif(abs(t-t_old $)<0.3)$

dl_tp=2*dl_tp;

end;

t_old $=t$;

$\mathrm{dl}=1 \_\mathrm{r}-1 \_\mathrm{sp}-1 \_\mathrm{tp}$;

if $(\mathrm{dl}<1 \mathrm{e}-4)$

disp(sprintf(>>507 Tube length reached, $\left.1=0.3 \mathrm{fm}, \mathrm{dl}=\% .4 \mathrm{fm} ., 1 \_\mathrm{r}, \mathrm{dl}\right)$ ); 
outlet $=1$;

if(choke iter $>1$ )

choke $=0$;

end;

break;

end;

if $(t<100)$

[h_sat,s_sat, v_sat,P_sat] $=t$ _P_x $(t, 0,1,0)$;

$\mathrm{dP}=\mathrm{P}-\mathrm{P}_{\text {_ sat; }}$

ratio=abs(dP_old/dP);

if(ratio $=0$ )

if(ratio $>5$ )

dl_tp $=0.05 * d 1 \_t p ;$

elseif(ratio $>3$ )

d1_tp $=0.1 * \mathrm{dl}$ ttp;

elseif(ratio $>1.5$ )

$\mathrm{dl} \_\mathrm{tp}=0.25 * \mathrm{dl} \_\mathrm{tp}$

elseif(ratio $>1.2$ )

dl_tp $=0.5 * \mathrm{dl}$ tp;

end;

end;

if $(\operatorname{abs}(\mathrm{dP})<0.05)$

dl_tp $=0.001 ;$

end;

end;

if $\left(\operatorname{abs}(d P)>0.2 \& \& d 1 \_t p>0.04\right)$

dl_tp $=0.04$;

end;

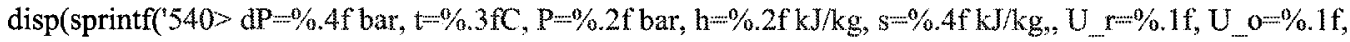
$\mathrm{x}_{\mathrm{O}} \mathrm{o}=\% .3 \mathrm{f}, \mathrm{Q}=\% .3 \mathrm{fW} \mathrm{dl} \mathrm{tp}_{\mathrm{p}}=\% .4 \mathrm{f} \mathrm{cm}, \mathrm{l}_{-} \mathrm{sp}=\% .4 \mathrm{fm} . \mathrm{f}^{\prime}, \ldots$

dP,t,P,h,s,U_r,U_outer,x_outer,Q,100*dl_sp,1_sp));

dP_old $=\mathrm{dP}$;

if $(\bmod (\mathrm{i}, 2)=0)$

t_out $(j)=t ; p \_o u t(j)=P ; h \_o u t(j)=h ; v \_o u t(j)=v ; \operatorname{miu} \_$out $(j)=\operatorname{miu} ; x \_$out $(j)=x$;

s_out $(j)=s ; Q$ out $(j)=Q ;$ fh_out $(j)=f h ; f \_o u t(j)=f ; f i \_o u t(j)=f i ; 1 \_o u t(j)=1$ tp;

t_outer_out $(j)=$ __outer; p_outer_out $(j)=P$ _outer; $h$ _outer_out $(j)=h$ _outer;

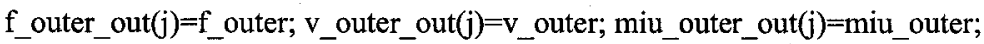

s_outer_out $(j)=s \_o u t e r ; x \_o u t e r \_o u t(j)=x \_o u t e r ;$

if $(x<0)$

$x_{-} \operatorname{out}(j)=0$;

end; 


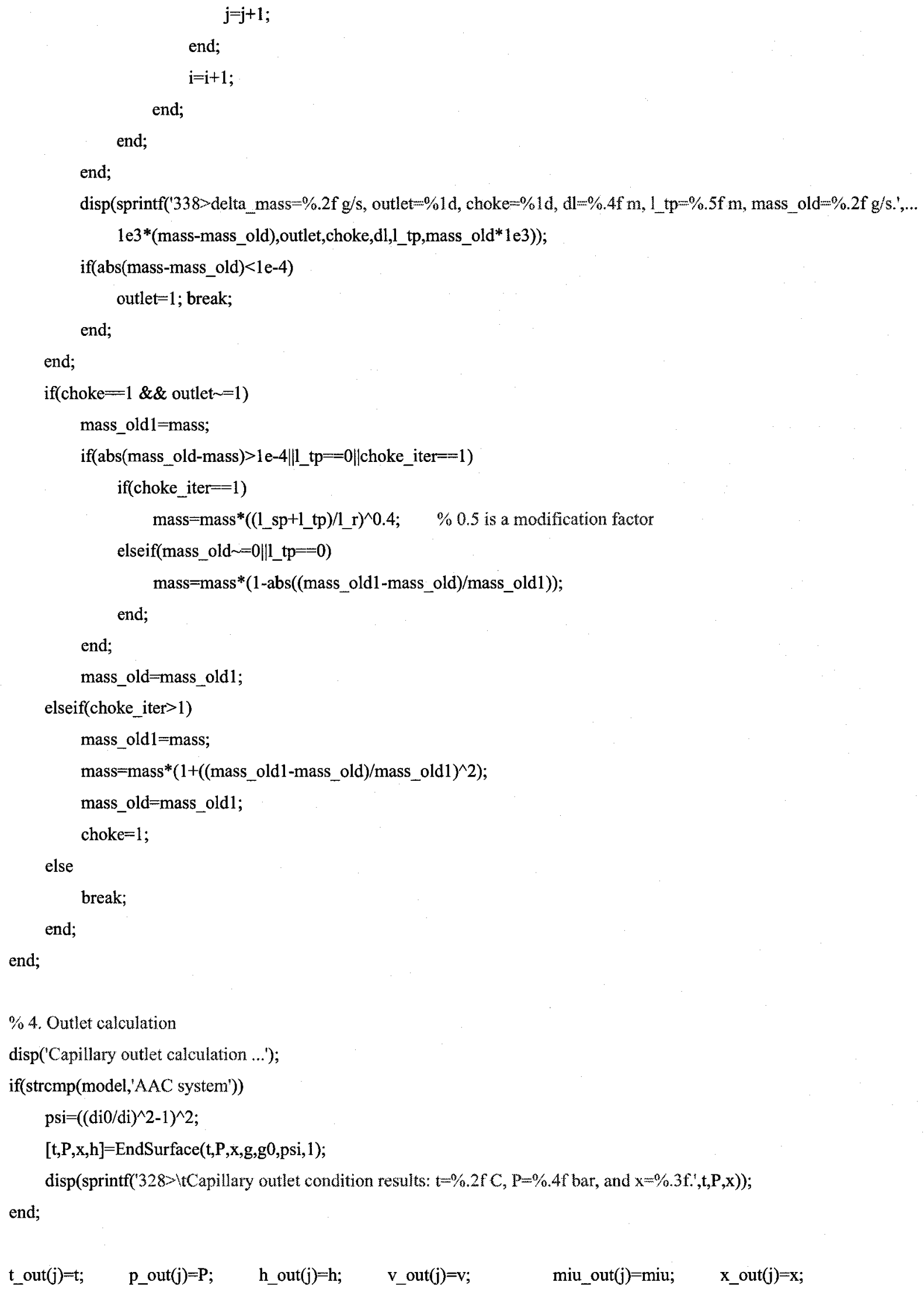




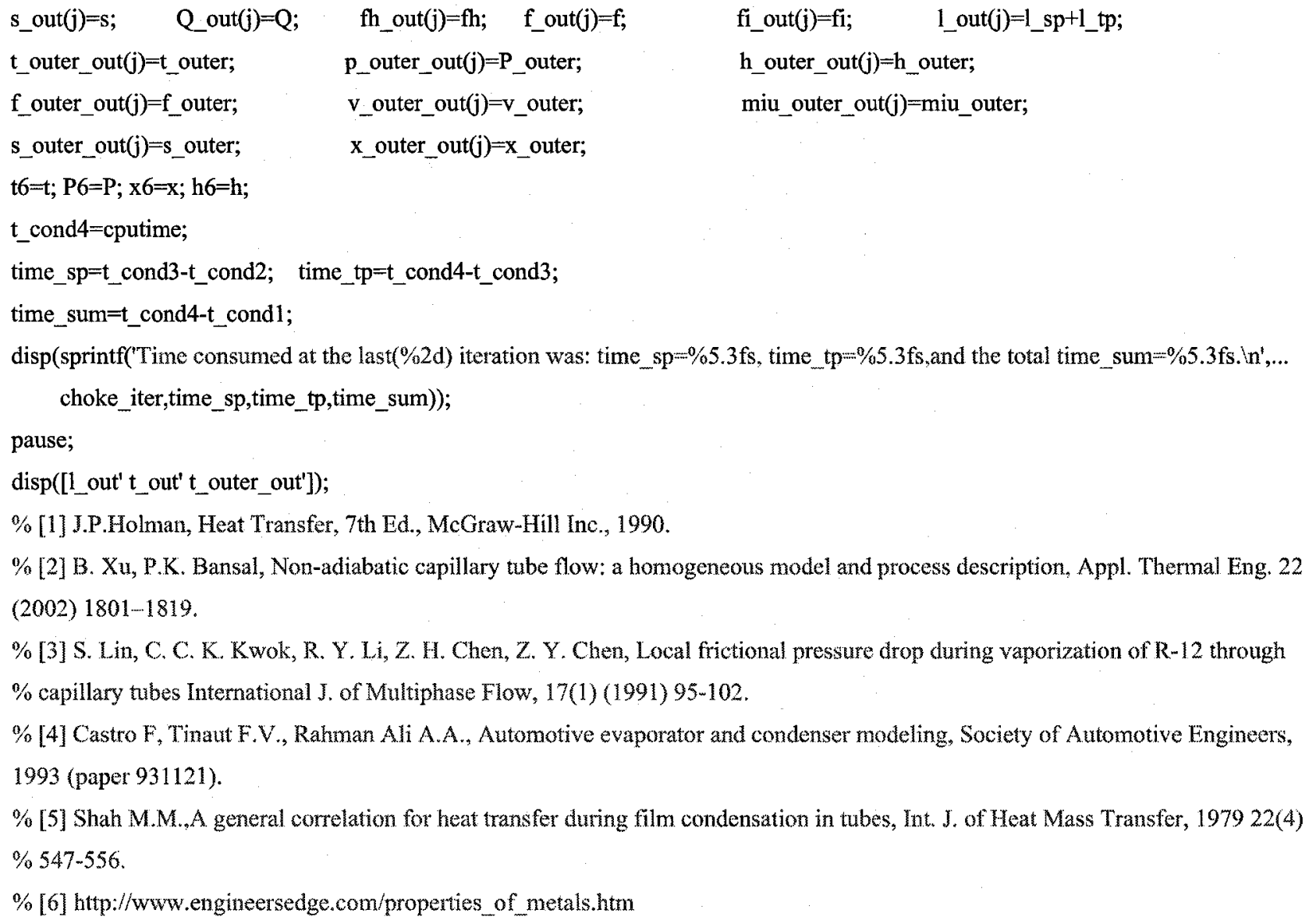

\section{A-2 Inlet and outlet calculation}

\section{This function takes care of sudden contractions and expansions.}

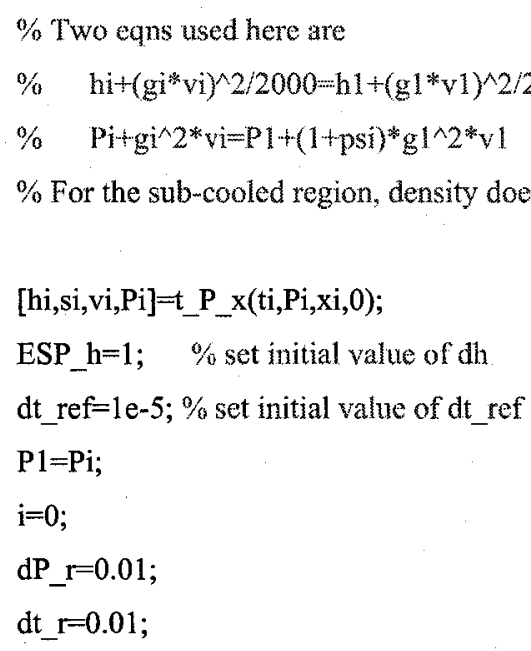




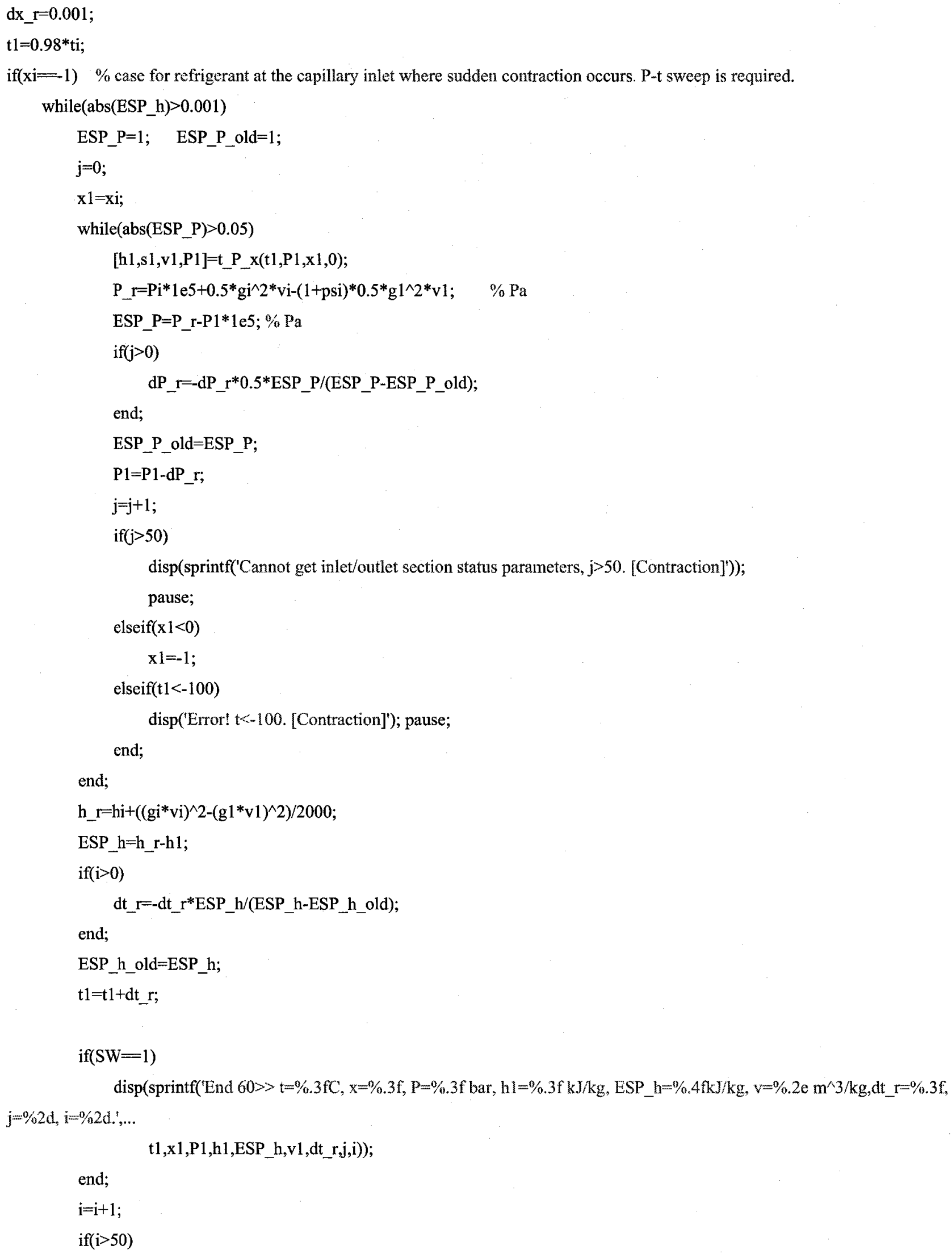




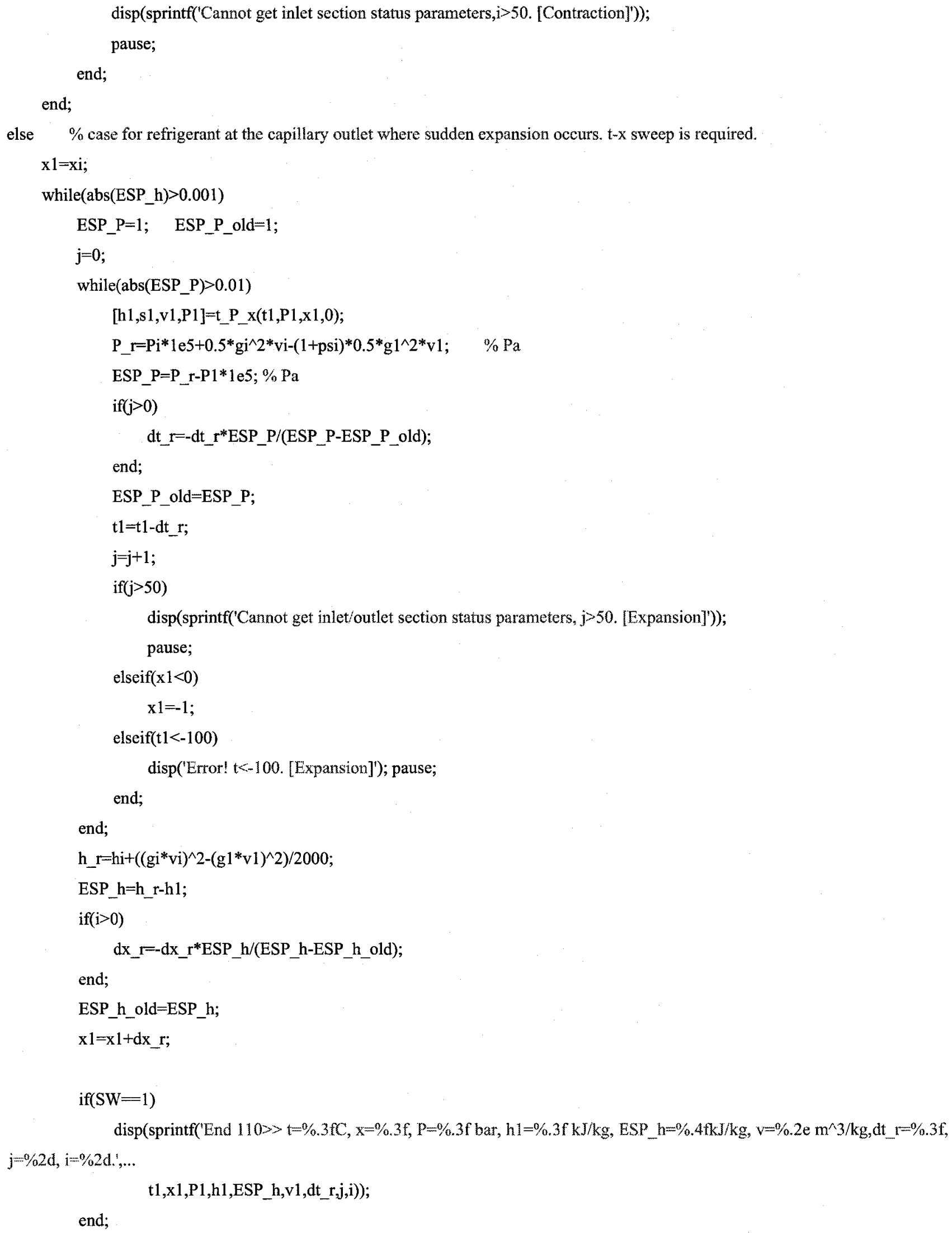


$\mathrm{i}=\mathrm{i}+1$

if $(\mathrm{i}>50)$

$\operatorname{disp(sprintf('Cannot~get~inlet~section~status~parameters,~} ;>50$. [Expansion]'));

pause;

end;

end;

end;

\section{A-3 Calculations of R134a properties knowing two temperatures}

This function provides the thermophysical properties of R134a using the PR equation. For a single-phase flow, two temperatures are required as inputs, one for determining saturated pressure and the other for the real temperature. For a two-phase flow, the temperature and the quality of the refrigerant are required.

function $[\mathrm{h}, \mathrm{s}, \mathrm{v}, \mathrm{P}, \mathrm{miu}, 1 \mathrm{ambda}, \mathrm{cp}]=\mathrm{R} 134 \mathrm{aProp}(\mathrm{tp}, \mathrm{t}, \mathrm{x}, \mathrm{trans})$

$\%$ t $\mathrm{p}$ is reference temperature, being used to calculate the corresponding saturated pressure, Celsius

$\% t$ is refrigerant temperature, Celsius

$\% \mathrm{x}$ is refrigerant quality

$\%$ trans is a sign for whether or not calculating the transport properties. trans $=0$, no; trans $=1$, yes.

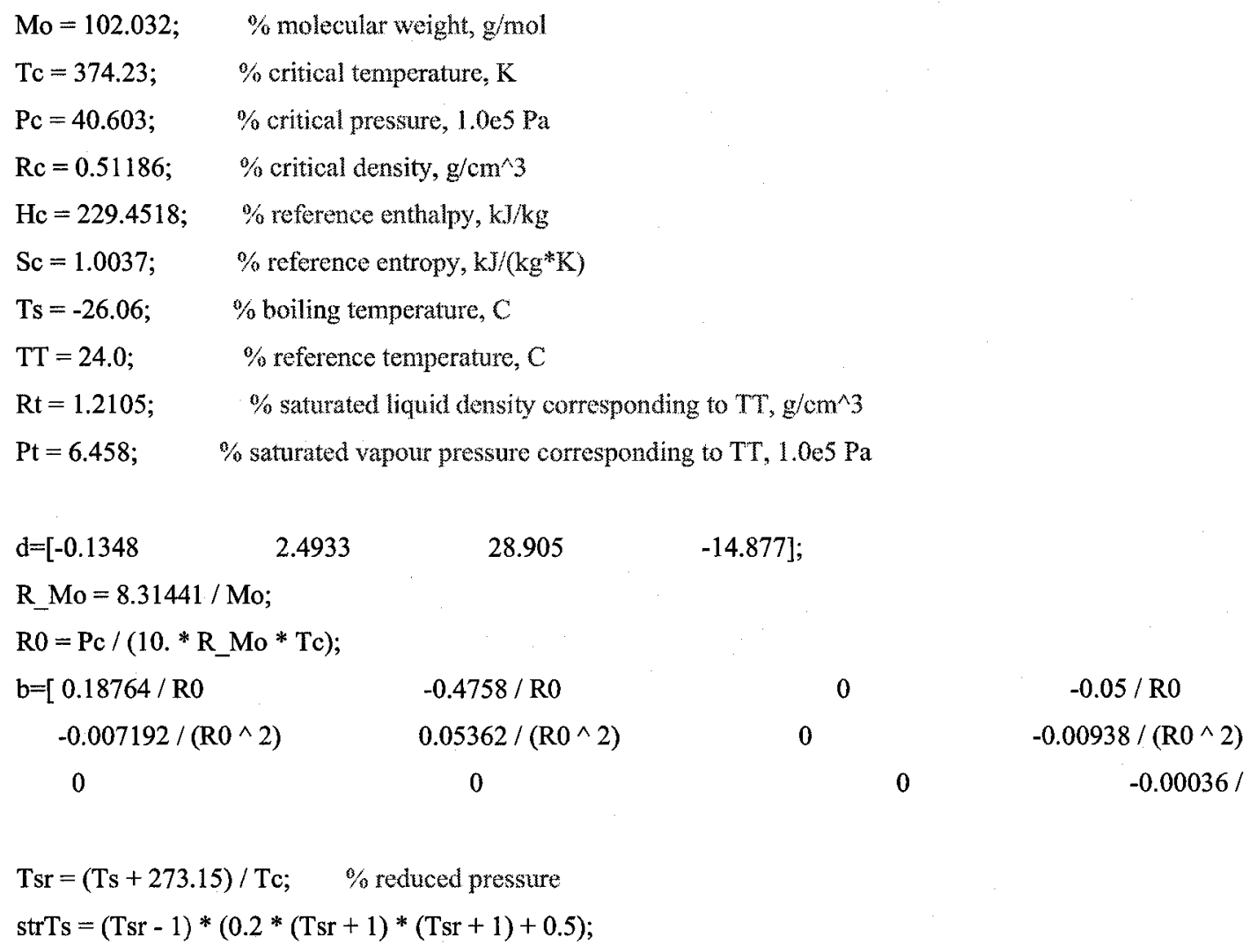




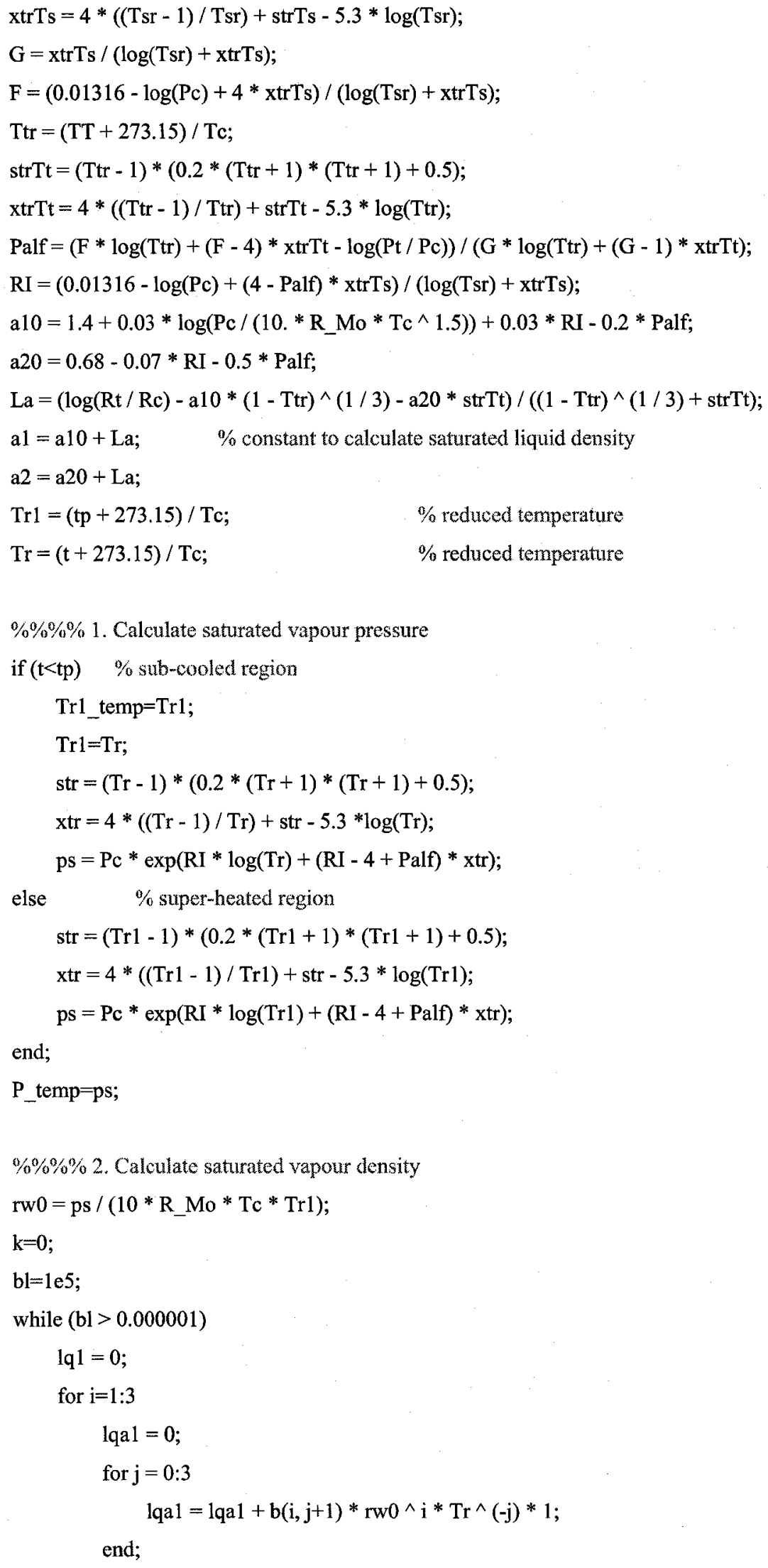




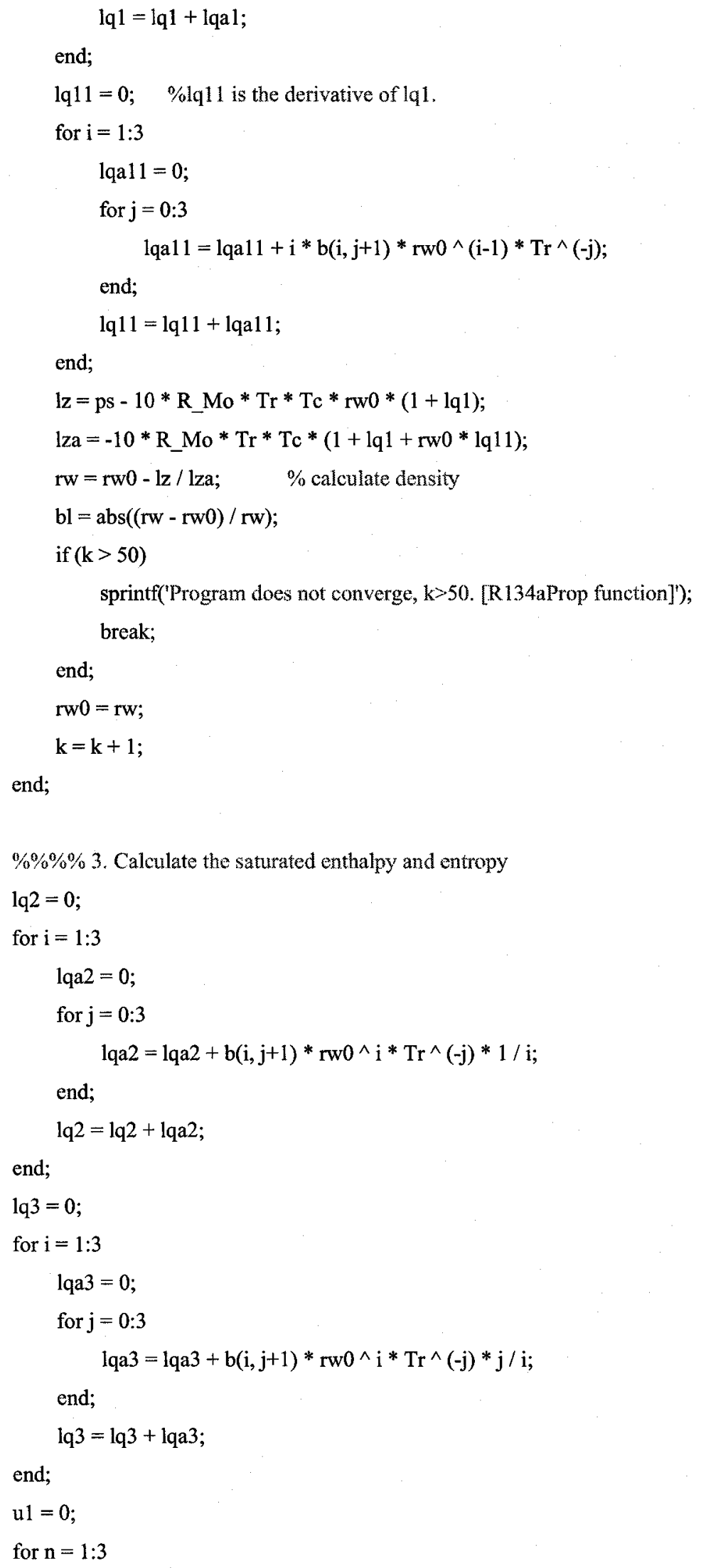


$\%$ calculate the reduced internal energy

$\mathrm{ul}=\mathrm{ul}+\mathrm{d}(\mathrm{n}+1) *(\operatorname{Tr} * \mathrm{Tc} / 1000 .)^{\wedge} \mathrm{n} / \mathrm{n}$

end;

$\mathrm{u} 0=1000 . * \mathrm{R} \_\mathrm{Mo} *(\mathrm{~d}(1) * \log (\mathrm{Tr} * \mathrm{Tc} / 1000)+.\mathrm{u} 1) ; \quad \%$ reduced internal energy

s_ref $=-\mathrm{d}(1) /(\operatorname{Tr} * \operatorname{Tc} / 1000)+.\mathrm{d}(2) * \log (\operatorname{Tr} * \mathrm{Tc} / 1000)+.\mathrm{d}(3) *(\operatorname{Tr} * \mathrm{Tc} / 1000$.

$\mathrm{s} 0=\mathrm{R}_{-} \mathrm{Mo} *\left(\mathrm{~s}_{-}\right.$ref $\left.+0.5 * \mathrm{~d}(4) *(\mathrm{Tr} * \mathrm{Tc} / 1000 .)^{\wedge} 2\right) ; \quad \%$ reduced entropy with constant volume

$\%$ Modify the saturated enthalpy and entropy to make them close to NIST results

$\%$ if $t=t p, t$ modi $=273.15 /(\mathrm{t}+273.15)$;

$\%$ if $t \mathrm{tp}=0 \mathrm{C}, \mathrm{t} \operatorname{modi}=1$.

if $(\mathfrak{t}<\mathrm{tp})$

t $\operatorname{modi}=1$;

else

n_t $=((\mathrm{tp}+273.15) / 273.15)^{\wedge} 4.5$;

t_modi $=273.15^{*}(\mathrm{t}+273.15)^{\wedge}\left(\mathrm{n}_{-} \mathrm{t}-0.62\right) /(\mathrm{tp}+273.15)^{\wedge}\left(\mathrm{n}_{-} \mathrm{t}+0.38\right)$;

end;

$\mathrm{hq}=\mathrm{t} \_$modi^ $0.19 *\left(\mathrm{R} \_\mathrm{Mo} * \mathrm{Tc} * \mathrm{Tr} *(1+\mathrm{lq} 1+\mathrm{lq} 3)+\mathrm{u} 0\right)+\mathrm{Hc} ; \%$ vapour enthalpy

$\mathrm{sq}=\mathbf{t} \_\operatorname{modi}{ }^{\wedge} 0.13 *\left(\mathbf{R} \_\mathrm{Mo} *(\mathrm{lq} 3-\operatorname{lq} 2-\log (\mathrm{rw} 0))+\mathrm{s} 0\right)+\mathrm{Sc} ; \%$ vapour entropy

$\mathrm{vq}=1 . /(1000 . * \mathrm{rw} 0)$;

if $(\operatorname{Tr}<=1)$

$\mathrm{rwl}=\mathrm{Rc} * \exp \left(\mathrm{al} *(1-\mathrm{Tr})^{\wedge}(1 / 3)+\mathrm{a} 2 * \operatorname{str}\right) ; \%$ liquid density

$\mathrm{vl}=1 /(1000 *$ rwl);

$\mathrm{pst}=(\mathrm{RI} / \operatorname{Tr} 1+(\mathrm{RI}-4+\operatorname{Palf}) *(4 . /(\operatorname{Tr} 1 * \operatorname{Tr} 1)-5.3 / \operatorname{Tr} 1+0.2 *(\operatorname{Tr} 1+1) *(\operatorname{Tr} 1+1)+0.4 *(\operatorname{Tr} 1+1) *(\operatorname{Tr} 1-1)+0.5)) *$

ps / Tc;

hq1 $=0.0965 * \mathrm{t}_{-} \operatorname{modi}{ }^{\wedge} 0.15^{*} \operatorname{Tc}{ }^{*} \operatorname{Tr} *(1 . /$ rw0 $-1 . /$ rwl $) *$ pst; \% latent heat

$\mathrm{hl}=\mathrm{hq}-\mathrm{hq1} ; \quad \%$ saturated liquid enthalpy

$\mathrm{sl}=\mathrm{sq}-0.0965 *(1 . / \mathrm{rw} 0-1 . / \mathrm{rwl}) * \mathrm{pst} ; \%$ saturated liquid entropy

else

$\mathrm{hl}=\mathrm{hq}$;

$\mathrm{sl}=\mathrm{sq}$;

$\mathrm{vl}=\mathrm{vq}$;

end;

$\%$ to return correct pressure, calculate ps again

if $(t<t p)$

$\operatorname{Tr} 1=\operatorname{Tr} 1$ _temp;

$\operatorname{str}=(\operatorname{Tr} 1-1) *(0.2 *(\operatorname{Tr} 1+1) *(\operatorname{Tr} 1+1)+0.5)$

$\mathrm{xtr}=4 *((\operatorname{Tr} 1-1) / \operatorname{Tr} 1)+\mathrm{str}-5.3 * \log (\operatorname{Tr} 1)$;

ps $=\mathrm{Pc} * \exp (\mathrm{RI} * \log (\mathrm{Tr} 1)+(\mathrm{RI}-4+$ Palf $) * \mathrm{xtr})$;

end;

$P=p s ;$ 


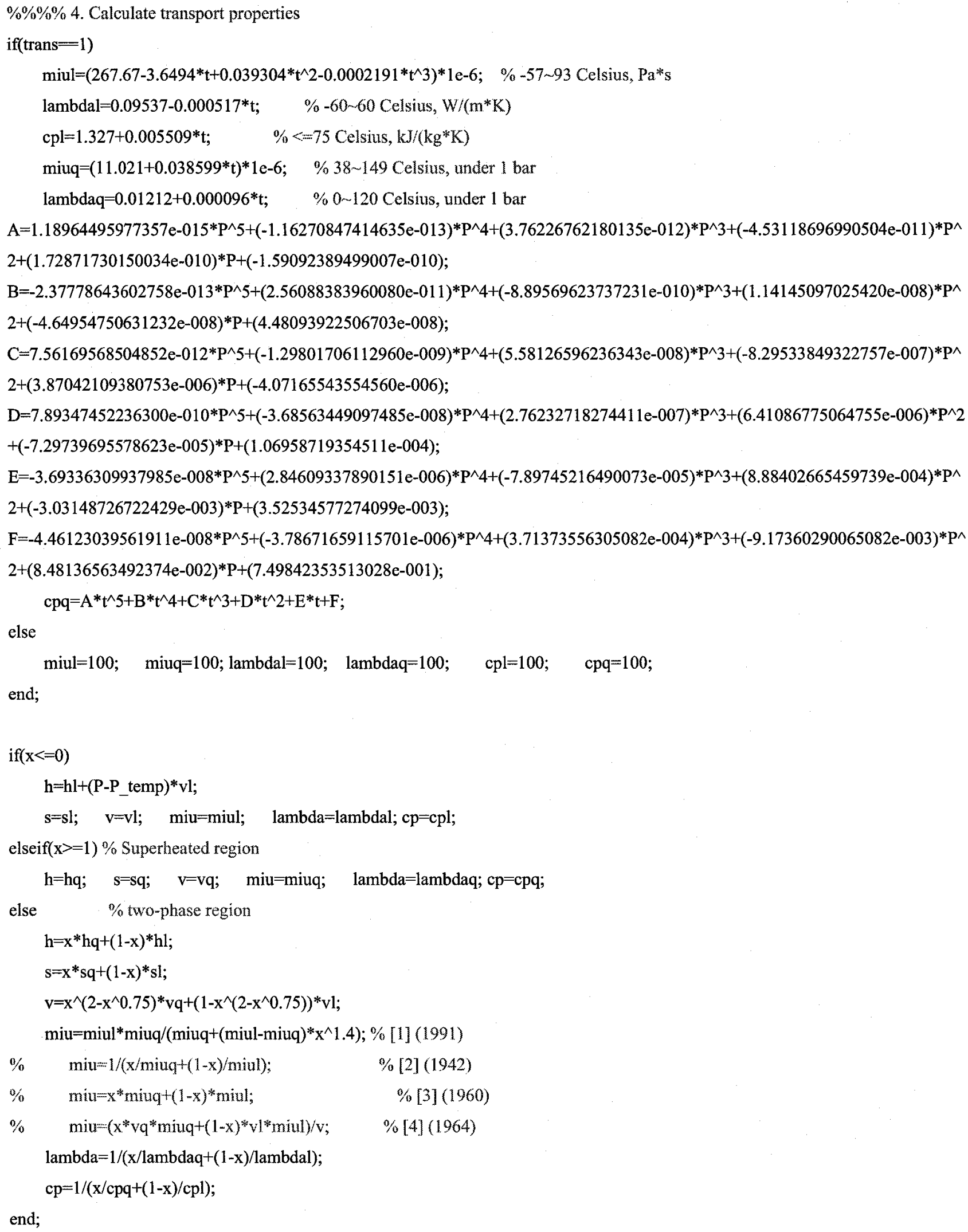


$\%$ [1] S. Lin, et. al, Local friction pressure drop during vaporization of R-12 through capillary tubes, Int. J. Multiphase Flow, Vol. 17 , No. 1, 95-102, 1991. Equation (22).

\% [2] W. H. MoAdams et. al., Vaporization inside horizontal tubes II, Benzene-oil mixture, Trans. ASME 64, $193,1942$.

$\%$ [3] A. Cicchitti, et. al., Two-phase cooling experimetns-pressure drop, heat transfer and burnout measurements, Energia Nucl.

Milano, 7, 407-425, 1960 .

\% [4] A. E. Dukler, M. Wicks, and R. G. Cleveland, Pressure drop and hold-up in two-phase flow: Part A-A comparison of existing $\%$ correlations; Part B-An approach through similarity analysis, AICHE Journal 10(1), 38-51.

\section{A-4 Calculation of the single-phase flow}

This function calculates the outlet condition for a capillary element $d l$ if inlet condition is given and the flow is single-phase.

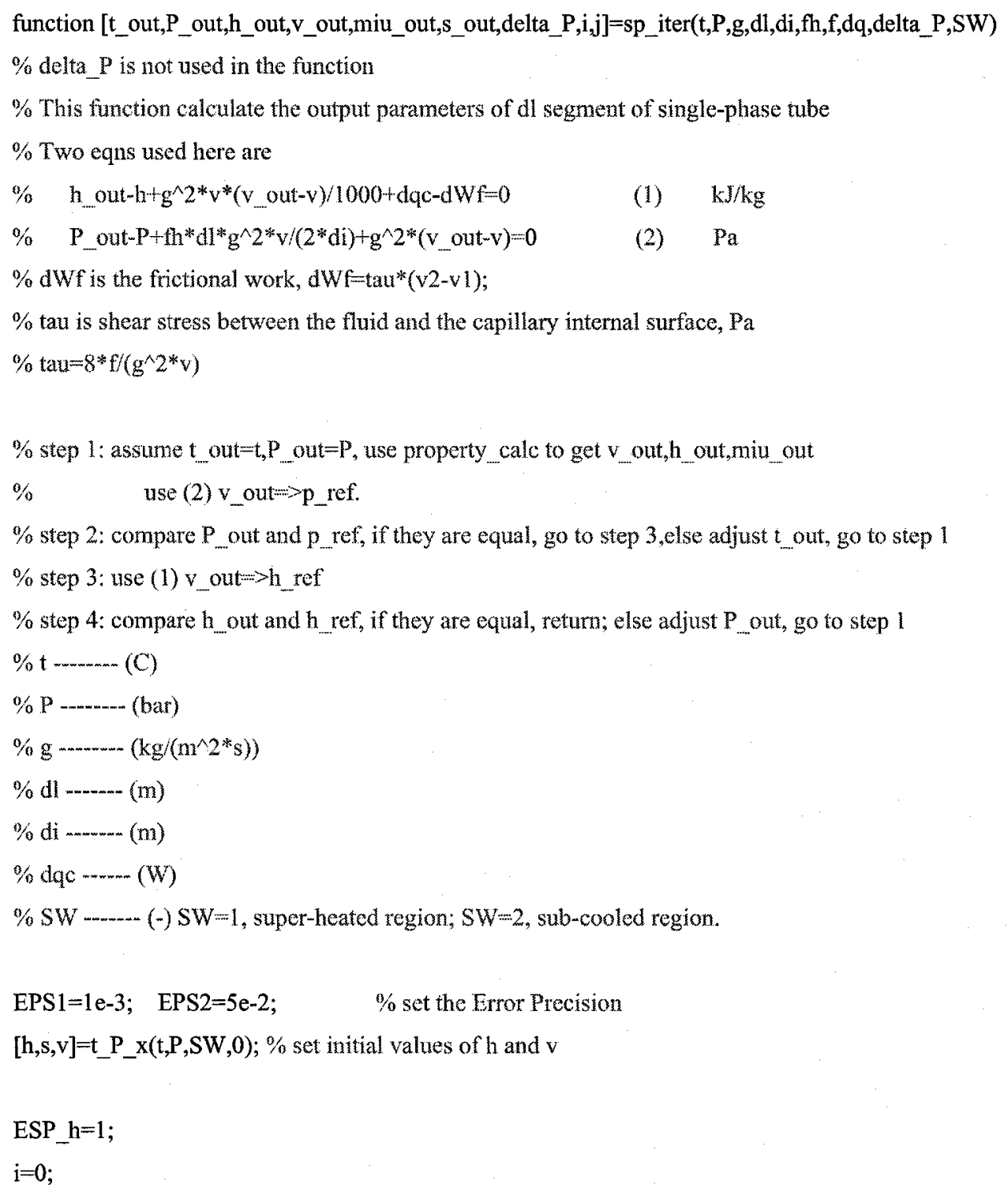




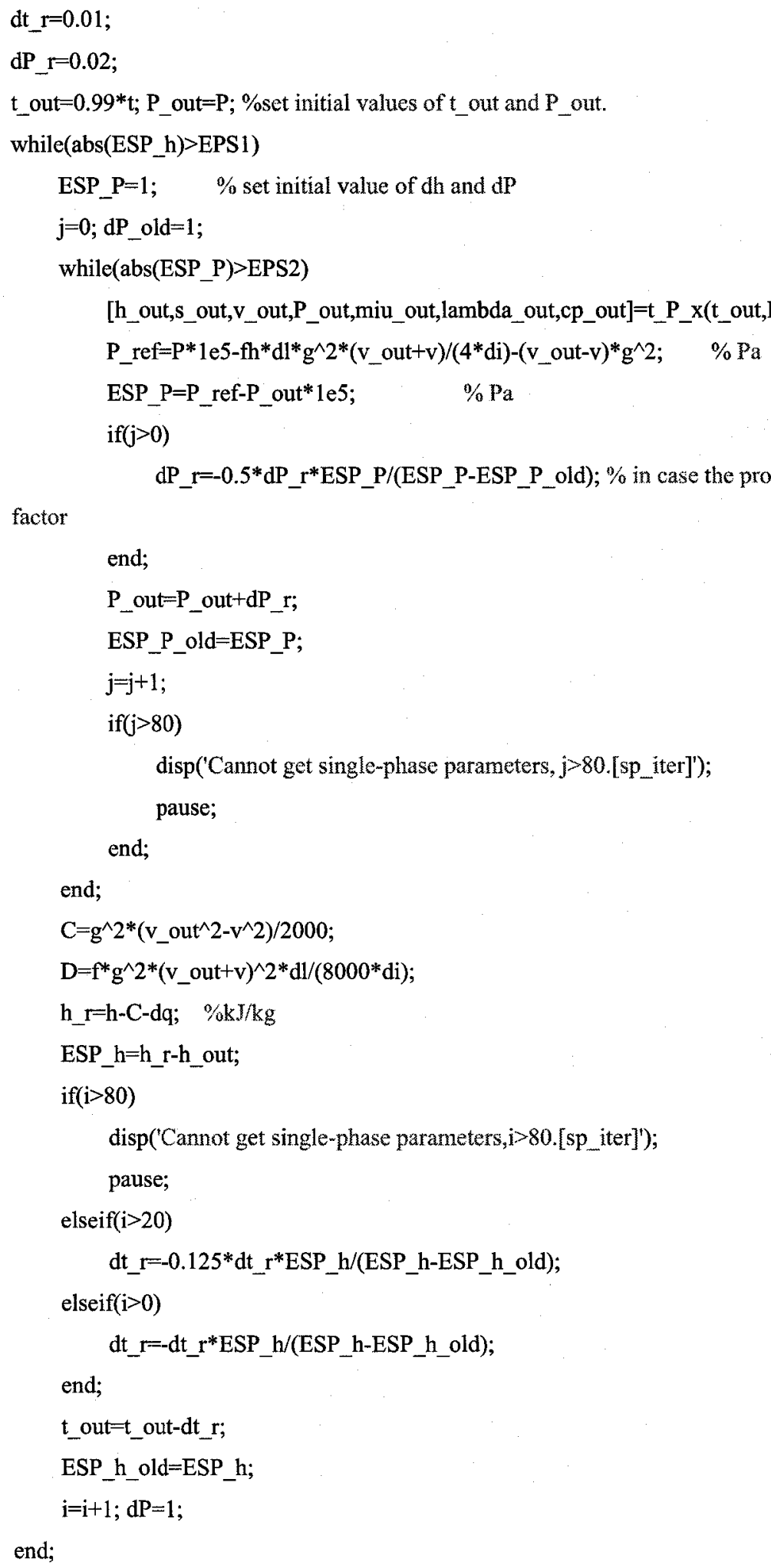

\section{A-5 Calculation of the two-phase flow}


This function calculates the outlet condition for a capillary element $d l$ if inlet condition is given and the flow is two-phase.

function [t_out,P_out,x_out,h_out,v_out,miu_out,s_out,i] $=\operatorname{tp}$ iter(t,P,x,g,dl,di,fh,f,dq,EPS_indicator,SW)

$\%$ This function calculate the output parameters of dl segment of single-phase tube

$\%$ Two eqns used here are
$\% \quad$ h_out $-\mathrm{h}+\mathrm{g} \wedge 2 * \mathrm{v}^{*}(\mathrm{v}$ out-v)/1000+Q $\mathrm{c}=0$
(1) $\mathrm{kJ} / \mathrm{kg}$

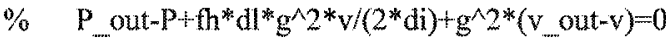
(2) $\mathrm{Pa}$

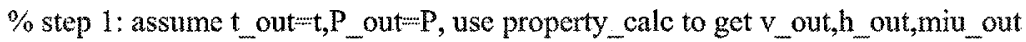

$\% \quad$ use $(2) \vee$ out $\Rightarrow \mathrm{P}$ ref.

$\%$ step 2: compare $P$ _out and $P$ _ef, if they are equal, go to step 3,else adjust $t$ _out, go to step 1

$\%$ step 3: use (1) v out $\Rightarrow$ h ref

$\%$ step 4: compare h_out and h_ref, if they are equal, return; else adjust $P$ _out, go to step 1

$\% \mathrm{t} \cdots(\mathrm{C})$

$\% \mathrm{P}$-...m-m.m- (bar)

$\% \mathrm{~g}-\left(\mathrm{kg} /\left(\mathrm{m}^{\wedge} 2 *^{*} \mathrm{~s}\right)\right)$

$\% \mathrm{~d} l-m-\cdots-m)$

$\%$ di -.--- (m)

$\% \mathrm{k}-\cdots(-)$

$\%$ dqf ----- $(\mathrm{kJ} / \mathrm{kg})$

$\% \mathrm{dqc} \cdots(\mathrm{kJ} / \mathrm{kg})$

$\% \mathrm{SW}-(-) \mathrm{SW}=1$, expansion; $\mathrm{SW}=2$, condensation/evaporation.

$\%[t, P, x, h, y$, miu,s,omega,i,j] $]=$ p iter $1(34.124,8.6581,0,20435,0.1,0.0018,0.020478,2.2565,0.01,2)$

$[h, s, v, P]=t+P \quad x(t, 0, x, 0) ; \%$ set initial values of $h$ and $v$

$\mathrm{ESP} \mathrm{h}=1 ; \%$ set initial value of ESP $\mathrm{h}$

dt_r=1e-4; \% set initial value of dt $\mathrm{r}$

$\mathrm{dx} r=1 \mathrm{e}-4$;

P_out=P;

if(EPS_indicator $=0) \quad \%$ EPS indicator $=0$, outer refrigerant flows.

$\mathrm{EPS} 1=1 \mathrm{e}-4$;

$\mathrm{EPS} 2=1 \mathrm{e}-3$;

else $\%$ EPS indicator $=1$, inner capillary flow

EPS1=1e-2; $\quad \%$ could be tightened to $5 \mathrm{e}-3$ to increase accuracy, but will leads to unconvergent problems

EPS2 $=1 \mathrm{e}-2 ; \quad \%$ set the Error Precision

end;

$t$ _out $=t ; \quad \%$ set initial values of $t$ out

$\mathrm{i}=\mathbf{0}$;

if $(x<0.1)$

$\mathrm{x} \_$out $=\mathrm{x}+0.001$; 


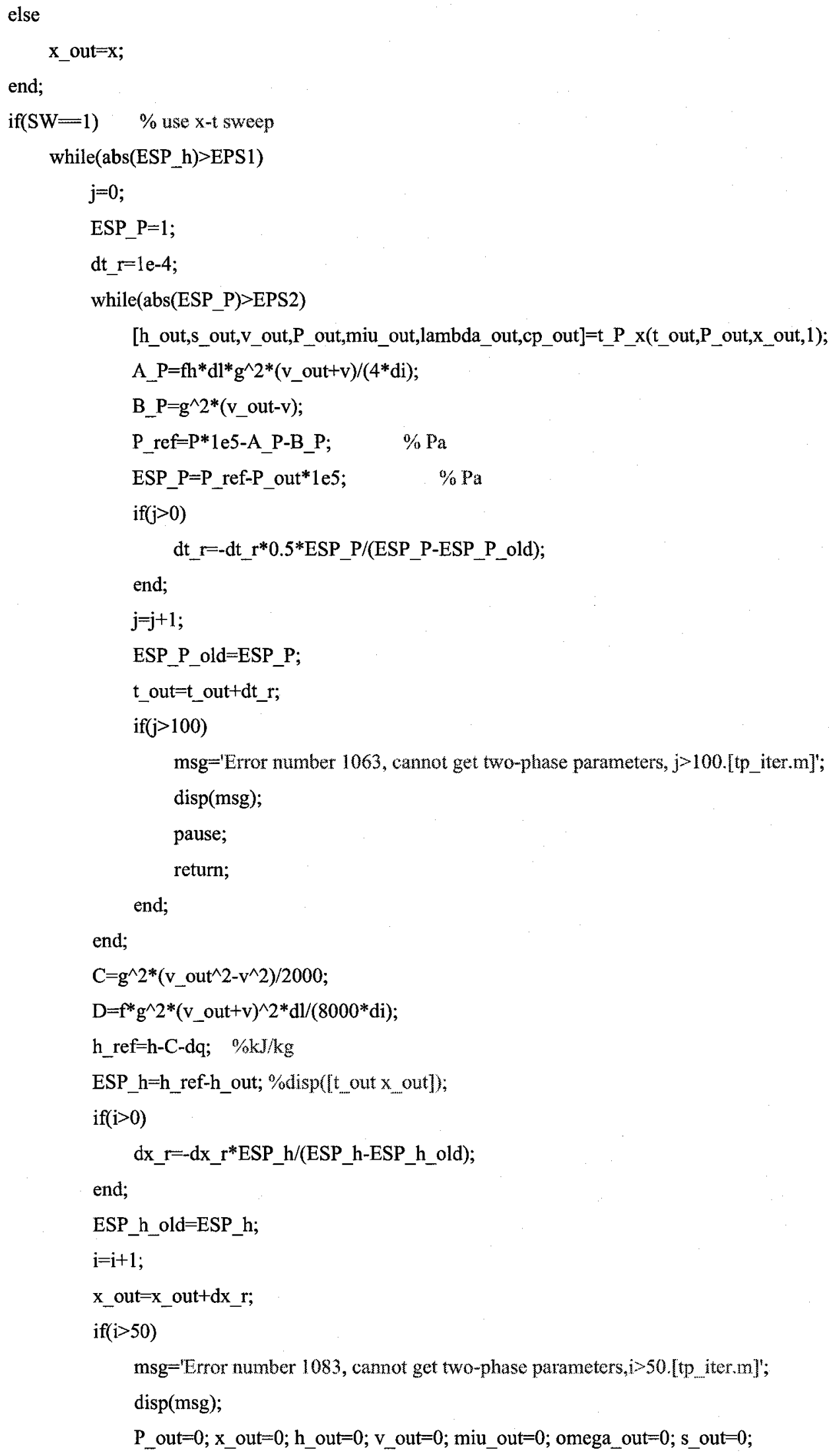




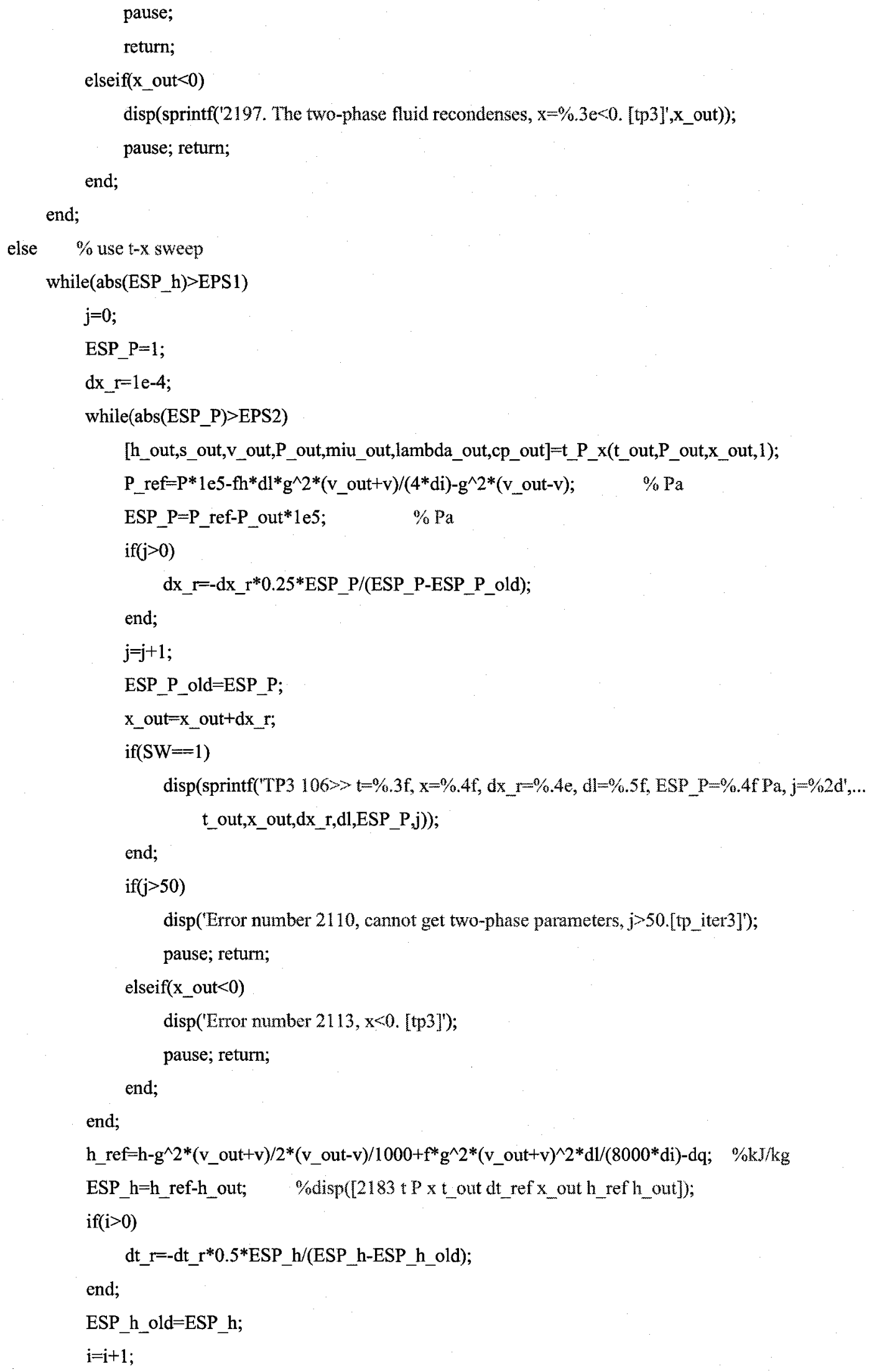

end;

if $(j>50)$

disp('Error number 2110, cannot get two-phase parameters, $j>50 .[\mathrm{tp}$ iter3]'); pause; return; 


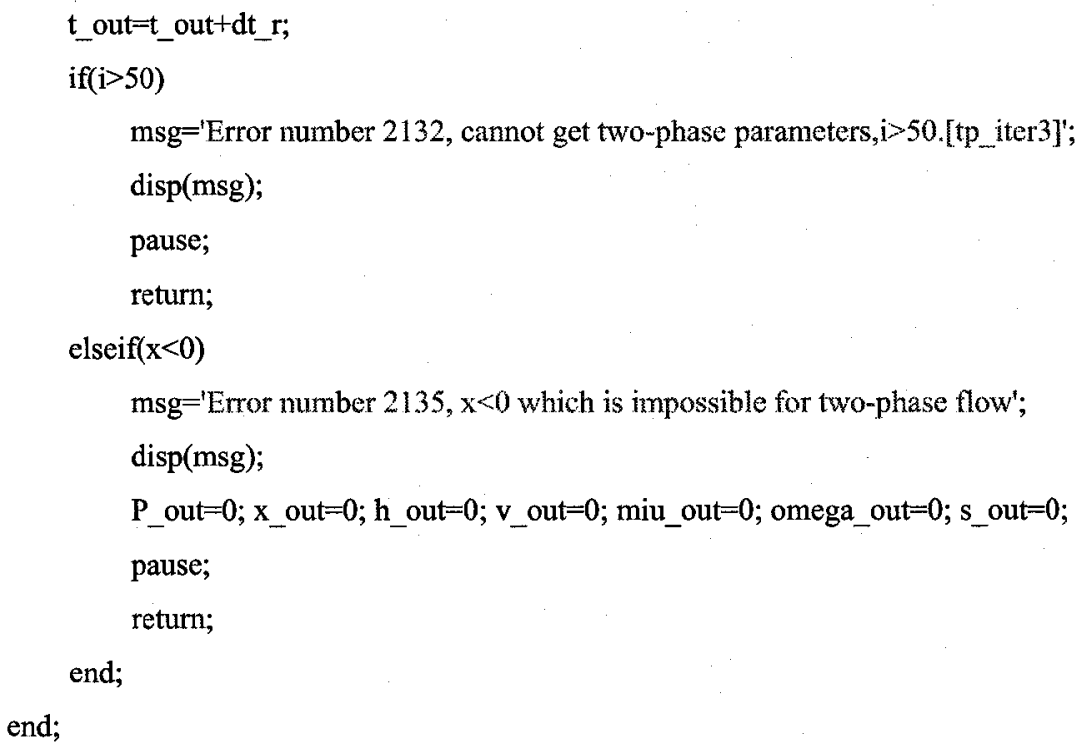

\section{A-6 Calculation of refrigerant properties knowing $t, P$ or $x$}

This function calculates the refrigerant properties with known temperature and pressure/quality. Depending on which flow region the flow lies in, either a pressure or quality is required to be provided with another parameter, a temperature.

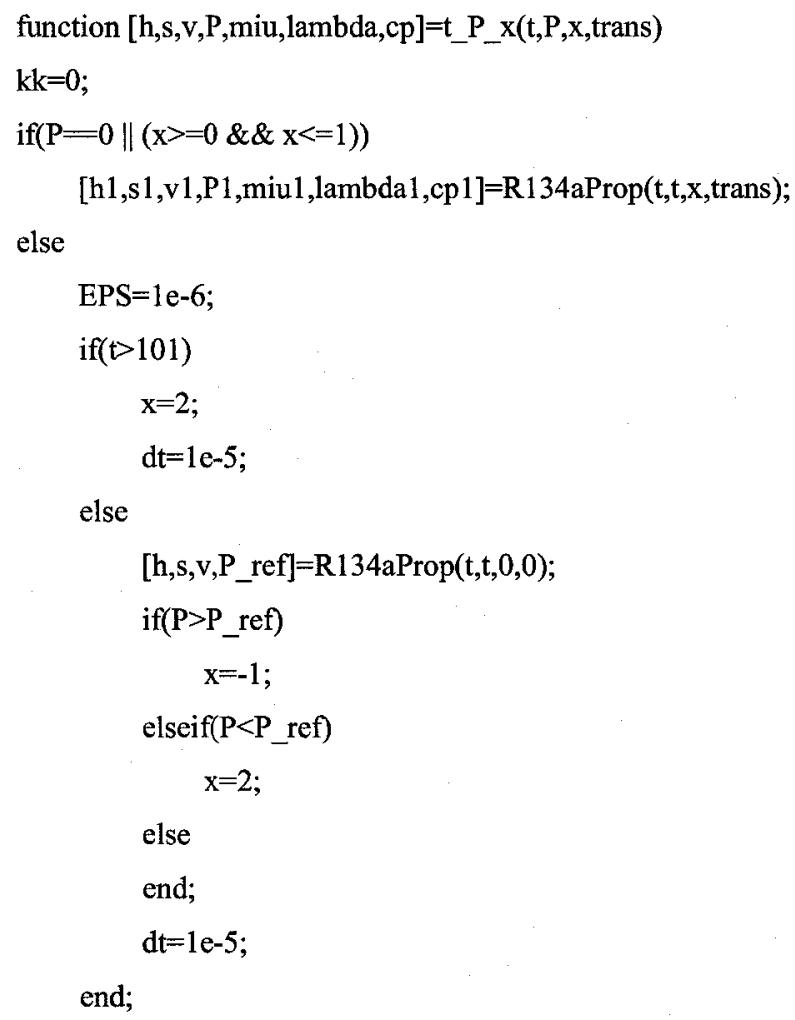




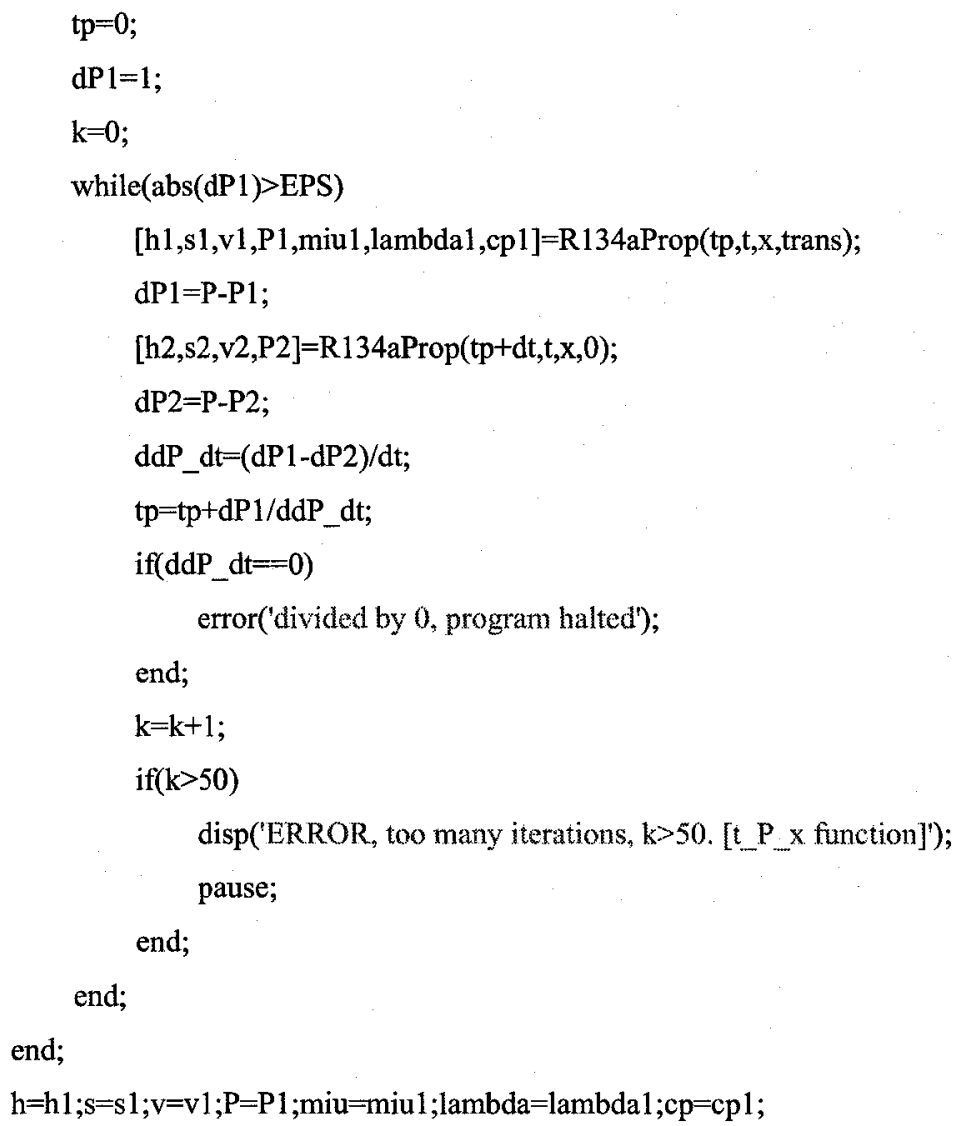

\section{A-7 Calculation of the heat transfer coefficient and pressure drop factor}

This function calculates the heat transfer coefficient and pressure drop factor with given parameters.

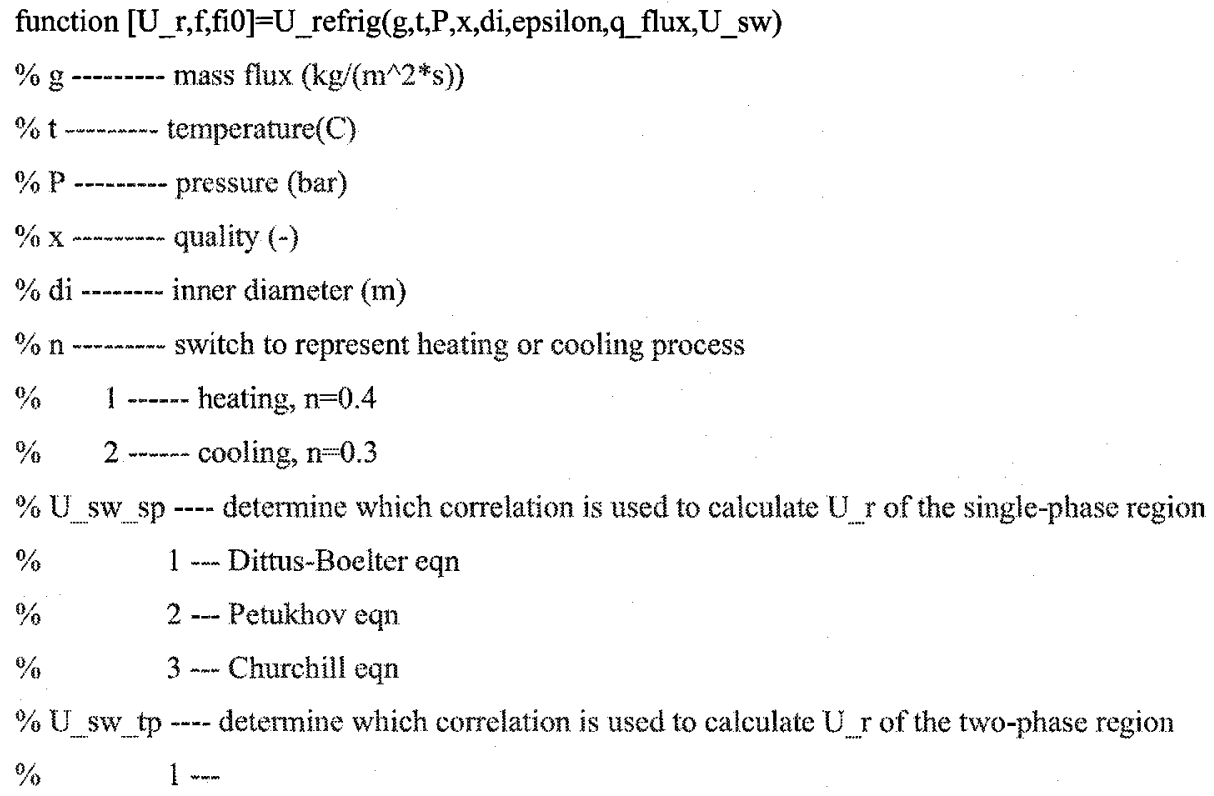




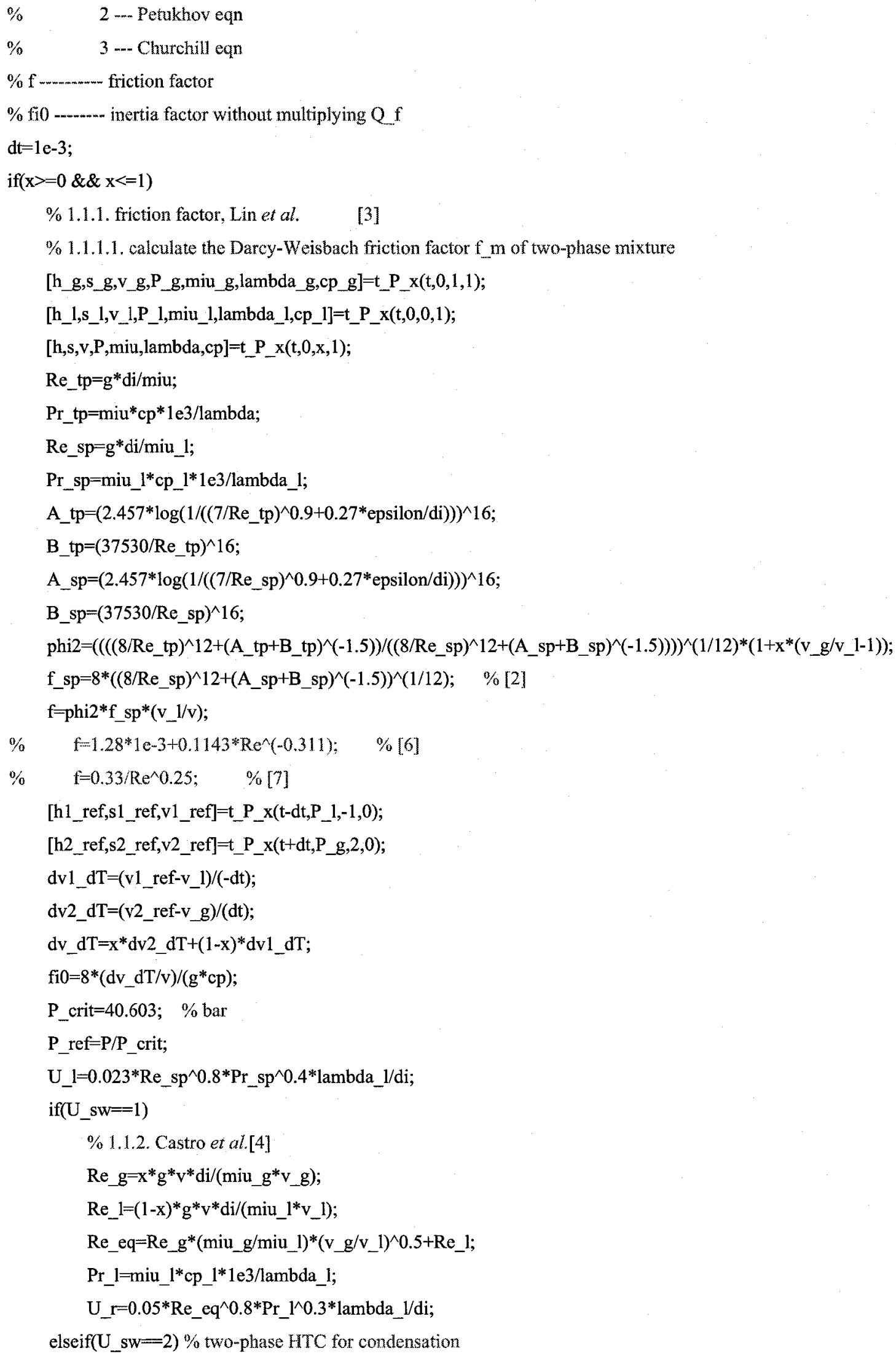




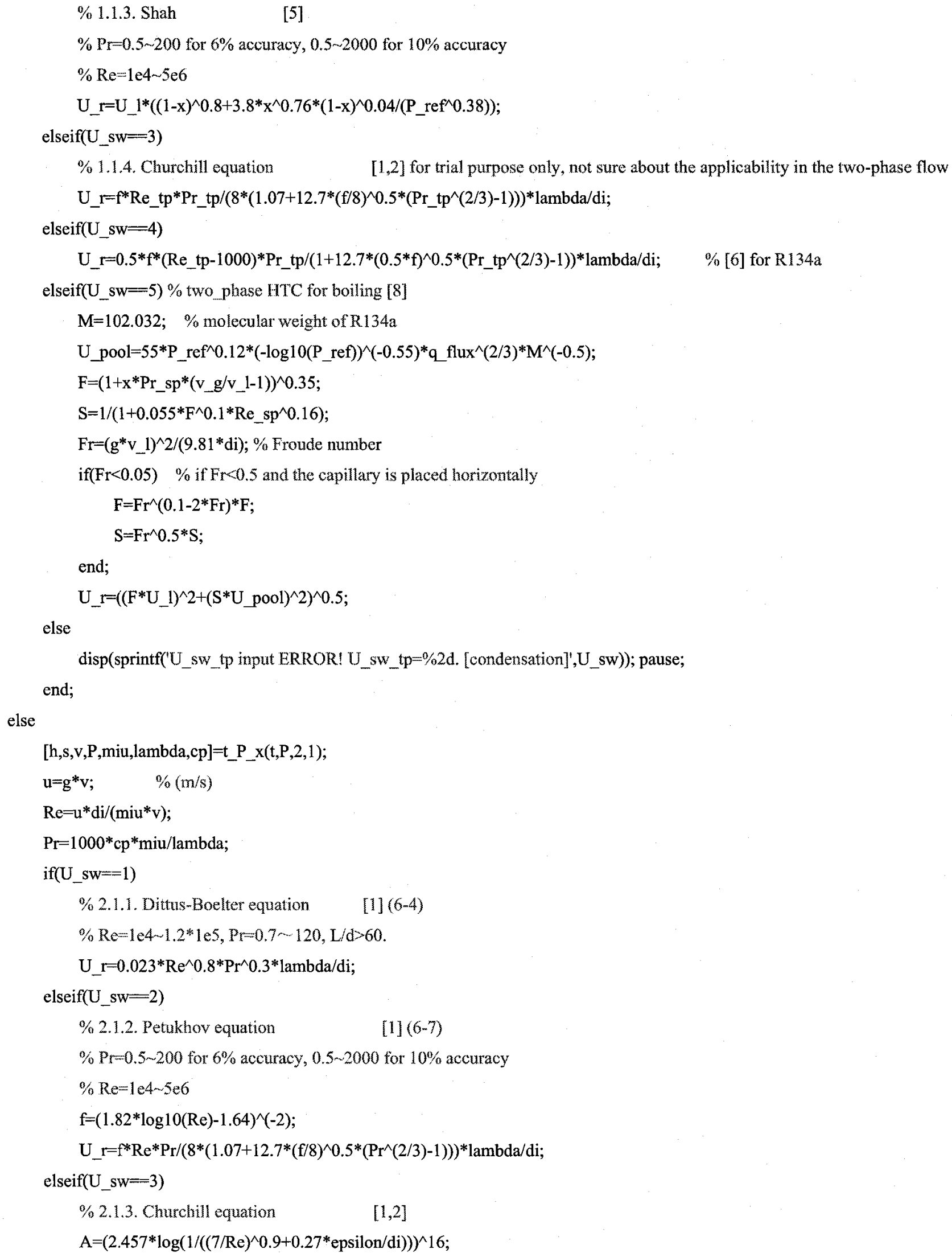




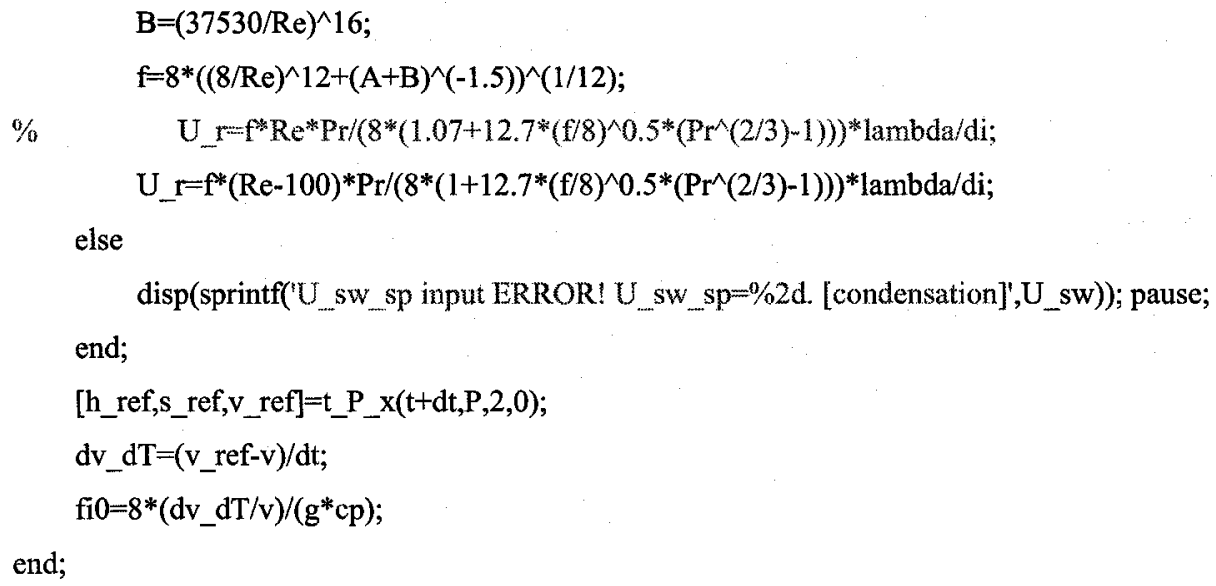

\section{Appendix B Program to correlate pressures and the compressor speed in an}

\section{R134a AAC test stand}

\section{B-1 Main code}

This program is developed to obtain the relationship between changes of system pressures and that of the compressor speed.

clear all; 
format short $\mathrm{g}$;

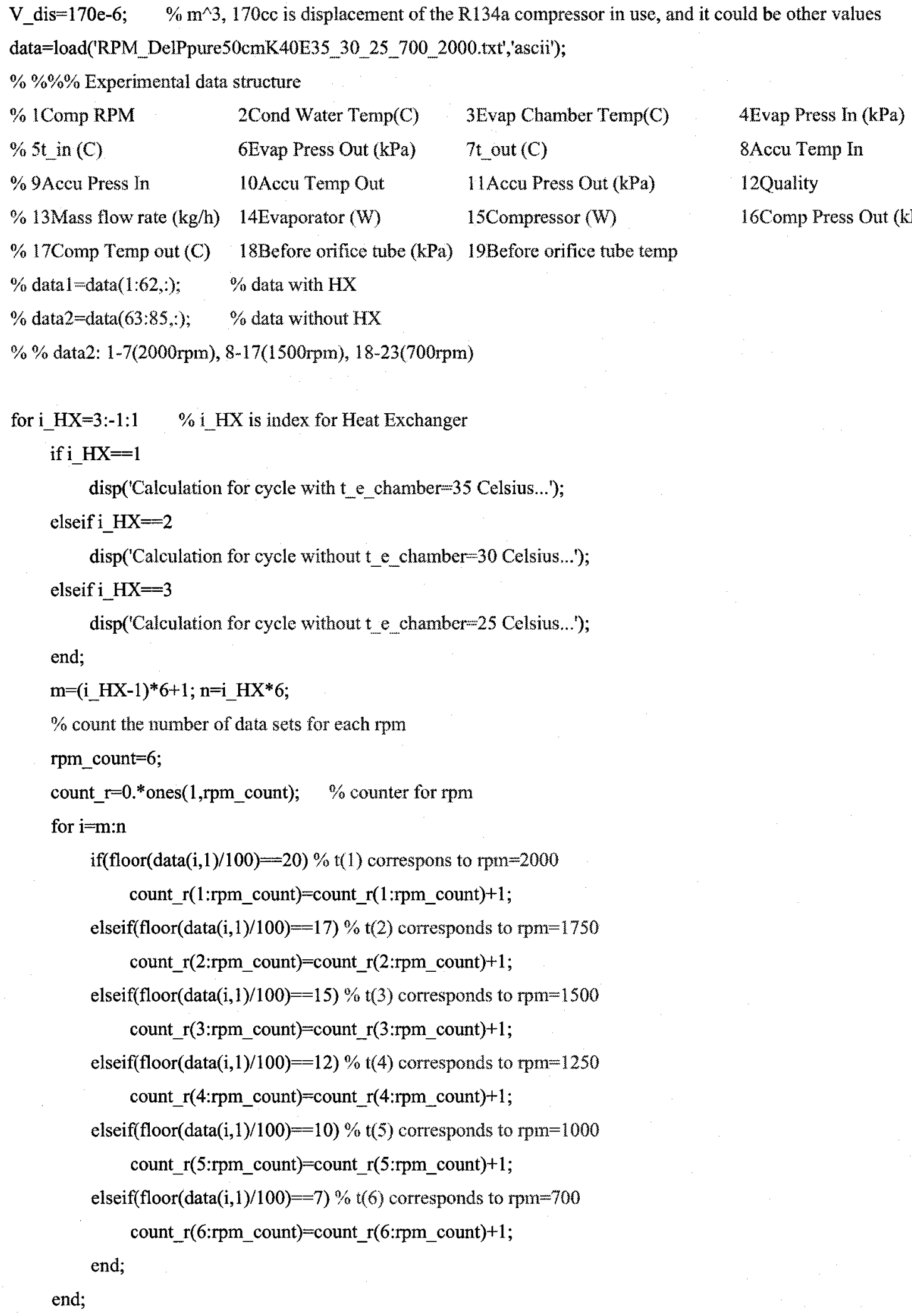




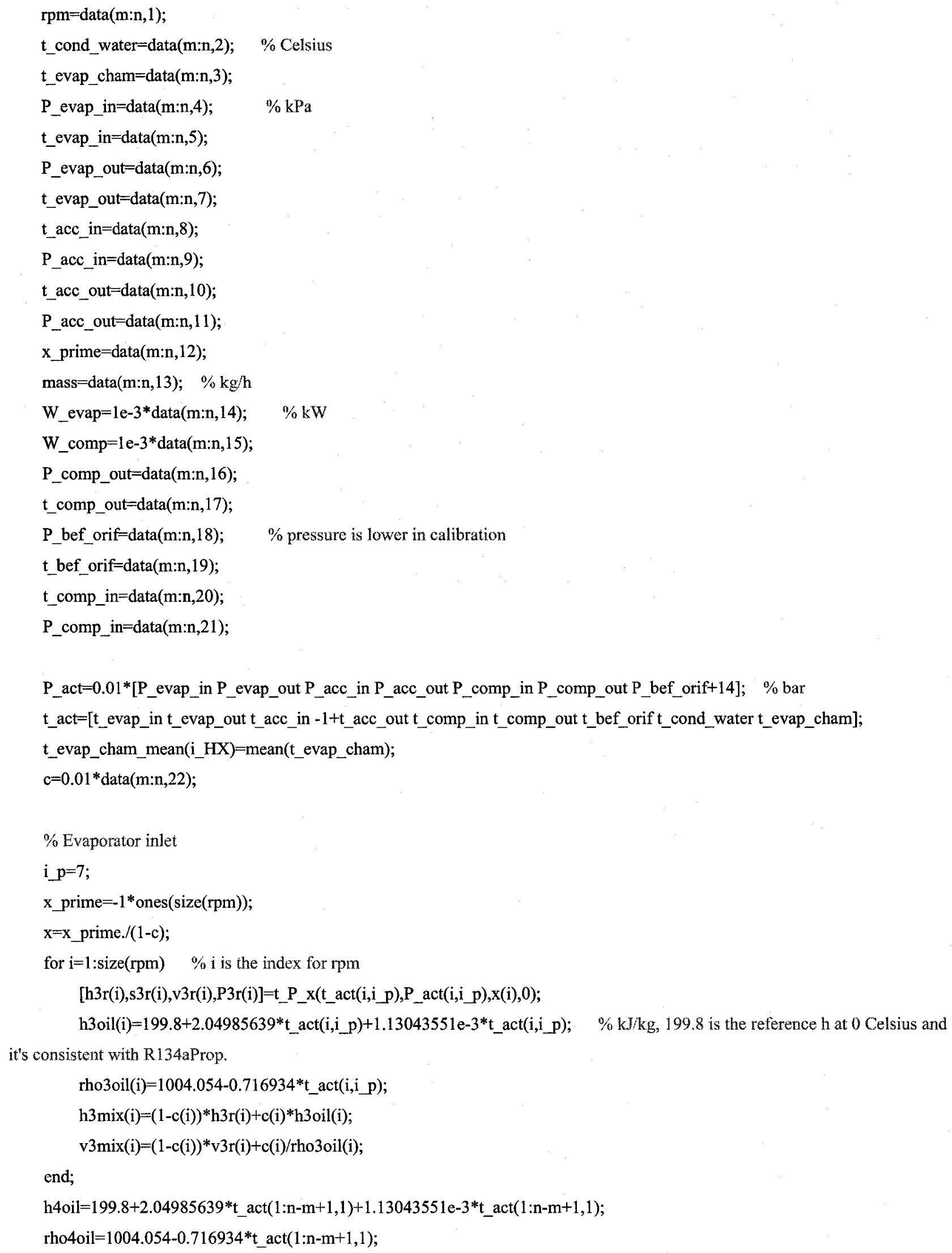




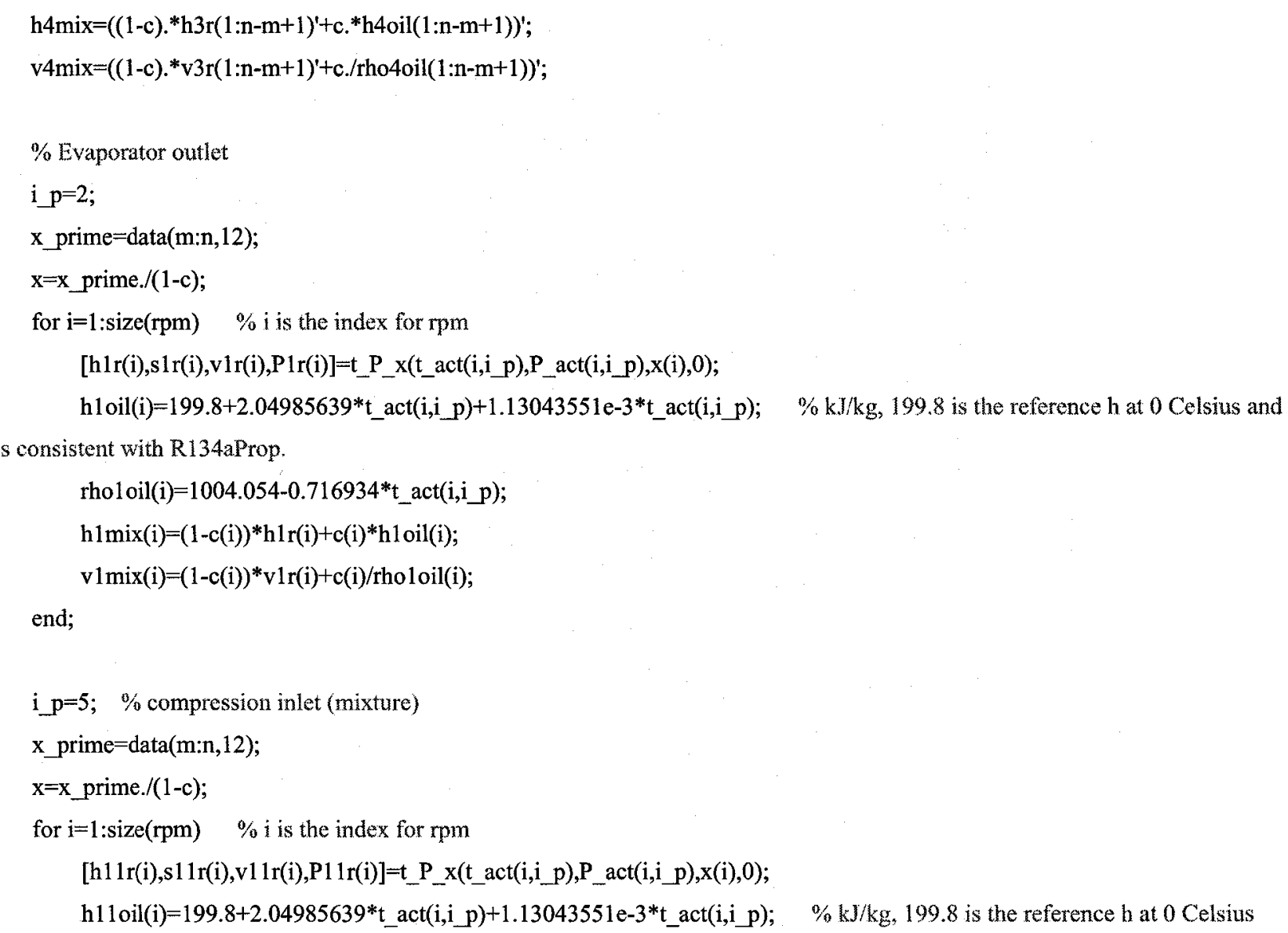
and it's consistent with R134aProp.

rho1 1 oil(i) $=1004.054-0.716934 *$ t_act $\left(i, i \_p\right)$;

h1 $1 \operatorname{mix}(\mathrm{i})=(1-\mathrm{c}(\mathrm{i}))^{*} \mathrm{~h} 11 \mathrm{r}(\mathrm{i})+\mathrm{c}(\mathrm{i})^{*} \mathrm{~h} 11$ oil(i);

v11 mix(i) $=(1-c(i))^{*} v 11$ r(i) $+c(i) /$ rhol 1oil(i);

end;

i_p $=6 ; \%$ compression outlet (mixture)

$\%$ Get resuls for refrigerant containing oil

$\%$ Known $\mathrm{P}$ and $\mathrm{t}$, find $w$ using iteration

$\mathrm{B}=\left[\begin{array}{lllll}-555.5660407994 & 1529.8308839129 & -1631.4871981140 & 849.3854993108 & -217.1646418392\end{array}\right.$

22.7862554254

$\begin{array}{llllll}9941.6002771189 & -27779.6767926228 & 29642.0579341856 & -15277.5529515402 & 3861.5694435505\end{array}$

$-383.4913577175$

$\begin{array}{lllll}-57338.0467646767 & 166134.4413974230 & -180687.1251094080 & 93312.8363512552 & -23313.6087782079\end{array}$

2281.0651153148

$\begin{array}{llllll}135583.8975459850 & -415835.1487935210 & 466329.3760186240 & -244495.8941000320 & 61297.8729089902\end{array}$

$-5973.4765674293$

$\begin{array}{llllll}-135357.3007350370 & 452121.6734588610 & -529159.5623516080 & 283692.8179295740 & -71819.1906150121\end{array}$

7021.5094881398 
-3027.6557035784];

$\mathrm{P}=\mathrm{P}$ _act(:,i_p);

w_origin $=0.5 *$ ones $(\operatorname{size}(\mathrm{rpm}))$;

$\mathrm{w}=\mathrm{w}$ _origin;

for $\mathrm{i}=1: \operatorname{size}(w)$

$\mathrm{ESP}=1$;

$\mathrm{dw}=0.002$;

while (abs(ESP)>1e-6)

$\mathrm{A}=\mathrm{B}(1,:)^{*} \mathrm{w}(\mathrm{i})^{\wedge}-1+\mathrm{B}(2,:)+\mathrm{B}(3,:)^{*} \mathrm{w}(\mathrm{i})+\mathrm{B}(4,:)^{*} \mathrm{w}(\mathrm{i}) .^{\wedge} 2+\mathrm{B}(5,:)^{*} \mathrm{w}(\mathrm{i})^{\wedge} 3+\mathrm{B}(6,:)^{*} \mathrm{w}(\mathrm{i})^{\wedge} 4$;

$\mathrm{A}_{-} \mathrm{r}=\mathrm{B}(1,:)^{*}(\mathrm{w}(\mathrm{i})+\mathrm{dw})^{\wedge}-1+\mathrm{B}(2,:)+\mathrm{B}(3,:)^{*}(\mathrm{w}(\mathrm{i})+\mathrm{dw})+\mathrm{B}(4,:)^{*}(\mathrm{w}(\mathrm{i})+\mathrm{dw}) .^{\wedge} 2+\mathrm{B}(5,:)^{*}(\mathrm{w}(\mathrm{i})+\mathrm{dw})^{\wedge} 3+\mathrm{B}(6,:)^{*}(\mathrm{w}(\mathrm{i})+\mathrm{dw})^{\wedge} 4$;

$\mathrm{t}(\mathrm{i})=\mathrm{A}(1)^{*} \mathrm{P}(\mathrm{i})^{\wedge}-1.5+\mathrm{A}(2)^{*} \mathrm{P}(\mathrm{i})^{\wedge}-1+\mathrm{A}(3)^{*} \mathrm{P}(\mathrm{i})^{\wedge}-0.5+\mathrm{A}(4)+\mathrm{A}(5)^{*} \mathrm{P}(\mathrm{i})^{\wedge} 0.5+\mathrm{A}(6)^{*} \mathrm{P}(\mathrm{i})$

$\mathrm{t} \_\mathrm{r}(\mathrm{i})=\mathrm{A} \_\mathrm{r}(1)^{*} \mathrm{P}(\mathrm{i})^{\wedge}-1.5+\mathrm{A} \_\mathrm{r}(2)^{*} \mathrm{P}(\mathrm{i})^{\wedge}-1+\mathrm{A} \_\mathrm{r}(3)^{*} \mathrm{P}(\mathrm{i})^{\wedge}-0.5+\mathrm{A} \_\mathrm{r}(4)+\mathrm{A} \_\mathrm{r}(5)^{*} \mathrm{P}(\mathrm{i})^{\wedge} 0.5+\mathrm{A} \_\mathrm{r}(6)^{*} \mathrm{P}(\mathrm{i})$;

$\mathrm{ESP}=\mathrm{t} \_\operatorname{act}\left(\mathrm{i}, \mathrm{i} \_\mathrm{p}\right)-\mathrm{t}(\mathrm{i})$;

ESP_r=t_act(i,i_p)-t_r(i);

$d w=d w^{*} E S P /\left(E S P-E S P \_r\right) ;$

$w(i)=w(i)+d w ;$

end;

end;

for $\mathrm{i}=1$ :size(P)

$\operatorname{if}(w(i)<0)$

$\mathrm{w}(\mathrm{i})=\mathrm{w}$ _origin(i);

$\mathrm{ESP}=1$;

$\mathrm{dw}=0.002$;

while $($ abs $(\mathrm{ESP})>1 \mathrm{e}-6)$

$\mathrm{A}=\mathrm{B}(1, ;)^{*} \mathrm{w}(\mathrm{i})^{\wedge}-1+\mathrm{B}(2,:)+\mathrm{B}(3,:)^{*} \mathrm{w}(\mathrm{i})+\mathrm{B}(4,:)^{*} \mathrm{w}(\mathrm{i}) .^{\wedge} 2+\mathrm{B}(5,:)^{*} \mathrm{w}(\mathrm{i})^{\wedge} 3+\mathrm{B}(6,:)^{*} \mathrm{w}(\mathrm{i})^{\wedge} 4 ;$

$A_{-} r=B(1,:)^{*}(w(i)+d w)^{\wedge}-1+B(2,:)+B(3,:)^{*}(w(i)+d w)+B(4,:)^{*}(w(i)+d w) .^{\wedge} 2+B(5,:)^{*}(w(i)+d w)^{\wedge} 3+B(6,:)^{*}(w(i)+d w)^{\wedge} 4$

$\mathrm{t}(\mathrm{i})=\mathrm{A}(1)^{*} \mathrm{P}(\mathrm{i})^{\wedge}-1.5+\mathrm{A}(2)^{*} \mathrm{P}(\mathrm{i})^{\wedge}-1+\mathrm{A}(3)^{*} \mathrm{P}(\mathrm{i})^{\wedge}-0.5+\mathrm{A}(4)+\mathrm{A}(5)^{*} \mathrm{P}(\mathrm{i})^{\wedge} 0.5+\mathrm{A}(6)^{*} \mathrm{P}(\mathrm{i})$

$\mathrm{t} \_\mathrm{r}(\mathrm{i})=\mathrm{A} \_\mathrm{r}(1)^{*} \mathrm{P}(\mathrm{i})^{\wedge}-1.5+\mathrm{A} \_\mathrm{r}(2)^{*} \mathrm{P}(\mathrm{i})^{\wedge}-1+\mathrm{A} \_\mathrm{r}(3)^{*} \mathrm{P}(\mathrm{i})^{\wedge}-0.5+\mathrm{A} \_\mathrm{r}(4)+\mathrm{A} \_\mathrm{r}(5)^{*} \mathrm{P}(\mathrm{i})^{\wedge} 0.5+\mathrm{A} \_\mathrm{r}(6)^{*} \mathrm{P}(\mathrm{i})$;

$E S P=t \_a c t\left(i, i \_p\right)-t(i)$;

ESP_r=t_act(i,i_p)-t_r(i);

$\mathrm{dw}=\mathrm{dw}^{*} 0.5 * \mathrm{ESP} /\left(\mathrm{ESP}-\mathrm{ESP} \mathrm{r}_{-}\right.$);

$\mathrm{w}(\mathrm{i})=\mathrm{w}(\mathrm{i})+\mathrm{dw}$;

end;

end;

end;

$\%$ Given $w$ and $c$, find $x$.

$\mathrm{c}=0.042 *$ ones $(\operatorname{size}(\mathrm{rpm}))$;

$\mathrm{x} \_$prime $=(1-\mathrm{w}-\mathrm{c}) . /(1-\mathrm{w})$; 
$\mathrm{x}=\mathrm{x} \_$prime. $/(1-\mathrm{c})$;

$\%$ Apply mixing rule to calculate mixture properties

for $\mathrm{i}=1$ :size(rpm) $\% \mathrm{i}$ is the index for $\mathrm{rpm}$

[h2r_l(i),s2r_l(i),v2r_l(i),P2r_l(i)]=R134aProp(t_act(i,i_p),t_act(i,i_p),0,0);

[h2r_v(i),s2r_v(i),v2r_v(i),P2r_v(i)]=t_P_x(t_act(i,i_p),P_act(i,i p),x(i),0);

$\mathrm{h} 20 \mathrm{il}(\mathrm{i})=199.8+2.04985639 * \mathrm{t} \_$act(i,i_p $)+1.13043551 \mathrm{e}-3 * \mathrm{t} \_$act(i,i_p); $\% \mathrm{~kJ} / \mathrm{kg}, 199.8$ is the reference $\mathrm{h}$ at 0 Celsius and it's consistent with R134aProp.

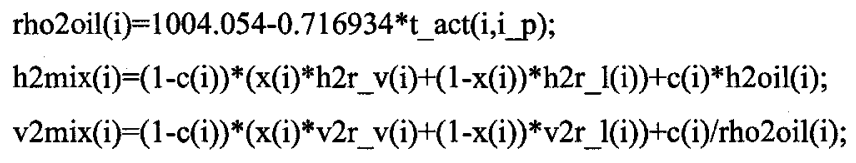


end;

end;

$\% t$ mean(:, 1$)$ is the average temperatures in evaporator

$\% \mathrm{t}$ mean $(: 2)$ is the average temperatures in condenser

del_t_e=t_act(:,9)-t_mean(1:n-m+1,1);

del_t_k=t_mean(1:n-m+1,2)-t_act(:,8);

$\mathrm{rpml}=\mathrm{rpm} ; \quad \%$ backup rpm information for use when $\mathrm{i}$ HX -3 where there is no IHX in system

hlmix=h1mix $(1: n-m+1)$;

h11 mix $=h 11 \operatorname{mix}(1: n-m+1)$;

$\mathrm{h} 3 \mathrm{mix}=\mathrm{h} 3 \mathrm{mix}(1: \mathrm{n}-\mathrm{m}+1)$;

$\mathrm{h} 4 \mathrm{mix}=\mathrm{h} 4 \mathrm{mix}(1: \mathrm{n}-\mathrm{m}+1)$;

$\mathrm{h} 2 \mathrm{mix}=\mathrm{h} 2 \mathrm{mix}(1: \mathrm{n}-\mathrm{m}+1)$;

mass $=$ mass. $/ 3600$;

Q_e=mass.*(h1 mix'-h4mix');

W_c =mass.* ${ }^{*}\left(\right.$ h2mix'-h1 $\left.1 \mathrm{mix}^{\prime}\right)$;

Q_k=Q_e+W_c;

UA_e=Q_e./del_t_e;

UA_k=Q_k./del_t k ;

eta_Qe=Q_e./W_evap; \% effectiveness of the cvaporator???

eta_Wc=W_c./W_comp; \% isentropic efficiency of the compressor???

del_P=P_k_m-P_e_m;

t_mean_e=t_mean $(1: \mathrm{n}-\mathrm{m}+1,1)$;

$\mathrm{t} \_$mean_k $=\mathrm{t} \_$mean $(1: \mathrm{n}-\mathrm{m}+1,2)$;

if $\mathrm{i}_{-} \mathrm{HX}=3$

$\%$ get relationship between $(\mathrm{hl} / \mathrm{h} 4) / \mathrm{UA}$ e and rpm, t evap-t e=mass $\%(\mathrm{~h} 1 \mathrm{~h} 4) / \mathrm{UA}$ e

Ratiol=1.3*(h1mix'-h4mix')./UA_e;

$\mathbf{x x}=[\operatorname{ones}(\operatorname{size}(\mathrm{rpm})) \mathrm{rpm}]$;

coef_R $=\mathrm{xx} \backslash$ Ratio 1

verify $=\mathrm{xx} *$ coef $\mathrm{R}$;

disp([verify Ratiol verify-Ratiol]);

$\%$ get relationship between $\left(\mathrm{t} k-\mathrm{t}\right.$ e) and $\mathrm{m}^{\wedge} 2$

D_t=t_mean_k-t_mean_e;

$\mathrm{xx}=\left[\right.$ mass mass. $\left.{ }^{\wedge} 2\right]$;

coef $D t=x x \backslash D t$

verify $=x x^{*}$ coef_D_t;

disp([verify D_t verify-D_t]); 
$\%$ get relationship between experimental mass flow rate and rpm

$\mathrm{xx}=[$ ones(size $\left.(\mathrm{rpm})) \mathrm{rpm} .{ }^{\wedge} 0.8\right]$

coef_m=xxไmass

verify $=x x^{*}$ coef_m;

$\operatorname{disp}\left(\left[\right.\right.$ verify mass $\left.\left.(1-\text { verify./mass })^{*} 100\right]\right)$;

end;

para $=[\operatorname{rpm}$ h1mix' h3mix' h1 1mix' h2mix' ...

Q_e Q_kUA_e UA_kP_e_m...

P_k_m del_P];

$\%$ find average value for each rpm

for $\mathrm{i}=1$ :size(para')

para_m(:,i) $=[\operatorname{mean}($ para $(1:$ count_r(1),i)) mean(para(count_r(1)+1:count_r(2),i)) mean(para(count_r(2)+1:count_r(3),i)) ... mean(para(count_r(3)+1:count_r(4),i)) mean(para(count_r(4)+1:count_r(5),i))

mean(para(count_r(5)+1:count_r(6),i))]';

end;

$\%$ get d_t_e and d_t_k

$\mathrm{nn}=\mathrm{rpm} ; \quad \%$ verify the model

$\mathrm{kk}=\operatorname{size}(\mathrm{nn})-1$;

for $\mathrm{i}=1: \mathrm{kk}$

$\operatorname{Del} n(i, 1)=n n(i+1)-n n(1)$;

end;

$\mathrm{R}=$ coef_R(1)+coef_R(2)*nn(1:kk); \% Ratio $=\mathrm{f}(\mathrm{nn})$

Del_R=coef_R(2)*Del_n;

m_calc $=$ coef_m $(1)+$ coef_m(2)*nn( $1: \mathrm{kk}) .^{\wedge} 0.8$;

Del_m $=\left(0.8^{*}\right.$ coef_m(2)*nn(1:kk).^-0.2). ${ }^{*} \operatorname{Del} \_\mathrm{n}(1: \mathrm{kk})$;

Del_t_e $=-$ Del_m.*R-m_calc(1:kk).*Del_R;

Del_t_k=Del_t_e+coef_D_t(1)*Del_m+coef_D_t $(2)^{*} 2 *$ m_calc. ${ }^{*}$ Del_m;

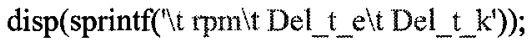

$\operatorname{disp}([\mathrm{nn}(1: \mathrm{kk})$ Del_t_e Del_t_k] $)$;

$\%$ compare the calculated results with experimental data

for $\mathrm{i}=1: \mathrm{kk}$

Del_t_e_exp(i)=t_mean_e(i+1)-t_mean_e(1); \% experimental temperature difference Del_t

Del_t_k_exp(i)=t_mean_k(i+1)-t_mean_k(1); \% experimental temperature difference Del_t_e end; 
disp([Del_t_e_exp' Del_t_e Del_t_e_exp'-Del_t_e Del_t_k_exp' Del_t_k Del_t_k_exp'-Del_t_k]); end;

\section{B-2 Program to improve the accuracy of Grebner's correlation}

This code improves the accuracy of Grebner's correlation based on his original model.

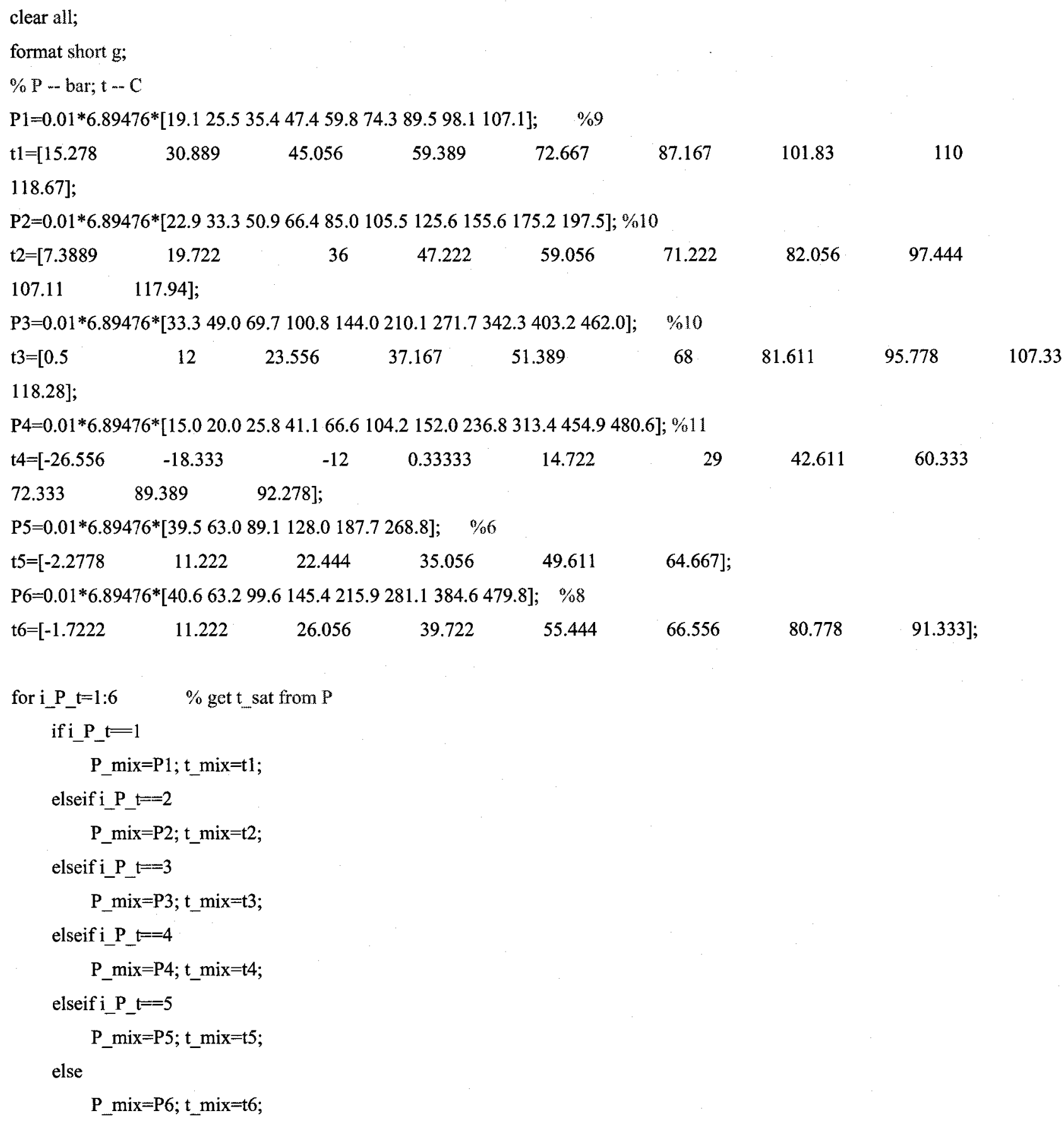




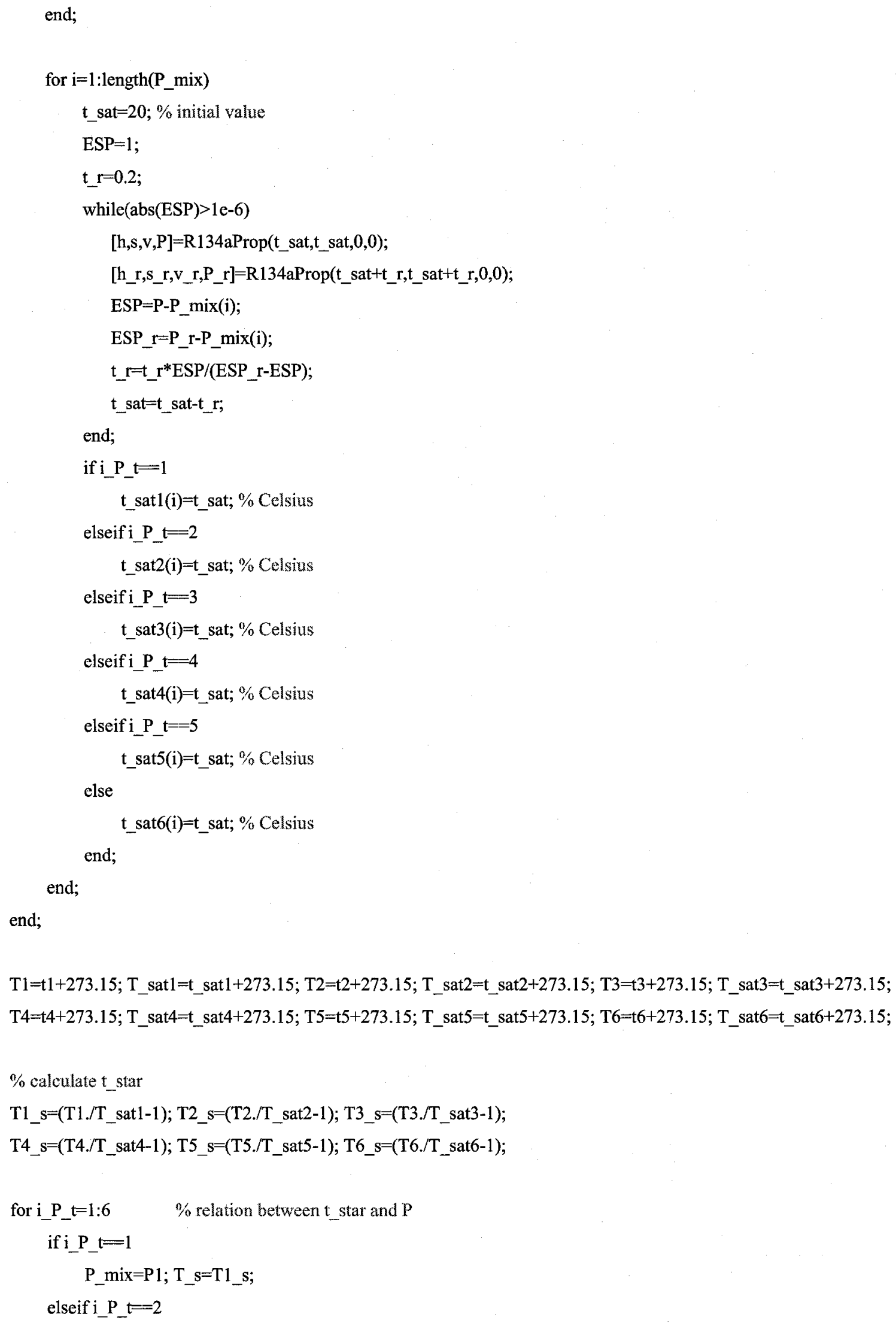




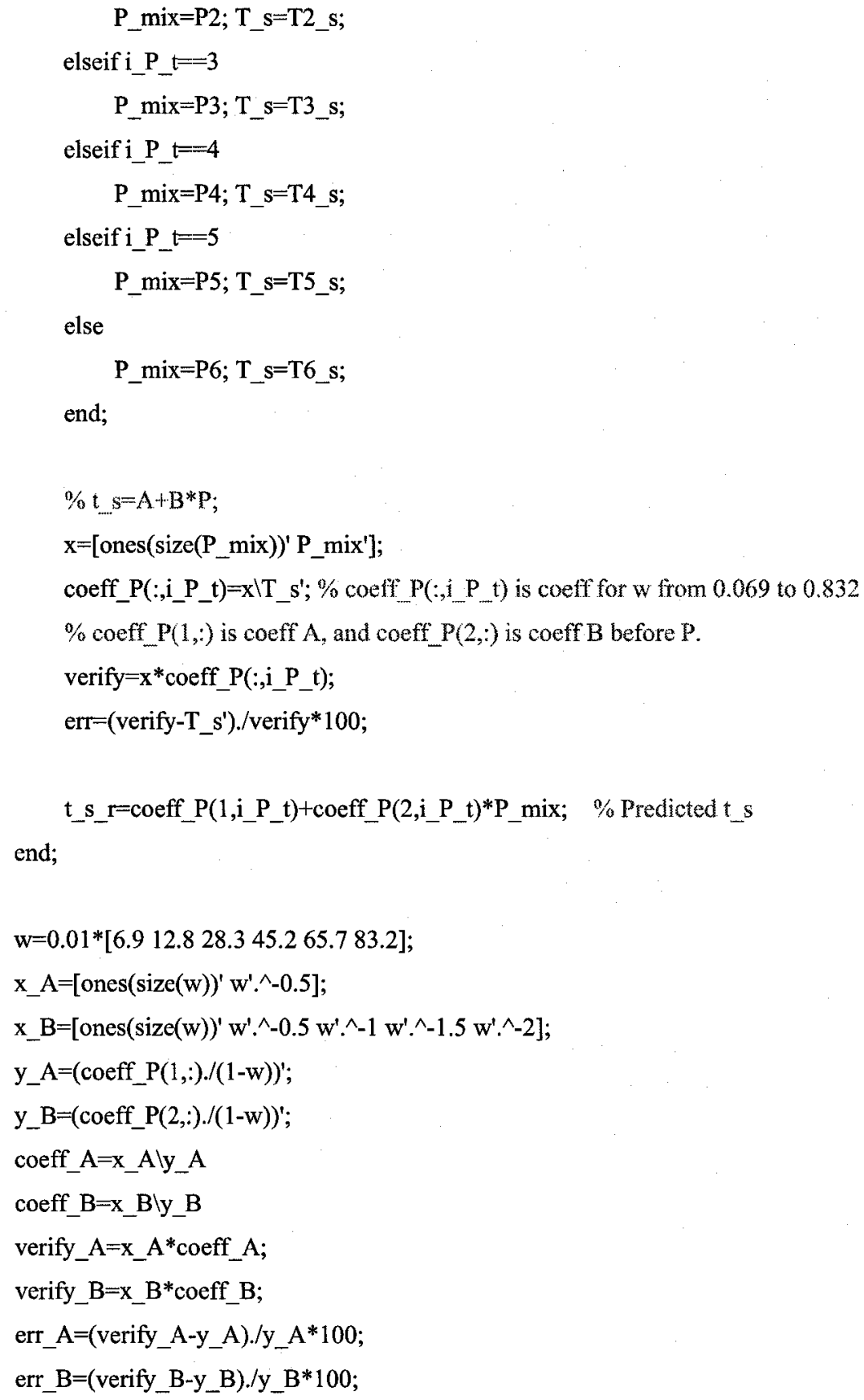

\section{Appendix C Program to calculate AXE flow in $\mathrm{CO}_{2}$-based systems}

The programs used to simulate $\mathrm{CO}_{2}$ flows are similar to those for R134a flow; some special techniques need to be used to handle the transcritical uncertainties. Therefore, only the main code 


\section{showing such techniques is shown below.}

\section{C-1 Main code}

This function calculates the outlet parameters of the non-adiabatic $\mathrm{CO}_{2}$ capillary flow if the inlet condition is given.

function [tf_inner, pf_inner, hf_inner, tf_outer, pf_outer, xf_outer,tube_length,

Q_tot] $=\mathrm{AHE}(\mathrm{Q} 0, \mathrm{t} 1, \mathrm{t} 11, \mathrm{t} 3, \mathrm{pk}$,do_inner,thick_inner,epsilon,do_outer,thick_outer,x_outer_origin,t_outer_origin)

$\% \mathrm{tf}$ - $\mathrm{t}$ means temperature, f means final (at the outlet)

$\%$ pf --..-.-...- p means pressure

$\%$ hf -.n-man-m.n-m h means enthalpy

$\% \times f$-..-.-.-.- $x$ means quality

$\%$ inner munnoms the refrigerant of the inner tube

$\%$ outer -........- means the refrigerant of the outer tube

$\%$ Q tot - _......m-m.m total heat transfer rate (W)

$\%$ QO -.......- cooling capacity $(\mathrm{kW})$

$\% \mathrm{t} 1$-................ evaporating temperature (degree)

$\% \mathrm{t} 11$-...........- suction temperature (degree)

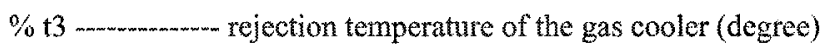

$\%$ pk ---.---.-- cooling pressure (bar)

$\%$ do inner m.mm outer diameter of the inner tube (enlarged capillary tube) (mm)

$\%$ thick inner --.-m thickness of the inner tube (mm)

$\%$ epsilon -........ roughness of the inner surface (mm)

$\%$ do outer --.-.-- outer diameter of the outer tube (mm)

$\%$ thick outer m-m- thickness of the outer tube (mm)

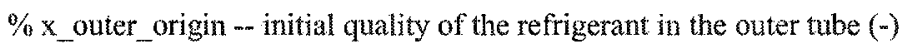

$\% t$ outer origin - initial temperature of the refrigerant in the outer tube (degree)

$\%$ This function calculates the length need for an enlarged capillary tube for $\mathrm{CO}_{2}$ refrigeration systems.

$\%$ There is no need to get high EPS because the property calc code is not accurate enough.

$\%$ When using formulae to get thermoproperties high EPS could be used.

$\%$ - hypercritical data are arranged as

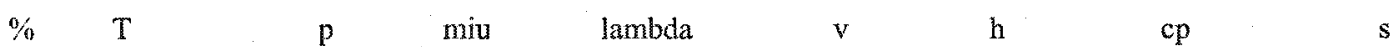

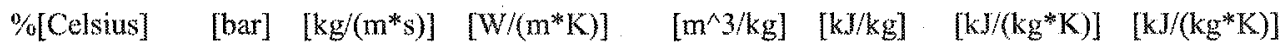

$\%$ temperature, pressure, viscosity, conductivity, spec. vol,enthalpy, spec. heat entropy

$\%$ subcritical data are arranged as

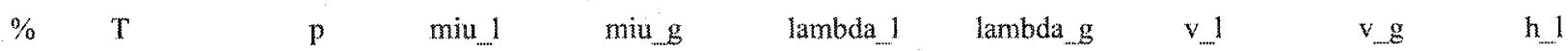

h_g cp_l cp_g $\quad$ r




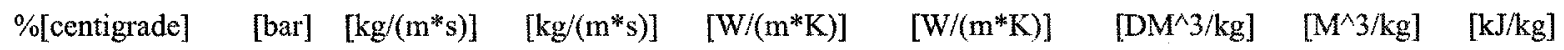

$[\mathrm{kJ} / \mathrm{kg}] \quad\left[\mathrm{kJ} /\left(\mathrm{kg}^{*} \mathrm{~K}\right)\right]\left[\mathrm{kJ} /\left(\mathrm{kg}^{*} \mathrm{~K}\right)\right] \quad[\mathrm{kJ} / \mathrm{kg}]$

$\%$ temperature, pressure, vis. of liq, vis. of gas cond. of l, cond. of g. $v$ of liquid, $v$ of gas, $h$ of liquid, h of gas, spec. heat, spec. heat latent heat

format short $\mathrm{g}$;

global tt_sp tt_tp pp_sp pp_tphh_sphh_tp vv_sp vv_tp mium_sp mium_tplambdal_sp lambdal_tp ss_sp ss_tp cpc_sp cpc_tp...

xx_tp errorlength iteration tmax 1 tmin 1 tmax 2 tmin 2 pmax pmin flux_critical;

singlephase=load('singlephase-10_160.dat','-ascii'); \% load thermodynamic property data

twophase=load('twophase-30-31.dat','-ascii');

flag $=0 ; \%$ FLAG is used for debugging. flag $=0$, single phase calculation is executed, flag $=1$, not executed.

do=do_inner; $\quad$ di $=$ do- $2 *$ thick_inner;

$\%$ Main input

$\operatorname{tmax} 1=160 ; \operatorname{tmin} 1=-10 ; \operatorname{tmax} 2=31 ; \operatorname{tmin} 2=-30 ; p \max =135 ; \mathrm{pmin}=25$;

$\mathrm{n} 1$ temp $=(\operatorname{tmax} 1-\mathrm{tmin} 1)+1 ; \quad \% \mathrm{n} 1$ _temp is the number of single-phase temperature values

$\mathrm{n} 1 \_$pres $=($pmax - pmin $)+1 ; \quad \% \mathrm{nl}$ pres is the number of single-phase pressure values

$\mathrm{n} 2=(\operatorname{tmax} 2-\operatorname{tmin} 2)+1 ; \%$ for two-phase status, number of temperature data equals to number of pressure data

EPS1 $=0.6 ;$ EPS2 $=2$;

interpoint $=4 ; \quad \%$ points between 33 Celsius and saturated temperature

errorlength $=-1000 ; \%$ set error judgement value

for $\mathrm{i}=1: \mathrm{n} 1$ temp $\%$ data mesh for single-phase region

tt_sp $(1, \mathbf{i})=$ singlephase $\left((\mathrm{i}-1){ }^{*} \mathrm{n} 1 \_\right.$pres $\left.+1,1\right)$;

for $\mathrm{j}=1: \mathrm{n} 1$ pres

hh_sp $(\mathrm{j}, \mathrm{i})=$ singlephase $((\mathrm{i}-1) * \mathrm{n} 1$ pres $+\mathrm{j}, 6)$;

vv_sp $(\mathrm{j}, \mathrm{i})=\operatorname{singlephase}\left((\mathrm{i}-1)^{*} \mathrm{n} 1\right.$ pres $\left.+\mathrm{j}, 5\right)$;

mium_sp $(\mathrm{j}, \mathrm{i})=\operatorname{singlephase}\left((\mathrm{i}-1)^{*} \mathrm{n} 1 \_\right.$pres $\left.+\mathrm{j}, 3\right)$;

lambdal_sp $(\mathrm{j}, \mathrm{i})=$ singlephase $\left((\mathrm{i}-1) * \mathrm{n} 1 \_\right.$pres $\left.+\mathrm{j}, 4\right)$;

ss_sp $(\mathrm{j}, \mathrm{i})=$ singlephase $\left((\mathrm{i}-1){ }^{*} \mathrm{n} 1 \_\right.$pres $\left.+\mathrm{j}, 8\right)$;

cpc_sp $(\mathrm{j}, \mathrm{i})=$ singlephase $\left((\mathrm{i}-1)^{*} \mathrm{n} 1 \_\right.$pres $\left.+\mathrm{j}, 7\right)$;

end;

end;

for $\mathrm{j}=1: \mathrm{n} 1 \_$pres

pp_sp $(1, j)=\operatorname{singlephase}\left((i-1)^{*} n 1 \_\right.$pres $\left.+\mathrm{j}, 2\right) ;$

end;

for $\mathrm{i}=1: \mathrm{n} 2 \quad \%$ data mesh for two phase region

tt_tp $(1, \mathrm{i})=$ twophase $(\mathrm{i}, 1)$;

for $\mathrm{j}=1: 11$

$x x_{\text {_ref }}=(\mathrm{j}-1) * 0.1$;

hh_tp $(j, i)=x x \_r e f * t w o p h a s e(i, 10)+\left(1-x x_{-}\right.$ref $) *$ twophase $(i, 9)$;

$v_{-}$tp $(j, i)=x x \_r e f^{*}$ twophase $(i, 8)+\left(1-x x_{-} \text {ref }\right)^{*}\left(\right.$ twophase $\left.(i, 7)^{*} 1 e-3\right)$;

mium_tp $(j, i)=1 /\left(x x \_r e f /\right.$ twophase $(i, 4)+\left(1-x x_{-}\right.$ref $) /$twophase $\left.(i, 3)\right)$; 
lambdal_tp(j,i)=1/(xx_ref/twophase $(\mathrm{i}, 6)+(1-\mathrm{xx}$ _ref $) /$ twophase $(\mathrm{i}, 5))$;

cpc_tp(j,i) $=x x \_r e f * t w o p h a s e(i, 12)+\left(1-x x \_r e f\right)^{*}$ twophase $(i, 11)$;

ss_tp(j,i) $=x x \_r e{ }^{*}$ twophase $(i, 15)+\left(1-x x \_r e f\right) * t w o p h a s e(i, 14)$;

pp_tp(j,i)=twophase $(\mathrm{i}, 2)$;

end;

end;

for $\mathrm{j}=1: 11 \%$ to save calculating time, mesh $\mathrm{xx}$ separately

$\mathrm{xx} \operatorname{tp}(1, \mathrm{j})=(\mathrm{j}-1) * 0.1$;

end;

alfa_outer $=1000$;

for counter $=1: 1$

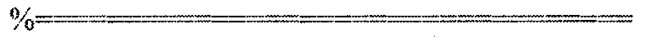

INPUT DATA

$\% \quad \% \mathrm{~kW} \quad \mathrm{C} \quad \mathrm{C} \quad \mathrm{C} \quad$ bar $\quad \mathrm{mm} \quad \mathrm{mm} \quad \mathrm{mm}$ (roughness of inner surface)

di_outer=do_outer- $2 *$ thick_outer;

if counter $==1 \quad \mathrm{t} 1=0$;

elseif counter $=2 \quad \mathrm{t} 1=5$;

elseif counter $=3 \quad \mathrm{tl}=10$

elseif counter $=4 \quad \mathrm{di}=1.9$;

elseif counter $==5 \quad \mathrm{di}=2.0$

end;

$\mathrm{psi}=0.5 ; \%$ psi is inlet correction coefficient

$\mathrm{t} 31=\mathrm{t} 3-(80 / \mathrm{pk})^{\wedge} 4 * 24 /\left(\mathrm{di}^{\wedge} 0.8\right) ; \quad \mathrm{t} 31=30 ; \%$ modify the initial value of $\mathrm{t} 31$ to decrease loop times

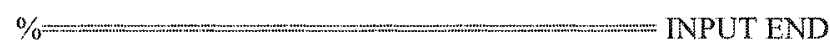

hl=property_calc(t_outer_origin,0,x_outer_origin);

if(hl==errorlength) \% crror handler

return;

end;

omega_outer_ $\mathrm{x}=0.5 ; \%$ omega_outer_ $\mathrm{x}$ is relax factor for calculating $\mathrm{x}$ of outer mbe

$\mathrm{Ai}=0.25^{*} \mathrm{pi}^{*} \mathrm{di}^{\wedge} 2 * 1 \mathrm{e}-6 ; \quad \% \mathrm{~m}^{\wedge} 2$

Ai_outer $=0.25^{*} \mathrm{pi}^{*} \mathrm{di}$ outer $2^{*} 1 \mathrm{e}-6-\mathrm{Ai} ; \%$ annular area of tube-in-tube $\mathrm{HX}, \mathrm{m}^{\wedge} 2$

di_outerl=di_outer;

di_outer=di_outer-do; \% characteristic diameter for outer tube, $\mathrm{mm}$

$\mathrm{dh} 4=1000 ; \mathrm{t} 31$ origin $=\mathrm{t} 31$; iteration $=1$;

while(abs(dh4)>EPS1)

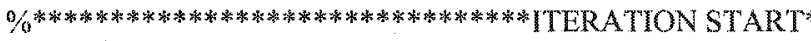

omega_ $\mathrm{t}=0.25 ; \quad \%$ omega $\mathrm{t}$ is relax factor for calculating $\mathrm{t}$ of inner tube

$\operatorname{disp}([$ counter iteration $])$;

$\mathrm{t}$ _outer=t_outer_origin; $\mathrm{x}$ _outer=x_outer_origin; $\%$ initialize status of outer tube two-phase flow

$\mathrm{dl}=\mathrm{di}^{\wedge} 2 ; \quad \%$ length of finite tube segment, $\mathrm{mm}$.

h4-property_calc(t31,pk,2); \% the value of the 3rd data doesn't change the output. NEED CHECK

if(h4==errorlength)

return; 
end;

$\mathrm{q} 0=\mathrm{h} 1-\mathrm{h} 4 ; \quad \% \mathrm{~kJ} / \mathrm{kg}$

$\mathrm{G}=\mathrm{Q} 0 / \mathrm{q} 0 ; \quad \% \mathrm{~kg} / \mathrm{s}$

$\mathrm{g}=\mathrm{G} / \mathrm{Ai} ; \quad \% \mathrm{~kg} /\left(\mathrm{m}^{\wedge} 2{ }^{*} \mathrm{~s}\right)$

flux_critical $=2.4 \mathrm{e} 4 ; \quad \%$ Set a value for whether calculating local sonic velocity or not, thus time can be saved.

if(pk $<=110 \| \mathrm{g}>$ flux_critical)

t_sp_min $=33.5$;

if $(\mathrm{pk}<=110 \& \& \mathrm{~g}>$ flux_critical $)$

t_sp_min $=35$;

if $(\mathrm{g}>1.2 *$ flux_critical $)$

t_sp_min= 35.5 ;

end;

end;

else

t_sp_min=32.5;

end;

if $(\mathrm{g}<1.5 \mathrm{e} 4) \quad \%$ define the criterium for calculation stop point if capillary is more like a $\mathrm{HX}$ (either di is big, or mass flow rate is small, or both)

t_sp_min=t1;

end;

if(t_sp_min $=\mathrm{t} 1)$

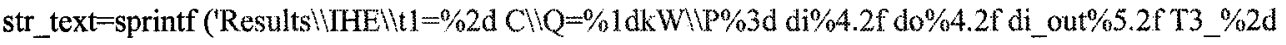

$\mathrm{x} \% 4.2 \mathrm{f}$ dQ..m',t1,Q0,pk,di,do,di_outer1,t3,x_outer_origin);

str_data $1=$ sprintf('Results $\backslash \mathrm{IHE} \backslash \mathrm{t} t \mathrm{l}=\% 2 \mathrm{~d} C \backslash \mathrm{Q}=\% 1 \mathrm{dkW} \backslash \mathrm{P} \% 3 \mathrm{~d}$ di $\% 4.2 \mathrm{f} \mathrm{do} \% 4.2 \mathrm{f} \mathrm{di}$ ont $\% 5.2 \mathrm{f} \mathrm{T} 3 \_\% 2 \mathrm{~d} \mathrm{x} \% 4.2 \mathrm{f} \_\mathrm{dQ}$ inner data.m',t1,Q0,pk,di,do,di_outerl,t3,x_outer_origin);

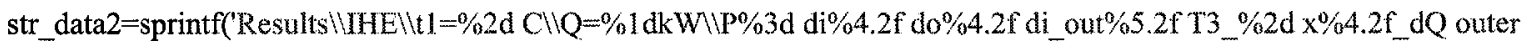
data.m',t1,Q0,pk,di,do,di_outer1,t3,x_outer_origin);

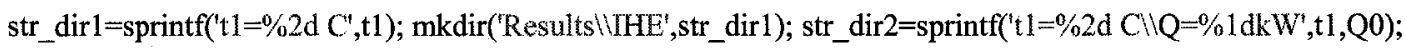

mkdir('ResultsiUHE',str_dir2);

else

str_text=sprintf ('ResultsilAHE

$\mathrm{x} \% 4.2 \mathrm{f}$ ddQ.m',t1,Q0,pk,di,do,di_outer1,t3,x_outer_origin);

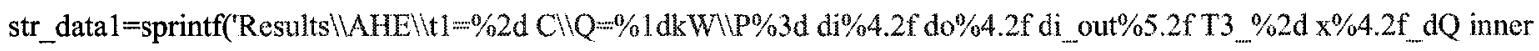
data.m',t1,Q0,pk,di,do,di_outerl,t3,x_outer_origin);

str_data2=sprintf('Results $\backslash A H E \backslash \backslash t 1=\% 2 \mathrm{~d} C \backslash \mathrm{Q}=\% 1 \mathrm{dkW} \backslash \mathrm{P} \% 3 \mathrm{~d}$ di $\% 4.2 \mathrm{f} \mathrm{do} \% 4.2 \mathrm{f} \mathrm{di}$ out $\% 5.2 \mathrm{f} \mathrm{T3} \% 2 \mathrm{~d} \mathrm{x} \% 4.2 \mathrm{f}$ dQ outer data.m',t1,Q0,pk,di,do,di_outer1,t3,x_outer_origin);

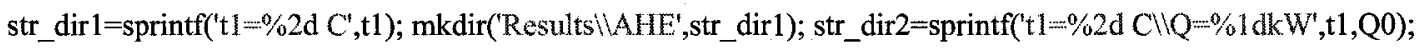

mkdir('Results\:AHE',str_dir2);

end;

fid=fopen(str_text,'w'); \% specify a file to save results

fprintf(fid,'The input parameters are: $\left.\mathrm{in}^{\prime}\right)$; 


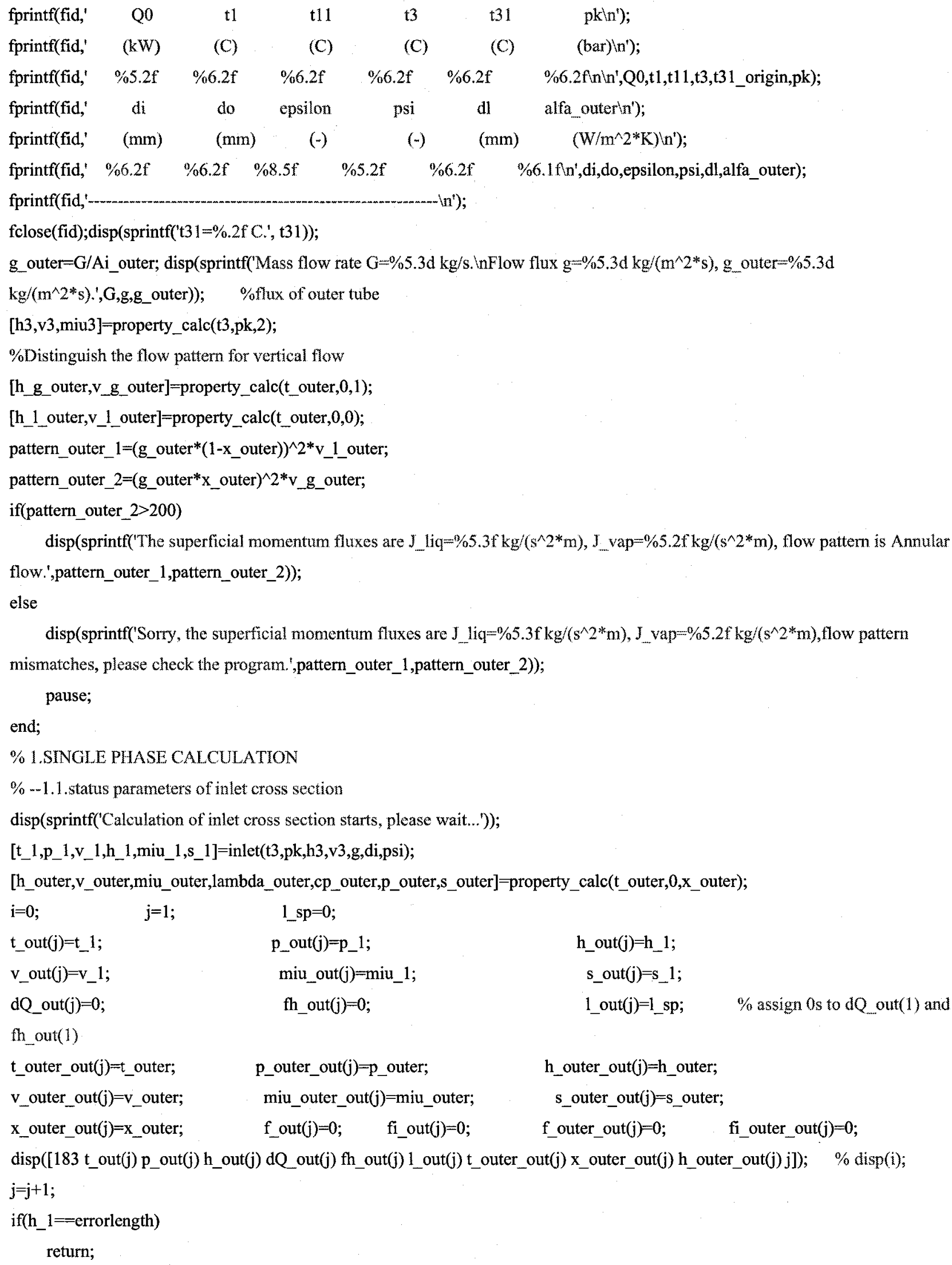


end;

disp('Calculation of inlet cross section ends.');

fid=fopen(str_text,'a'); \% specify a file to save results

fprintf(fid,'The status parameters at the end of inlet eross section are: $\left(\mathrm{n}^{\prime}\right)$;

fprintf(fid,' to $1 \quad$ p_1 $\quad$ v_1 $\left.\quad h_{-} 1 / \mathrm{n}^{\prime}\right)$;

fprintf(fid,' $\quad$ (C) $\quad$ (bar) $\left.\quad\left(\mathrm{m}^{\wedge} 3 / \mathrm{kg}\right) \quad(\mathrm{kJ} / \mathrm{kg}) \mathrm{kn}^{\prime}\right)$;

fprintf(fid,' $\% 6.2 \mathrm{f} \quad \% 8.3 \mathrm{f} \quad \% 7.4 \mathrm{e} \quad \% 7.2 \mathrm{fn}$ ',[t_l $\left.\left.\mathrm{p}_{-} 1 \mathbf{v}_{-} 1 \mathrm{~h}_{-} 1\right]\right)$;

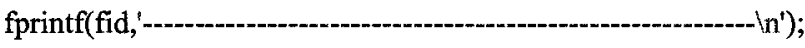

fclose(fid);

if(flag $=0$ )

$\%-1.2$. numerical calculation of single-phase tube length

disp('Calculation of single-phase tube length starts, please wait...');

$\mathrm{dh}=1 ; \mathrm{dp}=1 ; \mathrm{Cl}=0 ; \mathrm{Q} \mathrm{w}=0 ; \mathrm{t}=\mathrm{t} \_1 ; \mathrm{p}=\mathrm{p} \_1 ; \mathrm{t}$ _old_sp= $1 ; \mathrm{t}$ _outer_ref=t $1 ; \%$ set initial conditions.

$\%$ l sp is tube length for the single-phase flow; Q $w$ is heat transfer between the inner tube fluid and the ambient.

while $((t>t$ _sp_min $) \& \&(t-t$ _outer_ref $>0.03))$

if $\left(t \_s p \_m i n=t 1 \& \& t>30.1 \& \& t<32\right) \quad \%$ to stride over the transcritical area between $\sim 30-32$ degrees

for $k=1: j-1$

$\mathrm{CC}(\mathrm{k},:)=\left[\left(1 \_ \text {out(k) }\right)\right)^{\wedge} 1 \_$out(k) 1$] ; \quad \% \mathrm{CC}$ means coefficients

end;

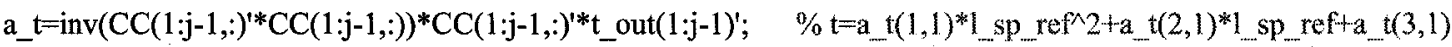

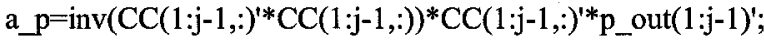

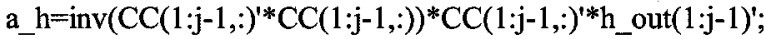

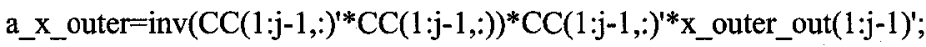

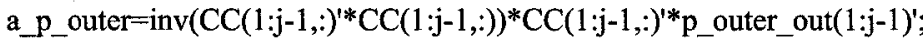

$\%$ a

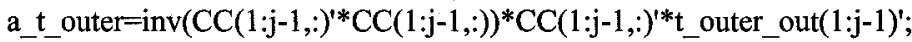

1_sp_ref $=\left(-a_{-} t(2,1)-\operatorname{sqrt}\left(\left(a_{-} t(2,1)\right)^{\wedge} 2-4 * a_{-} t(1,1) *\left(a_{-} t(3,1)-30\right)\right)\right) /\left(2 * a_{-} t(1,1)\right) ; \%$ trial calculation of 1 sp starts from where

$t=31$ celsius degree.

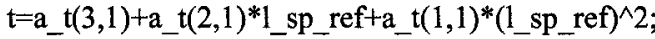

$\mathrm{p}=\mathrm{a} \_\mathrm{p}(3,1)+\mathrm{a} \_\mathrm{p}(2,1) * 1$ sp_ref+a_p $(1,1)^{*}\left(\mathbf{I}_{-} \mathrm{sp} \text { _ref }\right)^{\wedge} 2$;

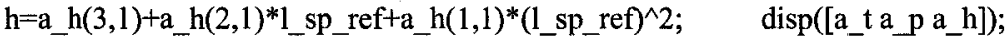

[h_ref,v_ref,miu_ref,lambda_ref,cp_ref,p_ref,s_ref] $=$ property_calc $(t, p, 2)$;

$[\mathrm{fh}, \mathrm{dQ}, \mathrm{f}, \mathrm{fi}]=\mathrm{fh} \_$calc(t,p,g,l_sp_ref-1_sp,t_outer,epsilon,di,do,1,alfa_outer,'sp'); $\%$ there is no difference for $\mathrm{x}=0$ or 1 for single-phase area

$\mathrm{x} \_$outer $=\mathrm{a} \_\mathrm{x} \_$outer $(3,1)+\mathrm{a} \_\mathrm{x} \_$outer $(2,1) * 1$ sp_ref+a $+\mathrm{x} \_$outer $(1,1) *\left(1 \_\mathrm{sp} \_ \text {ref }\right)^{\wedge} 2$;

p_outer $=\mathbf{a} \_$p_outer $(3,1)+\mathbf{a} \_$p_outer $(2,1)^{*} 1$ _sp_ref+a_p_outer $(1,1)^{*}(1 \text { _sp_ref })^{\wedge} 2$;

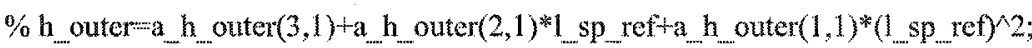

t_outer=a_t_outer(3,1)+a_t_outer(2,1)*1_sp_ref+a_t_outer $(1,1) *(1$ _sp_ref $) \wedge$;

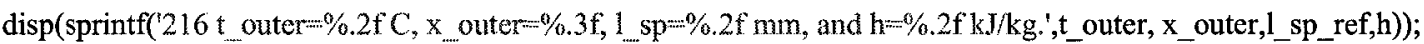

[h_outer,v_outer,miu_outer,lambda_outer,cp_outer,p_outer,s_outer]=property_calc(t_outer,p_outer,x_outer); 
[fh_outer,f_outer,fi_outer,alfa_outer]=alfa_outer_calc(t_outer,p_outer,g_outer,epsilon,1_sp_ref-1_sp,dQ,do,di_outer,x_outer,'alfa');

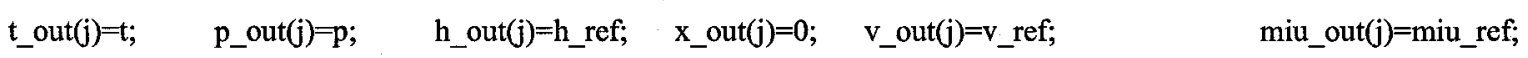
s_out $(j)=$ s_ref;
dQ_out $(j)=G^{*} d Q * 1 e 3$;
fh_out $(\mathrm{j})=\mathrm{fh}$;
f_out $(j)=f ;$
fi_out $(\mathrm{j})=\mathrm{fi}$;

1_out $(\mathrm{j})=1$ _sp_ref;

t_outer_out $(j)=t \_o u t e r ; \quad$ p_outer_out $(j)=p \_$outer; $\quad$ h_outer_out $(j)=h \_$outer; $\quad f \_$outer_out $(j)=f \_$outer;

fi_outer_out $(\mathrm{j})=$ fi_outer;

v_outer_out $(j)=$ v_outer;

miu_outer_out $(j)=$ miu_outer;

s_outer_out $(j)=$ s_outer;

fh_outer_out $(\mathrm{j})=\mathrm{fh} \_$outer;

$x \_o u t e r \_o u t(j)=x \_o u t e r ;$

alfa_outer_out $(\mathrm{j})=\mathrm{alfa} \_$outer;

$\operatorname{disp}\left(\left[236 \mathrm{t} \_\right.\right.$out $(\mathrm{j}) \mathrm{p} \_$out $(\mathrm{j}) \mathrm{h} \_$out $(\mathrm{j}) \mathrm{dQ}$ out $(\mathrm{j})$ 1_out(j) t_outer_out $(\mathrm{j}) \mathrm{x}$ _outer_out $(\mathrm{j}) \mathrm{h}$ _outer_out $(\mathrm{j})$ alfa_outer_out $\left.\left.(\mathrm{j}) \mathrm{j}\right]\right)$;

$j=j+1$; beep on; beep; pause $(0.5)$; beep off;

if $(\mathrm{t}-\mathrm{t}$ _outer_ref $<0.05)$

$\mathrm{d} \mathbf{l}=0.5 * \mathrm{dl}$;

elseif(t-t_outer_ref $<0.2)$

$\mathrm{dl}=0.5 * \mathrm{dl}$;

elseif(t-t_outer_ref $<0.5$ )

$\mathrm{dl}=0.5 * \mathrm{dl} ;$

elseif(t-t_outer_ref $<1)$

$\mathrm{dl}=0.5^{*} \mathrm{dl}$;

end;

if $(\mathrm{dl}<1)$

$\mathrm{dl}=1$;

end;

1_sp=1_sp_ref;

else

1_sp=1_sp+dl; i $=\mathrm{i}+1$;

if $(j=2) \quad \% g i v e$ an initial value for $d Q$. Since at beginning $d Q$ is useless for $x$ outer $<1$, so assign $d Q=0$.

$\mathrm{dQ}=\mathbf{0}$;

end;

[fh_outer,f_outer,fi_outer,alfa_outer]=alfa_outer_calc(t_outer,p_outer,g_outer,epsilon,dl,dQ,do,di_outer,x_outer,'alfa');

$[\mathrm{fh}, \mathrm{dQ}, \mathrm{f}, \mathrm{fi}]=\mathrm{fh} \_$calc(t,p,g,dl,t_outer,epsilon,di,do,x_outer,alfa_outer,'sp');

$\mathrm{Q} \_\mathrm{w}=\mathrm{Q} \_\mathrm{w}+\mathrm{dQ} ; \quad \% \mathrm{~kJ} / \mathrm{kg}$

if $(\mathrm{fh}==$ errorlength)

return;

end;

[t_ref,p_ref,h_ref,v_ref,miu_ref,omega_ref,s_ref] $=s p \_i t e r\left(t, p, g, d l, d i, f h, d Q, o m e g a \_t, 1 \_s p\right)$;

if(h_ref=errorlength)

return;

end;

[t_outer_ref,p_outer_ref,x_outer_ref,h_outer_ref,v_outer_ref,miu_outer_ref,omega_outer_ref,s_outer_ref] $=$...

outer_iter(t_outer,p_outer,x_outer,g_outer,dl,di_outer,fh_outer,-dQ,omega_outer_x,1_sp); 


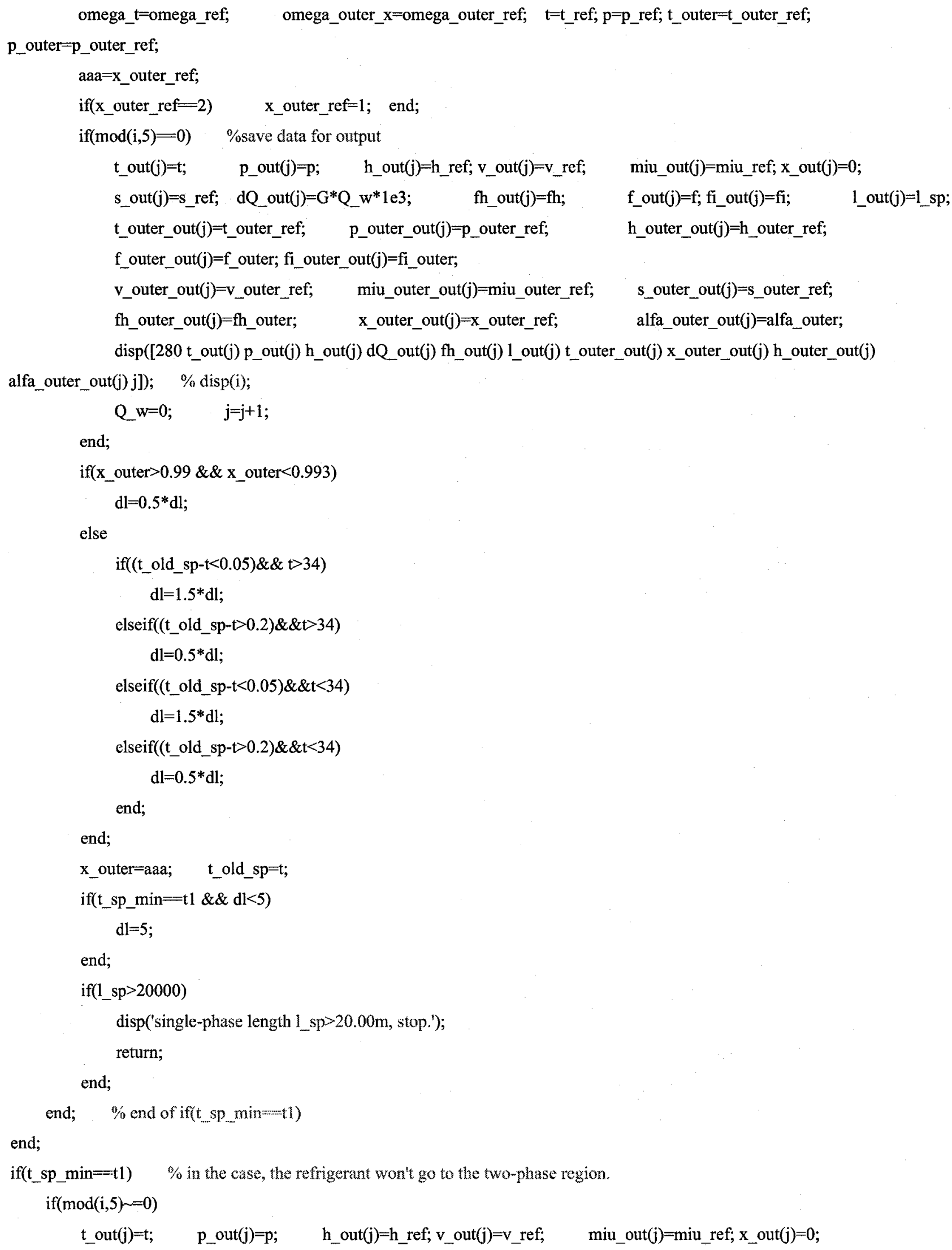


s_out $(j)=s \_r e f ; \quad$ dQ_out $(j)=G^{*} Q \_w^{*} 1 e 3 ; \quad$ fh_out $(j)=f h ; \quad f \_$out $(j)=f ;$

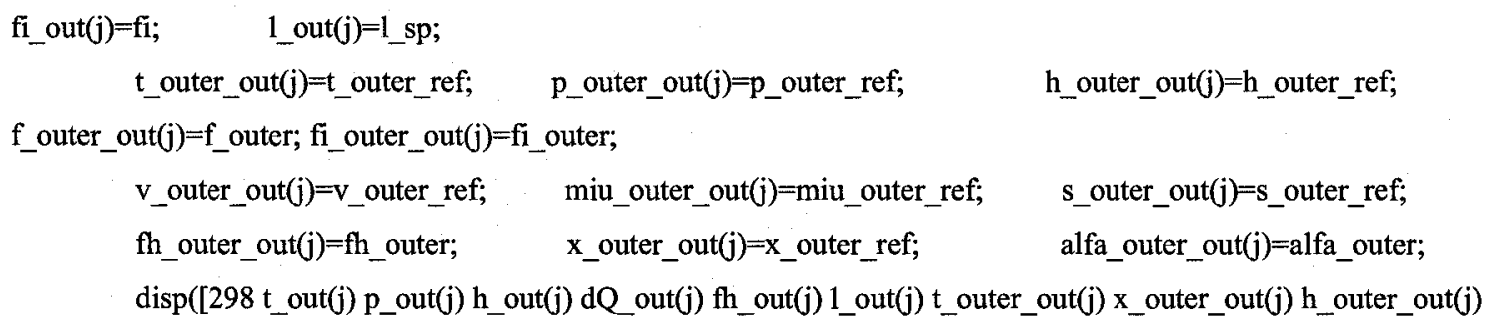


else

$\mathrm{x}=1 ; \quad \%$ if $\mathrm{CO} 2$ goes into tp area from super heated area

end;

delta_l=1e-3;1_sp_ref_old=1_sp_ref; \% to make sure $t$ starts at 31 Celsius exactly to prevent display error while calling property_calc

while $(\operatorname{abs}(\mathrm{dh})>0.01)$

$\mathrm{t}=\mathrm{a} \_\mathrm{t}(3,1)+\mathrm{a} \_\mathrm{t}(2,1)^{*}$ __sp_ref+a $\mathrm{t}(1,1) *(1 \text { sp_ref })^{\wedge} 2$;

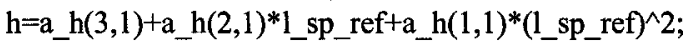

x_outer=a_x_outer(3,1)+a_x_outer(2,1)*1_sp_ref+a_x_outer(1,1)*(1_sp_ref) 2 ;

h_outer=a_h_outer $(3,1)+$ a_h_outer $(2,1)^{*}$ __sp_ref+a_h_outer $(1,1)^{*}(1 \text { _sp_ref })^{\wedge} 2$;

p_outer=a_p_outer $(3,1)+\mathbf{a} \_$_ _outer $(2,1)^{*} 1$ _sp_ref $+\mathbf{a} \_$_outer $(1,1)^{*}\left(1_{-} \text {sp_ref }\right)^{\wedge} 2$;

if $\left(1 \_s p \_r e f=1\right.$ _sp_ref_old $)$

$\mathrm{t}=31$;

end;

$[\mathrm{fh}, \mathrm{dQ}, \mathrm{f}, \mathrm{fi}]=\mathrm{fh} \_\mathrm{calc}\left(\mathrm{t}, \mathrm{p}, \mathrm{g}, \mathrm{l}_{-} \mathrm{sp}\right.$ ref-I_sp,t_outer,epsilon,di,do,x,alfa_outer,'sp'); $\%$ there is no difference for $\mathrm{x}=0$ or 1 for single-phase area

[h_ref,v_ref,miu_ref,lambda_ref,cp_ref,p_ref,s_ref]=property_calc $(\mathrm{t}, 0, \mathrm{x})$;

$\%$ NOTICE: if $\mathrm{CO} 2$ goes through liquid area, $\mathrm{x}=0$, else $\mathrm{x}=1$.

dh=h-h_ref;

$\mathrm{t} \_11=\mathrm{a} \_\mathrm{t}(3,1)+\mathrm{a} \_\mathrm{t}(2,1) *\left(1 \_s p \_r e f+\right.$ delta_ 1$)+\mathrm{a}_{-} \mathrm{t}(1,1)^{*}(1 \text { _sp_ref }+ \text { delta_ } 1)^{\wedge} 2$;

h_11_ref-property_calc(t_11,0,x);

dh_ref=h-h_11_ref;

ddh_dl=-(dh_ref-dh)/delta_l;

1_sp_ref=1_sp_ref+dh/ddh_dl;

end;

$\mathrm{p}=\mathrm{p}_{-}$ref;

dh_outer=1;

t_outer_ref=t_outer; p_outer_ref=p_outer; dt_outer $=1 \mathrm{e}-3$;

while $($ abs $(\mathrm{dh}$ _outer $)>0.01)$

[h_outer_ref,v_outer,miu_outer,lambda_outer,cp_outer,p_outer,s_outer]=property_calc(t_outer,p_outer_ref,x_outer);

[fh_outer,fouter,fi_outer,alfa_outer]=alfa_outer_calc(t_outer_ref,p_outer,g_outer,epsilon,dl,dQ,do,di_outer,x_outer,'alfa');

dh_outer=h_outer-h_outer_ref;

h_11_ref-property_calc(t_outer+dt_outer,p_outer_ref,x_outer);

dh_outer_ref $=$ h_outer-h_11_ref;

$\mathrm{ddh}$ dt $=-$-(dh_outer_ref-dh_outer)/dt_outer;

t_outer=t_outer+dh_outer/ddh_dt;

end;

x_outer_ref=x_outer; $\quad$ if $\left(x_{-}\right.$outer $\left.>1\right) x_{-}$outer $=1$; $\quad$ end;

1_sp=1_sp_ref; disp([385 t p h t_outer p_outer $x_{-}$outer h_outer 1_sp]);

$\%$ to make sure $t$ out is sorted in descending order, store datum in (j+interpoint) cell.

t_out $(j+$ interpoint $)=t$;

p_out(j+interpoint $)=p$;

h_out(j+interpoint) $=h \_$ref; 


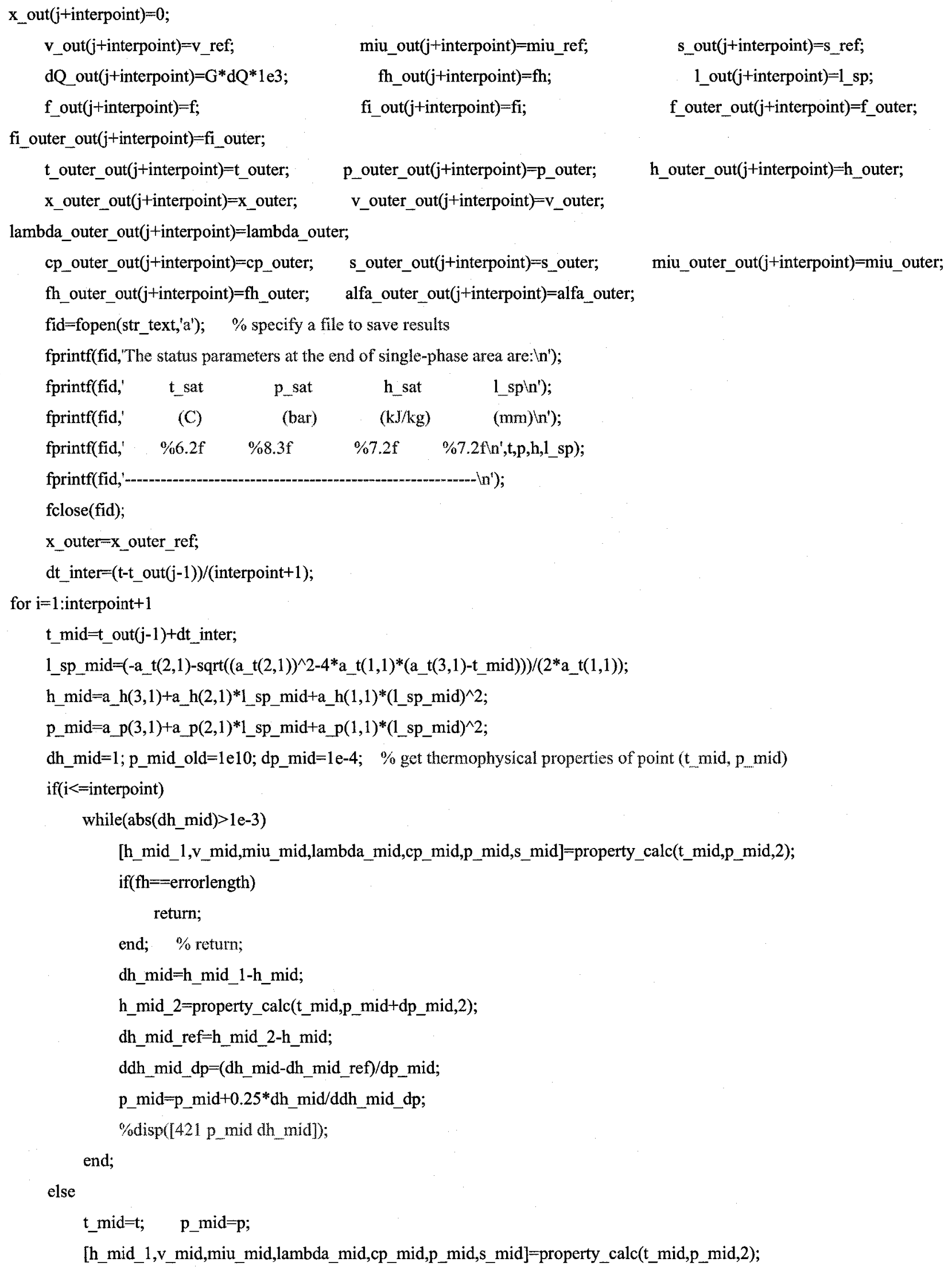


end;

p_mid=a_p $(3,1)+a \_p(2,1)^{*}$ __sp_mid+a_p $(1,1)^{*}\left(1 \_s p \_m i d\right)^{\wedge} 2 ; \%$ get back the value of $p \_$mid to make the data changing smooth

dl_sp=1_sp_mid-1_out(j-1); \% tube length from $t \_$out $(j-1)$ celsius degree to $t$ mid

[fh,dQ,f,fi]=fh_calc(t_mid,p_mid,g,dl_sp,t_outer,epsilon,di,do,1,alfa_outer,'sp');

h_outer_mid=a_h_outer $(3,1)+a \_h \_o u t e r(2,1)^{*}$ __sp_mid+a_h_outer $(1,1)^{*}(1 \text { _sp_mid })^{\wedge} 2$;

x_outer_mid=a_x_outer $(3,1)+\mathrm{a} \_$___outer $(2,1) * 1$ _sp_mid+a_x_outer $(1,1)^{*}(1 \text { _sp_mid })^{\wedge} 2$;

p_outer_mid=a_p_outer $(3,1)+a \_p \_o u t e r(2,1)^{*}$ __sp_mid+a_p_outer $(1,1)^{*}\left(1 \_s p \_m i d\right) \wedge$;

dh_outer $=1 ; \mathbf{t}$ _outer_mid_ref $-\mathrm{t}$ _outer_ref; $\mathrm{dt}$ _outer $=1 \mathrm{e}-3 ;$ con_ $\mathrm{i}=0 ; \quad \% \mathrm{con}$ _.. is a convergence factor

while(abs(dh_outer) $>0.01)$

[h_outer_mid_ref,v_outer_mid,miu_outer_mid,lambda_outer_mid,cp_outer_mid,p_outer_mid,s_outer_mid]=property_calc(t_outer_ mid_ref,p_outer_mid,x_outer_mid);

[fh_outer_mid,f_outer_mid,

fi_outer_mid,alfa_outer_mid]=alfa_outer_calc(t_outer_mid_ref,p_outer_mid,g_outer,epsilon,dl,dQ,do,di_outer,x_outer_mid,'alfa'); dh_outer=h_outer_mid-h_outer_mid_ref;

h_11_ref=property_calc(t_outer_mid_ref + dt_outer,p_outer_mid,x_outer_mid);

dh_outer_ref $=h \_o u t e r-h \_11 \_r e f ;$

$\mathrm{ddh} \_\mathrm{dt}=-$ (dh_outer_ref-dh_outer)/dt_outer;

if $\left(x \_\right.$outer_mid $\left.>1\right)$

ddh_dt $=0.1^{*}\left(\mathrm{dh} \_\right.$outer_ref-dh_outer $) / \mathrm{dt} \_$outer;

end;

t_outer_mid_ref-t_outer_mid_ref $+\mathrm{dh} \_$outer/ddh_dt;

con_i $\mathrm{i}=$ con_ $\mathrm{i}+1$;

end; $\quad$ __outer_ref $=$ t_outer_mid_ref;

t_outer=t_outer_mid_ref; alfa_outer=alfa_outer_mid;

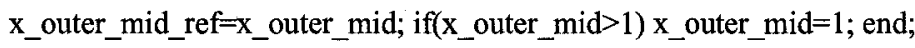

t_out $(\mathrm{j})=\mathrm{t} \_$mid; $\quad$ p_out $(\mathrm{j})=$ p_mid; $_{-} \quad$ h_out $(\mathrm{j})=\mathrm{h} \_$mid;

v_out $(j)=v \_$mid; $\quad$ miu_out $(j)=$ miu_mid; $\quad$ s_out $(j)=s \_m i d ; \quad$ x_out $(j)=0$;

dQ_out $(j)=G^{*} \operatorname{abs}(d Q)^{*} 1 e 3 ; \quad$ fh_out $(j)=\operatorname{abs}(f h) ; \quad$ 1_out $(j)=1 \_s p \_m i d ; \quad f \_o u t(j)=f ;$

fi_out $(\mathrm{j})=\mathrm{fi}$;

t_outer_out $(j)=$ __outer; $\quad$ p_outer_out $(j)=$ p_outer_mid; $\quad$ h_outer_out $(j)=h \_o u t e r \_m i d ;$

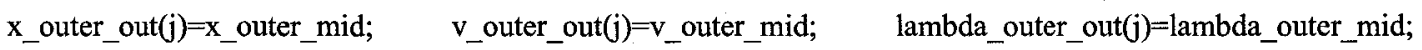

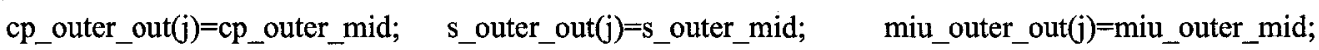

fh_outer_out $(j)=f h \_o u t e r \_m i d ; \quad f \_o u t e r \_o u t(j)=f \_o u t e r \_m i d ; \quad f i \_o u t e r \_o u t(j)=f i \_o u t e r \_m i d ;$

alfa_outer_out $(\mathrm{j})=$ alfa_outer_mid;

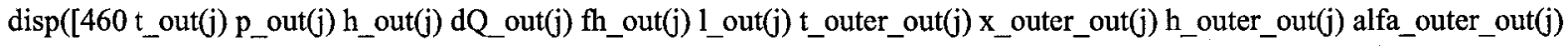

j]);

$\mathrm{j}=\mathrm{j}+1$; $\quad$ x_outer_mid=x_outer_mid_ref;

end; $\quad$ p_out $(\mathrm{j}-1)=\mathrm{p}$; beep on; beep; pause(0.5); beep off;

end; \% corresponding to if(t_sp_min $-\mathrm{t}=\mathrm{t}$ )

end; 


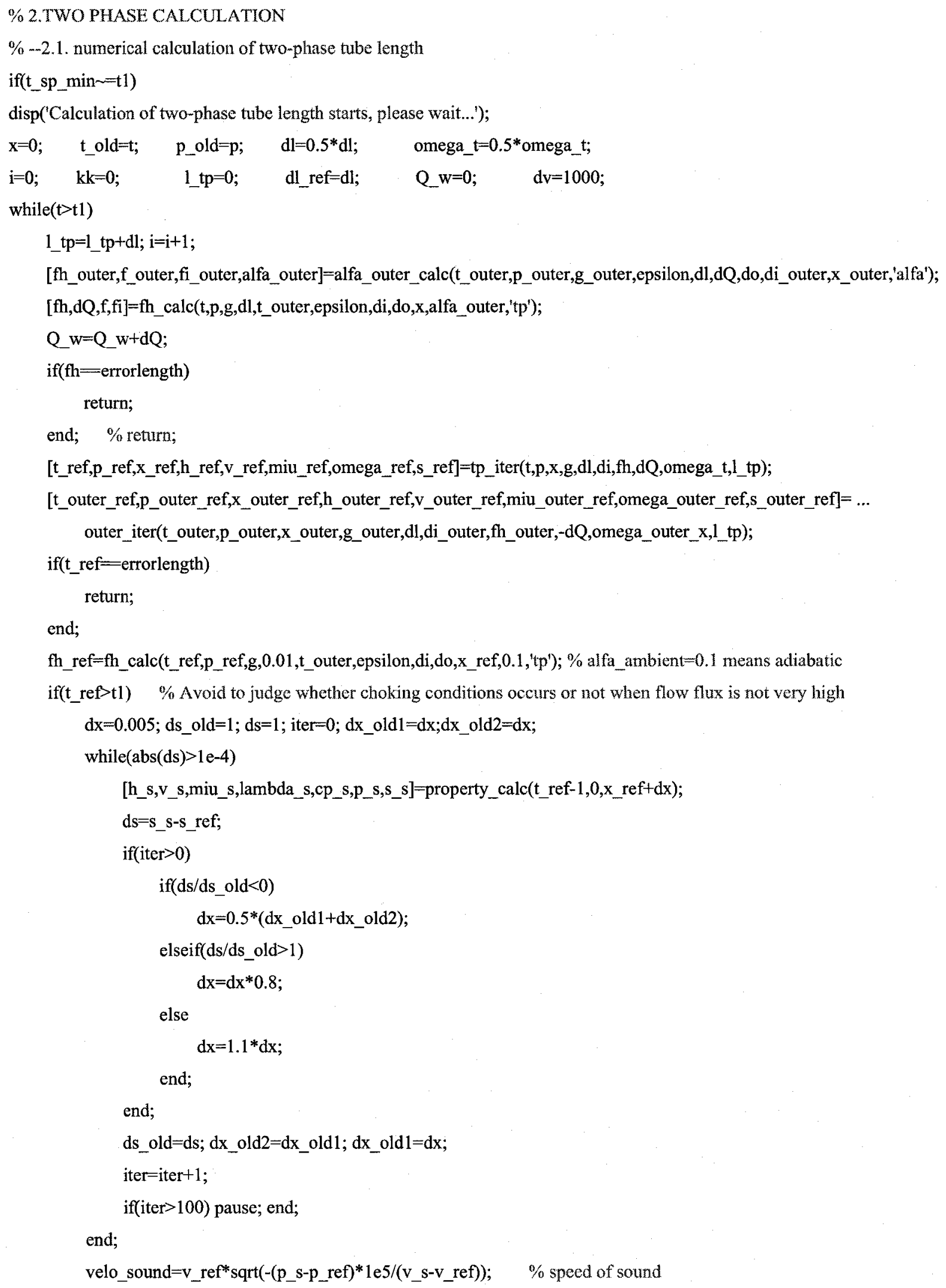




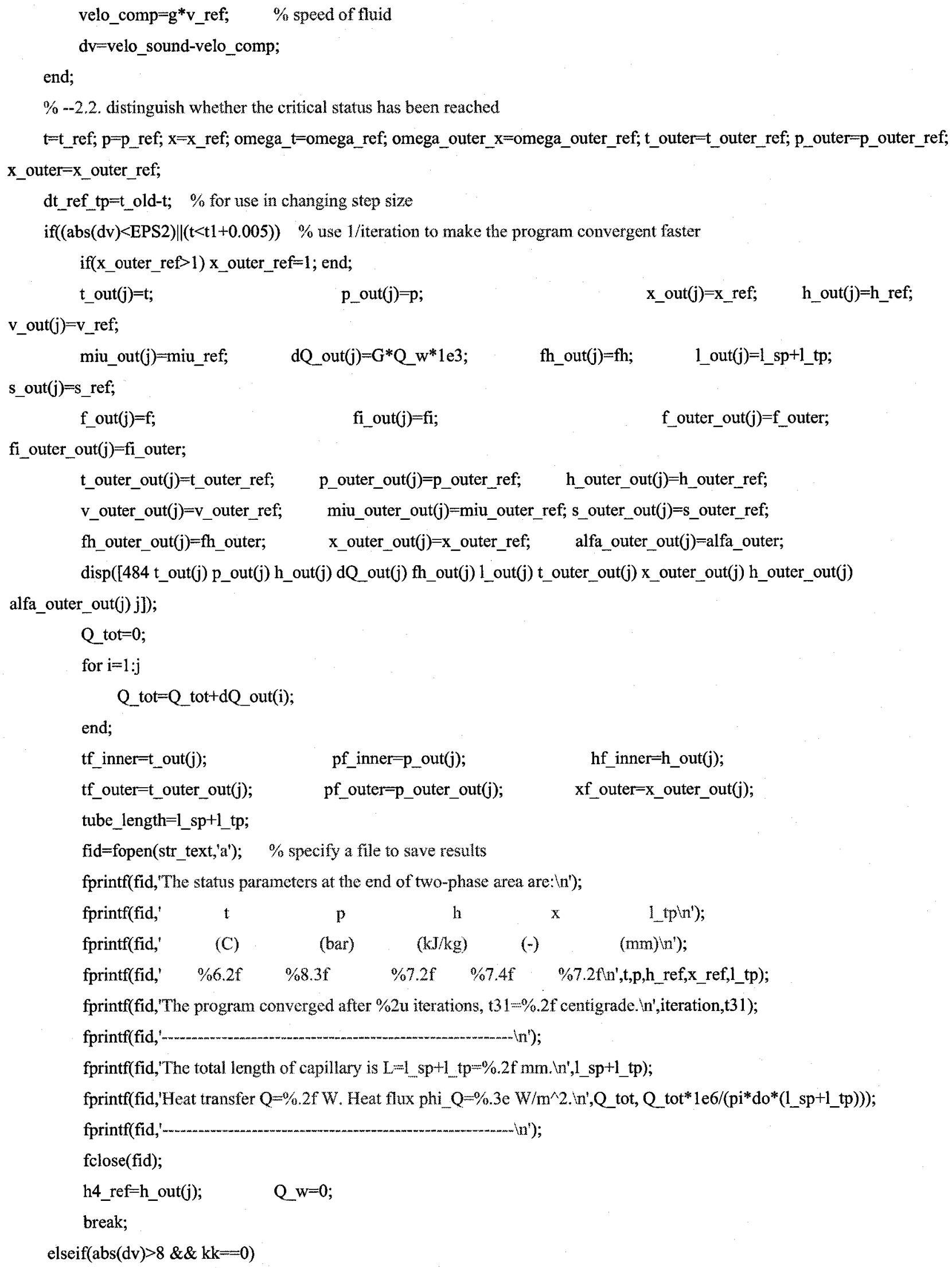




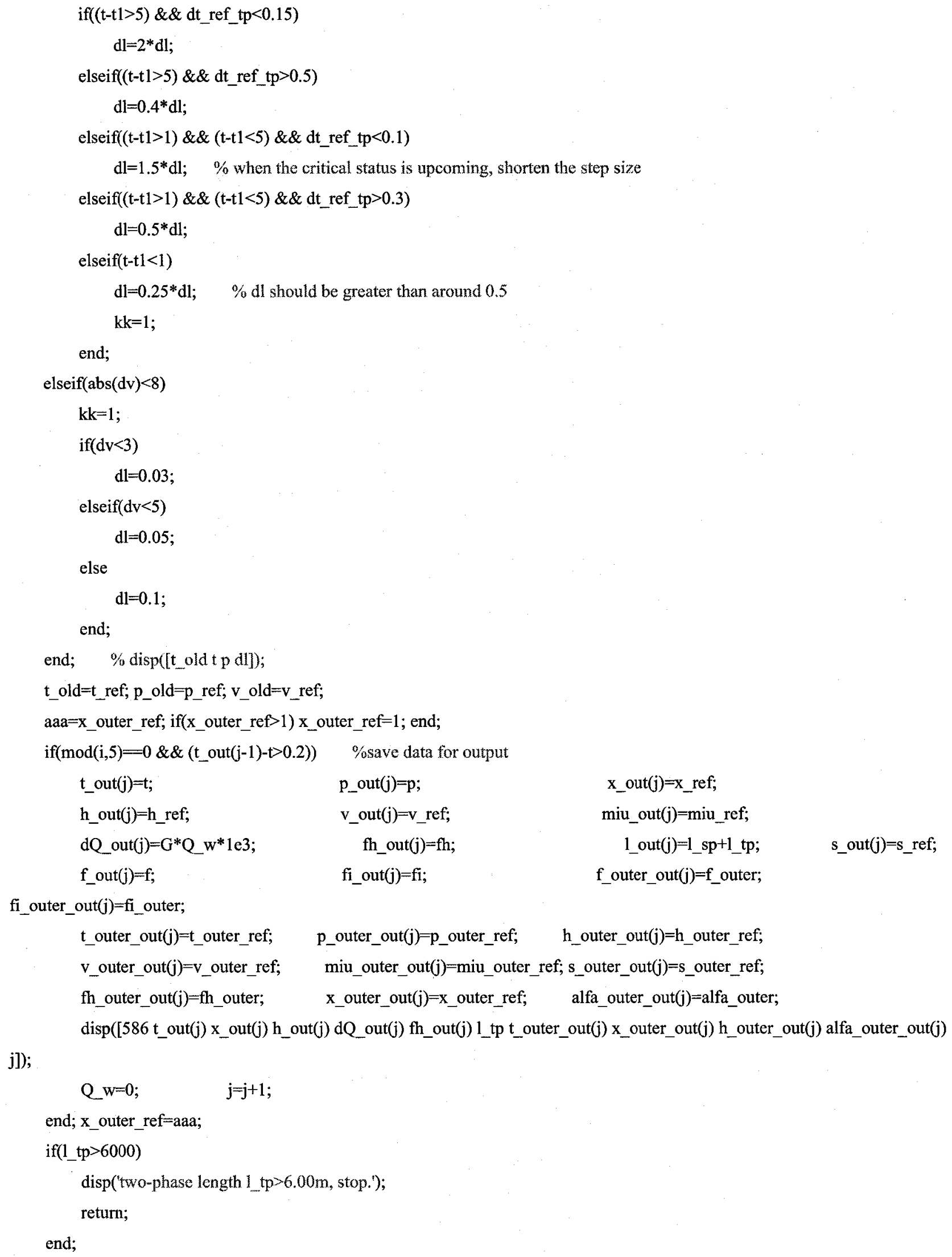




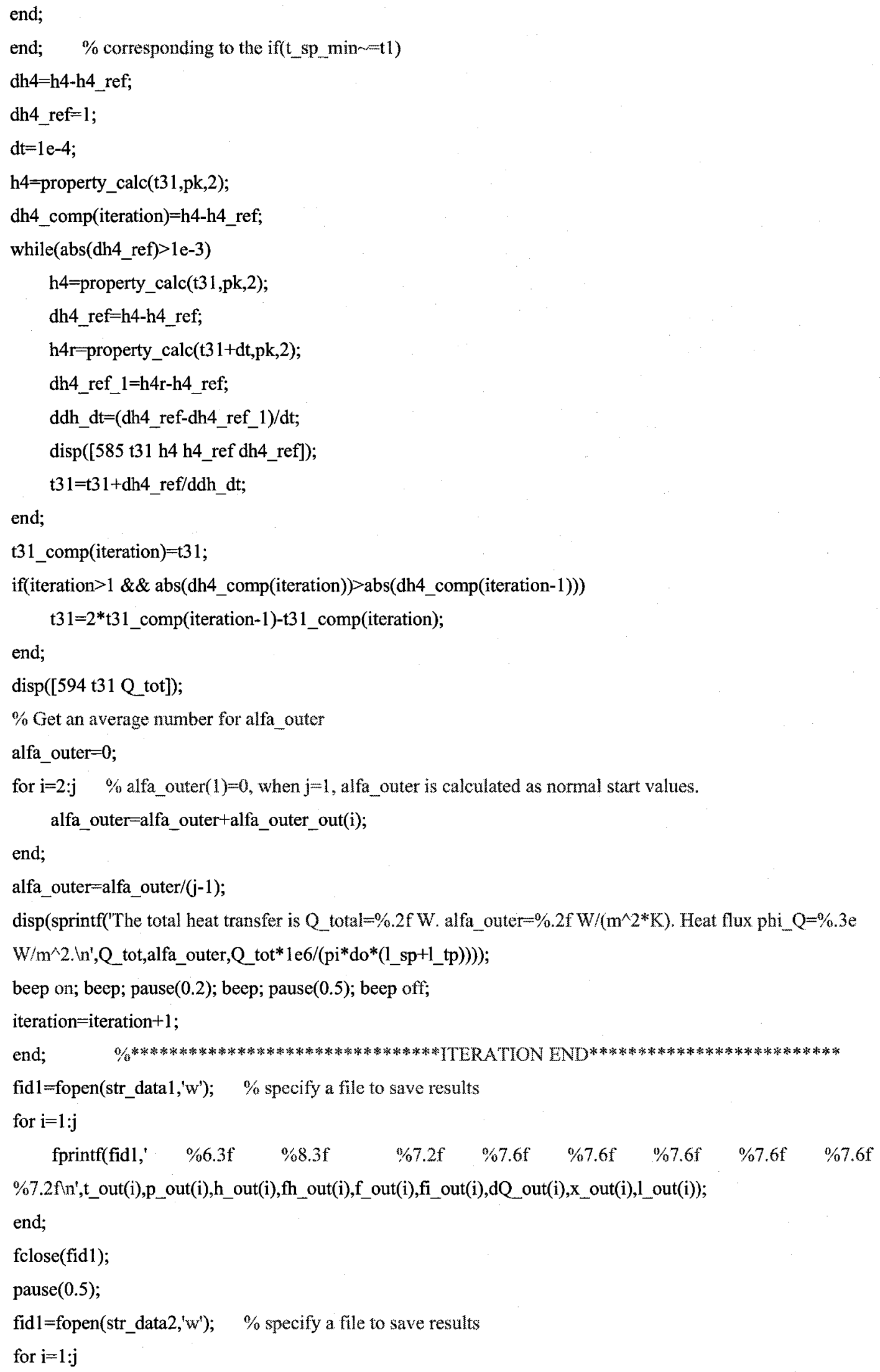




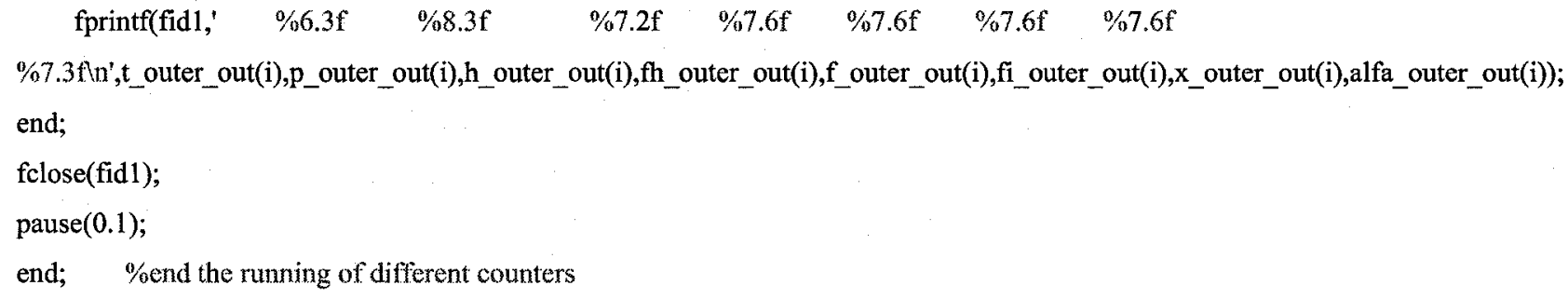

\section{Appendix D Program to calculate the optimum high pressure of a $\mathrm{CO}_{2}$-based}

\section{systems}

\section{D-1 Main code}

This program correlates the optimum high pressure with the ambient temperature, the effectiveness of the internal heat exchanger, and the suction-line inlet quality.

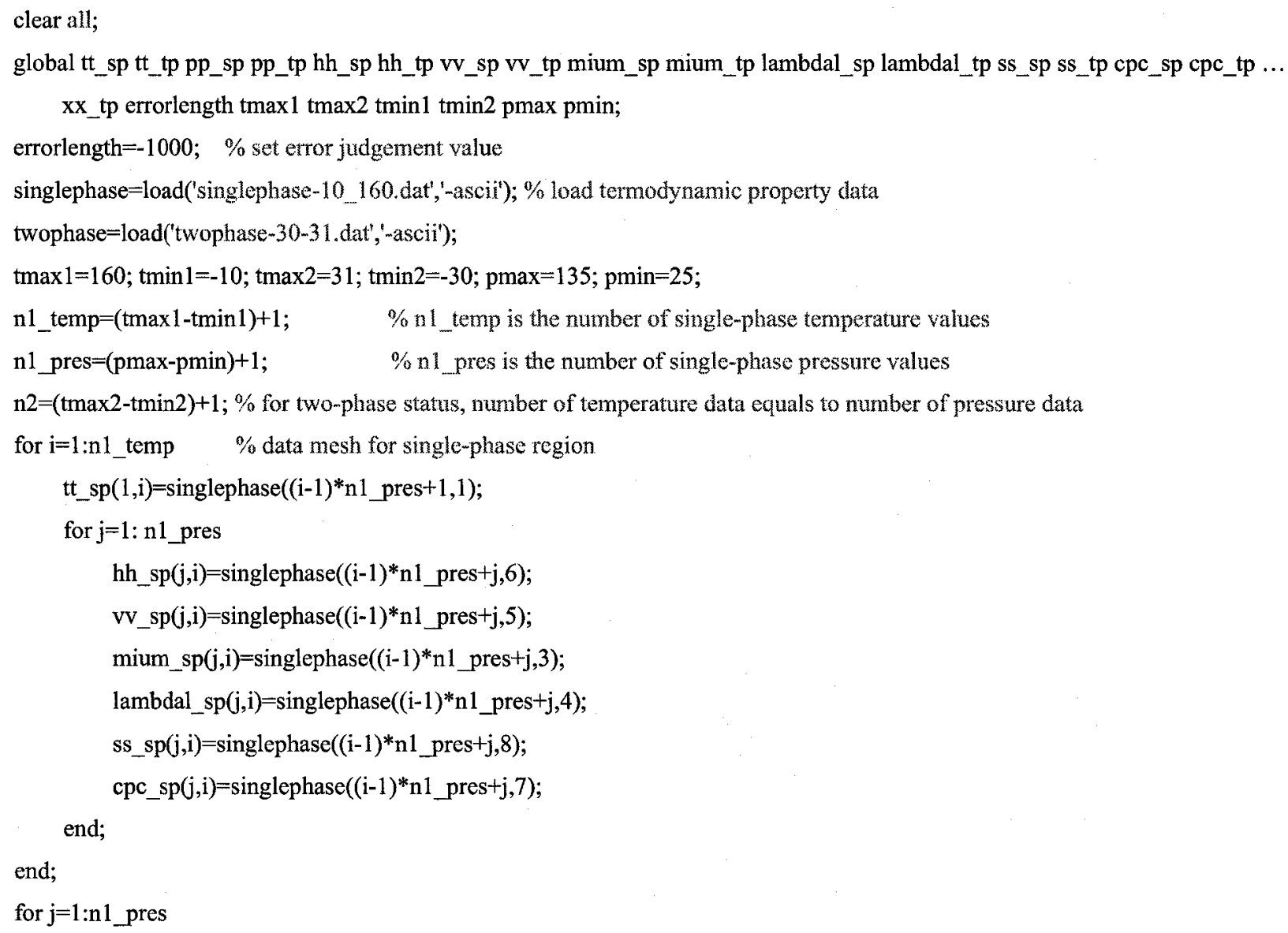


pp_sp $(1, j)=$ singlephase $\left((\mathrm{i}-1)^{*} \mathrm{n} 1 \_\right.$pres $\left.+\mathrm{j}, 2\right)$;

end;

for $\mathrm{i}=1: \mathrm{n} 2 \quad \%$ data mesh for two-phase region

tt_tp $(1, \mathbf{i})=$ twophase $(\mathrm{i}, 1)$;

for $\mathrm{j}=1: 11$

$x x \_r e f=(j-1) * 0.1$;

hh_tp $(\mathrm{j}, \mathrm{i})=\mathrm{xx}$ _ref*twophase $(\mathrm{i}, 10)+\left(1-\mathrm{xx} \_\right.$ref $) *$ twophase $(\mathrm{i}, 9)$;

vv_tp $(j, i)=x x \_r e f * t w o p h a s e(i, 8)+\left(1-x x \_r e f\right)^{*}\left(\right.$ twophase $\left.(i, 7)^{*} l e-3\right)$;

mium_tp $(\mathrm{j}, \mathrm{i})=1 /\left(\mathrm{xx} \_\right.$ref/twophase $(\mathrm{i}, 4)+(1-\mathrm{xx}$ _ref $) /$ twophase $\left.(\mathrm{i}, 3)\right)$;

lambdal_tp $(\mathrm{j}, \mathrm{i})=1 /\left(\mathrm{xx} \_\right.$ref $/$twophase $(\mathrm{i}, 6)+\left(1-\mathrm{xx} \_\right.$ref $) /$twophase $\left.(\mathrm{i}, 5)\right)$;

cpc_tp $(\mathrm{j}, \mathrm{i})=\mathrm{xx}$ _ref*twophase $(\mathrm{i}, 12)+\left(1-\mathrm{xx} \_ \text {ref }\right)^{*}$ twophase $(\mathrm{i}, 11)$;

ss_tp $(\mathrm{j}, \mathrm{i})=\mathrm{xx}$ _ref*twophase $(\mathrm{i}, 15)+(1-\mathrm{xx}$ _ref $) *$ twophase $(\mathrm{i}, 14)$;

$p p_{-} \operatorname{tp}(\mathrm{j}, \mathrm{i})=$ twophase $(\mathrm{i}, 2)$

end;

end;

for $\mathrm{j}=1: 11 \%$ to save calculating time, mesh $\mathrm{xx}$ separately

$x x_{-} \operatorname{tp}(1, j)=(j-1) * 0.1$

end;

$\mathrm{t} 1=[-10,0,10]^{\prime} ; \quad \% \mathrm{t}$ is the evaporating temperature (Celsius degree)

$\mathrm{x}=[0.7]$; $\quad \% \mathrm{x}$ is the outlet quality of the evaporator $(-)$

t_amb=[35:5:55]; $\quad \% \mathrm{t}$ anb is the ambient temperature at the outlet of gas cooler (Celsius degree)

$\mathrm{p} 3=[80: 0.5: 135]$; $\%$ high pressure (bar)

effi_IHX $=[1] ;$ \% efficiency of the AHE

for $\mathrm{ii}=1$ :size(t1)

for $\mathrm{jj}=1$ :size( $(x)$

h4sat=property_calc(t1(ii), 0,0$)$;

[h1_pref,s1_pref,pl_pref]-property_calc(t1(ii),0,1); \% referred enthalpy, use to judge whether 1_prime is in two-phase region or not.

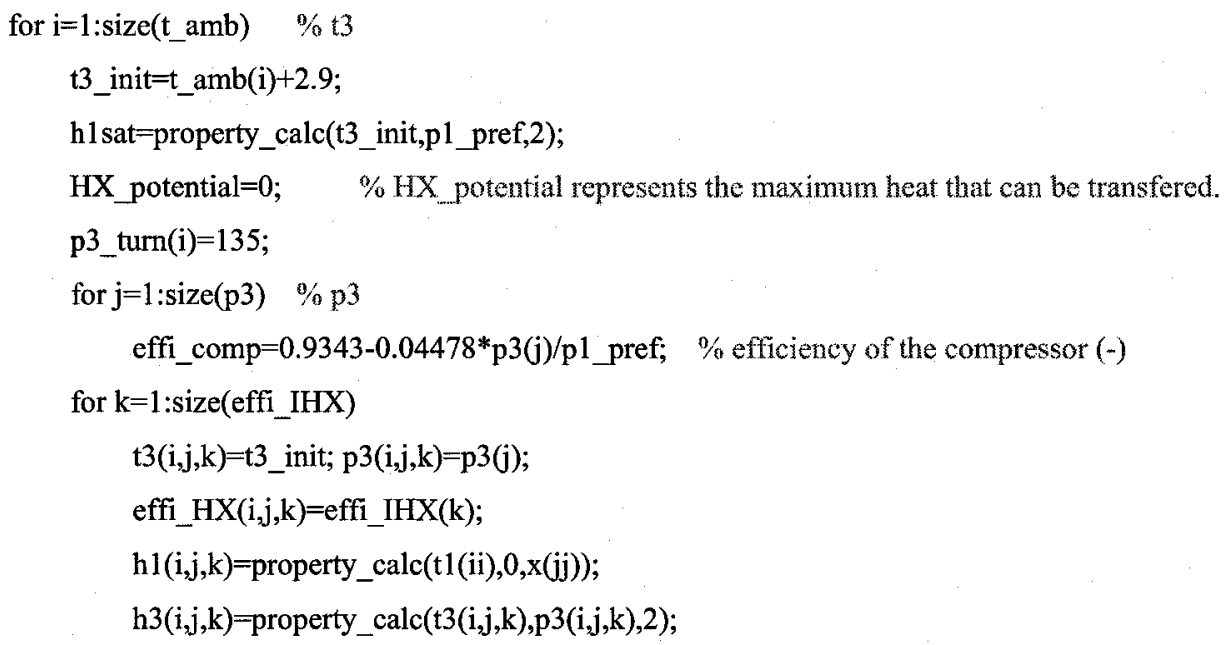




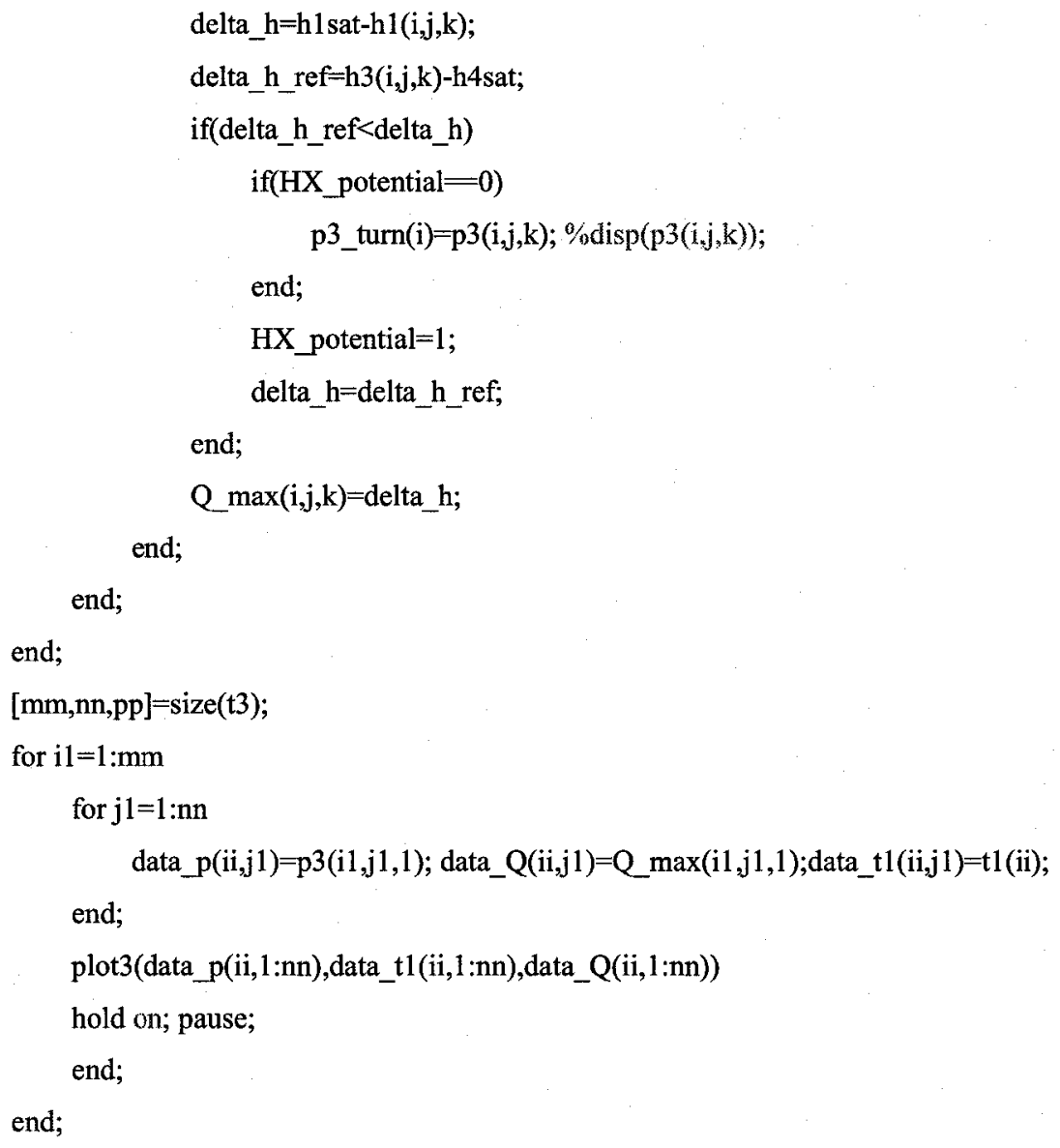

